\title{
A Practical Theory of Micro-Solar Power Sensor Networks
}

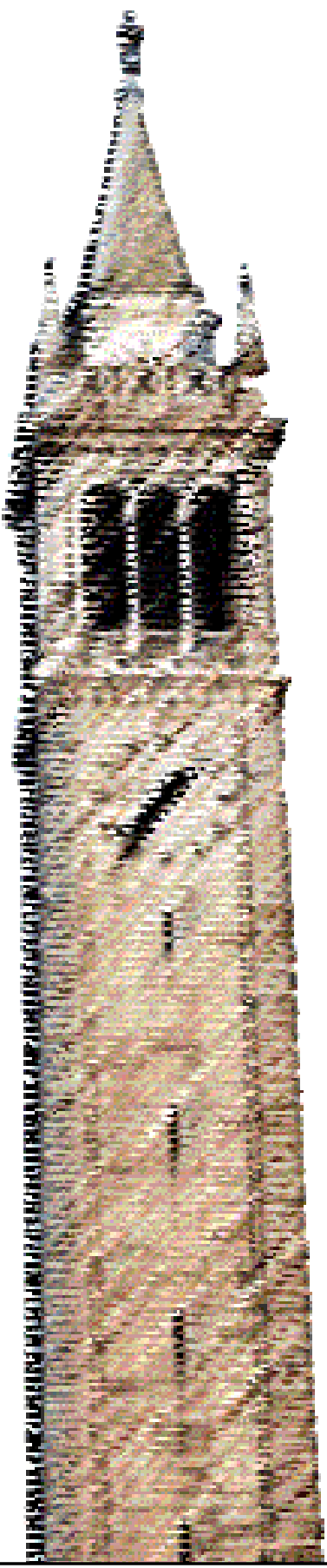

Jaein Jeong

Electrical Engineering and Computer Sciences University of California at Berkeley

Technical Report No. UCB/EECS-2009-49

http://www.eecs.berkeley.edu/Pubs/TechRpts/2009/EECS-2009-49.html

April 20, 2009 
Copyright 2009, by the author(s).

All rights reserved.

Permission to make digital or hard copies of all or part of this work for personal or classroom use is granted without fee provided that copies are not made or distributed for profit or commercial advantage and that copies bear this notice and the full citation on the first page. To copy otherwise, to republish, to post on servers or to redistribute to lists, requires prior specific permission. 


\title{
A Practical Theory of Micro-Solar Power Sensor Networks
}

\author{
by \\ Jaein Jeong \\ B.S.E. (Seoul National University) 1997 \\ M.S. (University of California, Berkeley) 2004 \\ A dissertation submitted in partial satisfaction of the \\ requirements for the degree of \\ Doctor of Philosophy \\ in \\ Computer Science \\ in the \\ GRADUATE DIVISION \\ of the \\ UNIVERSITY OF CALIFORNIA, BERKELEY
}

Committee in charge:

Professor David E. Culler, Chair

Professor Seth R. Sanders

Professor Paul K. Wright

Spring 2009 
The dissertation of Jaein Jeong is approved:

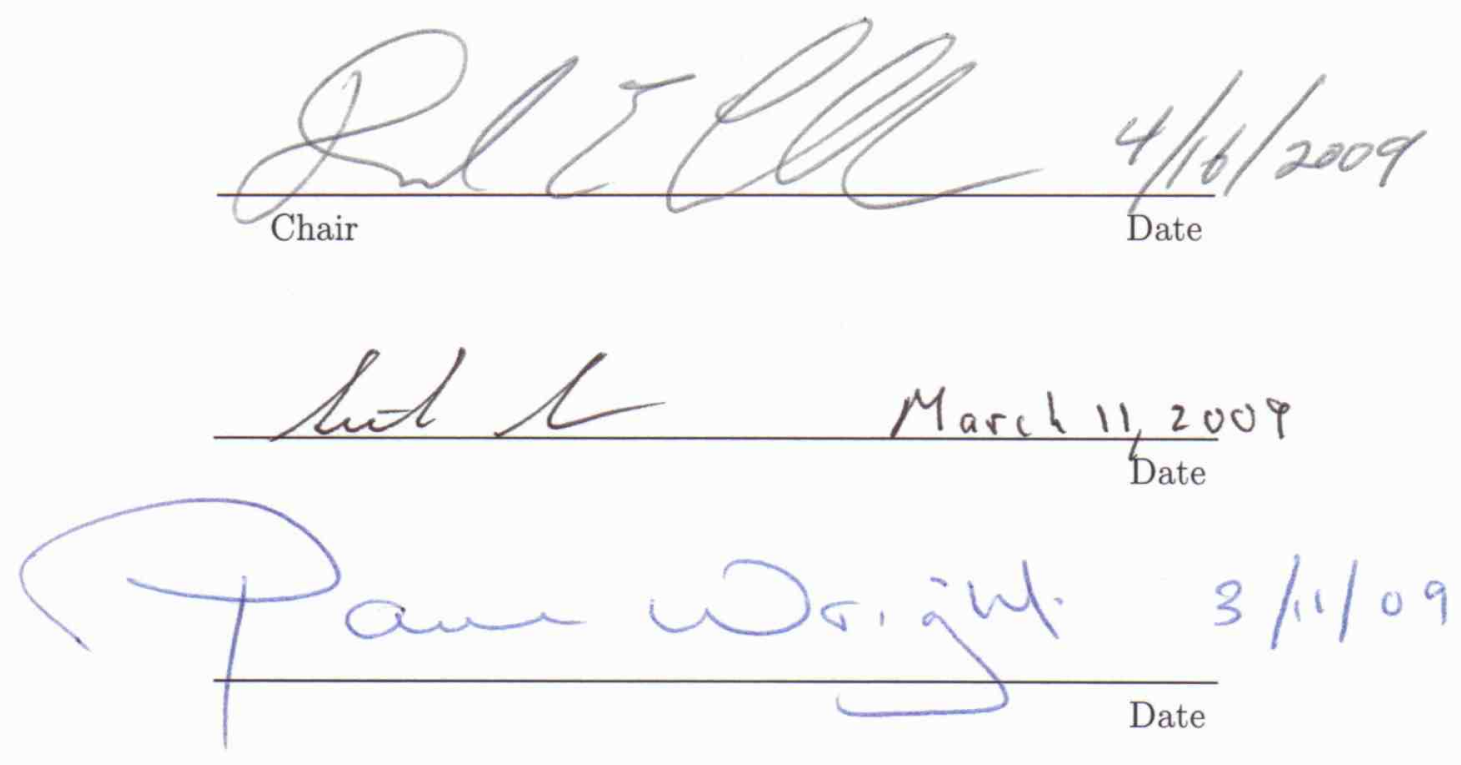

University of California, Berkeley

Spring 2009 
A Practical Theory of Micro-Solar Power Sensor Networks

Copyright 2009

by

Jaein Jeong 


\begin{abstract}
A Practical Theory of Micro-Solar Power Sensor Networks

by

Jaein Jeong

Doctor of Philosophy in Computer Science

University of California, Berkeley

Professor David E. Culler, Chair
\end{abstract}

Autonomous long-term monitoring is an essential capability of wireless sensor networks, and solar energy is a viable means of enabling this capability due to its high power density and wide availability. However, micro-solar power system design is challenging because it must address long-term system behavior under highly variable solar energy and consider a large space of design options. Several micro-solar power systems have been designed and implemented, validating particular points in the whole design space.

In this dissertation we develop a practical theory of micro-solar power systems that is materialized in a simulation suite that models component and system behavior over a long time-scale and in an external environment that depends on time, location, weather and local conditions. This simulation provides sufficient accuracy to guide specific design choices in a large design space. This design tool is very different from the many "macro-solar" calculators, which model typical behavior of kilowatt systems in the best conditions, rather than detailed behavior of milliwatt systems in the worst conditions. We provide a general architecture of micro-solar power systems, comprising key components and interconnections among the components, and formalize each component in an analytical or empirical model of its behavior. We incorporate these component models and their interconnections in the simulation suite.

Our discrete time-event simulation models the daily behavior and the long-term behavior by iteratively evaluating the state of the system in the context of its solar 
environment and internal loads. To model the variability of solar energy, it provides three solar radiation models depending on the degree of information available: an astronomical model for ideal conditions, an obstructed astronomical model for estimating solar radiation under the presence of shadows and obstructions, and a long-term measurement model for estimating solar radiation under weather variation. Our simulation suite is validated with a concrete design: the HydroWatch node which is a well-engineered climate monitoring node and network with a flexible power subsystem. A HydroWatch node is able to support various specific design points and provides visibility into solar performance in a real application setting.

With our simulation suite, micro-solar power systems can be designed in a systematic fashion. Putting the model and empirical vehicle together, the design choices in each component of a micro-solar power system are studied to reach a deployable candidate. The deployment is evaluated by analyzing the effects of different solar profiles across the network. The analysis from the deployment can be used to refine the next system-design iteration.

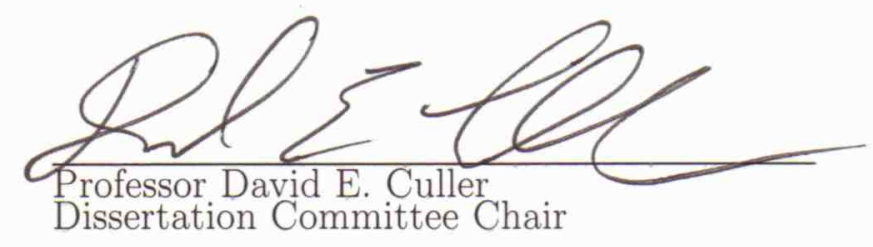




\section{Contents}

List of Figures $\quad$ v

List of Tables $\quad$ xi

\begin{tabular}{lll}
\hline & Introduction & 1
\end{tabular}

1.1 Motivation . . . . . . . . . . . . . . . . . . . . . 1

1.2 Problem Statement . . . . . . . . . . . . . . . . . . . . . . . . . . . . . . . . . . . . . . . . . . .

1.3 Contributions . . . . . . . . . . . . . . . . . . . 5

1.4 Roadmap . . . . . . . . . . . . . . . . . . . . . 5

$\begin{array}{lll}2 & \text { Background } & 7\end{array}$

$2.1 \quad$ Wireless Sensor Networks and Energy Sources . . . . . . . . . . . . . 7

2.1.1 Non-rechargeable Battery-Powered Nodes. . . . . . . . . . . . 8

2.1 .2 Wire-powered Nodes . . . . . . . . . . . . . . . . . . 8

2.1 .3 Renewable Energy Sources . . . . . . . . . . . . . . . . . . . . 10

2.2 Prior Work on Micro-solar Power Systems . . . . . . . . . . . . . . . 14

2.2.1 Micro-solar Power System Platforms . . . . . . . . . . . . . . 15

2.2 .2 Micro-solar Power System Models . . . . . . . . . . . . . . . . 18

2.2 .3 Relation to Macro-Solar Power Systems . . . . . . . . . . . . . 23

2.3 Refined Problem Statement . . . . . . . . . . . . . . . . . . . . 24

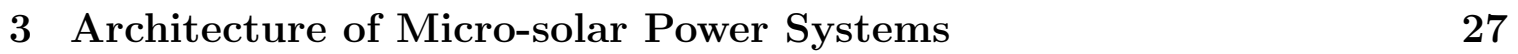

3.1 Overall Architecture of Micro-solar Power Systems . . . . . . . . . . . 27

3.1.1 External Environment . . . . . . . . . . . . . . . . . . . 29

3.1 .2 Solar Collector . . . . . . . . . . . . . . . . . . . . 31

3.1 .3 Input-power Conditioning . . . . . . . . . . . . . . . . . . . . 32

3.1 .4 Energy Storage . . . . . . . . . . . . . . . . . . . . 35

3.1 .5 Output Power Conditioning . . . . . . . . . . . . . . . . . . . . . . 37

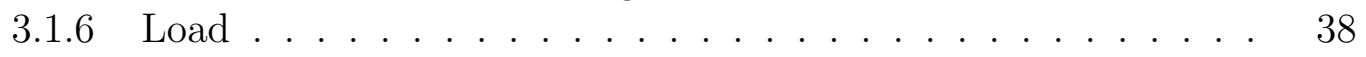

3.2 Energy Efficiency and Daily Power Cycle . . . . . . . . . . . . . . . . 39

$3.2 .1 \quad$ Energy Efficiency and Output Power . . . . . . . . . . . . . . . 39

3.2 .2 Energy Flow and Daily Power Cycle . . . . . . . . . . . . . . 42 
3.3 Validating the Energy Flow Relationship . . . . . . . . . . . . . . . . 43

3.3.1 HydroWatch Node with an Input Regulator . . . . . . . . . . 45

3.3.2 HydroWatch Node without an Input Regulator . . . . . . . . 46

3.3.3 System Efficiency . . . . . . . . . . . . . . . . . . . . . 48

3.4 Assessment of Prior Work . . . . . . . . . . . . . . . . . . . . 50

3.4 .1 Experimental Set-up . . . . . . . . . . . . . . . . . 50

3.4.2 $\quad$ Solar-collector Operation . . . . . . . . . . . . . . . . . . 54

3.4 .3 Energy Flow and Energy Efficiency . . . . . . . . . . . . . . . 57

3.5 Summary of Micro-solar Power System Architecture . . . . . . . . . . 62

\begin{tabular}{|lll}
4 & Design of Micro-Solar Power System Simulator & 65
\end{tabular}

4.1 Overall Architecture and Principles . . . . . . . . . . . . . . . 66

4.1 .1 Modularity . . . . . . . . . . . . . . . . . 66

4.1 .2 Time-Event Based Simulator . . . . . . . . . . . . . . . . . . 66

4.1.3 Defining a Component Using User-provided Data . . . . . . . 69

4.1 .4 Wiring Components . . . . . . . . . . . . . . . 71

4.2 Modeling Solar Radiation . . . . . . . . . . . . . . . . . . . . . . . . . . . . . . . . . .

4.2 .1 Astronomical Model . . . . . . . . . . . . . . . . . . . . . . 71

4.2 .2 Obstructed Astronomical Model . . . . . . . . . . . . . . . . . 78

4.3 Modeling Solar Panel . . . . . . . . . . . . . . . . . . . . 83

4.3 .1 Modeling IV Characteristic . . . . . . . . . . . . . . . . . . . 83

4.3.2 Modeling the Operating Point . . . . . . . . . . . . . . . . . . 86

4.4 Modeling Energy Storage . . . . . . . . . . . . . . . . . . 86

4.4 .1 Modeling NiMH Rechargeable Battery . . . . . . . . . . . . . 86

4.4 .2 Modeling Supercapacitors . . . . . . . . . . . . . . . . . . . . . . 88

4.5 Modeling Output Regulator . . . . . . . . . . . . . . . . . . . . . 90

4.5 .1 Modeling Operating Range and Output Voltage . . . . . . . . 91

4.5 .2 Modeling Power Efficiency . . . . . . . . . . . . . . . . . . 92

4.6 Modeling the Input Regulator . . . . . . . . . . . . . . . . . . . . . . 93

$4.6 .1 \quad$ Micro-Solar Power System without an Input Regulator . . . . 93

4.6.2 Micro-Solar Power System with an Input Regulator . . . . . . 94

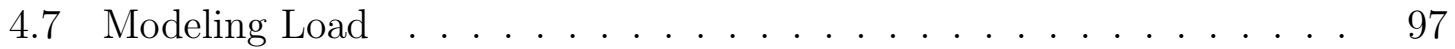

$4.7 .1 \quad$ Average Current-Based Model . . . . . . . . . . . . . . . . . . . . . 97

$4.7 .2 \quad$ Application Code Based Model . . . . . . . . . . . . . . . . 98

4.8 Composition . . . . . . . . . . . . . . . . . . . . 103

4.8 .1 Simulation under Constant Radiation . . . . . . . . . . . . . . 103

4.8 .2 Simulation under Daily Solar Radiation . . . . . . . . . . . . . 108

4.9 Summary of Micro-Solar Power System Simulator . . . . . . . . . . . 118 
5 Validating the Simulator Design Using a Reference Implementation 122

5.1 Node and Network Design of Reference Implementation . . . . . . . . 122

5.1 .1 Network Architecture . . . . . . . . . . . . . . . . . . . . 123

5.1 .2 Engineering the Node . . . . . . . . . . . . . . . . . . . . . . . 124

5.1 .3 Micro-Solar Power Subsystem of HydroWatch Node . . . . . . 127

5.1 .4 Modeling the HydroWatch node for the Simulator . . . . . . . 134

5.2 Evaluating the Reference Implementation . . . . . . . . . . . . . . . . 135

$5.2 .1 \quad$ A Sensor Network in an Urban Neighborhood . . . . . . . . . 135

5.2 .2 A Sensor Network in a Forest Watershed . . . . . . . . . . . . 141

5.3 Summary . . . . . . . . . . . . . . . . . . . . . . 150

$6 \quad$ Predicting the Long-term Behavior of a Micro-Solar Power System 153

6.1 Accounting for the Weather Effect. . . . . . . . . . . . . . . 153

6.1 .1 Experimental Set-up . . . . . . . . . . . . . . . . 153

6.1.2 Results. . . . . . . . . . . . . . . . . . . . 155

6.2 Developing Weather Effect Model . . . . . . . . . . . . . . . . . . . . 160

6.2 .1 Atmospheric Turbidity . . . . . . . . . . . . . . . . . . . . . 160

6.2 .2 Horizontal Visibility and Cloud Condition . . . . . . . . . . . 163

6.3 Evaluating Weather Effect Model . . . . . . . . . . . . . . . . . 165

6.3 .1 Defining Solar Radiation Estimators . . . . . . . . . . . . . . 165

6.3 .2 Calibrating Solar Radiation Estimators . . . . . . . . . . . . . 172

6.3.3 Predicting Solar Radiation using the History of a Weather Ef-

6.3 .4 Summary . . . . . . . . . . . . . . . . . . . 178

7 Extending the Simulator Beyond the Reference Design 184

7.1 Extending the Simulator for a Micro-Solar Power System with MultiLevel Energy Storage . . . . . . . . . . . . . . . . . . . . . . 184

7.1.1 A Simulation Model of Micro-Solar Power System with TwoLevel Energy Storage . . . . . . . . . . . . . . . . . . . 185

7.1.2 Simulation of Micro-Solar Power System with Two-Level Energy Storage under Constant Radiation . . . . . . . . . . . . 193

7.1.3 Summary of Simulation of Multi-Level Energy Storage . . . . 211

7.2 Extending the Simulator for Other Renewable Energy Sources . . . . 212

7.2.1 $\quad$ Extension for a Wind Energy Harvesting System . . . . . . . . . 212

7.2.2 Extension for Vibrational Energy Harvesting System . . . . . 214

7.3 Summary . . . . . . . . . . . . . . . . . 218

8 Extending the Simulator for a Meso-Solar Power System 220

8.1 System Architecture of Meso-Solar System . . . . . . . . . . . . . . . 220

8.2 Simulating Meso-Solar System . . . . . . . . . . . . . . . . . . . 228

8.2 .1 Simulation Set-up . . . . . . . . . . . . . . . . . . . . 228 
8.2 .2 Simulation Result . . . . . . . . . . . . . . . . . . . . . . . . . 229

8.3 Refining the Simulation of a Meso-Solar System Using Measurement 232

8.4 Predicting the Behavior of Meso-Solar Systems . . . . . . . . . . . . . 232

\begin{tabular}{lll}
\hline & Concluding Remarks & 238
\end{tabular}

\begin{tabular}{ll}
\hline Bibliography & 240
\end{tabular} 


\section{List of Figures}

2.1 Examples of sensornet applications that run on non-rechargeable bat-

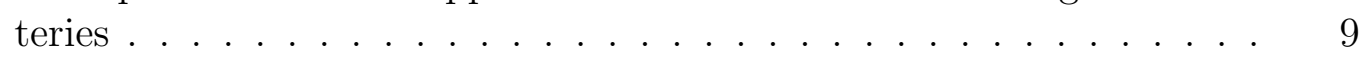

$2.2 \quad$ Different organizations of wire-powered node . . . . . . . . . . . . . 11

$2.3 \quad$ Comparison of renewable energy sources . . . . . . . . . . . . . . . . . 12

2.4 A general model for micro-solar power system . . . . . . . . . . . . . 14

2.5 Comparison of micro-solar power system platforms . . . . . . . . . . 19

2.6 Previous works on micro-solar power system models . . . . . . . . . . 23

2.7 Design space of micro-solar power systems and its relationship with a

3.1 Model for a solar-powered sensor system . . . . . . . . . . . . . . . . 28

3.2 Definition of the angles used in astronomical method . . . . . . . . . 29

3.3 Characteristics of a solar panel: (a) I-V curve, (b) P-V curve with MPP 31

3.4 A series of IV characteristics for a poly-crystalline solar panel . . . . 33

3.5 Comparison of solar panel output power and cumulative solar radiation for solar panel at operating point and MPP with a Trio node. . . . . 34

3.6 Current consumption of a Trio node with radio duty-cycle of $1.56 \%$. $\quad 39$

3.7 Energy flow and daily phases in our micro-solar model. . . . . . . . . 41

3.8 Architecture for HydroWatch Node . . . . . . . . . . . . . . . . . 43

3.9 Energy measurement set-up with HydroWatch Node. . . . . . . . . . 44

3.10 Energy flow of HydroWatch node with an input regulator . . . . . . . 47

3.11 Energy flow of HydroWatch node without an input regulator . . . . . 49

3.12 Block diagrams for Trio and Heliomote . . . . . . . . . . . . . . . 51

3.13 Experimental set-up for measuring solar panel output power. . . . . . 52

3.14 Experimental set-up for measuring energy efficiency . . . . . . . . . . 53

3.15 I-V characteristics of solar-panels . . . . . . . . . . . . . 55

3.16 Comparison of solar panel output power and cumulative solar radiation 56

3.17 Comparison of regulator designs . . . . . . . . . . . . . . . . . 57

3.18 Trio battery charging algorithm . . . . . . . . . . . . . . 58

3.19 The relation of operating voltage and power for Trio and Heliomote . 58

3.20 Daily energy flow of Trio and Heliomote . . . . . . . . . . . . . . . 60 
3.21 Energy flow of Trio and Heliomote at different phases . . . . . . . . . 61

4.1 Modular design of micro-solar power system simulator . . . . . . . . . 67

4.2 Time-event based simulator . . . . . . . . . . . . . . . . . . 68

4.3 Defining a component with curve-fitting method . . . . . . . . . . . 70

4.4 Defining a component using piecewise linear interpolation method . . 70

4.5 Matlab algorithm for estimating solar radiation using an astronomical

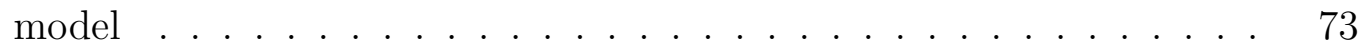

4.6 Matlab algorithm for estimating solar radiation using an astronomical model (continued) . . . . . . . . . . . . . . . 74

4.7 Astronomical model with effects of seasonal variation . . . . . . . . . 75

4.8 Astronomical model with effects of latitude and seasonal variation with

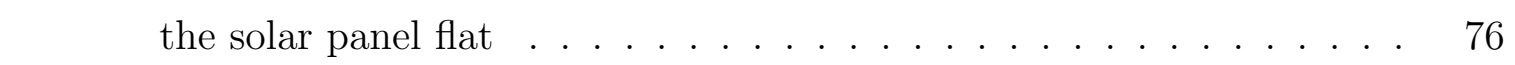

4.9 Astronomical model with effects of orientation and inclination on a particular day in mid-March . . . . . . . . . . . . . . . . . . . . . . . . . 77

4.10 Obstructed astronomical model . . . . . . . . . . . . . . . . . . . 80

4.11 Estimating the solar radiation using obstruction measurement . . . . 81

4.12 Daily solar radiation measurement with different estimation methods 82

4.13 Matlab algorithm for solar IV curve fitting . . . . . . . . . . . . . . 83

4.14 Matlab algorithm for estimating the solar panel IV and PV characteristics using measurement data. . . . . . . . . . . . . . . . . . 84

4.15 IV and PV graph derived from the measurement of $4 \mathrm{~V}-100 \mathrm{~mA}$ polycrystalline silicon solar panel . . . . . . . . . . . . . . . . 85

4.16 IV and PV graph derived from the measurement of $3.6 \mathrm{~V}-50 \mathrm{~mA}$ flexible solar panel . . . . . . . . . . . . . . . . . 85

4.17 Charging and discharging characteristics for the NH15-2500 NiMH bat-

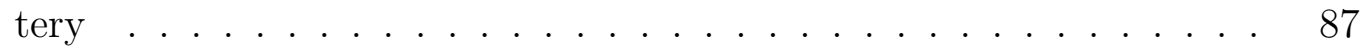

4.18 Algorithm that evaluates the state of a micro-solar power system with NiMH battery . . . . . . . . . . . . . . . . . . . . 88

4.19 Matlab algorithm for piecewise linear interpolation for the battery volt- 89

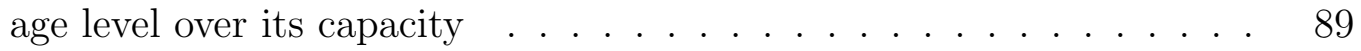

4.20 Voltage-Capacity Characteristic for $22 \mathrm{~F}$ supercapacitor . . . . . . . . 91

4.21 Leakage estimation model for supercapacitor . . . . . . . . . . . . . . 91

4.22 Output voltage of LTC1751 regulator as a function of input voltage . 92

4.23 Efficiency of LTC1751 regulator as a function of input voltage and load current with output voltage 3.3V . . . . . . . . . . . . . . . . . 92

4.24 Efficiency of MAX1724 regulator as a function of input voltage and load current with output voltage 3.3V . . . . . . . . . . . . . . 93

4.25 Configuration: a micro-solar power system with no input regulator. . 94

4.26 Operating range: a micro-solar power system with no input regulator 95

4.27 Configuration: a micro-solar power system with a buck converter as an input regulator. . . . . . . . . . . . . . . . . . . . 97 
4.28 Operating range: a micro-solar power system with a buck converter as an input regulator. . . . . . . . . . . . . . . . . . . 97

4.29 Structure of PowerTOSSIM . . . . . . . . . . . . . . . . . . . 100

4.30 Block diagram for CC2420 radio wrapper modules . . . . . . . . . . . 100

4.31 Solar-panel IV curve of the indoor lamp light . . . . . . . . . . . . . . 105

4.32 Trend of solar-panel and supercapacitor for a micro-solar power system with $22 \mathrm{~F}$ supercap under the indoor lamp light. No input regulator was used. . . . . . . . . . . . . . . ... 106

4.33 Trend of solar-panel and supercapacitor for a micro-solar power system with 22F supercap under the indoor lamp light with LM3671 step-down DC-DC converter as an input regulator. . . . . . . . . . . . . . 107

4.34 Trend of solar-panel and supercapacitor for a micro-solar power system with $140 \mathrm{~F}$ supercap under the indoor lamp light. No input regulator was used. . . . . . . . . . . . . . . . . . . . . . 109

4.35 Trend of solar-panel and supercapacitor for a micro-solar power system with 140F supercap under the indoor lamp light and LM3671 stepdown DC-DC converter as an input regulator. . . . . . . . . . . . . . 110

4.36 A micro-solar power system under average yearly solar radiation with HydroWatch workload (day of year $n=80$ ) . . . . . . . . . . . . . 112

4.37 A micro-solar power system under average yearly solar radiation with highest possible workload (day of year $n=80$ ) . . . . . . . . . . . . . 114

4.38 A micro-solar power system under worst-case yearly solar radiation with HydroWatch workload (day of year $n=356$ ) . . . . . . . . . . 115

4.39 A micro-solar power system under worst-case yearly solar radiation with highest possible workload (day of year $n=356$ ) $\ldots . . .117$

$5.1 \quad$ System architecture for the HydroWatch micro-climate network. . . . 123

5.2 Snapshot of the HydroWatch forest watershed deployment. . . . . . . 125

5.3 HydroWatch weather node. . . . . . . . . . . . . . . 125

5.4 HydroWatch micro-solar power subsystem. . . . . . . . . . . . . . . . 128

5.5 Current-Voltage and Power-Voltage performance of the Silicon Solar 4V-100mA solar panel. . . . . . . . . . . . . . . . 132

5.6 Urban neighborhood deployment site in Berkeley, CA . . . . . . . . . 135

5.7 Scatter plot of solar energy received in the urban neighborhood deploy-

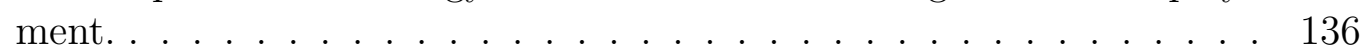

5.8 Comparison of solar panel output current and voltage on a sunny day (10/8/2007) for best, worst and middle mode in the urban neighborhood deployment. . . . . . . . . . . . . . . . . . . . 138

5.9 Comparison of solar panel output current and voltage on an overcast day (10/9/2007) for the urban neighborhood deployment. . . . . . . . 139

5.10 Scatter plot of solar energy received in the forest watershed deployment. 142 
5.11 Comparison of different radiation estimation methods for high radiation node (Router 78) on forest deployment. . . . . . . . . . . . . . . 143

5.12 Comparison of different radiation estimation methods for medium radiation node (Router 77) on forest deployment. . . . . . . . . . . . . 144

5.13 Comparison of different radiation estimation methods for low radiation node (Angelo 02) on forest deployment. . . . . . . . . . . . . . . . . . 145

5.14 Comparison of solar panel current and voltage on a sunny day (10/13/2007) in the forest watershed deployment. . . . . . . . . . . . . . . . . 147

5.15 Comparison of solar panel current and voltage on an overcast day $(10 / 16 / 2007)$ in the forest watershed deployment . . . . . . . . . . 148

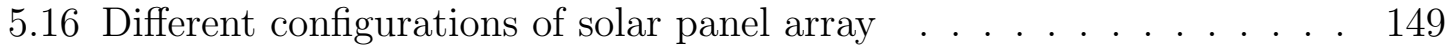

5.17 Effect of shading on polycrystalline silicon solar panel . . . . . . . . . 150

5.18 Effect of shading on flexible solar panel . . . . . . . . . . . . . . . . . 151

6.1 Solar profile for different weather conditions . . . . . . . . . . . . . . 154

6.2 Deployment map of HydroWatch nodes . . . . . . . . . . . . . . . . . 154

6.3 Seasonal solar radiation variation of HydroWatch weather nodes . . . 156

6.4 Deviation of the solar energy measurement from the estimate for the HydroWatch weather nodes deployment. . . . . . . . . . . . . . 157

6.5 Histogram and CDF of the estimation deviation from the measurement for the obstructed astronomical model . . . . . . . . . . . . . . . . . 159

6.6 Correlation of daily solar radiation estimation using turbidity and actual measurements at Golden, CO from 1/1/2008 to 12/31/2008 . . . 161

6.7 Histogram of correlation of turbidity and solar radiation with different window size at Golden, CO from 2002 to 2008 . . . . . . . . . . . . . 162

6.8 Correlation of solar radiation measurement with solar radiation estimation using visibility and cloud condition . . . . . . . . . . . . . 166

6.9 Correlation of solar radiation measurement with solar radiation estimation using visibility and cloud condition (continued) . . . . . . . . 167

6.10 Hourly weather data in Oakland, CA on 12/22/2007 . . . . . . . . . 168

6.11 Correlation of horizontal visibility and cloud conditions to the weather variation of the solar radiation using a linear scale mapping (Node A02 from $12 / 22 / 2007$ to $04 / 15 / 2008)$. . . . . . . . . . . . . . . . . 169

6.12 Correlation trends for each disjoint set of cloud conditions . . . . . . 170

6.13 Correlation trends for each disjoint set of visibility . . . . . . . . . . . 171

6.14 Occurences (frequency) of each disjoint set of visibility . . . . . . . . 172

6.15 Probability distribution of calibration factor for each disjoint set of cloud conditions . . . . . . . . . . . . . . . . 175

6.16 Probability distribution of calibration factor for each disjoint set of visibility . . . . . . . . . . . . . . . . . . 177

6.17 An exponential curve that fits the calibration factors with minimum errors. . . . . . . . . . . . . . . . 178 
6.18 Algorithm for predicting solar radiation with $k$-day history of weather effects . . . . . . . . . . . . . . . . . 179

6.19 Solar radiation prediction with the cloud-based model . . . . . . . . . 180

6.20 Solar radiation prediction with the visibility-based model . . . . . . . . 181

6.21 Deviation of solar radiation prediction with cloud-based and visibilitybased models . . . . . . . . . . . . . . . . . 182

7.1 A model for micro-solar power system with multi-level energy storage 185

$7.2 \quad$ Charging algorithm for two-level energy storage simulator . . . . . . . 189

7.3 Algorithm that determines the net power to the energy storage . . . . 191

7.4 Three cases for storage net charging power calculation. . . . . . . . . 192

7.5 Algorithm that determines the discrete states for the output power switch and the DC/DC converter . . . . . . . . . . . . . . . . 194

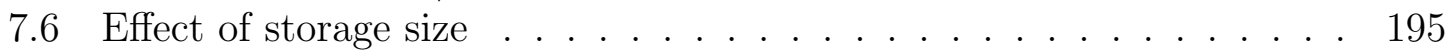

7.7 Narrowing the supercap charging range . . . . . . . . . . . . . . . . . 197

7.8 Shifting the supercap charging range . . . . . . . . . . . . . . . . . . 199

7.9 Comparison of solar panel operating voltage for Configuration 2a and 2c200

7.10 Effect of initial battery voltage level (with 22F supercap) . . . . . . . 200

7.11 Effect of initial battery voltage level (with 140F supercap) . . . . . . 201

7.12 Effect of initial battery voltage level . . . . . . . . . . . . . . . 203

7.13 Effect of maximum battery charging current (with 22F supercap) . . 204

7.14 Effect of maximum battery charging current (with 140F supercap) . . 205

7.15 Effect of efficiency of DC/DC converter . . . . . . . . . . . . . 207

7.16 Comparison with NiMH-only storage . . . . . . . . . . . . . . . 209

7.17 Comparison of solar panel operating voltage for Configuration $7 \mathrm{a}$ and $7 \mathrm{~b} 210$

7.18 Operation during darkness . . . . . . . . . . . . . . . . 210

7.19 A generic model for wind energy harvesting system . . . . . . . . . . 213

7.20 Output power from the wind generator for varying wind speed . . . . 215

7.21 Extension for the simulation model with wind energy harvesting . . . 216

7.22 A generic model for vibrational energy harvesting system . . . . . . . 216

7.23 Extension for the simulation model with vibrational energy harvesting 219

$8.1 \quad$ A solar-powered web server and its network architecture . . . . . . . 221

8.2 System architecture for a solar-powered web server . . . . . . . . . . 222

8.3 Characteristic for Kyocera KC50 solar panel . . . . . . . . . . . . . . 224

8.4 Voltage-to-capacity relationship for 12V-32Ah lead acid battery . . . 224

8.5 Characteristics of MorningStar Sunguard-4 regulator . . . . . . . . . 225

8.6 Characteristics of PowerStream PST-DC292 regulator . . . . . . . . . 226

8.7 Daily energy profile for the solar-powered web server with $n=80$. . 230

8.8 Daily energy profile for the solar-powered web server with $n=80$. . 231

8.9 Daily energy profile for the solar-powered web server with $n=356$. . 233 
8.10 Daily energy profile for the solar-powered web server with $n=356$,

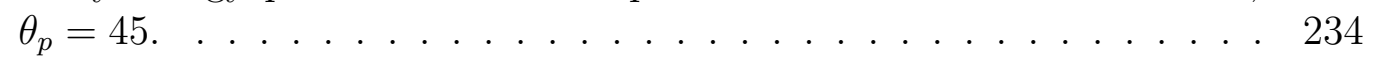

8.11 Layout of the solar-powered web server deployment . . . . . . . . . . 234

8.12 Estimation of daily solar panel output for the solar-powered web server 235

8.13 Yearly estimation for the solar-powered web server. . . . . . . . . . . 237 


\section{List of Tables}

2.1 Different types of energy storage elements for micro-solar power systems 16

2.2 Summary of sensor network power simulators . . . . . . . . . . . 21

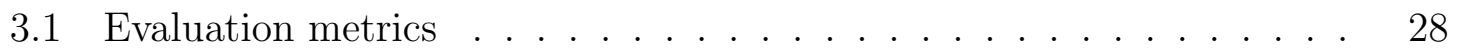

3.2 Operating range of energy storage, load, and output regulator . . . . 38

3.3 Current consumption of Trio at different duty-cycle rates . . . . . . . 39

3.4 Daily trends of system efficiency for HydroWatch node in two cases . 50

3.5 Component energy level for Trio and Heliomote . . . . . . . . . . . 57

$\begin{array}{lll}3.6 & \text { Energy efficiency of charging and discharging for Trio and Heliomote } 59\end{array}$

4.1 Comparing estimation from PowerTOSSIM with measurement . . . . 101

4.2 Power consumption model for TelosB node . . . . . . . . . . . . . . . 102

$4.3 \quad$ Average yearly solar radiation with HydroWatch workload . . . . . . 113

4.4 Average yearly solar radiation with highest possible workload . . . . . 113

4.5 Worst-case yearly solar radiation with HydroWatch workload . . . . . 116

4.6 Worst-case yearly solar radiation with highest possible workload . . . 116

5.1 Components for the HydroWatch board . . . . . . . . . . . . . . . . . 129

5.2 Estimated operating time of a node without energy storage recharging 130

5.3 Daily average of the solar panel output power for different estimation models and the deployment in an urban neighborhood. . . . . . . . . 137

6.1 Deviation of the obstructed astronomical model from the measurement 158

6.2 Correlation coefficient for the weather trend in VLSB deployment . . 158

6.3 Measurement sites used from the National Solar Radiation Data Base 164

6.4 Deviation for different nodes . . . . . . . . . . . . . . . . . . . . . . . 179

7.1 List of vibration sources . . . . . . . . . . . . . . . . . . . . . . . . . 217

8.1 Components for solar-powered web server . . . . . . . . . . . . . . . . 227

8.2 Comparison of a meso-solar system and a micro-solar system . . . . . 227 


\section{Acknowledgments}

I give special thanks to David Culler, my adviser and mentor during my $\mathrm{PhD}$ study. Under his guidance I was able to learn the fundamental lessons of being a researcher: finding interesting problems, doing the scientific study and presenting meaningful results. He guided me in determining the direction of my research and crystallized my efforts.

Professors Paul Wright, Seth Sanders, Ion Stoica and Kris Pister gave me valuable comments on this dissertation. Conversations with Paul, Seth, Ion and Kris allowed me to view the system from a broader perspective and solidify this work.

Jay Taneja is the main collaborator for the HydroWatch project, which provided concrete empirical data for micro-solar power system models. Jay participated in the design, testing and assembly of the energy subsystem of the HydroWatch nodes. He was in charge of building the sensing and mechanical elements of the HydroWatch nodes as well as deploying all the HydroWatch nodes in the field. Prabal Dutta has been very helpful when discussing hardware design and other technical problems. Prabal is the main designer of the Trio node, a project that led me to study the micro-solar power systems. He also provided the Epic node, which became the core component of the second generation HydroWatch node. Xiaofan Jiang participated in building the general model for a micro-solar power system and instrumenting Trio and Heliomote nodes.

Sukun Kim has always been a good friend with whom to discuss technical problems and school life. Gilman Tolle customized the gateway server software ArchRock Primer Pack for HydroWatch nodes and allowed me to use the rich monitoring capabilities of the Primer Pack. Jonathan Hui, Cory Sharp, Kamin Whitehouse and Robert Szewczyk have participated in the development and deployment of the Trio testbeds. Phoebus Chen, Songhwai Oh, Michael Manzo, Bruno Sinopoli, Shawn Shaffert, Bonnie Zhu, and Tanya Roosta, also participated in deploying the Trio testbeds. Stephen Dawson-Haggerty, Jorge Ortiz, Byung-Gon Chun, and Matt Caesar generously took their time and reviewed my works. 
I would like to thank Berkeley ERSO research staff members Albert Goto, Mike Howard and Eric Fraser for setting up and maintaining network infrastructure, without whom experiments would have taken more time and effort. ERSO staff members Angie Abbatecola, Lauren Bailey, Willa Walker, Damon Hinson, and Robert Miller helped me to do the experiments in less time by processing the purchasing requests and reimbursements.

This work was supported by the Defense Advanced Research Projects Agency (grant F33615-01-C-1895), the Keck Foundation (grant HydroWatch Center), the National Science Foundation (grant 0435454 "NeTS-NR", 0454432 "CNS-CRI"), This work was also supported by Korea Foundation for Advanced Studies Fellowship as well as generous gifts from the Hewlett-Packard Company, Intel Research, and California MICRO.

Finally, I am especially grateful to my family. My mother, Guinam Lim, has been caring and supportive throughout my life. My father, Ilgoo Jeong, has always inspired me with hope and vision. My sister, Jaehee, who has lived with me for the last few years, helped me to feel at home in Berkeley. 


\section{Chapter 1}

\section{Introduction}

\subsection{Motivation}

One of the visions of wireless sensor networks (WSNs) is autonomous long-term monitoring of the environment, and a key limiting factor is the ratio of power consumption and energy supply. Most sensornet applications in outdoor environment run on a battery since it is easily accessible in off-the-grid environments and is relatively inexpensive. However, a battery-powered application is not suitable for a longterm deployment due to the finite capacity of the energy storage $\left[\mathrm{SMP}^{+} 04, \mathrm{TPS}^{+} 05\right.$, Kim07], and the battery-capacity to power-consumption ratio. In order to address the limited-lifetime problem, many solutions have been proposed at the application level [MFHH02,NGSA04,PKR02,SS02] and networking level [PHC04,YHE04,YSH06, $\mathrm{ZZH}^{+}$07, CJBM01,Dus]. These solutions lengthen the lifetime of a sensor network by using various techniques to reduce power consumption, such as aggregation, data compression and radio duty-cycling, though the improvement is only a constant factor and does not solve the limited-lifetime problem. Renewable energy sources, such as solar radiation, vibration, human power and air flow can be used to solve this problem, as a renewable energy powered node can potentially run for a long period of time without requiring the replacement of the battery. Among these renewable energy sources, solar energy is the most promising for an outdoor wireless sensornet application. It has higher power-density than other renewable energy sources, and this allows a sensor node to collect sufficient energy with a small form factor. 
Also, it is available for several hours per day in most outdoor locations, whereas the availability of other renewable energy sources is very localized [Rou03, Par06, PC06].

Recognizing the possibility of long-term autonomous operation, several implementations have been made [ZSLM04, JPC05, $\mathrm{RKH}^{+}$05, SC06, $\mathrm{DHJ}^{+}$06, $\mathrm{PC06}, \mathrm{CVS}^{+}$07]. These implementations demonstrate that building a sensornet system with solar energy harvesting is possible. However, they address only particular points in the design space of micro-solar power systems, rather than providing a general model. These implementations do not provide guidance when they are placed in a setting different from their target environment, or if a different configuration of micro-solar power system is used. In order to explore possible choices in the design space of microsolar power systems, a general model is needed. A number of previous models have been made [JPC05, $\mathrm{RKH}^{+}$05, KPS04, KHZS07, VGB07, NLA ${ }^{+} 07, \mathrm{MBTB} 66 \mathrm{~b}, \mathrm{SKG}^{+} 07$, $\mathrm{SHC}^{+}$04, PSS00, PSS01, SVML03, SKA04, LWG05, Met, DHM75, VXSB07], but they have focused only on a particular component and not on the whole system.

The goal of this dissertation is to enable the systematic design of a micro-solar power system so that it will be possible to model and analyze hypothetical designs. We first provide a theory of micro-solar power systems, then, based on the theory, we develop simulation tools that reflect reality. The simulation tools enable us to predict the behavior of hypothetical designs and thus deploy only the working ones. Our simulation tools estimate the electrical behavior of micro-solar power systems in a similar way to Spice [New78], the de-facto standard circuit simulation tool. However, the time-scales are different, for while Spice is usually used for modeling the shortterm behavior of a circuit (microseconds to seconds), our simulation tools model the system behavior for a long period of time, such as months and years.

While our simulation tools are made for micro-solar power systems (small lowpower electronics with a solar panel measured in milliwatts of power), many tools and calculators are available for macro-solar and meso-solar installations in residential and commercial applications on the scale of kilowatts. Macro-solar and meso-solar systems have the same basic component categories and the same interconnection as a micro-solar power system, but they differ greatly in the sizes and relative sizes of these components, which lead to very different design and deployment issues. One difference is that with a micro-solar power system, the load is on the same scale 
as the management, and the system as a whole has to be very efficient and wellmatched. Another difference is that a micro-solar power system should be planned for the environment that we must deploy in, such as a deep dark forest, rather than the environment that is desired, such as a well-exposed rooftop.

\subsection{Problem Statement}

Hypothesis : A practical theory of micro-solar power systems can be developed and materialized in a simulation suite that models system behavior over a long timescale and in an external environment that depends on time, location, weather and local variations, with sufficient accuracy to guide specific design choices in a large design space.

Solar energy is considered a viable solution for powering outdoor wireless-sensornetwork applications due to its high power-density and wide availability. However, it has a few challenges that make the direct implementation of a micro-solar power system difficult: (i) large design space, (ii) long time-scale, (iii) different environments for development and deployment sites, (iv) variability of solar energy.

Typically, a micro-solar power system consists of several components, which collect the solar energy, buffer the energy in the energy storage or consume the energy for computation and communication. Given that there are $n$-different ways of building each component, the complexity of building a whole system increases as a polynomial in $n$; building and testing all these combinations is not a practical solution. Further, the problem of the large design space is exacerbated by the long time-scale of development and deployment, as developing the hardware and measuring the solar energy profile for verification of a design point may take months. One mistake in the development and deployment cycle requires repeating the entire cycle and delaying the actual deployment. The classic argument for a simulator works here: we can find a suitable design of micro-solar power systems by running simulations without having to implement all the possible designs. We can also keep the development and deployment cycle short with simulation by choosing only the designs that pass simulation tests and eliminating all others. 
A micro-solar power system is usually developed in an indoor environment, but it is deployed in an outdoor location whose solar energy profile varies greatly from one location to another. Thus, the development of a micro-solar power system requires modeling the solar energy profile for the deployment site. However, it is not easy to give a prediction of the solar energy in a straightforward way because solar radiation depends not only on astronomical factors (e.g. time and location) but also on local effects (e.g. weather and obstructions). Our simulation tools can predict the variability of solar energy due to astronomical factors, and can also refine the solar energy estimation using local factors when they are available. In order to evaluate our hypothesis, we have designed and implemented a reference hardware platform and a simulation tool suite for micro-solar power systems. The deployment results show that the estimate from our simulation tools is very close to the measurement in reasonably clear weather. This implies that our simulation tools can predict the solar profile well, even in the presence of obstructions. On cloudy or rainy days, the estimation error increases, but it was bounded to about $30 \%$. This implies that it is possible to design a micro-solar power system for long-term survival under varying weather conditions by having modest surplus in the solar collector.

Our simulation tools are based on a practical theory of micro-solar power systems which consists of two parts: (i) description of characteristics of each component and their relations, (ii) event driven temporal modeling of the interconnected whole. It is a common wisdom in Computer Science that a large, complex system can be easily described by smaller, more manageable components and their relations. This divide-and-conquer concept also applies to micro-solar power systems, so as a natural approach, we divide a micro-solar power system into multiple functional units whose characteristics are well-defined. These components are: the external environment, solar collector, input regulator, energy storage, output regulator and load. We model the relations of these six components in terms of energy flow, operating range, and efficiency, and have verified these based on the measurements of the reference platform. The second part of the theory builds a formal simulation model based on these relations and the descriptions of each component. Using this framework, we have built a formal model for several components, using analytical or empirical methods depending on how well its characteristics are defined. Our simulation tools 
are similar to Spice in that both estimate the electrical behavior of a system using a discrete time-event simulator, but they differ in which time-scales are used. While Spice is usually used for modeling the short-term behavior of a circuit (microseconds to seconds), our simulation tools model the system behavior for the long-term, such as months and years. Our simulation tools are configured with a coarse-grained timescale that is optimized for fast evaluation and long-term prediction without losing the accuracy within a diurnal behavior.

\subsection{Contributions}

The contributions of this dissertation are as follows:

- We provide an architecture of micro-solar power systems.

- We build a formal simulation model for micro-solar power systems.

- We develop a reference platform and provide a realistic validation of the simulation model based on it.

- We extend the simulation model for hypothetical designs of micro-solar power systems and meso-solar power systems

\subsection{Roadmap}

This dissertation is organized into nine chapters. Chapter 2 compares outdoor solar energy with other types of energy sources, and identifies it as a feasible solution for powering outdoor wireless sensor network applications. It then provides a background overview of solar energy harvesting in the domain of wireless sensor networks, defining the problems to be solved throughout this dissertation. Chapter 3 presents an architecture of micro-solar power systems, describing the characteristics of its key components and the relationships among its components. Chapter 4 develops the architecture of micro-solar power systems into a formal simulation model: first, by formalizing each component; then, by synthesizing different variations of models and 
validating the simulation model with benchtop experimental results. Chapter 5 describes a reference platform for micro-solar power systems, the HydroWatch node; then it develops a simulation model of the reference platform and validates the model using deployment data from urban and forest watershed environments. Chapter 6 predicts a long-term trend of reference implementation in an urban environment and compares it with the simulation model to validate the long-term prediction capability of the simulation model. Chapter 7 extends the simulation model for a hypothetical system to demonstrate that the simulation model can be used beyond the reference implementation. It first describes a model for a micro-solar power system with multi-level energy storage and presenting the simulation data. Then, it extends the simulation model for two other renewable energy sources: wind and vibrations. Chapter 8 gives a high-level comparison of micro-solar and meso-solar power systems by comparing the energy flow and efficiency of each component of the solar power system. For this, the simulation model for micro-solar power systems is extended to meso-solar power systems and is validated using a reference meso-solar power system platform, Solar-Powered Web Server. Finally, Chapter 9 concludes this dissertation. 


\section{Chapter 2}

\section{Background}

This chapter compares outdoor solar energy with other types of energy sources and shows that is is a feasible solution for powering outdoor wireless sensor network applications even with limited exposure at small panels. Then, it provides a background overview of solar energy harvesting in the domain of wireless sensor networks, and defines the problems to be solved throughout this dissertation.

\subsection{Wireless Sensor Networks and Energy Sources}

Typically, a wireless sensor node is composed of a micro-controller, communication subsystem, sensor/actuator subsystem, storage subsystem and power subsystem. The micro-controller, which is the processing unit of a wireless sensor node, takes input data from other subsystems and generates processed data. The communication subsystem consists of two parts: a radio transceiver for node-to-node communication over the air, and a serial communication controller for node-to-host communication. The sensor/actuator subsystem translates physical phenomenon such as light, temperature, humidity, etc. into an electrical signal (sensor), or converts signals from the

micro-controller into mechanical movement (actuator). The storage subsystem has a non-volatile storage device and provides an interface to the device so that the microcontroller can read and write data to and from it. The power subsystem provides power to the micro-controller and all other subsystems. Depending on the characteristic of the energy source, the power subsystem of a wireless sensor node can be 
categorized into three types: (a) non-rechargeable battery-powered, (b) wire-powered, and (c) renewable energy-powered. In this dissertation, we focus on the design space of the power subsystems, viewing the rest of the system as the load.

\subsubsection{Non-rechargeable Battery-Powered Nodes}

The power subsystem of a non-rechargeable battery-powered node is composed of an energy source and an optional regulator. Its energy source is a non-rechargeable battery such as an alkaline battery, and the optional regulator adjusts the voltage level from the battery to be within the operating voltage range of the sensor node. Using a non-rechargeable battery is the most common way of supplying energy to a sensor node. This is advantageous in that the non-rechargeable battery is relatively inexpensive and that the sensor node can be located anywhere without requiring the existing power infrastructure to be re-wired. However, using a non-rechargeable battery can be problematic in that the lifetime of the sensor nodes is limited due to its limited capacity. Figure 2.1 shows examples of sensornet applications that run on non-rechargeable batteries. A Great Duck Island node [SMP+ 04], designed to monitor the environmental characteristics of a bird's nest, has a small-capacity battery so as not to disrupt wildlife. Although sensing and transmission is done at a low rate, the small battery capacity limits its lifetime to about two months. A Golden Gate Bridge node [Kim07], is designed to monitor structural health data of a bridge using time-synchronized high frequency sampling of accelerometer signals. Even though a Golden Gate Bridge node has the freedom to use a larger battery, high power consumption of the accelerometer limits its lifetime to a little over one month. This limited lifetime of a non-rechargeable battery-based node motivates us to consider other types of energy sources for wireless sensor networks.

\subsubsection{Wire-powered Nodes}

Another way to supply energy to sensor nodes is through a wired back-channel. While wireless sensor networks were originally envisioned to be wire-free, a wired back-channel can be used for maintenance purposes, such as reprogramming and data downloading. A side effect of using a wired back-channel is that the sensor 


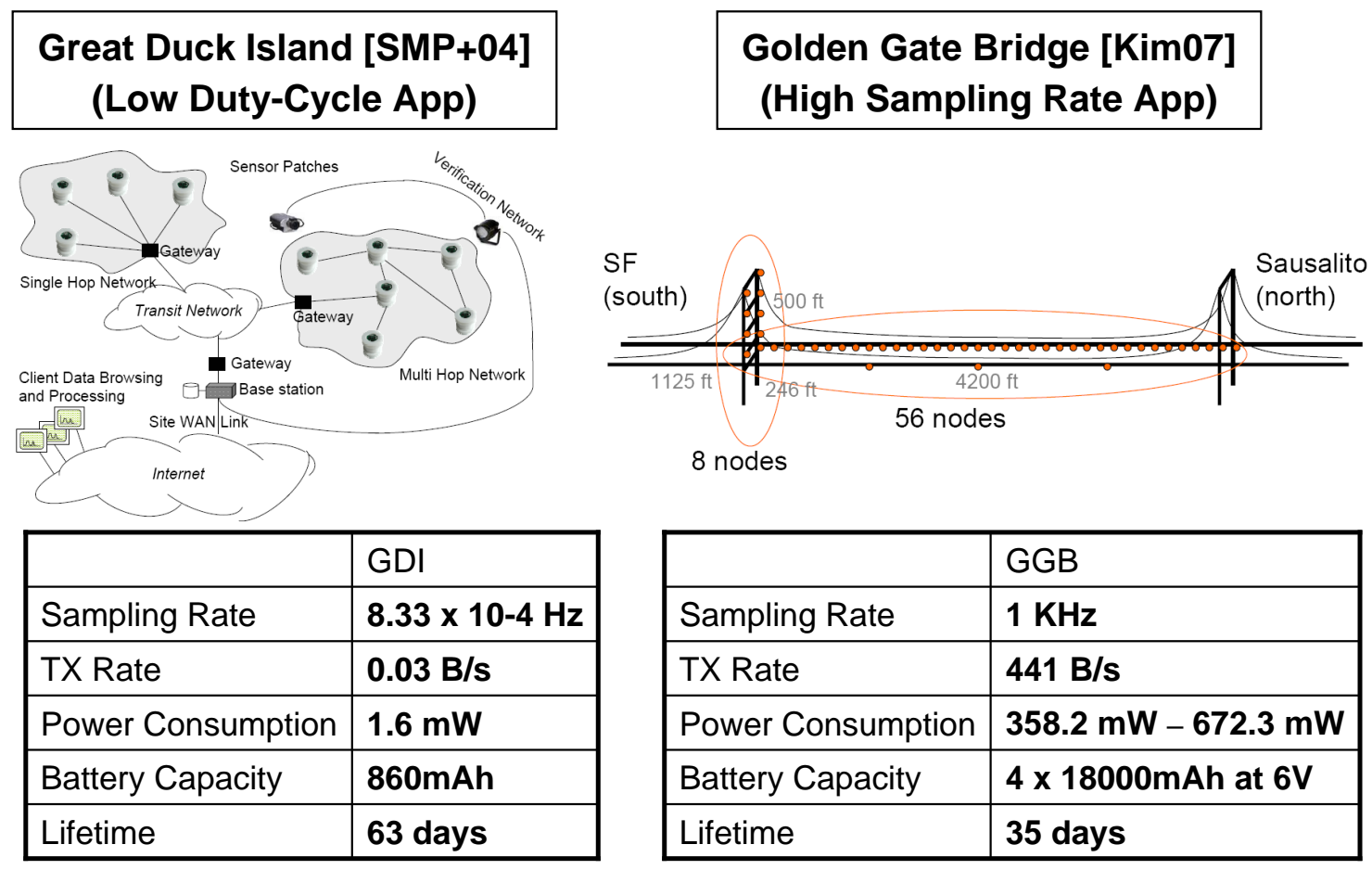

Figure 2.1: Examples of sensornet applications that run on non-rechargeable batteries

node can be powered through the wire. Many wireless sensor network testbeds utilize this in order to power the sensor nodes, as well as to reprogram or monitor the nodes. A wire-powered node consists of a wire power source and a regulator. There are a few variations of wire-powered nodes depending on the energy source: Power-Over-Ethernet(PoE)-powered nodes (Figure 2.2(a) and USB-powered nodes (Figure 2.2(b)]. A PoE-powered node can be powered through the wire if the programming board can take power from the PoE cable. Smote [Unib], MicaZ-based Motelab [WASW05], and Mirage [Int] are such examples. A USB-powered node can be powered through the USB host. In the Omega testbed [PSC05], a sensor node is plugged into the USB extension hub that is connected to the PC server in cascades. In TWIST [HKWW06] and Telos-based Motelab [Har], a sensor node is plugged into a USB-Ethernet converter from which it receives power. The USB-Ethernet converter communicates and delivers power to the sensor node through the USB interface while also communicating with the backbone network. While wire power makes it easy to maintain a testbed, it is limited to where a PoE or USB wiring is available. In 
an outdoor deployment, wire power may not be available, and making such devices weather-proof or wildlife-safe can add huge cost and complexity. It is the limiter in outdoor testbeds.

\subsubsection{Renewable Energy Sources}

A renewable energy-powered node runs on a renewable energy source such as solar radiation, vibrations, human power, or air flow, and is expected to run for a long period of time without requiring the replacement of the battery. Typically, a renewable energy-powered node consists of an energy source, energy collector, energy storage and regulator. The energy from the energy source is converted into electric energy through the energy collector, such as a solar cell, piezo electric material or wind generator. Then, the electric energy generated from the energy collector is stored in the energy storage, which stores energy and services the load even when the energy generation rate does not match the energy consumption rate. A regulator can be used to match the operating range between each component of the power subsystem.

Among the various renewable energy sources, we focus on outdoor solar energy in this dissertation for two reasons. First, outdoor solar energy has higher powerdensity than other renewable energy sources, and this allows us to build a solar-energy harvesting system with a small form factor (Figure 2.3). Second, the commercial availability of solar panels allows us to focus on the energy harvesting system from the perspective of computer science, which consists of synthesis, modeling and analysis, without the need to build the energy harvesting material itself.

\section{Solar Energy Harvesting}

Solar energy harvesting converts solar radiation (energy source) into electric energy using the photovoltaic effect (energy collector). A few types of semiconductors, such as polycrystalline silicon can emit electrons when hit by photons. A single solarcell outputs voltage between $0.5 \mathrm{~V}$ and $0.7 \mathrm{~V}$, depending on the material, and the current from the solar-cell is approximately proportional to the area of the solar-cell. Since the voltage and the current of a single solar-cell may not be large enough to meet the requirements of the energy consuming device, multiple solar cells are com- 


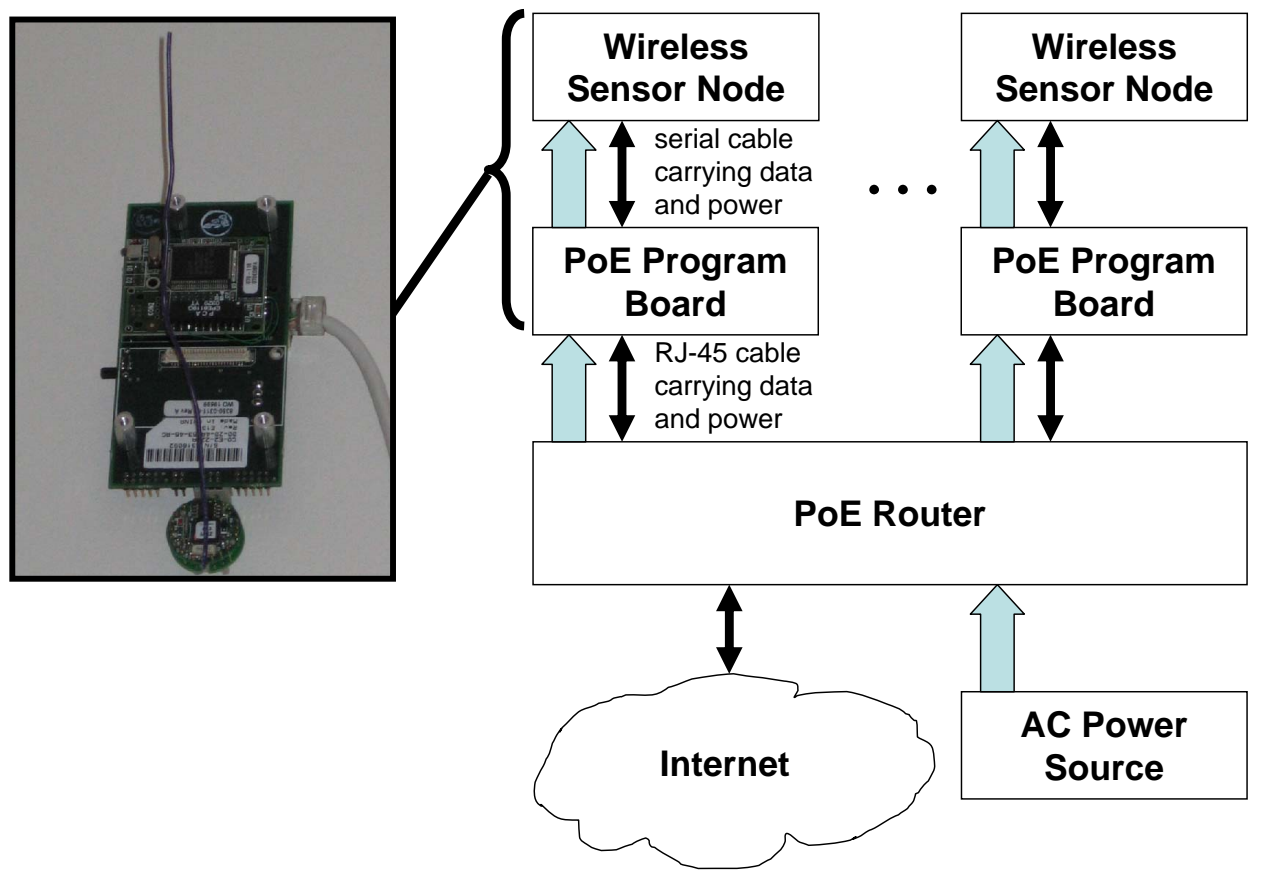

(a) Example of PoE-powered node: Smote [Unib]

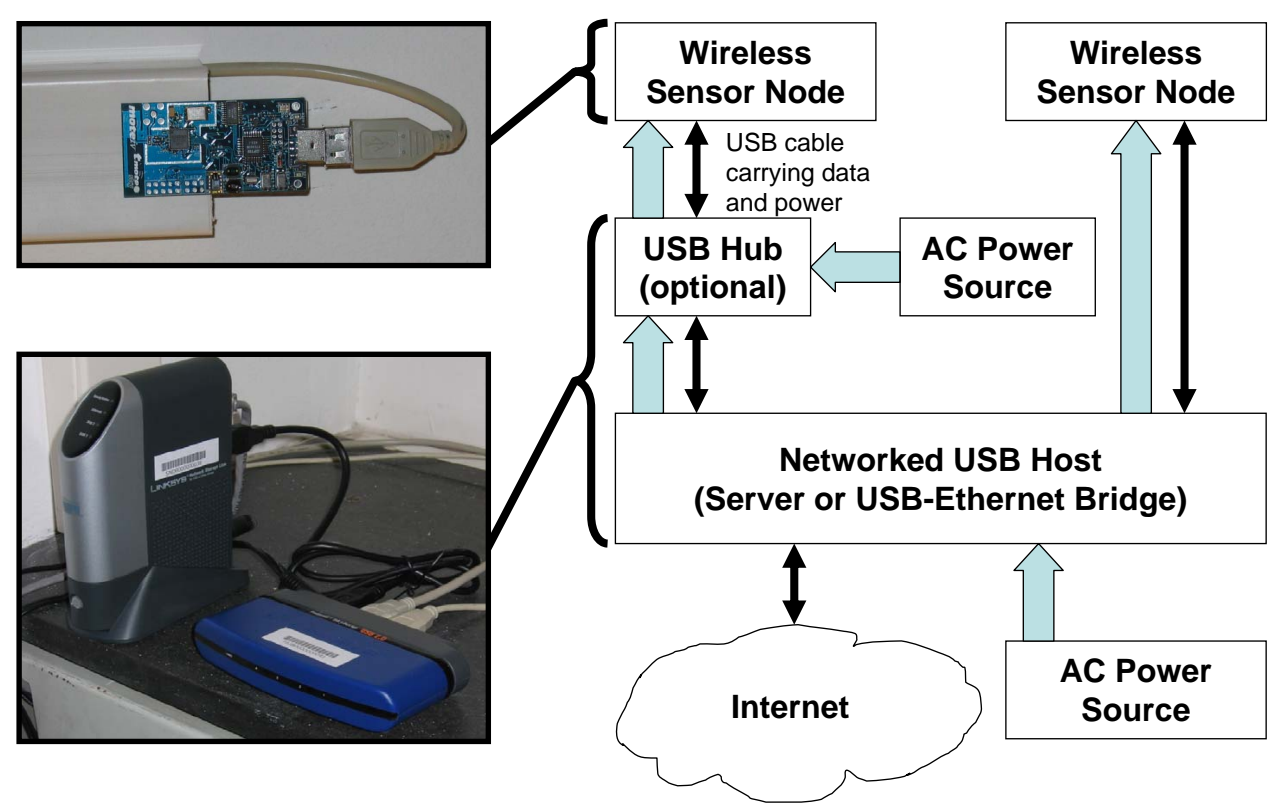

(b) Example of USB-powered node: TWIST [HKWW06]

Figure 2.2: Different organizations of wire-powered node 


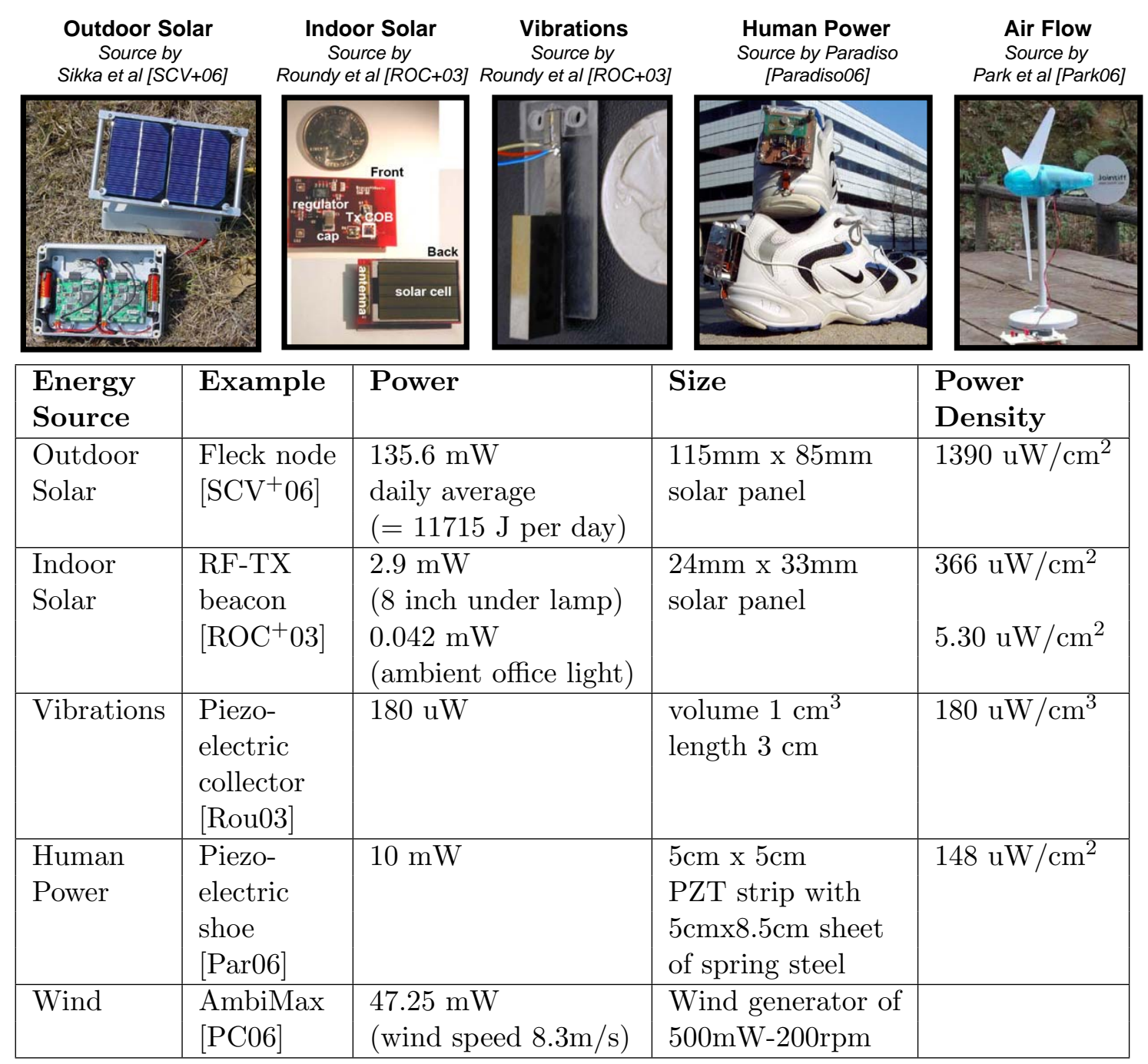

Figure 2.3: Comparison of renewable energy sources 
monly combined, in a series or parallel, into an array that is able to provide suitable voltage and current. Such a solar-cell array is called as solar panel.

\section{Vibration}

Solar energy has big advantages in the outdoors; it is available in most outdoor locations and has high power-density. However, it is not very useful in an indoor environment due to its low power-density. Figure 2.3 shows that the power-density of indoor light is three orders of magnitude smaller than that of outdoor sunlight; thus, other energy sources are more suitable depending on the situation. Vibrations can be a good source of energy when there is a source of continuous vibrations, such as frequently trodden doormats, motors in industrial facilities and washing machines are such examples. Roundy demonstrated that a piezoelectric material-based energy collector can generate $180 \mathrm{uW}$ of power [Rou03, $\left.\mathrm{RSF}^{+} 04\right]$.

\section{Human Power}

Paradiso [Par06] discusses several techniques for harvesting energy from human activity, such as push-button RF transmitter, magnetic generator based shoe, piezoelectric based shoe, and parasitic mobility node. For example, a piezoelectric-based shoe collects $10 \mathrm{~mW}$ of power. Human power has one advantage over other renewable energy sources: a small amount of energy can be generated quickly on demand by a human without depending on the environment. However, it is not suitable as a steady long-term energy source because intentional use of human power can disrupt normal human activity.

\section{Wind or Air Flow}

Park et al. demonstrated that a mote-scale device (AmbiMax node) can operate on wind-generated power [PC06]. The AmbiMax node stores the energy from a wind generator ${ }^{1}$ in a supercapacitor and performs maximum power point tracking (MPPT) between the wind generator and the supercapacitor. However, wind power has some drawbacks. Compared to solar power, its energy density is smaller $\left(380 \mu \mathrm{W} / \mathrm{cm}^{3}\right.$ vs.

\footnotetext{
${ }^{1}$ AmbiMax used a wind generator that outputs $1 \mathrm{~W}$ at $2000 \mathrm{rpm}$ and $0.25 \mathrm{~W}$ at $1000 \mathrm{rpm}$.
} 


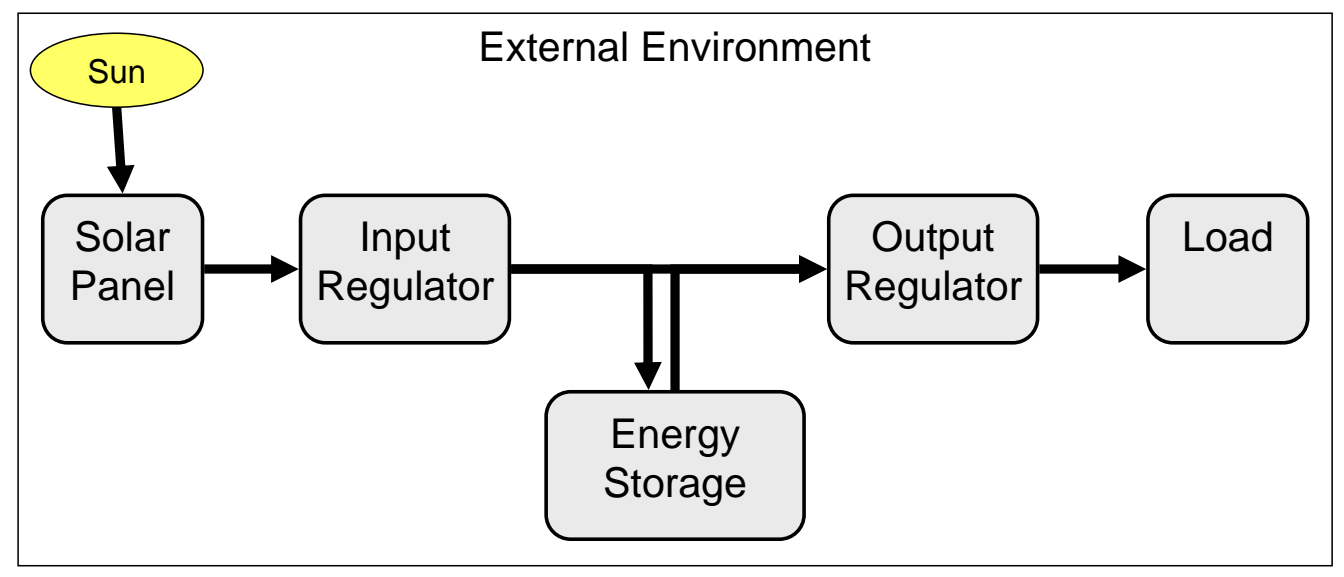

Figure 2.4: A general model for micro-solar power system

$\left.15000 \mu \mathrm{W} / \mathrm{cm}^{3}\left[\mathrm{RSF}^{+} 04\right]\right)$, and the available hours of wind power are shorter than those of solar power [PC06].

\subsection{Prior Work on Micro-solar Power Systems}

Typically, as shown in Figure 2.4, a micro-solar power system has the following organization: (a) an external environment that determines the amount of solar radiation available to the micro-solar power system, (b) a solar panel that collects solar energy, (c) energy storage, where extra energy from the solar panel is stored, (d) a load that will run on top of the power subsystem, consuming energy from the solar panel and the energy storage, (e) an input regulator that maximizes input power by matching the operating point between the solar panel and the energy storage, and (f) an output regulator that regulates the output voltage of the energy storage.

In this section, we develop a taxonomy of micro-solar power subsystems based on previously published systems, and we discuss strengths and weaknesses of each micro-solar power system design. From this, we distill a few designs of micro-solar power systems that are representative of different design points and have reasonable performance metrics. 


\subsubsection{Micro-solar Power System Platforms}

\section{Categorized by Storage and Charging Management}

First, we can categorize micro-solar power systems depending on the type of energy storage and its charging management. Table 2.1 lists lead acid battery, NiCd battery, NiMH battery, Li-ion battery, Li-polymer battery, and supercapacitor as energy storage for micro-solar power systems.

The Lead acid battery is the most commonly used energy storage in macro-solar power systems because it is easy to make in a large capacity, it can provide current high enough to drive residential electric devices, and its charging method is simple. But, at the scale of micro-solar power systems, it is not preferred due to its smaller energy density.

The NiMH battery is one of the most popular types of energy storage for microsolar power systems. This is because it has relatively high energy density, its charging method is simple, it is relatively inexpensive, and it can be replaced with a nonrechargeable battery when it is not charged. Among the micro-solar power systems we reviewed, Heliomote $\left[\mathrm{RKH}^{+} 05\right]$, Fleck $\left[\mathrm{CVS}^{+} 07\right]$ and HydroWatch [TJC08] used a NiMH battery as their energy storage. Since the current from the solar panel is relatively small compared to the maximum charging current, these platforms employ trickle charging, which can be done with a simple hardware-based controller.

The NiCd battery is similar to NiMH in a few cases: it has similar charging and discharging characteristics and it is available in standard battery form factors (e.g. AA, AAA, C) just as is the NiMH battery. This means that NiMH and NiCd are interchangeable in most cases. The differences are that the capacity of $\mathrm{NiCd}$ is smaller than NiMH (1100mAh vs. $2500 \mathrm{mAh}$ in Table 2.1), it has a memory effect that causes its capacity to decrease over multiple uses, and it contains cadmium that can be harmful when exposed to the environment. For this reason, NiMH is preferred over $\mathrm{NiCd}$ for micro-solar power systems.

The Li-ion (or Li-polymer) battery has the highest energy density and a high charge-to-discharge efficiency. This makes the Li-ion battery a good candidate for energy storage when the micro-solar power system needs a small form factor. However, the charging mechanism of a Li-ion battery is more complicated than that of other 
Table 2.1: Different types of energy storage elements for micro-solar power systems

\begin{tabular}{|l|r|r|r|}
\hline Type & Lead Acid & NiCd & NiMH \\
\hline Make & Panasonic & Sanyo & Energizer \\
Model No. & LC-R061R3P & KR-1100AAU & NH15-2500 \\
\hline
\end{tabular}

Characteristics of a single storage element

\begin{tabular}{|l|r|r|r|}
\hline Nominal voltage & $6.0 \mathrm{~V}$ & $1.2 \mathrm{~V}$ & $1.2 \mathrm{~V}$ \\
Capacity & $1300 \mathrm{mAh}$ & $1100 \mathrm{mAh}$ & $2500 \mathrm{mAh}$ \\
Energy & $7.8 \mathrm{Wh}$ & $1.32 \mathrm{Wh}$ & $3.0 \mathrm{Wh}$ \\
Weight energy density & $26 \mathrm{Wh} / \mathrm{Kg}$ & $42 \mathrm{Wh} / \mathrm{Kg}$ & $100 \mathrm{Wh} / \mathrm{Kg}$ \\
Volume energy density & $67 \mathrm{Wh} / \mathrm{L}$ & $102 \mathrm{Wh} / \mathrm{L}$ & $282 \mathrm{Wh} / \mathrm{L}$ \\
Weight & $300 \mathrm{~g}$ & $24 \mathrm{~g}$ & $30 \mathrm{~g}$ \\
Volume & $116.4 \mathrm{~cm}^{3}$ & $8.1 \mathrm{~cm}^{3}$ & $8.3 \mathrm{~cm}^{3}$ \\
Self-discharge (per month) & $3 \%-20 \%$ & $10 \%$ & $30 \%$ \\
Charge-discharge efficiency & $70 \%-92 \%$ & $70 \%-90 \%$ & $66 \%$ \\
Memory effect & No & Yes & No \\
Charging method & trickle & trickle/pulse & trickle/pulse \\
\hline
\end{tabular}

\begin{tabular}{|l|r|r|r|}
\hline Type & Li-ion & Li-polymer & Supercap \\
\hline Make & Ultralife & Ultralife & Maxwell \\
Model No. & UBP053048 & UBC433475 & BCAP0350 \\
\hline
\end{tabular}

Characteristics of a single storage element

\begin{tabular}{|l|r|r|r|}
\hline Nominal voltage & $3.7 \mathrm{~V}$ & $3.7 \mathrm{~V}$ & $2.5 \mathrm{~V}$ \\
Capacity & $740 \mathrm{mAh}$ & $930 \mathrm{mAh}$ & $350 \mathrm{~F}$ \\
Energy & $2.8 \mathrm{Wh}$ & $3.4 \mathrm{Wh}$ & $0.0304 \mathrm{Wh}$ \\
Weight energy density & $165 \mathrm{Wh} / \mathrm{Kg}$ & $156 \mathrm{Wh} / \mathrm{Kg}$ & $5.06 \mathrm{Wh} / \mathrm{Kg}$ \\
Volume energy density & $389 \mathrm{Wh} / \mathrm{L}$ & $296 \mathrm{Wh} / \mathrm{L}$ & $5.73 \mathrm{Wh} / \mathrm{L}$ \\
Weight & $17 \mathrm{~g}$ & $22 \mathrm{~g}$ & $60 \mathrm{~g}$ \\
Volume & $9.3 \mathrm{~cm}^{3}$ & $12.8 \mathrm{~cm}^{3}$ & $53.0 \mathrm{~cm}^{3}$ \\
Self-discharge (per month) & $<10 \%$ & $<10 \%$ & $5.9 \% /$ day \\
Charge-discharge efficiency & $99.9 \%$ & $99.8 \%$ & $97 \%-98 \%$ \\
Memory effect & No & No & No \\
Charging method & pulse & pulse & trickle \\
\hline
\end{tabular}


types of energy storage. This means that the system with a Li-ion battery needs either a dedicated charge management chip or software control to correctly control the battery. Among micro-solar power systems, ZebraNet [ZSLM04], Prometheus [JPC05] and Trio $\left[\mathrm{DHJ}^{+} 06\right]$ used a Li-ion battery as its energy storage, and it charged the battery using software control.

A supercapacitor is a capacitor whose capacity is high enough to be used as energy storage for low-power electronic devices (usually higher than $1 \mathrm{~F}$ to $10 \mathrm{~F}$ ). While its capacity is still much smaller than other types of batteries, its very high maximum recharge cycles allows it to be used for long-lifetime applications. EverLast [SC06] used a supercapacitor for its energy storage.

Micro-solar power systems such as Prometheus [JPC05], Trio [DHJ+06] and AmbiMax [PC06] used multiple levels of storage that consist of a supercapacitor and a Li-ion battery. Such a system can take the benefits of both the supercapacitor and Li-ion battery: a long lifetime and large capacity with high efficiency. The charging management is more complicated for a hybrid storage system because it has to choose which storage to charge and when to charge. While the charging controller can be made in hardware (AmbiMax), it can also be made in software (Prometheus and Trio) by using the sensing and actuation capability of a sensor node.

\section{Categorized by Solar-Panel Operation}

Since the output power of a solar panel bounds the available energy for a microsolar power system, a well-designed system should keep the operating point of the solar panel closely to the maximum power point so that the maximum output of power can be transferred from the solar panel. The operating point of the solar panel is determined by either an input regulator or energy storage depending on whether an input regulator is present. We can categorize published designs of micro-solar power systems depending on how the operating point of the solar panel is determined.

First, most NiMH battery-based designs have the solar panel operating point set to the voltage level of the energy storage (Heliomote $\left[\mathrm{RKH}^{+} 05\right]$, Fleck $\left[\mathrm{CVS}^{+} 07\right]$, HydroWatch [TJC08]). These designs use the fact that the voltage range of the NiMH while charging is relatively narrow. They can achieve near-optimal performance by choosing the solar panel that has its maximum power point in the charging range of 
the NiMH battery.

Second, the operating point of the solar panel can be set to a fixed range as in ZebraNet [ZSLM04]. Compared to other micro-solar power systems, ZebraNet uses a solar panel that has a relatively low open-circuit voltage $(1.55 \mathrm{~V})$ and this makes the solar panel operating range for maximum power transfer narrow. ZebraNet sets the operating point of the solar panel to a fixed range of $1.3 \mathrm{~V}$ to $1.5 \mathrm{~V}$ using a comparator and an input regulator.

Third, the maximum power point tracking should be used for a supercapacitorbased system. This is because the supercapacitor has a wide operating range and its energy transfer is not efficient when the operating point of the supercapacitor is far from the maximum power point of the solar panel. EverLast [SC06] dynamically adjusts the operating point of the solar panel by controlling the input regulator through the micro-controller. In Everlast, the micro-controller periodically reads the opencircuit voltage of the solar panel by turning off the input regulator, and calculates the maximum power point of the solar panel based on the open-circuit voltage. Then, the micro-controller reads the solar panel operating point through an ADC and turns on or off the input regulator so that the solar panel operating point is maintained around the maximum power point. AmbiMax [PC06] also dynamically adjusts the operating point of the solar panel, but it uses the photo sensor and the comparator to control the input regulator.

Figure 2.5 summarizes the characteristics of several micro-solar power system platforms.

\subsubsection{Micro-solar Power System Models}

\section{Adaptive Workload Scheduling}

Some of the previous research has shown that duty-cycle [JPC05, RKH ${ }^{+} 05, \mathrm{KPS} 04$, KHZS07, VGB07, NLA+ 07], task scheduling [MBTB06a], or energy harvesting-aware programming language $\left[\mathrm{SKG}^{+} 07\right]$ could be adjusted dynamically, depending on the environment, in order to achieve higher utilization and meet the scheduling deadlines.

Jiang et al. [JPC05] showed a simple duty-cycling scheme that changes the dutycycle depending on the solar level. However, their work just showed a proof of concept 


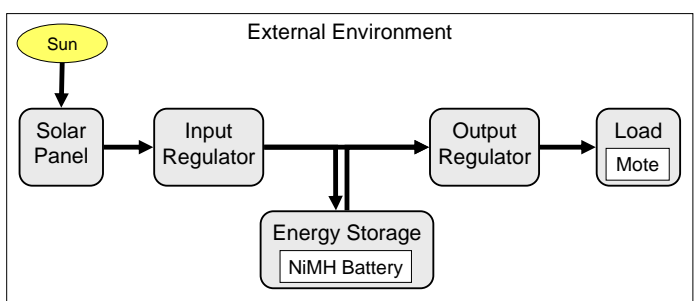

(a) System on NiMH battery

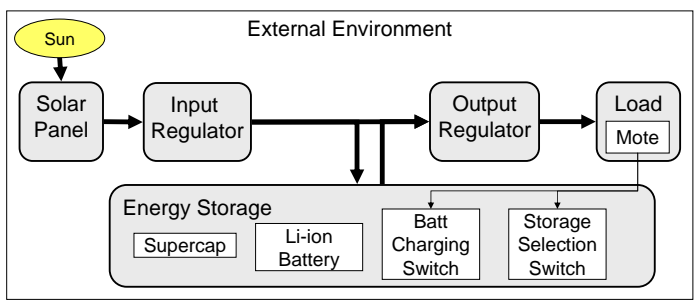

(c) System on supercap and Li-Ion battery

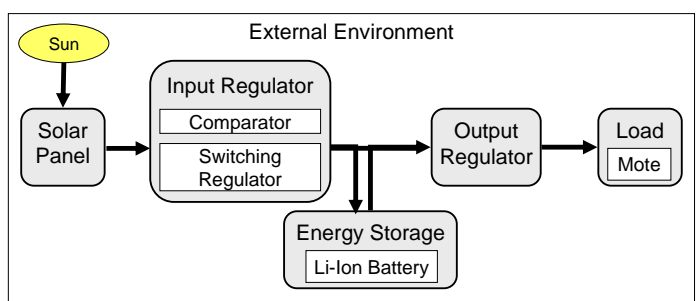

(b) System on Li-Ion battery

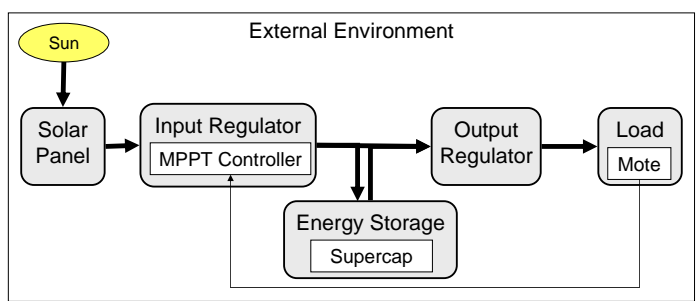

(d) System on supercap with SW-controlled MPPT

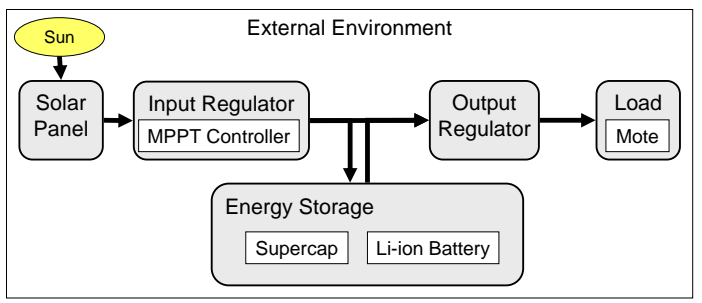

(e) System on supercap and Li-ion battery with HW-controlled MPPT

\begin{tabular}{|c|c|c|c|}
\hline & Examples & $\begin{array}{l}\text { Energy } \\
\text { Storage }\end{array}$ & $\begin{array}{lll}\text { Solar } & \text { Panel } & \text { Operating } \\
\text { Point } & & \\
\end{array}$ \\
\hline (a) & $\begin{array}{l}\text { Heliomote }\left[\mathrm{RKH}^{+} 05\right] \\
\text { Fleck }\left[\mathrm{CVS}^{+} 07\right] \\
\text { HydroWatch [TJC08] }\end{array}$ & $\mathrm{NiMH}$ & Depends on battery voltage \\
\hline (b) & ZebraNet [ZSLM04] & Li-ion & $\begin{array}{l}\text { Fixed range controlled by com- } \\
\text { parator and switching regulator }\end{array}$ \\
\hline (c) & $\begin{array}{l}\text { Prometheus [JPC05] } \\
\text { Trio }\left[\mathrm{DHJ}^{+} 06\right]\end{array}$ & $\begin{array}{l}\text { Supercap and } \\
\text { Li-ion }\end{array}$ & Operating range set by software \\
\hline (d) & Everlast [SC06] & Supercap & $\begin{array}{l}\text { MPPT controlled by software } \\
\text { and switching regulator }\end{array}$ \\
\hline (e) & AmbiMax [PC06] & $\begin{array}{l}\text { Supercap and } \\
\text { Li-ion }\end{array}$ & $\begin{array}{l}\text { MPPT controlled by compara- } \\
\text { tor and switching regulator }\end{array}$ \\
\hline
\end{tabular}

Figure 2.5: Comparison of micro-solar power system platforms 
without formulating the relation between the desired duty-cycle rate and the corresponding system parameters. Moser et al. [MBTB06b] showed an algorithm that schedules tasks to meet deadlines within the constraint of varying energy supply and storage. However, their algorithm has drawbacks in that it assumes non-ideal perfect storage (no leakage and non-ideal storage round-trip efficiency) and the results were shown only in simulation, with no consideration of realistic energy harvesting devices. Kansal et al. [KPS04] showed a bound rule for sustainable operation of energy-harvested nodes. They demonstrated the sustainable operation of a single node using Heliomote by setting its parameters according to the bound rule. But, in the case of multiple nodes, they just showed the concept without demonstration. Kansal et al. [KHZS07] proposed an adaptive duty-cycling that tries to achieve maximum utility of an energy harvesting system, and showed that their algorithm had its energy utilization very close to the optimum value that could be achieved with complete knowledge of energy supply and consumption in the future. This paper demonstrated adaptive duty-cycling using two example cases (field monitoring and event monitoring).

\section{Simulating Power Consumption of Load}

There are several sensor network simulators that can estimate power consumption of sensor nodes. PowerTOSSIM [SHC ${ }^{+}$4], SensorSim [PSS00, PSS01], Prowler [SVML03], SENS [SKA04] and AEON [LWG05] are such examples. These simulators are similar to each other in that they execute a sensor network application and estimate power consumption based on the pre-recorded energy consumption profile of primitive operations on the target sensor node platform. On the other hand, they differ in simulation platform, target sensor node platform, source base and extensibility. The characteristics of some sensor network power simulators are summarized in Table 2.2.

When we choose a sensor network power simulator, we are interested in two factors: reality and extensibility. The result from a simulator wouldn't be meaningful if the simulator did not reflect real sensor networks. In this sense, we prefer a simulator that takes an actual application program code and simulates the corresponding power consumption. PowerTOSSIM $\left[\mathrm{SHC}^{+} 04\right]$ and AEON [LWG05] are such examples. 
Table 2.2: Summary of sensor network power simulators

\begin{tabular}{|l|l|l|l|l|l|}
\hline & $\begin{array}{l}\text { Power- } \\
\text { TOSSIM } \\
{[\text { SHC }+4]}\end{array}$ & $\begin{array}{l}\text { Sensor- } \\
\text { SIM } \\
{[\text { PSS00, }} \\
\text { PSS01 }]\end{array}$ & $\begin{array}{l}\text { Prowler } \\
\text { [SVML03 }]\end{array}$ & $\begin{array}{l}\text { SENS } \\
{[\text { SKA04 }]}\end{array}$ & $\begin{array}{l}\text { AEON } \\
{[\text { LWG05] }}\end{array}$ \\
\hline $\begin{array}{l}\text { Simulation } \\
\text { Platform }\end{array}$ & $\begin{array}{l}\text { TOSSIM } \\
{[\text { LLWC03 }]}\end{array}$ & ns-2 & Matlab & C++ & $\begin{array}{l}\text { AVRORA } \\
{[\text { TLP05] }}\end{array}$ \\
\hline $\begin{array}{l}\text { Reference } \\
\text { Hardware }\end{array}$ & Mica2 & $\begin{array}{l}\text { WINS, } \\
\text { Medusa }\end{array}$ & Mica & $\begin{array}{l}\text { Mica2, } \\
\text { Medusa }\end{array}$ & Mica2 \\
\hline $\begin{array}{l}\text { Simulated } \\
\text { Entities }\end{array}$ & $\begin{array}{l}\text { Power } \\
\text { consumption }\end{array}$ & $\begin{array}{l}\text { Power } \\
\text { consumption } \\
\text { Network } \\
\text { throughput } \\
\text { Battery } \\
\text { lifetime }\end{array}$ & $\begin{array}{l}\text { Power } \\
\text { consumption } \\
\text { Network } \\
\text { stack }\end{array}$ & $\begin{array}{l}\text { Power } \\
\text { consumption } \\
\text { Radio propa- } \\
\text { gation }\end{array}$ & $\begin{array}{l}\text { Power } \\
\text { consumption }\end{array}$ \\
\hline Extensibility & $\begin{array}{l}\text { TinyOS } \\
\text { system }\end{array}$ & $\begin{array}{l}\text { Not } \\
\text { restricted }\end{array}$ & $\begin{array}{l}\text { Not } \\
\text { restricted }\end{array}$ & $\begin{array}{l}\text { Not } \\
\text { restricted }\end{array}$ & $\begin{array}{l}\text { Atmel-based } \\
\text { TinyOS sys- } \\
\text { tem }\end{array}$ \\
\hline
\end{tabular}

They take the same TinyOS application code that is used for real sensor nodes. Extensibility is also preferable because it allows a sensor network power simulator to be used for a set of evolving sensor network platforms. A simulator that is based on a generic sensor platform can easily accomplish this by changing the platform-specific parameters (e.g. SensorSIM [PSS00, PSS01], Prowler [SVML03], SENS [SKA04]). PowerTOSSIM, which is based on an event-level simulator TOSSIM, can be extended to a new platform by replacing the energy estimation mapping table. A simulator that is based on a specific instruction architecture (e.g. AEON) can estimate the power consumption more closely to real sensor nodes, but it requires major changes for different sensor platforms.

\section{Simulating Solar Radiation}

As a way to estimate solar radiation, a software suite called Meteonorm [Met] can be used. Its meteorological database covers over 30 years of solar radiation measurements from a number of locations around the world. If a location is not in the database, Meteonorm estimates its approximate solar radiation based on its geographic characteristics (latitude, longitude and altitude), and matches it to the data of previously known locations. Meteonorm provides different time granularity (month, day, hour) when it estimates solar radiation. Depending on the responsive- 
ness of the application, solar radiation estimates of suitable time granularity can be used.

With an astronomical model, we estimate the solar radiation using parameters that affect the angle between the sunlight and the solar panel. When the angle of sunlight from the normal to the solar panel is $\Theta$, the effective sunlight that shines on the solar panel is proportional to $\cos \Theta$ [DHM75]. The angle $\Theta$ depends on solar-panel inclination $\theta_{p}$, panel orientation $\phi_{p}$, latitude $L$, time of the day $t$, and day of the year $n$.

\section{Simulating the Battery}

A couple of research groups proposed a way to estimate the battery capacity or lifetime for WSNs [PSS00, PSS01, VXSB07]. Although these are built to model the discharge rate of a non-rechargeable battery, they can be extended for microsolar power systems by considering the charging profile of the battery as well as the discharging profile. These battery simulators vary in terms of their functions and complexity.

SensorSim by Park et al. [PSS00], which is a network-level simulator of a WSN, can also simulate energy-related metrics such as the capacity of a battery. It simulates the capacity of a battery using a linear model, which means the amount of energy that is drawn from the battery is proportional to the total aggregate power of a sensor node. SensorSim was extended to reflect different types of battery models in the follow-on paper [PSS01]: a linear model, a discharge rate-dependent model and a relaxation model. With the discharge-rate-dependent model, the battery capacity depends not only on the time duration, but also on the discharge rate. With this model, the charge the battery can drive is close to the initial charge at low current draw, and it becomes smaller as the current draw becomes higher. The extended version of SensorSim also reflects the relaxation model. With the relaxation model, the battery capacity decreases sharply under a heavy load, but recovers if the current draw becomes low again. sQualNet [VXSB07] by Varshney et al. is another networklevel simulator for WSN. sQualNet proposes two techniques for modeling battery capacity. First, they propose using polynomial functions to calculate the battery capacity. Second, they propose using a small recent history, rather than the entire 


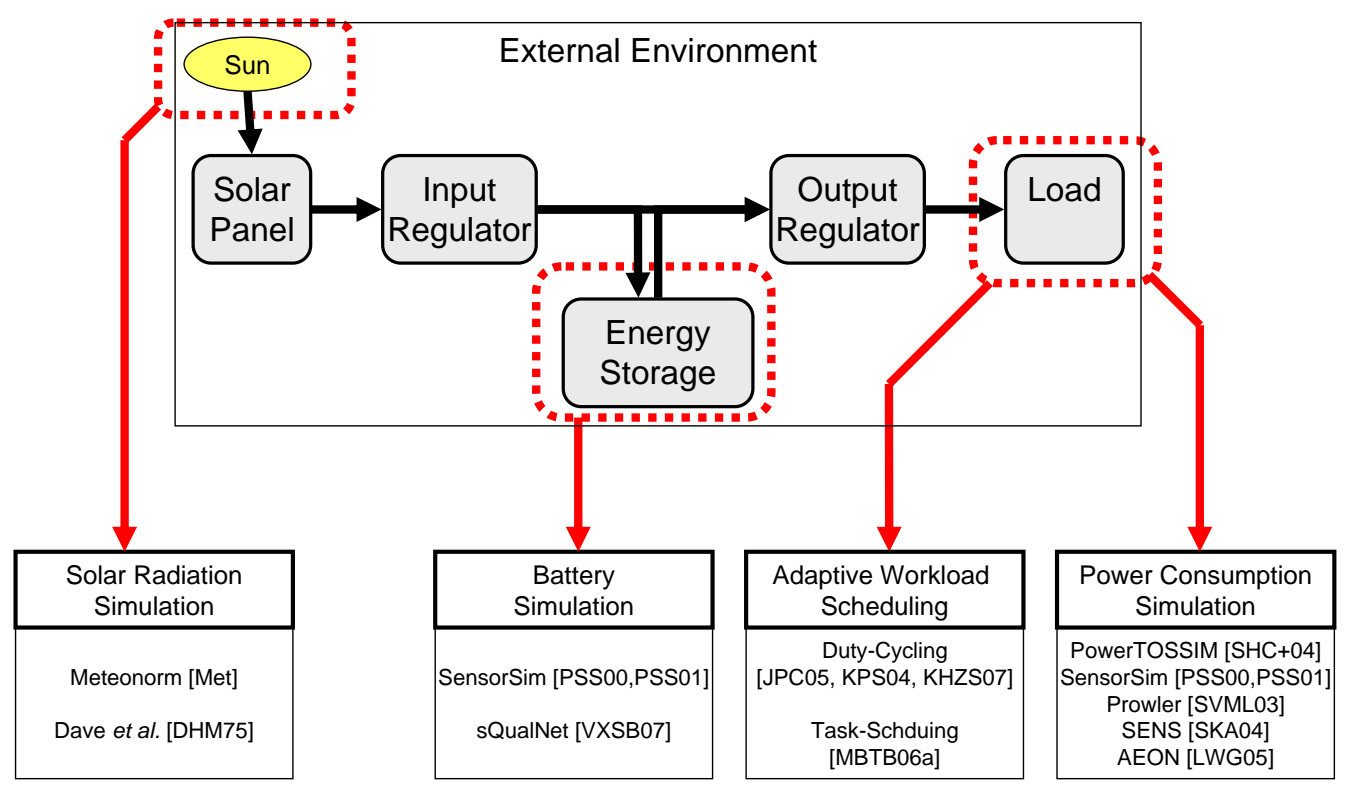

Figure 2.6: Previous works on micro-solar power system models

battery history. This reduces the memory requirements.

Figure 2.6 summarizes previous works on micro-solar power system models in relation to the general model of micro-solar power systems.

\subsubsection{Relation to Macro-Solar Power Systems}

We can categorize the solar energy harvesting systems by the output power of the solar panel. Macro-solar and meso-solar power systems are commercial and residential solar energy harvesting installations, and use a scale of kilowatts and watts. A micro-solar power system, however, is a small, low-power electronics with a solar panel of less than 1 Watt of power. Macro-solar and meso-solar systems have the same basic component categories and the same interconnection as a micro-solar power system. The difference is the relative sizes, which translates into differences in the design and deployment of a micro-solar power system. First, with a micro-solar power system, the load is on the same scale as the management of the system, and the system as a whole has to be very efficient and well-matched. Second, with a microsolar power system, the device is located where the measurement must be taken, not where the sun is best. Thus, a micro-solar power system should be planned for the 
environment that exists, rather than be planned for the environment that is desired. There are many calculators for macro-solar power systems [Nat,fin,Iow,Sun,Cal, Wea]. Concepts from these tools can be applied to micro-solar power systems: they compose a system as a collection of several components and their interconnections; and they estimate solar radiation using an astronomical model. However, they are not suitable for modeling the dynamics of micro-solar power systems due to the following reasons. First, each component is represented as a single number rather than a function or a curve. This approach may predict the average or maximum performance, but cannot predict the varying performance with different operating points. Second, due to economic reasons, these macro-solar tools assume that energy surpluses are sold to the grid rather than accumulated into energy storage. However, because micro-solar power systems are often placed where grid power is not available, micro-solar systems generally require energy storage, breaking the assumption used in these macro-solar tools.

\subsection{Refined Problem Statement}

As stated in the previous chapter, building a micro-solar power system has four main challenges: large design space, long time-scale, different environments for development and deployment sites, and variability of solar energy. In this dissertation, we provide a practical theory of micro-solar power systems to address these problems and guide specific design choices. It presents a general architecture of micro-solar power systems describing key components and interconnections among the components, and it formalizes each component of the architecture in either an analytical or empirical model of its behavior. Finally, it incorporates these component models and their interconnections in a simulation suite.

We take a modular approach to modeling micro-solar power systems over a large design space. As shown in Figure 2.7, a micro-solar power system consists of the six components - external environment, solar panel, energy storage, load, input regulator and output regulator - and its design space is a cross-product of all the possible choices for its components. In order to abstract such a large diversity in a single framework, the simulation suite represents each component of a micro-solar power 
system as a module, describing the behavior of each component with a formal model either analytically or empirically.

Our simulation suite has a discrete time-event architecture to simulate a microsolar power system in a long time-scale. With our simulation tools, the daily behavior of a micro-solar power system is simulated by iteratively evaluating the state of the system for the given initial condition and the time interval vector. Similarly, the longterm behavior is simulated by iteratively evaluating the daily behavior. Assuming that the complexity of each module being simulated is constant for a given architecture, the simulation time is determined by the granularity of the time interval vector. Since the goal is the estimation of a long-term behavior, our simulation suite sets the time-scale so that it can estimate the system behavior both efficiently and accurately.

Our simulation suite provides solar radiation models that can estimate solar radiation with sufficient accuracy even under long-term weather variation and obstruction effect to model the variability of solar energy and simulate the environment for deployment sites at development time. Our solar radiation models are based on an astronomical model that estimates solar radiation in an ideal condition using the angle between the sunlight and the normal to the solar panel. An obstructed astronomical model, which models the solar radiation under the obstruction effect, refines the estimation from the astronomical model using an empirical measurement on previous days. To model the solar radiation under long-term weather variation, we used the difference between the estimation from an obstructed astronomical model and a long-term measurement result from the reference platform. This difference, which is about $30 \%$ in an urban rooftop environment, implies that the solar radiation estimation needs to be adjusted by this amount for estimating the solar radiation under long-term weather variation.

With our simulation suite, a micro-solar power system can be designed in a systematic way. A design candidate of micro-solar power systems can be composed by putting together the corresponding components into a single simulation set. Then, it is simulated with the given constraints, and is considered as a plausible design only when it passes the simulation. A plausible design candidate can be built and evaluated. Additionally, this can be fed to the simulation tools to refine the estimation quality for the design. 
Hypothetical Design

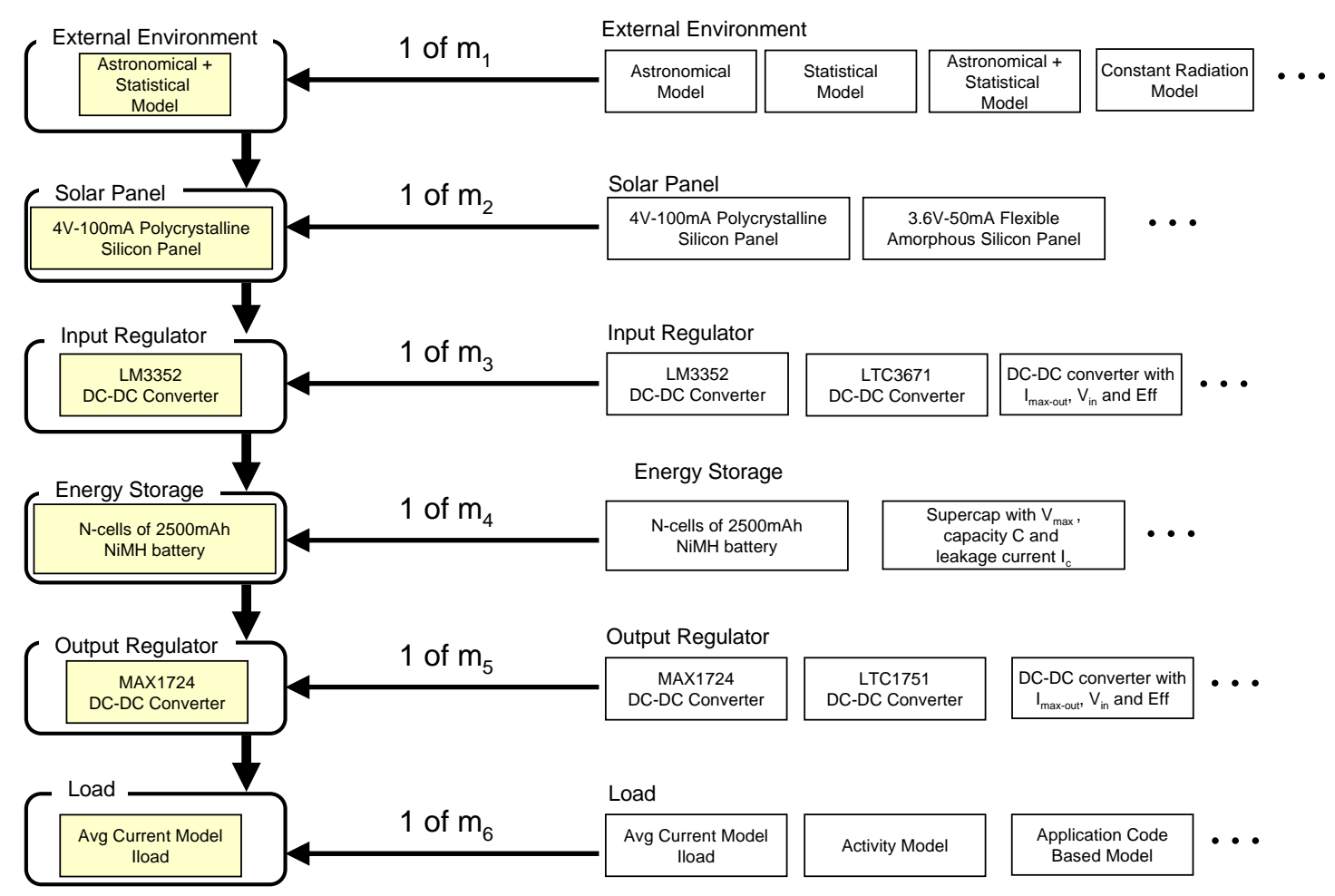

Figure 2.7: Design space of micro-solar power systems and its relationship with a hypothetical design 


\section{Chapter 3}

\section{Architecture of Micro-solar Power Systems}

This chapter presents an architecture of micro-solar power systems describing the characteristics of its key components and the relationships among the components. Then, it provides an empirical analysis of two well-studied designs as concrete examples of micro-solar power systems, and finally it summarizes the proposed architecture.

\subsection{Overall Architecture of Micro-solar Power Sys- tems}

In general, any solar-powered system consists of the following six components: external environment, solar panel, input regulator, energy storage, output regulator and load (Figure 3.1). The solar energy from the environment is collected by the solar collector and is made available for the operation of the load. The energy storage is used to buffer the varying energy income and distribute it to the load throughout the duration. The input regulator can be used to adjust the mismatch between the operating range of the solar panel and the energy storage, while the output regulator is used to shape the operating range of the energy storage to that of the load. The design decisions for each component will dictate the energy flow between them and the overall behavior of the system.

In the rest of this chapter, we describe the architecture of a micro-solar power 


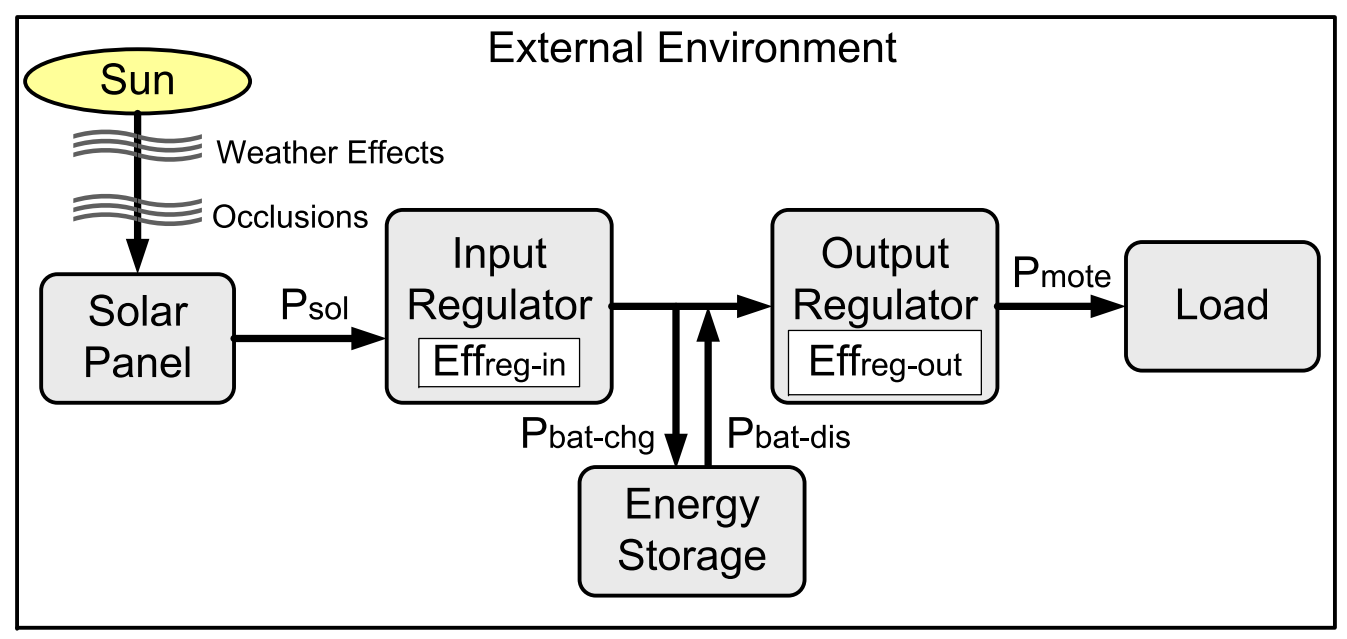

Figure 3.1: Model for a solar-powered sensor system

Table 3.1: Evaluation metrics

\begin{tabular}{|l|l|}
\hline Metric & Description \\
\hline$P_{\text {solar-in }}$ & Total incident solar radiation. \\
\hline$P_{\text {sol }}$ & Power produced by the solar panel. \\
\hline$P_{\text {storage-in }}$ & Power collected by the solar-collector. \\
\hline$P_{\text {bat-chg }}$ & Power stored in the energy storage. \\
\hline$P_{\text {bat-dis }}$ & Power extracted from the energy storage. \\
\hline$P_{\text {mote }}$ & Energy consumed by the mote. \\
\hline Eff $_{\text {reg-in }}$ & Efficiency of the input regulator. \\
\hline Eff $_{\text {reg-out }}$ & Efficiency of the output regulator. \\
\hline
\end{tabular}

system in terms of the energy flow of each component. Section 3.1.1 shows how to estimate solar radiation $\left(P_{\text {solar-in }}\right)$ using statistical and mathematical methods. Section 3.1 .2 discusses the factors that affect the solar panel operating point $\left(P_{\text {sol }}\right)$. Section 3.1.3 discusses the effect of input-power conditioning $\left(\right.$ Eff $\left._{r e g-i n}\right)$. Section 3.1.4 discusses the factors that affects the storage capacity and the lifetime. Section 3.1.5 discusses the effect of output-power conditioning $\left(\mathrm{Eff}_{r e g-o u t}\right)$. Section 3.1.6 explains how to model the energy consumption of a mote $\left(P_{\text {cons }}\right)$ with radio duty-cycling. Table 3.1 lists the metrics that will be used throughout this chapter. 


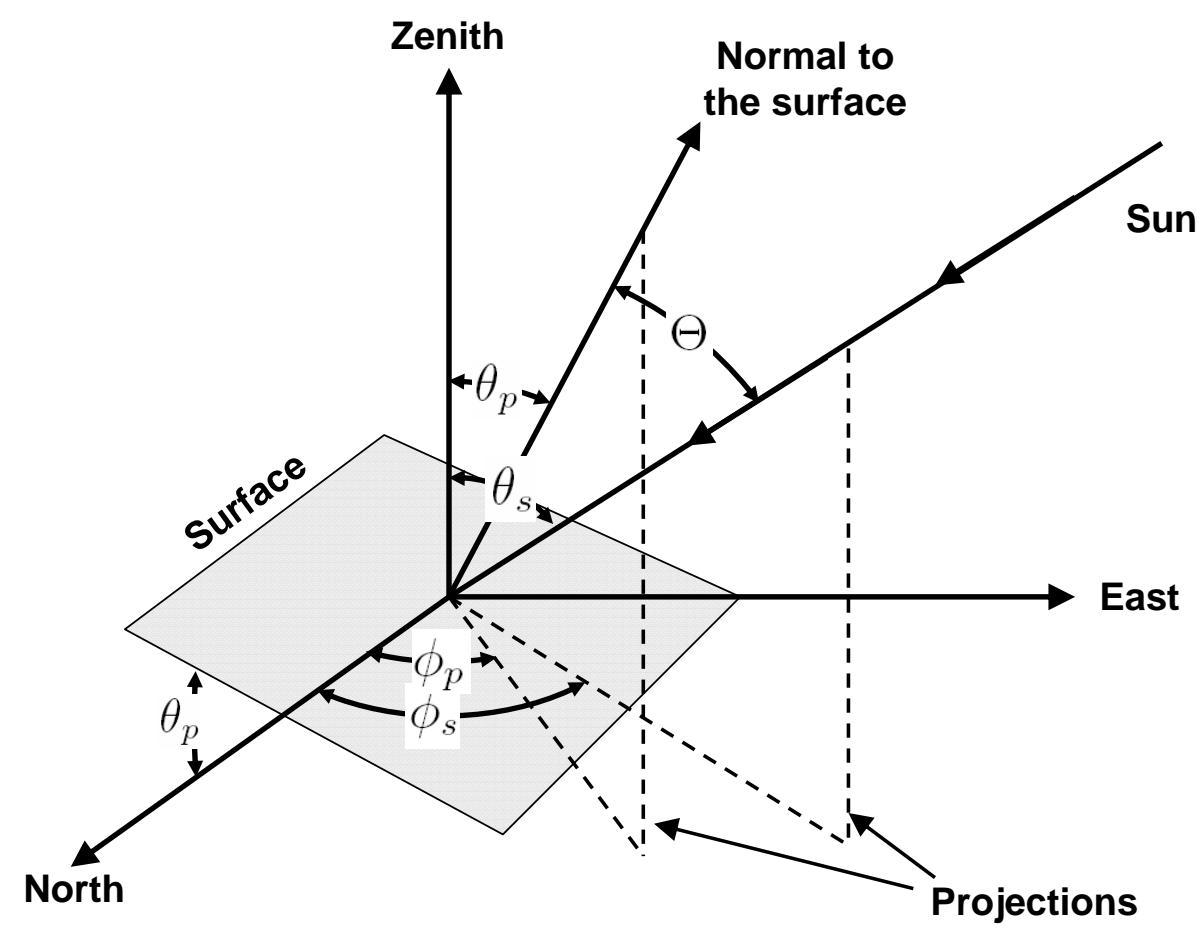

Figure 3.2: Definition of the angles used in astronomical method - courtesy of Dave et al.

\subsubsection{External Environment}

The amount of solar radiation $P_{\text {solar-in }}$ depends on the environment, and places an upper bound on the maximum energy output of the solar collector $P_{\text {sol }}$. Most previous designs of micro-solar power sensor systems did not carefully model solar radiation: they either paid little attention to the available solar energy or considered a constant average solar radiation $\left[\mathrm{ROC}^{+} 03, \mathrm{SC} 06, \mathrm{JPC} 5, \mathrm{DHJ}^{+} 06, \mathrm{SCV}^{+} 06, \mathrm{RKH}^{+} 05\right]$. Kansal et al. [KPS04] proposed a rule for perpetual operation, which stated a relationship between the node consumption and the energy budget. However, this rule considered a generic energy input and did not provide a way to estimate the energy budget for solar energy harvesting. In this dissertation, we describe three ways to estimate solar radiation: (a) an astronomical method, (b) an astronomical method with history of weather effects, (c) an astronomical method with local enhancements.

With an astronomical model, we estimate the solar radiation using the parameters that affect the angle between the sunlight and the solar panel. According to Dave 
et al. [DHM75] (Figure 3.2), the effective sunlight that shines on the solar panel is proportional to $\cos \Theta$ when the angle of sunlight from the normal to the solar panel is $\Theta$, The angle $\Theta$ depends on solar panel inclination $\theta_{p}$, panel orientation $\phi_{p}$, latitude $L$, time of the day $t$, and day of the year $n$ :

$$
\begin{aligned}
\cos \Theta= & \cos \theta_{p} \cdot \cos \theta_{s}+\sin \theta_{p} \cdot \sin \theta_{s} \cdot \cos \left(\phi_{p}-\phi_{s}\right) \\
\cos \theta_{s}= & \sin \delta \cdot \sin L+\cos \delta \cdot \cos L \cdot \cos h \\
\sin \phi_{s}= & -\cos \delta \cdot \sin h / \sin \theta_{s} \\
x= & 2 \pi n / 365 \\
h= & 15(t-12) \\
\delta= & 0.302-22.93 \cos x-0.229 \cos 2 x-0.243 \cos 3 x \\
& \quad+3.851 \sin x+0.002 \sin 2 x-0.055 \sin 3 x
\end{aligned}
$$

With an astronomical method with history of weather effects, we estimate the solar radiation under the influence of weather effects by using the history of solar radiation measurements. In order to get such a history, we can use a meteorological database software suite such as Meteonorm [Met], which estimates solar energy radiation as a monthly solar radiation $E_{\text {month }}\left(\mathrm{kWh} / \mathrm{m}^{2}\right)$. Using $E_{\text {month }}$, we can calculate the peak solar hours (PSH), which is the equivalent number of solar radiation hours per day assuming that the same amount of solar energy is given at an uniform intensity of $1 \mathrm{~kW} / \mathrm{m}^{2}$. Then, the available energy from a specific solar panel for one day, $E_{\text {sol-day }}$, can be estimated as the product of the PSH and the solar panel output power $P_{\text {panel }}$ at $1 \mathrm{~kW} / \mathrm{m}^{2}$ (usually provided by its manufacturer):

$$
E_{\text {sol-day }}=\mathrm{PSH} \cdot P_{\text {panel }}=\frac{E_{\text {month }} \cdot P_{\text {panel }}}{1 \mathrm{~kW} / \mathrm{m}^{2} \cdot \# \text { days }}
$$

As well as the weather effects, occlusions from local objects such as trees and buildings can affect solar radiation. With an astronomical method with local enhancements, we refine the solar radiation estimate from the astronomical method with a measurement of local obstructions. 

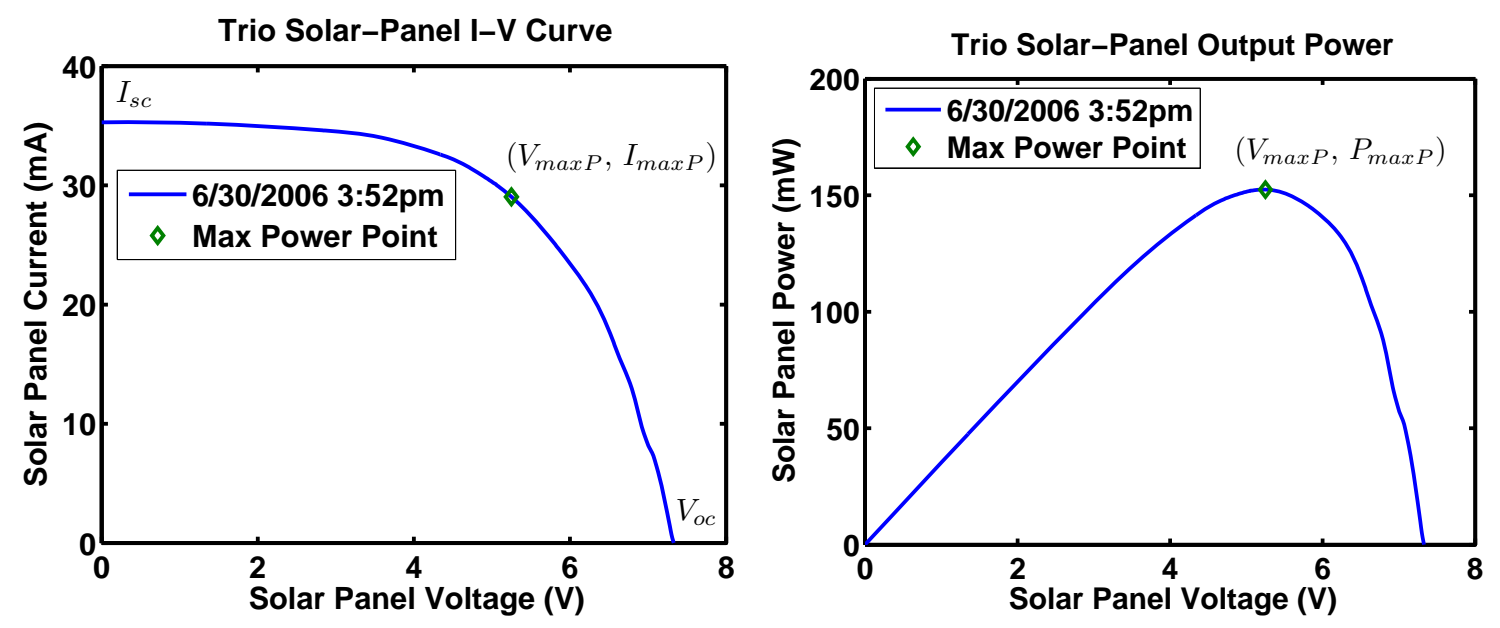

Figure 3.3: Characteristics of a solar panel: (a) I-V curve, (b) P-V curve with MPP

\subsubsection{Solar Collector}

Solar energy from the environment is converted to electric energy by the solar collector, which includes a solar panel and a regulator. The solar panel converts photons into electricity and the regulator conditions the output power of the solar panel for energy transfer to the storage. The amount of solar power out of the solar collector $P_{\text {sol }}$ is determined by the following factors: (1) solar radiation, (2) solar-panel characteristics, (3) the operating point of the solar-panel.

The solar panel I-V curve describes how the output current of a solar panel behaves at a certain radiation condition. (Figure 3.3). The current of a solar panel is at a maximum when the impedance across the two terminals of the solar panel is zero, and the current at the zero impedance is called the short circuit current $\left(I_{s c}\right)$. As the impedance increases, the current from the solar panel decreases and the voltage across the two terminals of the solar panel increases. When the impedance becomes infinite, the current becomes zero. The solar panel voltage at this point is called the open circuit voltage $\left(V_{o c}\right)$. In order to deliver the highest power from the solar panel, we need to operate the solar panel near the optimum point. This optimum point $\left(V_{\max P}, I_{\max P}\right)$ is called the maximum power point $(\mathrm{MPP})$, and the power at the maximum power point is no less than the power at any other points $(V, I)$ :

$$
V_{\max P} \cdot I_{\max P} \geq V \cdot I \text { for any }(V, I)
$$


The solar-panel I-V characteristic also depends on the radiation condition. As the solar irradiance increases or decreases, the I-V curve moves outwards or inwards. Thus, a solar panel can be described as a sequence of I-V curves with each I-V curve corresponding to a particular solar irradiance condition. Figure 3.4 shows I-V curves for a solar panel at different times of day. Each I-V curve in Figure 3.4 is drawn by fitting an exponential curve to 20 sample points. We notice that some of the I-V curves are non-monotonic even though they are not expected to overlap. This is an artifact of sampling errors and curve fitting. The current of an I-V curve around the open-circuit voltage is much lower than the peak value and even a small amount of sampling error in the open-circuit voltage region can change the shape of an I-V curve.

In order to maximize the power transfer from the solar panel, it is recommended that the regulating circuit operate near the maximum power point (MPP), which is the point in the $\mathrm{I}-\mathrm{V}$ curve where $P=I \cdot V$ is maximized. As a way of achieving maximum power transfer, a maximum power point tracker can be used (e.g. Everlast $[\mathrm{SC06}])$. An active circuit is usually required when the energy signal is near DC. While this may sound like an attractive option, care needs to be taken when using it in practice. Since the power a sensor node operates at is usually very small (in the $\mathrm{mW}$ range), the energy consumed by the maximum power point tracker becomes significant, and sometimes more than what it can save. Figure 3.5 compares the solar panel output power of the Trio node at its operating point with the output power that would have been achieved with the maximum power point tracking. Notice that at the end of day, the daily cumulative solar radiation for each case is 2,666 J and 3,055J. The difference, 389J, is $13 \%$ of the daily solar radiation at the MPP. Using a maximum power point tracker is meaningful when its daily energy consumption is smaller than this.

\subsubsection{Input-power Conditioning}

In a micro-solar power system, an input regulator can be used to set the specific operating point of the solar panel to meet the operational constraints of the particular energy storage using voltage limits, current limits, and charge duration. While matching the operating points of the solar panel and the energy storage is an advan- 


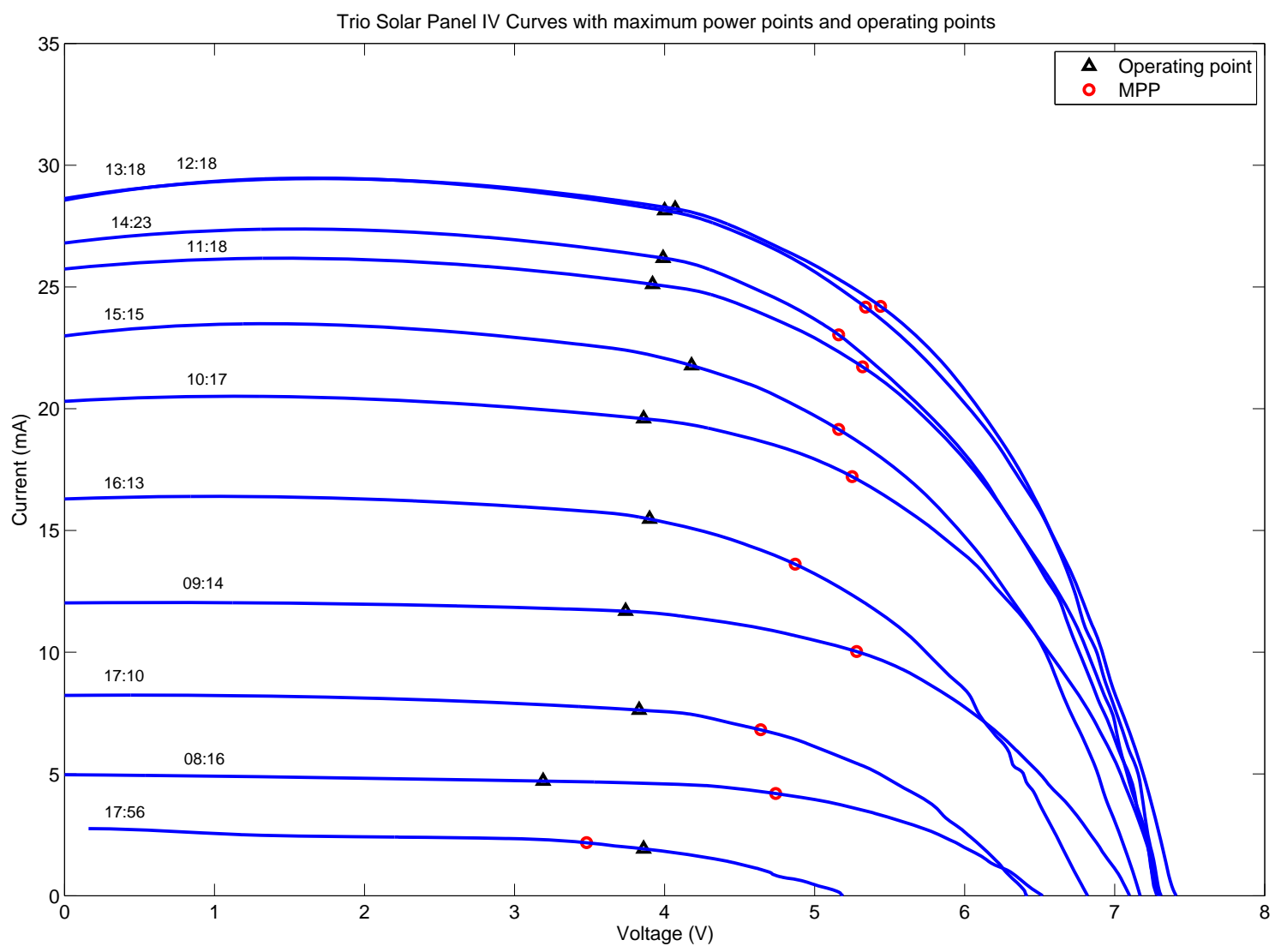

Figure 3.4: A series of IV characteristics for a poly-crystalline solar panel. The dimension of this panel is $67 \mathrm{~mm} \times 37 \mathrm{~mm}$ and its rated current and voltage are $30 \mathrm{~mA}$ and $6.7 \mathrm{~V}$. The operating point on each IV curve corresponds to the voltage and the current of the solar panel that is being used in a Trio node. 

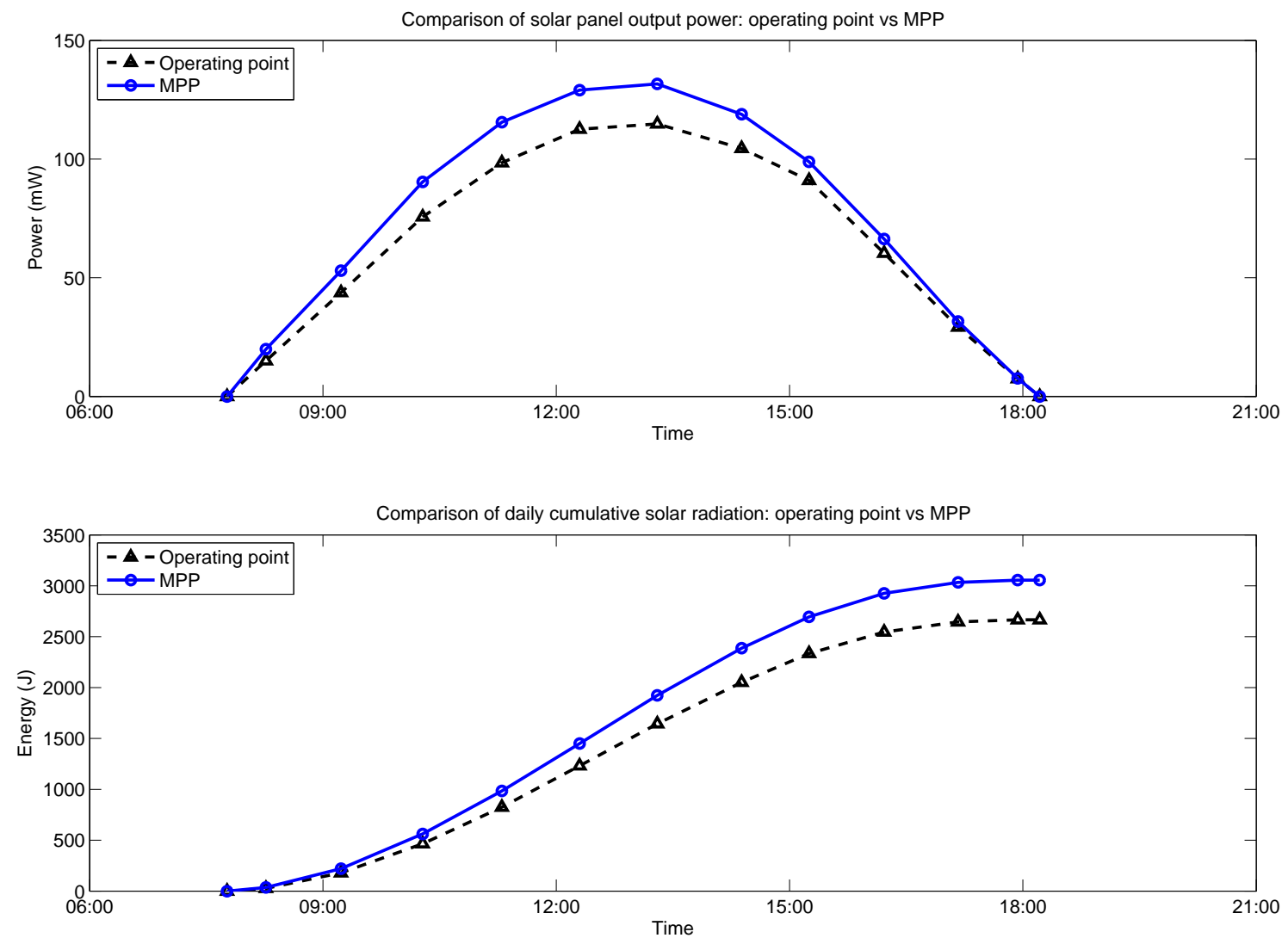

Figure 3.5: Comparison of solar panel output power and cumulative solar radiation for solar panel at operating point and MPP with a Trio node. 
tage of using an input regulator, the sub-unity efficiency of the input regulator is a

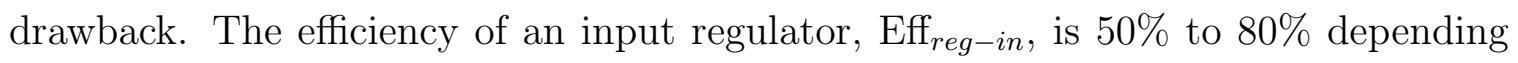
on the part being used. Thus, the analysis of the possible gain of the operating point matching against the inefficiency of the input regulator should be made before it is used in a micro-solar power system.

\subsubsection{Energy Storage}

Energy storage is the group of storage elements used to buffer the energy coming from the solar collector and to deliver it to the mote in a predictable fashion. Energy storage can consist of any number of storage elements grouped together in some configuration. The challenge is to find a desirable configuration and combination of storage elements so that the requirements are met.

In general, designing the energy storage involves choosing the storage elements and charging mechanism for correct operation and efficient energy transfer. As a first step of storage design, a system designer should consider system requirements as follows:

- Lifetime: The lifetime of the energy storage is determined by the maximum charge or discharge cycles and depth of discharge. The lifetime should be long enough to avoid frequent replacement.

- Capacity: The energy storage should be large enough to support the operation of the end device under the disruption of solar energy. The capacity of the storage should be proportional to the blackout period that the storage is desired to support. It is important to realize that the energy input is not a constant, and therefore the buffers need to be sized appropriately to accommodate environmental variations. Other than nominal capacity of the storage element, self discharge rate and memory effects also affect the storage capacity. The effective storage capacity should be corrected for these factors:

- Current draw: The energy storage should also be able to support a short burst of power (the maximum current draw of the end device). 
- Size and weight: A system designer should pick energy storage elements that meet the requirement of the application.

Table 2.1 in Section 2.2.1 lists the characteristics of different storage elements. For micro-solar power systems, a NiMH battery, Li-ion / Li-polymer or supercapacitor is desirable. The NiCd battery is not preferred due to its smaller energy density, memory effects and harmful environmental effects. Depending on the priority of requirements: NiMH battery (capacity and cost), Li-ion / Li-polymer (high energy density and capacity), or supercapacitor (lifetime) can be chosen.

Configuring the connection between the power supply and the energy storage, and the connection between the energy storage and the mote presents another opportunity for system optimization. More importantly, different configurations of energy storage elements allow greater freedom in how to best satisfy the various requirements stated above. Compared to using a single type of storage element, a combination of storage elements with different capacities has a desirable characteristic: short, frequent disruptions are buffered by a smaller capacity storage, while larger but less frequent disruptions are buffered by a larger capacity storage. In this way, small disruptions do not affect the lifetime of a larger storage element, which may often have fewer charge cycles. A side effect of two-level storage is additional energy loss caused by sub-unity round-trip efficiency of an energy storage element, so the charging mechanism should be designed to minimize this.

The charging mechanism is another factor that affects system performance. Depending on the decisions in the charging mechanism (e.g. when and how much), the amount of energy that can be transferred to the storage can vary. Different energy storage types have different charging profiles, and this affects the complexity of the charging mechanism. For example, a NiMH battery has a simple charging profile: it can be charged with continuous small current, whereas a Li-ion battery may require constant-current $(\mathrm{CC})$ followed by constant-voltage $(\mathrm{CV})$. Depending on the system requirement, a system designer can make a trade-off between complexity and more efficient energy transfer. 


\subsubsection{Output Power Conditioning}

In a micro-solar power system, an output regulator can be used to condition the output of the energy storage to meet the operational voltage range of the load. When the operational range of the load is different from that of the energy storage, there is a chance that the load may not run, even though some energy is still available in the energy storage. Let us take an example from the HydroWatch node, which will be explained in detail in Chapter 5. The load on the HydroWatch node, the TelosB node, operates between $2.1 \mathrm{~V}$ to $3.6 \mathrm{~V}$ for normal operation (micro-controller and radio communication) and between $2.7 \mathrm{~V}$ to $3.6 \mathrm{~V}$ for flash memory access. However, part of this range is outside the operating range of the energy storage. The HydroWatch nodes uses two NiMH batteries (NH15-2500) in series, and their operating voltage is $1.8 \mathrm{~V}$ to 2.8 during discharging and $2.566 \mathrm{~V}$ to $2.966 \mathrm{~V}$ during charging. This implies that the TelosB node cannot access its flash memory while most of the energy is still in the NiMH battery.

An output regulator has a wider operating input voltage than the load, and it shapes the output voltage of the energy storage to fit within the operating voltage of the load most of the time. For example, the LTC1751-3.3 regulator can operate with input voltage between $2 \mathrm{~V}$ to $4.4 \mathrm{~V}$ and the MAX1724-3.3 regulator can operate between $0.91 \mathrm{~V}$ to $5.5 \mathrm{~V}$; in both cases the output power of the output regulator is smaller than the output power from the energy storage. For example, the LTC1751-3.3 regulator has efficiency Eff $_{\text {reg-out }}$ of around 60\% and the MAX1724-3.3 regulator has efficiency of $80 \%$. This sub-unity efficiency is the cost for using an output regulator. Table 3.2 summarizes the operating range for some examples of the energy storage, the load, and the output regulator.

Another reason to use an output regulator is to provide a constant supply of voltage for sensing applications. The ADC readings, which depend on the supply voltage of the sensor node as an internal reference voltage, can vary greatly if the supply voltage swings a lot. Having an output regulator makes the supply of voltage to the sensor node near-constant, which can improve the quality of the ADC readings. 
Table 3.2: Operating range of energy storage, load, and output regulator

\begin{tabular}{|l|l|l|}
\hline & Min & Max \\
\hline TelosB & $2.1 \mathrm{~V}$ & $3.6 \mathrm{~V}$ \\
TelosB with flash memory access & $2.7 \mathrm{~V}$ & $3.6 \mathrm{~V}$ \\
\hline Two NH15-2500 during discharge & $1.8 \mathrm{~V}$ & $2.8 \mathrm{~V}$ \\
Two NH15-2500 during charge & $2.566 \mathrm{~V}$ & $2.966 \mathrm{~V}$ \\
\hline LTC1751-3.3 (Quiescent current 20uA) & $2 \mathrm{~V}$ & $4.4 \mathrm{~V}$ \\
MAX1724-3.3 (Quiescent current 1.5uA) & $0.91 \mathrm{~V}$ & $5.5 \mathrm{~V}$ \\
\hline
\end{tabular}

\subsubsection{Load}

The sensor node (mote) is the end consumer of energy in our micro-solar power system. The amount of energy a mote consumes $\left(P_{\text {cons }}\right)$ determines the capacity planning of a solar-powered sensor node. In order to size the mote energy consumption, we need to understand its main causes of energy consumption: radio communication and sensing. Since a mote draws a much higher current when its radio-chip is awake, radio duty-cycling is commonly used as a technique to lower the energy consumption of a mote. Power savings for the sensing device can be achieved in a similar way. A mote's current consumption rate $I_{\text {est }}$ can be estimated with the formula below if the current consumption rates for the sleep state and the active state $\left(I_{\text {sleep }}\right.$ and $\left.I_{\text {awake }}\right)$ are known:

$$
I_{\text {est }}=R \cdot I_{\text {awake }}+(1-R) \cdot I_{\text {sleep }}
$$

Table 3.3 compares the current consumption measurements $\left(I_{\text {avg }}\right)$ and the estimates for different radio duty-cycle rates. The small difference between the measurements and the estimates $(-1.62 \%$ to $0.61 \%)$ implies that we can estimate the current consumption of a mote as a simple function of the duty-cycle rate $R(0<R<1)$. While the effect of radio duty-cycling is given for a single sensor node, the concept is still valid for a network of sensor nodes. Low-duty cycle MAC protocols [vDL03, PHC04, YHE04, YSH06, EV06, ZZH ${ }^{+} 07$, Dus] can make a sensor node operate at low radio duty-cycle while maintaining network connectivity. In order to adjust the radio duty-cycle according to energy availability, we can use techniques in the literature [HZKS06, MBTB06b, JPC05, KPS04].

With duty-cycling, the power consumption of the load itself looks like the average value, but it can be more than the average value when the load is connected to the 
Table 3.3: Current consumption of Trio at different duty-cycle rates

\begin{tabular}{|c|r|r|r|r|r|}
\hline Duty-cycle rate & $\mathbf{1 . 5 6 \%}$ & $\mathbf{6 . 2 5 \%}$ & $\mathbf{1 2 . 5 \%}$ & $\mathbf{2 5 \%}$ & $\mathbf{5 0 \%}$ \\
\hline$I_{\text {avg }}(\mathrm{mA})$ & 0.528 & 1.327 & 2.408 & 4.569 & 8.854 \\
$I_{\text {sleep }}(\mathrm{mA})$ & 0.264 & 0.265 & 0.276 & 0.275 & 0.319 \\
$I_{\text {awake }}(\mathrm{mA})$ & 17.414 & 17.342 & 17.355 & 17.306 & 17.362 \\
$I_{\text {est }}(\mathrm{mA})$ & 0.536 & 1.338 & 2.405 & 4.541 & 8.812 \\
\hline$\left(I_{\text {avg }}-I_{\text {est }}\right) / I_{\text {est }}$ & $-1.62 \%$ & $-0.79 \%$ & $0.12 \%$ & $0.61 \%$ & $0.47 \%$ \\
\hline
\end{tabular}

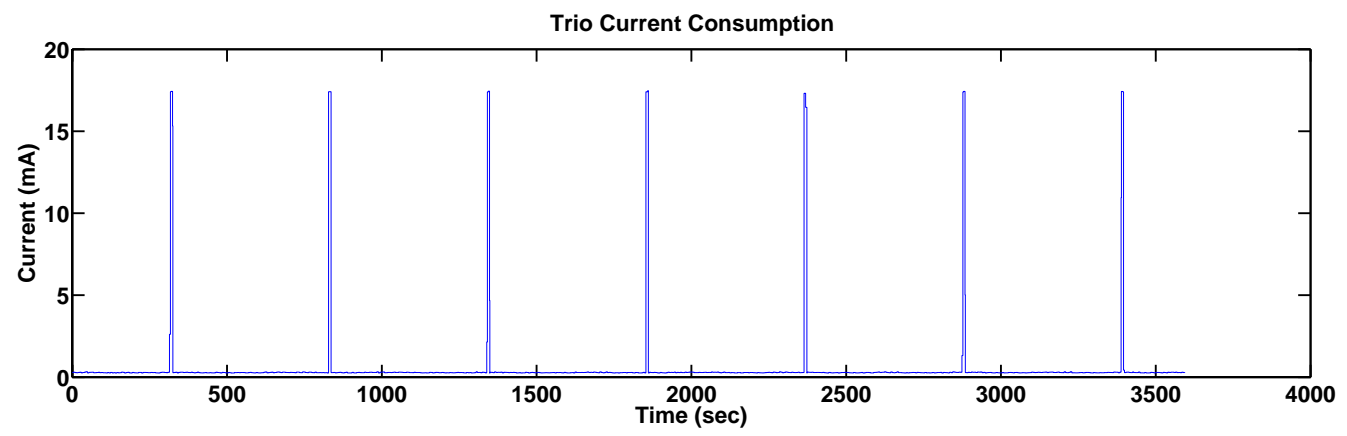

Figure 3.6: Current consumption of a Trio node with radio duty-cycle of $1.56 \%$

rest of system through the output regulator. Typically, the efficiency of an output regulator varies depending on the load current. A sensor node at low duty-cycle consumes tens of $\mathrm{mA}$ during the radio-on period, and less than $1 \mathrm{~mA}$ during the radio-off period (Figure 3.6). For more accurate modeling, the efficiency of the output regulator should be adjusted according to the operating modes and the frequency of the load.

\subsection{Energy Efficiency and Daily Power Cycle}

\subsubsection{Energy Efficiency and Output Power}

A solar panel transforms available incident solar radiation to electrical power. A given panel is characterized by its IV curve and, in particular, three points: the opencircuit voltage $\left(V_{o c}\right)$, short-circuit current $\left(I_{s c}\right)$, and maximum power point (MPP). Internally, these are determined by the serial and parallel composition of the solar cells and the total area of the panel. Increasing temperature depresses the IV curve somewhat, reducing the power output. For the large, expensive panels used in macro- 
solar installations, these factors are accurately characterized in data sheets and well validated. For the small, inexpensive panels used in micro-solar applications, empirical characterization is often required. More importantly, the operating point of the IV curve is determined by the load experienced at the panel, which is determined by the input regulator, storage facility, and downstream load. For most panels, the IV curve is nearly flat for voltages less than that of the MPP, so power increases nearly linearly with the voltage in this range.

The input regulator conditions the output of the panel to meet the operational constraints of the particular battery, including voltage limits, current limits, and charge duration. While macro-solar inverters operate in the neighborhood of $95 \%$ efficiency, in the sub-watt range, regulator efficiencies Eff $_{r e g-i n}$ of $70-80 \%$ are more typical. The product of such low efficiencies translates into a significant overall supply:demand ratio.

As for energy storage, a wide range of battery configurations and chemistries are available for storing charge, as well as supercapacitors, all with differing operating voltages, charge algorithms, and complexities. From a system design perspective, it is desirable for the power subsystem to be able to charge a fully discharged battery without software in the loop, so that when placed in sunlight the device is guaranteed to eventually become active. The portion of energy transferred into the battery during the day and discharged during the night incurs an additional round-trip transfer

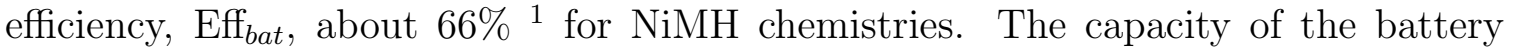
determines not only the potential lifetime in darkness, but also how much energy can be harvested while the sun shines, as discussed below.

The output regulator matches the battery characteristics to the requirements of the mote. It too is characterized by its efficiency, Eff ${ }_{r e g-o u t}$, and in particular its efficiency at two very different operating points: 10s of micro-watts most of the time and 10 s of milliwatts during short active periods. For a typical bimodal $P_{\text {mote }}$, an effective efficiency of $50 \%$ or less is expected. This determines the load experienced by the supply and storage components of the power subsystem.

As for system-wide efficiency, just a back-of-the-envelope calculation of solar availability during $2 \%$ of operation (i.e., a half hour of radiation during the day) and a

\footnotetext{
${ }^{1}$ With charging rate $0.1 \mathrm{C} /$ hour, where $\mathrm{C}$ is the capacity of the battery
} 

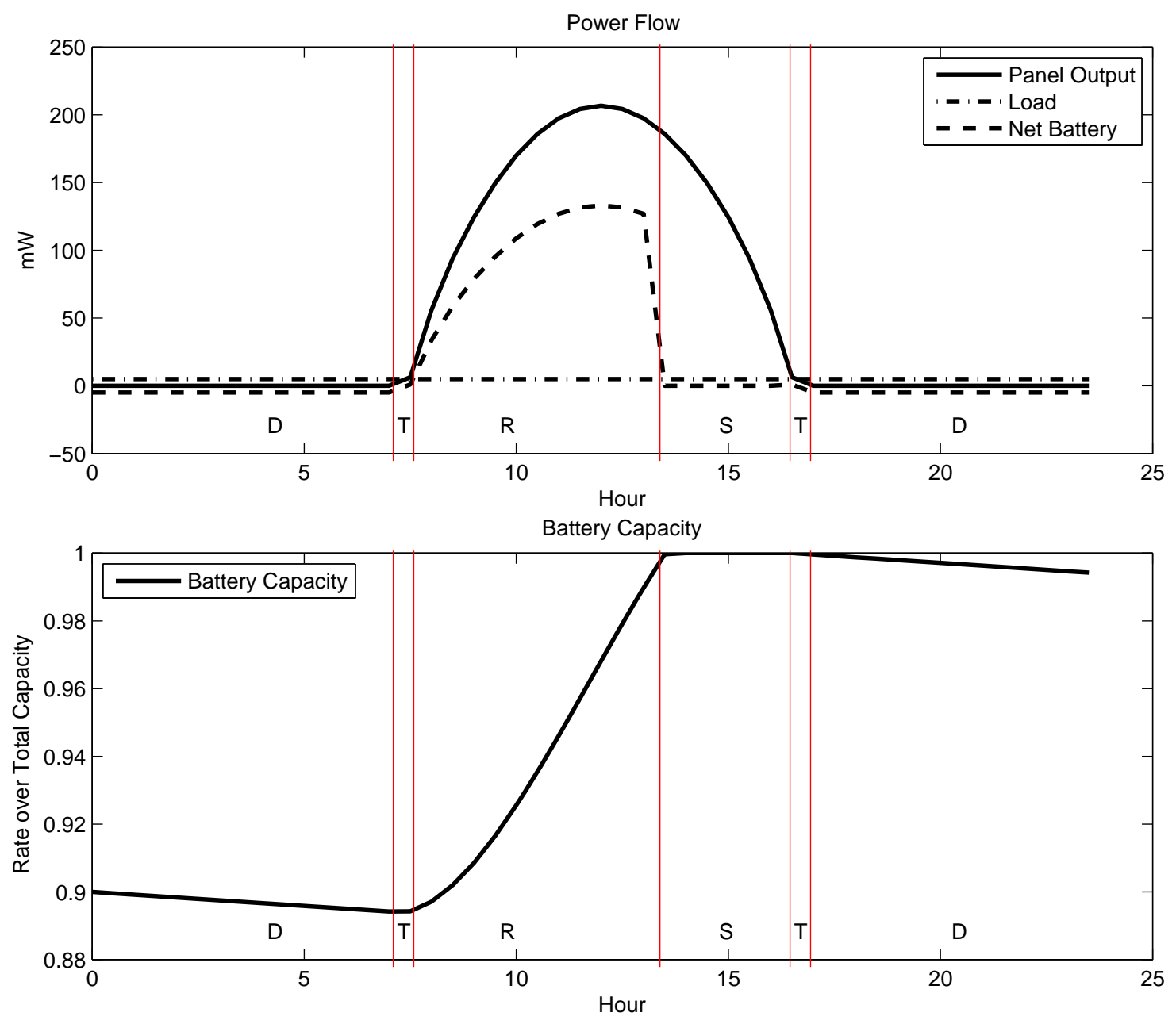

D: Discharge T: Transition R: Recharge S: Saturation

Figure 3.7: Energy flow and daily phases in our micro-solar model.

3:1 supply/demand ratio from the product of efficiencies Eff reg-out $\cdot \mathrm{Eff}_{\text {bat }}$ suggest that the solar panel needs to be sized at 150 times the average demand.

$$
\frac{1}{\text { solar-availability } \cdot \mathrm{Eff}_{\text {reg-out }} \cdot \mathrm{Eff}_{\text {bat }}}=\frac{1}{0.02 \cdot 0.66 \cdot 0.5}=\frac{1}{0.02 \cdot 0.33}=150
$$

This makes every aspect of the micro-solar subsystem design critical, and motivates the detailed design and analysis in the remainder of the chapter. 


\subsubsection{Energy Flow and Daily Power Cycle}

The behavior of the system has a roughly daily pattern; generally the daily power cycle has five phases, as illustrated in Figure 3.7. From sundown to sun up, the battery discharges, supplying the device load. As the panel is initially illuminated, a transition period occurs during which the battery provides only a portion of the device load. With sufficient illumination, the panel supports the entire load and delivers charge into the battery. If this recharge period is sustained sufficiently long, the battery becomes fully charged and the system operates in saturation, shunting power. Eventually, a dusk transition occurs similar to dawn. The efficiency coefficients dictate the net change in battery capacity over the daily cycle, given the starting capacity, supply power, and demand power. Our sizing guideline assumed that the recharge period would need to be no more than half an hour, possibly distributed throughout the day. Saturation merely preserves capacity. Of course, a series of overcast days may result in a progressive drop in battery capacity, which would then increase the recharge duration when the weather clears. In the micro-solar setting, given the ratio of mote load and typical battery capacities, it is even reasonable to consider design points that absorb entire seasonal variations in weather patterns.

In the discharge period, there is no solar energy available and the battery is discharged to run the load. If we assume constant load consumption, we can formulate the condition of each component of the micro-solar power system as follows:

$$
\begin{aligned}
P_{\text {sol }} & =0, P_{\text {bat-chg }}=0, P_{\text {bat-dis }}>0, P_{\text {mote }}=\mathrm{const} \\
P_{\text {mote }} & =P_{\text {bat-dis }} \cdot \mathrm{Eff}_{\text {reg-out }}
\end{aligned}
$$

In the transition period, there is solar radiation, but it is not high enough to charge the battery. Since the energy to run the load comes from both the solar radiation and the battery discharge, the following relationship can hold:

$$
\begin{aligned}
P_{\text {sol }} & >0, P_{b a t-c h g}=0, P_{b a t-d i s}>0, P_{\text {mote }}=\mathrm{const} \\
P_{\text {mote }} & =\left(P_{\text {sol }} \cdot \mathrm{Eff}_{r e g-i n}+P_{b a t-d i s}\right) \cdot \mathrm{Eff}_{r e g-o u t}
\end{aligned}
$$

This relationship can be further reduced when the micro-solar power system has an input regulator with $\mathrm{Eff}_{r e g-i n}=1$ :

$$
P_{\text {mote }}=\left(P_{\text {sol }}+P_{\text {bat-dis }}\right) \cdot \mathrm{Eff}_{\text {reg-out }}
$$




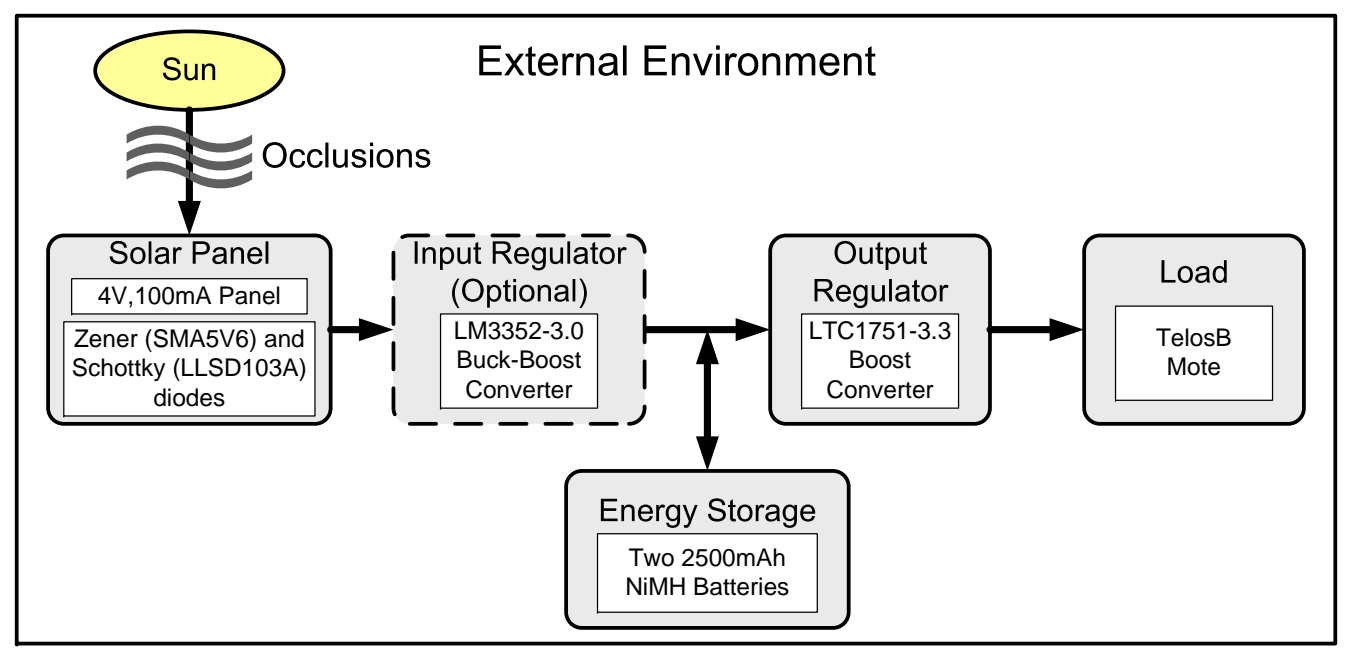

Figure 3.8: Architecture for HydroWatch Node

In the recharge period, the solar radiation is sufficiently high and the energy to run the mote comes from solar radiation. At the same time, the rest of the solar radiation is stored in the energy storage. Then, the following relationship can hold among each component:

$$
\begin{aligned}
P_{\text {sol }} & >0, P_{b a t-c h g}>0, P_{b a t-d i s}=0, P_{\text {mote }}=\mathrm{const} \\
P_{\text {sol }} \cdot \mathrm{Eff}_{r e g-i n} & =P_{b a t-c h g}+P_{\text {mote }} / \mathrm{Eff}_{r e g-o u t}
\end{aligned}
$$

In the saturation period, the solar radiation is sufficiently high, but the energy storage is fully charged. The energy to run the load comes from the solar radiation with the battery not being charged nor being discharged, and the rest of energy from solar radiation is shunted:

$$
\begin{aligned}
P_{\text {sol }} & >0, P_{b a t-c h g}=0, P_{b a t-d i s}=0, P_{\text {mote }}=\mathrm{const} \\
P_{\text {sol }} \cdot \mathrm{Eff}_{\text {reg-in }} & =P_{\text {shunted }}+P_{\text {mote }} / \mathrm{Eff}_{\text {reg-out }}
\end{aligned}
$$

\subsection{Validating the Energy Flow Relationship}

In this section, we empirically validate the energy flow relationship of a micro-solar power system. As an experimental platform, the HydroWatch node is used. More 


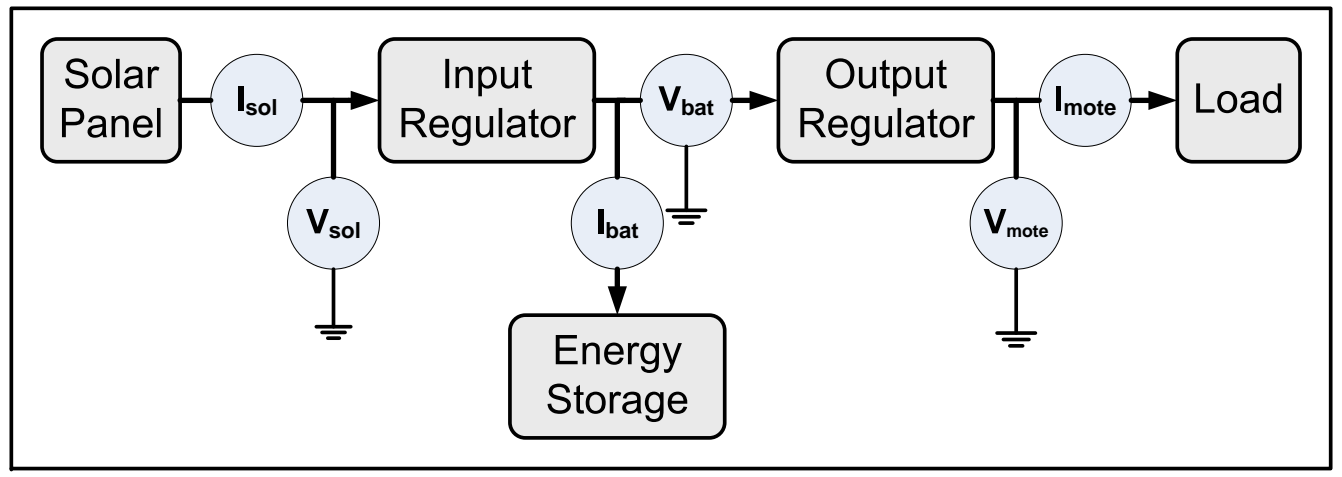

Figure 3.9: Energy measurement set-up with HydroWatch Node

detail of the HydroWatch node will be discussed later in Chapter 5. The summary of the HydroWatch node is below:

- Solar Panel: 4V-100mA polycrystalline silicon panel with Zener diode (SMA5V6) and Schottky diode (LLSD103A) for protection from high voltage and reverse flow

- Input Regulator: two configurations, one with LM3352-3.0 buck-boost converter as an input regulator and the other without an input regulator

- Energy Storage: Two 2500mAh NiMH batteries, series connected

- Output Regulator: LTC1751-3.3 boost converter

- Load: TelosB node running at 3mA average current

Figure 3.9 shows the experiment set-up for measuring the overall energy flow of a HydroWatch node. A few measurement points are set up with digital multimeters logging the voltage and the current - solar panel voltage $\left(V_{\text {sol }}\right)$, solar panel current $\left(I_{\text {sol }}\right)$, battery voltage $\left(V_{\text {bat }}\right)$, battery charging current $\left(I_{b a t}\right)$, load voltage $\left(V_{\text {mote }}\right)$ and load current $\left(I_{\text {mote }}\right)$. The experiment has two configurations: one with an input regulator, and the other without an input regulator. 


\subsubsection{HydroWatch Node with an Input Regulator}

Figure 3.10 shows the energy flow when the HydroWatch node was configured with an input regulator. Depending on the availability of solar radiation and the charging status of the battery, the daily trend can be divided into different periods: discharge before dawn (00:17:08 - 06:57:13), transition at dawn (06:57:13 - 08:47:16), recharge (08:47:16 - 17:27:16), transition at dusk (17:27:16 - 19:27:13), and discharge after dusk (19:27:13 - 23:57:20).

\section{Discharge Period}

During the discharge period, we can see that conditions (3.6) are met from Figure 3.10. The mote power consumption was expected to be constant, but was fluctuating a bit. This was because the device was in the outdoor environment without proper sealing: exposure to moisture increased the current consumption of the mote. From relation (3.7), we have calculated Eff $_{\text {out-reg }}$ of $57 \%$ for discharge before dawn and $51 \%$ for discharge after dusk. Compared to the manufacturer-provided value $65 \%$, our estimations of $\mathrm{Eff}_{\text {out-reg }}$ have a deviation of $8 \%$ and $14 \%$, respectively.

\section{Transition Period}

During the transition period, we can see that conditions (3.8) are met. In order to estimate the efficiency of the input regulator $\mathrm{Eff}_{i n-r e g}$ from relation (3.7), we assumed that the efficiency of the output regulator Eff $_{\text {out-reg }}$ is the same as the value in the adjacent discharge periods. The calculated input regulator efficiency is $70 \%$ for transition at dawn and $60 \%$ for transition at dusk. Compared to the manufacturer-provided efficiency $81 \%$, these estimations have a deviation of $11 \%$ and $21 \%$, respectively.

\section{Recharge Period}

During the recharge period, we can see that conditions (3.11) are met. In order to see whether the relation 3.12 holds, we take the mean values of Eff $_{\text {in-reg }}$ and Eff $_{\text {out-reg }}$ from the transition and discharge periods as the input regulator efficiency and the output regulator efficiency during the recharge period. From Figure 3.10, we 
can evaluate the two sides of relation $(3.12)$ : the solar radiation after the input regulator, $E_{\text {sol }} \cdot \mathrm{Eff}_{r e g-i n}$, is evaluated as $2604 \mathrm{~J}$ and the energy charged to the battery, and mote consumption, $E_{b a t-c h g}+E_{\text {mote }} / \mathrm{Eff}_{\text {reg-out }}$, is evaluated as $2459 \mathrm{~J}$. The difference between the two sides of the relation is $5 \%$, which implies that our hypothesis for relation 3.12 holds within an error of $5 \%$.

\section{Saturation Period}

In this experiment, we did not observe the situation for the saturation period, where the energy flow should meet relationships (3.13).

\subsubsection{HydroWatch Node without an Input Regulator}

Figure 3.11 shows the energy flow when the HydroWatch node was configured without an input regulator. As in the case with an input regulator, the daily trend can be divided into different periods depending on the availability of solar radiation and the charging status of the battery: discharge before dawn (00:52:20 - 07:15:32), transition at dawn (07:15:32 - 08:05:34), recharge (08:05:34 - 18:05:34), transition at dusk (18:05:34 - 18:25:32), and discharge after dusk (18:25:32 - 23:55:36).

\section{Discharge Period}

During the discharge period, we can see that conditions 3.6 are met from Figure 3.11. From relation (3.7), we have calculated Eff ${ }_{\text {out-reg }}$ of $50 \%$ for discharge before dawn and $47 \%$ for discharge after dusk. Compared to the manufacturer-provided value $65 \%$, our estimations of Eff $_{\text {out-reg }}$ have a deviation of $15 \%$ and $18 \%$, respectively.

\section{Transition Period}

The condition for the transition period (3.8) has a parameter for the efficiency of the input regulator, while this configuration does not have an input regulator. We can see that this condition is still met if we set the efficiency of the input regulator as $100 \%$ (no power loss). This can be also confirmed by solving relation (3.7) for the efficiency of the input regulator assuming that the efficiency of the output regulator is the same as the value in the adjacent discharge periods, which gives $100 \%$ and $96 \%$. 

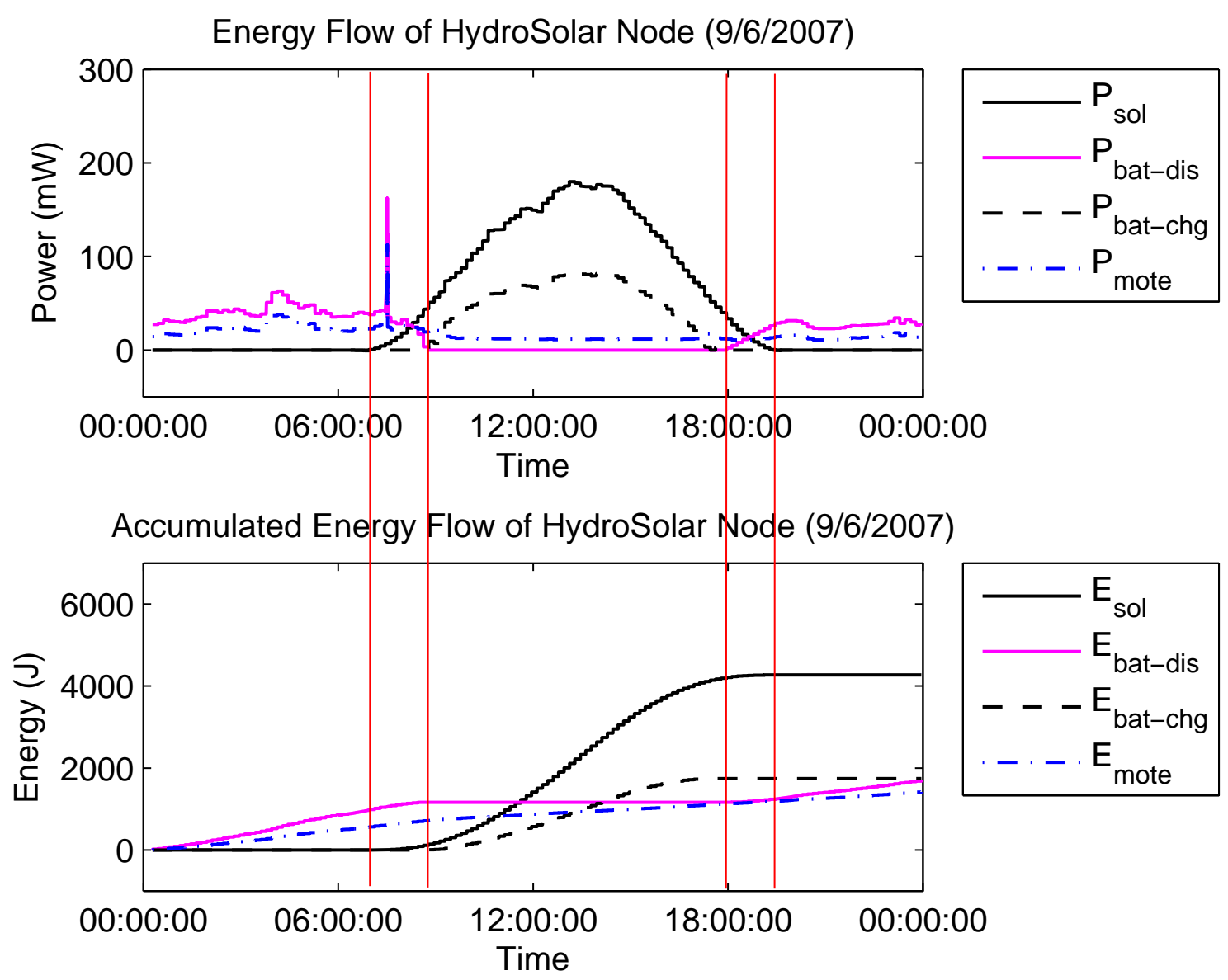

With an input regulator $(9 / 6 / 2007)$

\begin{tabular}{|l|r|r|r|r|r|r|}
\hline & $\begin{array}{r}E_{\text {mote }} \\
(\mathrm{J})\end{array}$ & $\begin{array}{r}E_{\text {bat-dis }} \\
(\mathrm{J})\end{array}$ & $\begin{array}{r}E_{\text {sol }} \\
(\mathrm{J})\end{array}$ & $\begin{array}{r}E_{\text {bat-chg }} \\
(\mathrm{J})\end{array}$ & Eff $_{\text {inreg }}$ & Eff $_{\text {outreg }}$ \\
\hline $\begin{array}{l}\text { Discharge before dawn } \\
(00: 17: 08-06: 57: 13)\end{array}$ & 553.424 & 967.267 & -0.761 & 0.000 & - & $57.22 \%$ \\
\hline $\begin{array}{l}\text { Transition at dawn } \\
(06: 57: 13-08: 47: 16)\end{array}$ & 157.547 & 195.819 & 113.002 & 0.019 & $70.37 \%$ & $57.22 \%$ \\
\hline $\begin{array}{l}\text { Recharge } \\
(08: 47: 16-17: 27: 16)\end{array}$ & 387.278 & 0.001 & 3998.227 & 1741.361 & $65.20 \%$ & $53.93 \%$ \\
\hline $\begin{array}{l}\text { Transition at dusk } \\
(17: 27: 16-19: 27: 13)\end{array}$ & 87.268 & 76.562 & 159.636 & 2.180 & $60.02 \%$ & $50.63 \%$ \\
\hline $\begin{array}{l}\text { Discharge after dusk } \\
(19: 27: 13-23: 57: 20)\end{array}$ & 232.957 & 460.131 & -0.286 & 0.000 & - & $50.63 \%$ \\
\hline
\end{tabular}

Figure 3.10: Energy flow of HydroWatch node with an input regulator (9/6/2007 in Richmond Field Station, Richmond, CA). The net battery charging energy is 4152J. 


\section{Recharge Period}

During the recharge period, we can see that conditions (3.11) are met. In order to see whether the relation (3.12) holds, we estimate the efficiencies of the input regulator and the output regulator as $98 \%$ and $49 \%$, which are the mean values during transition and discharge periods. From Figure 3.11, we can evaluate the two sides of relation (3.12): the solar radiation after the input regulator, $E_{\text {sol }} \cdot$ Eff $_{r e g-i n}$, is evaluated as 6490J and the energy charged to the battery, and mote consumption, $E_{\text {bat-chg }}+E_{\text {mote }} / \mathrm{Eff}_{\text {reg-out }}$, is evaluated as 5911J. The difference between the two sides of the relation is $9 \%$, which implies that our hypothesis for relation (3.12) holds within an error of $9 \%$. Also notice that this experiment shows a weather effect in the late afternoon, where the solar panel output power gets smaller than the envelope and becomes irregular.

\section{Saturation Period}

In this experiment, we did not observe the situation for the saturation period, where the energy flow should meet relationships (3.13).

\subsubsection{System Efficiency}

For a unit quantity of solar radiation,

$$
\mathrm{Eff}_{r e g-i n} \cdot \mathrm{Eff}_{b a t} \cdot \mathrm{Eff}_{r e g-o u t}
$$

is eventually used for the operation of the mote. We define (3.15) as the system efficiency of a micro-solar power system. Table 3.4 compares the daily trends of system efficiency for the HydroWatch node in two cases: case a with an input regulator, and case $\mathbf{b}$ without an input regulator. Both cases have a similar value for the efficiency of the output regulator ( $52.4 \%$ for case 1 and $48.3 \%$ for case 2 ), but the absence of the input regulator makes case 2 about $50 \%$ higher in system efficiency than case 1 ( $21.7 \%$ for case 1 and $32.6 \%$ for case 2 ). 


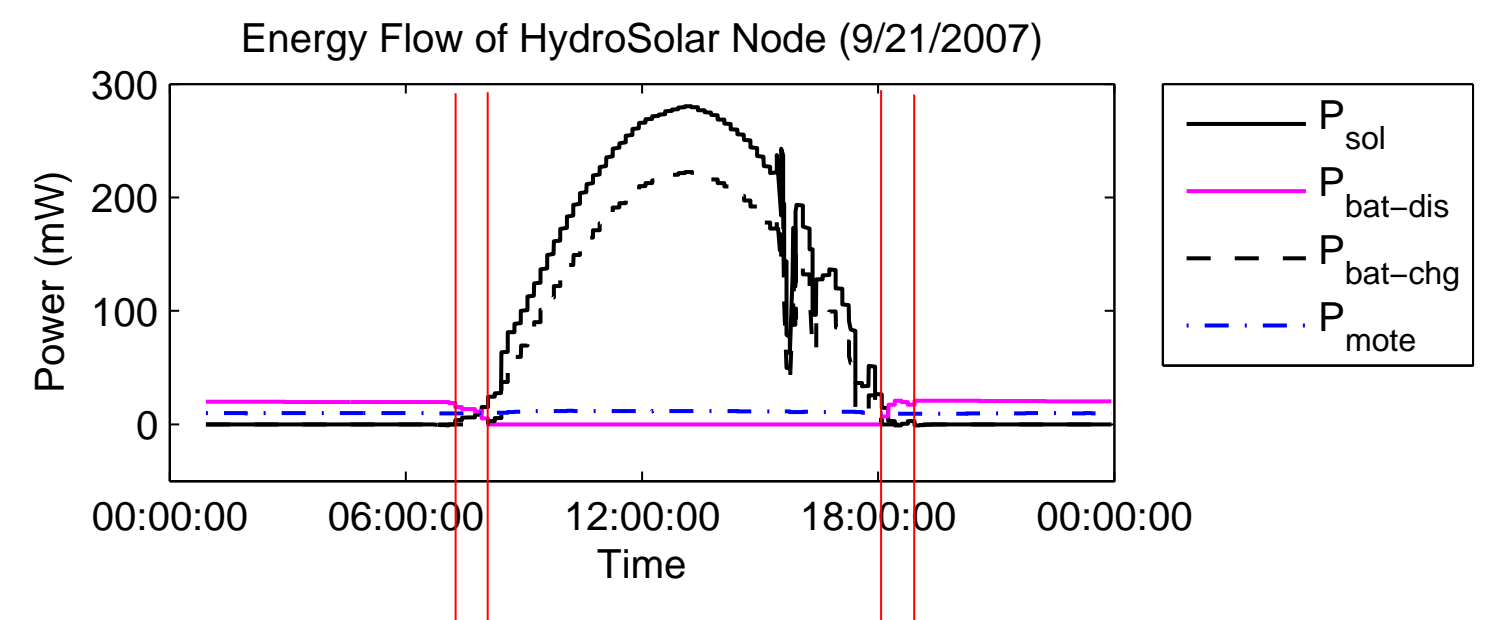

Accumulated Energy Flow of HydroSolar Node (9/21/2007)

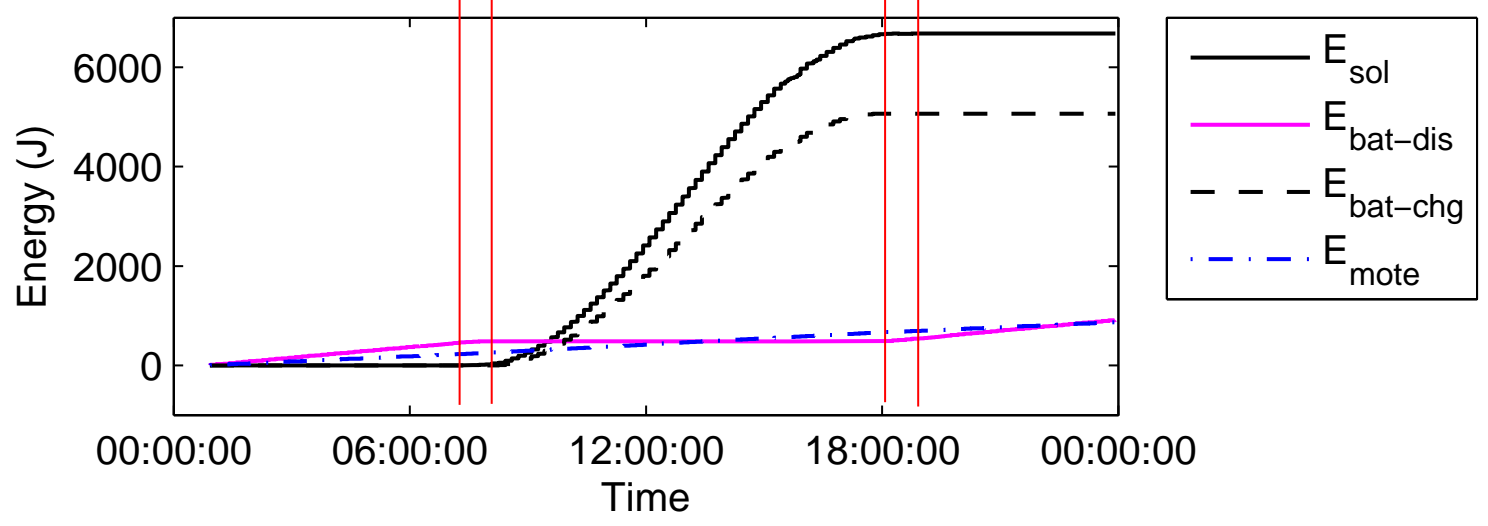

Without an input regulator $(9 / 21 / 2007)$

\begin{tabular}{|l|r|r|r|r|r|r|}
\hline & $\begin{array}{r}E_{\text {mote }} \\
(\mathrm{J})\end{array}$ & $\begin{array}{r}E_{\text {bat-dis }} \\
(\mathrm{J})\end{array}$ & $\begin{array}{r}E_{\text {sol }} \\
(\mathrm{J})\end{array}$ & $\begin{array}{r}E_{\text {bat-chg }} \\
(\mathrm{J})\end{array}$ & Eff $_{\text {inreg }}$ & Eff $_{\text {outreg }}$ \\
\hline $\begin{array}{l}\text { Discharge before dawn } \\
(00: 55: 20 \text { - 07:15:32) }\end{array}$ & 225.224 & 450.898 & -0.824 & 0.000 & - & $49.95 \%$ \\
\hline $\begin{array}{l}\text { Transition at dawn } \\
(07: 15: 32-08: 05: 34)\end{array}$ & 29.439 & 35.275 & 23.738 & 0.006 & $99.68 \%$ & $49.95 \%$ \\
\hline $\begin{array}{l}\text { Recharge } \\
(08: 05: 34-18: 05: 34)\end{array}$ & 409.010 & 0.014 & 6643.725 & 5068.786 & $97.68 \%$ & $48.59 \%$ \\
\hline $\begin{array}{l}\text { Transition at dusk } \\
(18: 05: 34-18: 25: 32)\end{array}$ & 11.653 & 14.531 & 10.603 & 0.000 & $95.69 \%$ & $47.22 \%$ \\
\hline $\begin{array}{l}\text { Discharge after dusk } \\
(18: 25: 32-23: 55: 36)\end{array}$ & 196.609 & 416.336 & 0.423 & 0.000 & - & $47.22 \%$ \\
\hline
\end{tabular}

Figure 3.11: Energy flow of HydroWatch node without an input regulator (9/21/2007 in Richmond Field Station, Richmond, CA). The net battery charging energy is 44J. 
Table 3.4: Daily trends of system efficiency for HydroWatch node in two cases

(a) Daily trends with input regulator

\begin{tabular}{|l|r|r|r|r|r|r|}
\hline & $\begin{array}{r}E_{\text {sol }} \\
(\mathrm{J})\end{array}$ & $\begin{array}{r}E_{\text {bat-chg }}(\mathrm{J}) \\
E_{\text {bat-dis }}\end{array}$ & $\begin{array}{r}E_{\text {mote }} \\
(\mathrm{J})\end{array}$ & Eff $_{\text {out-reg }}$ & Eff $_{\text {system }}$ \\
\hline $9 / 6 / 2007$ & 4270 & 1744 & 1700 & 1418 & $53.92 \%$ & $23.20 \%$ \\
\hline $9 / 7 / 2007$ & 3843 & 1590 & 1157 & 1015 & $50.92 \%$ & $20.18 \%$ \\
\hline
\end{tabular}

(a) Daily trends without input regulator

\begin{tabular}{|l|r|r|r|r|r|r|}
\hline & $\begin{array}{r}E_{\text {sol }} \\
(\mathrm{J})\end{array}$ & $\begin{array}{r}E_{\text {bat-chg }} \\
(\mathrm{J})\end{array}$ & $\begin{array}{r}E_{\text {bat-dis }} \\
(\mathrm{J})\end{array}$ & $\begin{array}{r}E_{\text {mote }} \\
(\mathrm{J})\end{array}$ & Eff $_{\text {out-reg }}$ & Eff $_{\text {system }}$ \\
\hline $9 / 21 / 2007$ & 6678 & 5069 & 917 & 871 & $48.59 \%$ & $31.32 \%$ \\
\hline $9 / 22 / 2007$ & 2126 & 1212 & 1117 & 908 & $48.83 \%$ & $31.73 \%$ \\
\hline $9 / 23 / 2007$ & 3782 & 2587 & 857 & 819 & $47.61 \%$ & $34.88 \%$ \\
\hline
\end{tabular}

\subsection{Assessment of Prior Work}

To illustrate the use of the model of micro-solar power systems, we compare Trio $\left[\mathrm{DHJ}^{+} 06\right]$ and Heliomote $\left[\mathrm{RKH}^{+} 05\right]$, as modeled in Figure 3.12. These two systems have been widely deployed and show different design points in this space. Trio uses two-level storage (supercapacitors and Li-ion battery), software-controlled charging and regulation in favor of flexibility and efficiency. Heliomote, on the other hand, uses single-level storage (NiMH battery), hardware-controlled charging and regulation for simplicity. In this section, we compare the empirical data of the two systems and analyze how system performance is affected by different design decisions.

\subsubsection{Experimental Set-up}

In order to evaluate the system performance of the two micro-solar power systems, we compare (a) solar-collector operation, and (b) system energy flow and efficiency.

\section{Solar-collector Operation}

We evaluate the solar collector performance of each system by comparing the daily cumulative solar radiation at the operating point $E_{o p}$ with the cumulative solar radiation at the maximum power point $E_{\max P}$. These two quantities can be determined from an experiment, as in Figure 3.13. In experiment (a), we measured the current and the voltage of the solar panel that is used for the Trio or the Heliomote node 

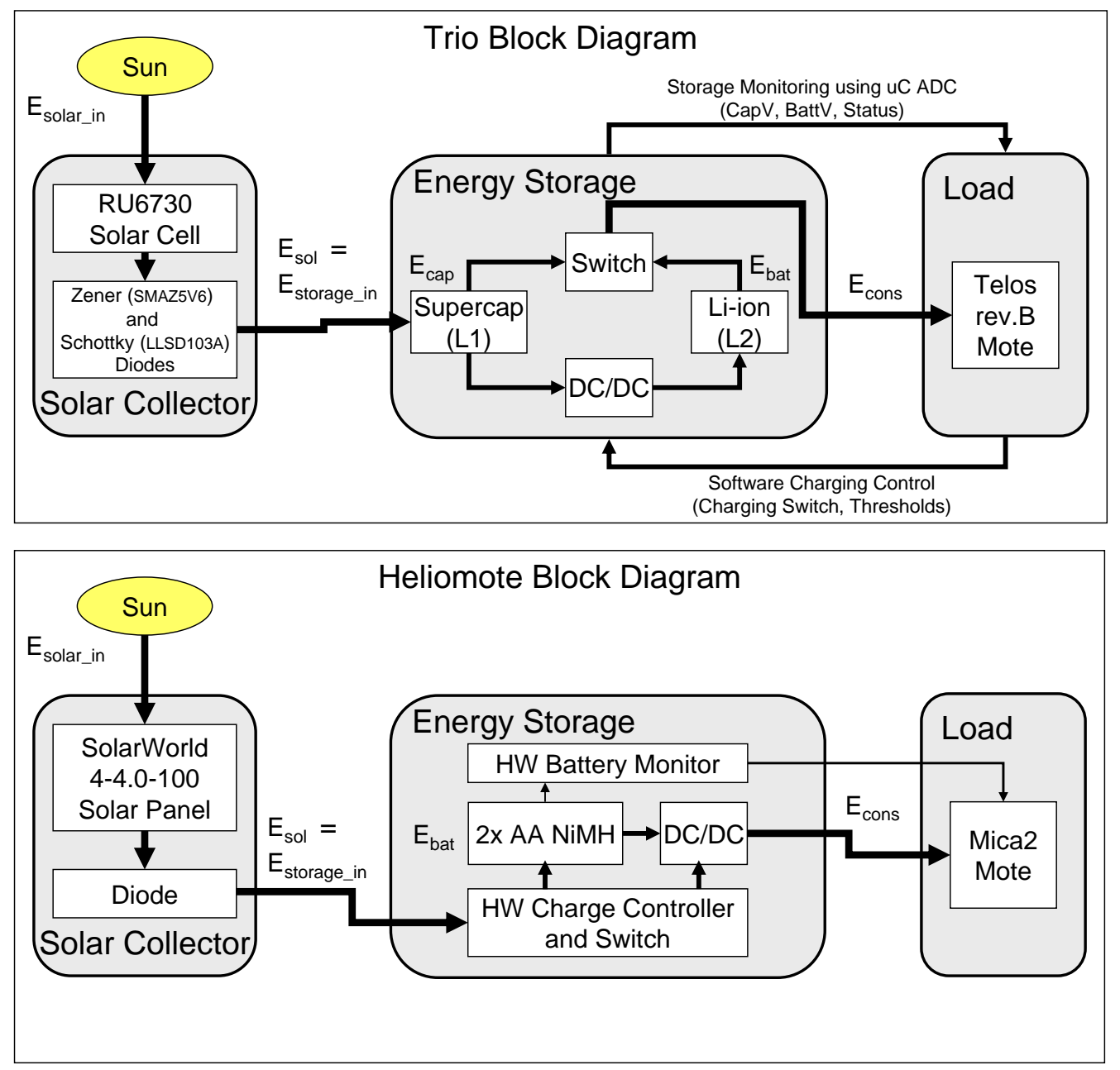

Figure 3.12: Block diagrams for Trio and Heliomote 


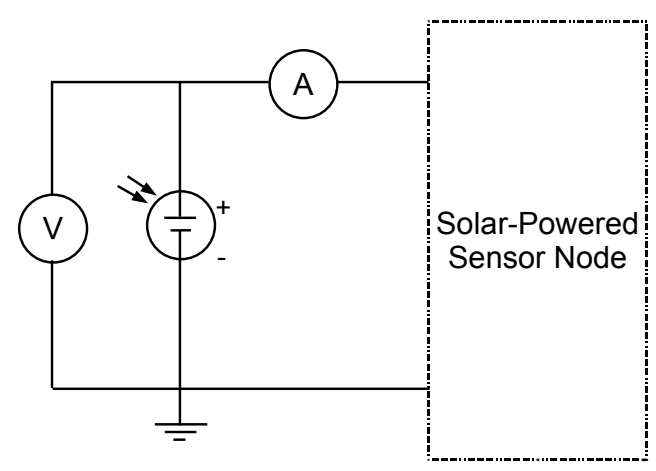

(a) Operating point $\left(I_{o p}, V_{o p}\right)$ is measured

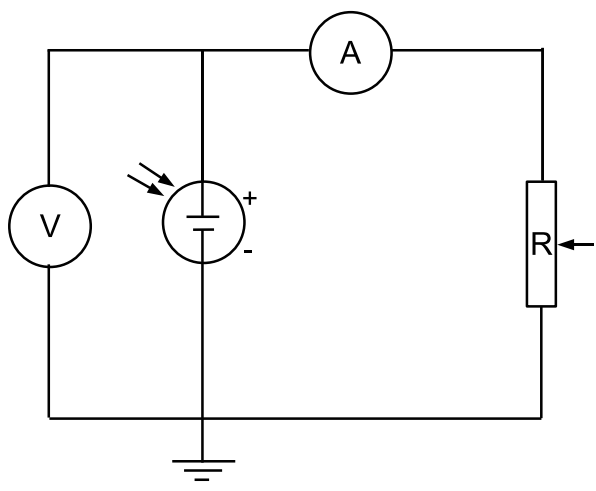

(b) I-V curve is measured

Figure 3.13: Experimental set-up for measuring solar panel output power.

$\left(I_{o p}, V_{o p}\right)$. This is characteristic of the solar panel at its operating point. In experiment (b), we measured a sequence of $(\mathrm{I}, \mathrm{V})$ values for a separate solar panel by adjusting the load impedance. Based on this I-V curve, the maximum power point $\left(I_{\max P}, V_{\max P}\right)$ can be found where the solar panel output power $P=I \cdot V$ is maximized.

\section{Energy Flow and Energy Efficiency}

We evaluate the performance of the overall system by comparing the energy efficiency of a micro-solar power system. Depending on system status, the system energy efficiency can be defined as follows:

\section{- Daily System-wide efficiency:}

Eff $_{\text {sys }}=\left(\Delta E_{\text {bat }}+\Delta E_{\text {cap }}+\Delta E_{\text {cons }}\right) / \Delta E_{\text {sol }}$

This metric represents how much of the daily solar radiation energy is used for battery charging, supercapacitor charging and mote consumption.

\section{- Battery discharging efficiency:}

$\mathrm{Eff}_{\text {bat-dis }}=\Delta E_{\text {cons }} / \Delta E_{\text {bat-dis }}$

In darkness, the system runs on the energy that is discharged from the battery or the supercapacitor. This metric represents how much of the energy discharged from the battery is used for the mote operation during a battery discharge period. 


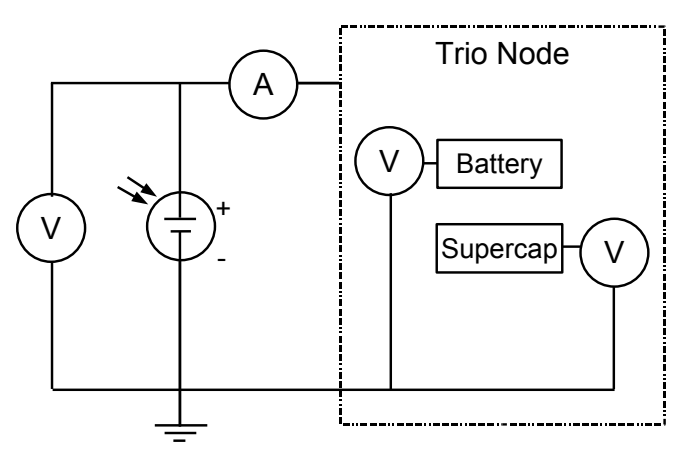

(a) Trio

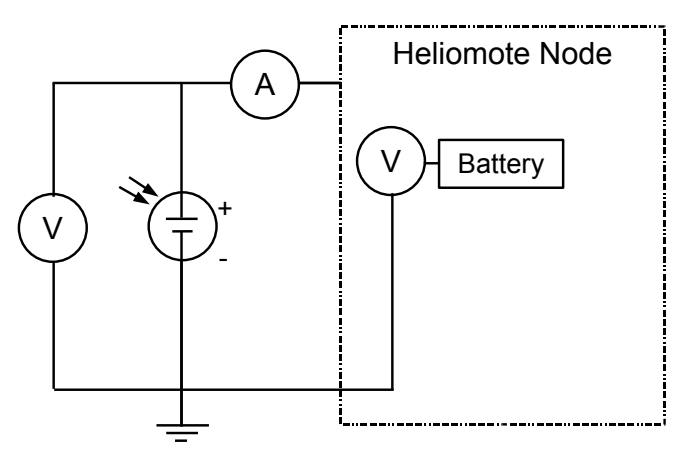

(b) Heliomote

Figure 3.14: Experimental set-up for measuring energy efficiency

\section{- Capacitor discharging efficiency:}

$\mathrm{Eff}_{\text {cap-dis }}=\Delta E_{\text {cons }} / \Delta E_{\text {cap-dis }}$

This metric represents how much of the energy discharged from the supercapacitor is used for the mote operation during a supercapacitor discharge period.

\section{- Charging efficiency:}

$\mathrm{Eff}_{\text {chg }}=\left(\Delta E_{\text {bat-chg }}+\Delta E_{\text {cap-chg }}+\Delta E_{\text {cons }}\right) / \Delta E_{\text {sol }}$

This metric represents how much of the daily solar radiation energy is used for battery charging, supercapacitor charging and mote consumption during recharge period.

In order to calculate these metrics, we set up the experiment as in Figure 3.14 by measuring the following characteristics of Trio and Heliomote: the solar panel voltage $V_{\text {sol }}(t)$, solar panel current $I_{\text {sol }}(t)$, and voltage levels of the energy storage elements $\left(V_{c a p}(t), V_{b a t}(t)\right)$. As for the mote consumption, we use the average current consumption $0.5448 \mathrm{~mA}$ (Trio) and $0.4031 \mathrm{~mA}$ (Heliomote), which is obtained by using a TelosB mote at $1.56 \%$ radio duty-cycle. As for the energy stored in the battery $E_{b a t}$, we estimated the capacity from the voltage using the manufacturer-provided voltage-to-capacity profile.

Both solar-panel operation and energy flow experiments were executed at the Richmond Field Station in Richmond, California with a non-obstructed view of sunlight. 
Fluke-189 data logging multimeters were used to record measurements. In comparing the two systems, we focus on high radiation hours (9AM to 5PM), because the solar panel output current is too small for practical use outside this window of time.

\subsubsection{Solar-collector Operation}

A series of solar panel I-V curves for Trio and Heliomote are shown in Figure 3.15 . and each I-V curve is marked with the corresponding operating point and the maximum power point. Figure 3.16(a) compares the solar panel output power at the operating point $P_{o p}$ and the maximum power point $P_{\max P}$, and it shows that the solar collector of Trio is better matched to the maximum power point than that of Heliomote. Figure 3.16(b) gives a more quantitative sense of the possible energy savings from operating point matching. The deviation of the daily cumulative energy at operating point $E_{o p}$ from the daily cumulative energy at MPP $E_{\max P}$ is $4.8 \%$ $\left(E_{o p}=3203 J\right.$ and $\left.E_{\max P}=3055 J\right)$ for Trio whereas it is $22.0 \%\left(E_{o p}=1712 J\right.$ and $\left.E_{\max P}=2195 J\right)$ for Heliomote.

This gap happens because the way the solar panel and the energy energy are matched is different for the two systems (Figure 3.17). For Trio, solar panel output is buffered in the supercapacitor before charging the battery. The supercapacitor operates between $3.2 \mathrm{~V}$ and $4.0 \mathrm{~V}$. This range is set by thresholds $V_{c a p-l b}$ and $V_{c a p-u b}$ in the software-based charging controller (Figure 3.18). The overload protection voltage $V_{\text {overload }}$ is set to $5.1 \mathrm{~V}$ by the reverse-biased Zener diode. Thus, the solar panel output can be transferred to the supercapacitor and the battery without being cut by the overload protection circuit. Heliomote, on the other hand, charges the solar-panel output to the battery without buffering, and the solar-panel operating voltage $V_{o p}$ is clipped to the overload protection voltage $V_{\text {overload }}(=2.8 \mathrm{~V})$. While this protects the battery from overload, more of the energy from the solar panel is wasted, causing the larger deviation from $P_{\max P}$.

Figure 3.19 shows the relationship between the solar panel output power, $P_{o p}$ and the operating voltage, $V_{o p}$ for the two systems. $P_{o p}$ is close to zero for a large range of voltage levels, but rises sharply once the voltage is past a certain threshold (3.7V for Trio and $2.8 \mathrm{~V}$ for Heliomote). This implies that the useful (most power produced) range of the solar panel in a particular system is very narrow. Therefore, power 


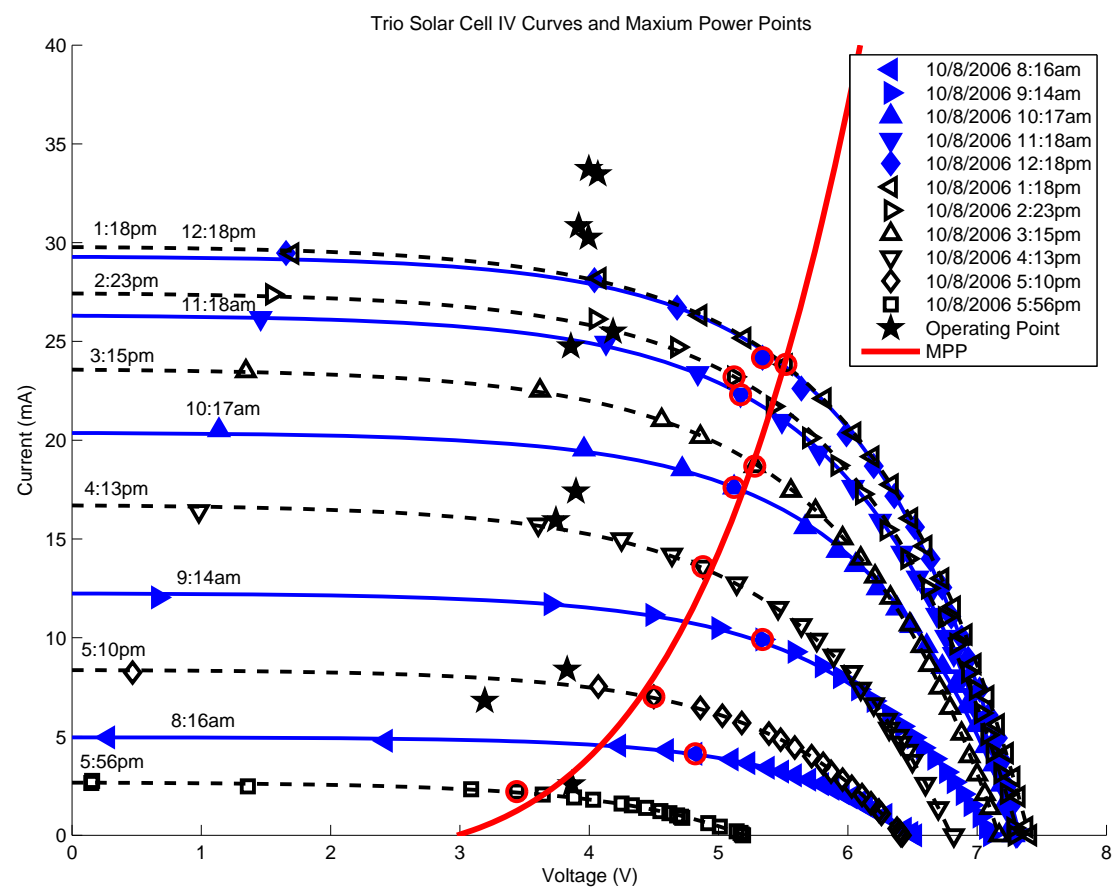

(a) Trio

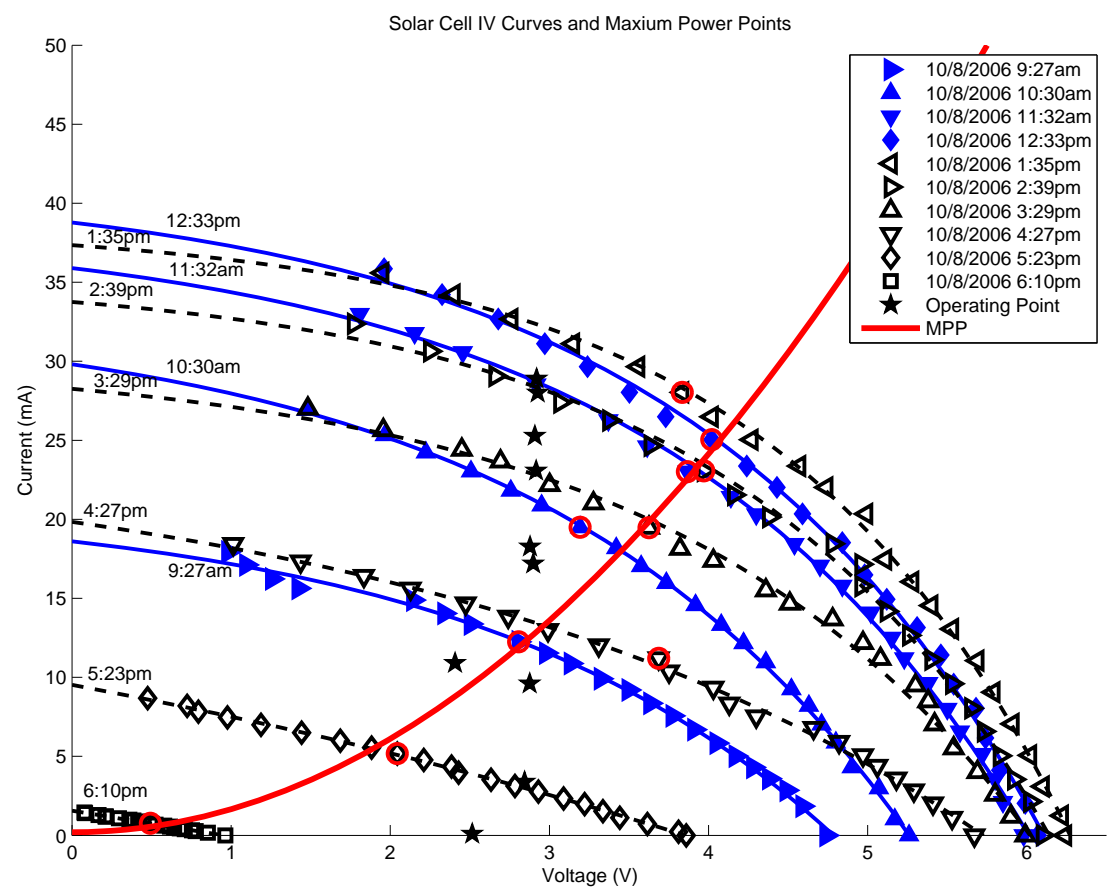

(b) Heliomote

Figure 3.15: I-V characteristics of solar-panels 

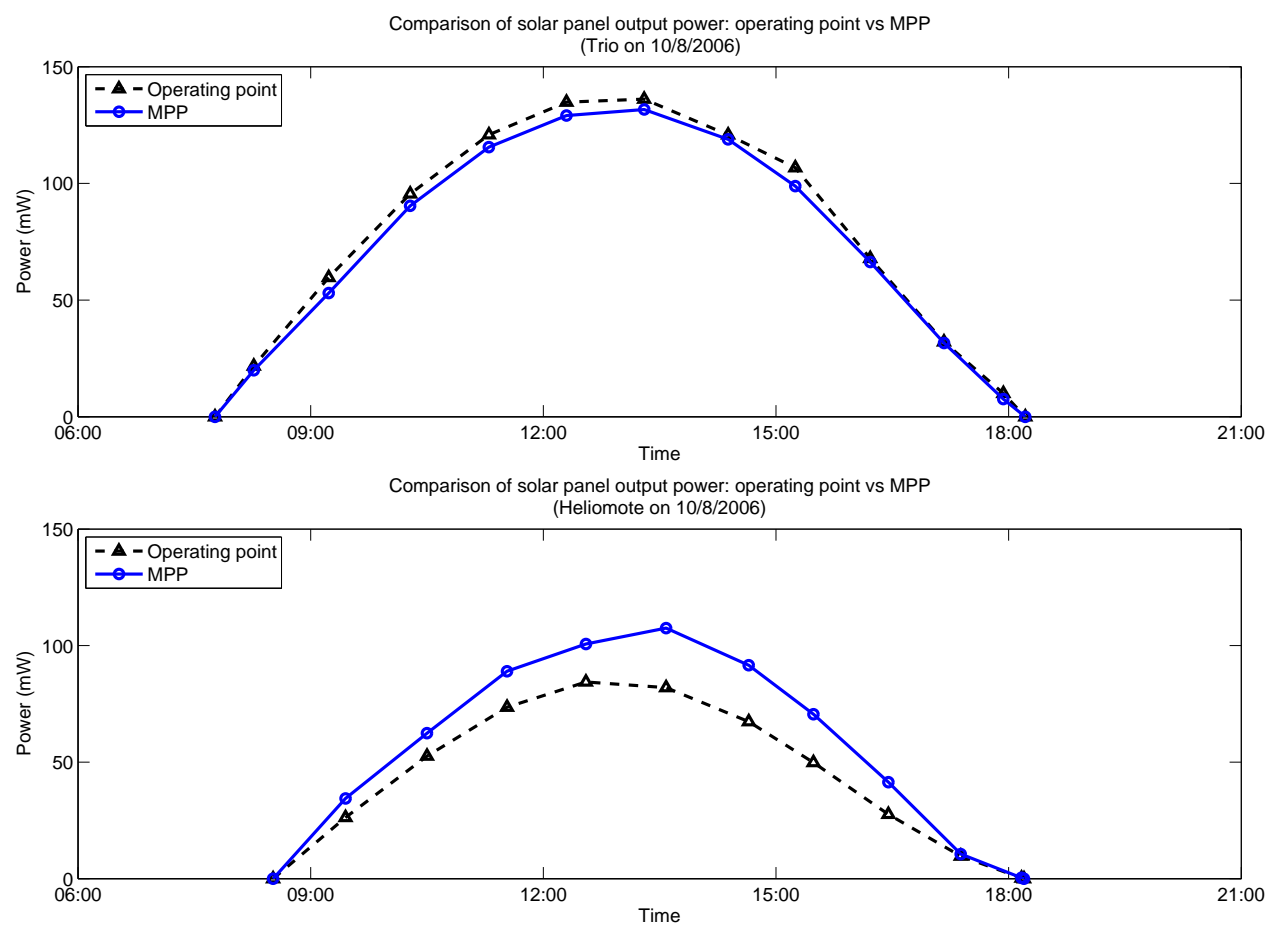

(a) Solar panel output power
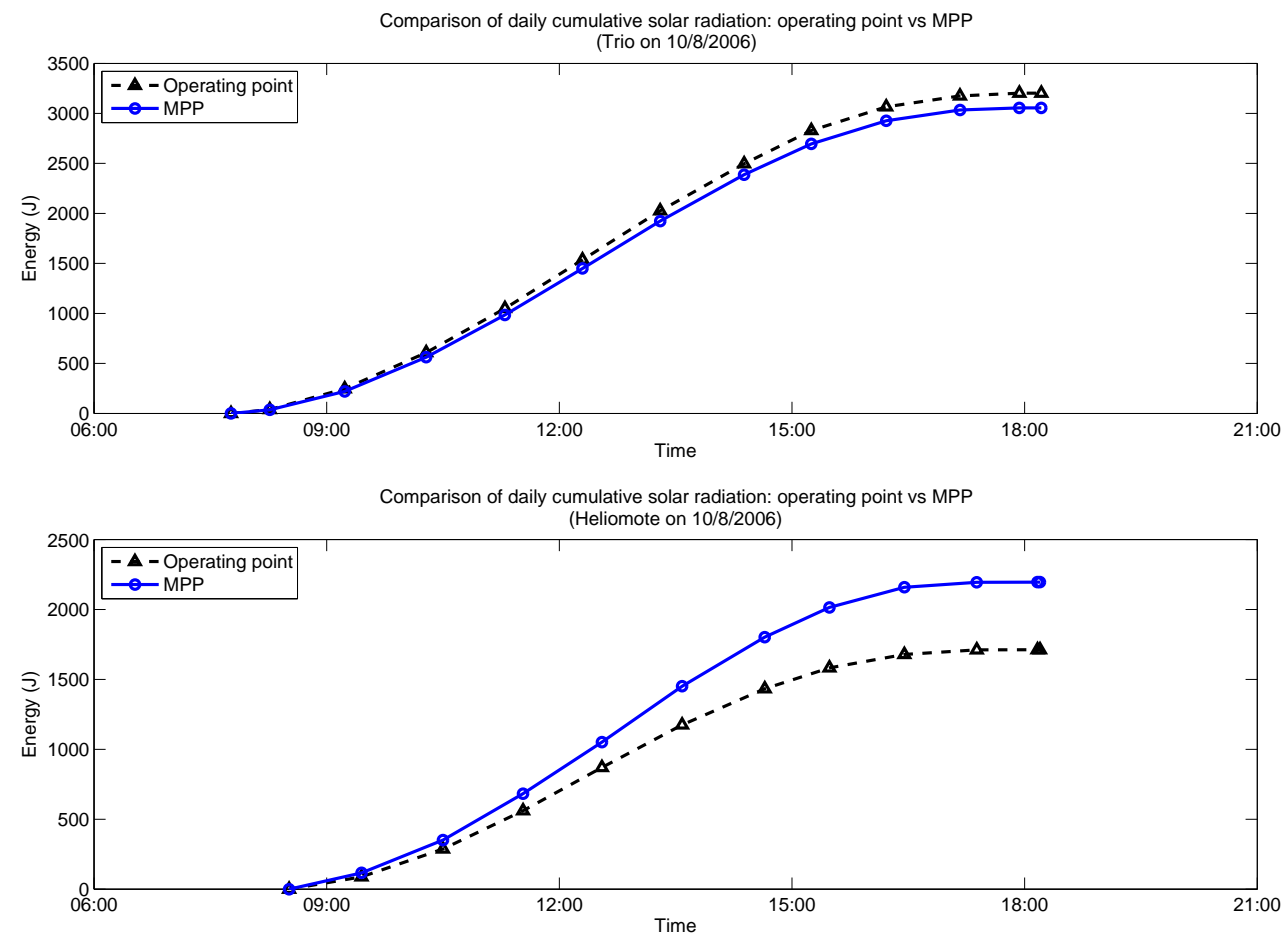

(b) Cumulative solar radiation

Figure 3.16: Comparison of solar panel output power and cumulative solar radiation 

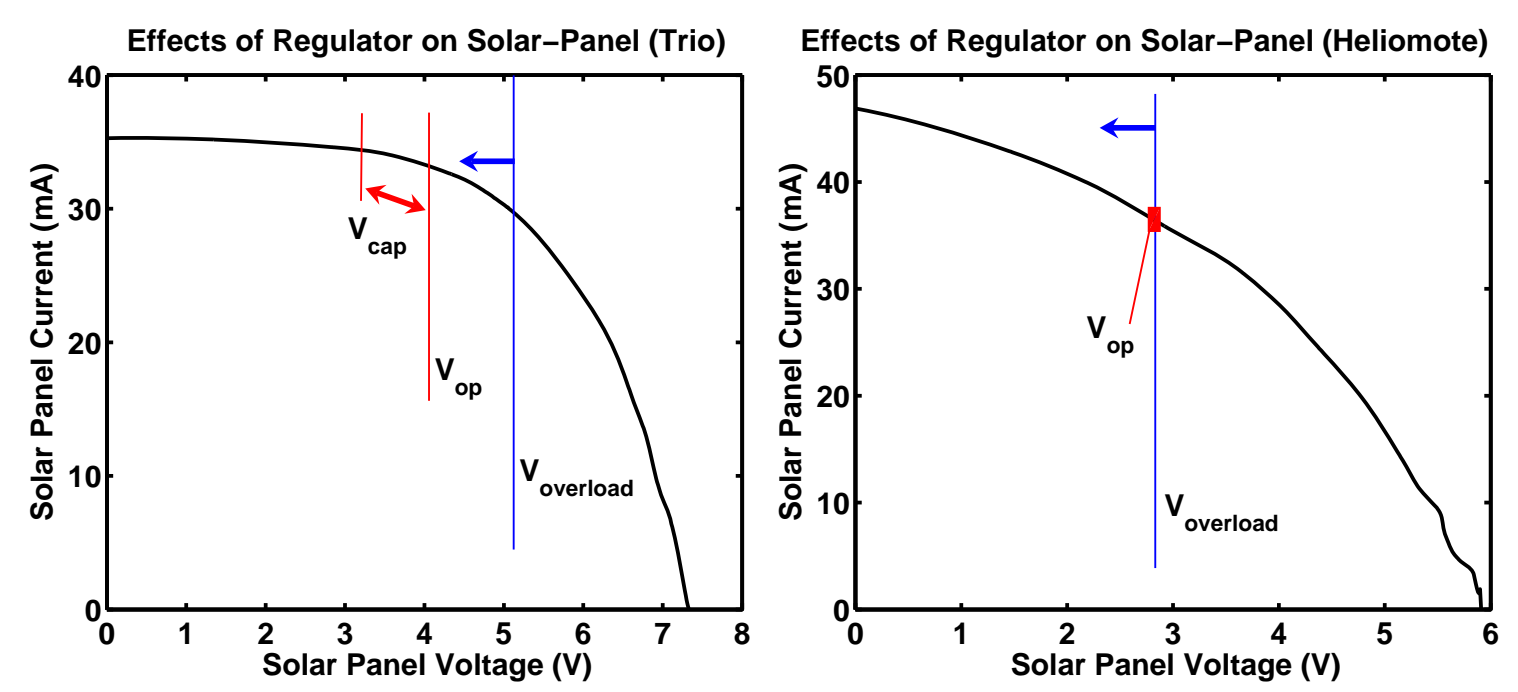

Figure 3.17: Comparison of regulator designs

Table 3.5: Component energy level and system energy efficiency for Trio and Heliomote

\begin{tabular}{|l|l|r|r|r|}
\hline Trio & Date & $10 / 9 / 06$ & $10 / 11 / 06$ & $10 / 12 / 06$ \\
\cline { 2 - 5 } & $\Delta E_{\text {sol }}$ & $3031.3 \mathrm{~J}$ & $2885.5 \mathrm{~J}$ & $2870.8 \mathrm{~J}$ \\
& $\Delta E_{\text {cap }}$ & $-0.05 \mathrm{~J}$ & $-0.04 \mathrm{~J}$ & $0.16 \mathrm{~J}$ \\
& $\Delta E_{\text {bat }}$ & $791.2 \mathrm{~J}$ & $342.1 \mathrm{~J}$ & $348.8 \mathrm{~J}$ \\
& $\Delta E_{\text {cons }}$ & $221.0 \mathrm{~J}$ & $220.3 \mathrm{~J}$ & $221.0 \mathrm{~J}$ \\
\cline { 2 - 5 } & Eff $_{\text {sys }}$ & $33.4 \%$ & $19.5 \%$ & $19.8 \%$ \\
\hline Heliomote & Date & $10 / 14 / 06$ & $10 / 16 / 06$ & $10 / 18 / 06$ \\
\cline { 2 - 5 } & $\Delta E_{\text {sol }}$ & $1237.6 \mathrm{~J}$ & $1449.9 \mathrm{~J}$ & $1641.4 \mathrm{~J}$ \\
& $\Delta E_{\text {bat }}$ & $27.0 \mathrm{~J}$ & $107.3 \mathrm{~J}$ & $10.1 \mathrm{~J}$ \\
& $\Delta E_{\text {cons }}$ & $10.0 \mathrm{~J}$ & $104.5 \mathrm{~J}$ & $103.0 \mathrm{~J}$ \\
\cline { 2 - 5 } & Eff $_{\text {sys }}$ & $10.5 \%$ & $14.6 \%$ & $6.9 \%$ \\
\hline
\end{tabular}

tracking circuits or algorithms are only meaningful within this small range.

\subsubsection{Energy Flow and Energy Efficiency}

The trend of daily energy flow and system energy efficiency for the two microsolar power systems is summarized in Table 3.5. From this result, we can observe the following: First, the net battery energy level has increased, which means that Trio and Heliomote have excess energy to store even after mote consumption and energy losses. Second, the supercapacitor of the Trio node stays at about the same energy 
1: if $\left(V_{b a t}<V_{b a t-u b}\right.$ and $\left.V_{c a p}<V_{c a p-l b}\right)$ then

2: Stop charging.

3: else if $\left(V_{b a t}<V_{b a t-u b}\right.$ and $\left.V_{c a p} \geq V_{c a p-u b}\right)$ then

4: $\quad$ Start charging.

5: else if $\left(V_{b a t} \geq V_{b a t-u b}\right)$ then

6: Stop charging.

7: end if

Figure 3.18: Trio battery charging algorithm
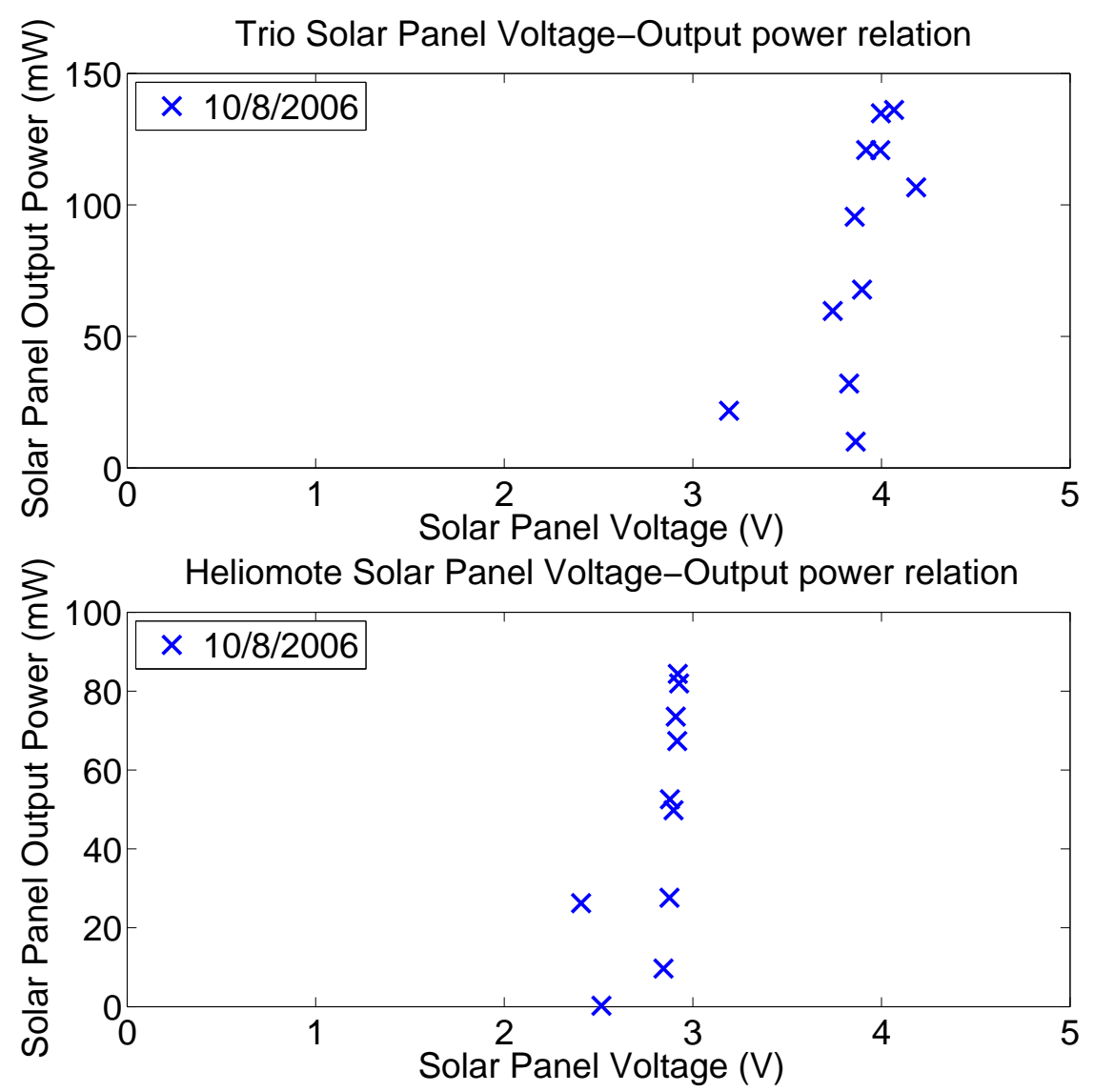

Figure 3.19: The relation of operating voltage and power for Trio and Heliomote 
Table 3.6: Energy efficiency of charging and discharging for Trio and Heliomote

\begin{tabular}{|l|l|r|r|r|}
\hline \multirow{3}{*}{ Trio } & Date & $10 / 9 / 06$ & $10 / 11 / 06$ & $10 / 12 / 06$ \\
\cline { 2 - 5 } & Eff $_{\text {chg }}$ & $46.9 \%$ & $31.2 \%$ & $28.5 \%$ \\
& Eff $_{\text {bat-dis }}$ & $24.5 \%$ & $32.7 \%$ & $34.9 \%$ \\
& Eff $_{\text {cap-dis }}$ & $57.2 \%$ & $54.3 \%$ & $68.6 \%$ \\
\hline \multirow{3}{*}{ Heliomote } & Date & $10 / 14 / 06$ & $10 / 16 / 06$ & $10 / 18 / 06$ \\
\cline { 2 - 5 } & Eff $_{\text {chg }}$ & $52.0 \%$ & $45.5 \%$ & $54.2 \%$ \\
& Eff $_{\text {bat-dis }}$ & $35.6 \%$ & $32.2 \%$ & $32.7 \%$ \\
\hline
\end{tabular}

level each day, with a net increase close to zero. This implies that the supercapacitor buffers the solar energy, and transfers the excess energy to the battery. Further, the net battery energy increase $\Delta E_{b a t}$ is positively correlated with the daily solar energy budget $\Delta E_{\text {sol }}$. Finally, our experimental data shows that for the Trio node, $19.5 \%$ to $33.4 \%$ of the available solar energy is charged to storage or consumed by the mote, while the Heliomote node has an energy efficiency between $6.9 \%$ to $14.6 \%$. As for the weather condition for the measurement on each day, we chose sunny days in midOctober so that the weather condition would be similar in all the measurements. One difference between the measurements of each day is the energy level of the storage elements, because we used the same experiment set-up over multiple days without depleting the energy storage. This explains the difference in the energy level of the storage elements $\left(\Delta E_{b a t}\right)$.

In order to calculate the charging and discharging efficiencies, we can divide the operating range of Trio and Heliomote into multiple phases as shown in Figure 3.20 . battery discharge period (D1), capacitor discharge period (D2), recharge period (C), and saturation period (S). The balance of the energy level of each component for these phases are shown in Figure 3.21 .

The charging and discharging efficiency results are summarized in Table 3.6. The Trio node has both a battery discharge period and a capacitor discharge period because it has two-level storage of a supercapacitor and Li-ion battery. Trio also has a recharge period, but not a saturation period. We observed a battery discharging efficiency of $24.5 \%$ to $34.9 \%$, capacitor discharging efficiency of $54.3 \%$ to $68.6 \%$, and charging efficiency of $28.5 \%$ to $46.9 \%$. The Heliomote node has a battery discharge period and a recharge period. It has a saturation period because the charging protection circuit is activated during its operation. We observed a battery discharging 

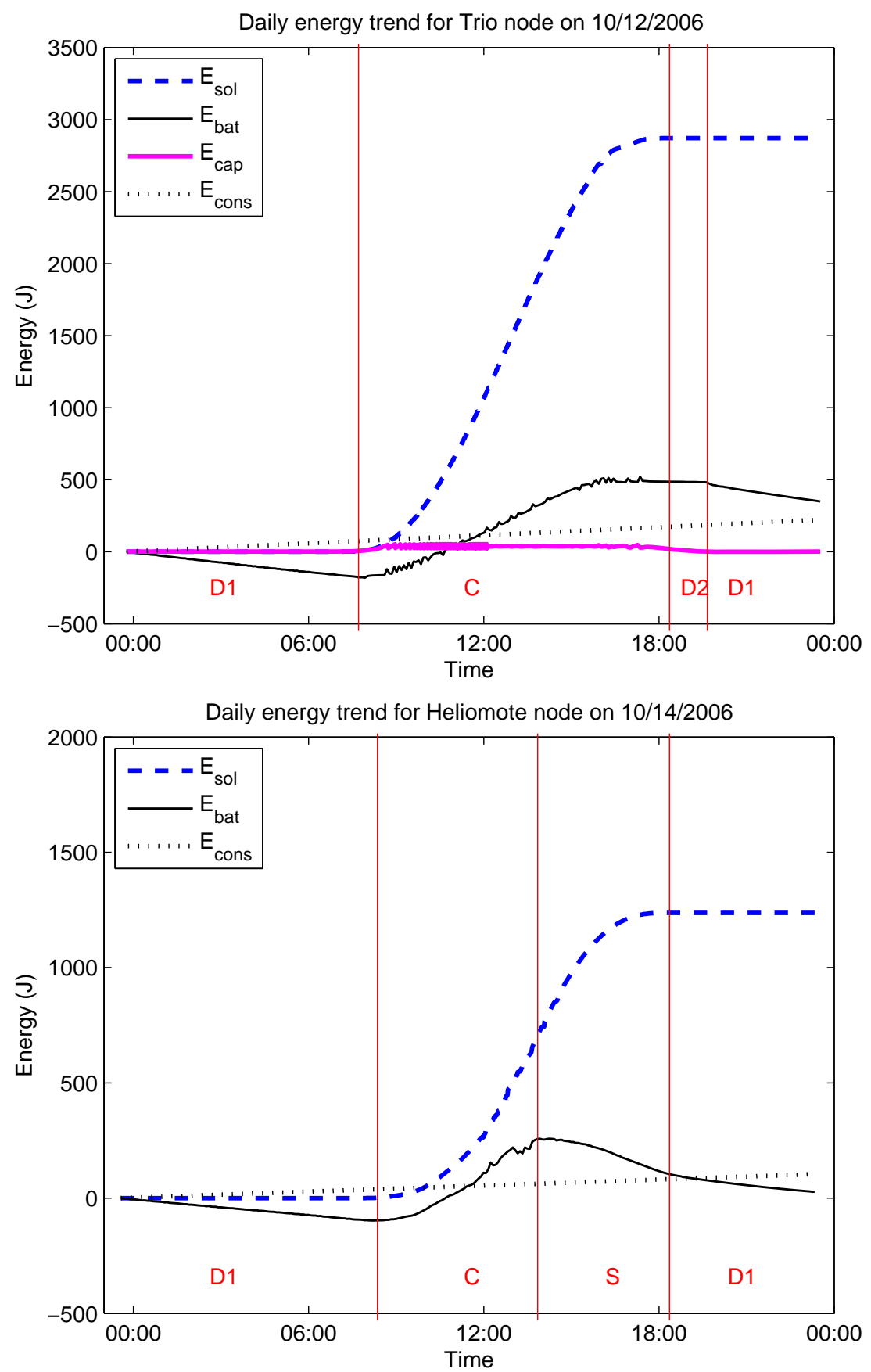

Figure 3.20: Daily energy flow of Trio and Heliomote 

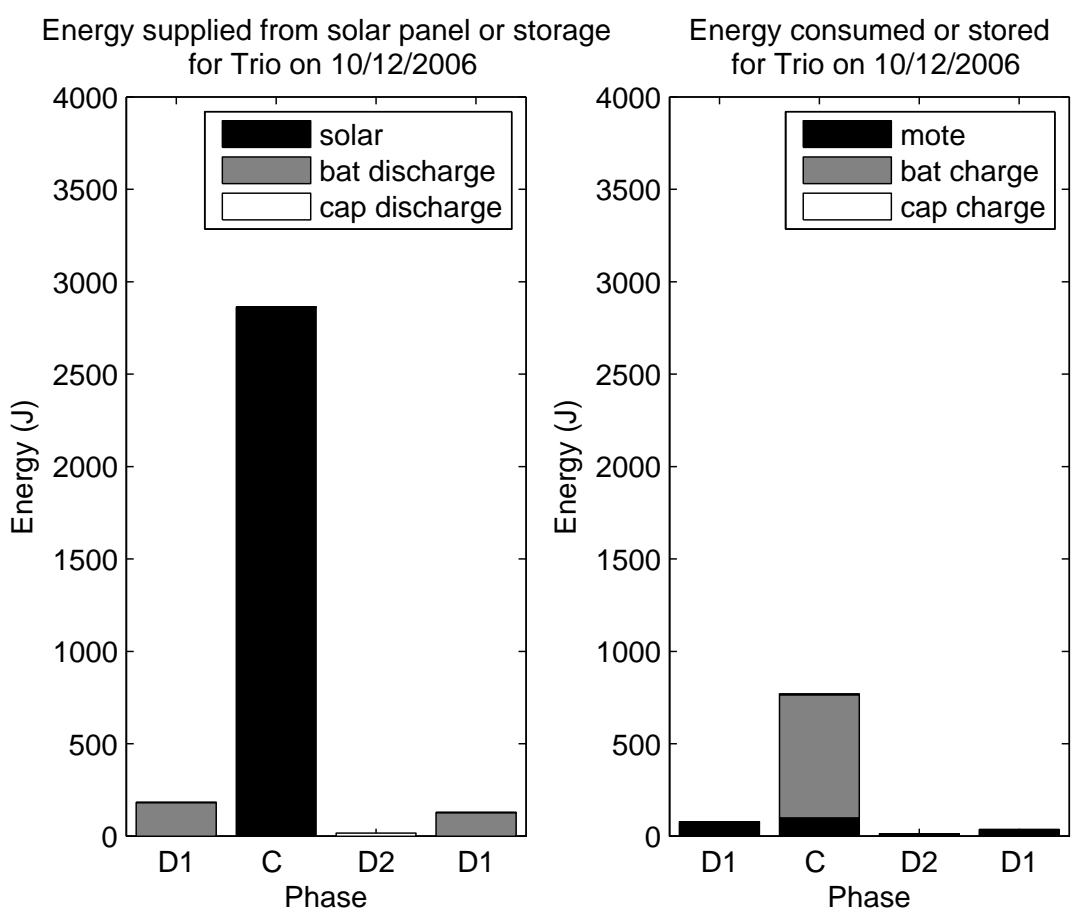

Energy supplied from solar panel or storage
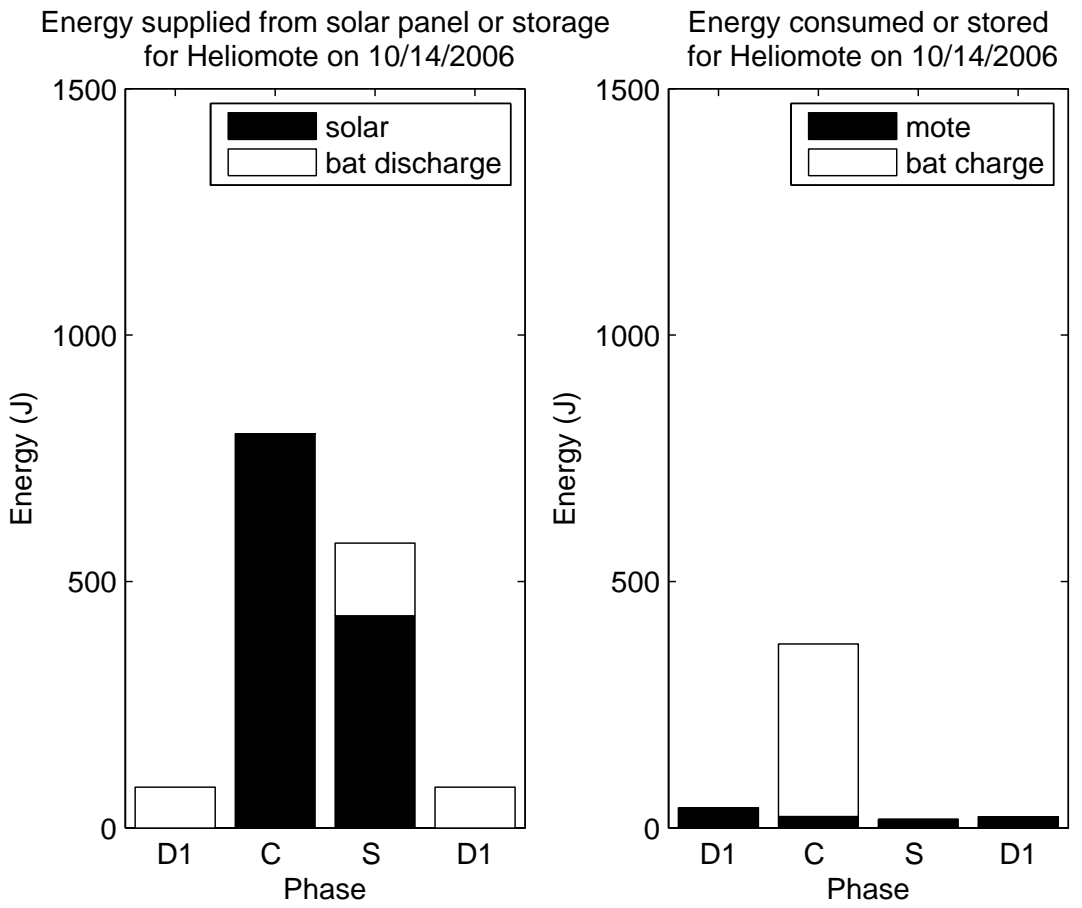

Figure 3.21: Energy flow of Trio and Heliomote at different phases 
efficiency of $32.2 \%$ to $35.6 \%$ and charging efficiency of $45.5 \%$ to $54.2 \%$.

We can see that the charging and discharging efficiency of Heliomote is as good as that of Trio, but its system efficiency $(6.9 \%$ to $14.6 \%)$ is much smaller than that of Trio (19.5\% to $33.4 \%$ ). This is because much of the solar energy is wasted during the saturation period; at a lower battery capacity, the system efficiency would be higher. If we assume that Heliomote does not go through the saturation period and utilizes the solar energy during saturation period at the same rate as in recharge period, then the system efficiency would be $31.9 \%$ to $41.9 \%$.

\subsection{Summary of Micro-solar Power System Archi- tecture}

In this chapter, we have introduced an architecture for micro-solar power systems by exploring the characteristics of each constituent component. The behavior of each component and its interactions with other components can be summarized in the following way:

\section{External Environment}

The radiation condition at a certain time and location is described by radiation rate $R R$, which is a real number between 0 and 1 . The radiation rate is determined by either an astronomical model or an astronomical model with local enhancements:

$$
R R \in[0,1]
$$

\section{Solar Collector}

The operating point of a solar panel is described by the $I$ - $V$ curve of the panel, which is determined by a sequence of $(\mathrm{I}, \mathrm{V})$ pairs taken from empirical measurement. The current of the solar panel under this radiation rate, $I_{r a d}$, is given by the product of the radiation rate $R R$ and the solar panel current in the I-V curve $I_{\text {panel }}$.

$$
\begin{aligned}
& I_{\text {panel }}=\operatorname{IVCurve}\left(V_{\text {panel }}\right) \\
& I_{\text {rad }}=R R \cdot I_{\text {panel }}
\end{aligned}
$$




\section{Energy Storage}

The energy storage is described by its voltage level, energy level and chargedischarge efficiency. The voltage-to-energy relationship, which is usually given by the manufacturer, describes the relationship between the voltage level and the energy level of the energy storage. The charge-discharge efficiency, which depends on the technology of the energy storage, describes how much energy can be discharged when a given energy is charged.

$$
\begin{aligned}
& V_{\text {stor }}=\mathrm{E} \text {-to- } \mathrm{V}\left(\mathrm{E}_{\text {stor }}\right) \Leftrightarrow \mathrm{E}_{\text {stor }}=\mathrm{V} \text {-to- } \mathrm{E}\left(V_{\text {stor }}\right) \\
& \mathrm{E}_{\text {discharged }}=\mathrm{Eff}_{\text {stor }} \cdot \mathrm{E}_{\text {charged }}
\end{aligned}
$$

\section{Input Regulator}

The operation of an input regulator is described by its power efficiency and a transfer function. The power delivered out of an input regulator is proportional to the power from the solar panel and the power efficiency of the input regulator, which depends on the solar panel voltage, the solar panel current and the energy storage voltage. The input regulator sets the operating point of the solar panel, interconnecting with the energy storage. Depending on the type of input regulator being used (e.g. no-input regulator or constant-input-voltage regulator), the voltage of the solar panel can follow the voltage of the energy storage, or can be set to a constant.

$$
\begin{aligned}
& P_{\text {in-reg }}=\mathrm{Eff}_{\text {in-reg }} \cdot P_{\text {panel }} \\
& V_{\text {panel }}=V_{\text {stor }}+V_{t h} \text { or const }
\end{aligned}
$$

\section{Output Regulator}

The operation of an output regulator is described by power efficiency and the output voltage. The power efficiency of an output regulator is dependent on the energy storage voltage and the load current. The output voltage of an output regulator is relatively constant over its operating range. The power consumption of an output regulator is proportional to the power consumption of the load and inversely 
proportional to the power efficiency.

$$
\begin{aligned}
& V_{\text {out }- \text { reg }}=\text { const } \\
& P_{\text {out-reg }}=\frac{1}{\text { Eff }_{\text {out-reg }}} \cdot P_{\text {load }}
\end{aligned}
$$

\section{Load}

The operation of the load is described by its average current and operating voltage. The average current is determined by the application, and the operating voltage is determined by the output regulator.

$$
\begin{aligned}
& I_{\text {load }}: \text { given by application } \\
& V_{\text {load }}=V_{\text {out }- \text { reg }}
\end{aligned}
$$

\section{Overall System Behavior}

Once the behavior of the components of a micro-solar power system and their interconnections are defined, the system behavior can be described as follows:

- Surplus power from the solar radiation after being used for load:

$$
P_{\text {net }}=P_{\text {in-reg }}-P_{\text {out }- \text { reg }}
$$

- If the surplus is positive $\left(P_{n e t}>0\right)$,

System is in recharge state with energy of $P_{n e t} \cdot \Delta t$ being charged.

- If the surplus is non-positive $\left(P_{\text {net }} \leq 0\right)$,

System is in discharge state with energy of $\left(-P_{n e t}\right) \cdot \Delta t$ being discharged.

- Change of the energy level and the voltage of the energy storage:

$$
\begin{aligned}
& E_{\text {stor }}=E_{\text {stor }}+P_{\text {net }} \cdot \Delta t \\
& V_{\text {stor }}=\mathrm{E}-\mathrm{to}-\mathrm{V}\left(E_{\text {stor }}\right)
\end{aligned}
$$

In the next chapter, we construct a micro-solar power system simulator based on this architecture. 


\section{Chapter 4}

\section{Design of Micro-Solar Power System Simulator}

One of the difficulties of building a network of micro-solar power systems is that the whole process of design, development and deployment takes a long time. Suppose a micro-solar power system is deployed in an outdoor environment. It may have an energy surplus for a few days or even several months, but, depending on the weather or other conditions that affect solar radiation, it can either keep accumulating solar energy or may lose stored battery energy, becoming unable to provide energy for perpetual operation. This is because the operation of a micro-solar power system depends greatly on the characteristics of the environment and each component. In order to have a reasonable estimation of system behavior we may take extensive measurements over time, but this can make the whole process of design, development and deployment even longer. What we need is a design and simulation tool that allows us to estimate the behavior of a micro-solar power system with sufficient accuracy over the long term without taking the time and effort of an actual development and deployment. In this chapter, we present a Matlab-based micro-solar power system simulator and describe its key ideas. The simulator takes a configuration of a micro-solar power system along with user-defined data and quickly generates behavior estimates for either a short period or a long period of time. The rest of this chapter is organized as follows: first, Section 4.1 states an overall architecture and principles for the micro-solar power system simulator; then, Sections 4.2 through 4.7 describe 
key implementation ideas that translate each component of a micro-solar power system into the framework of the simulator; finally, Section 4.8 validates the simulation model with benchtop experiments.

\subsection{Overall Architecture and Principles}

\subsubsection{Modularity}

The simulator is composed of the following modules: external environment module, solar panel module, input regulator module, energy storage module, output regulator module and load module. Each module of the simulator describes the behavior of a corresponding component of a micro-solar power system (Figure 4.1). Each simulator module can be exchanged with another module in the same class. For example, a definition of $3.6 \mathrm{~V}-50 \mathrm{~mA}$ flexible solar panel can be used instead of a definition of $4 \mathrm{~V}-100 \mathrm{~mA}$ solar panel without changing other parts of the simulator. In the simulator, the internal operation of each component is hidden, so in order to access the characteristic of each component, a user should use a function that describes the component.

\subsubsection{Time-Event Based Simulator}

Our simulator computes the state of each component of a micro-solar power system when the initial condition (e.g. initial battery capacity) and the time-event vector are given. (Figure $4.2(\mathrm{a})$ ). The time-event vector is a tuple of discrete times during the day at which the state of the micro-solar power system will be evaluated. For example, the time-event vector can be given as $\left[t_{0}, t_{1}, t_{2}, t_{3}, \cdots, t_{n}\right]=[0,0.25,0.5,0.75, \cdots, 24]$ for simulating system status on a particular day at 15 minute interval starting from midnight. Overall, the estimation accuracy increases as the interval between the evaluation times gets smaller, but, at the same time, the computation time increases in proportion to the inverse of the interval. Once the state of the micro-solar power system is computed for one day, the long-term trend can be computed iteratively as is illustrated in Figure 4.2(b); the state of the system is evaluated with the result of one day being the initial condition of the next day. 


\section{External Environment}

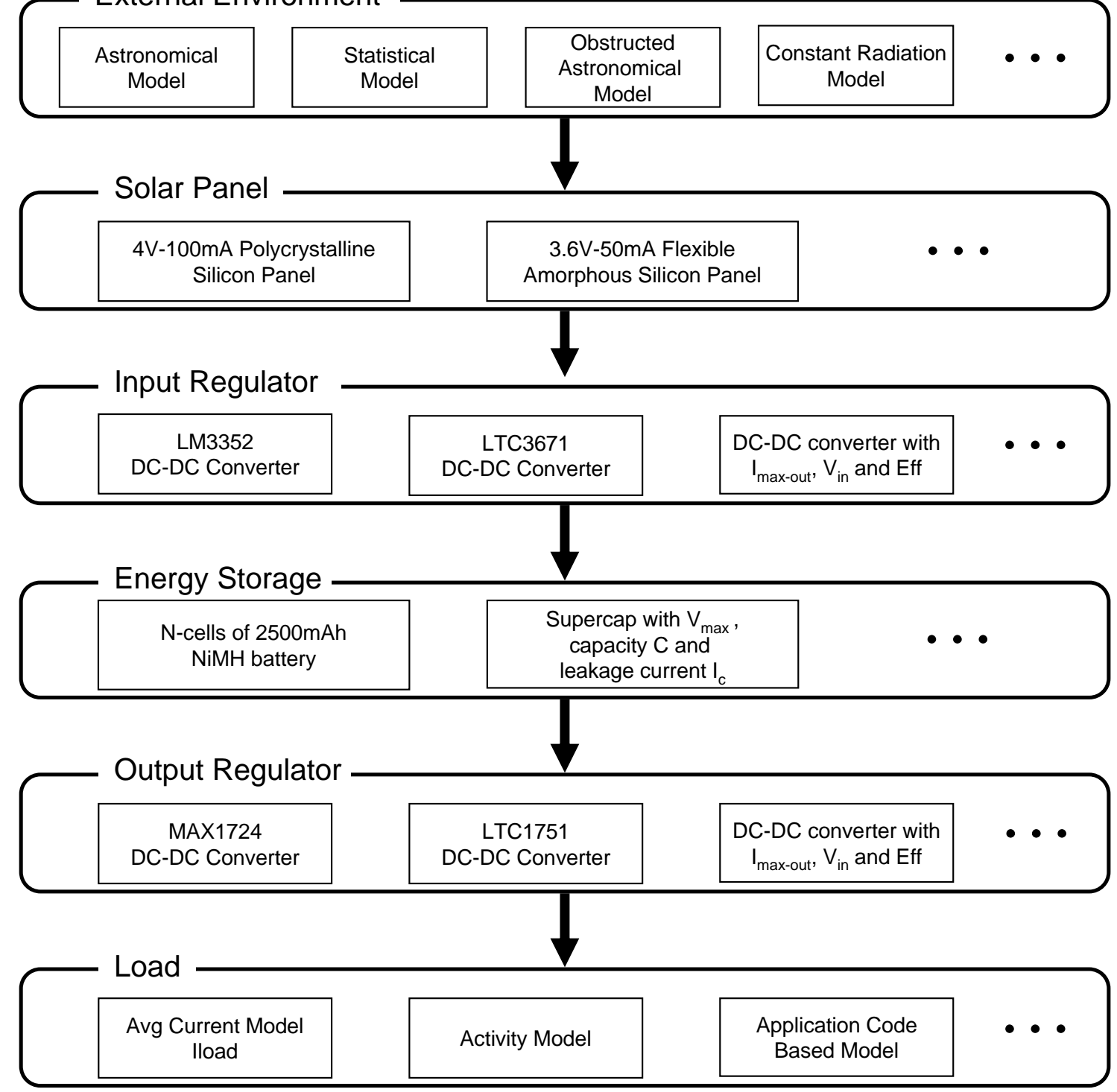

Figure 4.1: Modular design of micro-solar power system simulator 


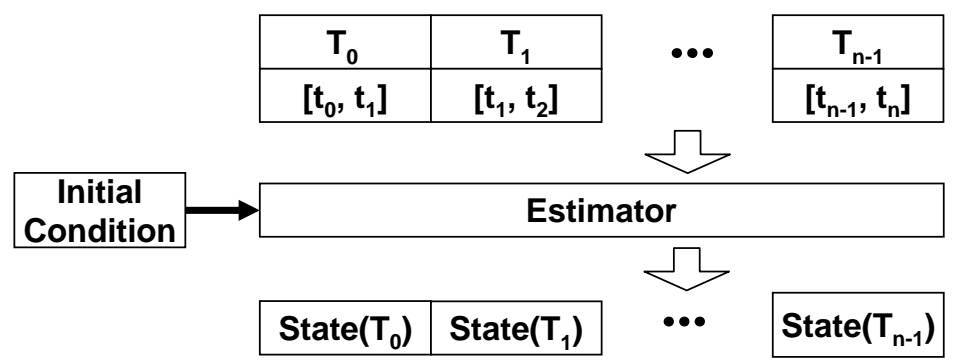

(a) Estimation of daily trend

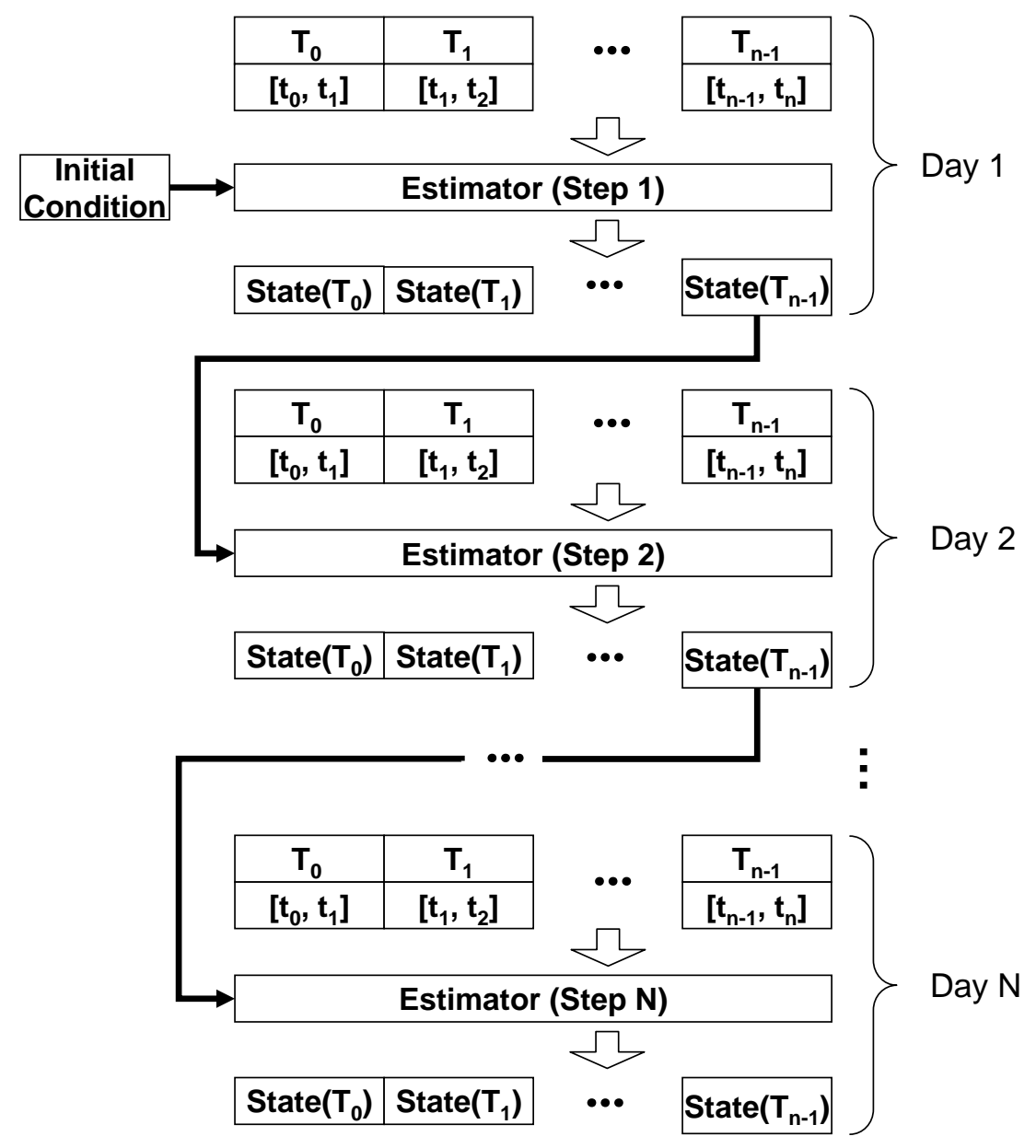

(b) Estimation of long-term trend

Figure 4.2: Time-event based simulator 


\subsubsection{Defining a Component Using User-provided Data}

A component of a micro-solar power system has a common generic behavior, but its detailed behavior may vary depending on what instance of a component is used. Our simulator provides two different ways to customize the behavior of a component: curve-fitting and piecewise linear interpolation.

The curve-fitting method is used when the behavior of a component can be described by a formula. A formula generally consists of a collection of variables, constants and operators. When measurements of the component are available, the curve-fitting algorithm determines the unknown constants that minimize the errors between the measured output and the estimated output. The solar-panel IV characteristic is one example where the curve fitting method is used. Figure 4.3 shows that the I-V characteristic of a solar panel can be described as a generic curve $I=I_{s c}-A \cdot(\exp (B \cdot V)-1)$. This formula describes the relationship between the solar panel voltage $V$ and the solar panel current $I$. Each instance of this solar panel model describes a different type of solar panel simply by altering the values for the parameters $I_{s c}, A$, and $B$.

Piecewise linear interpolation is used when the behavior of a component cannot be represented by a single formula. In order to represent the behavior of a component with a known formula, piecewise linear interpolation partitions the input domain. Suppose the input signal of a component operates in domain $D$. Then the domain can be partitioned into $D_{1}, D_{2}, D_{3}, \cdots, D_{n}$ so that the behavior of the component can be described with linear functions $f_{1}, f_{2}, f_{3}, \cdots, f_{n}$ in each partition of the domain (Figure 4.4). Here are a few examples that can be described by piecewise linear interpolation:

- The output voltage of a regulator over its load current

- The efficiency of a regulator over its load current

- The voltage level of a battery over its capacity

In order to define the domain partitions and the piecewise linear functions, the piecewise linear interpolation method takes a sequence of value pairs: $\left(x_{1}, y_{1}\right),\left(x_{2}, y_{2}\right)$, $\left(x_{3}, y_{3}\right), \cdots\left(x_{n}, y_{n}\right),\left(x_{n+1}, y_{n+1}\right)$. Then, the domain partitions $D_{i}$ and the piecewise 


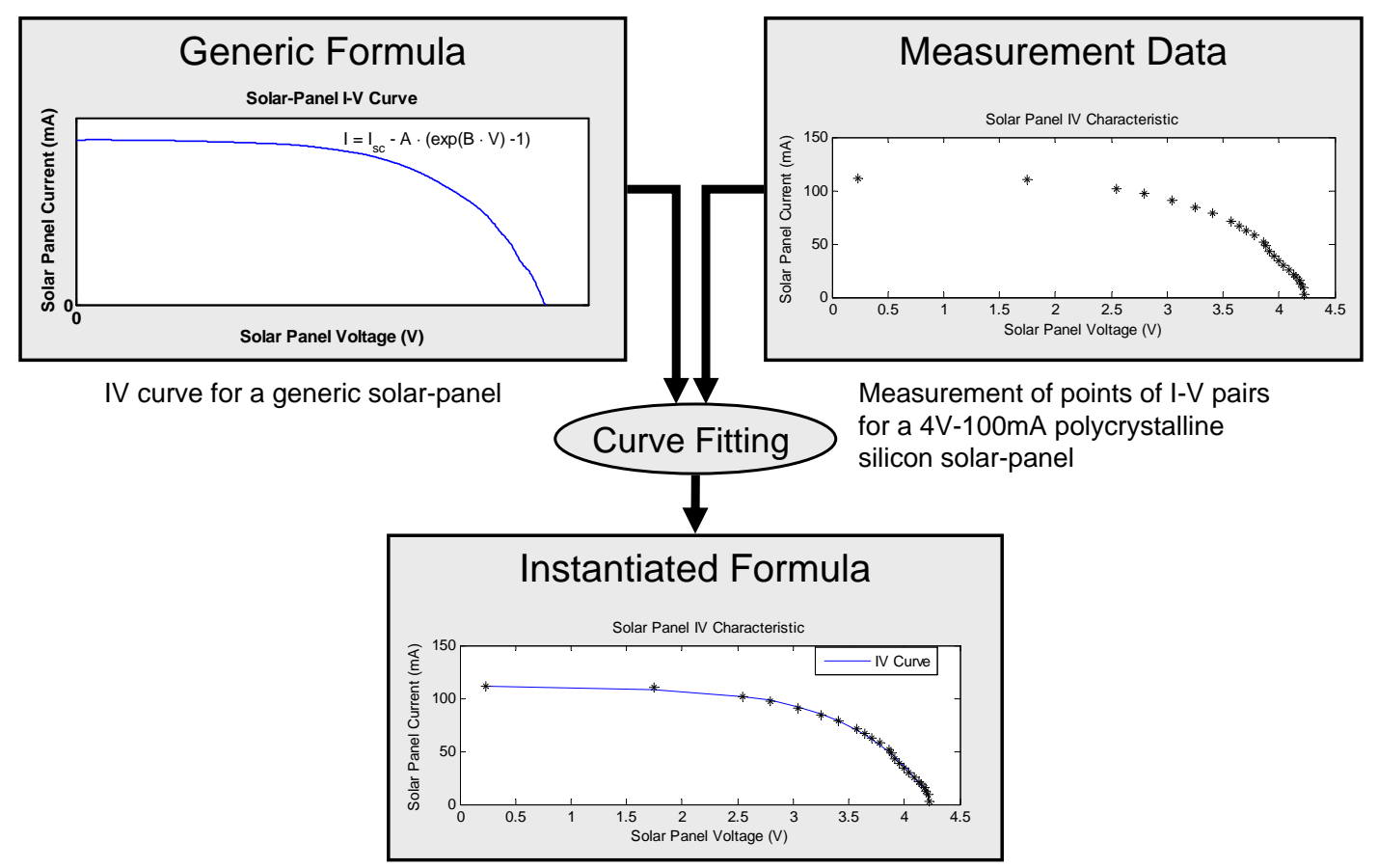

IV curve for a $4 \mathrm{~V}$-100mA polycrystalline silicon solar-panel with $A=0.2526, B=1.4255$ and $\mathrm{I}_{\mathrm{sc}}=111.160$

Figure 4.3: Defining a component using user-provided data: curve-fitting method

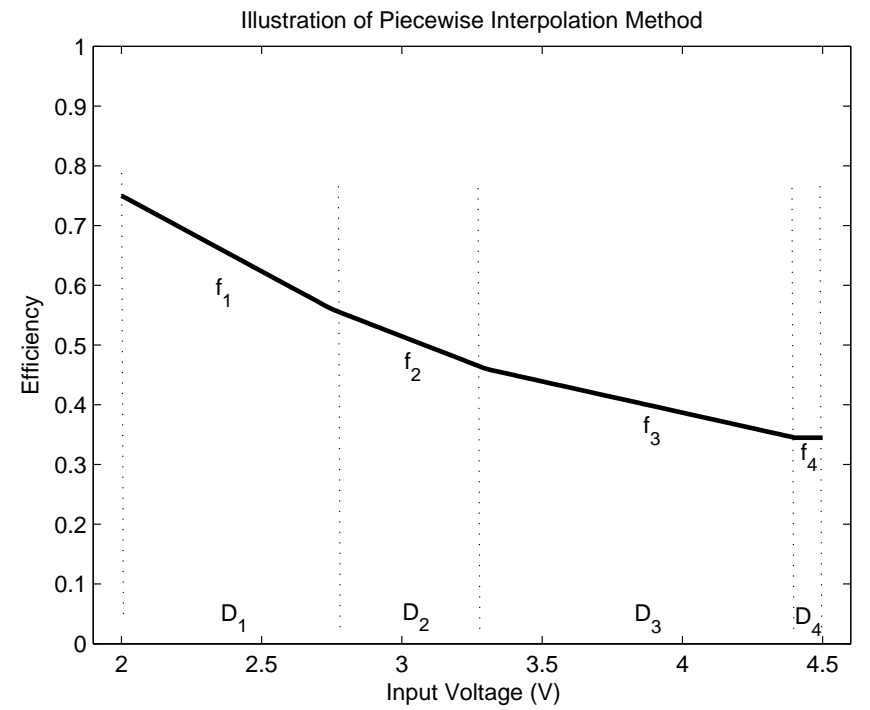

Figure 4.4: Defining a component using user-provided data: piecewise linear interpolation method 
linear function $f_{i}$ are determined as follows:

$$
\begin{aligned}
D_{i} & =\left[x_{i}, x_{i+1}\right] \\
f_{i} & =\frac{y_{i+1}-y_{i}}{x_{i+1}-x_{i}}\left(D-x_{i}\right)+y_{i}
\end{aligned}
$$

The algorithm in Figure 4.19 estimates the voltage level of a NiMH battery for a given capacity using piecewise linear interpolation by fitting chords to the curve.

\subsubsection{Wiring Components}

In our micro-solar power simulator, we use function calls and a sequence of statements to wire components to each other. We modularize this so that the wiring can be done by plugging components into each other, with all the constraints hidden inside the components.

\subsection{Modeling Solar Radiation}

Our simulator provides a few of ways to model solar radiation: an astronomical model, a statistical model and an astronomical model with obstruction vector.

\subsubsection{Astronomical Model}

The astronomical model, which is based on the algorithm by Dave et al. [DHM75] (Figure 3.2 in Chapter 3.1.1), estimates the solar radiation rate by calculating the angle $\Theta\left(0^{\circ} \leq \Theta \leq 180^{\circ}\right)$ between the sunlight and the normal to the solar panel. Depending on whether the sunlight is above or below the horizon, the solar radiation rate is given as $\cos \Theta$ (when $0^{\circ} \leq \Theta \leq 90^{\circ}$ ) or 0 (when $90^{\circ} \leq \Theta \leq 180^{\circ}$ ). Thus, the solar radiation rate can be represented as $\max (\cos \Theta, 0)$.

The solar radiation rate $\max (\cos \Theta, 0)$ is a function of several parameters that affect the time variation $(t, n)$ and installation variation $\left(L, \theta_{p}, \phi_{p}, \tau\right)$ :

- $t$ : Hour of the day $(0 \leq t \leq 24)$

- $n$ : Day of the year $(1 \leq n \leq 365)$ 
- $L$ : Latitude $\left(-90^{\circ} \leq L \leq 90^{\circ}\right)$

- $\theta_{p}$ : Tilted angle of solar panel $\left(0^{\circ} \leq \theta_{p} \leq 180^{\circ}\right)$

- $\phi_{p}$ : Azimuth angle, panel orientation from north $\left(0^{\circ} \leq \phi_{p} \leq 360^{\circ}\right)$. For example, 90 for the east, 180 for the south, and 270 for the west.

- $\tau$ : Atmospheric attenuation effect factor $(0 \leq \tau \leq 1)$

Then, $\cos \Theta$ can be given as follows:

$$
\cos \Theta=\cos \theta_{p} \cdot \cos \theta_{s}+\sin \theta_{p} \cdot \sin \theta_{s} \cdot \cos \left(\phi_{p}-\phi_{s}\right)
$$

The intermediate terms $\theta_{s}, \phi_{s}, \mathrm{~h}, \mathrm{x}, \delta$ are also given as follows:

- $\theta_{s}$ : Angle between solar radiation and local vertical $\left(0^{\circ} \leq \theta_{s} \leq 180\right)$ $\cos \theta_{s}=\sin \delta \cdot \sin L+\cos \delta \cdot \cos L \cdot \cos h$

- $\phi_{s}$ : Angle between solar radiation and local north $\left(-180^{\circ} \leq \phi_{s} \leq 180^{\circ}\right)$ $\sin \phi_{s}=-\cos \delta \cdot \sin h / \sin \theta_{s}$

- $h$ : Hour angle $\left(-180^{\circ} \leq h \leq 180^{\circ}\right)$

$h=15(t-12)$

- $x$ : Day of the year in radian $(0 \leq x \leq 2 \pi)$ $x=2 \pi n / 365$

- $\delta$ : Declination of sun $\left(-90^{\circ} \leq \delta \leq 90^{\circ}\right)$ $\delta=0.302-22.93 \cos x-0.229 \cos 2 x-0.243 \cos 3 x$ $+3.851 \sin x+0.002 \sin 2 x-0.055 \sin 3 x$

The algorithm for calculating the solar radiation rate is given in Figure 4.5 and 4.6.

Figures 4.7, 4.8 and 4.9 show the estimation of the daily solar radiation for different sets of parameters: time of the year $n$ (Figure 4.7), latitude $L$ (Figure 4.8), and inclination $\theta_{p}$ and panel orientation $\phi_{p}$ (Figure 4.9. 


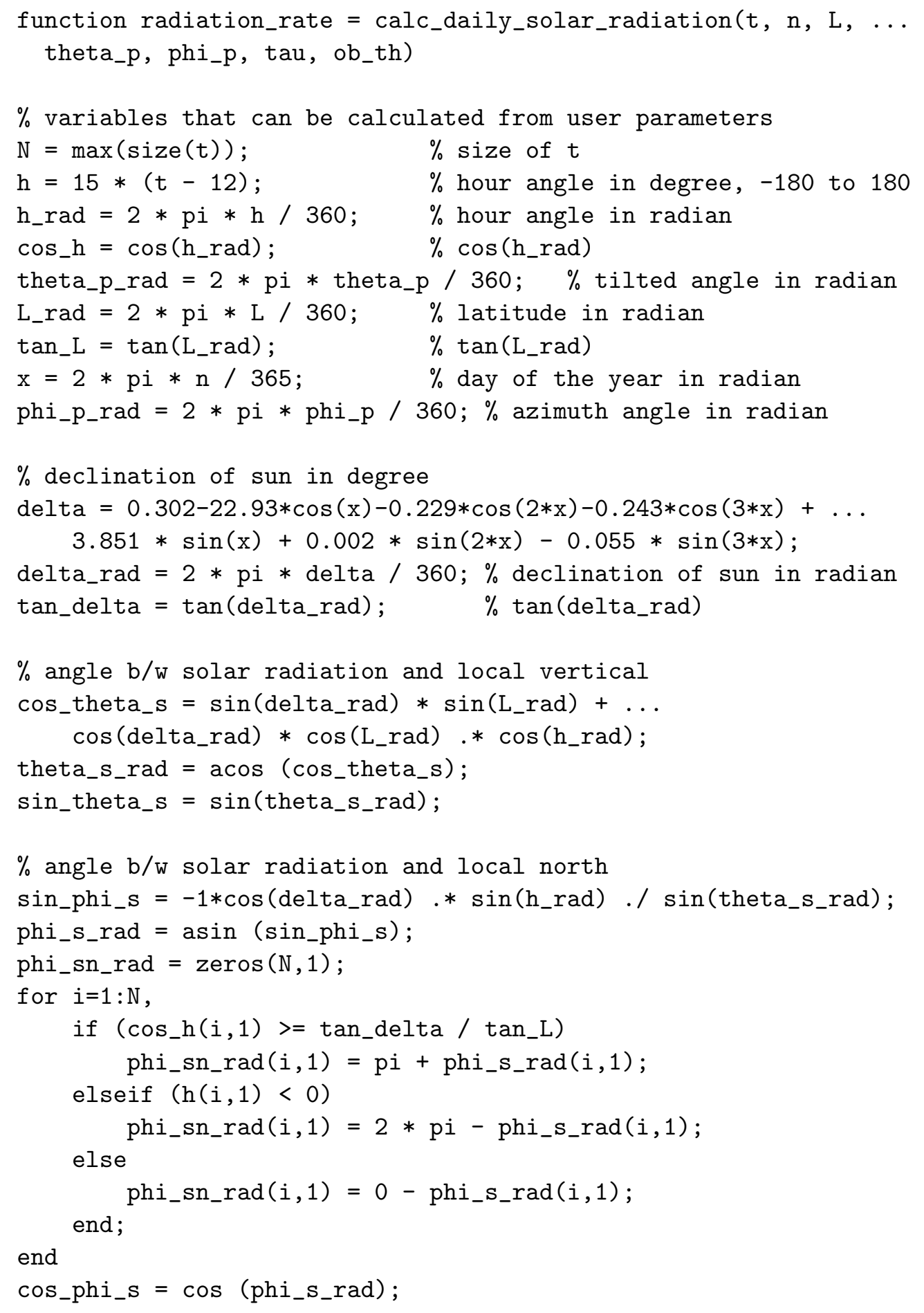

Figure 4.5: Matlab algorithm for estimating solar radiation using an astronomical model 


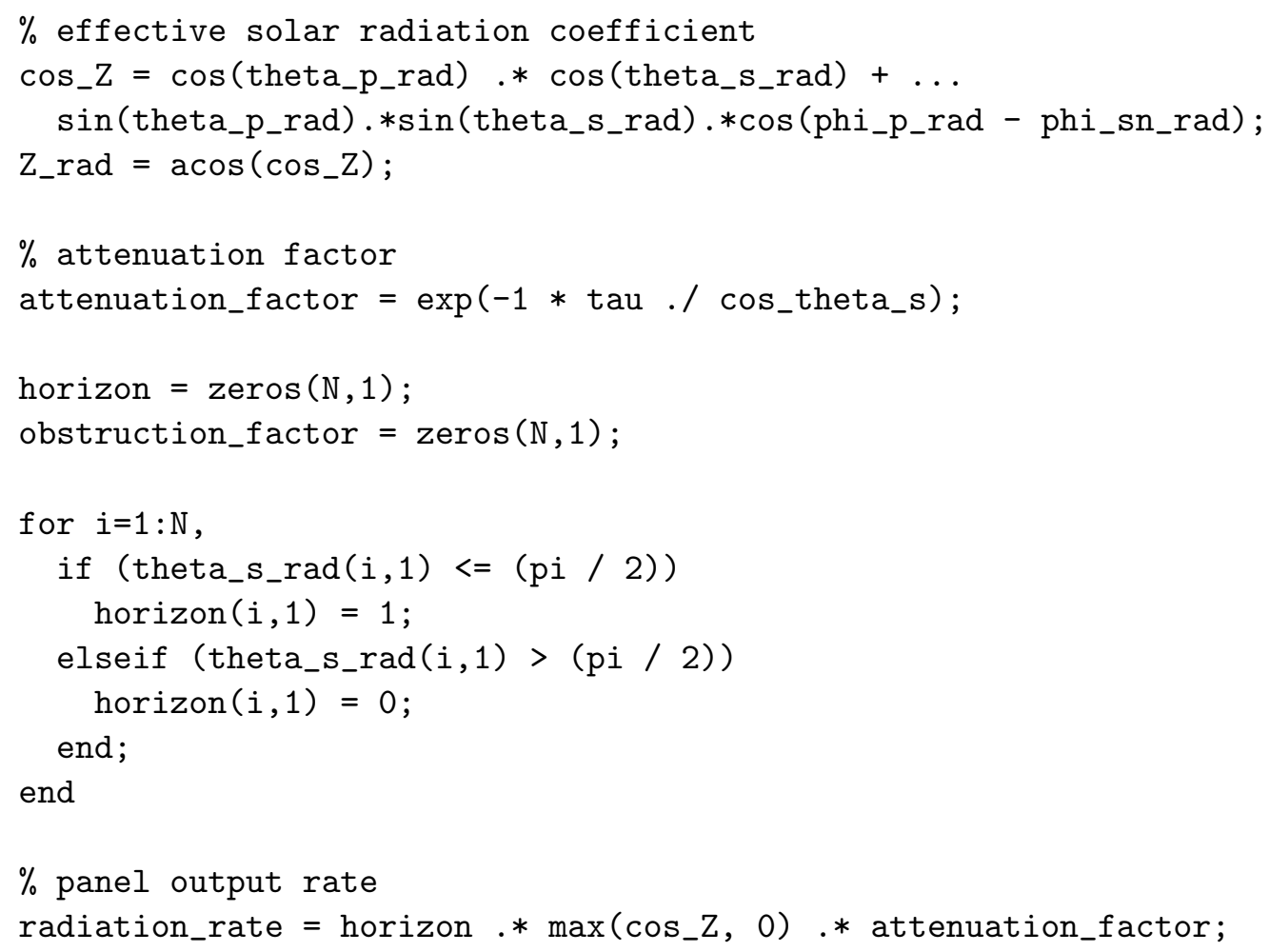

Figure 4.6: Matlab algorithm for estimating solar radiation using an astronomical model (continued) 


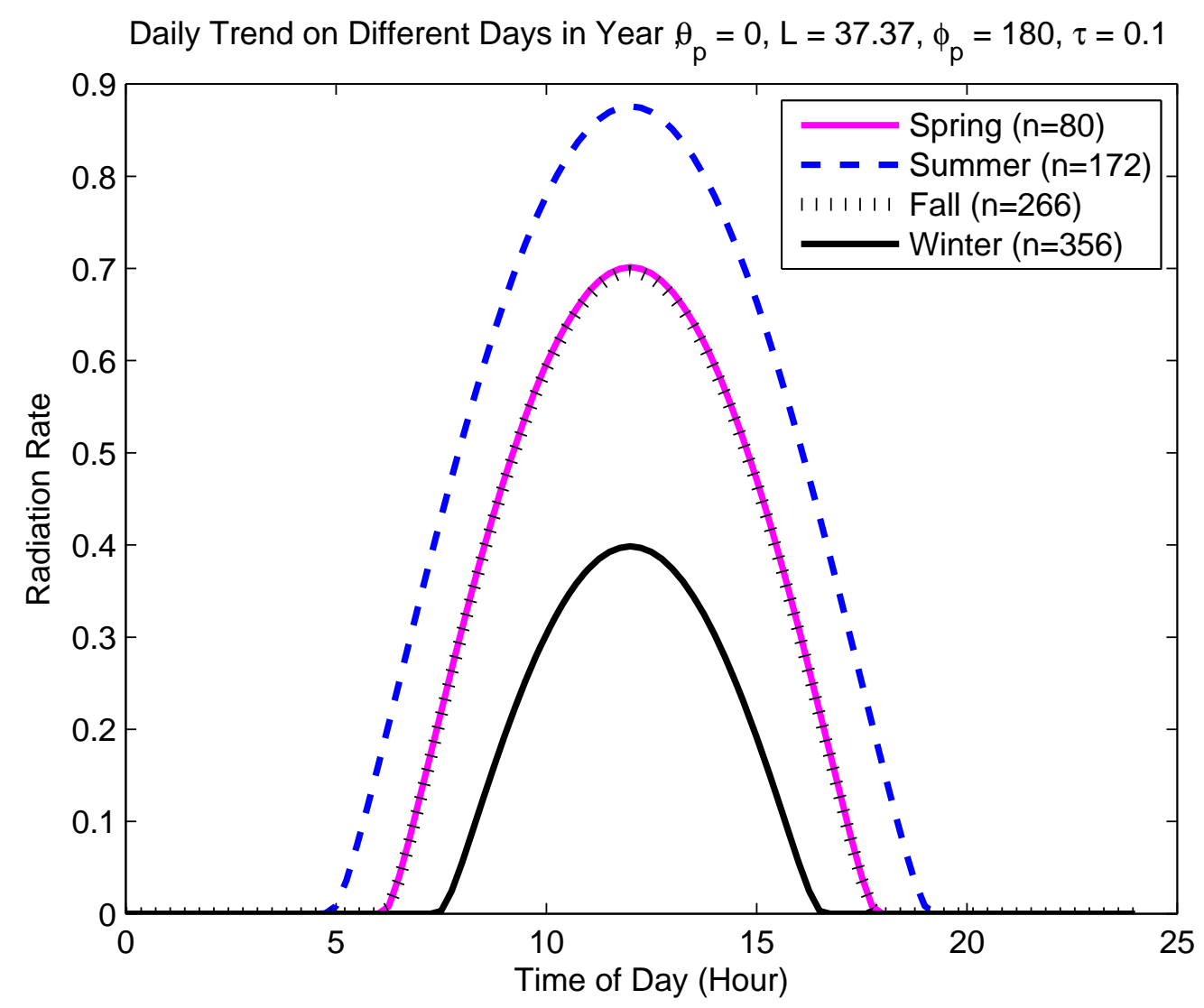

Figure 4.7: Astronomical model with effects of seasonal variation 


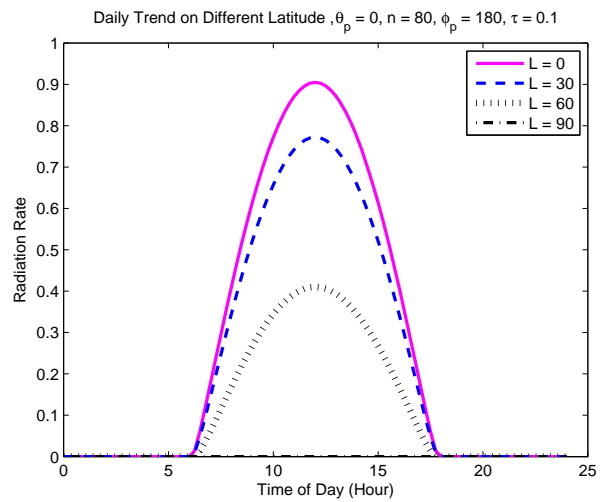

(a) Spring and fall

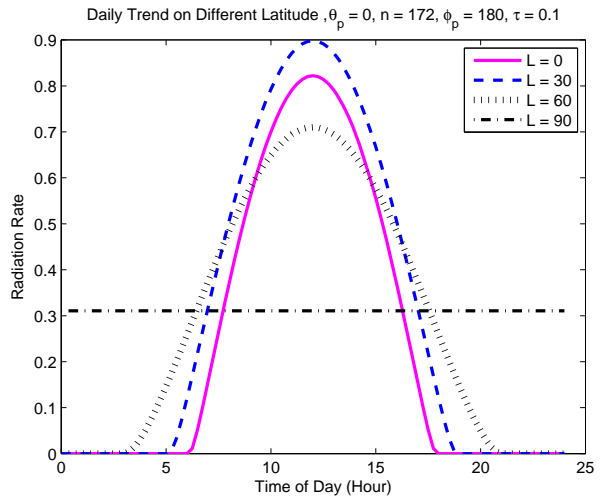

(b) Summer

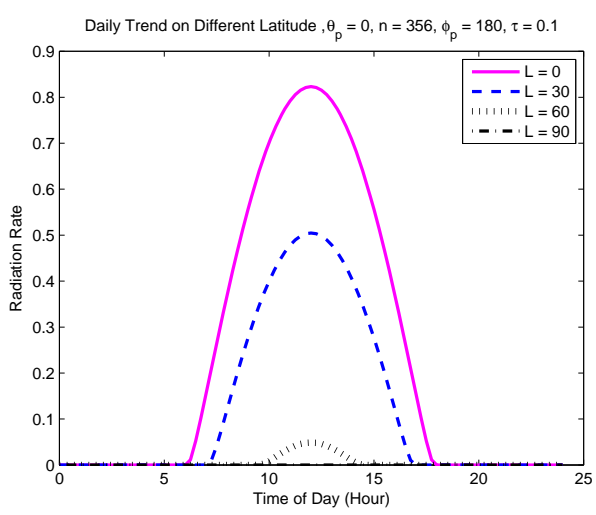

(c) Winter

Figure 4.8: Astronomical model with effects of latitude and seasonal variation with the solar panel flat $\left(\theta_{p}=0\right.$ and $\left.\phi_{p}=180\right)$. With each case, the day of year $n$ is fixed to 80 (spring), 172 (summer) or 356 (winter) while the latitude $L$ varies. 


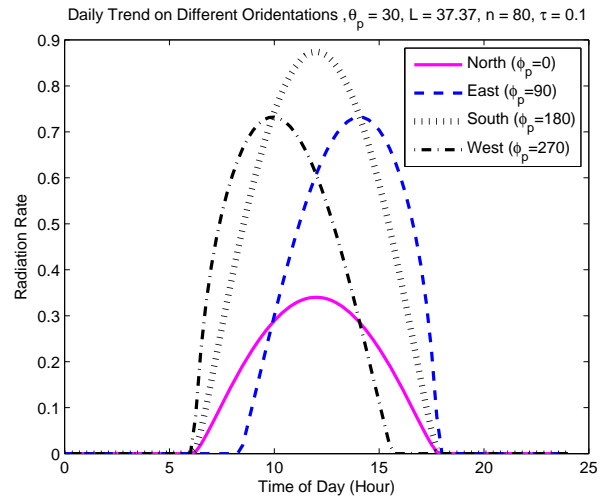

(a) Inclination $=30$

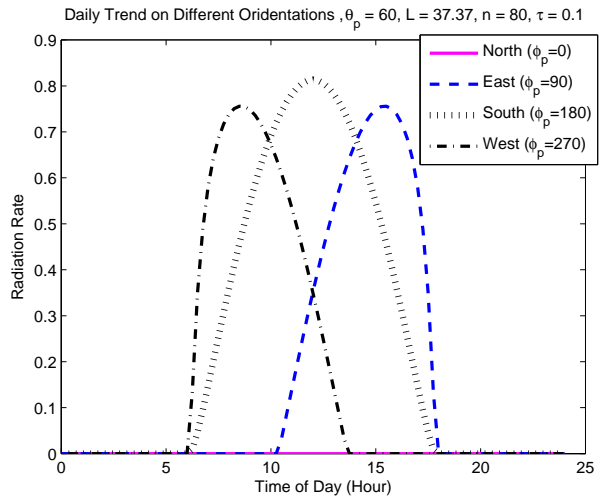

(b) Inclination $=60$

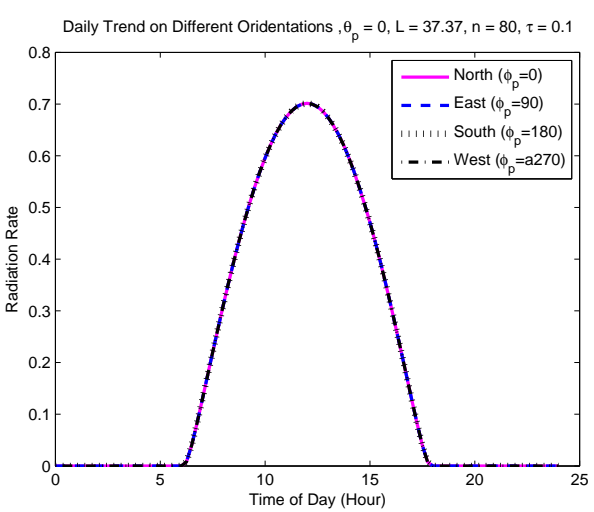

(c) Inclination $=0$

Figure 4.9: Astronomical model with effects of orientation and inclination on a particular day in mid-March $(n=80)$. With each case, the inclination $\theta_{p}$ is fixed to 30 , 60 or 0 while the orientation $\phi_{p}$ varies. 


\subsubsection{Obstructed Astronomical Model}

The estimation of solar radiation from the astronomical or statistical model can be a useful tool in understanding the long-term variation of solar radiation, but it deviates from the real measurement in many cases when the solar radiation is obstructed by objects or diffused by weather. In this subsection, we improve the accuracy of our simulator on the effects of obstructions using the previously measured obstruction profile.

When we estimate solar radiation under obstruction effects, we assume that objects that cause obstructions are stationary, and we can expect the same pattern of obstructions from one day to another. At time $t$ and day $n$, we can define the following variables:

- $R 1(t, n)$ : Estimation of solar radiation using an astronomical model.

- $M(t, n)$ : Measurement of solar radiation.

- $O b(t, n)$ : Obstruction factor.

- $R 2(t, n)$ : Scaled astronomical model. $R 1(t, n)$ is scaled to match the envelop of the measurement $M(t, n)$. When $S$ is such a scaling factor, $R 2(t, n)$ is given as $S \cdot R 1(t, n)$.

- $R 3(t, n)$ : Reconstructed astronomical model, where the solar radiation loss due to the obstruction factor is subtracted from the scaled astronomical model. $R 3(t, n)$ is given as $S \cdot R 1(t, n) \cdot(1-O b(t, n))$.

Suppose $t$ is defined over discrete time intervals $t_{1}$ through $t_{m}$, and the measurement of solar radiation $M(t, n)$ has a maximum at interval $t_{\max }$. Then, the scaling factor $S$ is defined as follows:

$$
S=\frac{M\left(t_{\max }, n\right)}{R 1\left(t_{\max }, n\right)}
$$

Using the maximum point $M\left(t_{\max }, n\right)$ for the scaling factor $S$ may produce a misleading result, depending on the profile of the solar radiation measurement. In 
order to remove the case when the maximum point is an outlier, we used a (100$\alpha / 2) \%$-percentile point to calculate the scaling factor:

$$
S=\frac{M\left(t_{(100-\alpha / 2) \% \max }, n\right)}{R 1\left(t_{(100-\alpha / 2) \% \max }, n\right)}
$$

This removes outliers that exist outside $(100-\alpha / 2) \%$ of the node distribution. For example,

$$
S=\frac{M\left(t_{97.5 \% \max }, n\right)}{R 1\left(t_{97.5 \% \max }, n\right)}
$$

is an scaling factor estimate that removes $5 \%$ of outliers.

The obstruction factor $O b(t, n)$ is the relative difference between the scaled astronomical model $R 2(t, n)$ and the measurement $M(t, n)$.

$$
O b(t, n)= \begin{cases}\frac{R 2(t, n)-M(t, n)}{R 2(t, n)} & \text { if } R 2(t, n)>0 \\ 1 & \text { otherwise. }\end{cases}
$$

The reconstructed astronomical model at time $t$ and date $n^{\prime}, R 3\left(t, n^{\prime}\right)$ is given as $S \cdot R 1\left(t, n^{\prime}\right) \cdot\left(1-O b\left(t, n^{\prime}\right)\right)$. Since we assume that obstructions are stationary, $O b\left(t, n^{\prime}\right)=O b(t, n)$. Thus,

$$
R 3\left(t, n^{\prime}\right)=S \cdot R 1\left(t, n^{\prime}\right) \cdot(1-O b(t, n))
$$

While the example above used samples from a single day to estimate the scaling factor and the obstruction vector, estimates from multiple days can be used to reduce the error. The process of creating a reconstructed astronomical model is summarized in Figure 4.10 .

Figure 4.11 shows the measurement $M(t, n)$ with three different solar radiation estimations: the astronomical model $R 1(t, n)$, the scaled astronomical model $R 2(t, n)$ and the reconstructed astronomical model $R 3(t, n)$. A sensor node was placed in a meadow under a tree, and we used the measurement gathered on 10/13/2007 to calculate the scaling factor $S$ and the obstruction factor $O b(t, n)$. Figure 4.12 compares the solar radiation of different estimation methods with the measured value. We can see that the reconstructed astronomical model $R 3(t, n)$ is very close to the measurement $M(t, n)$ when the weather is sunny. In Figure 4.12, the estimation error of $R 3(t, n)$ is less than $25 \%$ on average. 


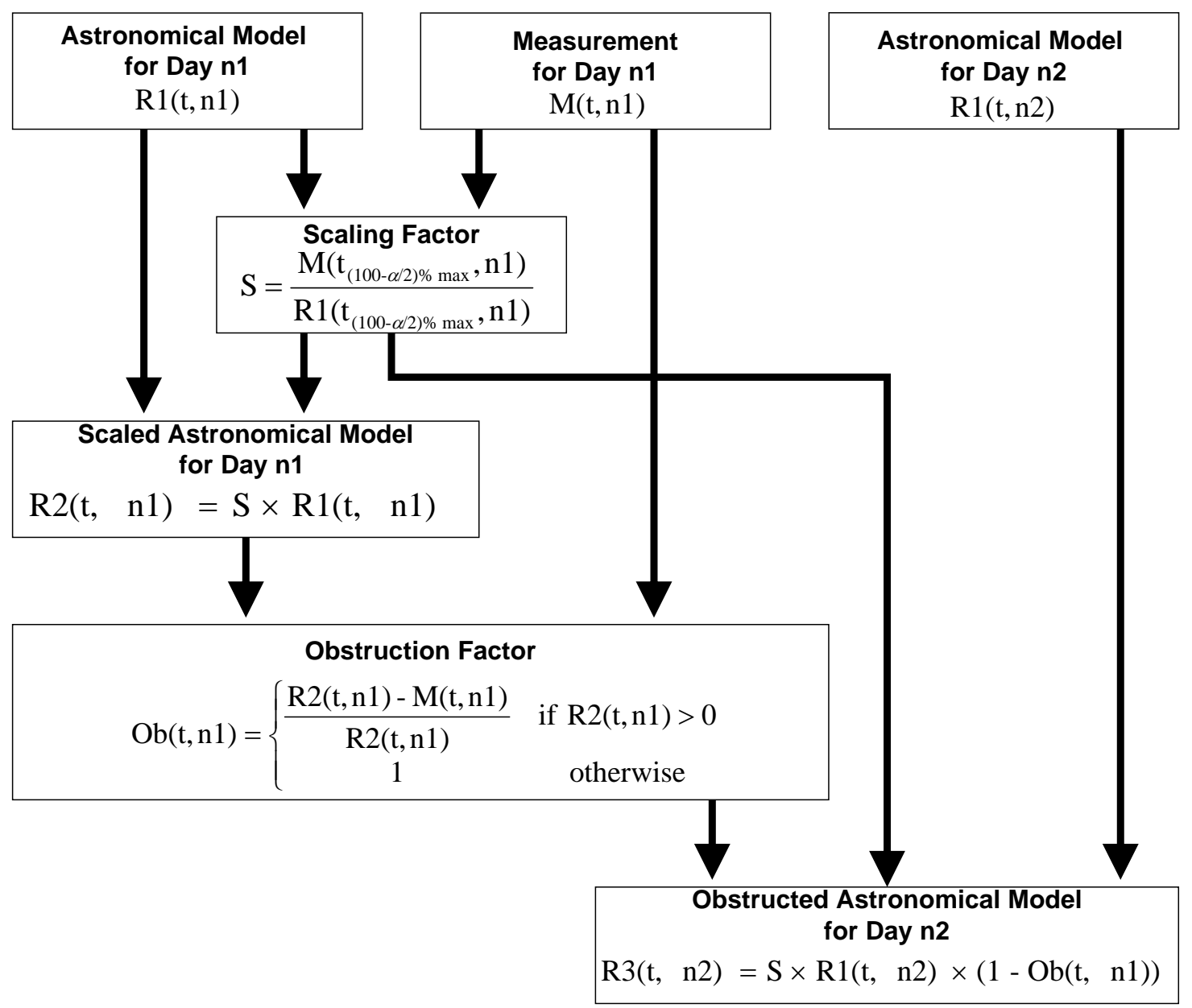

Figure 4.10: Obstructed astronomical model 


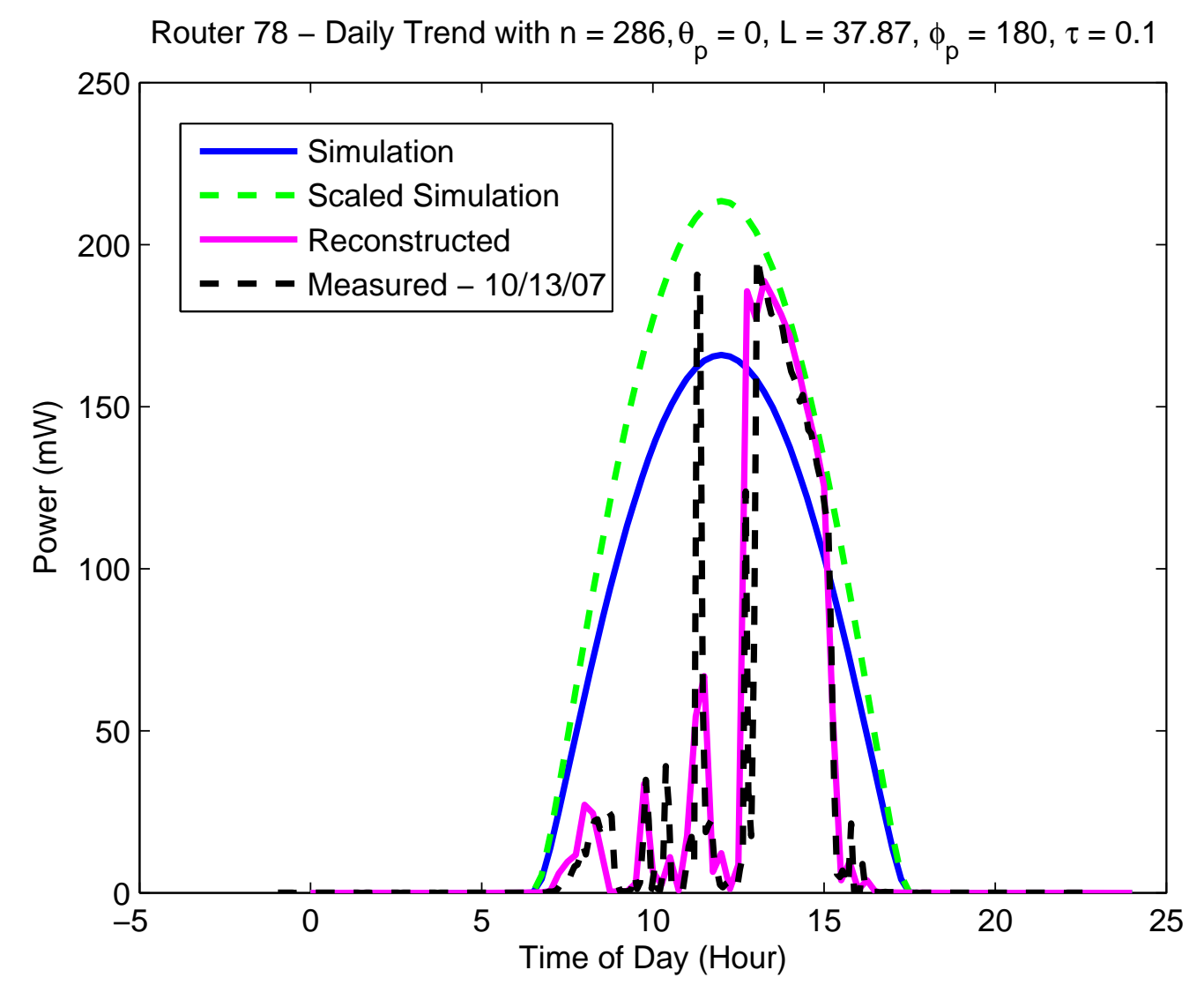

Figure 4.11: Estimating the solar radiation using obstruction measurement 


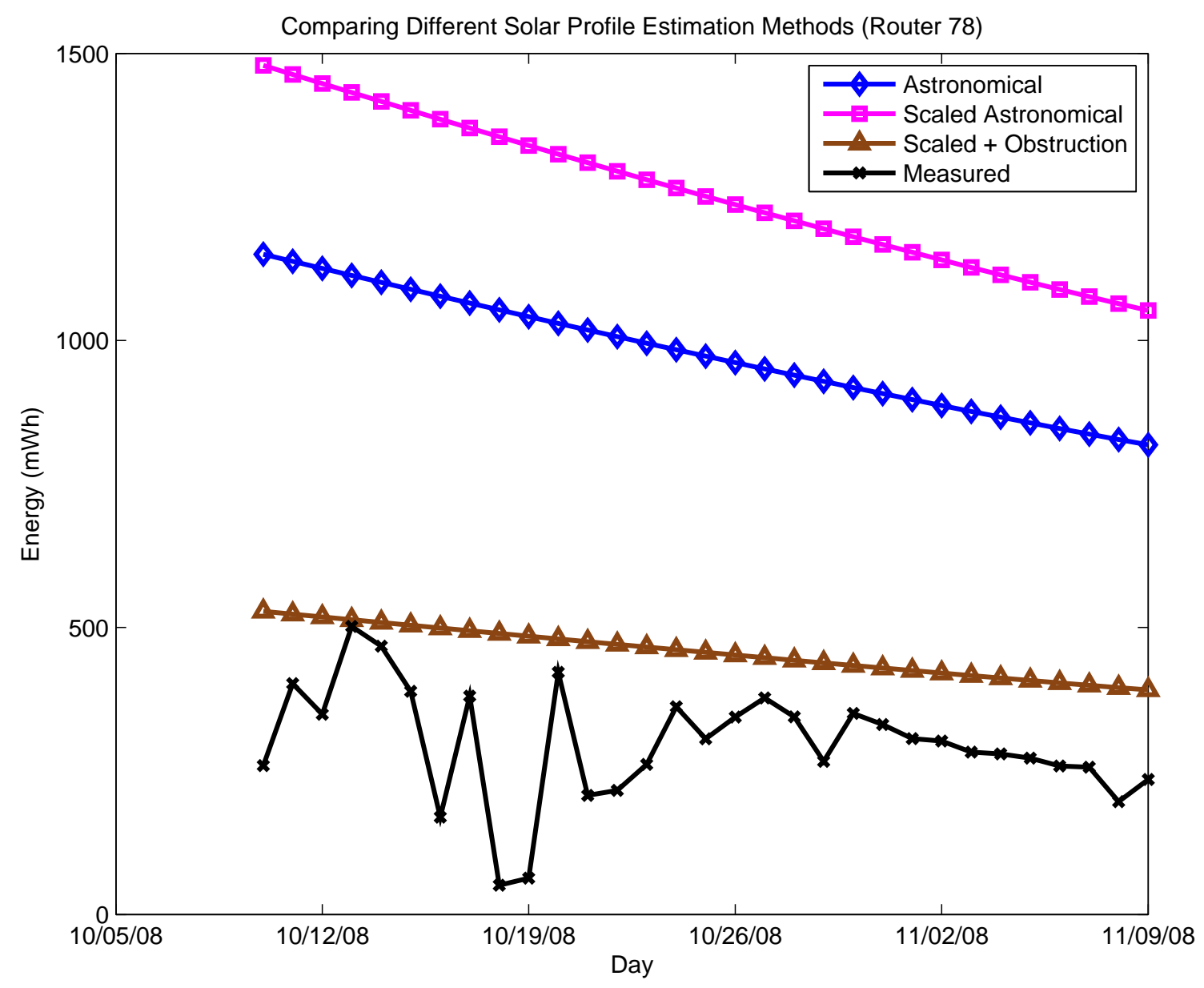

\begin{tabular}{|c|c|c|c|}
\hline \multicolumn{4}{|c|}{ Deviation of estimation from the measurement } \\
\hline & Astronomical & Scaled Astronomical & Reconstructed \\
\hline Mean & $68.3 \%$ & $73.3 \%$ & $24.8 \%$ \\
\hline
\end{tabular}

Figure 4.12: Comparing daily solar radiation measurement with different estimation methods 
function[estimates, model] = get_IVcurve(xdata_V,ydata_mA,Isc_mA)

start_point $=\operatorname{rand}(1,2)$;

model = Qexpfun;

estimates $=$ fminsearch(model, start_point);

function [sse, FittedCurve] = expfun (params)

$A=\operatorname{params}(1)$;

$B=\operatorname{params}(2)$;

FittedCurve $=$ Isc_mA $-\mathrm{A} \cdot *(\exp (\mathrm{B} *$ xdata_V $)-1)$;

ErrorVector $=$ FittedCurve - ydata_mA;

sse $=\operatorname{sum}($ ErrorVector .`2);

end

end

Figure 4.13: Matlab algorithm for solar IV curve fitting

\subsection{Modeling Solar Panel}

\subsubsection{Modeling IV Characteristic}

The I-V characteristic of a solar panel can be described with a generic formula:

$$
I=I_{s c}-A \cdot(\exp (B \cdot V)-1)
$$

Having a generic formula allows us to determine the I-V characteristic of a solar cell using the curve-fitting method, and the algorithm shown in Figure 4.13. As input parameters, this algorithm takes a sequence of IV measurement points (xdata_V, ydata_mA) and the short circuit current measurement Isc_mA. As a return value, the algorithm returns a parameter pair $(\mathrm{A}, \mathrm{B})$ that minimizes the error between the measured output and the estimated output. After the I-V characteristic of a solar panel is determined, our model can find the current or power of a solar panel at a given operational voltage (Figure 4.14). Figure 4.15 and 4.16 show IV characteristics for two different types of solar panels. The first one is a $4 \mathrm{~V}-100 \mathrm{~mA}$ polycrystalline silicon solar panel, and the second is a $3.6 \mathrm{~V}-50 \mathrm{~mA}$ amorphous silicon solar panel. 


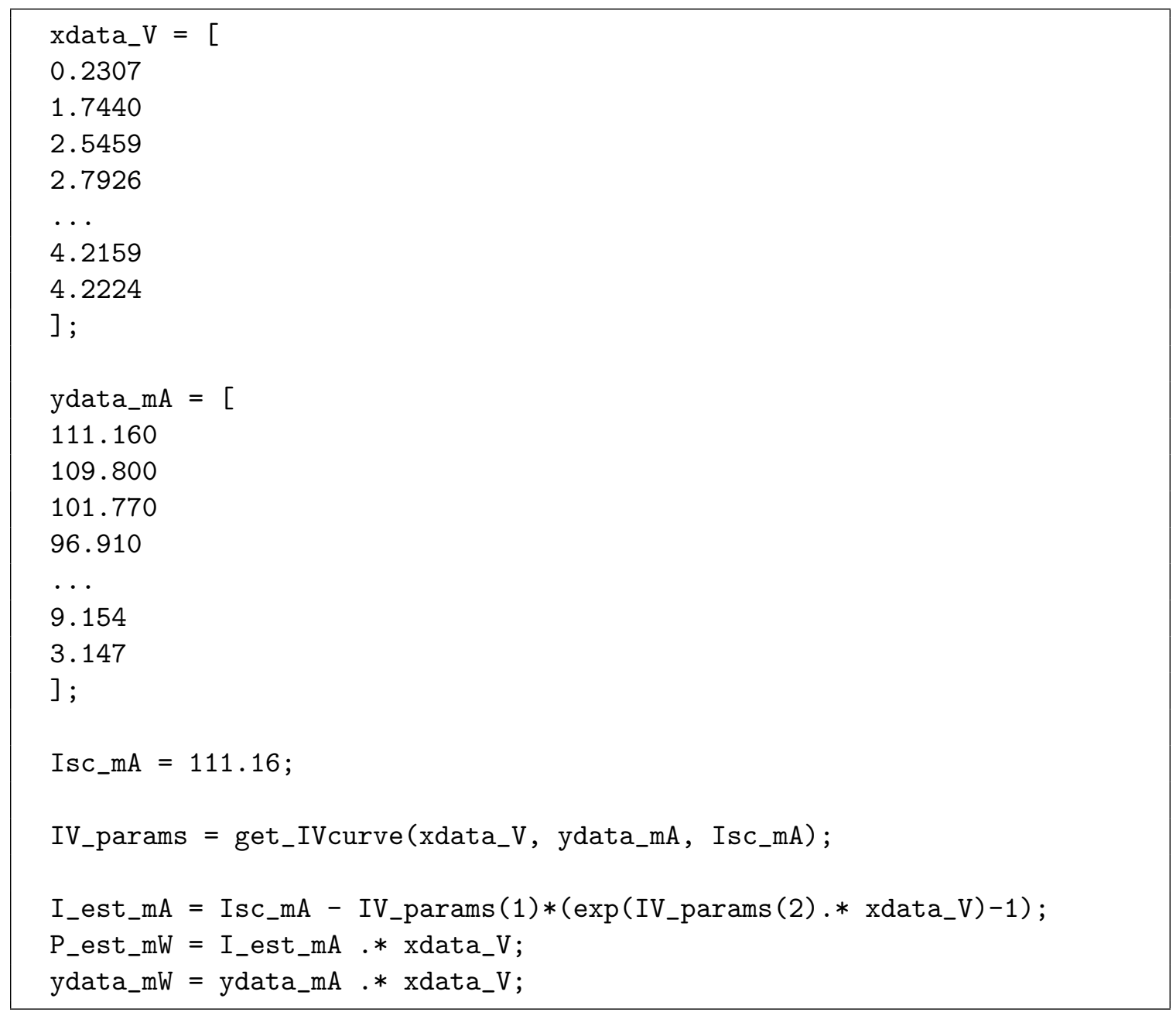

Figure 4.14: Matlab algorithm for estimating the solar panel IV and PV characteristics using measurement data. The units for voltage and current are $\mathrm{V}$ and $\mathrm{mA}$ respectively. 

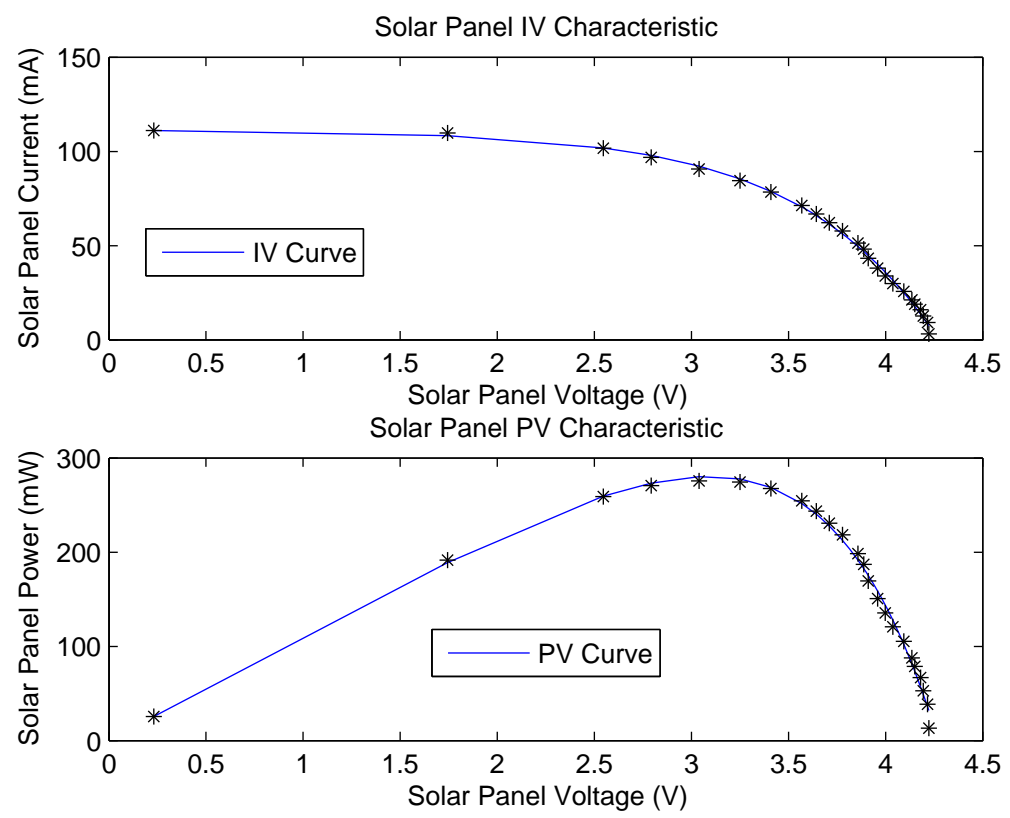

Figure 4.15: IV and PV graph derived from the measurement of 4V-100mA polycrystalline silicon solar panel with IV curve fitting constants $\mathrm{A}=0.2526$ and $\mathrm{B}=$ 1.4255
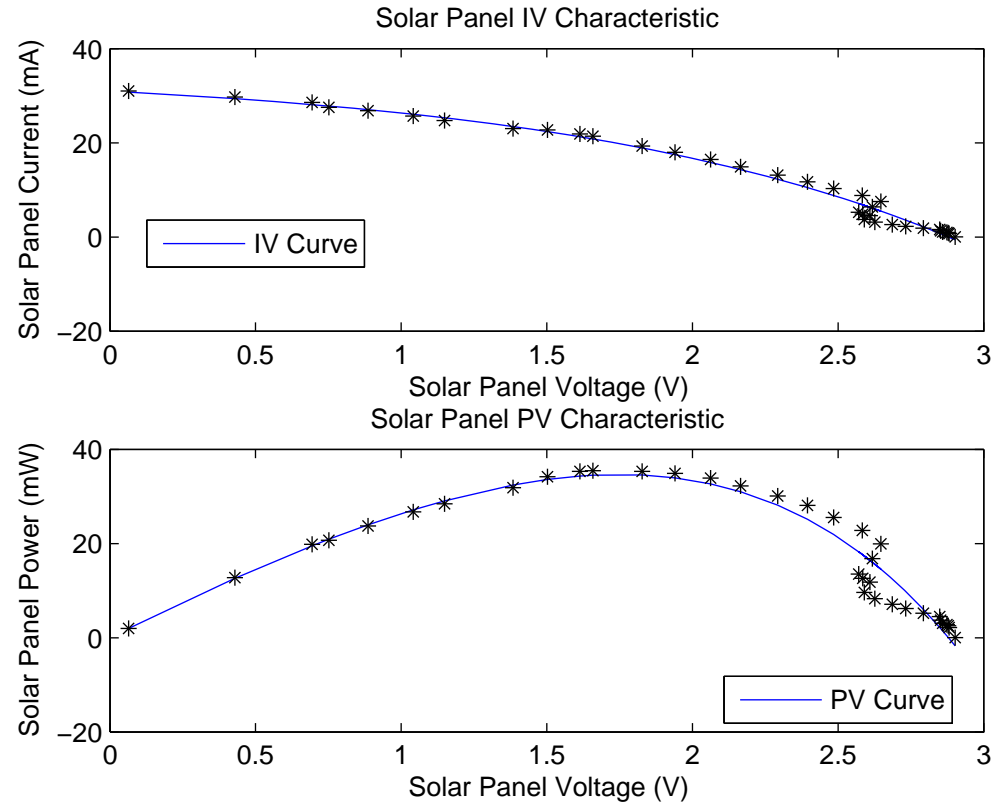

Figure 4.16: IV and PV graph derived from the measurement of $3.6 \mathrm{~V}-50 \mathrm{~mA}$ flexible solar panel with IV curve fitting constants $\mathrm{A}=4.328$ and $\mathrm{B}=0.729$. We notice that IV and PV relation implied by the actual data points are non-monotonic for the solar panel voltage above 2.5 and this irregularity is is caused by a sampling error. 


\subsubsection{Modeling the Operating Point}

The solar panel operates as a voltage-controlled current source. The voltage of the solar panel is determined by the output of the solar panel, which is either a battery or a regulating diode.

$$
V_{\text {panel }}= \begin{cases}V_{\text {batt }}+V_{t h-s c h o t t k y} & \text { with diode } \\ V_{\text {batt }} & \text { otherwise }\end{cases}
$$

Once the voltage of the solar panel is determined, its current and power can be determined by its IV curve.

\subsection{Modeling Energy Storage}

\subsubsection{Modeling NiMH Rechargeable Battery}

From the perspective of the micro-solar power simulator, NiMH rechargeable batteries have two characteristics:

First, NiMH has different characteristics depending on whether it is in charging mode or discharging mode, which makes the problem more complicated. This is because the output voltage of the solar panel depends on the battery voltage, and the battery can be either in charging mode or discharging mode, depending on the solar panel output power. We address this problem by solving the states of all components for a few possible modes and choosing the mode that meets the constraints of the system. Figure 4.17 shows the capacity-to-voltage profiles for a NH15-2500 NiMH battery.

Second, while a NiMH rechargeable battery maintains a stable voltage level for a large operating range, its voltage level changes over the stored charge. This capacityto-voltage characteristic is non-linear and is not easily represented by a simple formula. In order to represent this characteristic, we use the piecewise linear interpolation method, using the user-provided data for charging and discharging modes (Figure 4.19).

Figure 4.18 describes an algorithm that evaluates the system state depending on whether the battery is either in discharge or in charge. The system variables, initial 

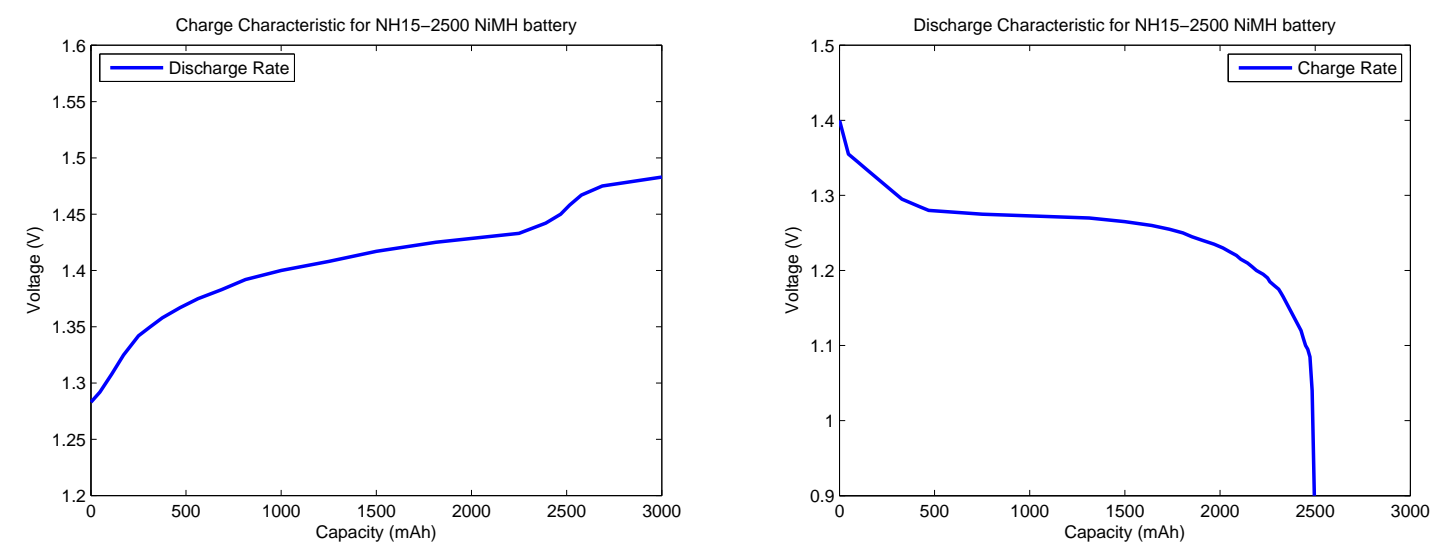

Figure 4.17: (a) Charging characteristic for the NH15-2500 NiMH battery, (b) Discharging characteristic for the NH15-2500 NiMH battery

conditions, functions for this algorithm are listed below:

- $t[i]$ : Starting time for $i$-th iteration

- $C_{\text {next-bat }}$ : Capacity of the battery for next iteration

- $C_{b a t-i n i t}$ : Initial value for capacity of the battery

- $C_{b a t}[i]$ : Capacity of the battery

- $V_{\text {bat-dis }}[i]$ : Voltage of the battery at discharge

- $V_{b a t-c h g}[i]$ : Voltage of the battery at charge

- $S_{d i s}[i]$ : System state at discharge, which is a collection of variables for each component of the system

- $S_{\text {chg }}[i]$ : System state at charge

- $P_{n e t-d i s}[i]$ : Net power being charged to the battery at discharge

- $P_{\text {net-chg }}[i]$ : Net power being charged to the battery at charge

- $S[i]$ : System state

- $P_{\text {net-bat }}[i]$ : Net power being charged to the battery

- $V_{b a t}[i]$ : Voltage of the battery

- Cap-Vbat-Dis( ) : Function that gives battery voltage at discharge for given battery capacity 


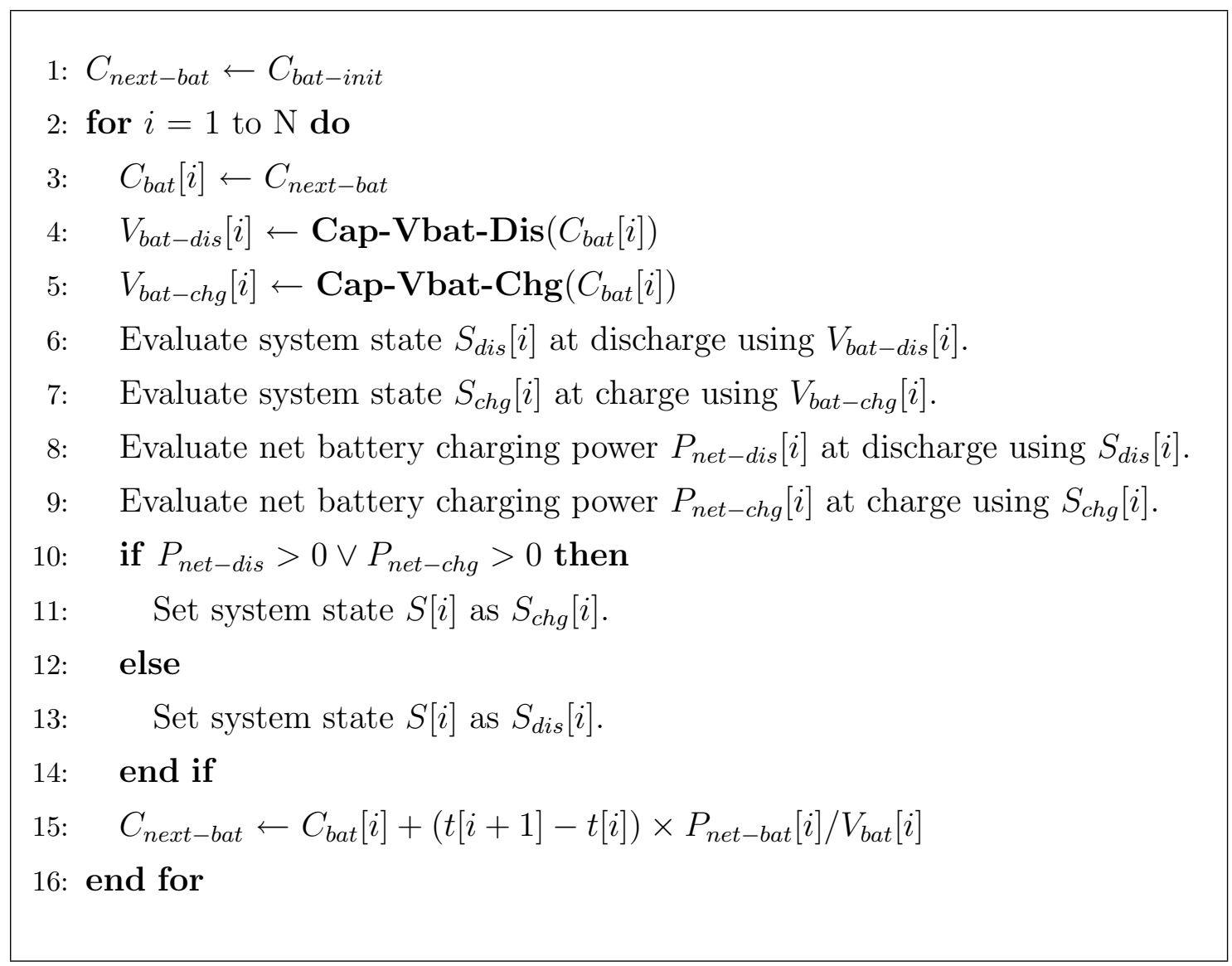

Figure 4.18: Algorithm that evaluates the state of a micro-solar power system with NiMH battery

- Cap-Vbat-Chg( ) : Function that gives battery voltage at charge for given battery capacity

\subsubsection{Modeling Supercapacitors}

For a given voltage level, the energy level of a supercapacitor can be calculated using formula (4.10), and the voltage level can be calculated from the inverse equation:

$$
E=\frac{1}{2} C V^{2} \Longleftrightarrow V=\sqrt{\frac{2 E}{C}}
$$

Figure 4.20 shows the voltage-to-capacity relationship for a $22 \mathrm{~F}$ supercapacitor. 


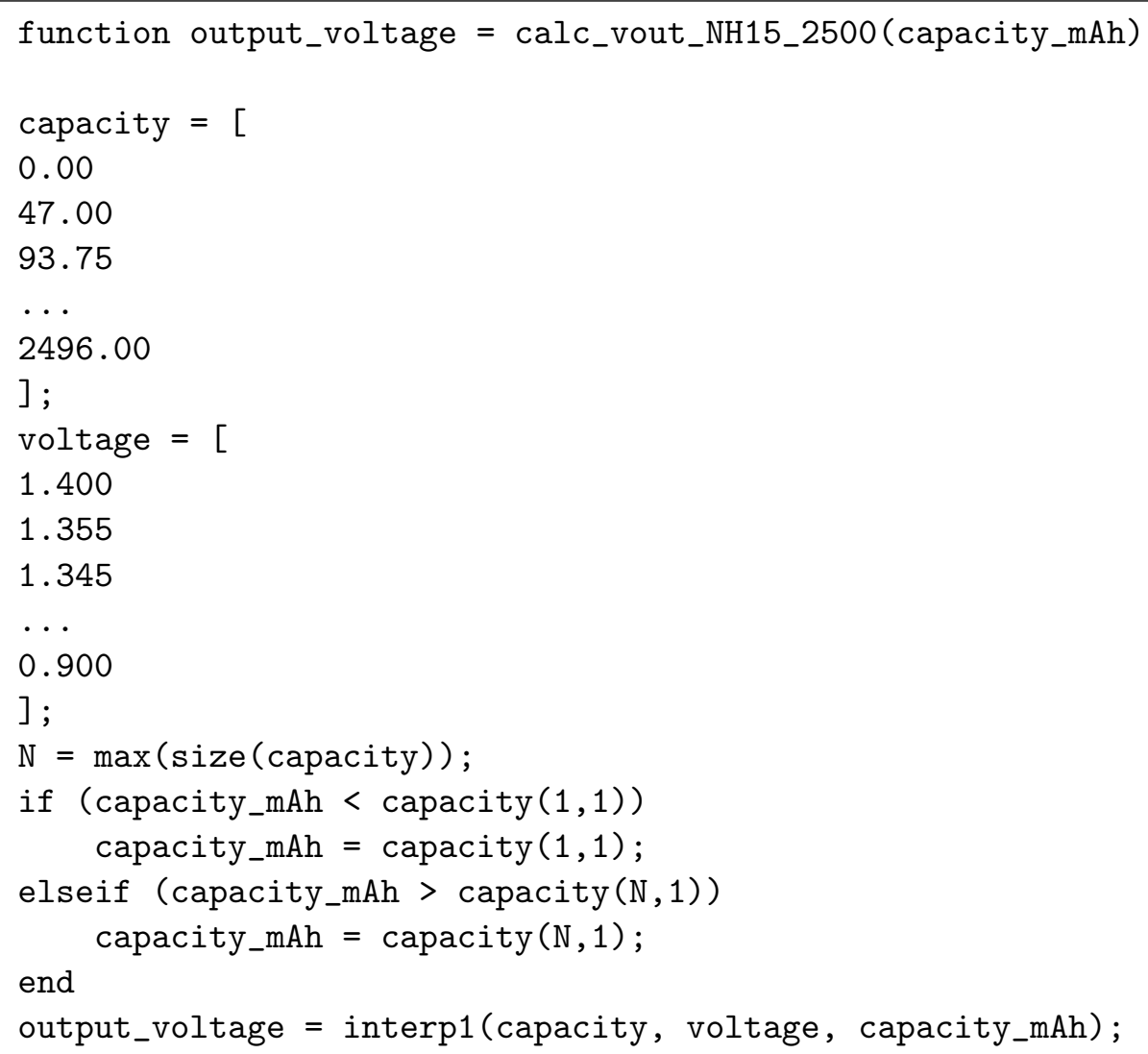

Figure 4.19: Matlab algorithm for piecewise linear interpolation for the battery voltage level over its capacity 
Our simulator also considers the effect of energy leakage when modeling a supercapacitor, because a supercapacitor has a higher energy leakage rate than a battery, and its effect is noticeable on a large time-scale.

Using the manufacturer-provided leakage current is one way of modeling the energy leakage of a supercapacitor. When the leakage current is given as $I_{c}$ and the supercapacitor operates at voltage $V(t)$, the energy leakage $E_{\text {leak }}(t)$ at time $t$ for the time duration of $\Delta t$ can be given as 4.11 :

$$
E_{\text {leak }}(t)=I_{c} \cdot V(t) \cdot \Delta t
$$

Using this leakage model, we can simulate the energy level of a supercapacitor for an arbitrary time period $T$. Suppose the initial energy level of the supercapacitor is $E_{\text {init }}$ and the time period $T$ can be divided into discrete time intervals $\left[t_{0}, t_{1}\right],\left[t_{1}, t_{2}\right]$, $\cdots,\left[t_{k-1}, t_{k}\right]$ where $t_{i+1}-t_{i} \leq \Delta t$. Then, the energy level of a supercapacitor at time $t_{i}, E\left(t_{i}\right)$, can be given by the following equations:

$$
\begin{aligned}
E\left(t_{0}\right) & =E_{\text {init }} \\
V\left(t_{i}\right) & =\sqrt{2 E\left(t_{i}\right) / C} \\
E_{\text {leak }}\left(t_{i}\right) & =I_{c} \cdot V\left(t_{i}\right) \cdot\left(t_{i+1}-t_{i}\right) \\
E\left(t_{i+1}\right) & =E\left(t_{i}\right)-E_{\text {leak }}\left(t_{i}\right)
\end{aligned}
$$

Another way of estimating the energy leakage of a supercapacitor is to use an energy-time graph as illustrated in Figure 4.21. This method uses the property that the energy level of a supercapacitor monotonically decreases. If the energy level of a supercapacitor at time $t_{0}$ is $E\left(t_{0}\right)$ and the time duration is $T$, the energy level of a supercapacitor becomes $E\left(t_{0}+T\right)$. Thus, the leakage can be given as $E\left(t_{0}\right)-E\left(t_{0}+T\right)$.

\subsection{Modeling Output Regulator}

The behavior of an output regulator can be described by the following three characteristics:

- Operating Range: At what input voltage and output current can an output regulator operate? 


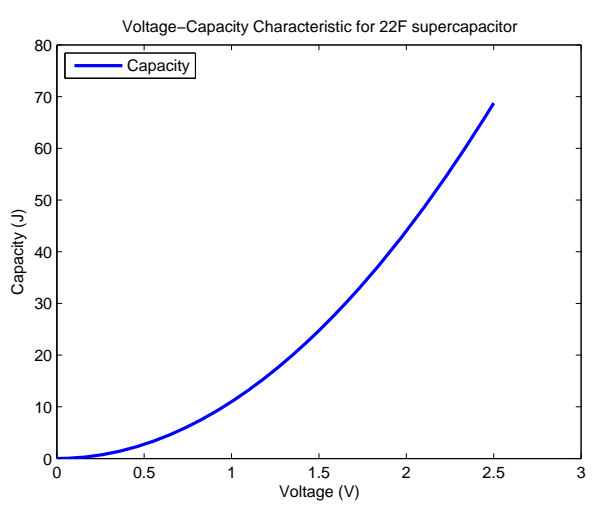

Figure 4.20: Voltage-Capacity Characteristic for $22 \mathrm{~F}$ supercapacitor

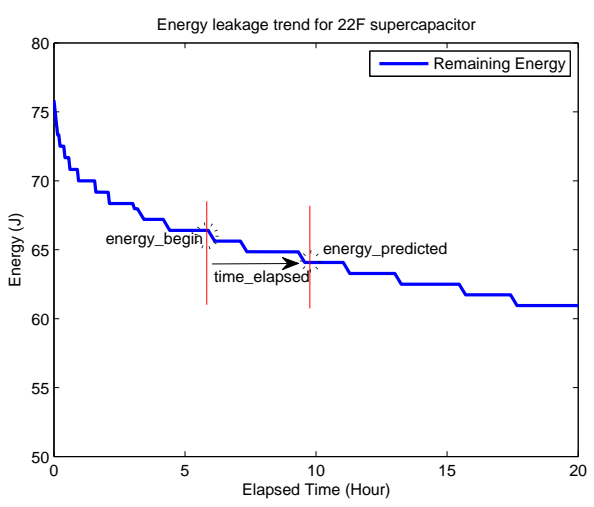

Figure 4.21: Leakage estimation model for supercapacitor

- Output Voltage: What is the output voltage of an output regulator for a given input voltage and output load?

- Power Efficiency: How much power is transferred?

While these characteristics are provided by the manufacturer, they are mostly nonlinear and have different forms among different parts, thus we use the piecewise linear interpolation method to model the behavior of an output regulator.

\subsubsection{Modeling Operating Range and Output Voltage}

The operating range of the input voltage can be easily confirmed by checking whether the given voltage lies in the manufacturer-provided input voltage range. The maximum load current of an output regulator varies depending on its input voltage. This can also be modeled using interpolation when the maximum load current is provided for a sampling of input voltages.

Figure 4.22 shows the estimation of output voltage of a LTC1751 regulator based on the manufacturer-provided data. We can see that this regulator has a relatively stable output voltage within its operating voltage range. As for the MAX1724 regulator, we modeled the output voltage as a constant value of $3.3 \mathrm{~V}$ because it has a stable output voltage (3.267V minimum, 3.333V maximum and 3.3V typical). 


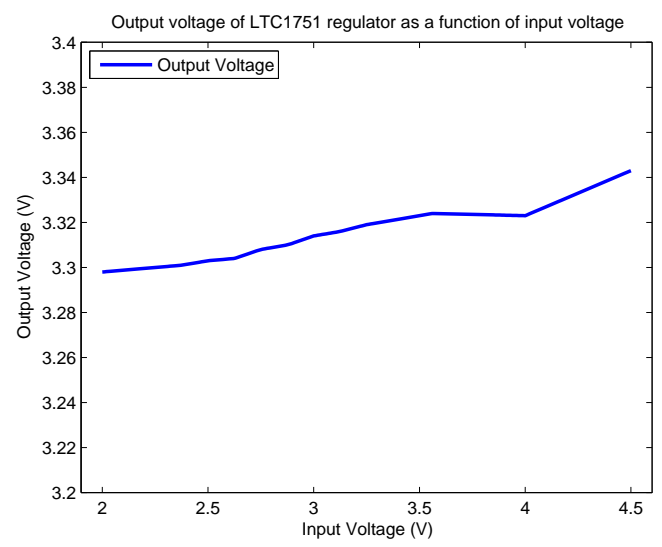

Figure 4.22: Output voltage of LTC1751 regulator as a function of input voltage
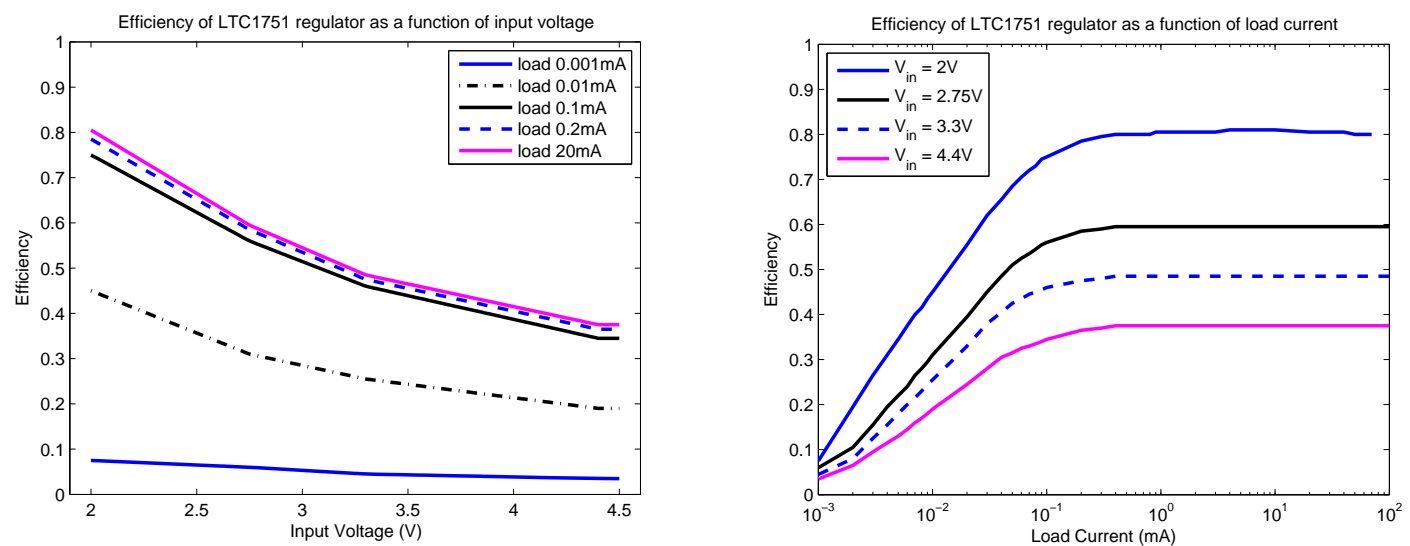

Figure 4.23: Efficiency of LTC1751 regulator as a function of input voltage and load current with output voltage $3.3 \mathrm{~V}$

\subsubsection{Modeling Power Efficiency}

Figure 4.23 shows the estimation of efficiency of a LTC1751 regulator based on the manufacturer-provided data. We can see that this regulator has a higher efficiency, as the load current increases and the input voltage decreases. At a typical operating point of a micro-solar power sensor system with two NiMH batteries in series, the input voltage is around $2.75 \mathrm{~V}$ and the load current is between $0.1 \mathrm{~mA}$ to $10 \mathrm{~mA}$. The figure shows that the LTC1751 regulator has an efficiency between $55 \%$ to $60 \%$ within this operating range.

Figure 4.24 shows the estimation of efficiency of a MAX1724 regulator based on 

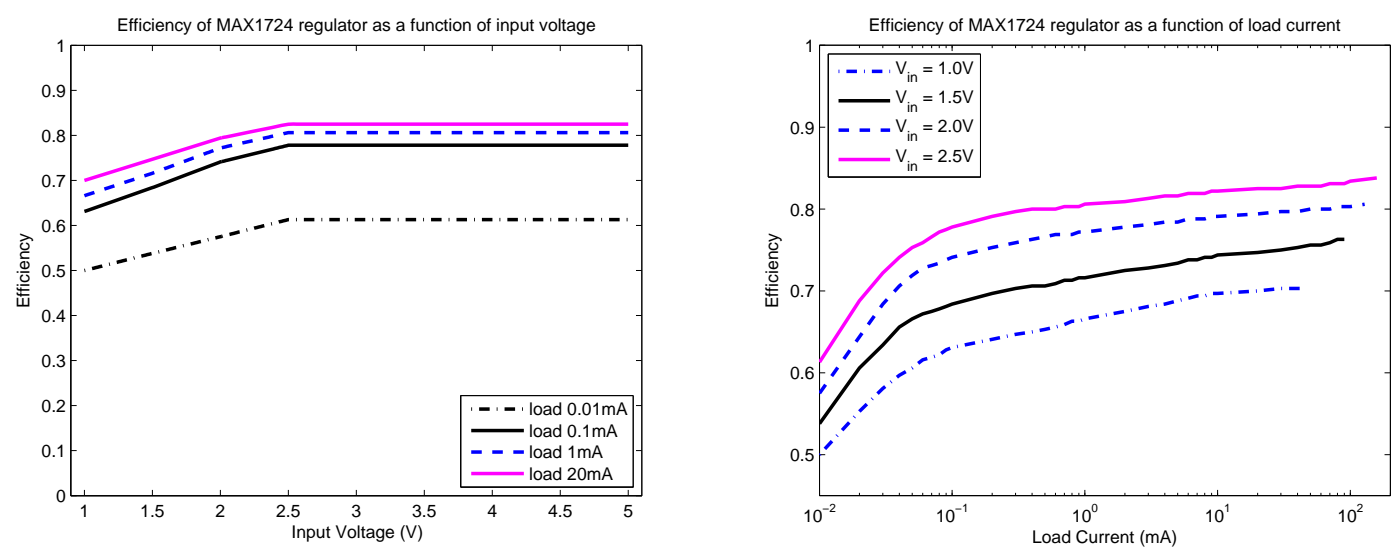

Figure 4.24: Efficiency of MAX1724 regulator as a function of input voltage and load current with output voltage $3.3 \mathrm{~V}$

the manufacturer-provided data. As is the case with the LTC1751, the efficiency of the MAX1724 increases as the load current increases, but the voltage-to-efficiency of the MAX1724 has a different characteristic from the LTC1751. As the input voltage increases, the efficiency of the MAX1724 increases and it reaches the saturation point at around $2.5 \mathrm{~V}$.

The MAX1724 regulator is more efficient than the LTC1751 regulator. To make a fair comparison, we can look up the efficiency of the MAX1724 at the same input voltage level $(2.75 \mathrm{~V})$ and the load current range $(0.1 \mathrm{~mA}$ to $10 \mathrm{~mA})$. Figure 4.24 shows that the MAX1724 regulator has an efficiency between $75 \%$ to $80 \%$ within this operating range. Whereas the efficiency of the LTC1751 is 55\% to $60 \%$.

\subsection{Modeling the Input Regulator}

\subsubsection{Micro-Solar Power System without an Input Regulator}

Before we model the input regulator, we can consider the behavior of a micro-solar power system with no input regulator, as is illustrated in Figure 4.25. In this type of micro-solar power system, the solar panel voltage $V_{\text {sol }}$ is determined by the voltage level of the energy storage, $V_{\text {stor }}$, and the threshold voltage of the diode, $V_{\text {th-schottky }}$, 


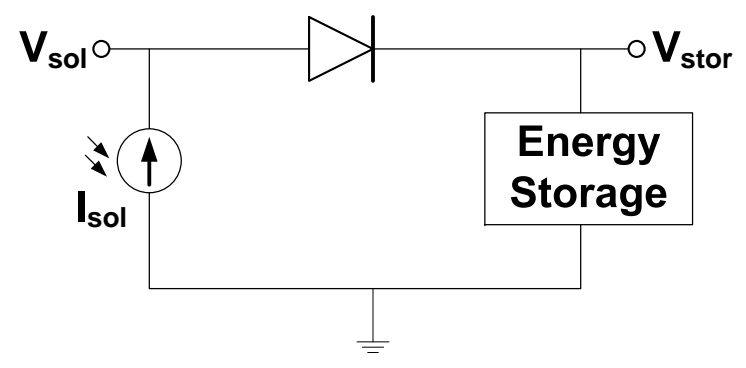

Figure 4.25: Configuration: a micro-solar power system with no input regulator

with the following equation:

$$
V_{\text {sol }}=V_{\text {stor }}+V_{\text {th-schottky }}
$$

While the threshold voltage of the diode $V_{t h-s c h o t t k y}$ is relatively constant, the voltage level of the energy storage, $V_{\text {stor }}$ varies as the energy storage is being charged or

discharged. Thus, the operating point of the solar panel $V_{\text {sol }}$ changes depending on the voltage level of the energy storage $V_{\text {stor }}$. This variability of the solar panel operating point is different for each type of energy storage.

For example, we can consider the operation of two different micro-solar power system designs, one with two AA NiMH batteries and the other with a $22 \mathrm{~F}$ supercapacitor as the energy storage (Figure 4.26). While the energy storage is in the charging state, the NiMH based system has its solar-panel output power between $97.3 \%$ to $100 \%$ of the MPP of the solar panel, the supercap-based system has a wider swing for its solar-panel output power, between $13.9 \%$ to $98.3 \%$ of MPP of the solar panel.

An input regulator, which matches the operating point of the solar panel and the energy storage, can be considered when the solar panel output power can deviate far from the MPP due to the wide operating range.

\subsubsection{Micro-Solar Power System with an Input Regulator}

We can consider a micro-solar power system with an input regulator; in this system, there is an input regulator between the output of the diode and the input of the energy storage (Figure 4.27). 

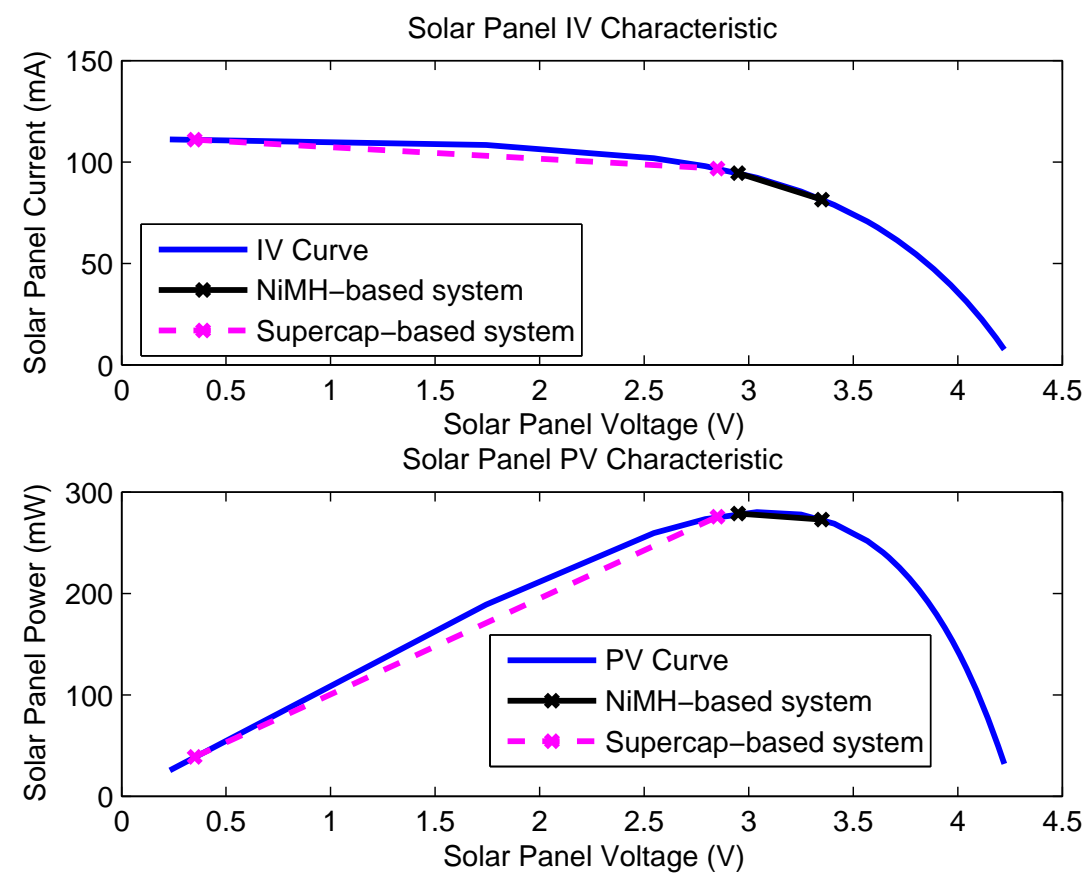

\begin{tabular}{|l|l|l|}
\hline & 2AA NiMH & 22F supercap \\
\hline$V_{\text {stor }}$ & $2.6 \mathrm{~V}$ to $3.0 \mathrm{~V}$ & $0 \mathrm{~V}$ to $2.5 \mathrm{~V}$ \\
\hline$V_{\text {sol }}$ & $2.95 \mathrm{~V}$ to $3.35 \mathrm{~V}$ & $0.35 \mathrm{~V}$ to $2.85 \mathrm{~V}$ \\
\hline$P_{\text {sol }}$ & $272.9 \mathrm{~mW}$ to $280.4 \mathrm{~mW}$ & $38.8 \mathrm{~mW}$ to $275.7 \mathrm{~mW}$ \\
& $(97.3 \%$ to $100 \%$ of $\mathrm{MPP})$ & $(13.9 \%$ to $98.3 \%$ of $\mathrm{MPP})$ \\
\hline
\end{tabular}

Figure 4.26: Operating range: a micro-solar power system with no input regulator

In order to model the behavior of the micro-solar power system with an input regulator, we can consider a simple case for the sake of easy analysis: a buck converter (step-down DC-DC converter) as an input regulator and a supercapacitor as energy storage. In this system, the buck converter operates in two modes:

1. Constant input-voltage mode $\left(V_{\text {stor }}<V_{\text {reg-in-th }}\right)$ :

If the output voltage of the input regulator $V_{\text {stor }}$ is below a certain level $V_{\text {reg-in-th }}$, the buck converter is turned on and its input voltage locks to the threshold value. The threshold voltage $V_{\text {reg-in-th }}$ is a property of a particular type of input regulator. Thus, in constant input-voltage mode, the solar panel operating point remains constant even though the voltage level of the storage changes due to 
charge and discharge.

$$
\begin{aligned}
V_{\text {reg-in }} & =V_{\text {th-reg-in }}=\text { const } \\
V_{\text {sol }} & =V_{\text {reg-in }}+V_{\text {th-schottky }} \\
& =V_{\text {th-reg-in }}+V_{\text {th-schottky }}=\text { const } \\
I_{\text {sol }} & =I \operatorname{Virve}\left(V_{\text {sol }}\right)=\text { const }
\end{aligned}
$$

2. Pass-through mode $\left(V_{\text {stor }}>V_{\text {reg-in-th }}\right)$ :

If the output voltage of the input regulator $V_{\text {stor }}$ is above the threshold $V_{\text {reg-in-th }}$, the buck converter is turned off and looks like a pass-through wire from the outside. In pass-through mode, the solar panel operating point changes along the solar panel IV curve, following the voltage of the storage.

$$
\begin{aligned}
V_{\text {reg-in }} & =V_{\text {stor }} \\
V_{\text {sol }} & =V_{\text {reg-in }}+V_{\text {th-schottky }} \\
& =V_{\text {stor }}+V_{\text {th-schottky }} \\
I_{\text {sol }} & =I V \operatorname{Curve}\left(V_{\text {sol }}\right)
\end{aligned}
$$

Figure 4.28 shows the case where a depleted supercapacitor is charged from a solar panel through a buck converter. In the beginning, the voltage level of the supercapacitor $V_{\text {stor }}$ is low meeting the condition for the constant-input-voltage mode, thus the buck converter is turned on and its input voltage $V_{\text {reg-in }}$ becomes constant due to the characteristic of constant input impedance of the device $R_{\text {reg-in }}$. Then, the solar panel current also becomes constant.

As time goes on, the supercapacitor is charged and its voltage level $V_{\text {stor }}$ rises. When $V_{\text {stor }}$ becomes high enough to meet the condition for the pass-through mode, the buck converter is turned off and the solar panel voltage $V_{\text {reg }}$ increases, following the supercapacitor voltage $V_{\text {stor }}$. The solar panel voltage $V_{\text {sol }}$ also increases as $V_{\text {reg }}$ increases. At the same time, the current of the solar panel $I_{\text {sol }}$ decreases along the solar panel IV curve.

When the amount of energy that comes into the buck converter is given as $P_{i n}=$ $V_{\text {reg-in }} \cdot I_{\text {sol }}$, the amount of energy that comes out of the buck converter can be given as $P_{\text {out }}=\mathrm{Eff}_{r e g-i n} \cdot P_{i n}$. The power efficiency Eff ${ }_{r e g-i n}$ is provided by the manufacturer and varies among parts. 


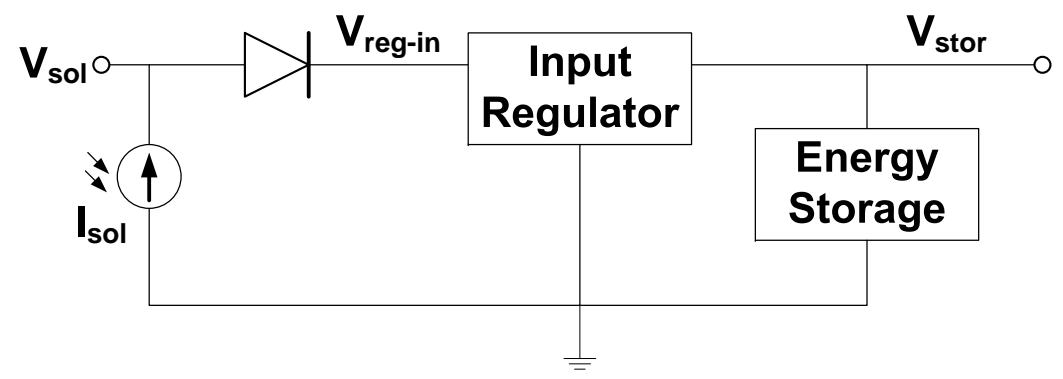

Figure 4.27: Configuration: a micro-solar power system with a buck converter as an input regulator

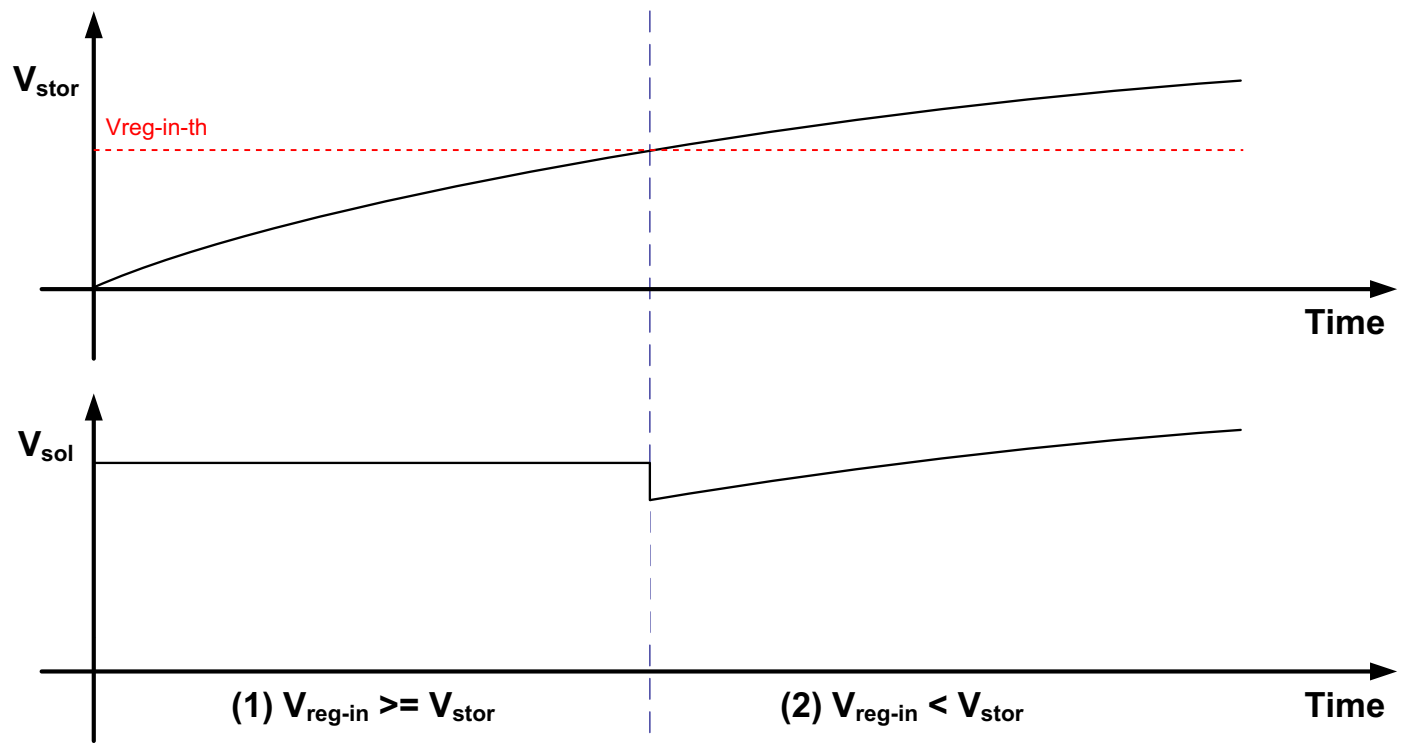

Figure 4.28: Operating range: a micro-solar power system with a buck converter as an input regulator

\subsection{Modeling Load}

\subsubsection{Average Current-Based Model}

For modeling the long-term behavior of the load, we can use the average current consumption measured by a scope or a multimeter. This is because most sensornet applications have periodic behavior of sensing and communication and any surge in power consumption becomes averaged over a long period of time. In our simulator, we use the average current-based model. 


\subsubsection{Application Code Based Model}

For modeling the short-term behavior of the load, we can use a power consumption simulator that takes application code. In the remainder of this subsection, we describe this methodology based on PowerTOSSIM [SHC $\left.{ }^{+} 04\right]$, which is a representative application-code-level power-consumption simulator.

\section{Structure of PowerTOSSIM}

Power profiling with PowerTOSSIM is done in three steps: application monitoring, logging and post-processing (Figure 4.29). First, in the application monitoring step, each TinyOS component that can influence the power consumption of a mote is modified to call the power monitoring module PowerStateM through the PowerState interface. Such TinyOS components are HPLADCC, PhotoTempM, CC1000ControlM, LedsM, SnoozeM and EEPROMM. In the logging step, the power monitoring module PowerStateM logs the power profile, such as component name, status and event time, into an output file as the sensor component calls the power monitoring module. Lastly in the post-processing step, the power status log file is associated with the power consumption model for the mote platform to provide more meaningful results.

\section{Extending PowerTOSSIM to a New Platform}

Extending PowerTOSSIM beyond the reference platform requires changing program code, as well as power consumption measurement for the power consumption model. Even when the same program code is used, we may need new power consumption measurement results because different hardware parts produce different power consumption results (e.g. LedsM). The TinyOS modules in the reference implementation of PowerTOSSIM with the Mica2 platform can be divided into five categories: (1)

radio module, (2) LED module, (3) sensing module, (4) power management module and (5) flash memory module.

As for the radio module, there is a platform dependency issue. The original PowerTOSSIM is based on the CC1000 radio chip and the Atmel micro-controller, whereas our reference platform, the HydroWatch node, runs on a different radio and microcontroller: the CC2420 radio and MSP430 micro-controller. While we may write 
a simulator module based on this reference platform, we decided not to for several reasons.

First, TOSSIM requires the radio module communicate in bits, whereas the CC2420 radio is a packet-level radio. Thus, emulating CC2420 native code requires us to change a major part of the TOSSIM simulator. Second, the applications that will use the CC2420 radio need to access only the high-level interface, not the low-level implementation details. Most CC2420 radio-based applications use the generic interface. Although they need the CC2420 radio specific interface, they access only a small number of functions, such as transmission power control. Thus, we have decided to implement the simulator by writing a CC2420 radio wrapper module on top of the simulator code for the CC1000 radio (Figure 4.30). The idea of using a wrapper module provides the same programming interface to the applications that run on the CC2420 radio, while minimizing the effort to write simulator code.

The LED module for PowerTOSSIM is quite straight-forward, but we corrected a problem when we extended it for the TelosB platform. The original version of PowerTOSSIM, built for the Mica2 platform, assumes the same power consumption for three different types of LED. This is not the case for the TelosB mote (see RED_LED, GREEN_LED and YELLOW_LED in Table 4.2), and we corrected this problem by making the post-processing script handle the current consumption for each LED differently.

Other modules, such as sensing, power management and flash memory modules aren't yet implemented for the TelosB mote. But, this can be done in a straightforward way by identifying the TinyOS code that accesses these modules and building the power consumption model for these modules from measurement.

In order to build the power consumption model for the TelosB node, we used two instruments to measure the power consumption of a TelosB node: a Fluke-189 multimeter and a Tektronics TDS7104 digital phosphor oscilloscope. The measurement result is shown in Table 4.2 .

\section{Evaluating the Modified PowerTOSSIM}

To evaluate the operation of PowerTOSSIM, we ran PowerTOSSIM for the TelosB platform and compared it with the power consumption measured from a TelosB node 


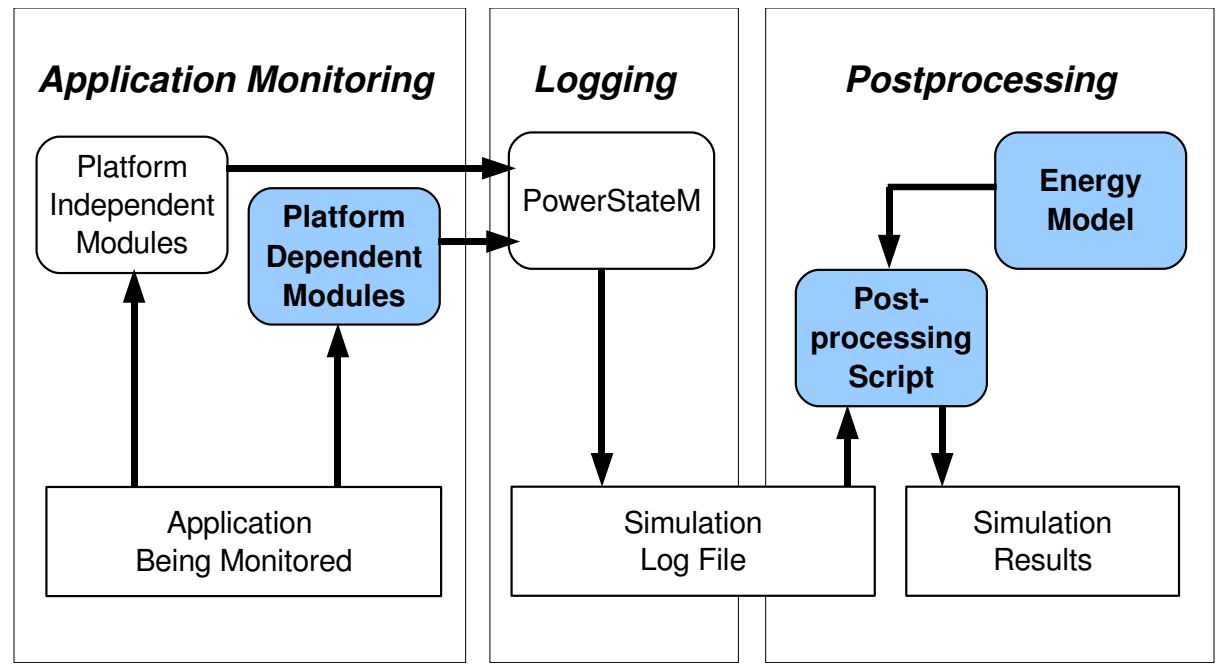

Figure 4.29: Structure of PowerTOSSIM. Highlighted blocks are the modules to be rewritten for different platforms.

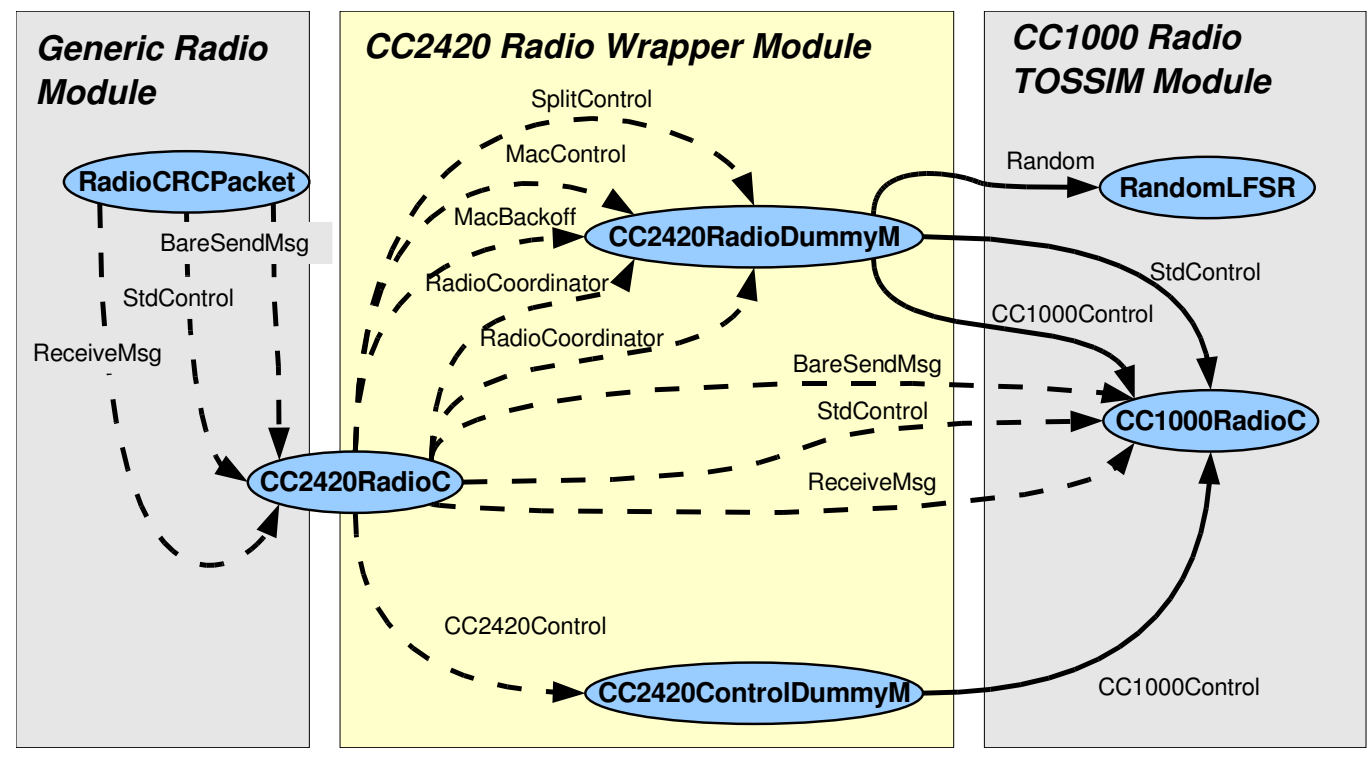

Figure 4.30: Block diagram for CC2420 radio wrapper modules 
Table 4.1: Comparison of the power consumption estimation from PowerTOSSIM with measurement

\begin{tabular}{|l|r|r|r|r|}
\hline & RF3 & RF31 & Oscope & CntLed \\
\hline PowerTOSSIM & $19.900 \mathrm{~mA}$ & $19.937 \mathrm{~mA}$ & $1.619 \mathrm{~mA}$ & $4.779 \mathrm{~mA}$ \\
Multimeter & $19.637 \mathrm{~mA}$ & $19.640 \mathrm{~mA}$ & $1.717 \mathrm{~mA}$ & $4.780 \mathrm{~mA}$ \\
\hline Difference & $1.34 \%$ & $1.51 \%$ & $5.71 \%$ & $0.02 \%$ \\
\hline
\end{tabular}

using a Fluke-189 multimeter. We compared the simulation results with multimeter measurements for four different applications: RF3, RF31, Oscope and CntLed. Table 4.1 shows the current consumption estimation from PowerTOSSIM and the multimeter measurement. The difference between the estimation and the measurement is within $6 \%$ of the measurement result. 
Table 4.2: Power consumption model for TelosB node. The unit for each entity is $\mathrm{mA}$ except for VOLTAGE, the unit of which is $\mathrm{V}$

\begin{tabular}{|ll||ll|}
\hline Keyword & Value & Keyword & Value \\
\hline RADIO_RX & 19.405 & VOLTAGE & 3.0 \\
RADIO_OFF & 0 & CPU_ACTIVE & 1.986 \\
RADIO_TX_03 & 8.392 & CPU_IDLE & 0.175 \\
RADIO_TX_04 & 8.956 & CPU_ADC_NOISE_REDUCTION & 0.0 \\
RADIO_TX_05 & 9.040 & CPU_POWER_DOWN & 0.0 \\
RADIO_TX_06 & 9.081 & CPU_POWER_SAVE & 0.0 \\
RADIO_TX_07 & 9.452 & CPU_STANDBY & 0.0037 \\
RADIO_TX_08 & 10.384 & CPU_EXTENDED_STANDBY & 0.0037 \\
RADIO_TX_09 & 10.277 & CPU_INIT & 1.986 \\
RADIO_TX_0A & 10.252 & CPU_FREQ & 4000000 \\
RADIO_TX_0B & 10.774 & LED & 2.2 \\
RADIO_TX_0C & 11.388 & LED_INIT & 0 \\
RADIO_TX_0D & 11.448 & RED_LED & 2.804 \\
RADIO_TX_0E & 11.323 & RED_LED_INIT & 0 \\
RADIO_TX_0F & 11.883 & GREEN_LED & 4.461 \\
RADIO_TX_10 & 12.553 & GREEN_LED_INIT & 0 \\
RADIO_TX_11 & 12.578 & YELLOW_LED & 2.002 \\
RADIO_TX_12 & 12.644 & YELLOW_LED_INIT & 0 \\
RADIO_TX_13 & 13.052 & EEPROM_READ & 1.75 \\
RADIO_TX_14 & 13.596 & EEPROM_WRITE & 2.69 \\
RADIO_TX_15 & 13.602 & ADC & 0.459 \\
RADIO_TX_16 & 13.572 & SENSOR_BOARD & 0.69 \\
RADIO_TX_17 & 14.180 & SENSOR_PHOTO & 0 \\
RADIO_TX_18 & 14.808 & SENSOR_TEMP & 0 \\
RADIO_TX_19 & 14.859 & SENSOR_INTERNAL_TEMP & 0.332 \\
RADIO_TX_1A & 14.828 & & \\
RADIO_TX_1B & 15.388 & & \\
RADIO_TX_1C & 15.911 & & \\
RADIO_TX_1D & 15.861 & & \\
RADIO_TX_1E & 15.929 & & \\
RADIO_TX_1F & 16.442 & & \\
RADIO_DEFAULT_POWER & 31.00 & & \\
\hline & & & \\
\hline
\end{tabular}




\subsection{Composition}

In this section, we construct a model of the whole micro-solar power system and simulate it based on the description of each component and its interconnection with others. We simulate the model of a micro-solar power system in two steps: first, under constant radiation, and second, under the daily solar radiation cycle.

\subsubsection{Simulation under Constant Radiation}

Here, we simulate the model of a micro-solar power system under constant radiation using a controllable light source. This makes it easy to verify the simulation model of the solar panel and the energy storage with the model of the external environment set to the measurement data.

\section{Effect of Input Regulator}

We simulate the effect of an input regulator and verify it with the measurement.

- External Environment and Solar Panel: For a controlled external environment, we used an indoor lamp light to make the radiation constant throughout the experiments. As for the solar panel, we used a 4.0V-100mA solar panel. The IV and PV curves of the solar panel under this radiation condition are shown in Figure 4.31 .

- Input Regulator: We consider two cases. In the first case, no input regulator is used where the output of the solar panel is directly connected to the energy storage through a Schottky diode (Figure 4.25). In the second case, an LM3671 step-down DC-DC converter is used as an input regulator (Figure 4.27). The LM3671 has a power efficiency between $80 \%$ and $90 \%$ within its operating range. In simulating the LM3671, we assume an efficiency of $80 \%$.

- Energy Storage: For the energy storage, we used a 22F supercapacitor from Aerogel. We used a supercapacitor for this test because a supercapacitor has a larger voltage swing (0V to $3 \mathrm{~V}$ ) from empty to full capacity than a rechargeable battery such as a NiMH. This allowed us to test the system over a wider operating range. 
- Output Regulator and Load: Since this is to test the charging process of a micro-solar power system, we did not use any output regulator or load.

The simulation and measurement result for the first case (not using an input regulator) is shown in Figure 4.32. From this, we can draw the following observations:

1. As the supercapacitor is being charged, the solar panel voltage increases and the solar panel current decreases. This is because the solar panel voltage follows the supercap voltage, and the solar panel voltage and the solar-panel IV curve determine the solar panel current. We can see that the simulation captures the effect well.

2. From empty capacity $(0.1 \mathrm{~V})$ to near-full capacity $(2.9 \mathrm{~V})$, it took approximately 17 minutes in measurement and 20 minutes in simulation until the supercap was fully charged.

3. The simulation closely predicts the behavior of the actual system. The correlation coefficient and estimation error between the simulation result and the measurement are shown in Figure 4.32. As for the supercapacitor voltage, the correlation coefficient is 0.9904 . The mean and the standard deviation of the simulation error is $-0.2376 \mathrm{~V}$ and $0.1203 \mathrm{~V}$.

The simulation and measurement result for the second case (using an LM3671 step-down DC-DC converter as an input regulator) is shown in Figure 4.33. From this, we can draw the following observations:

1. As the supercapacitor is being charged, the solar panel voltage and the solar panel current change in two phases: first, the constant-input-voltage mode, and second, the pass-through mode. We can see that the simulation captures this two-phase operation well.

2. From empty capacity $(0.1 \mathrm{~V})$ to near-full capacity $(2.9 \mathrm{~V})$, it took approximately 19 minutes in measurement and 20 minutes in simulation until the supercapacitor was fully charged. 

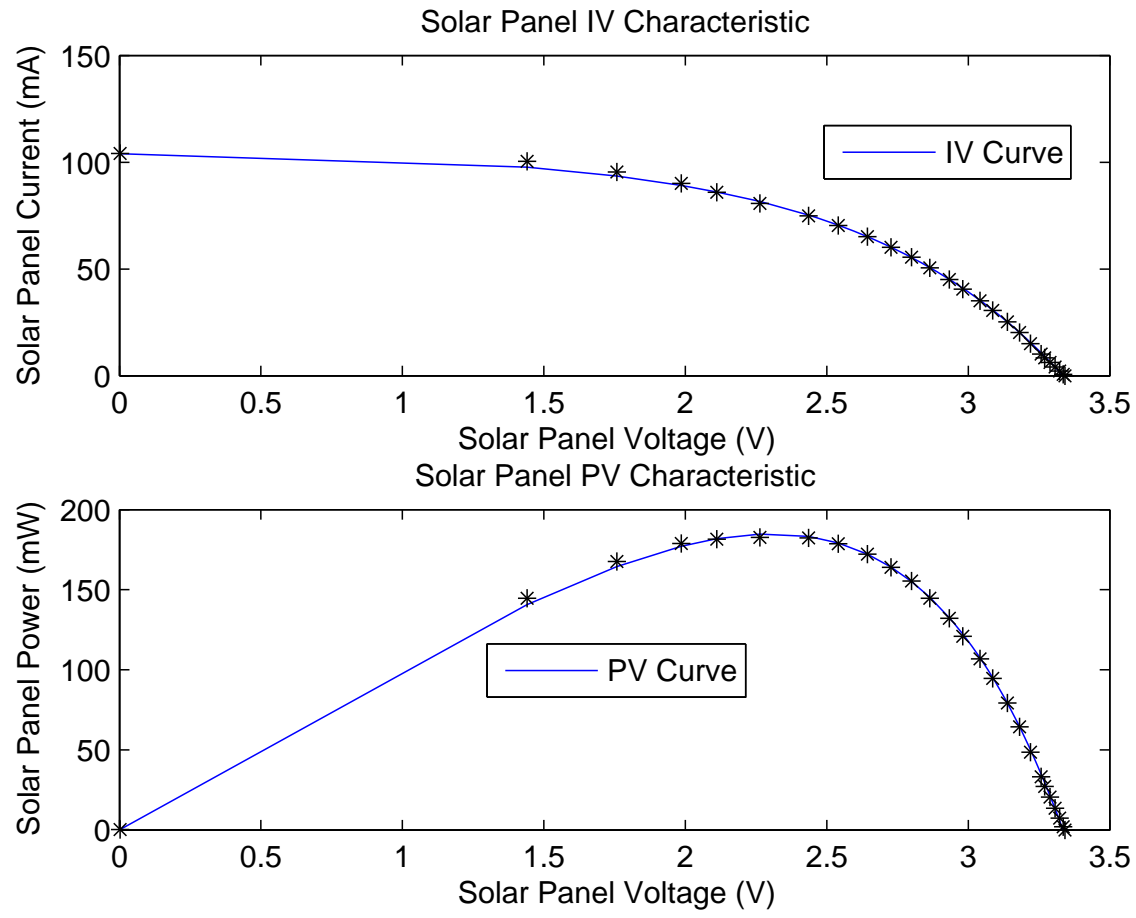

Figure 4.31: Solar-panel IV curve of the indoor lamp light

3. The simulation closely predicts the behavior of the actual system. The correlation coefficient, mean of measurement error, and the standard deviation of the measurement error are $0.9980,-0.0818 \mathrm{~V}$ and $0.0708 \mathrm{~V}$ respectively.

\section{Effect of Storage Capacity}

We simulate the effect of different energy storage capacities. For this purpose, we simulate the charging time of supercap-based systems with different energy storage sizes. To make a fair comparison, we made the other parameters the same.

- External Environment and Solar Panel: An indoor lamp light was used to make the radiation constant throughout the experiment. A $4.0 \mathrm{~V}-100 \mathrm{~mA}$ solar panel was used as the solar panel.

- Input Regulator: We considered two configurations for the input regulator: (a) no input regulator is used, (b) an LM3671 step-down DC-DC converter is 

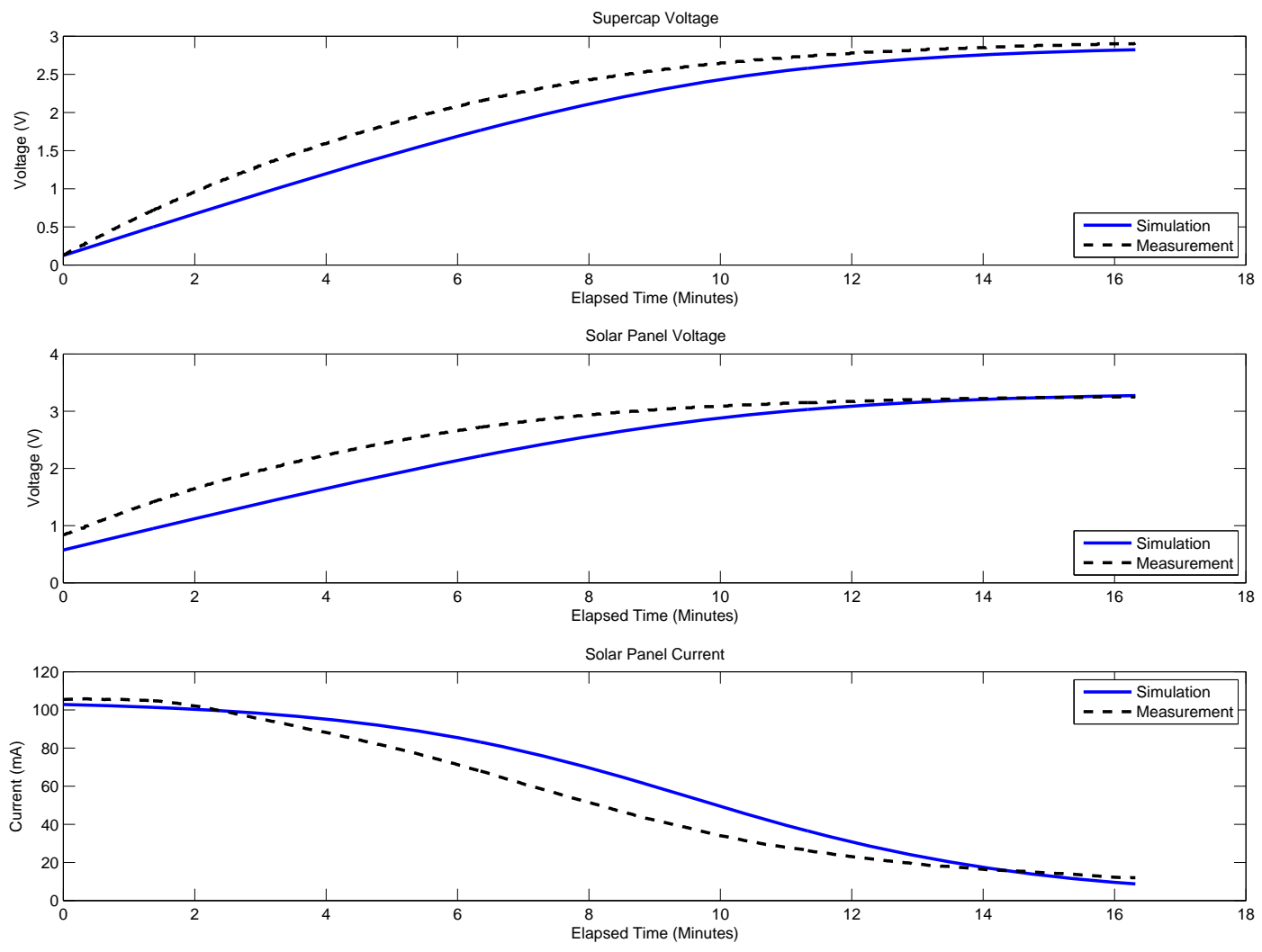

Correlation Coefficient and Estimation Error

(X: estimation, Y: measurement)

\begin{tabular}{|l|l|l|l|}
\hline & $\operatorname{corrcoef}(\mathrm{X}, \mathrm{Y})$ & $\operatorname{mean}(\mathrm{X}-\mathrm{Y})$ & stdev $(\mathrm{X}-\mathrm{Y})$ \\
\hline Supercap Voltage & 0.9904 & $-0.2376 \mathrm{~V}$ & $0.1203 \mathrm{~V}$ \\
\hline Solar-panel Voltage & 0.9802 & $-0.3027 \mathrm{~V}$ & $0.2175 \mathrm{~V}$ \\
\hline Solar-panel Current & 0.9736 & $7.1366 \mathrm{~mA}$ & $7.6078 \mathrm{~mA}$ \\
\hline
\end{tabular}

Figure 4.32: Trend of solar-panel and supercapacitor for a micro-solar power system with $22 \mathrm{~F}$ supercap under the indoor lamp light. No input regulator was used. No load or output regulator was attached. $V_{\text {cap-init }}=0.124 \mathrm{~V}$. We notice that there is a gap between the simulation and the measurement, and this is caused by an idealistic modeling of the Schottky diode. The threshold voltage of the Schottky diode is modeled as a fixed value in our simulator, however, the threshold voltage changes depending on the current in a real system. 

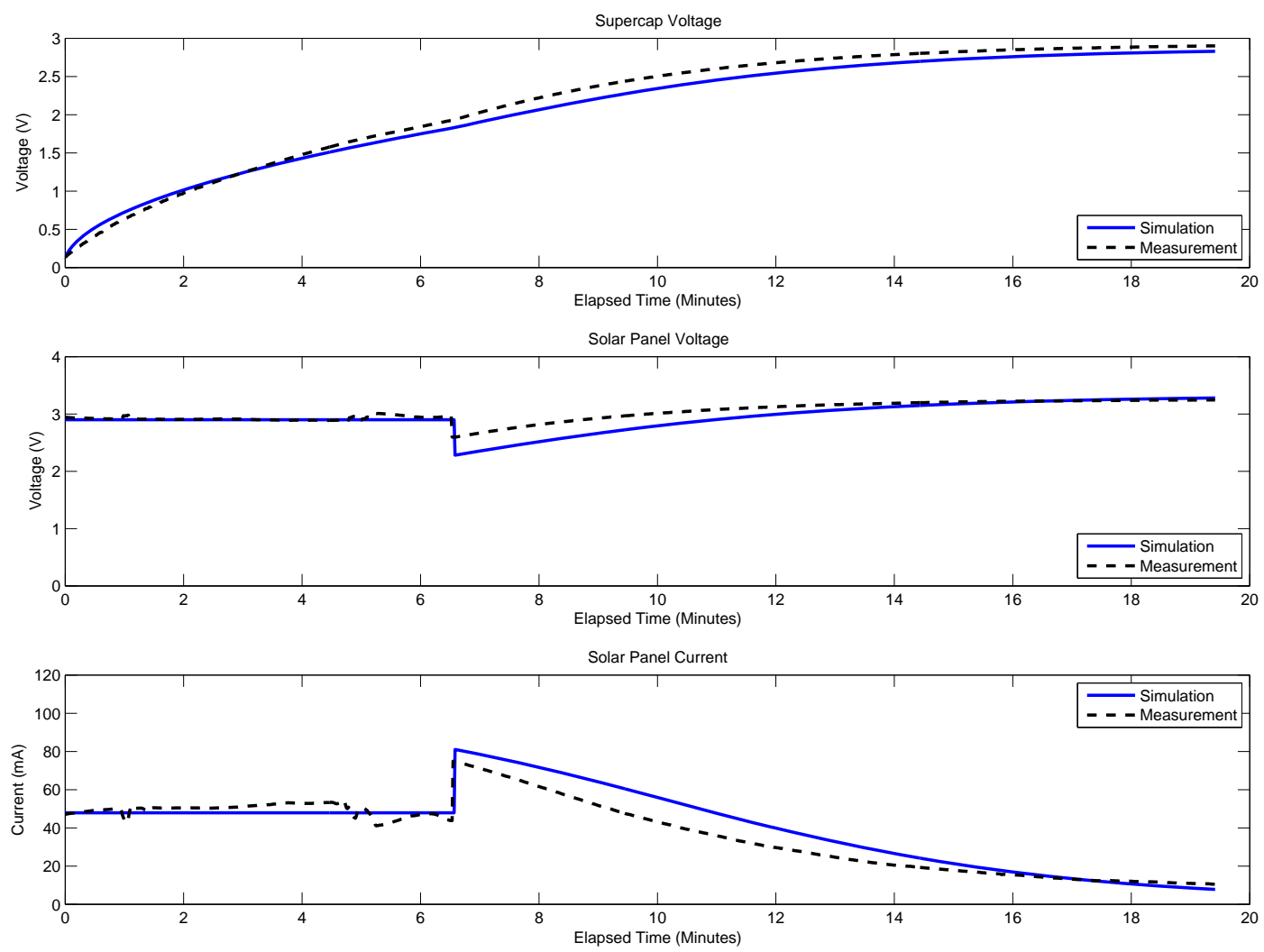

Correlation Coefficient and Estimation Error

(X: estimation, $\mathrm{Y}$ : measurement)

\begin{tabular}{|l|l|l|l|}
\hline & $\operatorname{corrcoef}(\mathrm{X}, \mathrm{Y})$ & $\operatorname{mean}(\mathrm{X}-\mathrm{Y})$ & stdev $(\mathrm{X}-\mathrm{Y})$ \\
\hline Supercap Voltage & 0.9980 & $-0.0818 \mathrm{~V}$ & $0.0708 \mathrm{~V}$ \\
\hline Solar-panel Voltage & 0.9125 & $-0.0851 \mathrm{~V}$ & $0.1098 \mathrm{~V}$ \\
\hline Solar-panel Current & 0.9522 & $3.5611 \mathrm{~mA}$ & $5.9328 \mathrm{~mA}$ \\
\hline
\end{tabular}

Figure 4.33: Trend of solar-panel and supercapacitor for a micro-solar power system with $22 \mathrm{~F}$ supercap under the indoor lamp light. LM3671 step-down DC-DC converter was used as an input regulator. No load or output regulator was attached. $V_{\text {cap-init }}=$ $0.130 \mathrm{~V}$. 
used as an input regulator.

- Energy Storage: We considered two different supercapacitor sizes: (a) one 22F supercapacitor by Aerogel, (b) one 140F supercapacitor by Maxwell.

- Output Regulator and Load: No output regulator or load was used.

The simulation and measurement results with the $140 \mathrm{~F}$ supercapacitor (Figure 4.34 and 4.35 show that the simulation also holds when we increase the energy storage size from $22 \mathrm{~F}$ to $140 \mathrm{~F}$. We can also see that the result captures the trend of the system for two different input regulator configurations. As for the charging time from empty capacity $(0.045 \mathrm{~V})$ to near-full capacity $(2.766 \mathrm{~V})$, it took 138 minutes for the case with no regulator and 149 minutes for the case with the LM3671 step-down converter. If we compare this with the charging time of the $22 \mathrm{~F}$ supercap, we can see that the charging time of a supercapacitor roughly increases in proportion to the capacity of the supercapacitor.

\subsubsection{Simulation under Daily Solar Radiation}

In this subsection, we simulate a micro-solar power system under a daily solar radiation cycle. By comparing daily energy accumulation and consumption, we can evaluate whether a particular design of a micro-solar power system can achieve sustainable operation.

As for the simulation, the following parameters are considered:

- External Environment: For the external environment, we consider a solar astronomical model with $\theta_{p}=0, L=37.87, \phi_{p}=180, \tau=0.1$ and no obstructions are assumed. We simulate the system for a few different values of $n$ to see the effect of seasonal variation in the operation of a micro-solar power system: (a) $n=80$ for spring, (b) $n=172$ for summer and (c) $n=356$ for winter.

- Solar Panel: A 4.0V-100mA solar panel

- Input Regulator: Not used. The energy storage was wired to the solar panel through the Schottky diode without an input regulator. 

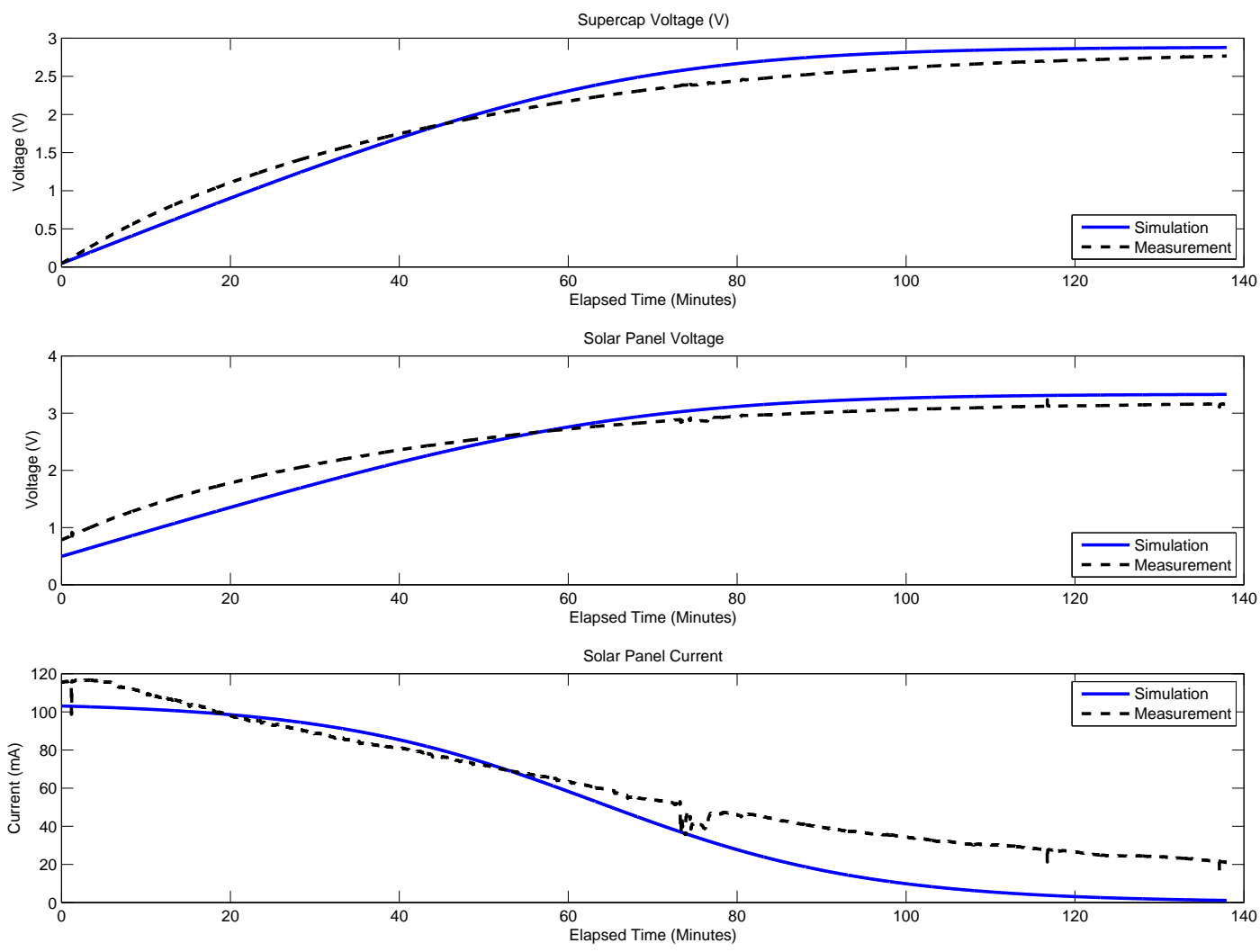

Correlation Coefficient and Estimation Error

(X: estimation, $\mathrm{Y}$ : measurement)

\begin{tabular}{|l|l|l|l|}
\hline & corrcoef $(\mathrm{X}, \mathrm{Y})$ & $\operatorname{mean}(\mathrm{X}-\mathrm{Y})$ & stdev $(\mathrm{X}-\mathrm{Y})$ \\
\hline Supercap Voltage & 0.9960 & $0.0659 \mathrm{~V}$ & $0.1496 \mathrm{~V}$ \\
\hline Solar-panel Voltage & 0.9945 & $-0.0235 \mathrm{~V}$ & $0.2452 \mathrm{~V}$ \\
\hline Solar-panel Current & 0.9818 & $-11.5760 \mathrm{~mA}$ & $11.5760 \mathrm{~mA}$ \\
\hline
\end{tabular}

Figure 4.34: Trend of solar-panel and supercapacitor for a micro-solar power system with $140 \mathrm{~F}$ supercap under the indoor lamp light. No input regulator was used. No load or output regulator was attached. $V_{\text {cap-init }}=0.045 \mathrm{~V}$. The gap between the simulation and the measurement is caused by an idealistic modeling of the Schottky diode. 

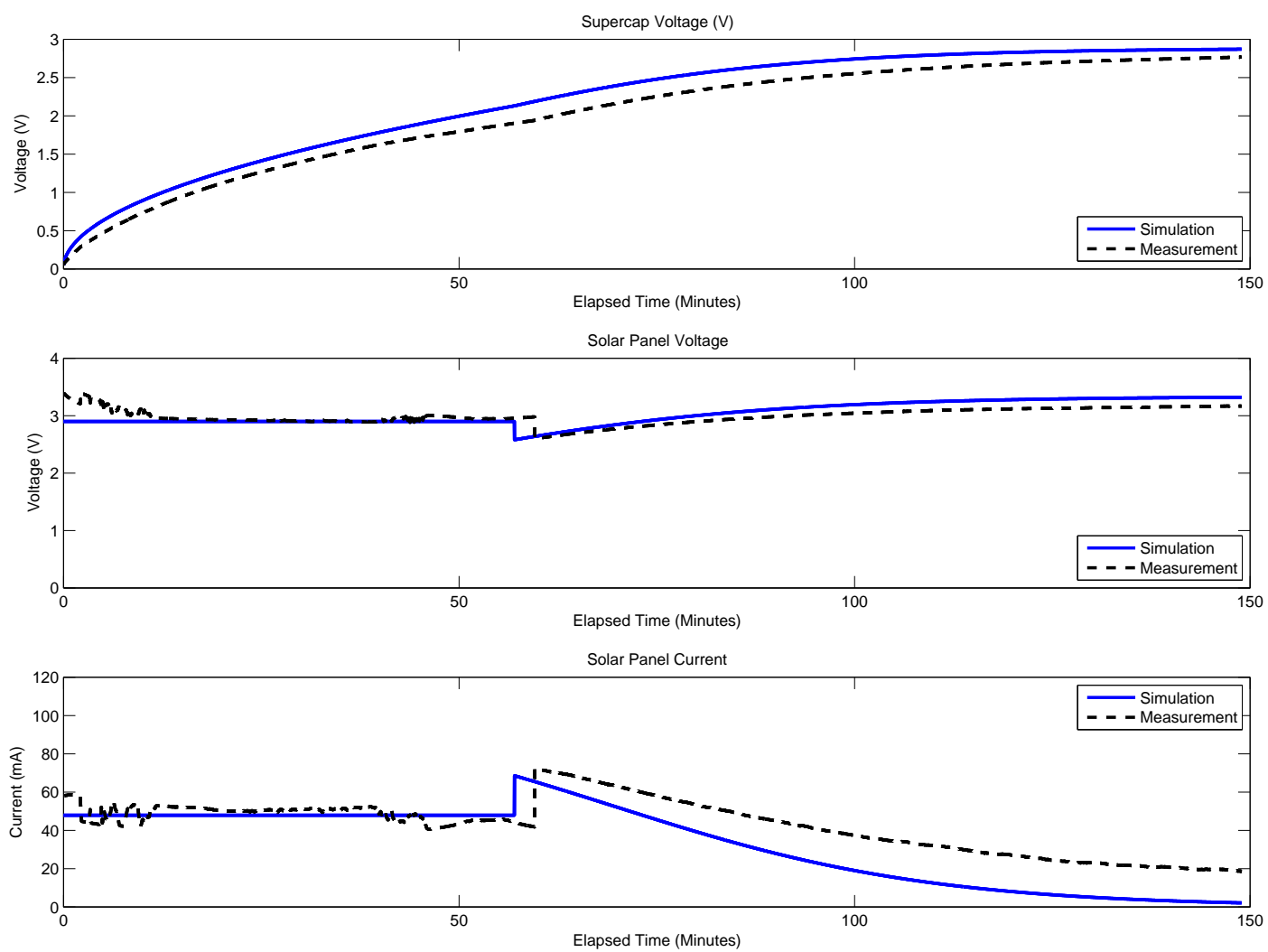

Correlation Coefficient and Estimation Error

(X: estimation, $\mathrm{Y}$ : measurement)

\begin{tabular}{|l|l|l|l|}
\hline & corrcoef $(\mathrm{X}, \mathrm{Y})$ & $\operatorname{mean}(\mathrm{X}-\mathrm{Y})$ & stdev $(\mathrm{X}-\mathrm{Y})$ \\
\hline Supercap Voltage & 0.9985 & $0.1736 \mathrm{~V}$ & $0.0387 \mathrm{~V}$ \\
\hline Solar-panel Voltage & 0.7036 & $0.0417 \mathrm{~V}$ & $0.1387 \mathrm{~V}$ \\
\hline Solar-panel Current & 0.9069 & $-9.6766 \mathrm{~mA}$ & $9.2987 \mathrm{~mA}$ \\
\hline
\end{tabular}

Figure 4.35: Trend of solar-panel and supercapacitor for a micro-solar power system with 140F supercap under the indoor lamp light. LM3671 step-down DC-DC converter was used as an input regulator. No load or output regulator was attached. $V_{\text {cap-init }}=0.045 \mathrm{~V}$. 
- Energy Storage: Three configurations are used: (a) one 22F supercap, (b) one 140F supercap, (c) Two 2500mAh NiMH batteries.

- Output Regulator: A MAX1724 step-up DC-DC converter.

- Load: A TelosB node was used. As a workload, the HydroWatch workload (0.53mA average) was used. We also considered the highest workload with energy surplus.

\section{Simulation under Average Yearly Solar Radiation (Spring)}

Figure 4.36 and Table 4.3 show the case when the HydroWatch workload $\left(I_{l o a d}=\right.$ $0.53 \mathrm{~mA}$ ) is used. With the HydroWatch workload, one $22 \mathrm{~F}$ supercap cannot provide enough storage for daily operation under average yearly solar radiation, whereas either one $140 \mathrm{~F}$ supercap or two $2500 \mathrm{mAh} \mathrm{NiMH}$ batteries can provide enough storage for daily operation. For these two cases, the charging time $T_{c}$, the discharging time $T_{d}$ and discharged energy $E_{d}$ are about the same. This is because these two cases have the same load current and radiation condition, which determine the energy consumption and energy supply rate, so the effects of using different energy storage types are not as high.

We notice that the discharging time $T_{d}$ is limited by the capacity of the energy storage for the case that does not survive an overnight operation (one $22 \mathrm{~F}$ supercap). For the cases that survive an overnight operation (one 140F supercap and two $2500 \mathrm{mAh}$ NiMH batteries), the discharging time $T_{d}$ is limited by the nighttime extent. We also notice that the discharging time of the first case is about two thirds of the second case ( 8 hour 15 minutes over 12 hour 15 minutes), and this implies that the minimum capacity for a supercapacitor that can survive an overnight operation is about $33 \mathrm{~F}(=22 \mathrm{~F} * 3 / 2)$.

Figure 4.37 and Table 4.4 show the case when the highest possible workload is used. We can see that the charging time $T_{c}$ increases as the load current $I_{\text {load }}$ increases (0.3mA for one $22 \mathrm{~F}$ supercap, $2.5 \mathrm{~mA}$ for one $140 \mathrm{~F}$ supercap and $7.15 \mathrm{~mA}$ for two $2500 \mathrm{mAh}$ NiMH batteries), whereas the discharging time $T_{d}$ is about the same. This is because the charging time $T_{c}$ is determined by the load current and the radiation condition while the discharging time $T_{d}$ is determined by the nighttime extent. We 


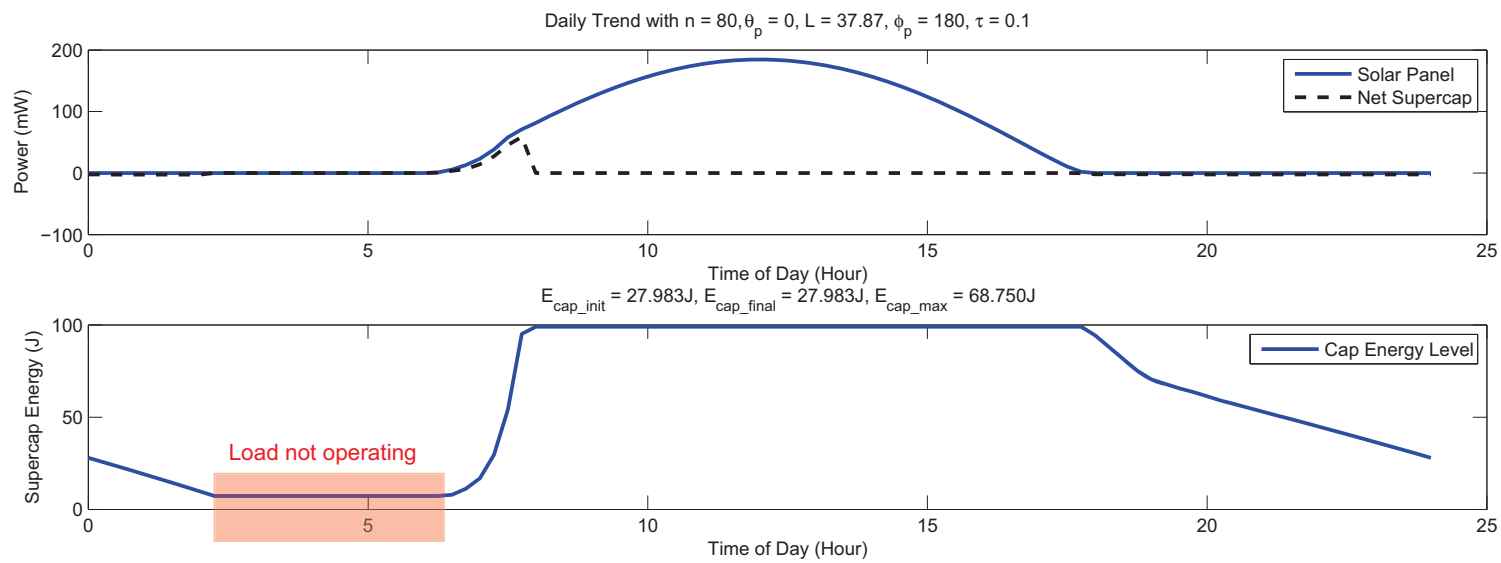

(a) $22 \mathrm{~F}$ supercapacitor, $I_{\text {load }}=0.53 \mathrm{~mA}$
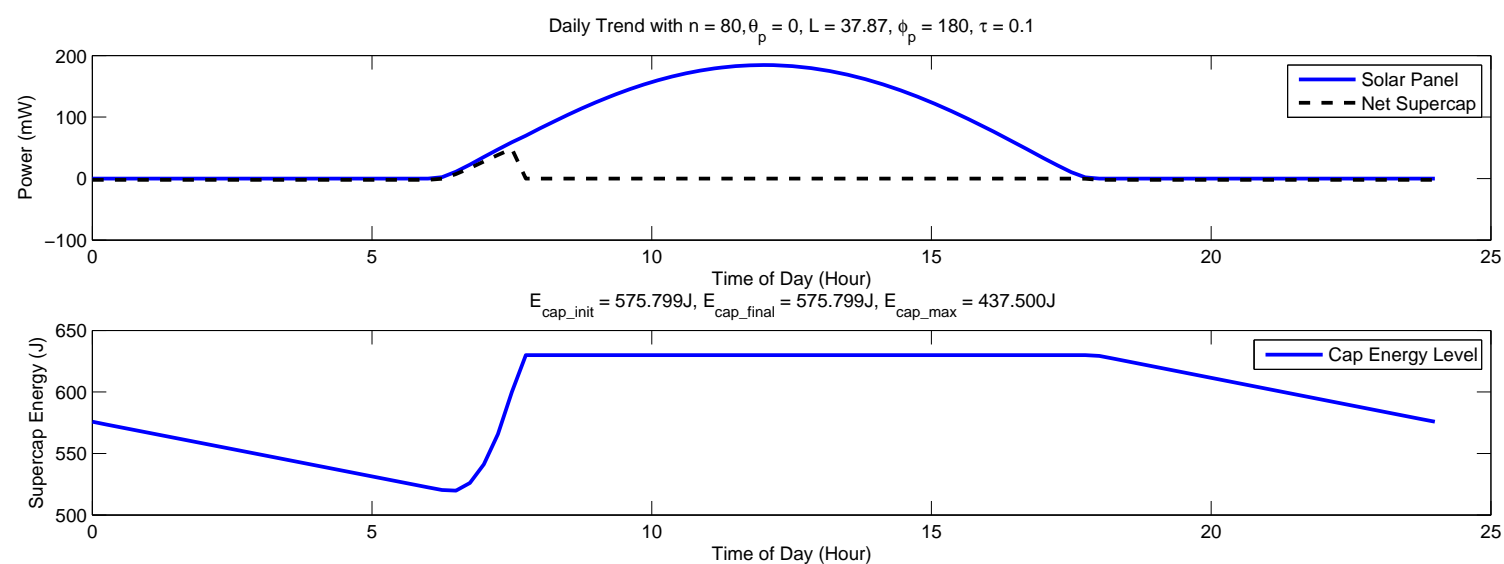

(b) $140 \mathrm{~F}$ supercapacitor, $I_{\text {load }}=0.53 \mathrm{~mA}$
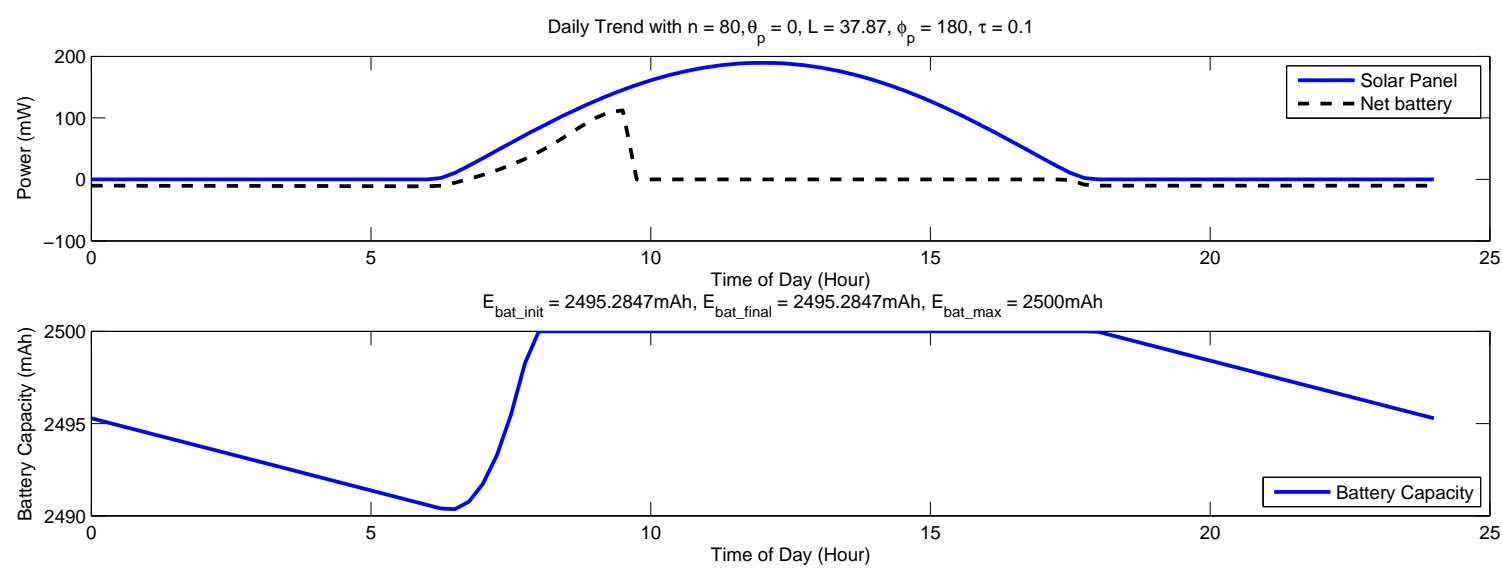

(c) Two $2500 \mathrm{mAh}$ NiMH batteries, $I_{\text {load }}=0.53 \mathrm{~mA}$

Figure 4.36: A micro-solar power system under average yearly solar radiation with HydroWatch workload (day of year $n=80$ ) 
Table 4.3: Simulation under average yearly solar radiation with HydroWatch workload (day of year $n=80$ ).

\begin{tabular}{|l|l|l|l|}
\hline Energy Storage & $\begin{array}{l}\text { One 22F super- } \\
\text { cap }\end{array}$ & $\begin{array}{l}\text { One 140F super- } \\
\text { cap }\end{array}$ & $\begin{array}{l}\text { Two 2500mAh } \\
\text { NiMH batteries }\end{array}$ \\
\hline $\begin{array}{l}\text { Load Current } \\
I_{\text {load }}\end{array}$ & $0.53 \mathrm{~mA}$ & $0.53 \mathrm{~mA}$ & $0.53 \mathrm{~mA}$ \\
\hline $\begin{array}{l}\text { Charging Time } \\
T_{c}\end{array}$ & $\begin{array}{l}1 \text { hour } 45 \text { minutes } \\
(06: 15 \text { to 08:00) }\end{array}$ & $\begin{array}{l}1 \text { hour } 30 \text { minutes } \\
(06: 15 \text { to } 07: 45)\end{array}$ & $\begin{array}{l}1 \text { hour } 45 \text { minutes } \\
(06: 15 \text { to 08:00) }\end{array}$ \\
\hline $\begin{array}{l}\text { Discharging } \\
\text { Time } T_{d}\end{array}$ & $\begin{array}{l}8 \text { hour } 15 \text { minutes } \\
(18: 00 \text { to 02:15) }\end{array}$ & $\begin{array}{l}12 \text { hour } 15 \text { minutes } \\
(18: 00 \text { to 06:15) }\end{array}$ & $\begin{array}{l}12 \text { hour } 15 \text { minutes } \\
(18: 00 \text { to 06:15) }\end{array}$ \\
\hline $\begin{array}{l}\text { Discharged En- } \\
\text { ergy } E_{d}\end{array}$ & $91.684 \mathrm{~J}$ & $\begin{array}{l}110.238 \mathrm{~J} \\
9.636 \mathrm{mAh} \\
97.130 \mathrm{~J} \text { at } 2.8 \mathrm{~V})\end{array}$ \\
\hline
\end{tabular}

Table 4.4: Simulation under average yearly solar radiation with highest possible workload (day of year $n=80$ )

\begin{tabular}{|l|l|l|l|}
\hline Energy Storage & $\begin{array}{l}\text { One 22F super- } \\
\text { cap }\end{array}$ & $\begin{array}{l}\text { One 140F super- } \\
\text { cap }\end{array}$ & $\begin{array}{l}\text { Two 2500mAh } \\
\text { NiMH batteries }\end{array}$ \\
\hline $\begin{array}{l}\text { Load Current } \\
I_{\text {load }}\end{array}$ & $0.30 \mathrm{~mA}$ & $2.5 \mathrm{~mA}$ & $7.15 \mathrm{~mA}$ \\
\hline $\begin{array}{l}\text { Charging Time } \\
T_{c}\end{array}$ & $\begin{array}{l}1 \text { hour } 30 \text { minutes } \\
(06: 15 \text { to } 07: 45)\end{array}$ & $\begin{array}{l}3 \text { hour } 30 \text { minutes } \\
(06: 15 \text { to 09:45) }\end{array}$ & $\begin{array}{l}5 \text { hour } 15 \text { minutes } \\
(06: 15 \text { to } 13: 30)\end{array}$ \\
\hline $\begin{array}{l}\text { Discharging } \\
\text { Time } T_{d}\end{array}$ & $\begin{array}{l}12 \text { hour } 30 \text { min- } \\
\text { utes }(18: 00 \text { to }\end{array}$ & $\begin{array}{l}12 \text { hour } 30 \text { minutes } \\
(17: 45 \text { to 06:15) }\end{array}$ & $\begin{array}{l}12 \text { hour } 45 \text { minutes } \\
17: 30 \text { to 06:15) }\end{array}$ \\
\hline $\begin{array}{l}\text { Discharged En- } \\
\text { ergy } E_{d}\end{array}$ & $84.316)$ & $493.798 \mathrm{~J}$ & $\begin{array}{l}140.157 \mathrm{mAh} \\
1412.8 \mathrm{~J} \text { at 2.8V })\end{array}$ \\
\hline
\end{tabular}

also notice that the charging time does not increase linearly with the load current, which is because the solar radiation is low at dawn and gets higher until noon.

\section{Simulation under Worst-case of Yearly Solar Radiation (Winter)}

Figure 4.38 and Table 4.5 show the case when the HydroWatch workload $\left(I_{l o a d}=\right.$ $0.53 \mathrm{~mA})$ is used during winter $(n=356)$, which is the worst case for yearly solar radiation. With the HydroWatch workload, one 22F supercap cannot provide enough storage for daily operation under average yearly solar radiation, whereas, either one 140F supercap or two $2500 \mathrm{mAh}$ NiMH batteries can provide enough storage for daily operation. For these two cases, we notice that the charging time and the discharging time are longer than the case with average yearly solar radiation $(n=80)$, and this is due to the fact that there is a smaller energy budget available during the winter. 

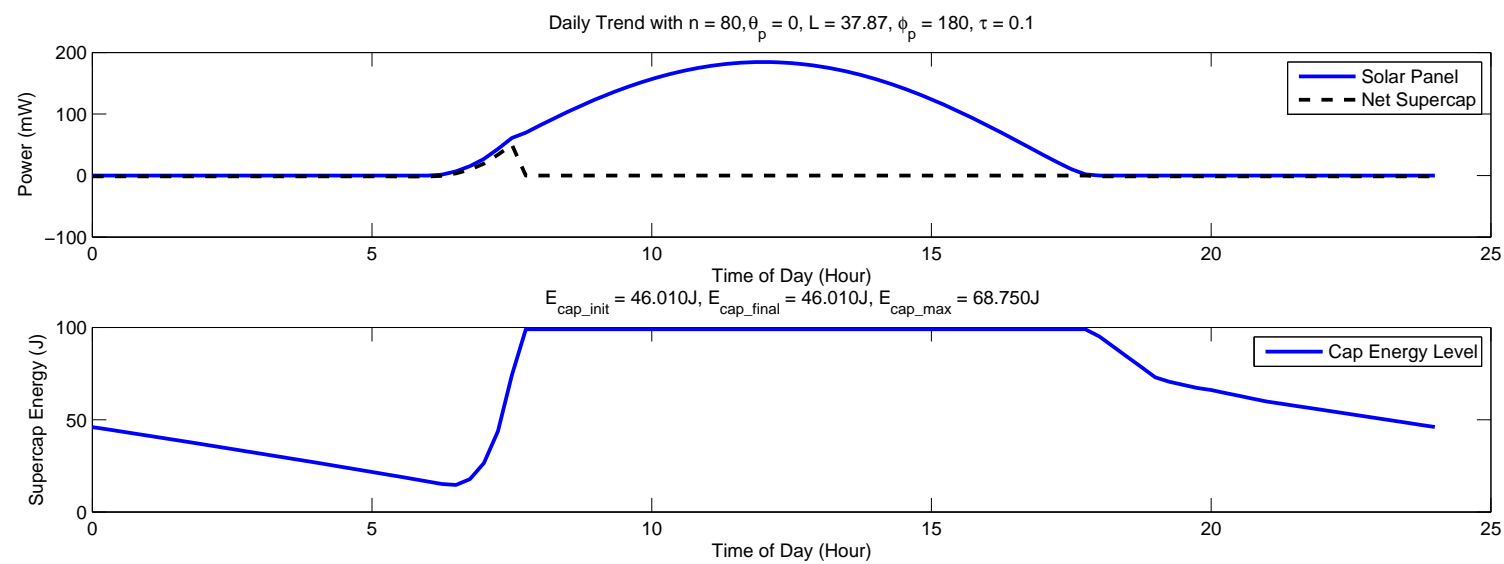

(a) 22F supercapacitor, $I_{\text {load }}=0.3 \mathrm{~mA}$
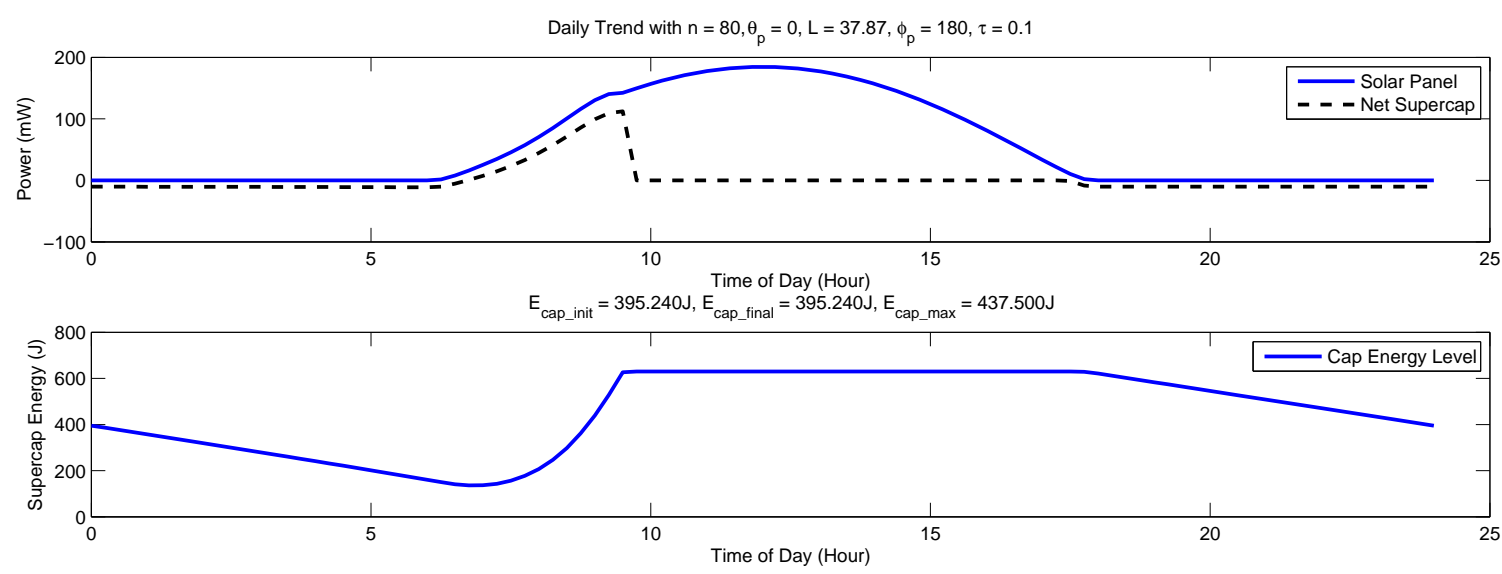

(b) $140 \mathrm{~F}$ supercapacitor, $I_{\text {load }}=2.5 \mathrm{~mA}$
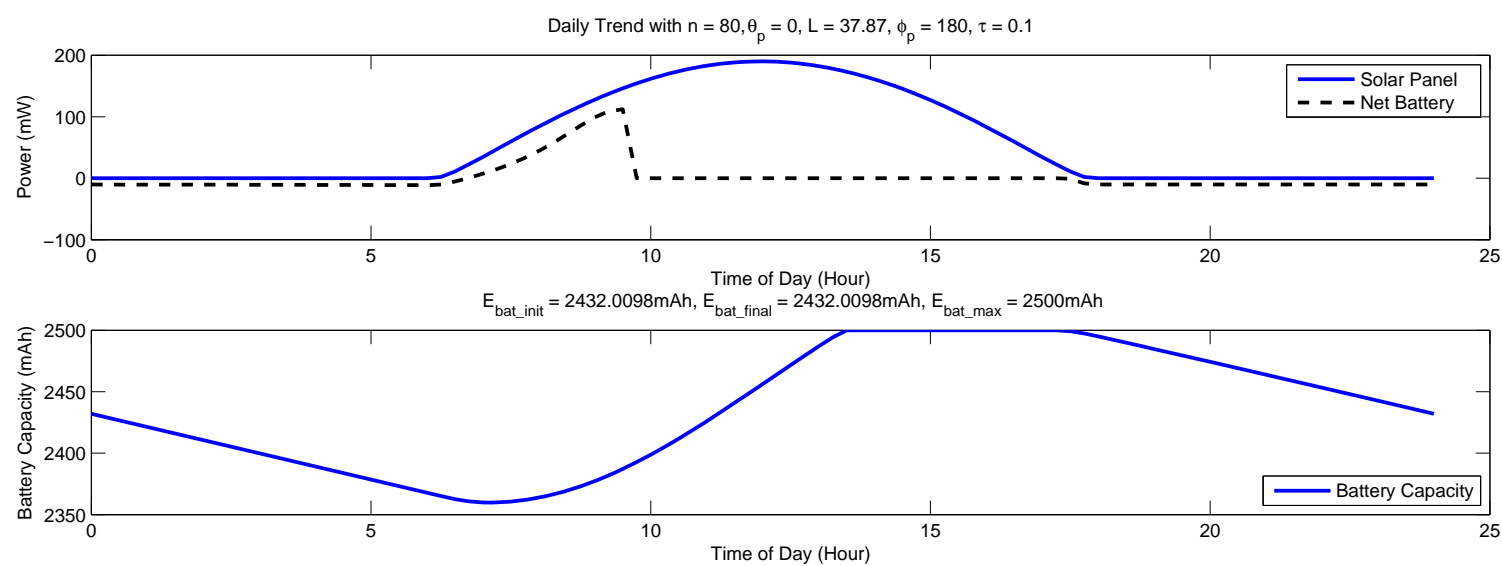

(c) Two $2500 \mathrm{mAh}$ NiMH batteries, $I_{\text {load }}=7.15 \mathrm{~mA}$

Figure 4.37: A micro-solar power system under average yearly solar radiation with highest possible workload (day of year $n=80$ ) 

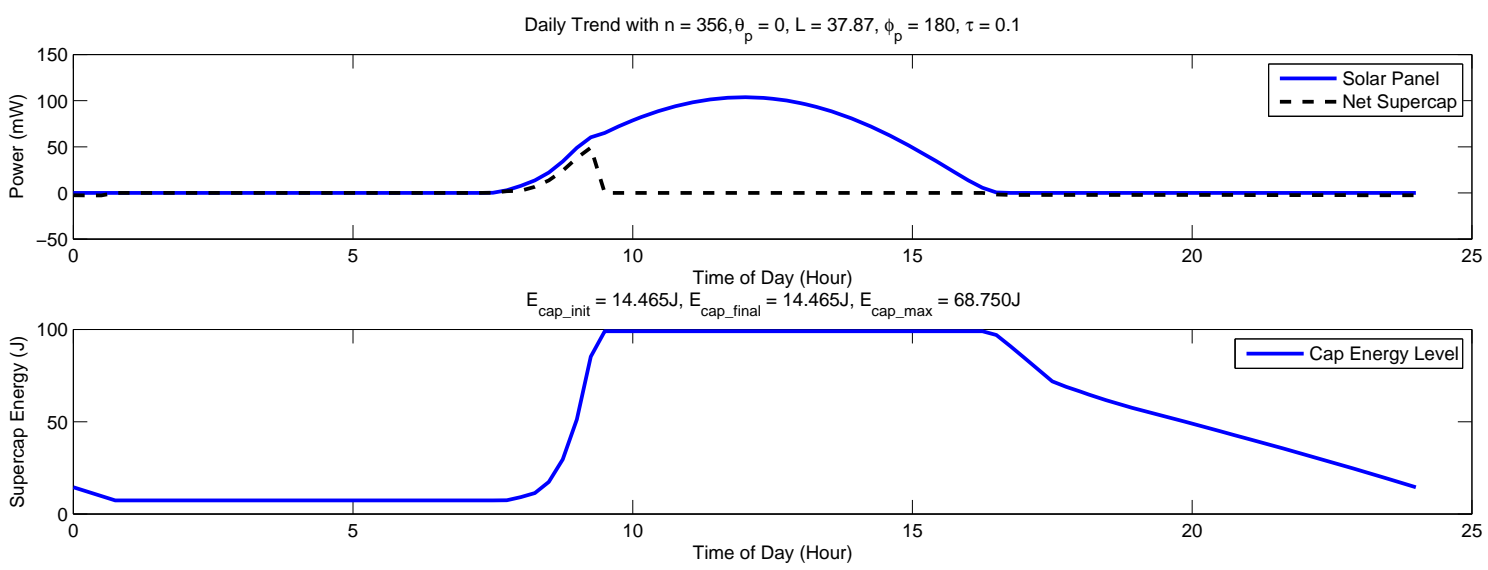

(a) $22 \mathrm{~F}$ supercapacitor, $I_{\text {load }}=0.53 \mathrm{~mA}$
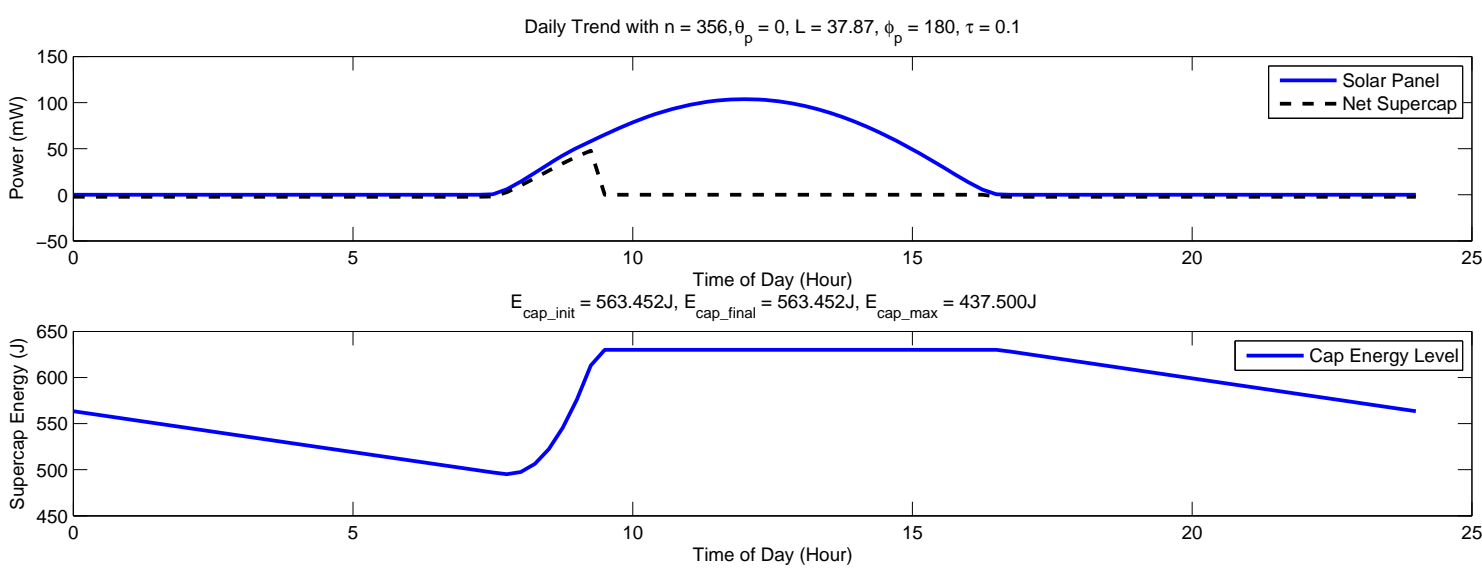

(b) $140 \mathrm{~F}$ supercapacitor, $I_{\text {load }}=0.53 \mathrm{~mA}$
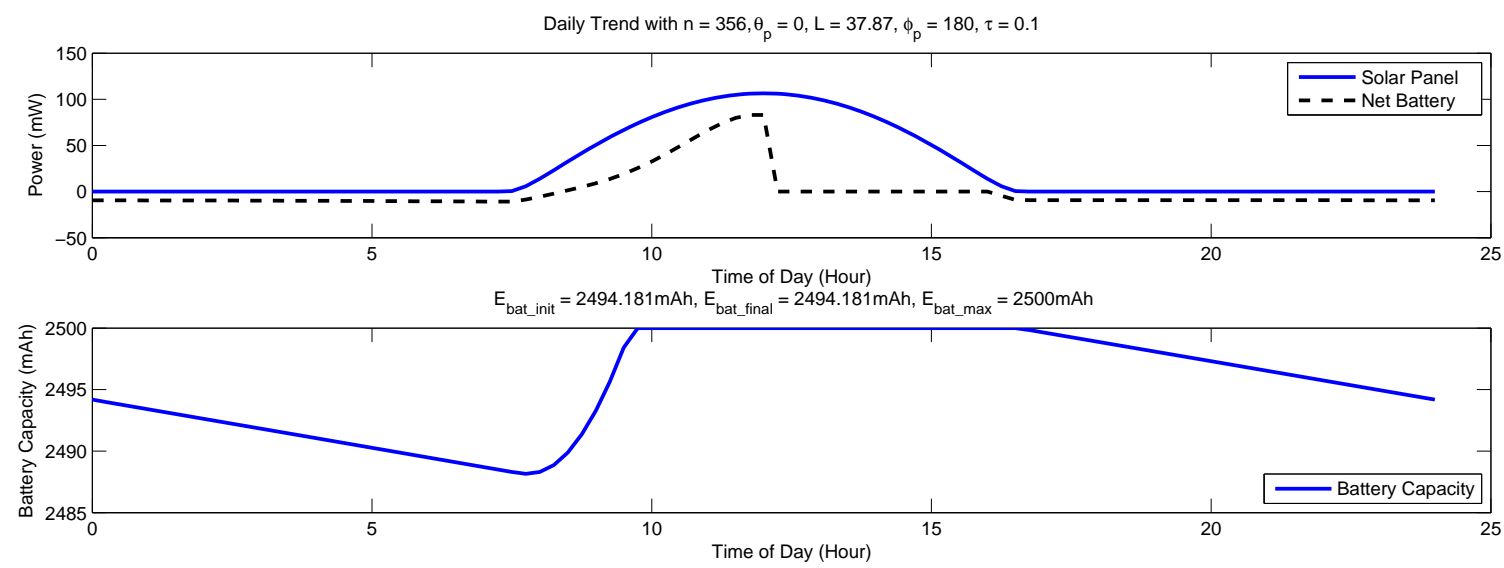

(c) Two $2500 \mathrm{mAh}$ NiMH batteries, $I_{\text {load }}=0.53 \mathrm{~mA}$

Figure 4.38: A micro-solar power system under worst-case yearly solar radiation with HydroWatch workload (day of year $n=356$ ) 
Table 4.5: Simulation under worst-case yearly solar radiation with HydroWatch workload (day of year $n=356$ )

\begin{tabular}{|l|l|l|l|}
\hline Energy Storage & $\begin{array}{l}\text { One 22F super- } \\
\text { cap }\end{array}$ & $\begin{array}{l}\text { One 140F super- } \\
\text { cap }\end{array}$ & $\begin{array}{l}\text { Two 2500mAh } \\
\text { NiMH batteries }\end{array}$ \\
\hline $\begin{array}{l}\text { Load Current } \\
I_{\text {load }}\end{array}$ & $0.53 \mathrm{~mA}$ & $0.53 \mathrm{~mA}$ & $0.53 \mathrm{~mA}$ \\
\hline $\begin{array}{l}\text { Charging Time } \\
T_{c}\end{array}$ & $\begin{array}{l}2 \text { hour (07:30 to } \\
09: 30)\end{array}$ & $\begin{array}{l}2 \text { hour }(07: 30 \text { to } \\
09: 30)\end{array}$ & $\begin{array}{l}2 \text { hour 15 minutes } \\
(07: 30 \text { to 09:45) }\end{array}$ \\
\hline $\begin{array}{l}\text { Discharging } \\
\text { Time } T_{d}\end{array}$ & $\begin{array}{l}8 \text { hour 15 minutes } \\
(16: 30 \text { to 00:45) }\end{array}$ & $\begin{array}{l}14 \text { hour } 45 \text { minutes } \\
(16: 45 \text { to 07:30) }\end{array}$ & $\begin{array}{l}14 \text { hour } 45 \text { minutes } \\
(16: 45 \text { to 07:30) }\end{array}$ \\
\hline $\begin{array}{l}\text { Discharged En- } \\
\text { ergy } E_{d}\end{array}$ & $91.685 \mathrm{~J}$ & $\begin{array}{l}134.836 \mathrm{~J} \\
11.852 \mathrm{mAh} \quad(=\end{array}$ \\
\hline
\end{tabular}

Table 4.6: Simulation under worst-case yearly solar radiation with highest possible workload (day of year $n=356$ )

\begin{tabular}{|l|l|l|l|}
\hline Energy Storage & $\begin{array}{l}\text { One 22F super- } \\
\text { cap }\end{array}$ & $\begin{array}{l}\text { One 140F super- } \\
\text { cap }\end{array}$ & $\begin{array}{l}\text { Two 2500mAh } \\
\text { NiMH batteries }\end{array}$ \\
\hline $\begin{array}{l}\text { Load Current } \\
I_{\text {load }}\end{array}$ & $0.25 \mathrm{~mA}$ & $2.3 \mathrm{~mA}$ & $3.5 \mathrm{~mA}$ \\
\hline $\begin{array}{l}\text { Charging Time } \\
T_{c}\end{array}$ & $\begin{array}{l}1 \text { hour } 45 \text { minutes } \\
(07: 30 \text { to 09:15) }\end{array}$ & $\begin{array}{l}5 \text { hour }(07: 30 \text { to } \\
12: 30)\end{array}$ & $\begin{array}{l}7 \text { hour }(07: 30 \quad \text { to } \\
14: 30)\end{array}$ \\
\hline $\begin{array}{l}\text { Discharging } \\
\text { Time } T_{d}\end{array}$ & $\begin{array}{l}15 \text { hour }(16: 30 \text { to } \\
07: 30)\end{array}$ & $\begin{array}{l}15 \text { hour }(16: 30 \text { to } \\
07: 30)\end{array}$ & $\begin{array}{l}14 \text { hour } 45 \text { minutes } \\
(16: 15 \text { to 07:30) }\end{array}$ \\
\hline $\begin{array}{l}\text { Discharged En- } \\
\text { ergy } E_{d}\end{array}$ & $87.964 \mathrm{~J}$ & $565.781 \mathrm{~J}$ & $\begin{array}{l}80.883 \mathrm{mAh} \quad(= \\
815.298 \mathrm{~J} \text { at } 2.8 \mathrm{~V})\end{array}$ \\
\hline
\end{tabular}

Figure 4.39 and Table 4.6 show the case when the highest possible workload is used during the winter. We can see that the highest possible workload $I_{\text {load }}$ is smaller than the case with average yearly solar radiation $(0.25 \mathrm{~mA}$ for one $22 \mathrm{~F}$ supercap, $2.3 \mathrm{~mA}$ for one $140 \mathrm{~F}$ supercap and $3.5 \mathrm{~mA}$ for two $2500 \mathrm{mAh}$ NiMH batteries), and this is because the energy surplus is smaller during the winter. 

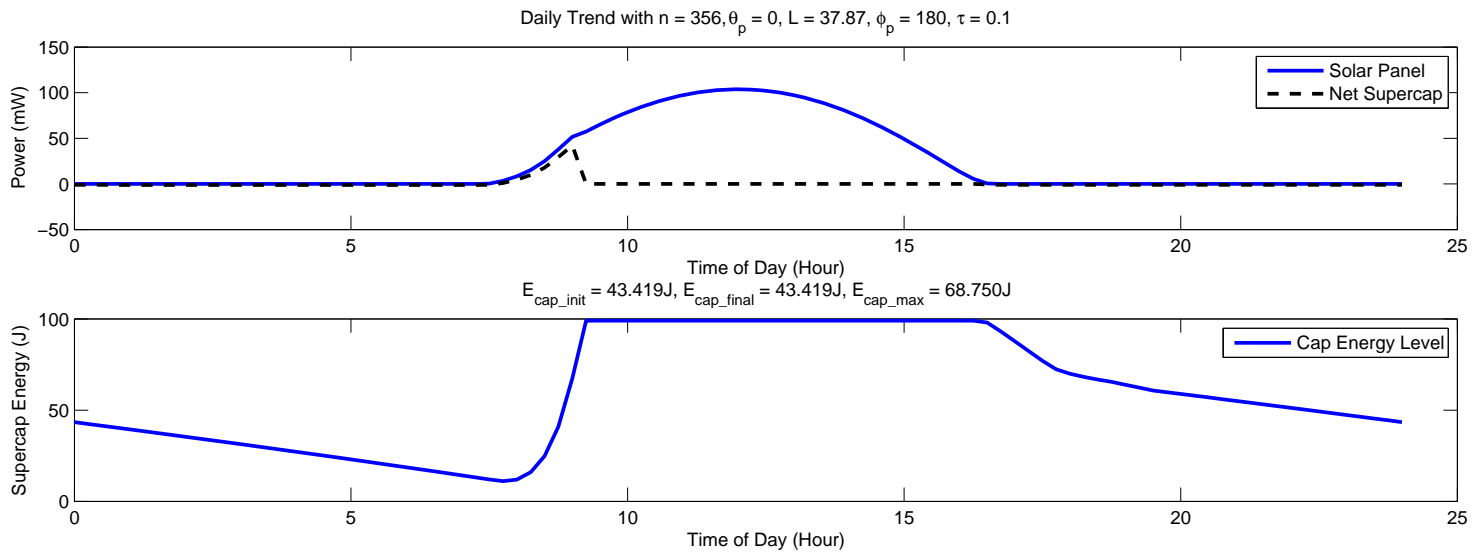

(a) 22F supercapacitor, $I_{\text {load }}=0.25 \mathrm{~mA}$
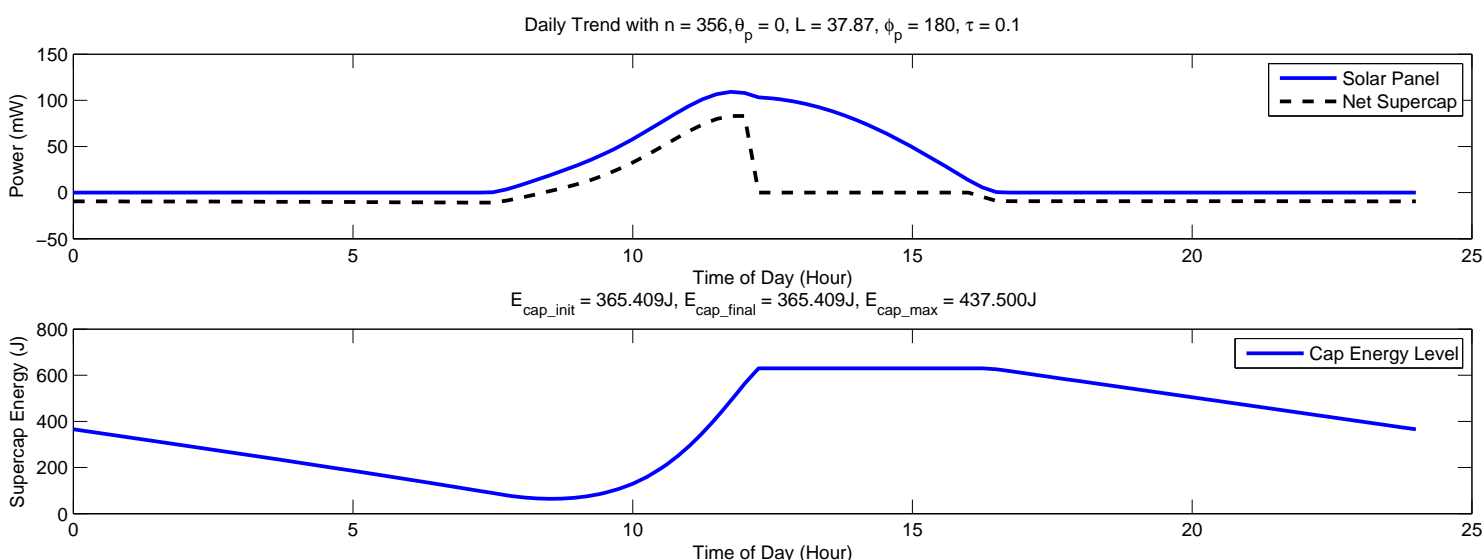

(b) $140 \mathrm{~F}$ supercapacitor, $I_{\text {load }}=2.3 \mathrm{~mA}$
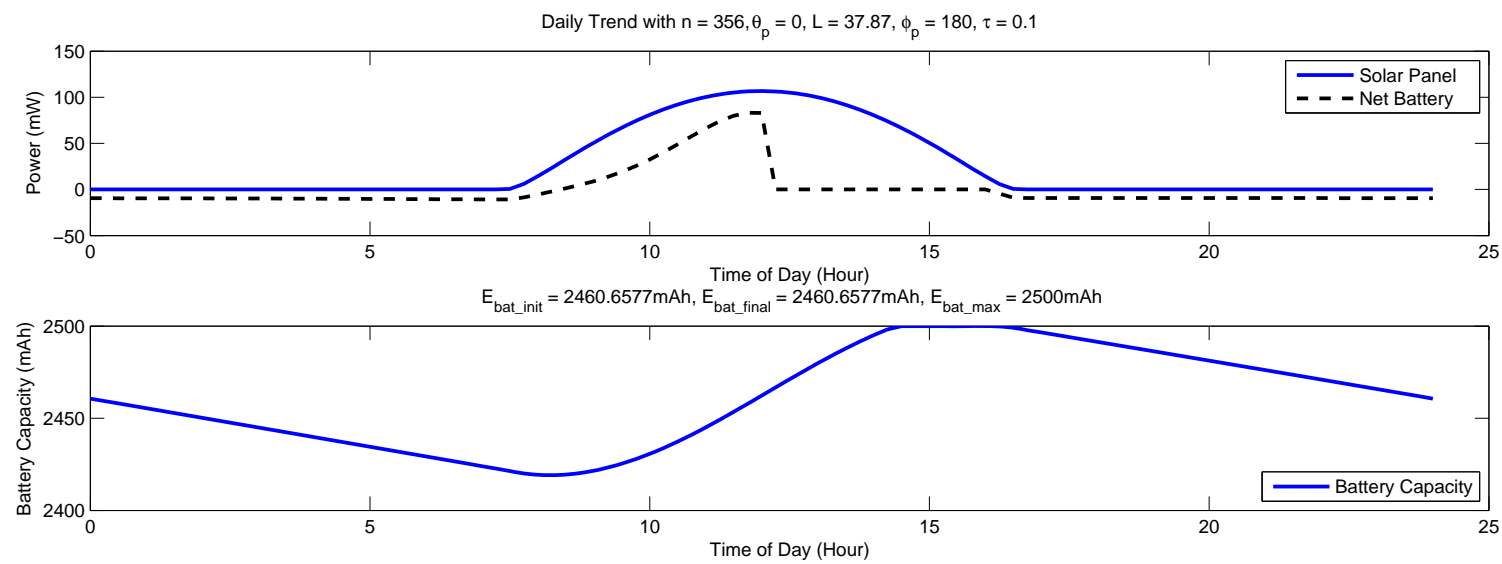

(c) Two $2500 \mathrm{mAh}$ NiMH batteries, $I_{l o a d}=3.5 \mathrm{~mA}$

Figure 4.39: A micro-solar power system under worst-case yearly solar radiation with highest possible workload (day of year $n=356$ ) 


\subsection{Summary of Micro-Solar Power System Simu- lator}

In this chapter, we have presented a Mablab-based simulator of micro-solar power systems. Below we summarize its architecture, principles and design methodology.

\section{Overall Architecture and Principles}

Our simulator is a time-event based simulator that has a modular architecture. The simulator is composed of the six modules, each of which describes the corresponding component in a micro-solar power system - external environment module, solar panel module, input regulator module, energy storage module, output regulator module and load module.

Our simulator has a modular and time-event architecture. First, our simulator is designed in a modular way. The simulator is composed of the six modules, each of which describes the corresponding component in a micro-solar power system external environment module, solar panel module, input regulator module, energy storage module, output regulator module and load module. With the simulator, a hypothetical system can be composed by plugging these modules together. Second, our simulator is a time-event based simulator. The daily behavior is simulated by iteratively evaluating the state of a system for the given initial condition and the time interval vector, and similarly, the long-term behavior is simulated by iteratively evaluating the daily behavior. Third, our simulator defines each module either analytically or empirically depending on the availability of the well-defined characteristic.

\section{Modeling Solar Radiation}

Our simulator provides two ways to model solar radiation. An astronomical model, which estimates solar radiation by calculating the angle between the sunlight and the normal to the sunlight, is used when the weather is clear and the solar panel is not obstructed. However, its estimation becomes significantly different from the actual measurement under obstruction. An obstructed astronomical model, which is a refinement of the astronomical model, can estimate solar radiation with relatively high 
accuracy even under obstruction using an empirical measurement on previous days. The obstructed astronomical model is based on the following two assumptions: first, the estimation from the astronomical model bounds the envelope of the solar radiation measurement; second, nodes and local obstruction objects are stationary, and the obstruction pattern changes little over days. From the first assumption, the obstructed astronomical model calculates an obstruction factor by taking a linear difference of the measurement and the estimation of the astronomical model on a relatively clear day $n 1$. From the second assumption, the model estimates the solar radiation on a different day $n 2$ by multiplying the obstruction factor with the estimation of the astronomical model on $n 2$.

\section{Modeling Solar Panel}

Our simulator models the behavior of a solar panel in two steps: first it defines the I-V characteristic of a panel; then it determines the operating point of the panel in a whole system. The simulator models the I-V characteristic of a solar panel with a generic curve $I=I_{s c}-A \cdot(\exp (B \cdot V)-1)$ and instantiates the generic curve into a specific curve using a Matlab curve-fitting algorithm when a sequence of $(I, V)$ measurement points are given. The simulator sets the operating voltage of the solar panel in a whole system as the voltage of the output of the solar panel using the fact that the solar panel operates as a voltage-controlled current source. Once the voltage of the solar panel is determined, its current and power can be determined by its I-V curve.

\section{Modeling Energy Storage}

Our simulator models two types of energy storage that are widely used for microsolar power systems: a NiMH battery and a supercapacitor. In order to simulate the behavior of these two types of energy storage, our simulate models capacity-to-voltage relation, charge-and-discharge relation, and self-discharge effect.

Capacity-to-voltage relation: For the case of a NiMH battery, the simulator models capacity-to-voltage relation using a manufacturer-provided capacity-to-voltage graph. Since a NiMH battery has different characteristics for the charging mode and the discharging mode, the simulator models its capacity-to-voltage relation differently 
depending on whether the system is in the charging mode or not. For the case of a supercapacitor, the simulator models capacity-to-voltage relation using a well-known

formula, $E=\frac{1}{2} C V^{2}$. Unlike a NiMH battery, a supercapacitor has the same operating mode for charging and discharging.

Charge-and-discharge relation: At each time interval, the simulator estimates energy increment, which tells how much energy is charged into or discharged from the energy storage during the time interval. At the end of a time interval, this increment is added to the current energy level of the energy storage. In the next iteration, the voltage level of the energy storage is evaluated from the updated energy level using the capacity-to-voltage relation.

Self-discharge effect: Our simulator models the self-discharge effect for a supercapacitor, which has a high leakage rate compared to its energy capacity. The selfdischarge effect is modeled either analytically with a manufacturer-provided leakage current or empirically with an energy-time graph.

\section{Modeling Output Regulator}

Our simulator models the behavior of an output regulator using a piecewise-linear interpolation method. This is because it is difficult to describe the behavior of various types of output regulator in a simple formula: their characteristics are different depending on the underlying technology and their behaviors are non-linear. For the given input voltage and the output current, the simulator determines the output voltage and the power efficiency by interpolating them from the pre-measured data points - either from a manufacturer-provided data sheet or from empirical measurement.

\section{Modeling Input Regulator}

Our simulator models two different configurations for an input regulator. The first configuration is when the solar panel is connected to the energy storage through a Schottky diode without an input regulator being used, and it is modeled by setting the voltage of the solar panel as the voltage of the energy storage offseted by the threshold voltage of the Schottky diode. In this configuration, the voltage of the solar panel follows the voltage of the energy storage. The second configuration is when the solar panel is connected to the energy storage through a buck converter, 
and it is modeled in a two-mode operation. In constant input voltage mode where the voltage of the energy storage is below a certain threshold, the input voltage of the input regulator stays constant and the voltage of the solar panel becomes also constant. In pass-through mode where the voltage of the energy storage is above the threshold, the input voltage of the input regulator becomes equal to the voltage of the energy storage. As the energy storage is being charged or discharged, the voltage of the solar panel follows the voltage of the energy storage.

\section{Modeling Load}

For modeling the power consumption of the load, we have introduced two methods: an average current based model and an application code based model. An average current based model, which is used in our simulator, abstracts the behavior of the load as average current consumption. It is suitable for modeling the long term behavior because most sensornet applications have periodic behavior of sensing and communication, and any surge in power consumption becomes averaged over a long period of time. An application code based model estimates the power consumption of the load over varying time using a source-level simulator and a power consumption look-up table. Since the time scale of this method can be as small as the timer interval of the simulator, it is suitable for modeling a short-term behavior of the load.

\section{Composition}

Using the component models described above, we have composed and simulated several designs of micro-solar power systems varying the following parameters: solar radiation, input regulator type, energy storage type and energy storage capacity. The simulation fits well with the measurement from actual implementations of microsolar power systems, and this shows that our simulator can estimate the behavior of different variations of micro-solar power system quite effectively with sufficient accuracy. 


\section{Chapter 5}

\section{Validating the Simulator Design Using a Reference Implementation}

The simulation becomes meaningful only after it is supported by reality. We have shown its accuracy in controlled settings in the lab. In this chapter, the simulation model of micro-solar power systems developed in the previous chapter is validated in realistic outdoor experiments using a reference implementation. This chapter first describes the reference implementation of a micro-solar power system, the HydroWatch node, in Section 5.1 focusing on its key design ideas. Then, in Section 5.2, it validates the simulation model using energy flow measurements taken from urban neighborhood and forest watershed deployments.

\subsection{Node and Network Design of Reference Imple- mentation}

The HydroWatch Project [Unia] aims to collect widespread, high-frequency, and automated observations of the life cycle of water as it progresses through a forest ecosystem. To gather this data, we aimed to deploy a robust network of lowmaintenance sensor nodes that could collect scientifically-relevant data indefinitely, while withstanding a challenging wet forest environment. This section details the network architecture, node mechanical design, and micro-power solar subsystem present on each node. 


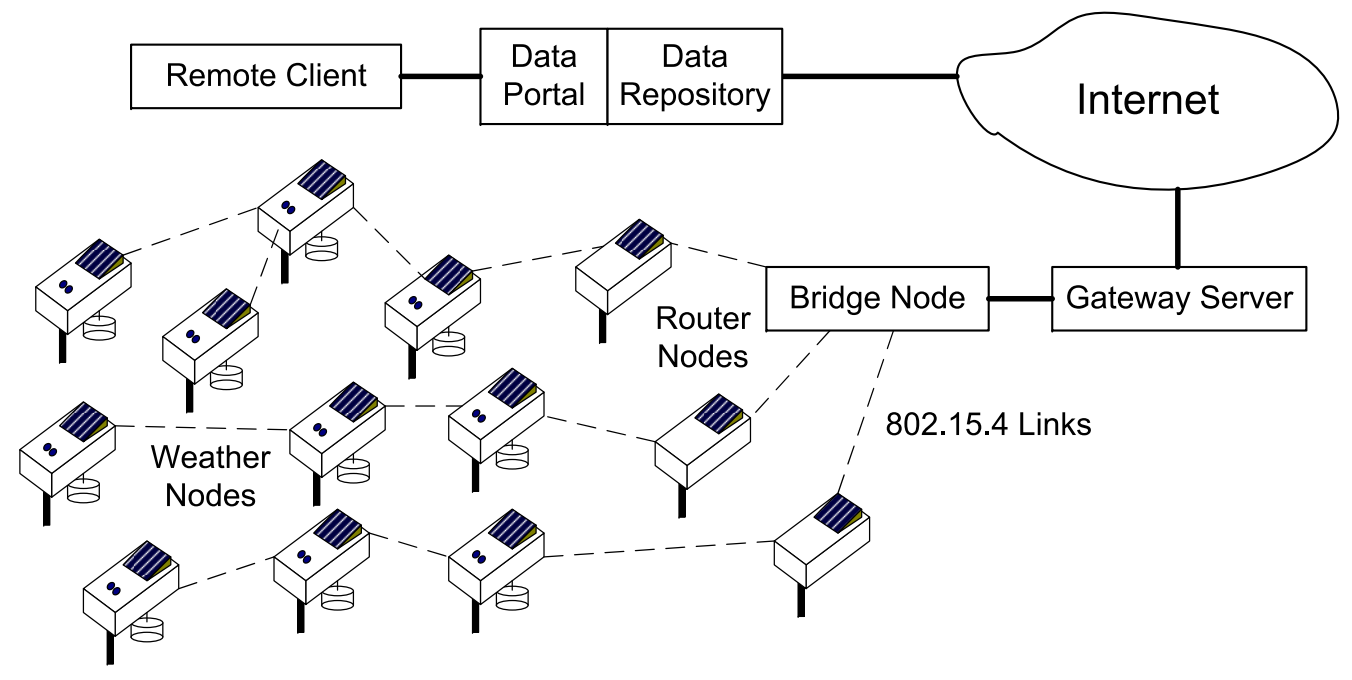

Figure 5.1: System architecture for the HydroWatch micro-climate network.

\subsubsection{Network Architecture}

The sensor network architecture follows the canonical habitat monitoring form described in $\left[\mathrm{SOP}^{+} 04\right]$, but is somewhat of a second-generation sensor network utilizing several commercially available elements. The sensor node is built around the TelosBcompatible Tmote Sky [Sen], and is described in detail below. The mote software, which provides periodic data acquisition, thresholding, power management, remote command processing, and health monitoring, is a modified Primer Pack/IP [Arc] based on TinyOS 2.0. The patch network is an implementation of IPv6 using 6LoWPAN over IEEE 802.15.4 radios [MKHC07]. It utilizes a packet-based form of lowpower listening [PHC04] to minimize idle listening. Data collection is implemented as UDP packets with the routing layer using hop-by-hop retransmissions and dynamic rerouting in a redundant mesh (up to three potential parents) to provide path reliability on lossy links. It utilizes Trickle-based [LPCS04] route updates for topology maintenance. Source-based IPv6 routing is used to communicate directly to specific nodes and dissemination is performed as a series of IPv6 link-local broadcasts.

In the initial HydroWatch deployment at the Angelo Reserve in Northern California, the sensor patch contained 19 nodes over a $220 \mathrm{~m}$ x 260m area stretching across a deep ravine formed by Elder Creek, up the deeply forested north slope of 
the watershed area, and bending to the east to a particular tall stand of Douglas Fir trees.

The transit network between the base-station and the patch was implemented using the same node and link technology as the patch, so there is no specific gateway node in the patch. To provide redundancy in the transit network, multiple microsolar router nodes cover a $120 \mathrm{~m}$ stretch from a shed housing the gateway across an old apple orchard. These nodes are just patch nodes without the environmental sensors. The network depth is 5 hops or greater. The IEEE 802.15.4 bridge node attached to the base-station uses a high-gain $(19 \mathrm{dBi})$ parabolic antenna pointed out through a window in the shed. The back haul network is a WiFi-based IP network with repeaters on peaks and tree tops to reach a $\mathrm{T} 1$ line.

The base-station is a Linux-class gateway server that provides a web services frontend, a PostgreSQL database for information storage and retrieval, and a web-based management console. It is also an IP router, permitting end-to-end connectivity to the patch nodes. The server facilitates such tasks as monitoring overall network health remotely, diagnosing misreporting or missing nodes, and checking the quality of links a node has to its neighbors, a function which proved critically important during the deployment phase.

The initial physical deployment of this architecture is illustrated in Figure 5.2. The base-station appears at the left (west) side of the image. The router nodes form a sparse stretch reaching southeast, and a fairly rich interconnection is provided among the several nodes up the watershed. Node sites were chosen to achieve both vertical (up the hill) and horizontal (across the hill) profiles to enable an initial understanding of the micro-climates in the watershed.

\subsubsection{Engineering the Node}

While the mote platform (micro-controller, radio, and flash), system software, and networking are fairly common across many applications, the sensor suite, power subsystem, and mechanical design of the node tend to be application-specific and highly interrelated.

The sensor suite for this micro-climate monitoring application was essentially developed for tracking weather fronts in Redwoods $\left[\mathrm{TPS}^{+} 05\right]$ and available natively on 


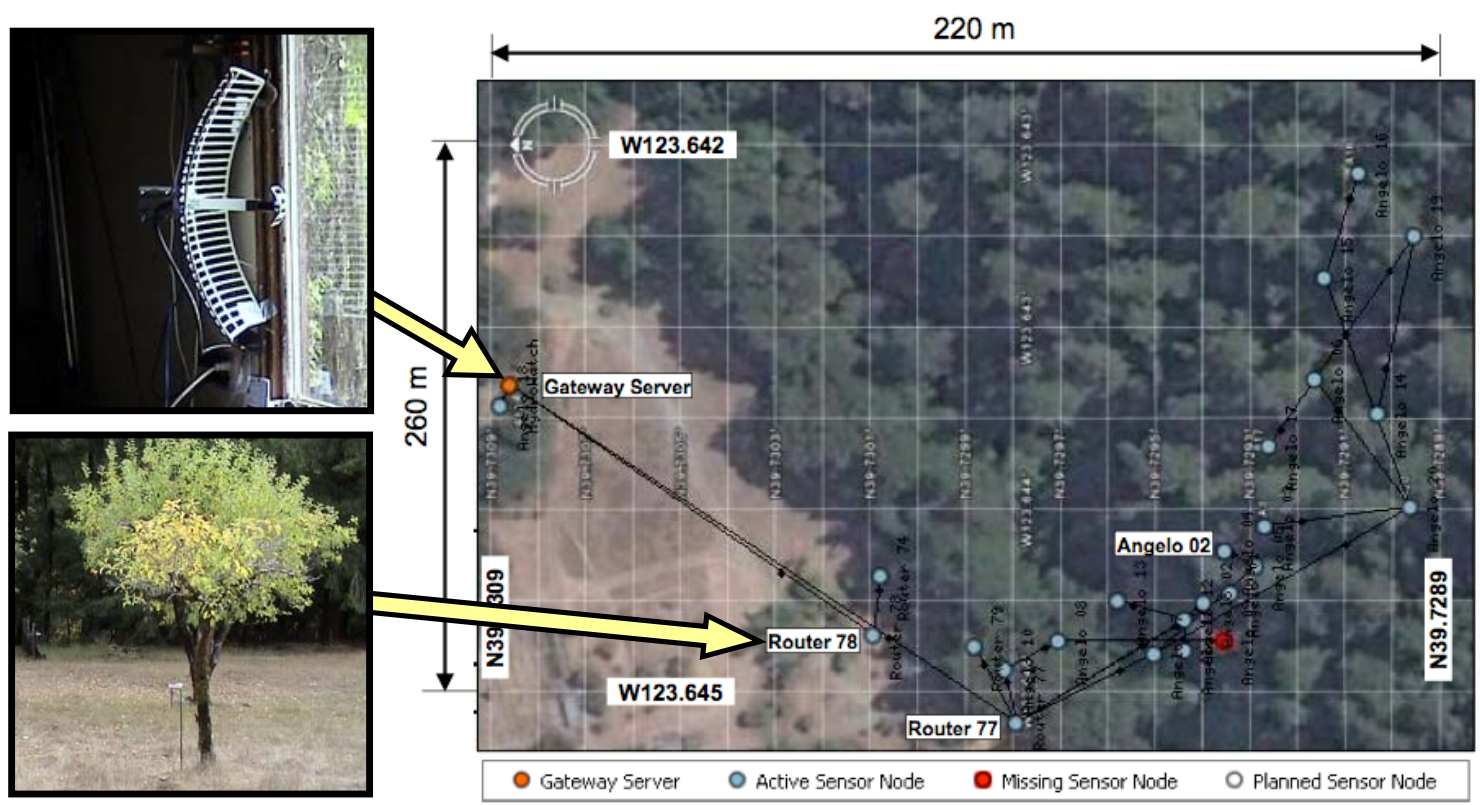

Figure 5.2: Snapshot of the HydroWatch forest watershed deployment.
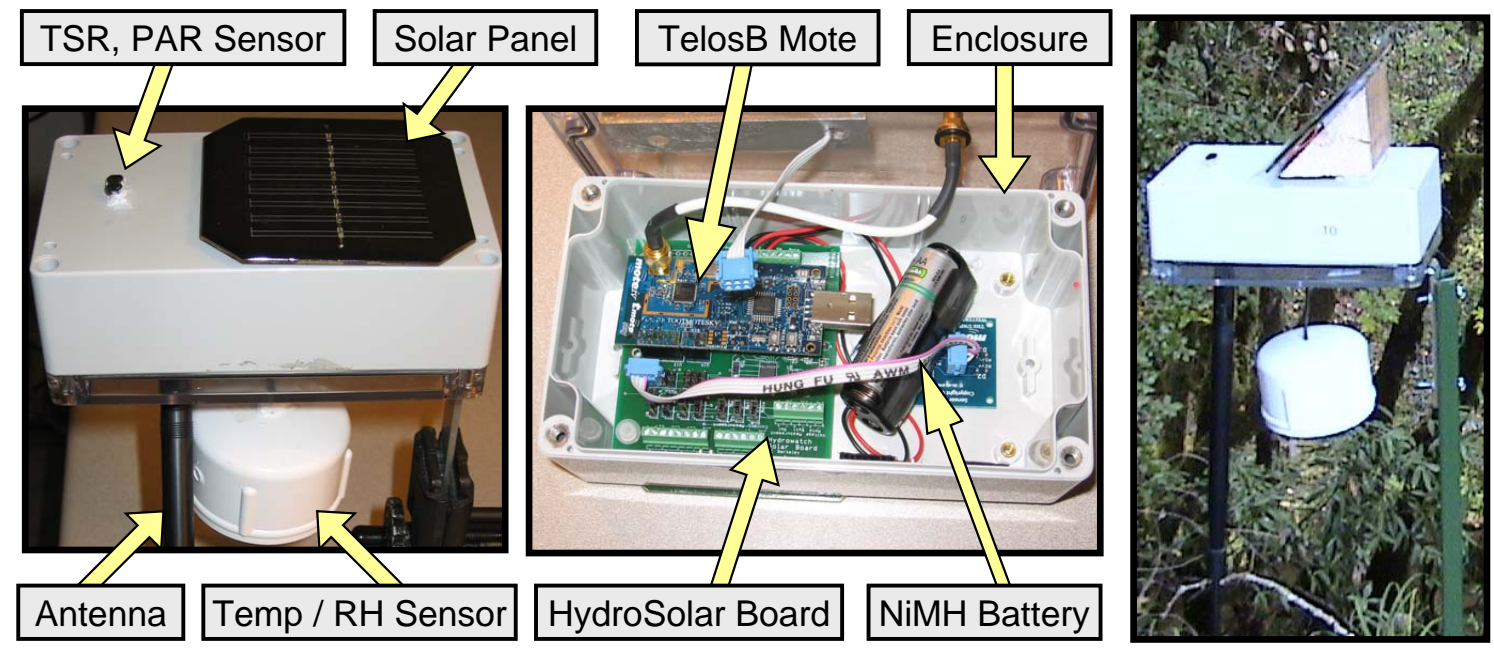

Figure 5.3: HydroWatch weather node. 
the TelosB platform - total solar radiation (TSR), photosynthetically active radiation (PAR), temperature, and relative humidity. However, to provide high-quality data, the sensors must be exposed properly to the environment while the rest of the electronics are protected. We used the TelosB-compatible Tmote Sky mote [PSC05] with an attached SMA connector for an external antenna and no on-board sensors. We connected the mote to external sensor boards using custom cables with IDC connectors, providing a degree of freedom to determine sensor orientation on the node.

The two photo-diodes used to measure incident PAR (Hamamatsu S1087) and TSR (Hamamatsu S1087-01) are tiny discs connected to two long leg-like contacts. Previous experience $\left[\mathrm{TPS}^{+} 05\right]$ recommends that these sensors should be elevated from their surroundings to avoid collecting water on the sensing surface and to obtain unobstructed indications of solar illumination.

A Sensirion SHT15 sensor measures relative humidity $(\mathrm{RH})$ and temperature, factory calibrated to exhibit a maximum $+/-2 \% \mathrm{RH}$ and $+/-0.3$ degrees Celsius error. To accurately measure humidity requires the sensor be exposed to naturallyaspirated air flow, whereas to measure temperature it should be shaded and decoupled from large thermal masses and sources of self-heating. To meet these demands, the SHT15 was placed within a 2-in PVC cap and suspended from the underside of the node. To prevent electrical malfunction, the hole in the top of the PVC cap for the interface wire was sealed and conformal coating was applied to the sensor board. Though we recognize that a hanging design may be prone to connection disturbances caused by curious wildlife, the accuracy of sensor data was worth the risk of a small number of unavailable nodes.

The RF environment was expected to pose a critical challenge for our network, due to interactions of foliage and water vapor with $2.4 \mathrm{GHz}$ radio connectivity. Evident from past deployments $\left[\mathrm{SMP}^{+} 04, \mathrm{LBV}_{06}, \mathrm{WAJR}^{+}\right.$05, ZSLM04,DHJ $\left.{ }^{+} 06\right]$ and more specifically forest deployments [TPS ${ }^{+} 05$, COSKM05], natural environments are capable of eliciting tremendous swings in link quality, causing wireless networks to fail drastically and unexpectedly. To address this issue, we provisioned our nodes with a $7 \mathrm{dBi}$ omnidirectional antenna with a flexible orientation. Nodes were attached to the top of $3 \mathrm{ft}$ and $4 \mathrm{ft}$ metal fence posts by a simple angle bracket.

Overall, the mechanical design of the node sought to provide accurate sensor 
readings over a long duration. To protect the internal electronic components from environmental damage, we limited the number of node features that required holes in the enclosure; every opening in the enclosure is a potential opportunity for a leak. Additionally, packets of indicating desiccant were placed inside each enclosure box, preventing water vapor from damaging interior electronic components.

\subsubsection{Micro-Solar Power Subsystem of HydroWatch Node}

The core of the node design is a flexible power subsystem board that ties together a solar panel, an optional input regulator, a battery, and a switching output regulator - as shown in Figure 5.4. It provides measurement points for a number of electrical parameters that can be connected to the mote ADCs, sampled and recorded along with the environmental measurements. In our configuration, these monitoring features produce time-series logs of solar panel voltage, solar panel current, and battery voltage, in addition to the logs of sensor data from the application and link/neighbor data. All of these are collected and stored by the gateway server, enabling deeper analysis of the performance of the node and network under varying solar conditions. The solar board also provides the mechanical structure that attaches the mote to the enclosure.

The HydroWatch board was designed to permit the study of a variety of power subsystem options. The solar panel and the battery are attached through screw terminals. Headers and mounting holes permit direct attachment of motes of the TelosB form factor, but a mote of any other type can be attached to the board through screw terminals. Additionally, the board has a prototyping area which can be used to change power subsystem configuration. In fact, we were able to change any of the circuit elements originally used in the board schematic by simply changing jumper settings and populating the prototyping area. We used this flexibility to evaluate candidate parts for each component and quantify their contribution to the efficiency of the entire system.

This section provides the rationale and key criteria for selecting specific components as seen through the lens of our experience designing the HydroWatch board. We begin with an analysis of application load - this directly impacts the selection of the other components in the design. The components ultimately selected for the 

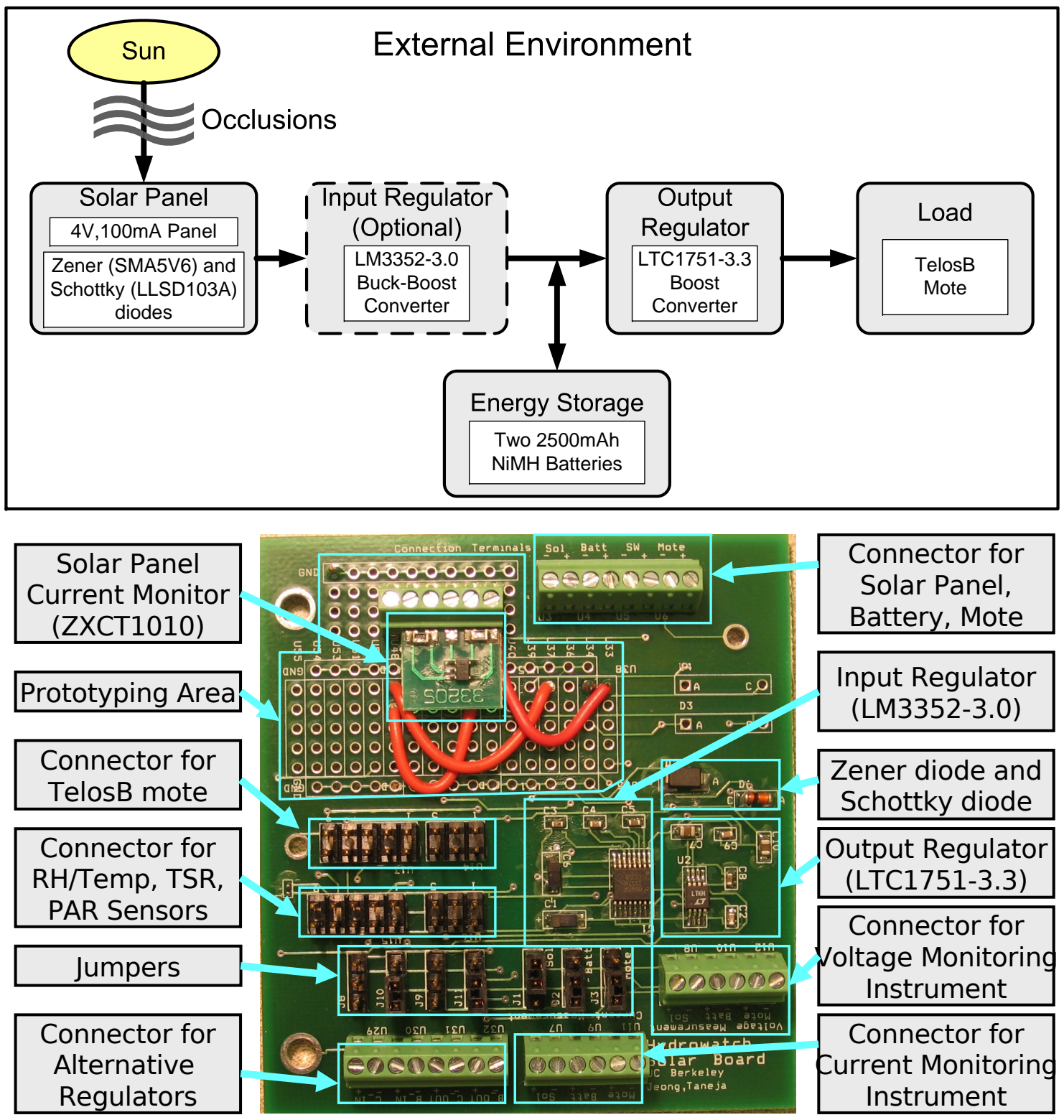

Figure 5.4: HydroWatch micro-solar power subsystem. 
HydroWatch micro-solar board are summarized in Table 5.1.

Table 5.1: Components for the HydroWatch board

\begin{tabular}{|c|c|}
\hline \multicolumn{2}{|c|}{ (a) Solar Panel (Silicon Solar \#16530) } \\
\hline$V_{o c}, I_{s c}$ & $4.23 \mathrm{~V}, 111.16 \mathrm{~mA}$ \\
\hline MPP & $276.0 \mathrm{~mW}$ at $3.11 \mathrm{~V}$ \\
\hline I-V curve & Shown in Figure 5.5 \\
\hline Dimension & $2.3 \mathrm{in} \times 2.3 \mathrm{in}$ \\
\hline Material, Efficiency & Polycrystalline silicon, $13 \%$ \\
\hline \multicolumn{2}{|c|}{ (b) Input Regulator (LM3352-3.0: Optional) } \\
\hline $\begin{array}{l}\text { Manufacturer- } \\
\text { provided efficiency }\end{array}$ & $\begin{array}{l}65 \%-83 \%\left(I_{\text {out }}=5 \mathrm{~mA}-100 \mathrm{~mA}, V_{\text {out }}=3.0 \mathrm{~V}, V_{\text {in }}\right. \\
=2.5 \mathrm{~V}-3 \mathrm{~V})\end{array}$ \\
\hline Measured efficiency & $\begin{array}{l}54.71 \%-65.40 \%\left(I_{\text {solar }}=0 \mathrm{~mA}-100 \mathrm{~mA}, V_{\text {out }}=\right. \\
3.0 \mathrm{~V})\end{array}$ \\
\hline \multicolumn{2}{|r|}{ (c) Energy Storage } \\
\hline Configuration & Two AA NiMH batteries in series \\
\hline Voltage & $2.4 \mathrm{~V}$ nominal, $2.6 \mathrm{~V}-3.0 \mathrm{~V}$ at charge \\
\hline Capacity & $2 \times 1.2 \mathrm{~V} \times 2500 \mathrm{mAh}=6000 \mathrm{mWh}$ \\
\hline \multicolumn{2}{|c|}{ (d) Output Regulator (LTC1751-3.3) } \\
\hline $\begin{array}{l}\text { Manufacturer- } \\
\text { provided efficiency }\end{array}$ & $\begin{array}{l}55 \%-60 \% \quad\left(I_{\text {out }}=0.1 \mathrm{~mA}-20 \mathrm{~mA}, \quad V_{\text {in }}=2.75 \mathrm{~V},\right. \\
\left.V_{\text {out }}=3.3 \mathrm{~V}\right)\end{array}$ \\
\hline Measured efficiency & $\begin{array}{l}49.69 \%-52.15 \% \quad\left(I_{\text {out }}=3 \mathrm{~mA}-6 \mathrm{~mA}, \quad V_{\text {in }}=2.55 \mathrm{~V}-\right. \\
\left.2.71 \mathrm{~V}, V_{\text {out }}=3.3 \mathrm{~V}\right)\end{array}$ \\
\hline \multicolumn{2}{|r|}{ (e) Load } \\
\hline Mote platform & Tmote Sky / TelosB mote \\
\hline Vec & $2.1 \mathrm{~V}-3.6 \mathrm{~V}, 2.7 \mathrm{~V}-3.6 \mathrm{~V}$ with flash \\
\hline Average current & App.-Dependent; 0.53mA for ours \\
\hline Maximum current & $23 \mathrm{~mA}$ with $\mathrm{MCU}$ on, radio $\mathrm{RX}$ \\
\hline
\end{tabular}

\section{Load}

To get a notion of the power requirements of a node, we empirically measured the load created by our application. As is typical of sensor networks for environmental data collection, nodes alternate between a low-power state roughly $99 \%$ of the time and brief higher-power active periods. The gateway server provides estimates of the duty cycle for the MCU $(0.4 \%)$ and the radio $(1.2 \%)$. The peak active current is 23 $\mathrm{mA}$ with the MCU on and the radio in RX mode, the sleep current is around $15 \mathrm{uA}$, and the RMS average current is $0.53 \mathrm{~mA}$. We use our application load requirement 
Table 5.2: Estimated operating time of a node without energy storage recharging

\begin{tabular}{|l|l|}
\hline Type & Lifetime \\
\hline Lead Acid (LC-R061R3P) & 98.5 days $\left(=\frac{7800 \mathrm{mWh}}{79.2 \mathrm{Wh} / \text { day }}\right)$ \\
Two NiCd (KR-1100AAU) & 33.3 days $\left(=\frac{2 \times 1320 \mathrm{mWh}}{79.2 \mathrm{mWh} / \text { day }}\right)$ \\
Two NiMH (NH15-2500) & 75.8 days $\left(=\frac{2 \times 3000 \mathrm{mWh}}{79.2 \mathrm{mWh} / \text { day }}\right)$ \\
Li-ion (UBP053048) & 35.4 days $\left(=\frac{2800 \mathrm{mWh}}{79.2 \mathrm{mWh} / \text { day }}\right)$ \\
Li-polymer (UBC433475) & 42.9 days $\left(=\frac{3400 \mathrm{mWh}}{79.2 \mathrm{mWh} / \text { day }}\right)$ \\
Supercap (BCAP0350) & 3.8 days $\left(=\frac{304 m \mathrm{mh}}{79.2 \mathrm{mWh} / \text { day }}\right)$ \\
\hline
\end{tabular}

to guide our selection of the rest of the components.

\section{Energy Storage}

Table 2.1 in Chapter 2.2.1 lists a number of possible rechargeable energy storage options that can be used for micro-solar power systems. We considered a number of characteristics including capacity, operating range, energy density and charging method.

Employing the measured average consumption of our application of $0.53 \mathrm{~mA}$ at $3.3 \mathrm{~V}$ and the efficiency of the output regulator estimated at $50 \%$, the daily energy requirement from the energy storage element is $79.2 \mathrm{mWh}$. This energy requirement drives the storage selection process. First, we compared each type of storage based on capacity in Table 5.2. All options except the supercapacitor can provide energy for more than 30 days of operation without recharging, long enough to operate for a number of days in the absence of solar radiation.

For our application, even with loose physical sizing constraints, lead-acid batteries are not plausible because of low energy density. NiCd batteries have a similar footprint and charging method as NiMH batteries, but with a much smaller capacity. Additionally, NiCd chemistries are less environmentally-friendly and far more susceptible to the memory effect, which can significantly reduce battery capacity over time.

For the decision between Lithium-based chemistries and NiMH, we drew on previous experience from the Trio deployment $\left[\mathrm{DHJ}^{+} 06\right]$. Our desire to avoid having software in the charging loop (ultimately to allow nodes to simply charge when placed in the sun entirely independent of their software state), coupled with the complexity of 
integrating a hardware Li-ion charger, dictated the selection of $\mathrm{NiMH}$, as it operates with more straightforward charging logic. This choice does present some drawbacks, however. This chemistry suffers from a self-discharge rate of $30 \%$ per month and an input-output efficiency of roughly $66 \%$, both worse than for any other battery chemistry considered. The practical implication of this is that for every 3 units of energy that are input to a battery, only 2 units of energy are output. We felt this cost was overcome by the simplicity of the charging logic.

A 2-cell configuration would enable the potential to operate without an input regulator; this choice is further discussed later in this section. For increased capacity, it would be possible to put 2-cell packs in parallel. Additionally, since the discharge curve of NiMH batteries is relatively flat, most of the discharge cycle produces a near-constant voltage.

\section{Solar Panel}

In selecting an appropriate panel for a micro-solar subsystem, the critical factors are the panel's IV curve (specifically, the MPP), its cell composition, and its physical dimensions. Care should be taken in selecting a panel that will operate near its MPP given the load it is expected to support, be it a combination of an input regulator and energy storage or energy storage alone. The cell composition - that is, how many cells are present and their serial/parallel arrangement - becomes a factor when the solar panel is partially occluded. Last, the physical dimensions of the panel should be compatible for the choice of enclosure.

For the HydroWatch power subsystem, we selected a $4 \mathrm{~V}-100 \mathrm{~mA}$ panel from Silicon Solar Inc., whose characteristics are summarized in Table 5.1(a) and whose IV and PV curves are in Figure 5.5. The MPP of this panel occurs at 3.11V, which makes it appropriate for charging $2 \mathrm{NiMH}$ cells directly. Additionally, using our rule of thumb of 30 minutes of sunlight per day, the solar energy generated by this panel at its MPP is $139 \mathrm{mWh}$, satisfying the $120 \mathrm{mWh}(=79.2 \mathrm{mWh} / 66 \%$ NiMH charge-discharge efficiency) per day requirement of our application. 

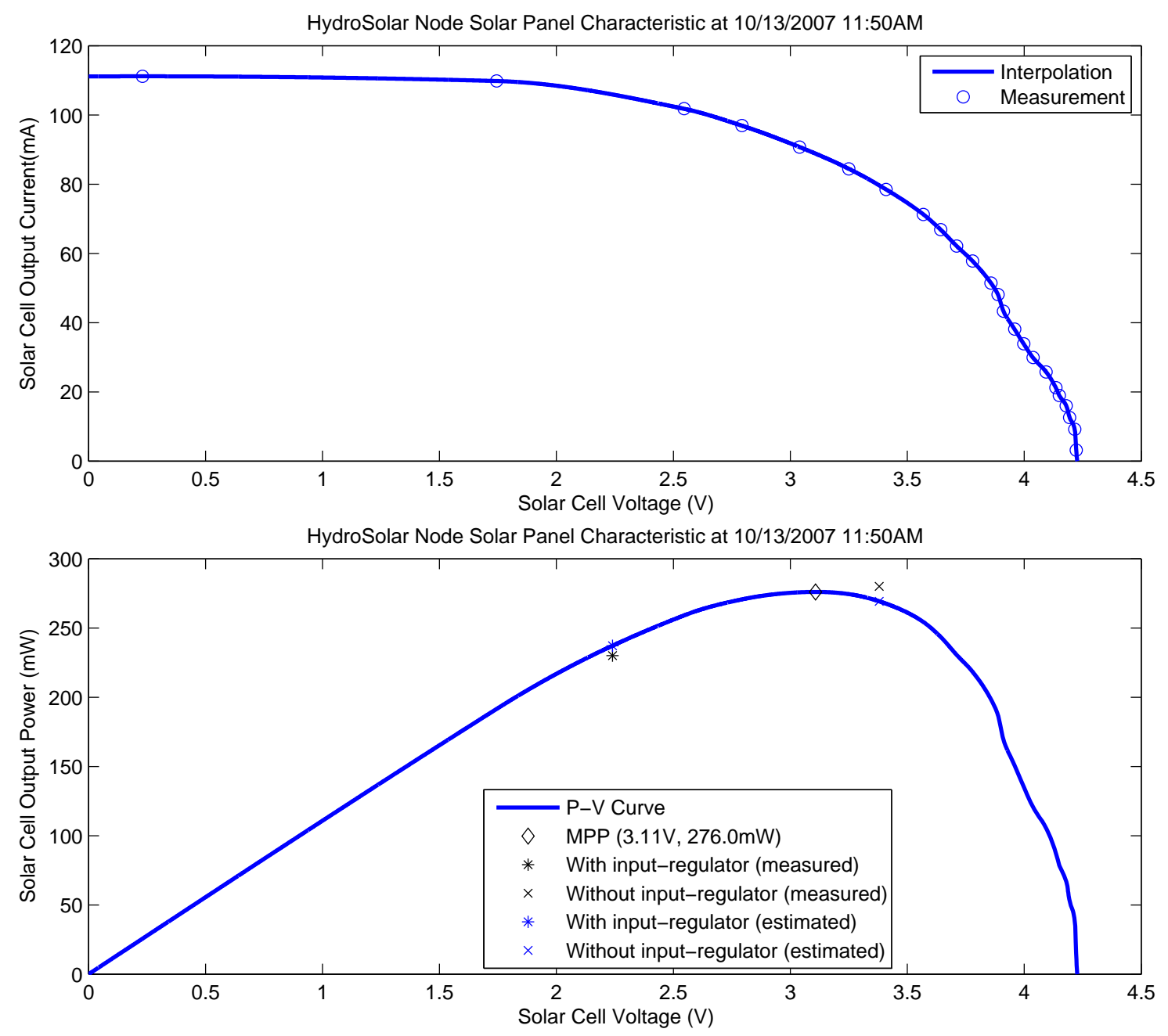

Figure 5.5: Current-Voltage and Power-Voltage performance of the Silicon Solar 4V$100 \mathrm{~mA}$ solar panel. 


\section{Input Regulator}

In selecting the input regulator, the important parameters are the operating range of the solar panel and batteries and the method and logic used to charge the battery. In our design, we chose to trickle charge the batteries because it requires only a simple circuit and no software control. For trickle charging, the solar panel and the battery should be sized to meet the following condition

$$
I_{\text {max }- \text { solar }} \leq 0.1 C / \text { hour }
$$

where $\mathrm{C}$ is the nominal capacity of the battery. The HydroWatch node has a solar panel of peak current $100 \mathrm{~mA}$ and NiMH batteries of capacity $2500 \mathrm{mAh}$, and this gives $I_{\text {max }- \text { solar }}=100 \mathrm{~mA}$ and $0.1 \mathrm{C} /$ hour $=250 \mathrm{~mA}$. Thus, the design of the HydroWatch node meets the trickle charging condition.

In our initial design of the HydroWatch board, we used an input regulator to limit the voltage to the battery. However, we observed that the existence of the input regulator forced the solar panel to operate at a point far from its MPP. Not using the input regulator results in significantly more energy harvested from the solar panel, because the input impedance of the regulator is less than that of the battery - see the bottom graph of Figure 5.5. In addition to this increase, energy is no longer consumed by the input regulator, which empirically has about a $60 \%$ efficiency factor. This substantial gain in total system energy as well as efficiency led us to remove the input regulator from our design; removing the input regulator is only an option because the operating voltage of the solar panel matches the charging voltage of the batteries.

\section{Output Regulator}

The key criteria for choosing an output regulator are the operating ranges of the batteries and the load, as well as the efficiency of the regulator over the range of the load. With our choice of 2 NiMH AA batteries, the nominal voltage of the energy storage is $2.4 \mathrm{~V}$ so a boost converter is required to match the $2.7-3.6 \mathrm{~V}$ operating range of TelosB motes (Table 5.1(e)). The output regulator also has the important responsibility to provide a stable supply voltage to ensure the fidelity of sensor data. Though DC-DC converters introduce high-frequency noise from the switching process into the output signal, the amplitude of the noise does not negatively affect the sensor 
readings. If noise were a critical factor, either a low-pass filter or a higher voltage energy supply in combination with a linear drop out (LDO) regulator could be used instead. We chose the LTC1751 regulator [Tec], which had an efficiency of around $50 \%$. It requires very few discrete parts and has low, constant switching noise.

\subsubsection{Modeling the HydroWatch node for the Simulator}

Based on the methodology described in Chapter 4, we modeled the operation of the HydroWatch node as follows:

- External Environment: As for the external environment, we considered three models: the astronomical model, scaled astronomical model and obstructed astronomical model.

- Solar Collector: We used a 4V-100mA polycrystalline solar panel. We modeled the solar panel using a generic form of I-V characteristic:

$$
I=I_{s c}-A \cdot(\exp (B \cdot V)-1)
$$

where $I_{s c}$ is the short circuit current of the solar panel and $A$ and $B$ are the panel-specific constants. Using the curve-fitting method discussed in Section 4.3.1, we have determined these constants as $I_{s c}=111.160, A=0.2526$ and $B=1.4255$.

- Input Regulator: The HydroWatch node has no input regulator. The solar panel voltage $V_{\text {sol }}$ is determined by the voltage level of the energy storage $V_{\text {stor }}$ and the threshold voltage of the diode $V_{t h-s c h o t t k y}$ with the following equation:

$$
V_{\text {sol }}=V_{\text {stor }}+V_{\text {th-schottky }}
$$

The threshold voltage of the diode $V_{t h-s c h o t t k y}$ is modeled as a single value $0.45 \mathrm{~V}$, which is taken as an average for a number of possible current draws for the diode.

- Energy Storage: A HydroWatch node uses two 2500mAh NiMH battery as its energy storage. In order to model the capacity of a NiMH battery, we used a battery capacity-to-voltage graph taken from the manufacturer provided data (Figure 4.17). 


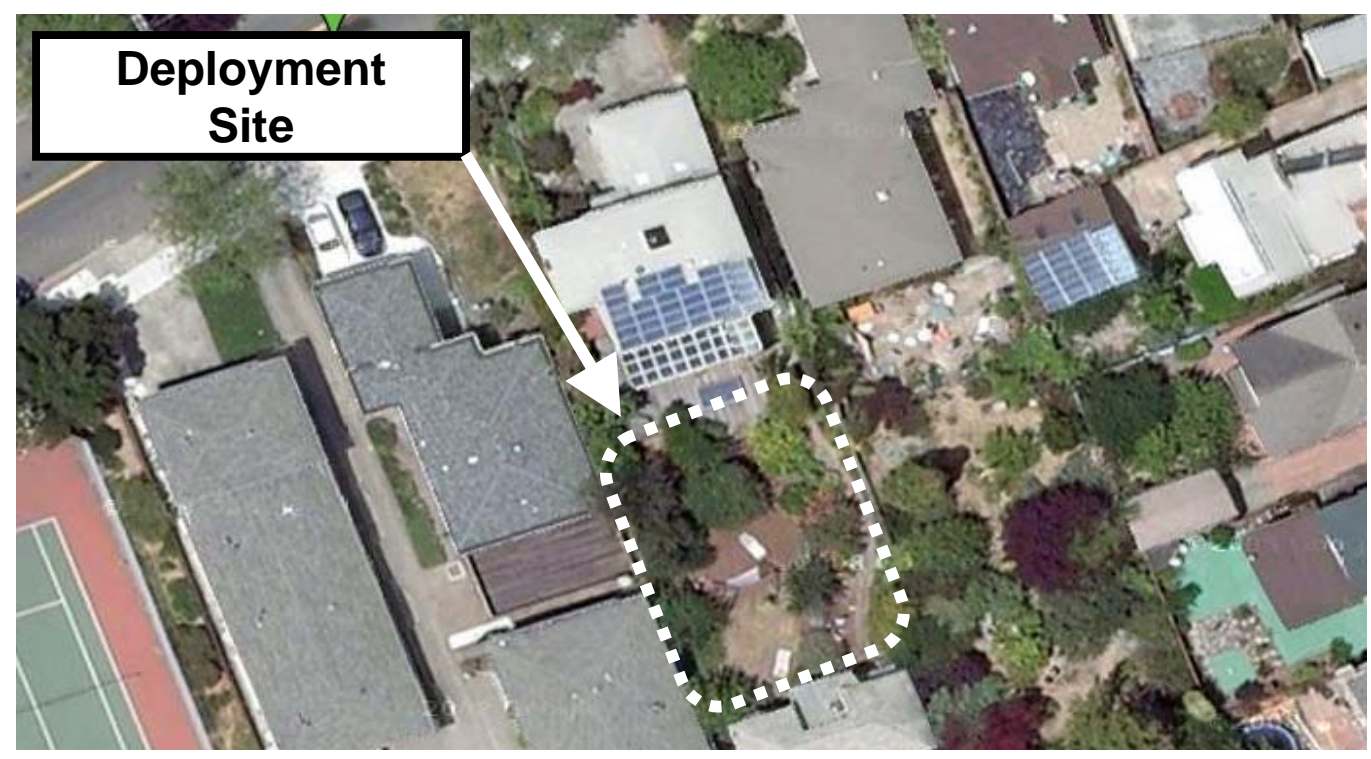

Figure 5.6: Urban neighborhood deployment site in Berkeley, CA

- Output Regulator: A HydroWatch node uses an LTC1751 regulator as its output regulator, and we have modeled its characteristics for output voltage and power efficiency based on the manufacturer provided data. We modeled it as a function of input voltage (output voltage - Figure 4.22) or a function of input voltage and load current (power efficiency - Figure 4.23).

- Load: We modeled the load with an average current consumption rate of $0.53 \mathrm{~mA}$.

\subsection{Evaluating the Reference Implementation}

In this section, we validate the operation of the simulator using the deployment data of two test networks of HydroWatch nodes.

\subsubsection{A Sensor Network in an Urban Neighborhood}

The purpose of our first deployment was to confirm that nodes could sense, charge, and operate continuously for a period of days, as well as assess whether the model we developed accurately estimated the generation and consumption of energy in a 


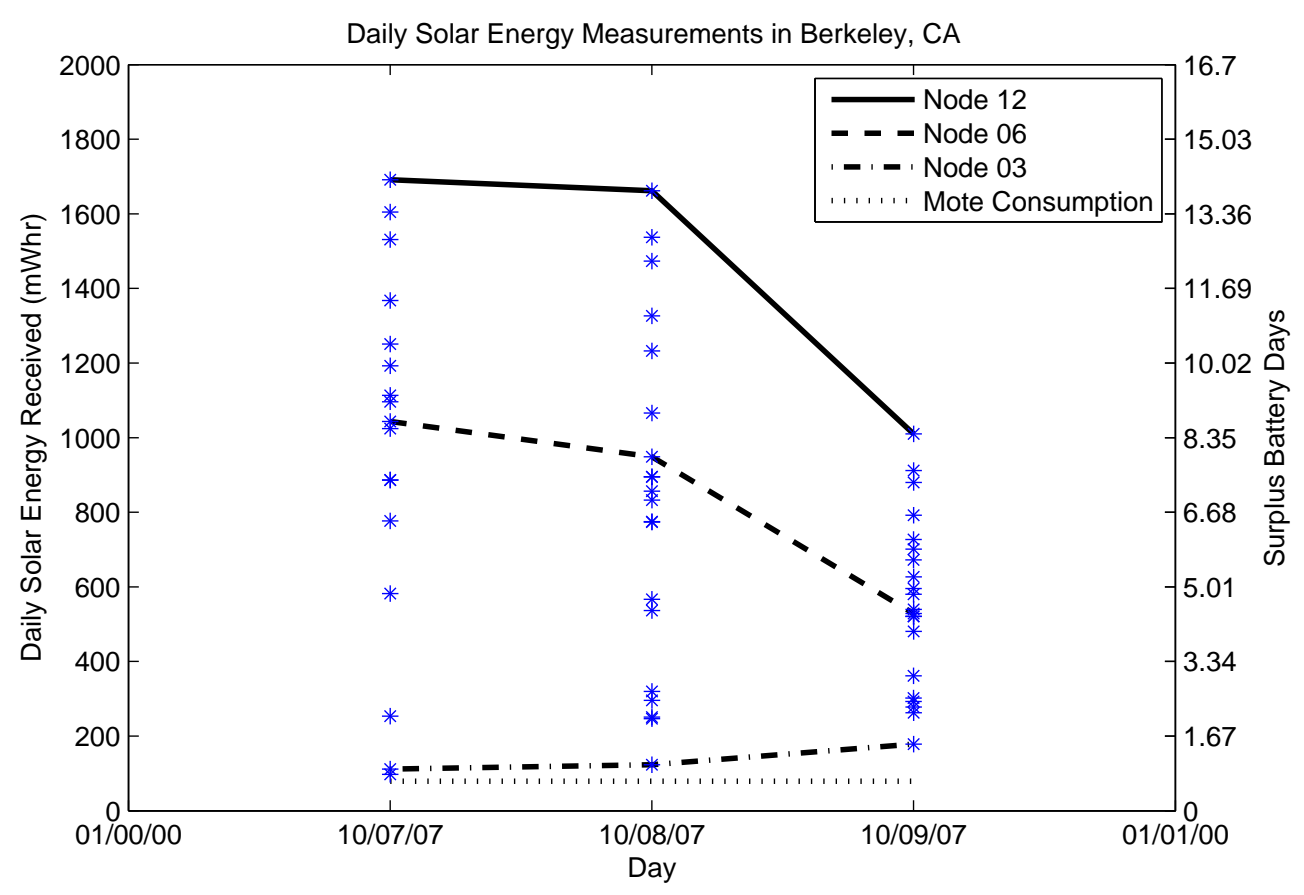

Figure 5.7: Scatter plot of solar energy received in the urban neighborhood deployment. Three representative nodes are highlighted.

variety of solar conditions. We deployed 22 nodes in an urban neighborhood in Berkeley; nodes were placed in varied locations, including on a house gutter, in and under trees, among shrubbery, and in a grassy yard. To emulate the situation in the forest watershed, we placed them in the vicinity of significant obstructions and varied the orientation of the solar panels: some were flat while others faced south, east, and west at a 45 degree inclination.

\section{Overall Trend}

The range of daily solar energy via $P_{\text {sol }}$ by each node over a period of three days can be seen in Figure 5.7. The lines on the graph show the behavior of the node that received the highest (Node 12), median (Node 06), and lowest (Node 03) amount of solar energy. The fourth line on the graph shows a constant $79.2 \mathrm{mWh}$ break-even point. The first day (10/07/2007) was a fairly sunny day, resulting in the widest distribution of received solar energy (roughly 100-1700 mWh). However, as the days 
Table 5.3: Daily average of the solar panel output power for different estimation models and the deployment in an urban neighborhood

(a) Node with highest solar radiation (Node 12)

\begin{tabular}{|l|l|l|l|l|}
\hline & $\begin{array}{l}\text { Astronomical } \\
\text { Model }\end{array}$ & $\begin{array}{l}\text { Scaled Astro } \\
\text { Model }\end{array}$ & $\begin{array}{l}\text { Obstructed } \\
\text { Astro Model }\end{array}$ & Measurement \\
\hline On a sunny day & $1691.2 \mathrm{~mW}$ & $2217.6 \mathrm{~mW}$ & $1721.8 \mathrm{~mW}$ & $1649.7 \mathrm{~mW}$ \\
$(10 / 8 / 2007)$ & $(2.4 \%)$ & $(25.6 \%)$ & $(4.2 \%)$ & \\
\hline On an overcast day & $1691.2 \mathrm{~mW}$ & $2217.6 \mathrm{~mW}$ & $1721.7 \mathrm{~mW}$ & $1034.5 \mathrm{~mW}$ \\
$(10 / 9 / 2007)$ & $(38.8 \%)$ & $(53.4 \%)$ & $(39.9 \%)$ & \\
\hline
\end{tabular}

(b) Node with median solar radiation (Node 06)

\begin{tabular}{|l|l|l|l|l|}
\hline & $\begin{array}{l}\text { Astronomical } \\
\text { Model }\end{array}$ & $\begin{array}{l}\text { Scaled Astro } \\
\text { Model }\end{array}$ & $\begin{array}{l}\text { Obstructed } \\
\text { Astro Model }\end{array}$ & Measurement \\
\hline $\begin{array}{l}\text { On a sunny day } \\
(10 / 8 / 2007)\end{array}$ & $\begin{array}{l}1691.2 \mathrm{~mW} \\
(44.1 \%)\end{array}$ & $\begin{array}{l}2079.1 \mathrm{~mW} \\
(54.5 \%)\end{array}$ & $\begin{array}{l}1137.7 \mathrm{~mW} \\
(16.8 \%)\end{array}$ & $946.1 \mathrm{~mW}$ \\
\hline On an overcast day & $1691.2 \mathrm{~mW}$ & $2079.1 \mathrm{~mW}$ & $1137.7 \mathrm{~mW}$ & $561.9 \mathrm{~mW}$ \\
$(10 / 9 / 2007)$ & $(66.8 \%)$ & $(73.0 \%)$ & $(50.6 \%)$ & \\
\hline
\end{tabular}

(c) Node with lowest solar radiation (Node 03)

\begin{tabular}{|l|l|l|l|l|}
\hline & $\begin{array}{l}\text { Astronomical } \\
\text { Model }\end{array}$ & $\begin{array}{l}\text { Scaled Astro } \\
\text { Model }\end{array}$ & $\begin{array}{l}\text { Obstructed } \\
\text { Astro Model }\end{array}$ & Measurement \\
\hline On a sunny day & $\begin{array}{l}1691.2 \mathrm{~mW} \\
(10 / 8 / 2007)\end{array}$ & $\begin{array}{l}235.0 \mathrm{~mW} \\
(92.5 \%)\end{array}$ & $\begin{array}{l}127.0 \mathrm{~mW} \\
(0.1 \%)\end{array}$ & $126.9 \mathrm{~mW}$ \\
\hline On an overcast day & $1691.2 \mathrm{~mW}$ & $235.0 \mathrm{~mW}$ & $127.1 \mathrm{~mW}$ & $173.7 \mathrm{~mW}$ \\
$(10 / 9 / 2007)$ & $(89.7 \%)$ & $(26.1 \%)$ & $(36.6 \%)$ & \\
\hline
\end{tabular}

became cloudier, the variance of the distribution lessened; nodes at the high end of the distribution received slightly more than half the solar energy when cloudy compared to a sunny day. Interestingly, nodes on the lower end of the distribution received more solar energy on cloudier days; this is presumably because the diffusion of light caused by the layer of clouds scatters the light source and enhances the opportunity of the normally-occluded solar panel to harvest solar energy $!_{1}^{1}$ Nonetheless, every node harvests a surplus of energy on both sunny and cloudy days; the number of surplus battery days this energy creates is also in Figure 5.7. Surplus battery days are calculated by multiplying the surplus of energy flowing into the battery by the charge-discharge efficiency $(66 \%)$ and dividing by the daily consumption $(79.2 \mathrm{mWh})$. 


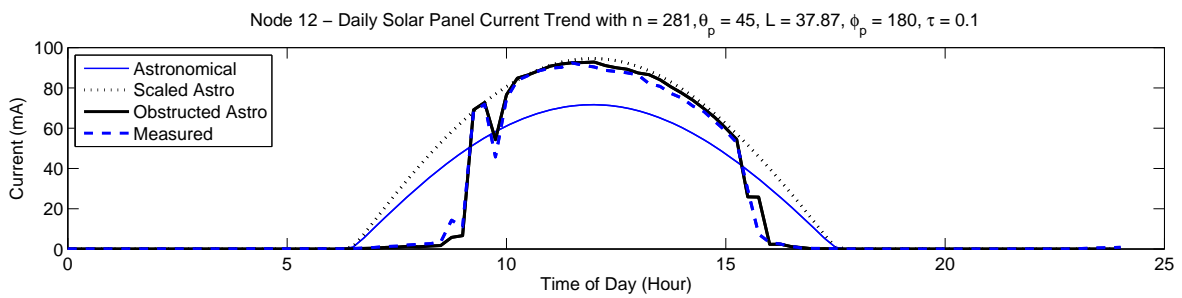

Node 12 - Daily Solar Panel Voltage Trend with $n=281, \theta_{p}=45, L=37.87, \phi_{p}=180, \tau=0.1$

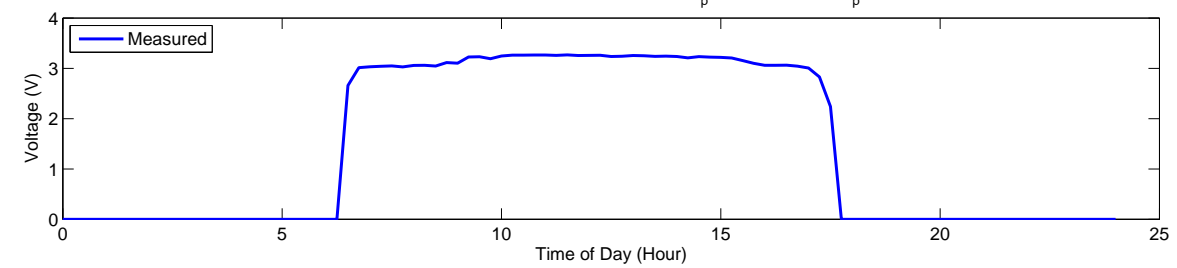

Node 06 - Daily Solar Panel Current Trend with $n=281, \theta_{p}=45, L=37.87, \phi_{p}=180, \tau=0.1$

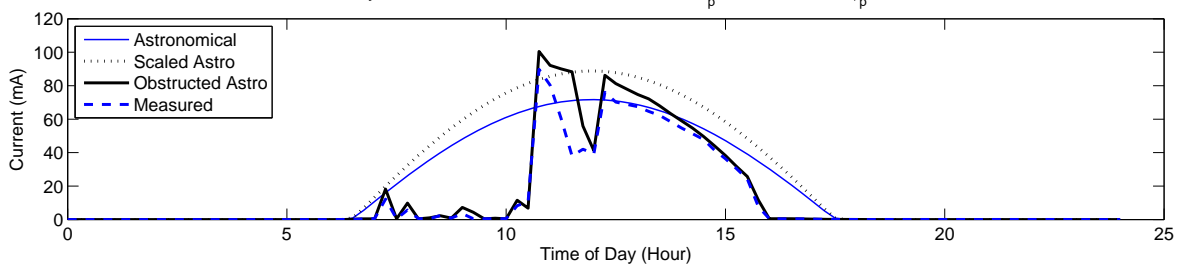

Node 06 - Daily Solar Panel Voltage Trend with $n=281, \theta_{p}=45, L=37.87, \phi_{p}=180, \tau=0.1$

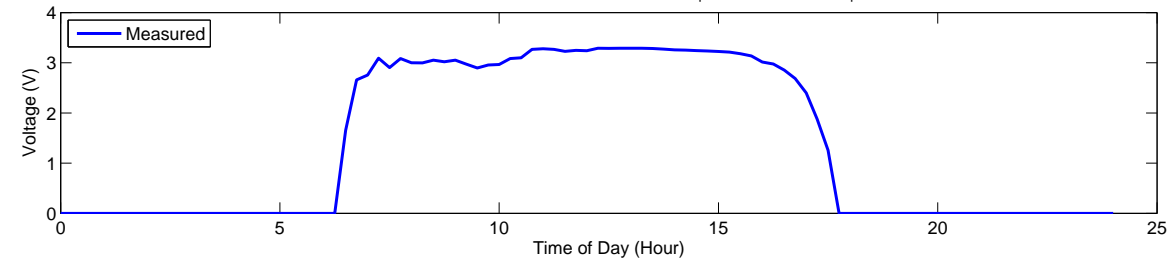

Node 03 - Daily Solar Panel Current Trend with $n=281, \theta_{p}=45, L=37.87, \phi_{p}=180, \tau=0.1$

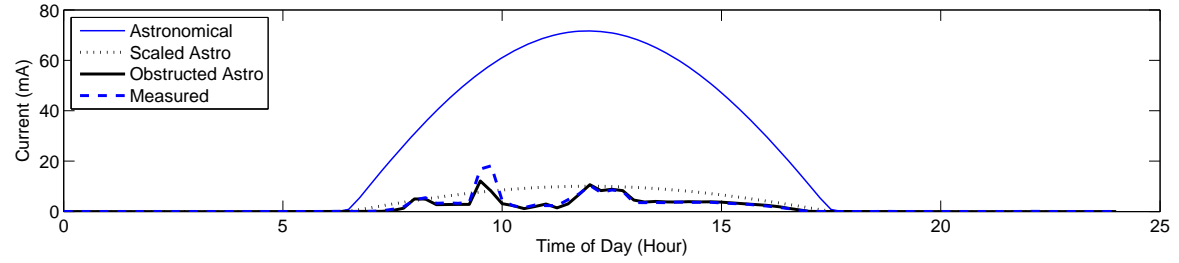

Node 03 - Daily Solar Panel Voltage Trend with $n=281, \theta_{p}=45, L=37.87, \phi_{p}=180, \tau=0.1$

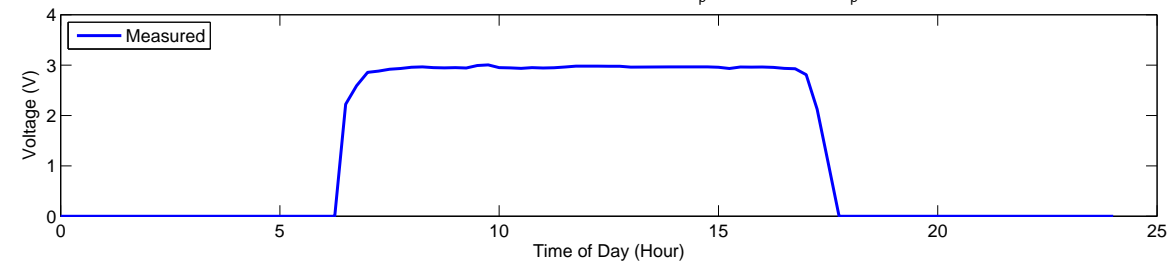

Figure 5.8: Comparison of solar panel output current and voltage on a sunny day $(10 / 8 / 2007)$ for best, worst and middle mode in the urban neighborhood deployment. Notice the differences in scale of the graphs. 

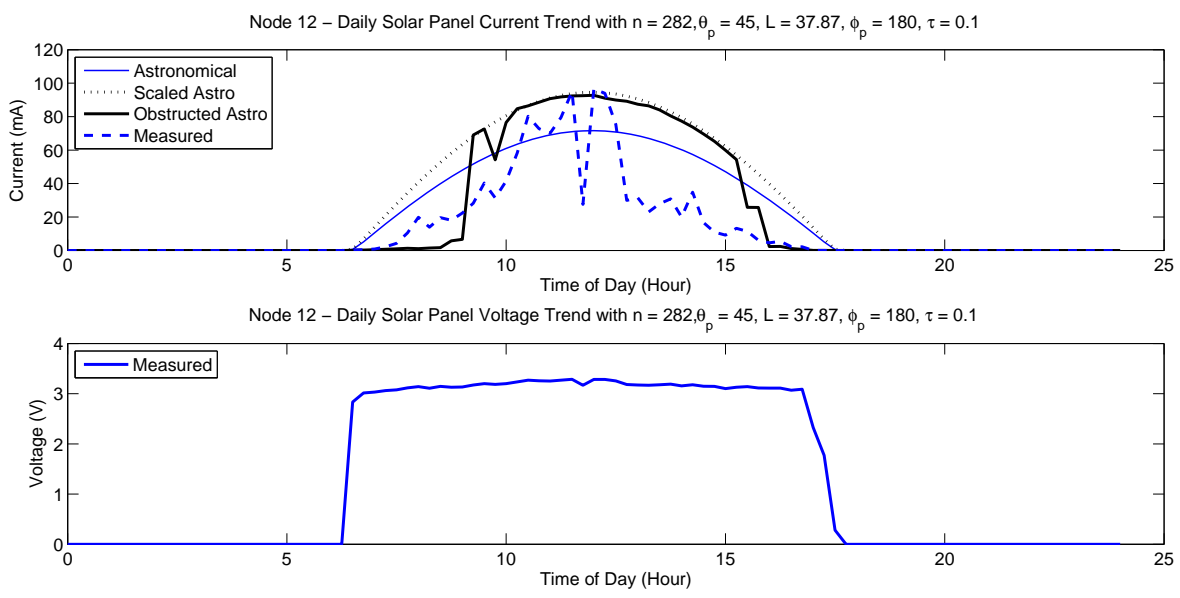

Node 06 - Daily Solar Panel Current Trend with $n=282, \theta_{p}=45, L=37.87, \phi_{p}=180, \tau=0.1$

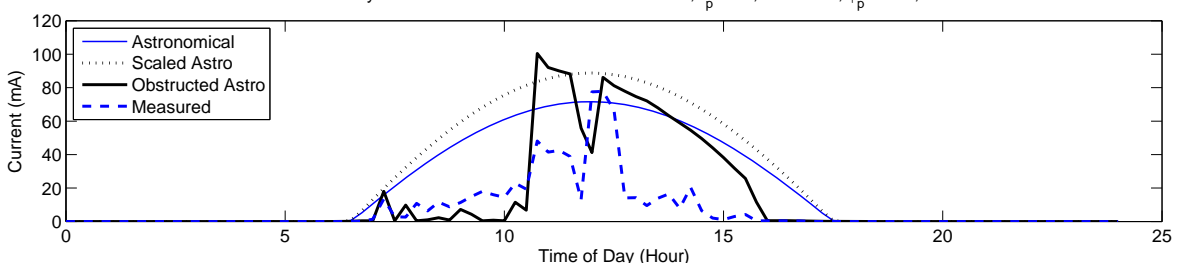

Node 06 - Daily Solar Panel Voltage Trend with $n=282, \theta_{p}=45, L=37.87, \phi_{p}=180, \tau=0.1$
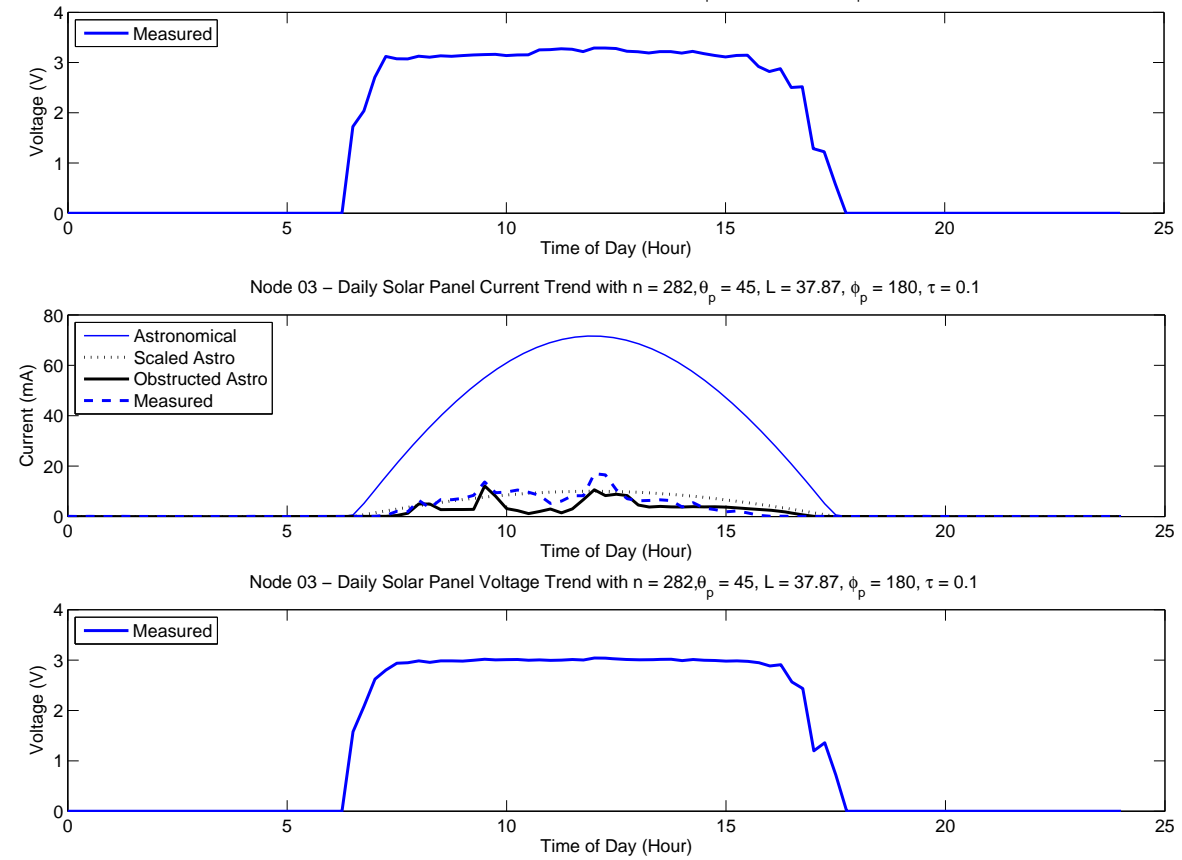

Figure 5.9: Comparison of solar panel output current and voltage on an overcast day (10/9/2007) for the urban neighborhood deployment. 


\section{Comparison with Estimation Models}

Looking at the daily graph of solar current and voltage experienced at each of the three representative nodes on a sunny day - shown in Figure 5.8 - we can see the variations in available solar energy inputs among nodes throughout a day. Nodes that generated very little solar energy still had a solar panel voltage above 3 volts for the light portion of the day. This voltage is limited by the load - in this case, the batteries. Thus, the solar voltage exhibits near binary behavior between 0 volts when there is no incident light and its maximum voltage (as dictated by its load) any time between dawn and dusk.

Additionally, these current graphs are plotted alongside the three estimation models (astronomical, scaled astronomical and obstructed astronomical) as a basis for comparison. The measurement of the solar profile fits the astronomical model when the solar panel has an unobstructed view of sunlight for a certain period of time. For other cases, whether the solar panel is obstructed during part of the day (Node 12 and Node 06) or the whole day (Node 03), the estimation from the astronomical model deviates far from the measurement. The obstructed astronomical model captures the shading effect and gives a better fitting to the measurement than the astronomical model. On an overcast day, the gap between the measurement and the estimation of each model becomes higher, because the estimation models capture time variation and obstruction effects, but not the weather effects. Table 5.3 summarizes the solar panel output measurement and estimations for both a sunny day (10/8/2007) and an overcast day (10/9/2007). We can see that the astronomical model has higher estimation error, as the node is obstructed for longer hours, thus the obstruction model fits the measurement on any obstruction conditions.

We can also notice that the sporadic pattern of the solar energy received throughout the day has implications for the daily power cycle introduced in Figure 3.7 as well; the progression through the daily model may instead oscillate among the recharge, saturation, and discharge phases during the daylight hours.

The urban neighborhood deployment demonstrated that even nodes with severe arboreal and other occlusions received enough sunlight to sustain operation; that is,

\footnotetext{
${ }^{1}$ This effect is most pronounced in this figure (the solar energy doubles on a cloudy day for Node 03), but appeared in other observations as well.
} 
the nodes in the most shade still received at least 30 minutes of sunlight on both sunny and cloudy days validating the prediction of our model and making us (falsely) confident that our design would succeed in the forest watershed.

\subsubsection{A Sensor Network in a Forest Watershed}

\section{Overall Trend}

The blend of solar profiles seen by the nodes in the forest watershed was far less diverse than the urban neighborhood as shown in Figure 5.10. Most of the nodes received no more than $50 \mathrm{mWh}$ of energy on any of the days of the deployment. Just as in Figure 5.7, the lines represent nodes chosen to show the range of the solar distribution. However, in Figure 5.10, the middle line represents the second-best performing node (not the median), and the lowest line is for a node representative of those that are receiving very limited energy. The stunning difference between the two deployments is how much less solar energy was harvested in the forest watershed - the best-performing node on a sunny day in the forest did not receive as much solar energy as the median node on a cloudy day in the urban neighborhood. Additionally, Angelo 02 (and other sun-starved nodes like it) harvested less than the node consumption each day. This daily energy deficit results in a negative number of surplus battery days. It is important to note that these nodes are experiencing different degrees of sun starvation - some are only consuming about half a day's worth of battery energy daily, while others are consuming a full day's worth of energy daily. Still, a majority of the nodes were not receiving sufficient solar energy to operate sustainably, causing a finite lifetime for the network.

\section{Comparison with Estimation Models}

Figure $5.11,5.12$ and 5.13 show the trends of solar radiation and battery capacity for the three representative nodes. In this forest deployment, most nodes are heav-

ily occluded and the astronomical model, which assumes unobstructed view of the sky, does not effectively estimate the solar radiation, with estimation error $80.9 \%$ to 99.9\% . The obstructed astronomical model fits the measurement within around $30 \%$ of error for nodes with high radiation condition (Router 78) and medium radiation 


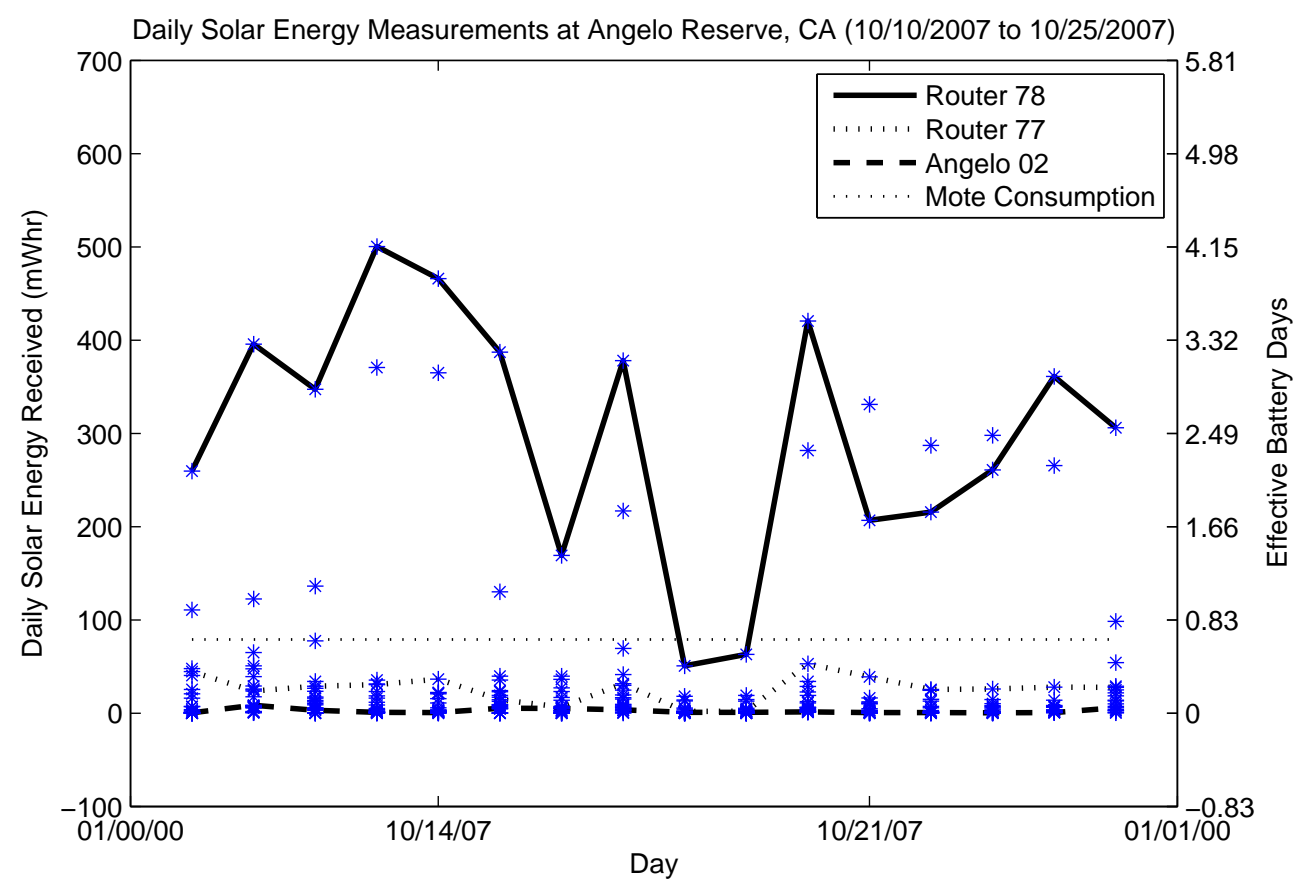

Figure 5.10: Scatter plot of solar energy received in the forest watershed deployment. Three representative nodes are highlighted. 


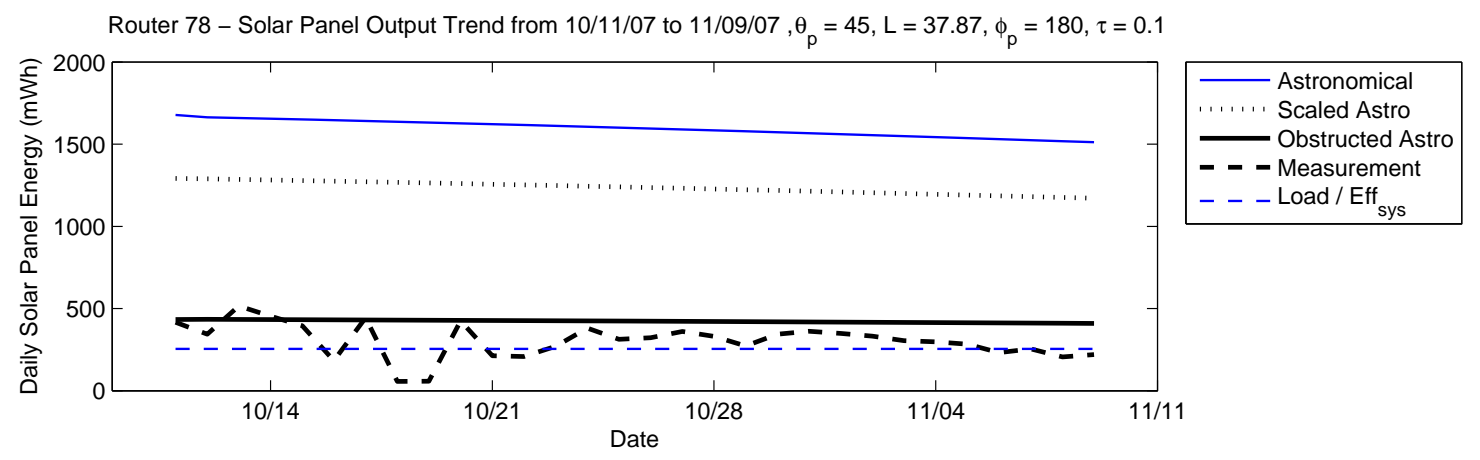

Router 78 - Solar Panel Output Trend (Enlarged) from 10/11/07 to 11/09/07, $\theta_{p}=45, L=37.87, \phi_{p}=180, \tau=0.1$

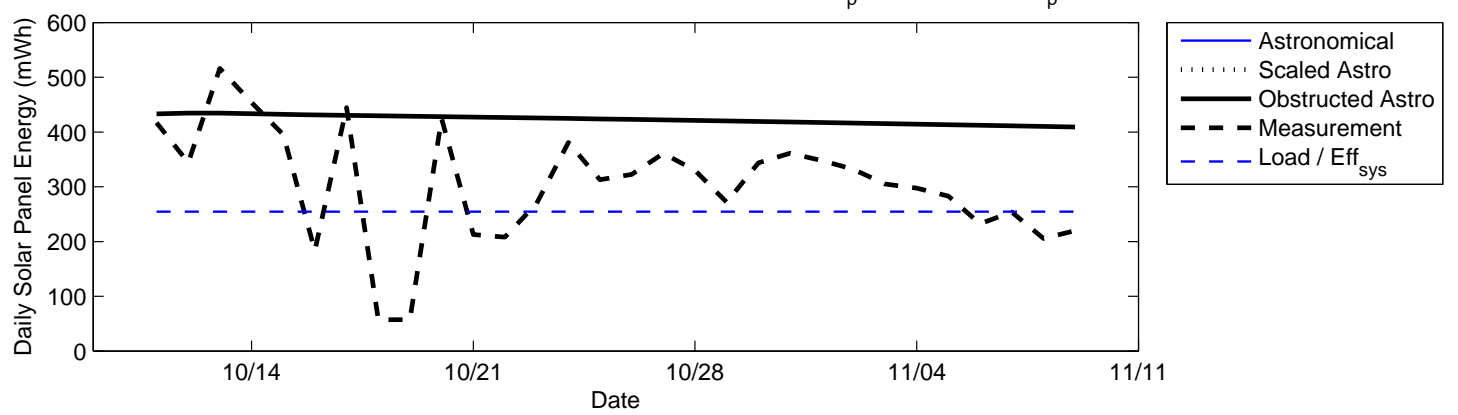

Router 78 - Battery Level Trend from 10/11/07 to 11/09/07, $\theta_{p}=45, L=37.87, \phi_{p}=180, \tau=0.1$

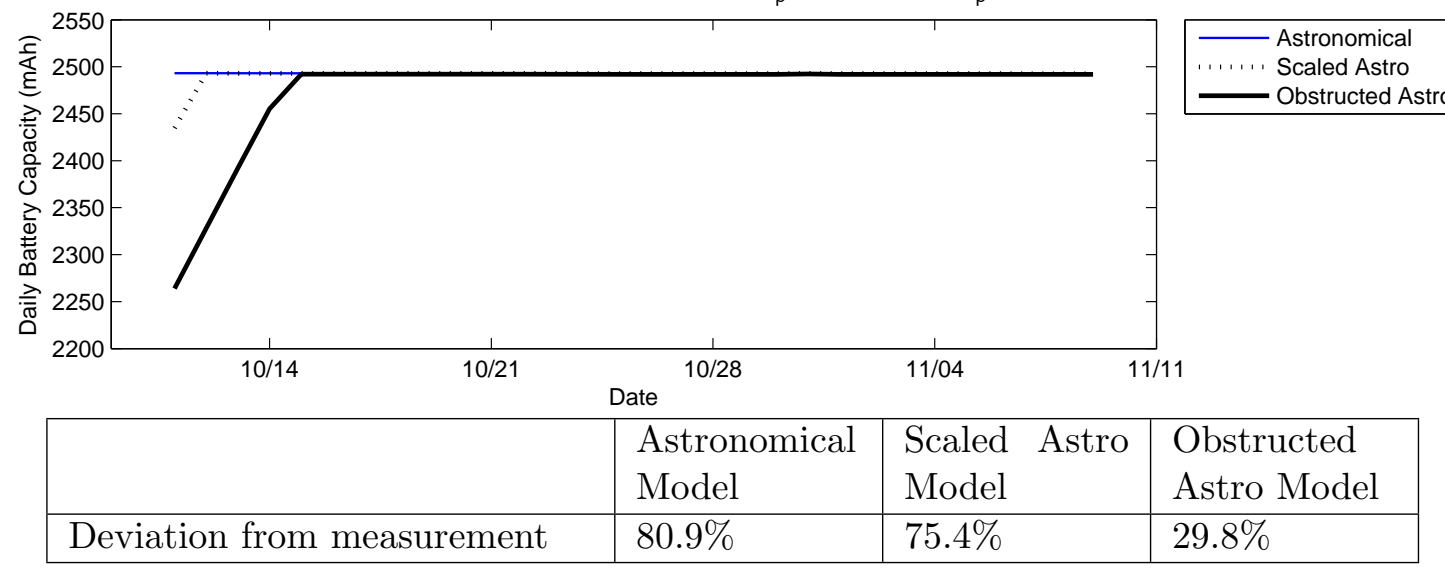

Figure 5.11: Comparison of different radiation estimation methods for high radiation node (Router 78) on forest deployment. 


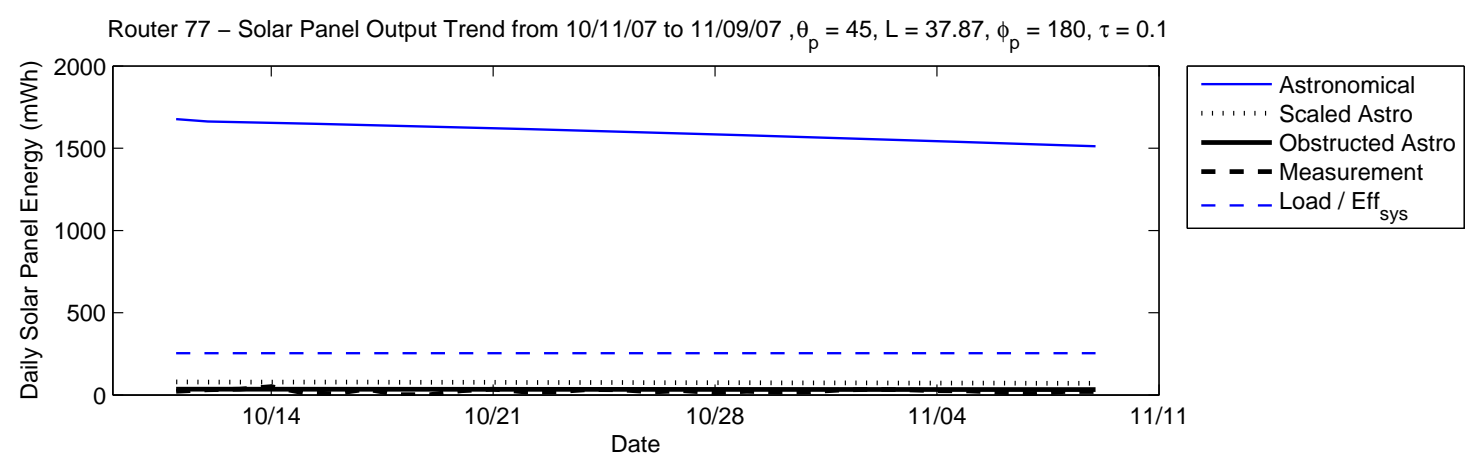

Router 77 - Solar Panel Output Trend (Enlarged) from 10/11/07 to 11/09/07, $\theta_{p}=45, L=37.87, \phi_{p}=180, \tau=0.1$
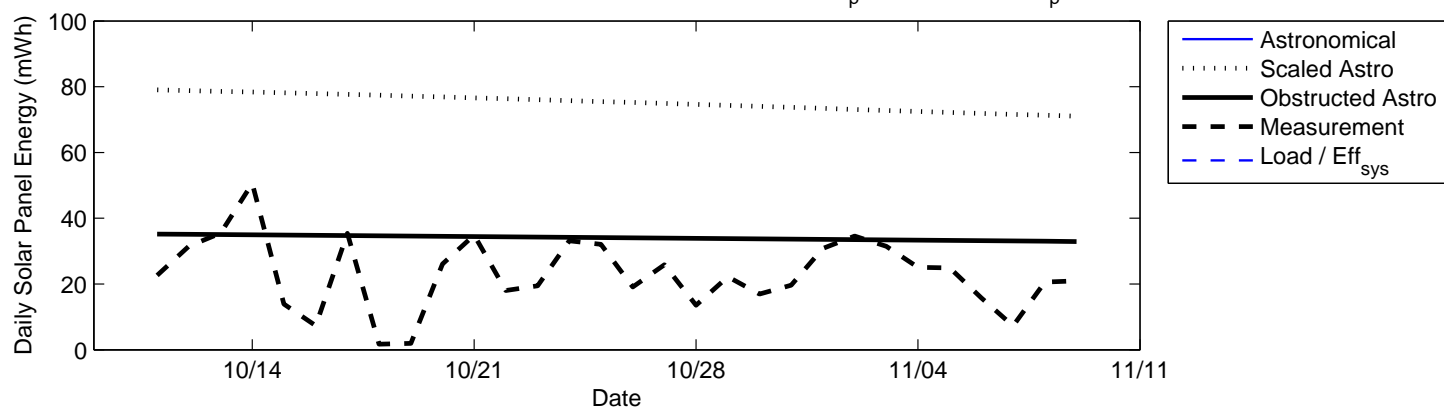

Router 77 - Battery Level Trend from 10/11/07 to 11/09/07, $\theta_{p}=45, L=37.87, \phi_{p}=180, \tau=0.1$
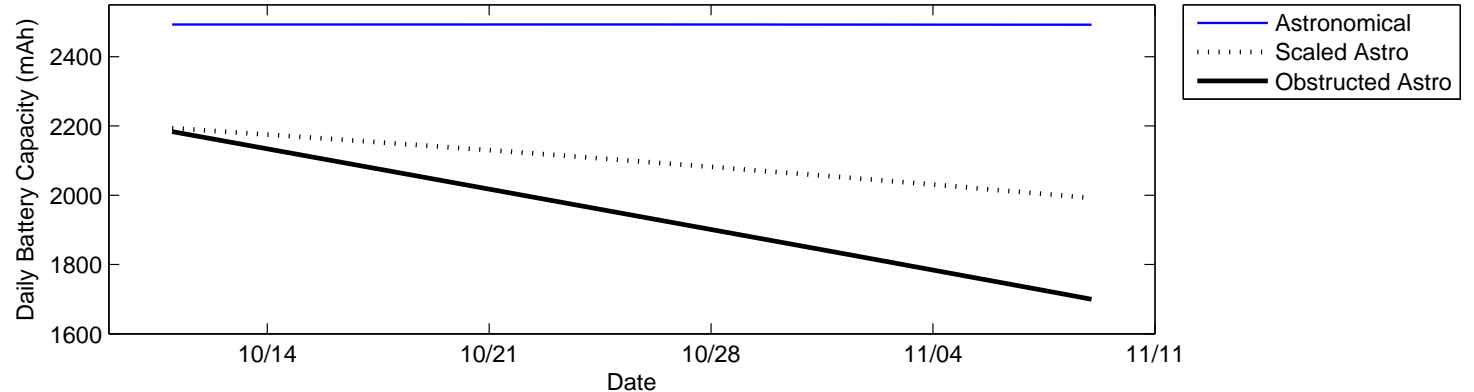

\begin{tabular}{|l|l|l|l|}
\hline & $\begin{array}{l}\text { Astronomical } \\
\text { Model }\end{array}$ & $\begin{array}{l}\text { Scaled Astro } \\
\text { Model }\end{array}$ & $\begin{array}{l}\text { Obstructed } \\
\text { Astro Model }\end{array}$ \\
\hline Deviation from measurement & $98.6 \%$ & $69.3 \%$ & $35.8 \%$ \\
\hline
\end{tabular}

Figure 5.12: Comparison of different radiation estimation methods for medium radiation node (Router 77) on forest deployment. 

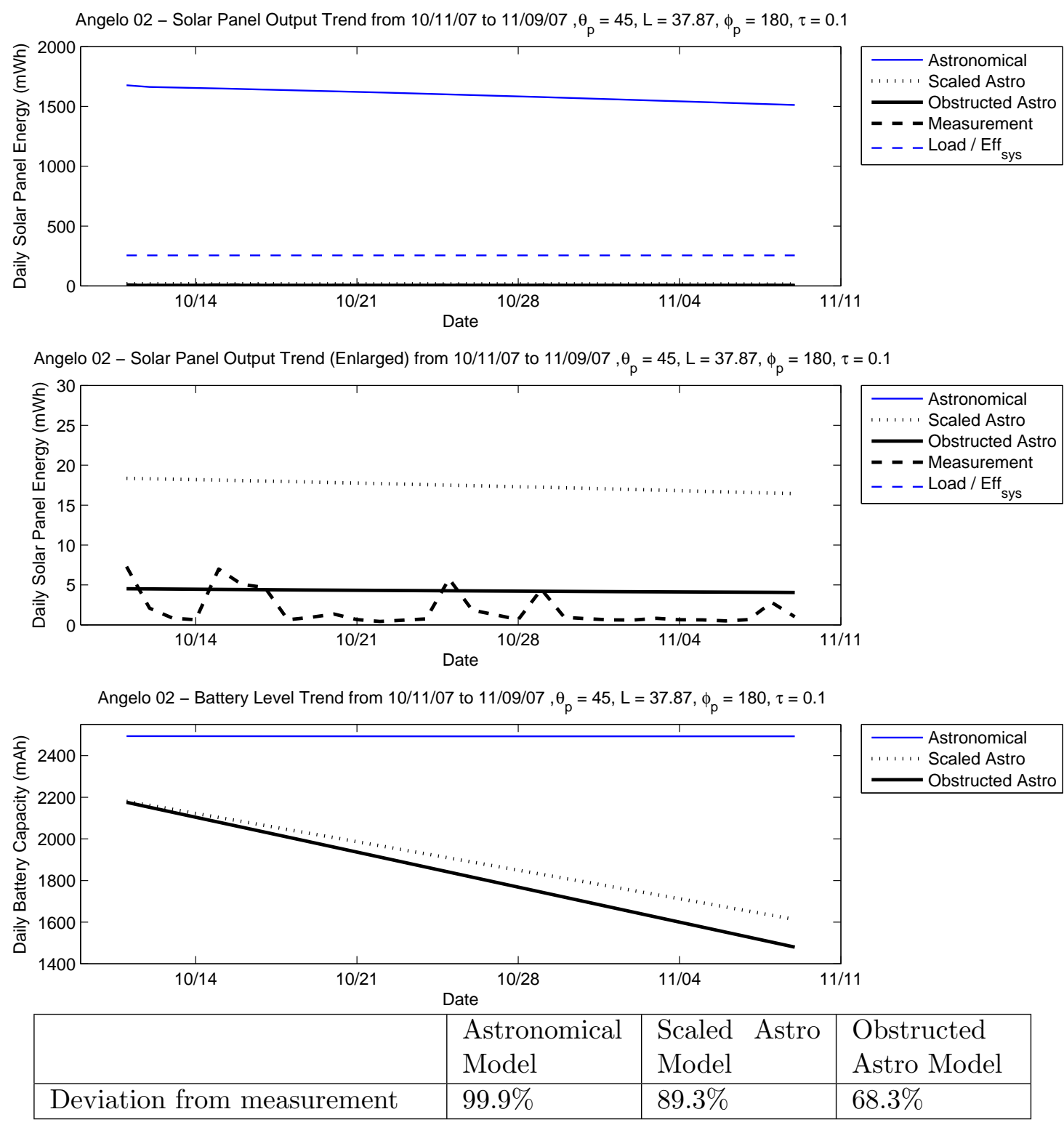

Figure 5.13: Comparison of different radiation estimation methods for low radiation node (Angelo 02) on forest deployment. 
condition (Router 77). This 30\% difference is due to the non-stationary changes such as a weather effect. For low radiation node (Angelo 02), the effect of non-stationary changes becomes more important with the estimation error $68 \%$.

\section{Lessons from Forest Deployment}

Figures 5.14 and 5.15 show the solar current and voltage of the three representative nodes on a sunny and overcast day, respectively. The solar voltages exhibit the familiar binary behavior in both cases. The solar currents noticeably suffer on the overcast day, but the heavily shaded node slightly improves its energy harvesting. Perhaps the most important observation is how spiky the solar profile is for the nodes that receive reasonable amounts of solar energy.

It appears that the primary limitation of available solar energy in the forest context is not the amount of light, but the speckled nature of the light that is present. Rarely is the spot of light that falls on even our small panels large enough to illuminate the entire panel. Overcast days diffuse the shadows, reducing the spotting. An individual solar cell produces about 0.5 volts, so several are placed in series within the panel to provide a useful output voltage. For example, our panels have two chains of eight cells in series (Figure 5.16(a) . The current of the cell is determined by its area, and cells can be interconnected in various serial-parallel networks. The problem is that when a single cell in a serial chain is not well-illuminated, it limits the current flow through the entire chain.

In order to see the effect of shading in a solar panel that consists of an array of solar cells in series or parallel, we built a solar panel array with four solar panels in three different configurations: (i) four panels in parallel, (ii) two parallel chains of two panels in series, (iii) four panels in series. For each configuration, we measured the short circuit current of the solar panel array. We also measured the short circuit current when we covered one of the panels to emulate shading (Figure 5.16(b), 5.16(c) and $5.16(\mathrm{~d})$. Figure 5.17 shows the result for the experiment. When all panels are connected in parallel, the decrease in short circuit current of the solar panel array is directly proportional to the covered panels (first figure). When panels are connected in two parallel chains of two panels in series, covering one panel caused one of the two chains to shutdown. This is the case for the $4.0 \mathrm{~V}-100 \mathrm{~mA}$ polycrystalline silicon 

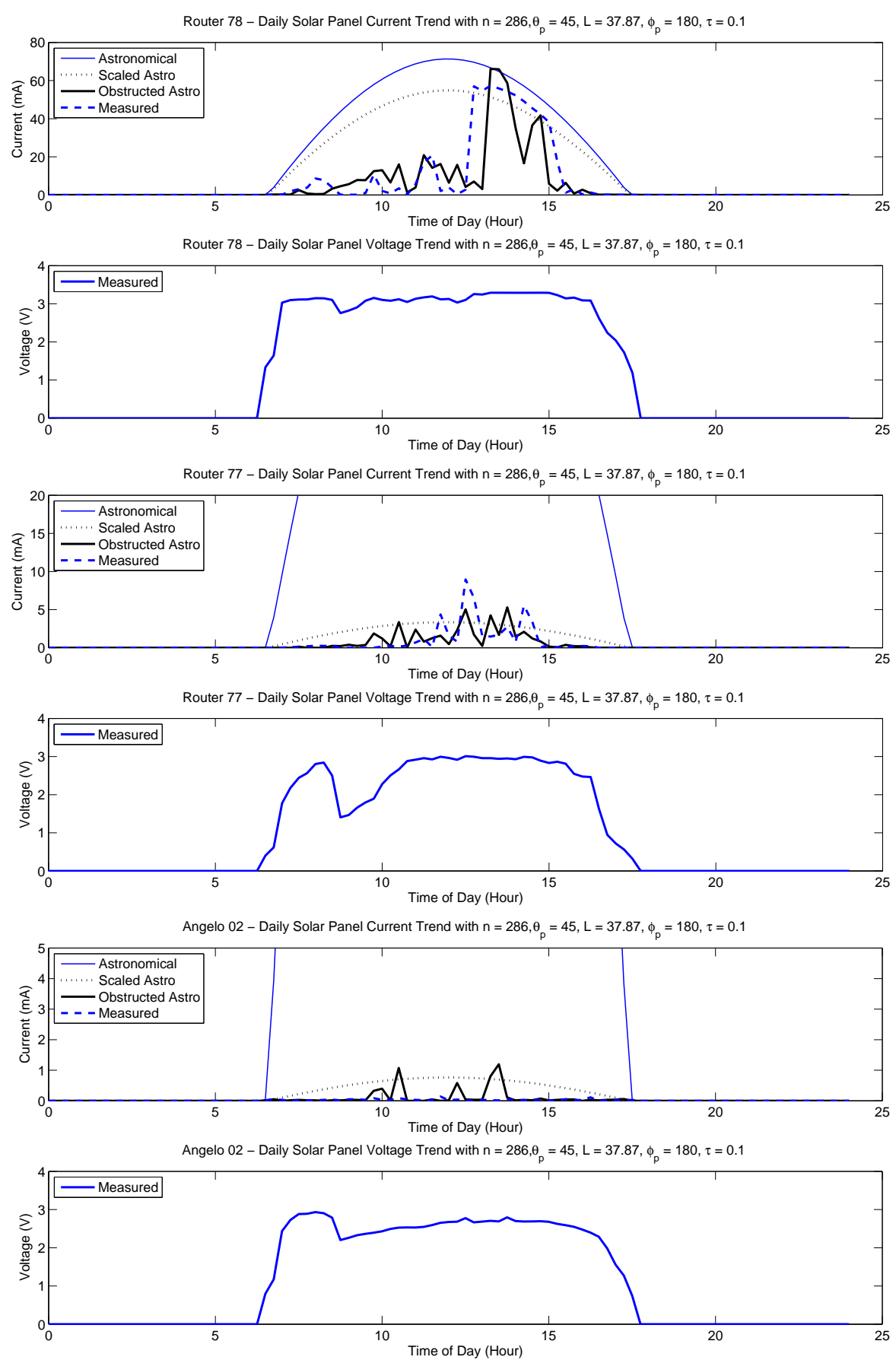

Figure 5.14: Comparison of solar panel current and voltage on a sunny day $(10 / 13 / 2007)$ in the forest watershed deployment. Notice the differences in the scale of the graphs. 


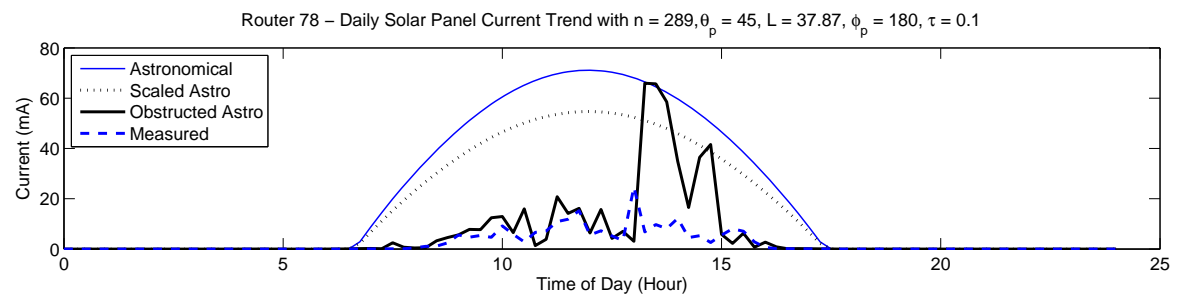

Router 78 - Daily Solar Panel Voltage Trend with $n=289, \theta_{p}=45, L=37.87, \phi_{p}=180, \tau=0.1$

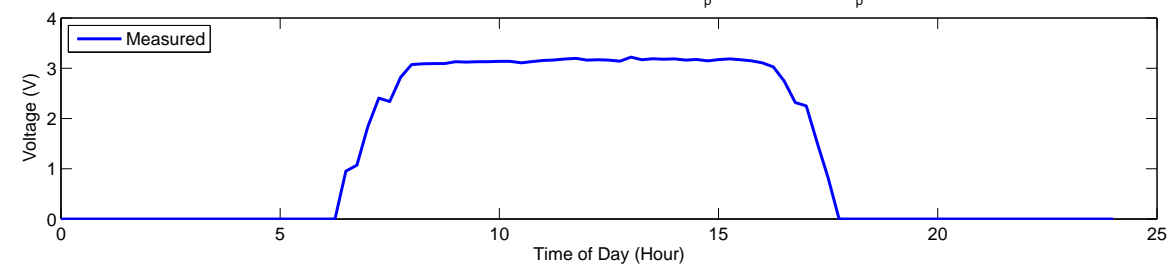

Router 77 - Daily Solar Panel Current Trend with $n=289, \theta_{p}=45, L=37.87, \phi_{p}=180, \tau=0.1$

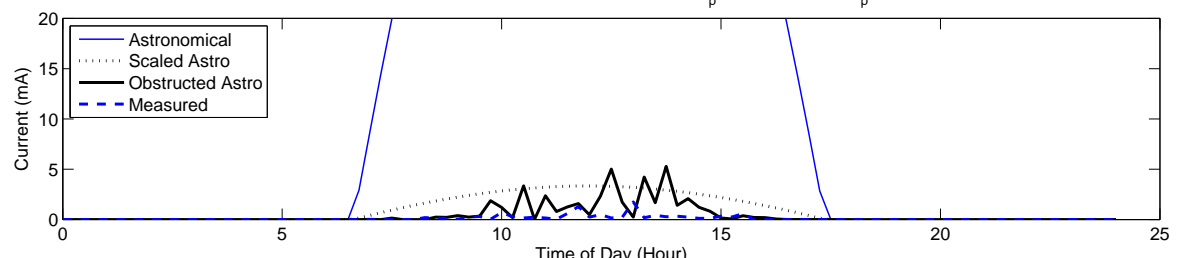

Router 77 - Daily Solar Panel Voltage Trend with $n=289, \theta_{p}=45, L=37.87, \phi_{p}=180, \tau=0.1$

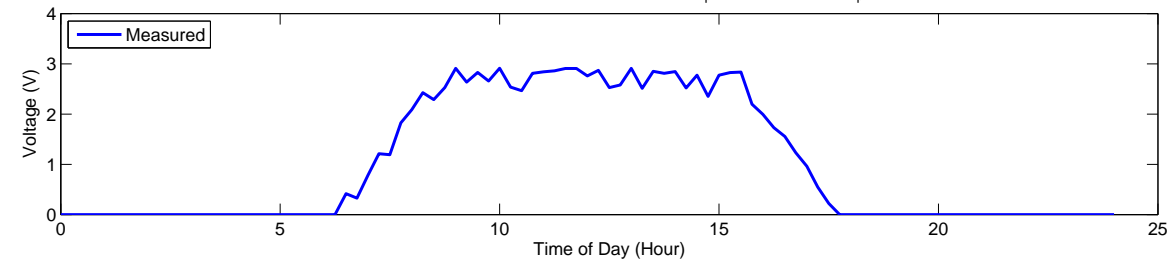

Angelo 02 - Daily Solar Panel Current Trend with $n=289, \theta_{p}=45, L=37.87, \phi_{p}=180, \tau=0.1$

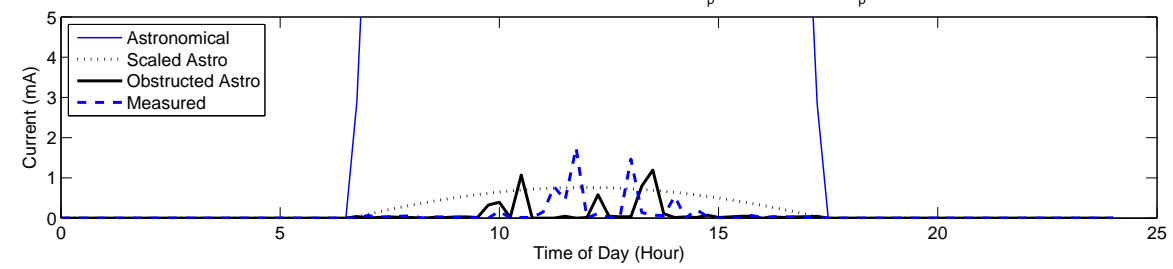

Angelo 02 - Daily Solar Panel Voltage Trend with $n=289, \theta_{p}=45, L=37.87, \phi_{p}=180, \tau=0.1$

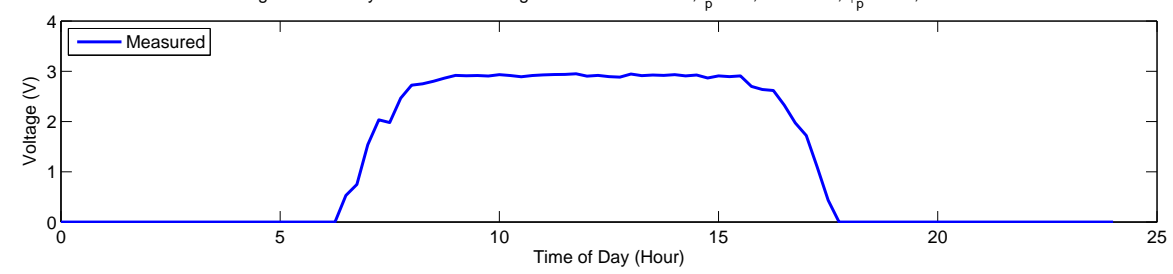

Figure 5.15: Comparison of solar panel current and voltage on an overcast day $(10 / 16 / 2007)$ in the forest watershed deployment. Notice the differences in the scale of the graphs. 


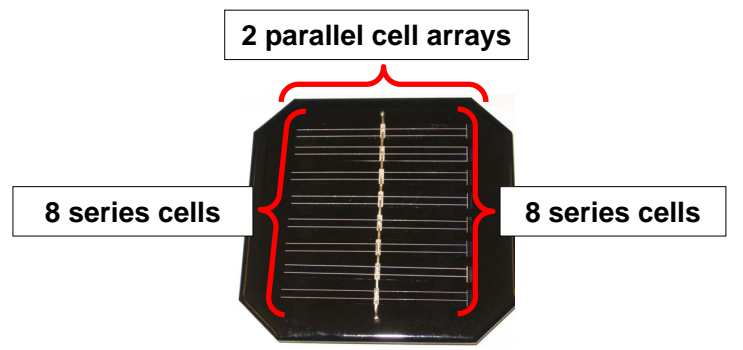

(a) Cell configuration of $4 \mathrm{~V}-100 \mathrm{~mA}$ polycrystalline silicon solar panel

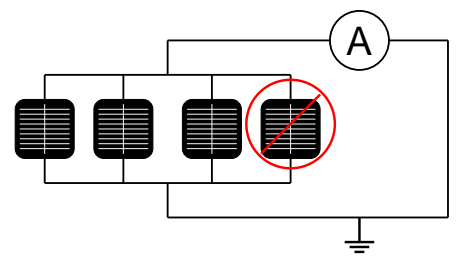

(b) 4 parallel panels with one panel covered

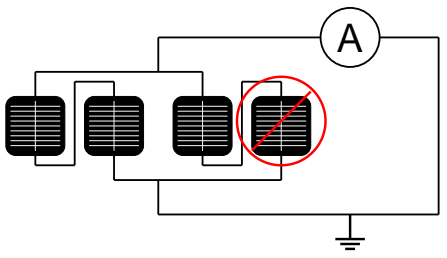

(c) 2-parallel and 2-series panels with one panel covered

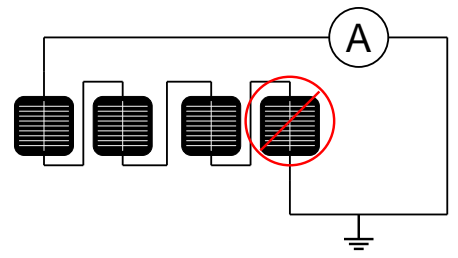

(d) 4 parallel panels with one panel covered

Figure 5.16: Different configurations of solar panel array

panel we used (second figure). When panels are connected in series, covering one panel caused the whole array to shutdown (third figure).

Thus, enlarging the panel does not necessarily increase the power output in speckled light. Instead, many small panels should be connected in a highly parallel configuration. Large residential and commercial arrays have this characteristic because of the sheer number of panels involved. One possibility of a highly parallel configuration in micro-solar power systems is to use a flexible solar panel (Figure 5.18(a)). Since each cell of a flexible solar panel is connected in parallel, covering $x$-percent of the whole cells causes only $x$-percent drop in the solar panel current (Figure 5.18(b) and $5.18(\mathrm{c})$. We find this has the same trend as the parallel solar panel configuration (Figure 5.16). One drawback of using a flexible solar panel is its lower material efficiency, which implies that we need a larger area of flexible solar panel than the polycrystalline silicon panel to output the equivalent amount of current under unobstructed radiation condition. However, resilience to the shading effect compensates the cost of lower material efficiency in a forest environment. 

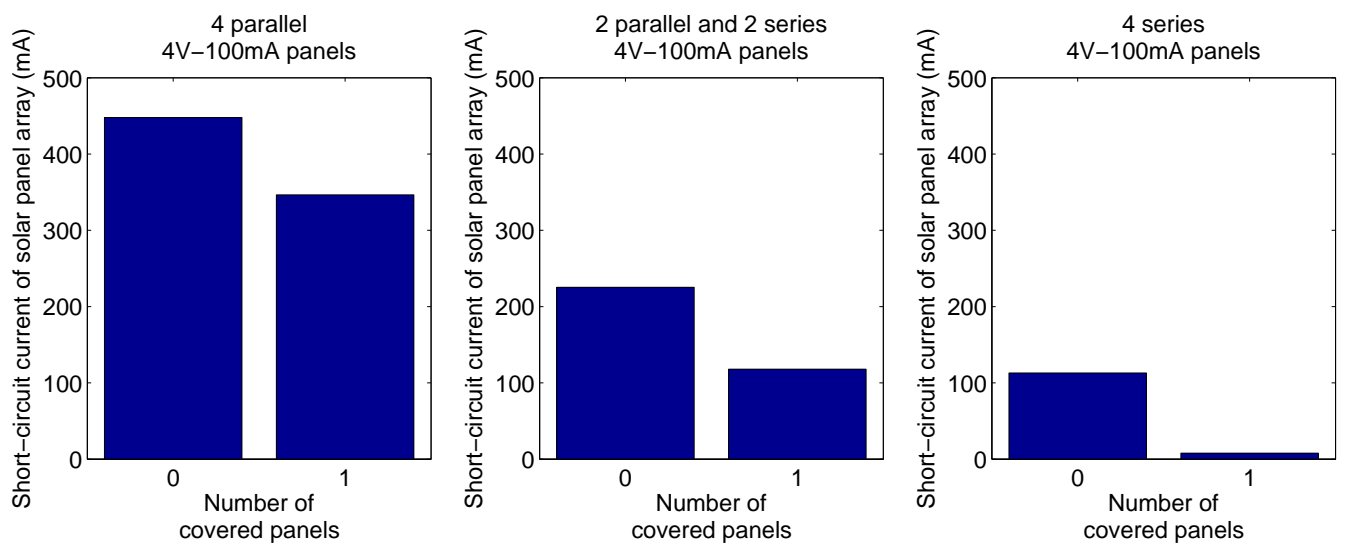

Figure 5.17: Effect of shading on polycrystalline silicon solar panel

Increasing the battery size also has surprising implications. With the low daily consumption of a well-engineered environmental monitoring application, it is reasonable to size batteries to last for several seasons. In deciduous forests, this would allow nodes to store up all their energy after the leaves fall. Even in coniferous forests, it means that energy can be collected when the interaction of the canopy and the sun angle are most favorable. Additional improvements are possible through utilizing more efficient regulators with somewhat more complex circuit requirements. Exploration of novel collectors and storage profiles for important solar-challenged environments will drive further improvements in the models as well as the physical design.

\subsection{Summary}

In this chapter, we have developed a reference platform, the HydroWatch node, to validate the simulation model of micro-solar power systems. It is designed to operate perpetually even on half an hour of sunlight per day while sensing the environment and networking over multiple hops in low-power mode. From the evaluation of over twenty HydroWatch nodes that were deployed in an urban neighborhood and forest watershed environment, we were able to observe the following. First, the obstructedastronomical model estimated solar radiation with modest error while each sensor node was under various obstruction and weather conditions. Second, all the sensor 


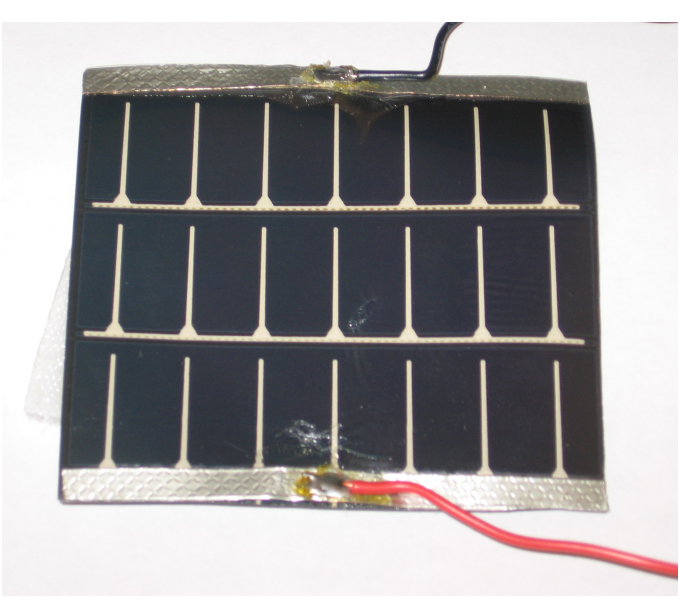

(a) $3.6 \mathrm{~V}-50 \mathrm{~mA}$ flexible solar panel

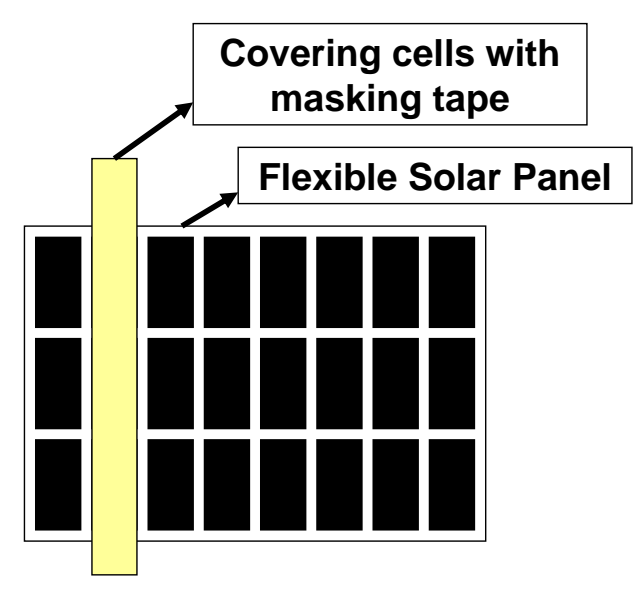

(b) Covering cells of flexible solar panel

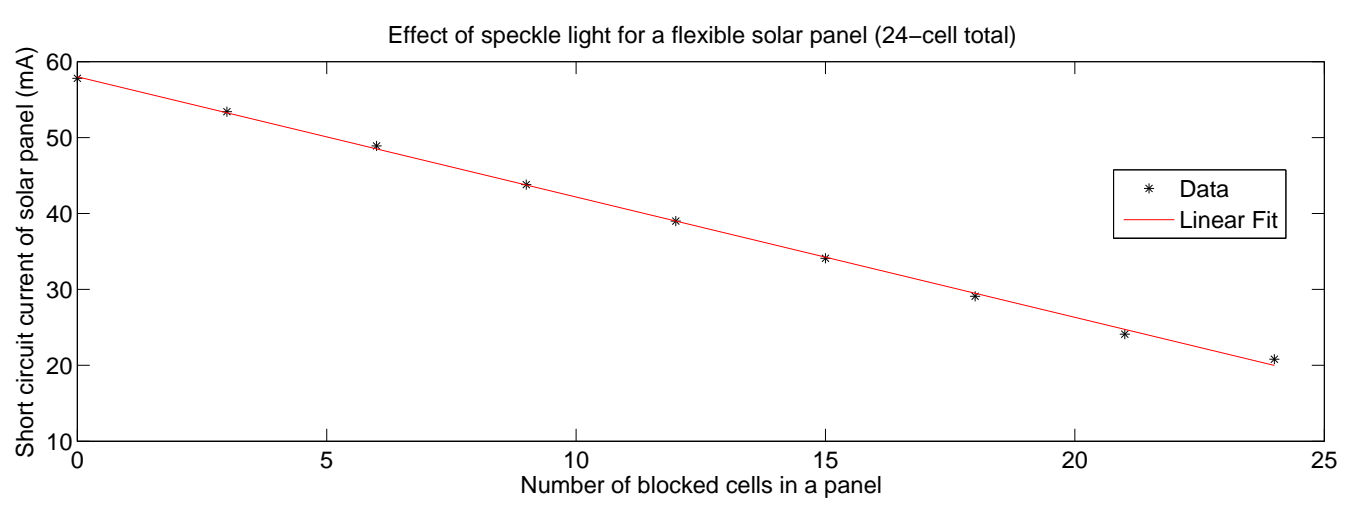

(c) Relation between number of covered cells and short circuit current for a $3.6 \mathrm{~V}-50 \mathrm{~mA}$ flexible solar panel. The $y$-value of the trend line is not zero, even though it is expected to be zero. It is because the masking tape did not completely block the sunlight, making the blocked cells of the solar panel still receive some amount of photons.

Figure 5.18: Effect of shading on flexible solar panel 
nodes in the urban neighborhood deployment accumulated sufficient solar energy for perpetual operation, validating the capacity planning for the HydroWatch node. Third, most nodes in the forest deployment did not collect sufficient solar energy, showing that a different solar panel should be used depending on radiation conditions: a flexible solar panel is more suitable for the forest environment though it has a lower efficiency than a polycrystalline silicon panel; its cells are connected in parallel and this allows the flexible panel to produce electricity even when part of the panel is shadowed. 


\section{Chapter 6}

\section{Predicting the Long-term Behavior of a Micro-Solar Power System}

In Chapter 4, we introduced an obstructed astronomical model that estimates solar radiation under obstructions. This model fits into the measurements well on a sunny day (Figure 6.1 (a)), but does not on an overcast day. The solar radiation becomes smaller due to the weather effect and the obstructed model does not catch this variation well with the estimation error getting higher (Figure 6.1 (b)).

In this chapter, we first study the effect of weather variations on solar radiation (Section 6.1). We use a long-term measurement of HydroWatch weather nodes that is taken in an obstructed environment, and compare the measurement data with the estimation models. Then, we develop a possible model for the solar radiation under weather variations using commercially available weather data (Section 6.2). We evaluate its validity by comparing the solar radiation estimate with the long-term measurement data of the HydroWatch weather nodes (Section 6.3).

\subsection{Accounting for the Weather Effect}

\subsubsection{Experimental Set-up}

In order to see the effect of weather variations, we deployed five HydroWatch weather nodes on the rooftop of the Valley Life Science Building (VLSB) in UC 


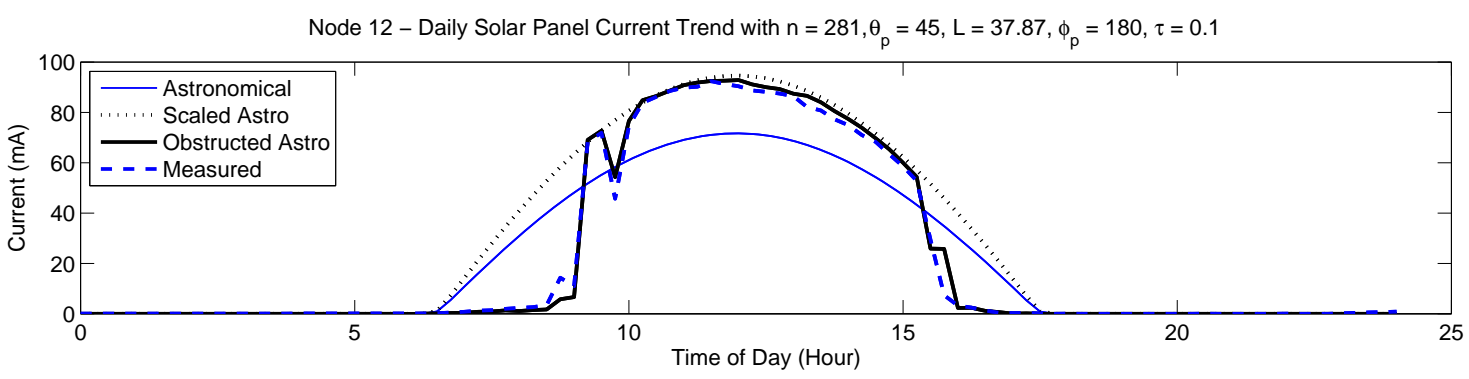

(a) Daily Solar Profile on a Sunny Day

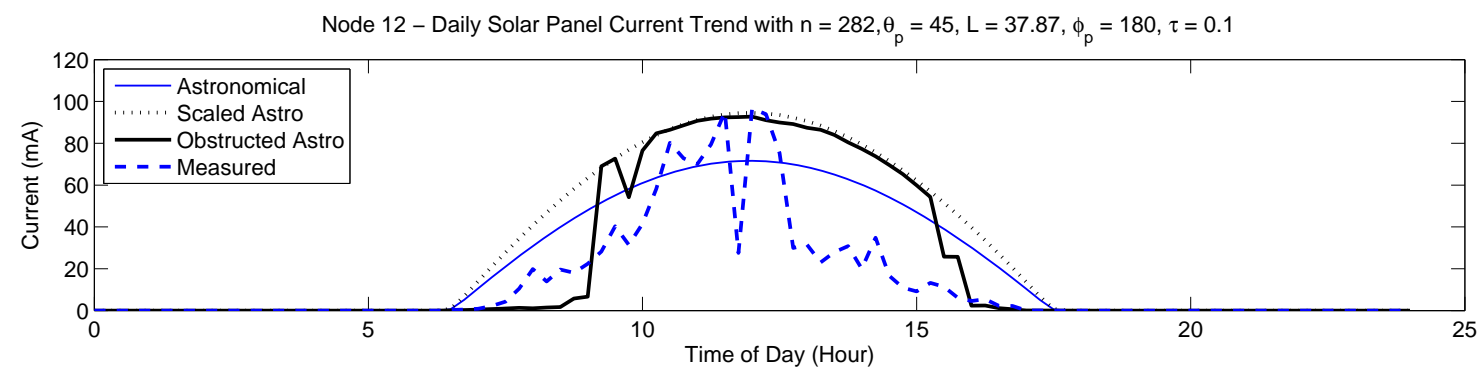

(b) Daily Solar Profile on an Overcast Day

Figure 6.1: Solar profile for different weather conditions

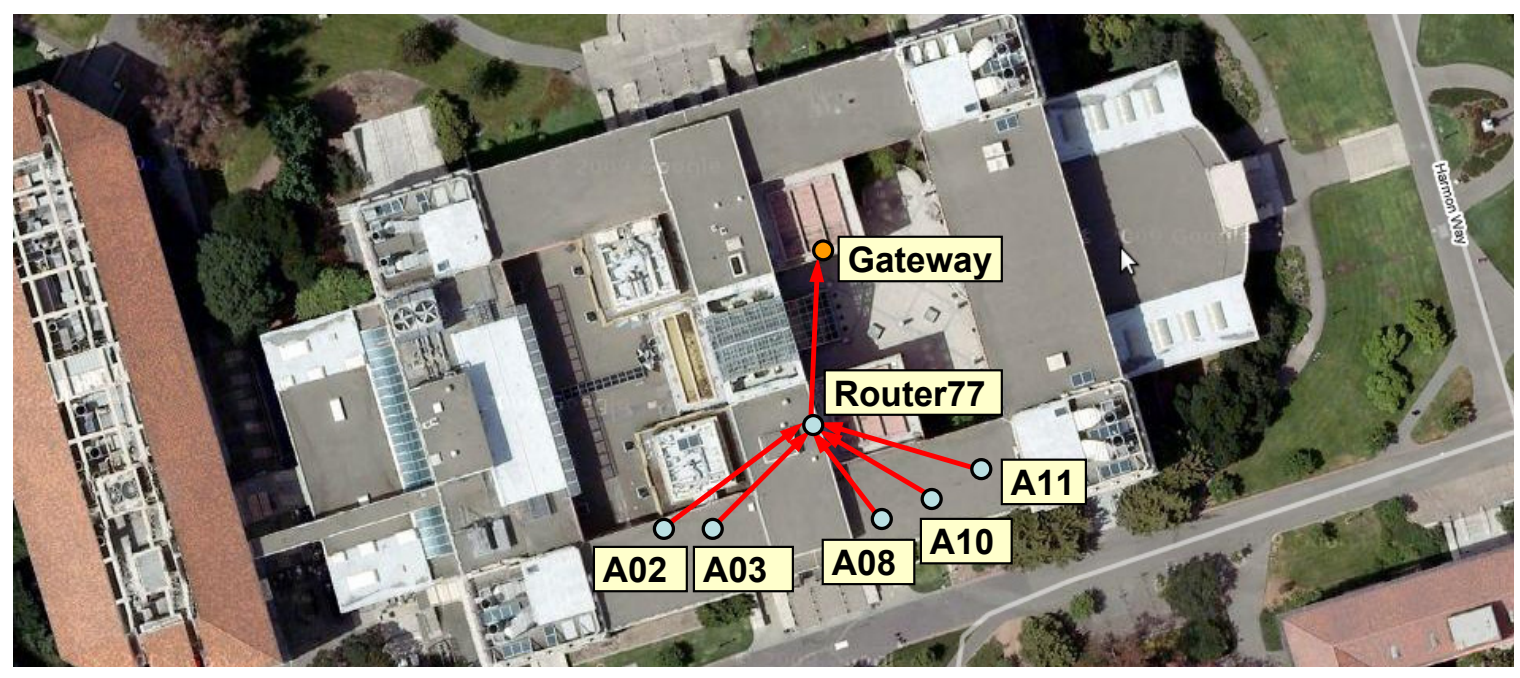

Figure 6.2: Deployment map of HydroWatch nodes on the rooftop of Valley Life Science Building in UC Berkeley. 
Berkeley where micro-solar nodes can get solar radiation without any obstructions from trees or other buildings (Figure 6.2). Since there are no obstructions between each node and the sun, the measurement of the solar panel output depends only on the diurnal and seasonal variation of solar radiation and the weather variation. The astronomical model, which is explained in Chapter 4 , gives an estimation of diurnal and seasonal variation of the solar radiation. We use the obstructed astronomical model in order to fit the astronomical model to the measurement, then we account for the weather effect by comparing the solar panel output measurement with the prediction from the obstructed astronomical model. For this experiment, five HydroWatch weather nodes were deployed, and the following parameters are used for the external environment:

- Day of the Year (n) : The experiment was conducted from 12/22/2007 (n = $356)$ to $4 / 15 / 2008(\mathrm{n}=365+106)$.

- Latitude (L) : The experiment was done in Berkeley, CA, which is at $37.87^{\circ} \mathrm{N}$.

- Solar Panel Inclination $\left(\theta_{p}\right)$ and Orientation $\left(\phi_{p}\right)$ : Depending on the solar panel inclination, the five HydroWatch weather nodes were sorted into two groups. Nodes A02 and A08 had their panels tilted $45^{\circ}\left(\theta_{p}=45\right)$ facing south $\left(\phi_{p}=180\right)$. And nodes A03, A10 and A11 had their panels flat to the ground $\left(\theta_{p}=0\right)$.

\subsubsection{Results}

Figure 6.3 compares the daily solar panel energy measurement with a few estimation models. From this plot, we can observe the following:

- Seasonal Variation: The three estimation models (astronomical, scaled astronomical and obstructed astronomical) capture the seasonal variation well and the obstructed astronomical model tracks the peak of the measurement.

- Weather Effect: The obstructed astronomical model deviates from the measurement within about $30 \%$ due to weather. 

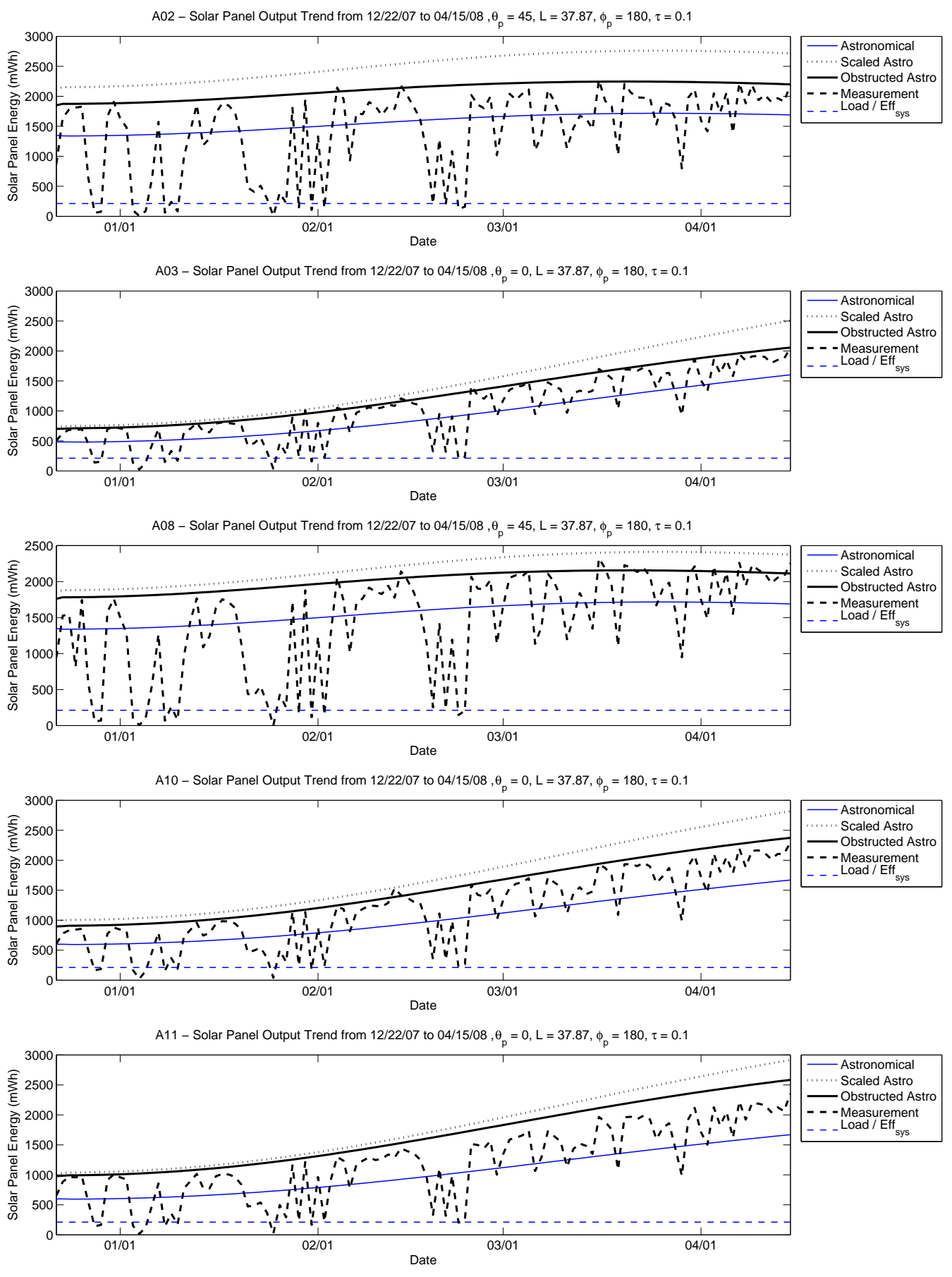

Figure 6.3: Seasonal solar radiation variation of HydroWatch weather nodes on the rooftop of Valley Life Science building in UC Berkeley 

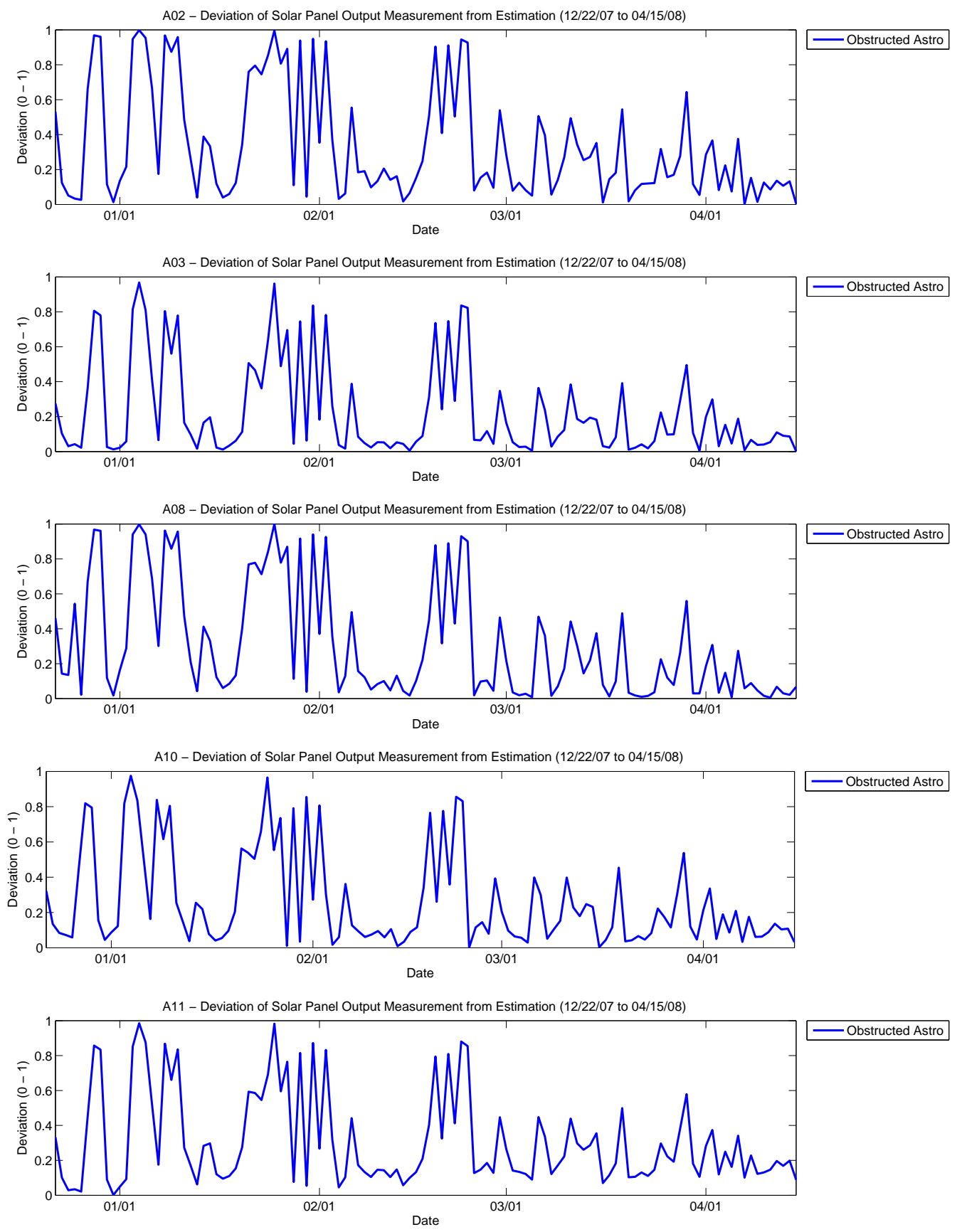

Figure 6.4: Deviation of the solar energy measurement from the estimate for the HydroWatch weather nodes deployment on the rooftop of Valley Life Science building in UC Berkeley 
Table 6.1: Deviation of the obstructed astronomical model from the measurement $(12 / 22 / 2007$ to $4 / 15 / 2008)$

\begin{tabular}{|c|c|c|c|c|c|}
\hline & $\mathrm{A} 02$ & $\mathrm{~A} 03$ & $\mathrm{~A} 08$ & $\mathrm{~A} 10$ & $\mathrm{~A} 11$ \\
\hline Deviation & $34.0 \%$ & $23.2 \%$ & $31.4 \%$ & $26.7 \%$ & $31.1 \%$ \\
\hline
\end{tabular}

Table 6.2: Correlation coefficient for the weather trend in VLSB deployment

\begin{tabular}{|c|c|c|c|c|c|}
\hline & A02 & A03 & A08 & A10 & A11 \\
\hline A02 & 1.0 & 0.97073 & 0.97644 & 0.98154 & 0.98055 \\
\hline A03 & & 1.0 & 0.95558 & 0.99355 & 0.99114 \\
\hline A08 & & & 1.0 & 0.96877 & 0.95503 \\
\hline A10 & & & & 1.0 & 0.99244 \\
\hline A11 & & & & & 1.0 \\
\hline
\end{tabular}

In order to see whether this weather effect is only for these particular nodes or for all the nodes in the deployment, we did the following analysis for all the nodes:

First, as shown in Figure 6.4, we calculated the deviation of the obstructed astronomical model $D V(n, t)$ as a relative difference between the estimation $\operatorname{AST}(n, t)$ and the measurement $M(n, t)$ :

$$
D V(n, t)=\frac{A S T(n, t)-M(n, t)}{A S T(n, t)}
$$

The average of the deviation for all the nodes are shown in Table6.1. We can see that there is slight variation, but the deviation of the estimation from the measurement is about $30 \%$.

Second, we calculated the correlation coefficient of the deviation for all the two pairs of the nodes in the deployment (Table 6.2). The correlation coefficient is between $95 \%$ to $99 \%$, and this implies that all the nodes in the deployment experienced the same weather variation.

Figure 6.5 shows the distribution of the deviation of the obstructed astronomical model to the measurement. This distribution has 0.29 as its average. We can also see that about half of the deviations are within 0.15 and the other half has a long tail and is spread from 0.15 to 1 . This distribution implies that much of the solar radiation measurement deviates within a small fraction of the estimate, but a non-negligible amount of measurement deviates a large fraction of the estimate. 


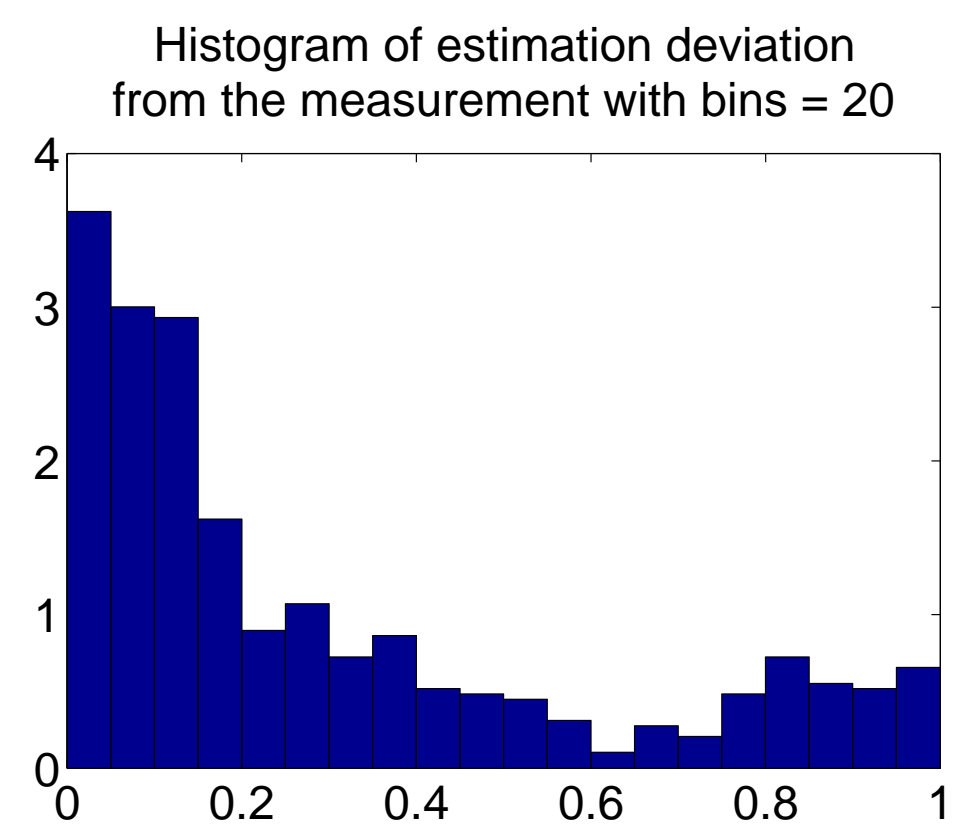

(a) Histogram

CDF of estimation deviation

from the measurement

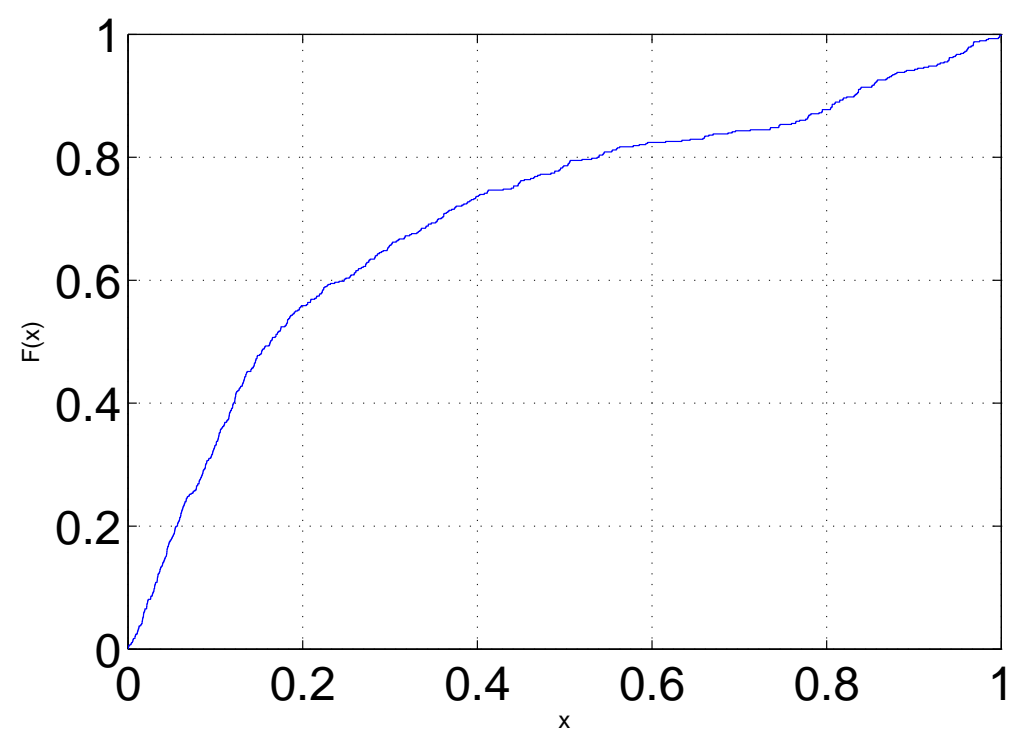

(b) Cumulative Distribution Function (CDF)

Figure 6.5: Histogram and CDF of the estimation deviation from the measurement for the obstructed astronomical model. The data is sampled over fives nodes of the Valley Life Science Building deployment 


\subsection{Developing Weather Effect Model}

In the previous section, we have shown that solar radiation estimation using astronomical and obstruction models has a variation of about $30 \%$ from the actual measurement due to weather effects. A natural question to ask is whether it is possible to predict solar radiation under weather variations. In order to answer this question, we develop a weather effect model using publicly available data and evaluate whether the model can predict the variation of solar radiation with sufficient accuracy. Among the various kinds of data measured in weather stations, there are several metrics related to the transfer rate of solar radiation under weather effects: atmospheric turbidity, horizontal visibility, and cloud cover. In this section, we model solar radiation under weather effects using these metrics and validate each model against extensive data from publicly available weather stations.

\subsubsection{Atmospheric Turbidity}

Atmospheric turbidity, or simply turbidity is used as a measure of air pollution in the atmospheric science community and is known to be highly correlated with solar radiation [PFR78, RV82, CH88]. As sunlight traverses the atmosphere, solar irradiance is degraded by several causes: sunlight can be scattered by air molecules (Rayleigh scattering); it can be absorbed by atmospheric gases such as ozone, water vapor and carbon dioxide; or it can be absorbed by aerosols such as clouds, fog and smog. Turbidity accounts for the degradation of solar radiation due to aerosols, and it is defined as the ratio of solar irradiance degraded by aerosols and extraterrestrial solar irradiance, which can be estimated using an astronomical model of solar radiation. Since solar irradiance and Rayleigh scattering varies depending on wavelength of sunlight, turbidity is typically defined at a particular wavelength. Turbidity at wavelength $\lambda, B(\lambda)$, is a non-negative number and is logarithmically related to the ratio of actual solar irradiance $J(\lambda)$ and extraterrestrial solar irradiance $J_{0}(\lambda)$ [RV82]:

$$
J(\lambda) / J_{0}(\lambda)=10^{-(R(\lambda)+Z(\lambda)+B(\lambda)) m}
$$

where $R$ is the Rayleigh scattering coefficient, $Z$ is the gaseous absorption coefficient, and $m$ is the optical air mass, which is the relative length of sunlight through 


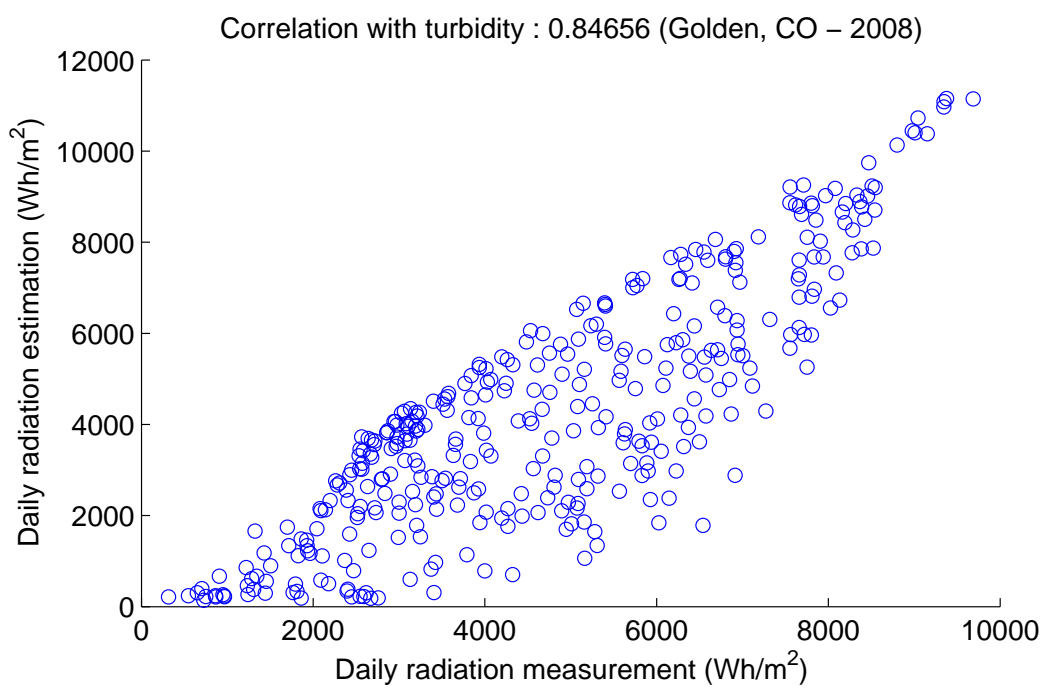

Figure 6.6: Correlation of daily solar radiation estimation using turbidity and actual measurements at Golden, CO from 1/1/2008 to $12 / 31 / 2008$

the atmosphere compared to when sunlight is normal to the Earth's surface. While turbidity is defined at a particular wavelength, we consider modeling solar radiation under weather variation using turbidity at $500 \mathrm{~nm}$, which has the highest intensity across the solar radiation spectrum. Suppose turbidity and the solar radiation estimate from the astronomical model are given as $B(n, t)$ and $A S T(n, t)$ on day $n$ and time $t$, we can estimate the daily solar radiation under the influence of turbidity, $R B(n, t)$ using the logarithmic relation of solar radiation and turbidity:

$$
\begin{aligned}
R B(n) & =\int_{0 h}^{24 h} R B(n, t) d t \\
& =\int_{0 h}^{24 h} k \cdot A S T(n, t) \cdot e^{-B(n, t)} d t
\end{aligned}
$$

where $k$ is a constant.

In order to validate this turbidity-to-radiation model, we compare the correlation coefficient between the solar radiation under the influence of turbidity and the solar radiation measurement using a publicly availble database provided by the Measurement and Instrumentation Data Center (MIDC) [BMS]. The MIDC database provides data for solar radiation measurement, turbidity and astronomical estimation of solar radiation at a per-minute or per-hour scale. From this raw data, we can 


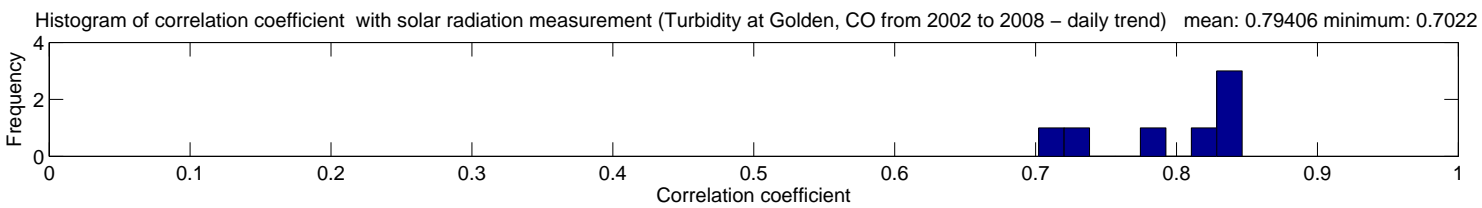

Histogram of correlation coefficient with solar radiation measurement (Turbidity at Golden, CO from 2002 to 2008 - 7-day trend) mean: 0.87926 minimum: 0.8307
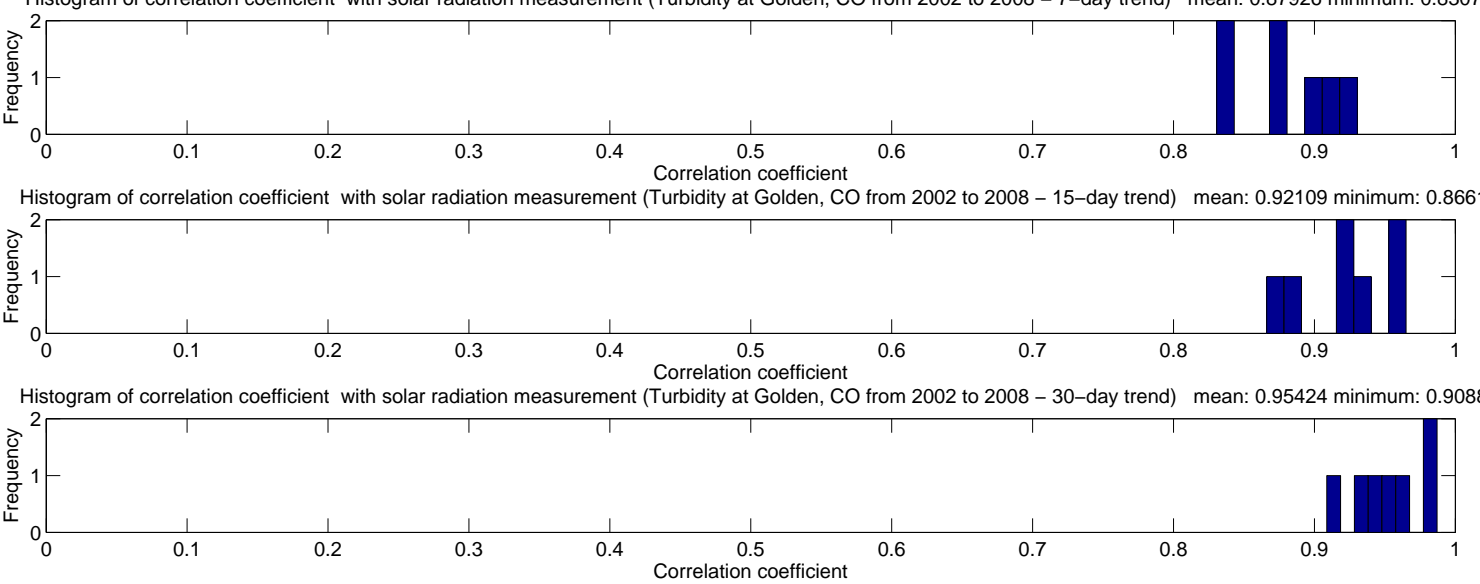

Figure 6.7: Histogram of correlation of turbidity and solar radiation with different window size at Golden, CO from 2002 to 2008

calculate actual solar radiation and the estimation over a $n$-day time window, and use them to validate the turbidity-to-radiation model. Figure 6.6 shows the correlation trend between the daily solar radiation estimation using turbidity and actual measurements at Golden, CO from 1/1/2008 to 12/31/2008 with the correlation coefficient 0.847 . We can see that turbidity and solar radiation has a strong correlation. The turbidity-to-radiation model can be further refined by covering solar radiation of $k$-days instead of a single day. Figure 6.7 shows the correlation trend between solar radiation using turbidity and actual measurements at Golden, CO from 2002 to 2008 when the comparison window size is changed from a single day to 7, 15 and 30 days. We can see that the correlation gets higher as the window size gets larger and the correlation coefficient becomes greater than $90 \%$ when the window size is set to 15 days.

While turbidity is a good estimator of solar radiation, the availability of finegrained turbidity measurement is very limited. Thus, there is a need to develop a weather effect model using more widely available data. Possible candidates are horizontal visibility and cloud conditions, which are available from measurement data 
at most airports and other weather stations.

\subsubsection{Horizontal Visibility and Cloud Condition}

Horizontal visibility, which is monitored at most airports to ensure air traffic safety, is the distance one can see horizontally with a maximum of 10.0 miles or 16.1 $\mathrm{km}$. With horizontal visibility and a solar radiation estimate from the astronomical model being given, we can estimate daily solar radiation under the influence of the visibility using the observations of Peterson et al. on the relationship between horizontal visibility and the ultra-violet (UV) spectrum of solar irradiance [PFR78]: Peterson et al. stated that visibility and UV irradiance had a strong correlation below $10 \mathrm{~km}$ of visibility while UV irradiance was only slightly correlated with visibility of $12 \mathrm{~km}$ or above. While their observation was about visibility and UV irradiance, we apply it to develop a weather effect model that will estimate solar radiation (not just UV spectrum) from horizontal visibility.

When the horizontal visibility and solar radiation estimate from the astronomical model are given as $V(n, t)$ and $A S T(n, t)$ on day $n$ and time $t$, we can estimate the daily solar radiation under the influence of visibility $R V(n)$ as follows:

$$
\begin{aligned}
& R V(n)=\int_{0 h}^{24 h} \frac{\widehat{V}(n, t)}{V_{\text {knee }}} \cdot A S T(n, t) d t \\
& \widehat{V}(n, t)= \begin{cases}V_{\text {knee }} & V(n, t) \geq V_{\text {knee }} \\
V(n, t) & \text { otherwise }\end{cases}
\end{aligned}
$$

where we set $V_{\text {knee }}$ as $12.0 \mathrm{~km}$.

Cloud condition is the percentage of the sky covered by clouds, and it is used to determine the cloudiness of the sky at a particular location. Depending on whether we count all visible clouds or only opaque clouds, cloud condition can be further divided into total cloud cover and opaque cloud cover. For a given cloud condition $W(n, t)$ and solar radiation estimate from the astronomical model $A S T(n, t)$ on day $n$ and time $t$, we can estimate $R W(n)$, the daily solar radiation under the influence of cloud condition on day $n$, as follows:

$$
R W(n)=\int_{0 h}^{24 h} W(n, t) \cdot A S T(n, t) d t
$$


Table 6.3: Measurement sites used from the National Solar Radiation Data Base

\begin{tabular}{|l|l|}
\hline \multicolumn{2}{|c|}{ Location } \\
\hline Denver Centennial Airport, CO & Broomfield / Jeffco Airport, CO \\
Tallahassee Regional Airport, FL & Boise Air Terminal, OR \\
Joslin Field Magic Valley Airport, ID & Univ. of Illinois Willard Airport, IL \\
Wolf Point International Airport, MT & Mercury Desert Rock Airport, NV \\
Albuquerque International Airport, NM & Elizabeth City Coast Guard Airport, NC \\
Bismarck Municipal Airport, ND & Ponca City Municipal Airport, OK \\
Burns Municipal Airport, OR & Eugene Mahlon Sweet Airport, OR \\
Klamath Falls Intl Airport, OR & Medford Rogue Valley International Airport, OR \\
State College Airport, PA & Abilene Regional Airport, TX \\
Amarillo International Airport, TX & Austin Mueller Municipal Airport, TX \\
Corpus Christi Intl Airport, TX & Del Rio Airport, TX \\
El Paso International Airport, TX & Houston Ellington Air Force Base, TX \\
Laredo International Airport, TX & Longview Gregg County Airport, TX \\
McAllen Miller International Airport, TX & Washington DC Dulles International Airport, VA \\
Salt Lake City International Airport, UT & Moab / Canyonlands Airport, UT \\
Seattle Boeing Field, WA & Spokane International Airport, WA \\
BluefieldMercer Co, WV & Madison Dane Co Regional Airport, WI \\
Rock Springs Airport, WY & \\
\hline
\end{tabular}

In order to validate weather effect models with horizontal visibility and cloud condition, we compare the solar radiation estimate using weather effect models and the actual solar radiation measurements from 35 sites during 1991 to 2005 using historical measurements from the National Solar Radiation Data Base [NSR]. Table 6.3 lists the sites whose measurement data are used in our study.

Figure 6.8 and 6.9 show the correlation of solar radiation measurement and estimate when we use visibility, total cloud cover and opaque cloud cover with the sliding window size as 1, 7, 15 and 30 days. For the weather effect models using horizontal visibility, total cloud cover and opaque cloud cover, we can observe the following:

First, the weather effect models show high correlation with the actual measurements when the estimation is based on a sufficient number of samples. For example, weather effect models with total cloud cover, opaque cloud cover and horizontal visibility have mean correlation of $89 \%, 93 \%$ and $96 \%$ for a window size of 15 days.

Second, the estimation quality of the weather effect models improves as we increase the window size, but it reaches a sweet spot at 15 days. When we increased the window size from 15 days to 30 days, the improvement in the correlation coefficient was marginal.

Third, horizontal visibility is a better predictor of solar radiation than total cloud cover or opaque cloud cover. The mean and the minimum of the correlation coefficient of horizontal visibility is $96 \%$ and $79 \%$ with a 15 -day window. Whereas, total cloud 
cover and opaque cloud cover have a much wider distribution. While the mean of their correlation coefficients are about $90 \%$, the minimum of the correlation coefficients are much smaller, making long tails (9\% and 10\%).

\subsection{Evaluating Weather Effect Model}

In the previous section, we have shown that horizontal visibility and cloud condition can be used to estimate the solar radiation under the weather effect because they have a high correlation with solar radiation when being averaged over multiple days. Also the data is widely available from many weather stations. In this section, we validate this idea with a concrete example. We will develop a solar radiation weather effect model using the historical data of horizontal visibility and cloud conditions from a publicly available weather station and compare it with the 5-month long solar radiation measurements from our reference implementation of a micro-solar power system.

\subsubsection{Defining Solar Radiation Estimators}

In order to develop a weather effect component, we use data from a publicly available online weather station, Wunderground [Wun]. Wunderground provides a live and archived view of several weather metrics for a number of weather stations across the United States. We have used archived data of horizontal visibility and cloud conditions for the Oakland International Airport, which is the nearest weather station to the measurement site at UC Berkeley, that provides fine-grained data of horizontal visibility and cloud conditions.

Suppose the solar radiation estimation without weather effect is given as $A S T(t)$ and the weather factor $W(t)$ can be represented as a number between 0 and 1 . Then, the solar radiation with weather effect $R W(t)$ can be represented as a product of $A S T(t)$ and $W(t)$

$$
R W(t)=W(t) \cdot A S T(t)
$$

Figure 6.10 shows a snapshot of an hourly weather data report from Wunderground which includes horizontal visibility and cloud conditions. Horizontal visibility is re- 

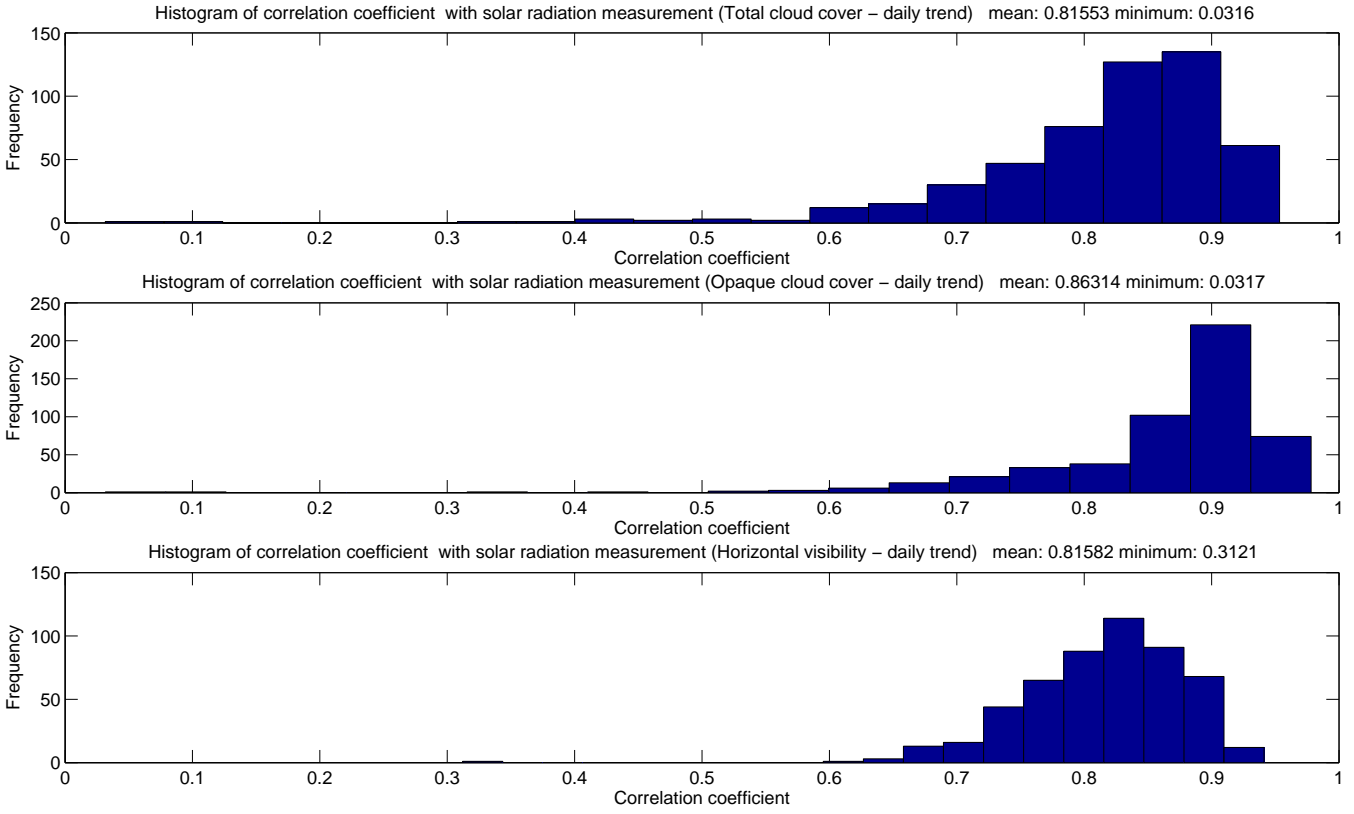

(a) Trend with 1-day sliding window
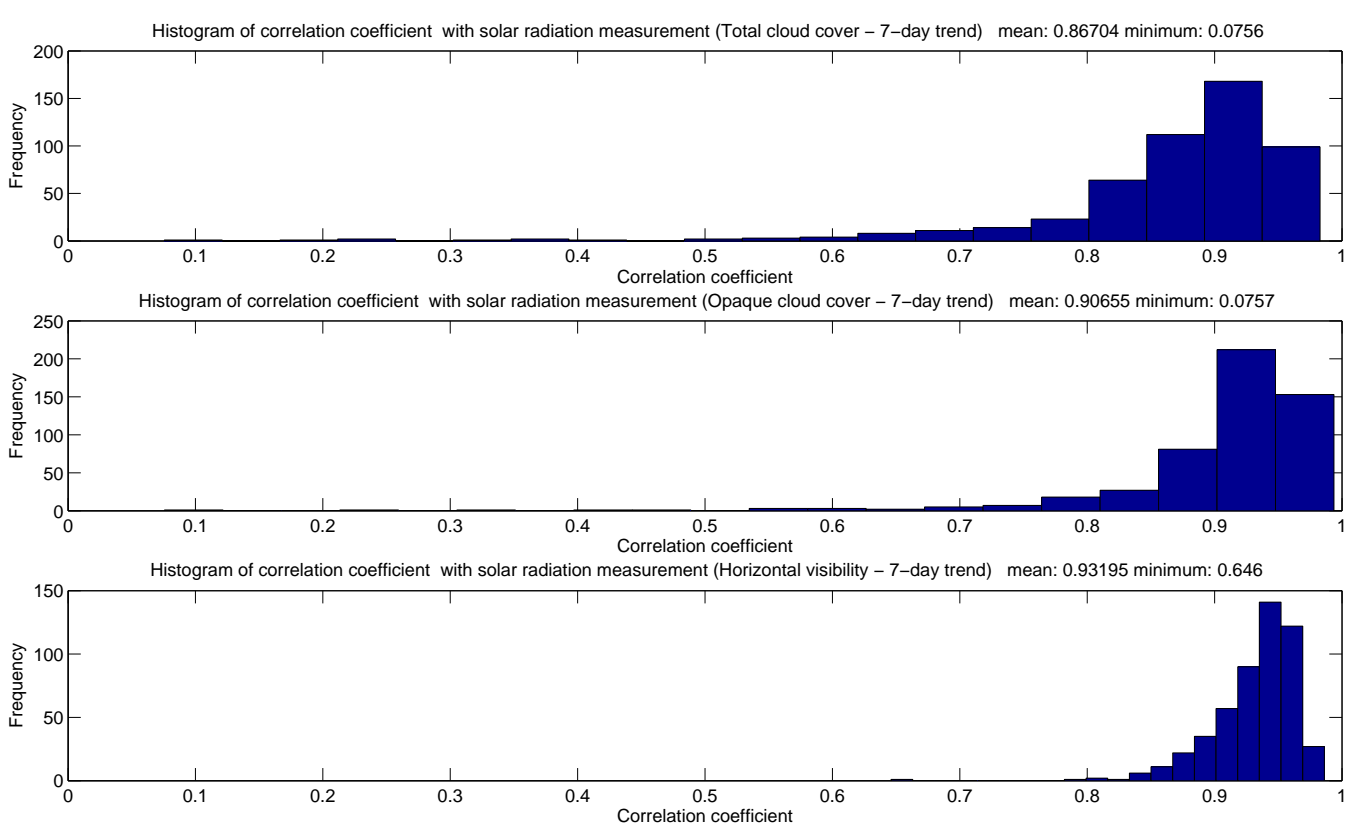

(b) Trend with 7-day sliding window

Figure 6.8: Correlation of solar radiation measurement with solar radiation estimation using visibility and cloud condition 

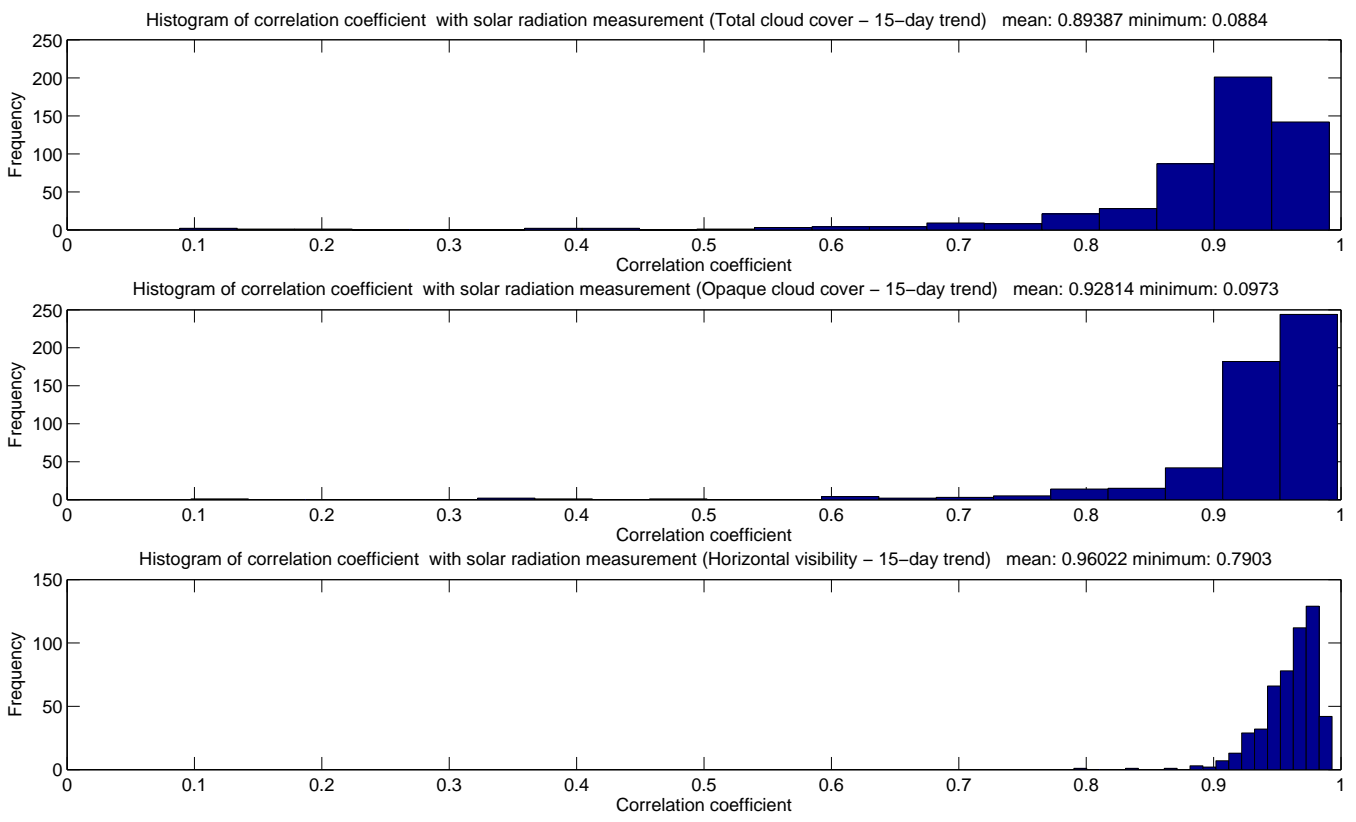

(a) Trend with 15-day sliding window
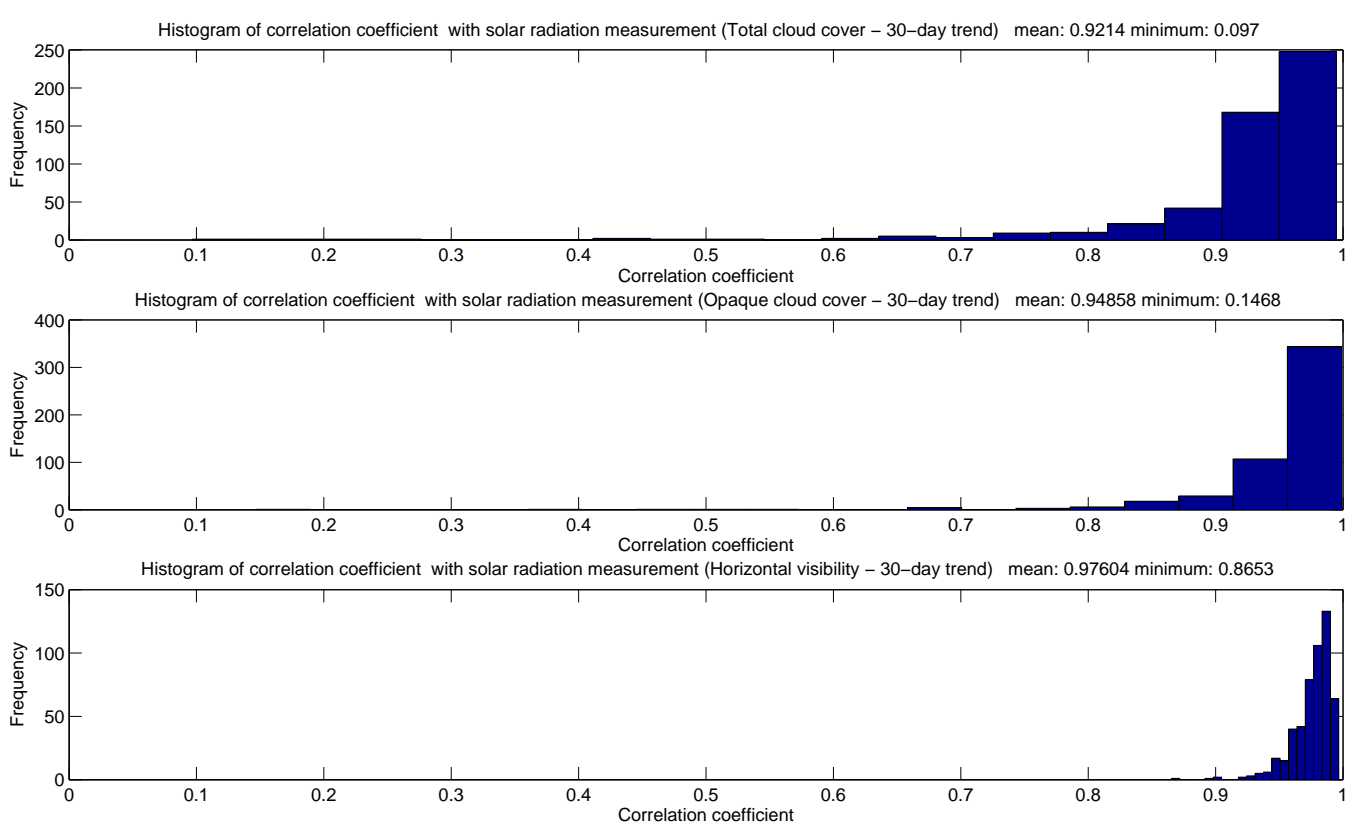

(b) Trend with 30-day sliding window

Figure 6.9: Correlation of solar radiation measurement with solar radiation estimation using visibility and cloud condition (continued) 


\begin{tabular}{|c|c|c|c|c|c|c|c|c|c|c|}
\hline Time (PST): & Temp.: & Dew Point: & Humidity: & Sea Level Pressure: & Visibility: & Wind Dir: & Wind Speed: & Gust Speed: & Precip: Events: & Conditions: \\
\hline 12:53 AM & $37.9^{\circ} \mathrm{F} / 3.3^{\circ} \mathrm{C}$ & $30.0^{\circ} \mathrm{F} /-1.1^{\circ} \mathrm{C}$ & $73 \%$ & 30.40 in / $1029.5 \mathrm{hPa}$ & $\mathbf{1 0 . 0}$ miles / $\mathbf{1 6 . 1}$ kilometers & Calm & Calm & - & N/A & Clear \\
\hline 1:53 AM & $35.1^{\circ} \mathrm{F} / 1.7^{\circ} \mathrm{C}$ & $30.0^{\circ} \mathrm{F} /-1.1^{\circ} \mathrm{C}$ & $82 \%$ & 30.41 in / $1029.8 \mathrm{hPa}$ & 9.0 miles / 14.5 kilometers & ENE & $4.6 \mathrm{mph} / 7.4 \mathrm{~km} / 2 / 2.1 \mathrm{~m} / \mathrm{s}$ & - & N/A & Clear \\
\hline 2:53 AM & $35.1^{\circ} \mathrm{F} / 1.7^{\circ} \mathrm{C}$ & $30.0^{\circ} \mathrm{F} /-1.1^{\circ} \mathrm{C}$ & $82 \%$ & $30.42 \mathrm{in} / 1029.9 \mathrm{hPa}$ & $\mathbf{1 0 . 0}$ miles / 16.1 kilometers & ENE & $4.6 \mathrm{mph} / 7.4 \mathrm{~km} / 2.1 \mathrm{~m} / \mathrm{s}$ & - & N/A & Clear \\
\hline 3:53 AM & $36.0^{\circ} \mathrm{F} / 2.2^{\circ} \mathrm{C}$ & $30.0^{\circ} \mathrm{F} /-1.1^{\circ} \mathrm{C}$ & $79 \%$ & $30.43 \mathrm{in} / 1030.3 \mathrm{hPa}$ & $\mathbf{1 0 . 0}$ miles / 16.1 kilometers & NE & $3.5 \mathrm{mph} / 5.6 \mathrm{~km} / \mathrm{h} / 1.5 \mathrm{~m} / \mathrm{s}$ & - & N/A & Clear \\
\hline 4:53 AM & $35.1^{\circ} \mathrm{F} / 1.7^{\circ} \mathrm{C}$ & $28.9^{\circ} \mathrm{F} /-1.7^{\circ} \mathrm{C}$ & $78 \%$ & 30.43 in / $1030.4 \mathrm{hPa}$ & 10.0 miles / 16.1 kilometers & NE & $3.5 \mathrm{mph} / 5.6 \mathrm{~km} / \mathrm{h} / 1.5 \mathrm{~m} / \mathrm{s}$ & - & N/A & Clear \\
\hline 5:53 AM & $36.0^{\circ} \mathrm{F} / 2.2^{\circ} \mathrm{C}$ & $30.0^{\circ} \mathrm{F} /-1.1^{\circ} \mathrm{C}$ & $79 \%$ & $30.45 \mathrm{in} / 1031.2 \mathrm{hPa}$ & 9.0 miles / 14.5 kilometers & Calm & Calm & - & N/A & Partly Cloudy \\
\hline 6:53 AM & $35.1^{\circ} \mathrm{F} / 1.7^{\circ} \mathrm{C}$ & $30.0^{\circ} \mathrm{F} /-1.1^{\circ} \mathrm{C}$ & $82 \%$ & 30.48 in / $1031.9 \mathrm{hPa}$ & 10.0 miles / 16.1 kilometers & ENE & $4.6 \mathrm{mph} / 7.4 \mathrm{~km} / \mathrm{h} / 2.1 \mathrm{~m} / \mathrm{s}$ & - & N/A & Partly Cloudy \\
\hline 7:53 AM & $36.0^{\circ} \mathrm{F} / 2.2^{\circ} \mathrm{C}$ & $30.9^{\circ} \mathrm{F} /-0.6^{\circ} \mathrm{C}$ & $82 \%$ & $30.49 \mathrm{in} / 1032.3 \mathrm{hPa}$ & $\mathbf{1 0 . 0}$ miles / 16.1 kilometers & Calm & Calm & - & N/A & Partly Cloudy \\
\hline 8:53 AM & $39.9^{\circ} \mathrm{F} / 4.4^{\circ} \mathrm{C}$ & $33.1^{\circ} \mathrm{F} / 0.6^{\circ} \mathrm{C}$ & $77 \%$ & 30.51 in / $1032.9 \mathrm{hPa}$ & 10.0 miles / 16.1 kilometers & Calm & Calm & - & N/A & Scattered Clouds \\
\hline 9:53 AM & $46.0^{\circ} \mathrm{F} / 7.8^{\circ} \mathrm{C}$ & $30.9^{\circ} \mathrm{F} /-0.6^{\circ} \mathrm{C}$ & $56 \%$ & 30.52 in / $1033.4 \mathrm{hPa}$ & $\mathbf{1 0 . 0}$ miles / 16.1 kilometers & Calm & Calm & - & N/A & Scattered Clouds \\
\hline 10:53 AM & $46.0^{\circ} \mathrm{F} / 7.8^{\circ} \mathrm{C}$ & $33.1^{\circ} \mathrm{F} / 0.6^{\circ} \mathrm{C}$ & $61 \%$ & 30.51 in / $1033.1 \mathrm{hPa}$ & 10.0 miles / 16.1 kilometers & South & $3.5 \mathrm{mph} / 5.6 \mathrm{~km} / \mathrm{h} / 1.5 \mathrm{~m} / \mathrm{s}$ & - & N/A & Scattered Clouds \\
\hline $11: 53 \mathrm{AM}$ & $48.9^{\circ} \mathrm{F} / 9.4^{\circ} \mathrm{C}$ & $33.1^{\circ} \mathrm{F} / 0.6^{\circ} \mathrm{C}$ & $54 \%$ & 30.47 in / $1031.7 \mathrm{hPa}$ & $\mathbf{1 0 . 0}$ miles / 16.1 kilometers & South & $6.9 \mathrm{mph} / 11.1 \mathrm{kmhh} / 3.1 \mathrm{~m} / \mathrm{s}$ & - & N/A & Scattered Clouds \\
\hline 12:53 PM & $48.9^{\circ} \mathrm{F} / 9.4^{\circ} \mathrm{C}$ & $34.0^{\circ} \mathrm{F} / 1.1^{\circ} \mathrm{C}$ & $56 \%$ & 30.46 in / $1031.5 \mathrm{hPa}$ & $\mathbf{1 0 . 0}$ miles / $\mathbf{1 6 . 1}$ kilometers & West & $5.8 \mathrm{mph} / 9.3 \mathrm{~km} / 2.2 .6 \mathrm{~m} / \mathrm{s}$ & - & N/A & Mostly Cloudy \\
\hline 1:53 PM & $48.9^{\circ} \mathrm{F} / 9.4^{\circ} \mathrm{C}$ & $33.1^{\circ} \mathrm{F} / 0.6^{\circ} \mathrm{C}$ & $54 \%$ & 30.45 in / $1031.0 \mathrm{hPa}$ & $\mathbf{1 0 . 0}$ miles / 16.1 kilometers & WNW & $5.8 \mathrm{mph} / 9.3 \mathrm{~km} / 2 / 2.6 \mathrm{~m} / \mathrm{s}$ & - & N/A & Mostly Cloudy \\
\hline 2:53 PM & $51.1^{\circ} \mathrm{F} / 10.6^{\circ} \mathrm{C}$ & $37.0^{\circ} \mathrm{F} / 2.8^{\circ} \mathrm{C}$ & $59 \%$ & $30.45 \mathrm{in} / 1030.9 \mathrm{hPa}$ & $\mathbf{1 0 . 0}$ miles / $\mathbf{1 6 . 1}$ kilometers & WNW & $4.6 \mathrm{mph} / 7.4 \mathrm{~km} / \mathrm{h} / 2.1 \mathrm{~m} / \mathrm{s}$ & - & N/A & Scattered Clouds \\
\hline 3.53 PM & $51.1^{\circ} \mathrm{F} / 10.6^{\circ} \mathrm{C}$ & $33.1^{\circ} \mathrm{F} / 0.6^{\circ} \mathrm{C}$ & $50 \%$ & 30.45 in / $1031.0 \mathrm{hPa}$ & $\mathbf{1 0 . 0}$ miles / $\mathbf{1 6 . 1}$ kilometers & NW & $3.5 \mathrm{mph} / 5.6 \mathrm{~km} / \mathrm{h} / 1.5 \mathrm{~m} / \mathrm{s}$ & - & N/A & Mostly Cloudy \\
\hline 4:53 PM & $48.9^{\circ} \mathrm{F} / 9.4^{\circ} \mathrm{C}$ & $37.0^{\circ} \mathrm{F} / 2.8^{\circ} \mathrm{C}$ & $64 \%$ & $30.45 \mathrm{in} / 1030.9 \mathrm{hPa}$ & $\mathbf{1 0 . 0}$ miles / $\mathbf{1 6 . 1}$ kilometers & NNM & $3.5 \mathrm{mph} / 5.6 \mathrm{~km} / 1 / 1.5 \mathrm{~m} / \mathrm{s}$ & - & N/A & Mostly Cloudy \\
\hline $5: 53 \mathrm{PM}$ & $46.0^{\circ} \mathrm{F} / 7.8^{\circ} \mathrm{C}$ & $33.1^{\circ} \mathrm{F} / 0.6^{\circ} \mathrm{C}$ & $61 \%$ & $30.48 \mathrm{in} / 1032.2 \mathrm{hPa}$ & 10.0 miles / 16.1 kilometers & NMW & $5.8 \mathrm{mph} / 9.3 \mathrm{~km} / 2 / 2.6 \mathrm{~m} / \mathrm{s}$ & - & N/A & Mostly Cloudy \\
\hline 6:53 PM & $45.0^{\circ} \mathrm{F} / 7.2^{\circ} \mathrm{C}$ & $30.9^{\circ} \mathrm{F} /-0.6^{\circ} \mathrm{C}$ & $58 \%$ & $30.48 \mathrm{in} / 1032.1 \mathrm{hPa}$ & $\mathbf{1 0 . 0}$ miles / $\mathbf{1 6 . 1}$ kilometers & East & $4.6 \mathrm{mph} / 7.4 \mathrm{~km} / \mathrm{h} / 2.1 \mathrm{~m} / \mathrm{s}$ & - & N/A & Mostly Cloudy \\
\hline 7:53 PM & $43.0^{\circ} \mathrm{F} / 6.1^{\circ} \mathrm{C}$ & $34.0^{\circ} \mathrm{F} / 1.1^{\circ} \mathrm{C}$ & $71 \%$ & 30.50 in / $1032.6 \mathrm{hPa}$ & $\mathbf{1 0 . 0}$ miles / 16.1 kilometers & ENE & $5.8 \mathrm{mph} / 9.3 \mathrm{~km} / 2 / 2.6 \mathrm{~m} / \mathrm{s}$ & - & N/A & Mostly Cloudy \\
\hline 8:53 PM & $45.0^{\circ} \mathrm{F} / 7.2^{\circ} \mathrm{C}$ & $33.1^{\circ} \mathrm{F} / 0.6^{\circ} \mathrm{C}$ & $63 \%$ & 30.49 in / $1032.3 \mathrm{hPa}$ & 10.0 miles / 16.1 kilometers & East & $3.5 \mathrm{mph} / 5.6 \mathrm{~km} / \mathrm{h} / 1.5 \mathrm{~m} / \mathrm{s}$ & - & N/A & Mostly Cloudy \\
\hline 9:53 PM & $43.0^{\circ} \mathrm{F} / 6.1^{\circ} \mathrm{C}$ & $32.0^{\circ} \mathrm{F} / 0.0^{\circ} \mathrm{C}$ & $65 \%$ & 30.50 in / $1032.8 \mathrm{hPa}$ & $\mathbf{1 0 . 0}$ miles / $\mathbf{1 6 . 1}$ kilometers & North & $6.9 \mathrm{mph} / 11.1 \mathrm{kmh} / 3.1 \mathrm{~m} / \mathrm{s}$ & - & N/A & Mostly Cloudy \\
\hline 10:53 PM & $44.1^{\circ} \mathrm{F} / 6.7^{\circ} \mathrm{C}$ & $32.0^{\circ} \mathrm{F} / 0.0^{\circ} \mathrm{C}$ & $63 \%$ & $30.50 \mathrm{in} / 1032.8 \mathrm{hPa}$ & 10.0 miles / 16.1 kilometers & East & $3.5 \mathrm{mph} / 5.6 \mathrm{~km} / \mathrm{h} / 1.5 \mathrm{~m} / \mathrm{s}$ & - & N/A & Scattered Clouds \\
\hline \multirow[t]{2}{*}{ 11:53 PM } & $45.0^{\circ} \mathrm{F} / 7.2^{\circ} \mathrm{C}$ & $30.0^{\circ} \mathrm{F} /-1.1^{\circ} \mathrm{C}$ & $56 \%$ & 30.49 in / $1032.3 \mathrm{hPa}$ & $\mathbf{1 0 . 0}$ miles / 16.1 kilometers & Calm & Calm & - & N/A & Scattered Clouds \\
\hline & & & & Show full METARS & METAR FAQ & & omma Delinited File & & & \\
\hline
\end{tabular}

Figure 6.10: Hourly weather data in Oakland, CA on 12/22/2007 from a publicly available weather station

ported as number of miles between 0 and 10. The cloud condition is reported in five different grades depending on the percentage of sky being covered: clear (0), partly cloudy (1/8 - 2/8), scattered clouds (3/8 - 4/8), mostly cloudy (5/8 - 7/8), and overcast or fog (1). To estimate solar radiation under weather effect, we need to translate a weather metric into the weather factor $W(t)$. The simplest way is to translate these metrics in a linear scale.

The weather factor for cloud conditions can be defined using the percentage of sky covered by clouds:

$$
W(t)= \begin{cases}1 \quad=1-0) & \text { clear } \\ 0.8125 \quad(=1-3 / 16) & \text { partly cloudy } \\ 0.5625(=1-7 / 16) & \text { scatter clouds } \\ 0.25(=1-3 / 4) & \text { mostly cloudy } \\ 0(=1-1) & \text { overcast or fog }\end{cases}
$$

The weather factor for horizontal visibility $\operatorname{Vis}(t)$ can be defined as follows using 


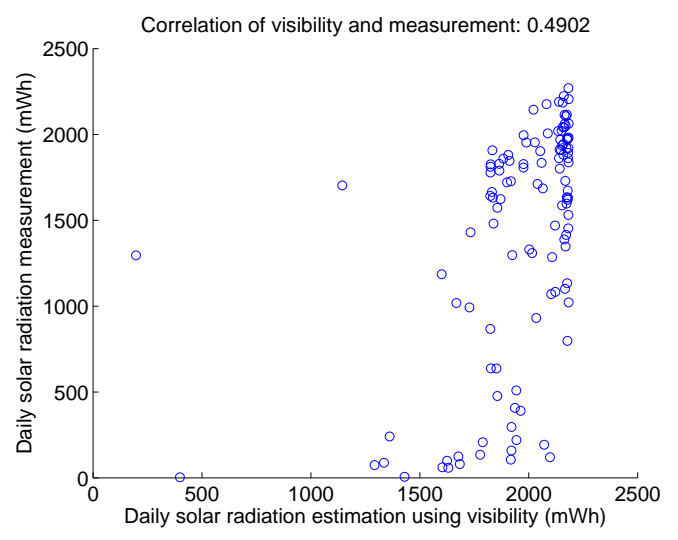

(a) Horizontal Visibility

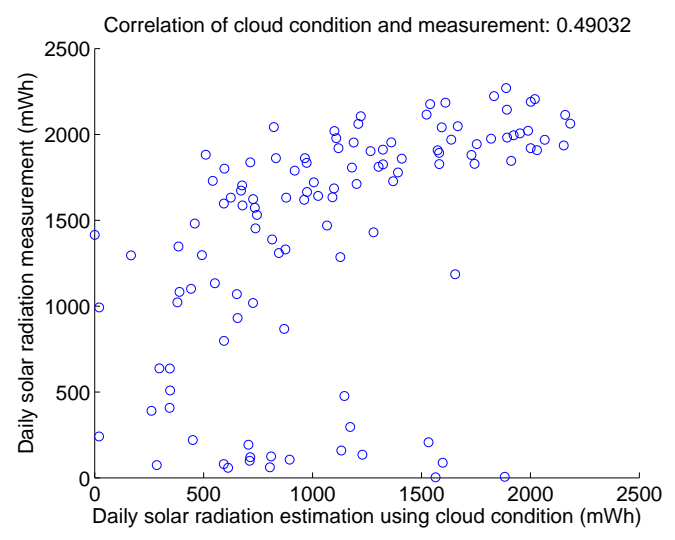

(b) Cloud Condition

Figure 6.11: Correlation of horizontal visibility and cloud conditions to the weather variation of the solar radiation using a linear scale mapping (Node A02 from $12 / 22 / 2007$ to $04 / 15 / 2008$ )

the observation by Peterson et al. [PFR78] that solar radiation is highly correlated with visibility for visibility of less than or equal to a threshold of $12 \mathrm{~km}\left(=\mathrm{Vis}_{k n e e}\right)$ :

$$
W(t)= \begin{cases}1 & \operatorname{Vis}(t) \leq \operatorname{Vis}_{k n e e} \\ \operatorname{Vis}(t) / \operatorname{Vis}_{\text {knee }} & \text { otherwise }\end{cases}
$$

Figure 6.11(a) and 6.11(b) shows a correlation between the estimation and measurement of solar radiation when we use horizontal visibility and cloud conditions with linear scale mapping. In both cases, correlations are not very high with correlation coefficients being less than 0.5. This implies that estimating solar radiation with linear scale mapping is not very meaningful.

However, these weather metrics show different trends if they are divided into smaller subgroups. Figure 6.12 shows a correlation between the solar radiation estimation and measurement when the cloud condition is divided into five different subgroups. We can see that there is a high correlation between the solar radiation estimation and measurement with a correlation coefficient of each subgroup between 0.79 and 0.99. Figure 6.13 shows a correlation between the solar radiation estimation and measurement when horizontal visibility is divided into 11 different groups: $W_{1}=[0,1), W_{2}=[1,2), W_{3}=[2,3), \cdots, W_{10}=[9,10), W_{11}=\{10\}$. We can see that with horizontal visibility the correlation between the solar radiation estimation 

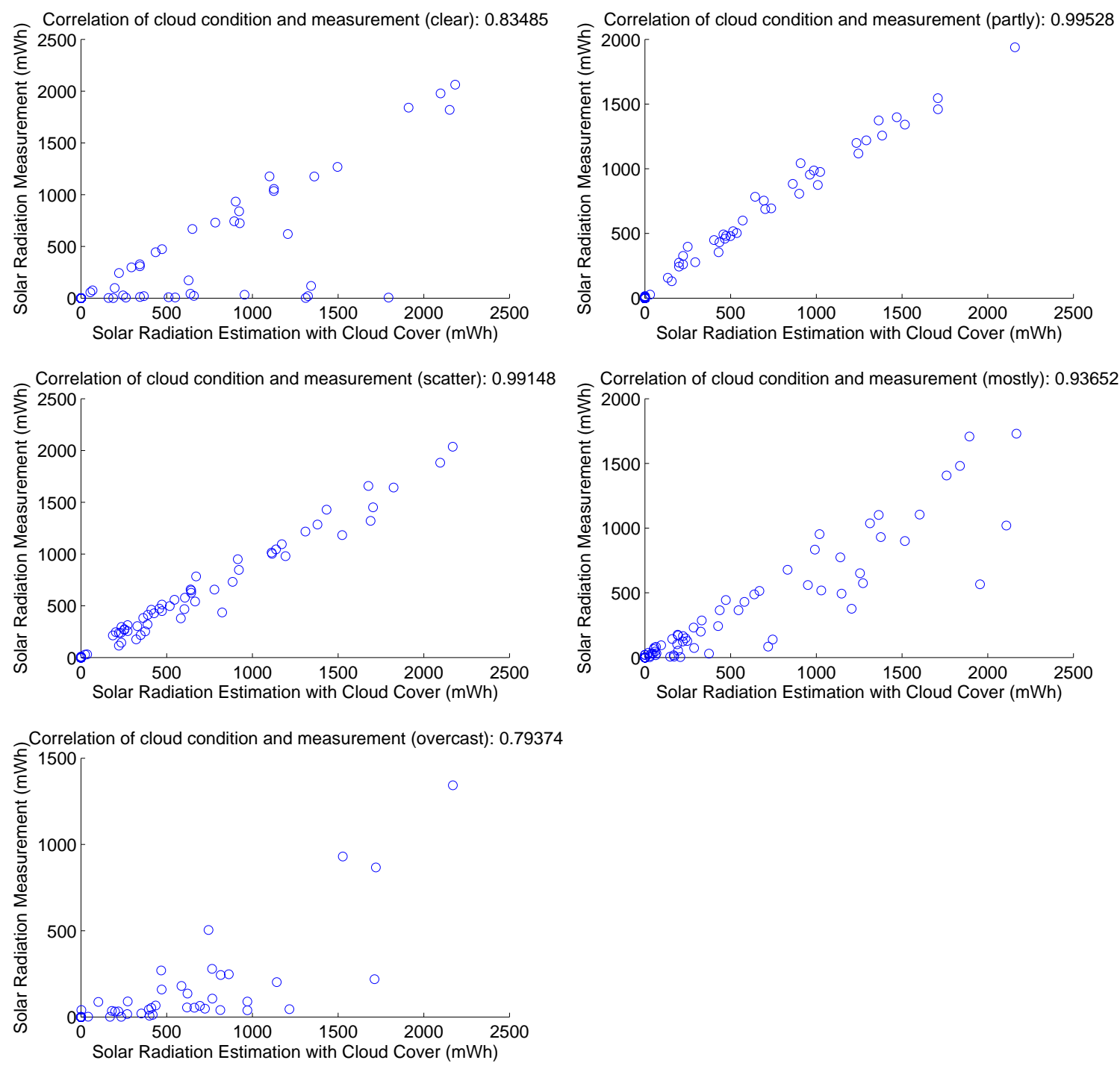

Figure 6.12: Correlation trends for each disjoint set of cloud conditions 

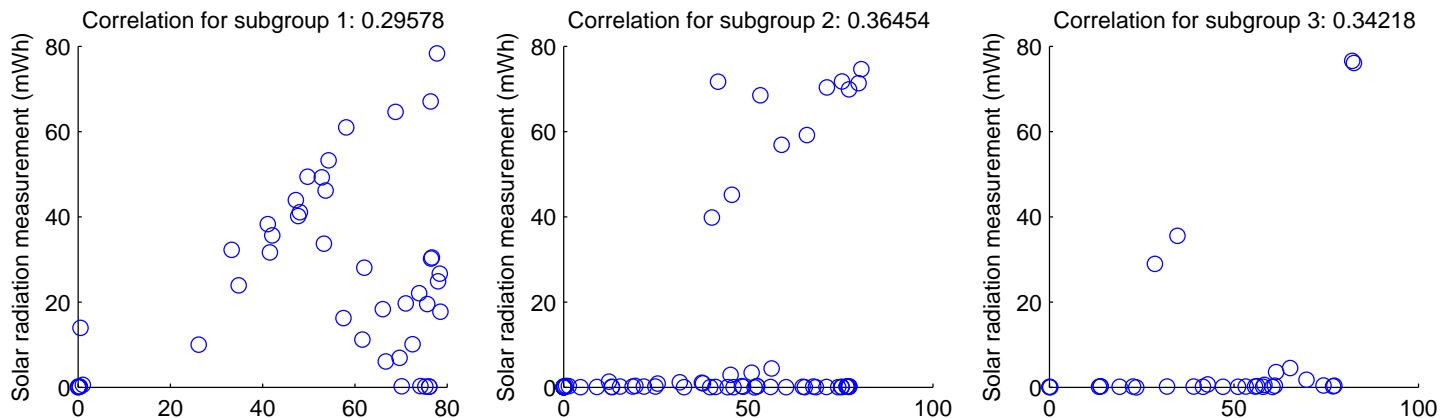

Solar radiation estimation with visibility $(\mathrm{mWh})$ Solar radiation estimation with visibility $(\mathrm{mWh})$ Solar radiation estimation with visibility (mWh)
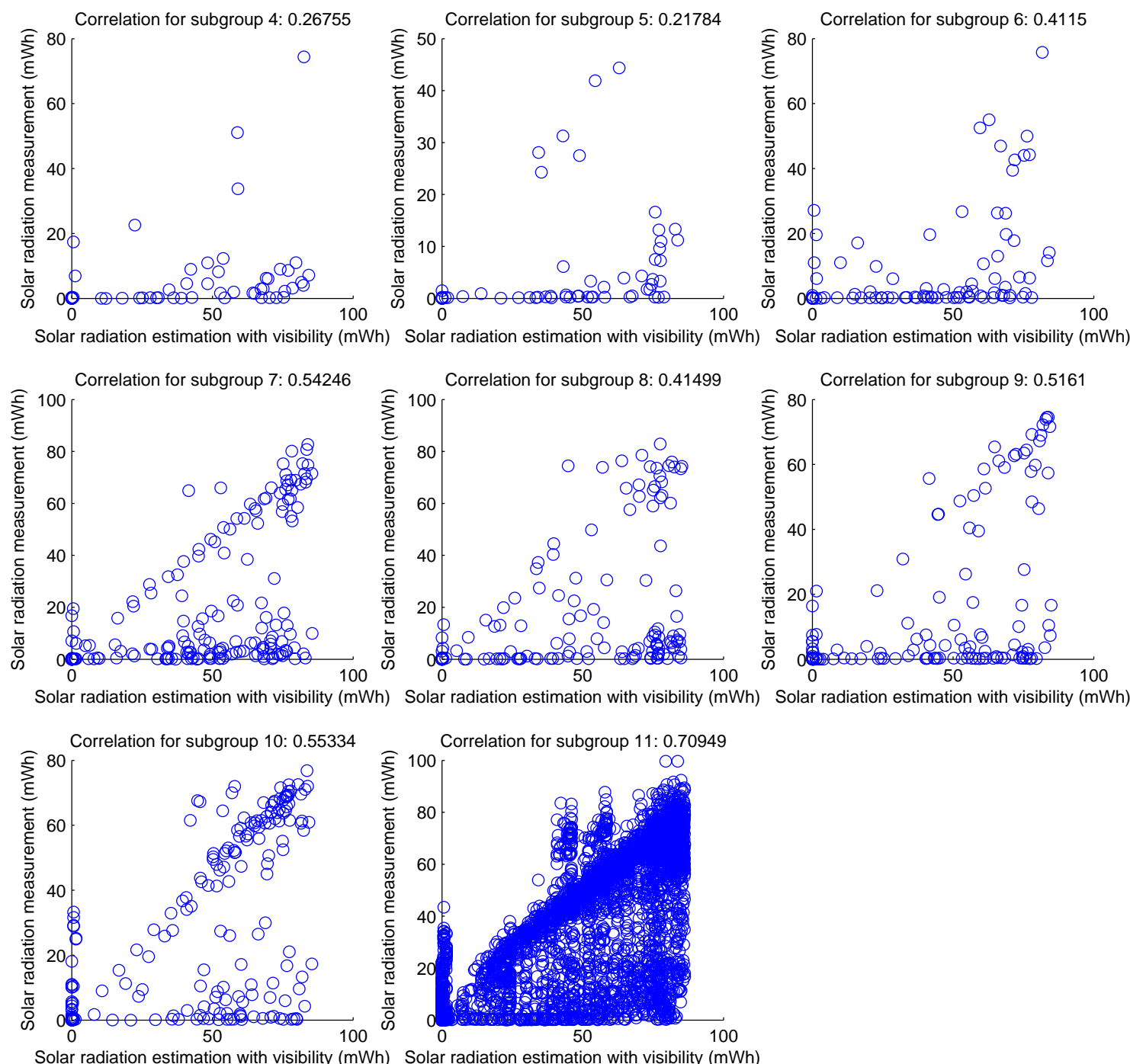

Figure 6.13: Correlation trends for each disjoint set of visibility 


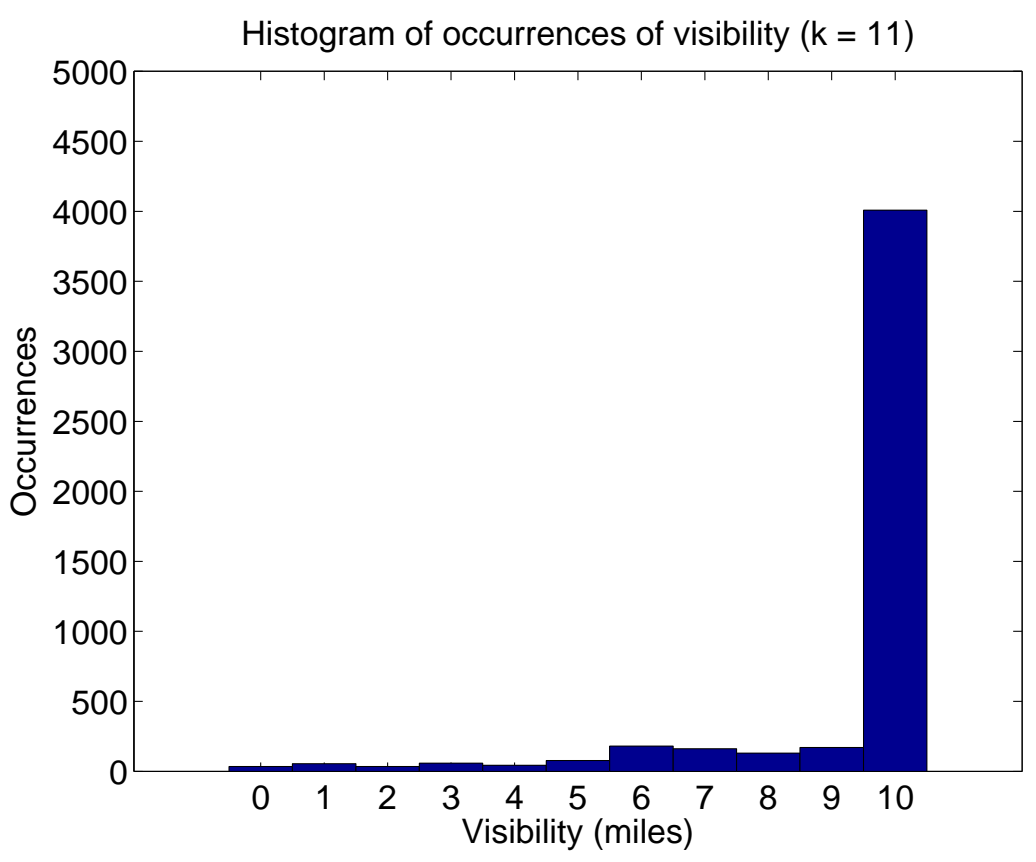

Figure 6.14: Occurences (frequency) of each disjoint set of visibility

and measurement is low except for the group with the highest visibility, $W_{11}$, which has a correlation coefficient of 0.7 . While horizontal visibility has a low correlation for groups with lower visibility, the histogram in Figure 6.14 shows that groups with low correlation is insignificant in terms of occurences.

\subsubsection{Calibrating Solar Radiation Estimators}

We have shown that we can achieve a relatively high correlation between the solar radiation estimation and measurement by dividing a weather metric into multiple subgroups. Since a weather metric has a different correlation coefficient depending on which subgroup it belongs to, the weather factor also needs to be defined for each subgroup.

The weather factor $W(t)$ can be calibrated using the probability distribution of the ratio between measurements and estimations for sample points of each subgroup. Suppose

- The values of weather metrics can be partitioned into $k$-disjoint subsets $W_{1}$, 
$W_{2}, \cdots, W_{k}$

- $r_{i}$, the ratio of the solar radiation measurements over solar radiation estimations using an obstructed astronomical model for subset $W_{i}$ on day $n$, is given by

$$
r_{i}(n)=\frac{Y_{i}(n)}{X_{i}(n)}=\frac{\int_{W(t) \in W_{i}} M(n, t) d t}{\int_{W(t) \in W_{i}} A S T(n, t) d t}
$$

- $r_{i}$ 's belong to $\left[0, r_{\max }\right]$, and the set of $r_{i}$ 's, $\left\{r_{i}\right\}$, is divided into $(m+1)$ equallyspaced bins $B_{0}, B_{1}, \cdots, B_{j}, \cdots, B_{m}$, where

$$
\begin{aligned}
B_{j} & =\left\{\frac{Y_{i}}{X_{i}} \mid \frac{j}{m} \cdot r_{\max } \leq \frac{Y_{i}}{X_{i}}<\frac{j+1}{m} \cdot r_{\max }\right\} \quad \text { for } j=0, \cdots,(m-1) \\
B_{m} & =\left\{\frac{Y_{i}}{X_{i}} \mid \frac{Y_{i}}{X_{i}} \geq r_{\max }\right\} \quad \text { for } j=m
\end{aligned}
$$

Then, the probability that the ratio $r_{i}$ belongs to a bin $B_{j}$ is

$$
P\left(r_{i} \in B_{j}\right)=\frac{\left|B_{j}\right|}{\left|B_{\text {all }}\right|}
$$

where $B_{\text {all }}$ is a union of $B_{0}$ through $B_{m}$. As a calibration factor, we use the expectation of $r_{i}, E\left(r_{i}\right)$ for each subset $W_{i}$.

We can describe this process in a more concrete way for cloud conditions and visibility. For cloud conditions, the $k$-disjoint subsets can be defined as follows:

$$
\begin{aligned}
& W_{1}=\{\text { clear }\} \\
& W_{2}=\{\text { partly cloudy }\} \\
& W_{3}=\{\text { scatter clouds }\} \\
& W_{4}=\{\text { mostly cloudy }\} \\
& W_{5}=\{\text { overcast }\}
\end{aligned}
$$

A set of $\left(X_{i}, Y_{i}\right)$ pairs for subset $i$ are the $(\mathrm{x}, \mathrm{y})$ coordinates in $i$-th scatter plot of Figure 6.12. If we partition a set $\left\{\frac{Y_{i}}{X_{i}}\right\}$ into 21 bins over domain $[0,2]$, then we can get a probability distribution as shown in Figure 6.15. Figure 6.15 also shows that the mean value of $\frac{Y_{i}}{X_{i}}$ for each subset. The overall trend is that the calibration factor becomes smaller as weather becomes more overcast. One exception is the calibration factor 
for the clear condition. The calculation from the sample points gives a calibration factor of 0.5341 . Since we expect calibration factors to be monitonically descreasing as cloud conditions become more overcast, we set the calibration factor for the clear condition as 0.9683 , which is the calibration factor for the partly cloudy condition.

For horizontal visibility, which is a real number between 0 and $10, k$-disjoint subsets can be defined by partitioning the domain as follows:

$$
\begin{aligned}
W_{1} & =\left[0, \frac{10}{k-1}\right) \\
W_{2} & =\left[\frac{10}{k-1}, \frac{20}{k-1}\right) \\
W_{3} & =\left[\frac{20}{k-1}, \frac{30}{k-1}\right) \\
& \vdots \\
W_{k-1} & =\left[\frac{10 \cdot(k-2)}{k-1}, \frac{10 \cdot(k-1)}{k-1}\right) \\
W_{k} & =\{10\}
\end{aligned}
$$

If we consider possible values for $k=11, k$-disjoint sets can be given as follows: $[0,1)$, $[1,2),[2,3), \cdots,[9,10),\{10\}$.

A set of $\left(X_{i}, Y_{i}\right)$ pairs for subset $i$ are plotted in $i$-th scatter plot of Figure 6.13. If we partition a set of $\left\{\frac{Y_{i}}{X_{i}}\right\}$ into 21 bins over domain [0,2], then we can get a probability distribution as shown in Figure 6.16. As a calibration factor for each subset $W_{i}$, we can use the expectation of $\frac{Y_{i}}{X_{i}}$, which is shown as a vertical line in each subplot. Since horizontal visibility is given as a real number between 0 and 10 rather than a discrete set of numbers, the calibration factor should be a continuous calibration function. The desirable properties for a calibration function are to minimize the difference between the measurements and the estimations of solar radiation and to monotonically increase as horizontal visibility increases. We have considered an exponential curve and a polynomical curve as a possible calibration function, and curve fitting with measurements and estimations of solar radiation at the Valley Life Science Building deployment has shown that an exponential curve is the best candidate. The exponential calibration function that converts a given horizontal visibility $x$ to a number 
Probability distribution of measurement over estimation ratio for cloud condition (clear)

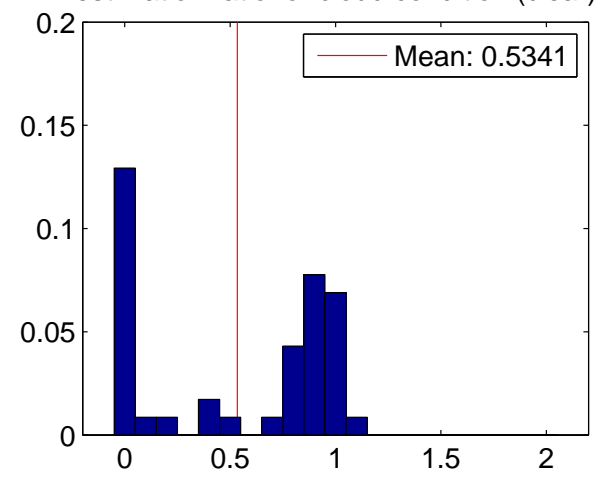

Probability distribution of measurement over estimation ratio for cloud condition (scatter)

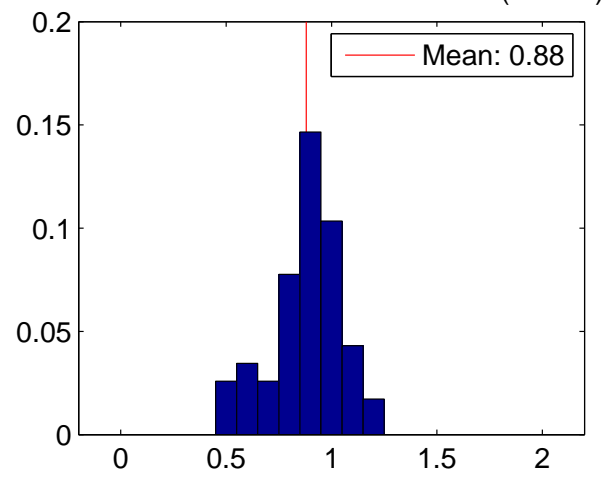

Probability distribution of measurement over estimation ratio for cloud condition (partly)

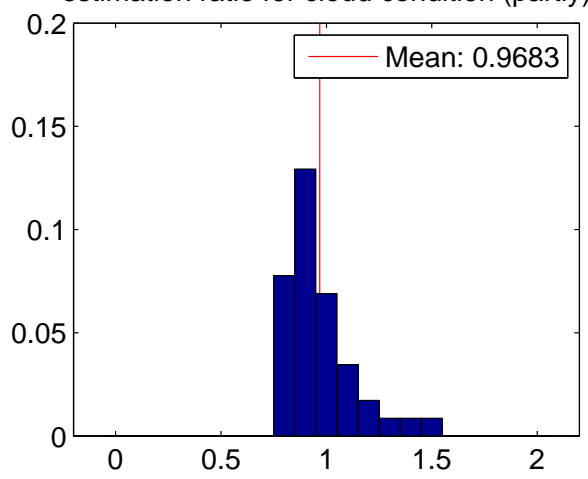

Probability distribution of measurement over estimation ratio for cloud condition (mostly)

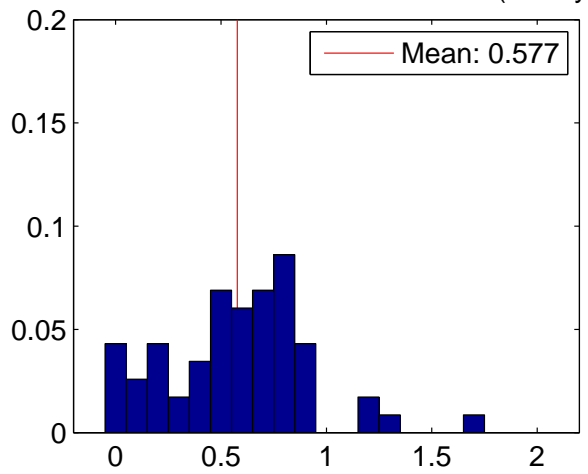

Probability distribution of measurement over estimation ratio for cloud condition (overcast)

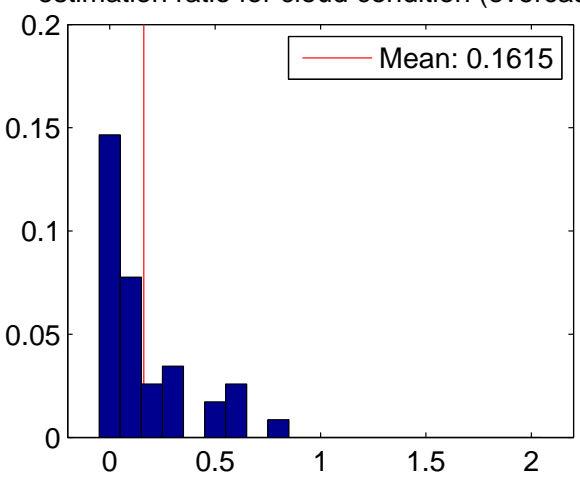

\begin{tabular}{|c|c|c|c|c|c|}
\hline & $c_{1}$ & $c_{2}$ & $c_{3}$ & $c_{4}$ & $c_{5}$ \\
\hline Calibration factor & 0.9683 & 0.0983 & 0.8800 & 0.5770 & 0.1615 \\
\hline
\end{tabular}

Figure 6.15: Probability distribution of calibration factor for each disjoint set of cloud conditions 
$y \in[0,1]$ is given as follows:

$$
y=a \cdot \exp (b \cdot x) \quad a: 0.06759 \text { and } b: 0.2324
$$

Figure 6.17 shows this exponential calibration function.

\subsubsection{Predicting Solar Radiation using the History of a Weather Effect Component}

We have shown how to estimate solar radiation using a weather metric and how to calibrate such a solar radiation estimator when a sample of measurements are given for solar radiation and the weather metrics. However, these measurements of solar radiation and weather metrics are given only for the past and the present. In order to predict solar radiation under a weather effect for the future, we need a different model.

A possible model is to predict solar radiation using a recent history of weather metrics. Figure 6.18 shows an algorithm that estimates the solar radiation for day $n$ using the astronomical model and the recent $k$-day history of weather metrics. As a weather factor for day $n$, this algorithm uses the ratio of RW_HIST(n) and AST_HIST(n), which are the recent $k$-day average of the solar radiation estimation using the weather effect and solar radiation estimation using an obstructed astronomical model. At the end of each iteration, the algorithm calculates RW(n), which is the solar radiation estimation using a weather effect for day $n$, and updates the history file with RW(n) so that a later iteration can look it up as history.

We estimated solar radiation using the cloud-based model and the visibility-based model for the nodes in the VLSB deployment. Figures 6.19 and 6.20 show the trend of solar radiation prediction with the models with different window sizes $(1,3,7$ and 30 days). Figure 6.21 shows the trend of the deviation between the prediction and the measurement as we increase the window size. We can see that the deviation is relatively high with a window size of 1 day, but it stabilizes after 3 days and converges after 7 days.

Table 6.4 shows the deviation of the prediction from the measurement when we use a window size of 3 and 7 days. We can see that with the cloud-based model, we can estimate the trend of solar radiation within $6 \%$ of the measured value whereas 
Probability distribution of ratio of measurement over estimation for $\mathrm{W}_{1}$

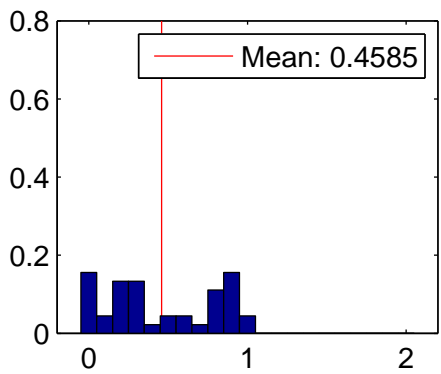

Probability distribution of ratio of measurement over estimation for $\mathrm{W}_{4}$

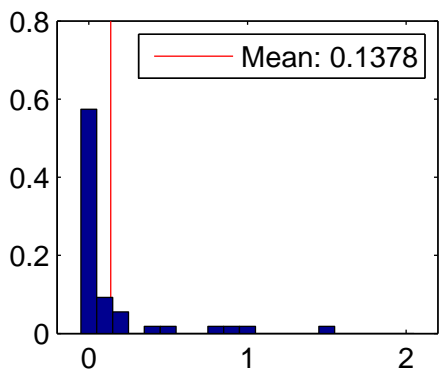

Probability distribution of ratio of measurement over estimation for $\mathrm{W}_{2}$

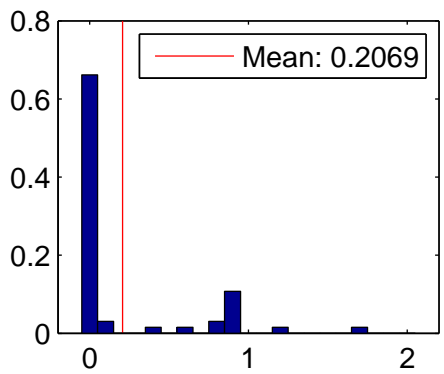

Probability distribution of ratio of measurement over estimation for $\mathrm{W}_{5}$

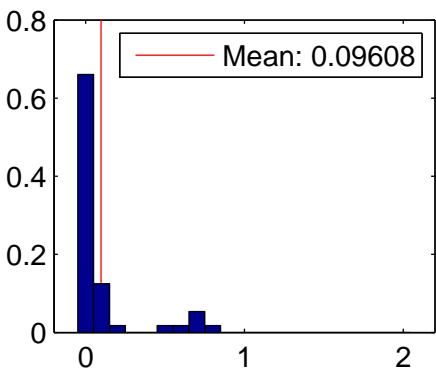

Probability distribution of ratio of measurement over estimation for $\mathrm{W}_{3}$

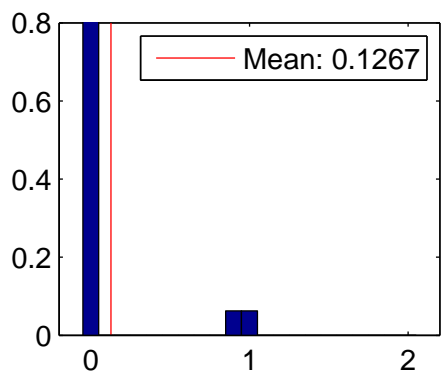

Probability distribution of ratio of measurement over estimation for $\mathrm{W}_{7}$

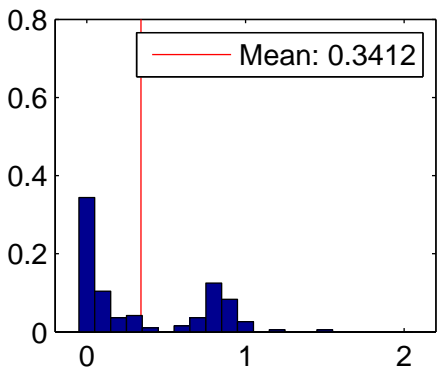

Probability distribution of ratio of measurement over estimation for $\mathrm{W}_{8}$

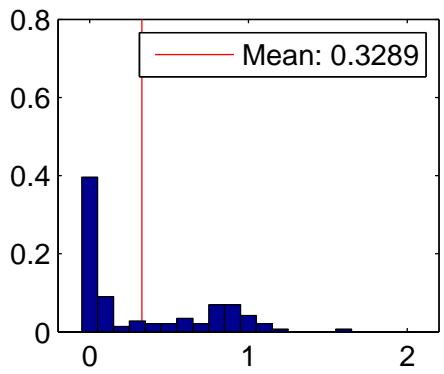

Probability distribution of ratio of measurement over estimation for $\mathrm{W}_{6}$

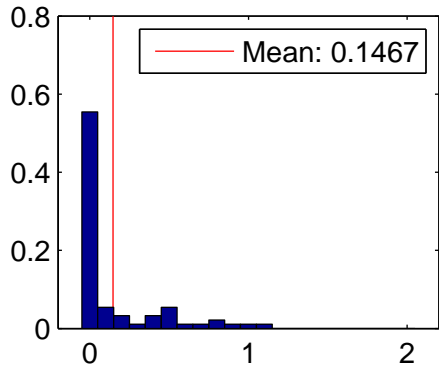

Probability distribution of ratio of measurement over estimation for $\mathrm{W}_{9}$

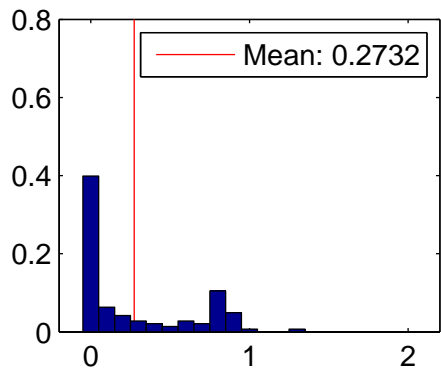

Probability distribution of ratio of measurement over estimation for $\mathrm{W}_{10}$

Probability distribution of ratio of

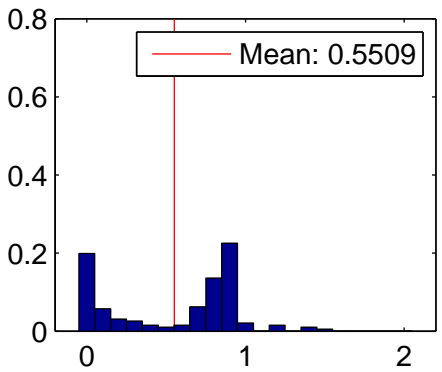
measurement over estimation for $\mathrm{W}_{11}$

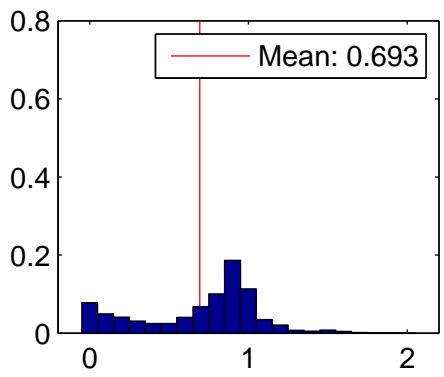

Figure 6.16: Probability distribution of calibration factor for each disjoint set of visibility 


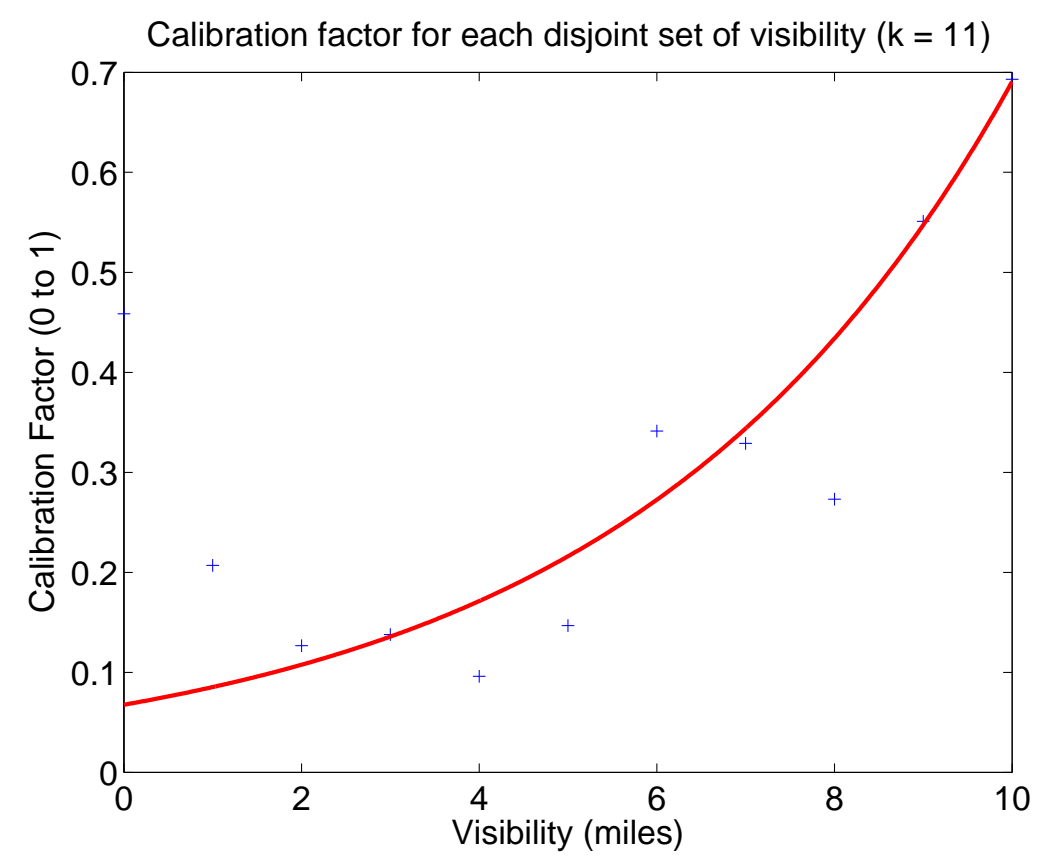

Figure 6.17: An exponential curve that fits the calibration factors with minimum errors.

not using a weather model gives an approximate $26 \%$ estimation error. We can also see that with the visibility-based mode, the estimation error is about $20 \%$ and the predictability is not as high as that of the cloud-based model.

\subsubsection{Summary}

In general, solar radiation can be estimated with three different models depending on their level of fidelity: an astronomical model estimates solar radiation in an ideal situation considering only the geometry of the sun and the solar panel; an obstructed astronomical model refines the astronomical model when the solar panel is blocked by local objects; and a weather-metric model further refines the obstructed astronomical model by adjusting the solar radiation estimation by the amount of weather effects.

In this section, we have developed and evaluated a weather-metric based model using cloud conditions and horizontal visibility, which are commonly measured in a number of weather stations and widely available. With the condition of a weather metric given, our weather metric model adjusts the solar radiation estimation of 
Require: $n$ : day to estimate solar radiation of

hist: history of solar radiation estimation

$k$ : number of days to look up the history (window size)

1: Evaluate $\operatorname{AST}(\mathrm{n})$

AST(n) $\leftarrow$ Estimation of solar radiation using an obstructed astronomical model for day $n$

2: Evaluation AST_HIST(n) from hist

$\operatorname{AST} \_H I S T(n) \leftarrow \frac{1}{k} \sum_{i=n-k}^{n-1} A S T(i)$

3: Evaluate RW_HIST(n) from hist

RW_HIST(n) $\leftarrow \frac{1}{k} \sum_{i=n-k}^{n-1} R W(i)$

4: Report RW_PRDT(n) as a solar radiation prediction for day $n$

RW_PRDT(n) $\leftarrow$ AST(n) $\times($ RW_HIST(n) / AST_HIST(n)

5: Evaluate RW(n)

RW(n) $\leftarrow$ Estimation of solar radiation using a weather factor for day $n$

6: Update hist with AST(n) and RW(n)

Figure 6.18: Algorithm for predicting solar radiation with $k$-day history of weather effects

Table 6.4: Deviation for different nodes

(a) With a 3-day window

\begin{tabular}{|c|r|r|r|r|r|r|r|}
\hline Models & A02 & A03 & A08 & A10 & A11 & Mean & Median \\
\hline Obstructed & $32.02 \%$ & $20.25 \%$ & $28.68 \%$ & $20.66 \%$ & $28.67 \%$ & $26.06 \%$ & $28.67 \%$ \\
\hline Cloud & $4.39 \%$ & $12.59 \%$ & $0.11 \%$ & $10.98 \%$ & $0.63 \%$ & $5.74 \%$ & $4.39 \%$ \\
Visibility & $11.61 \%$ & $31.63 \%$ & $16.60 \%$ & $30.56 \%$ & $17.67 \%$ & $21.61 \%$ & $17.67 \%$ \\
\hline
\end{tabular}

(b) With a 7-day window

\begin{tabular}{|c|r|r|r|r|r|r|r|}
\hline Models & A02 & A03 & A08 & A10 & A11 & Mean & Median \\
\hline Obstructed & $32.02 \%$ & $20.25 \%$ & $28.68 \%$ & $20.66 \%$ & $28.67 \%$ & $26.06 \%$ & $28.67 \%$ \\
\hline Cloud & $5.97 \%$ & $10.15 \%$ & $1.69 \%$ & $9.01 \%$ & $1.44 \%$ & $5.65 \%$ & $5.97 \%$ \\
Visibility & $9.69 \%$ & $31.63 \%$ & $14.73 \%$ & $28.47 \%$ & $15.61 \%$ & $19.57 \%$ & $15.61 \%$ \\
\hline
\end{tabular}



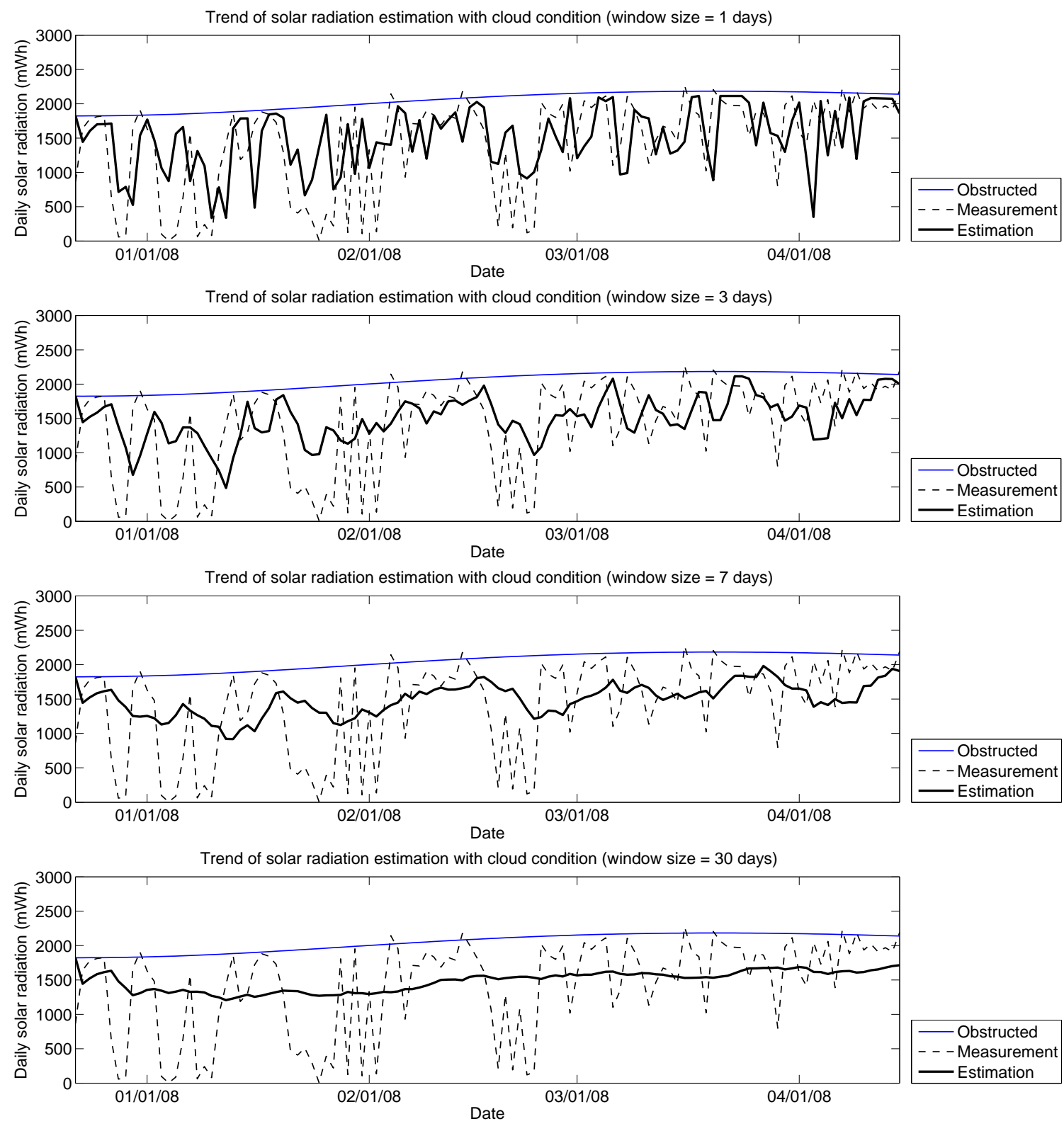

Figure 6.19: Solar radiation prediction with the cloud-based model with different window sizes (node A02 in the VLSB deployment from 12/22/2007 to 04/15/2008) 

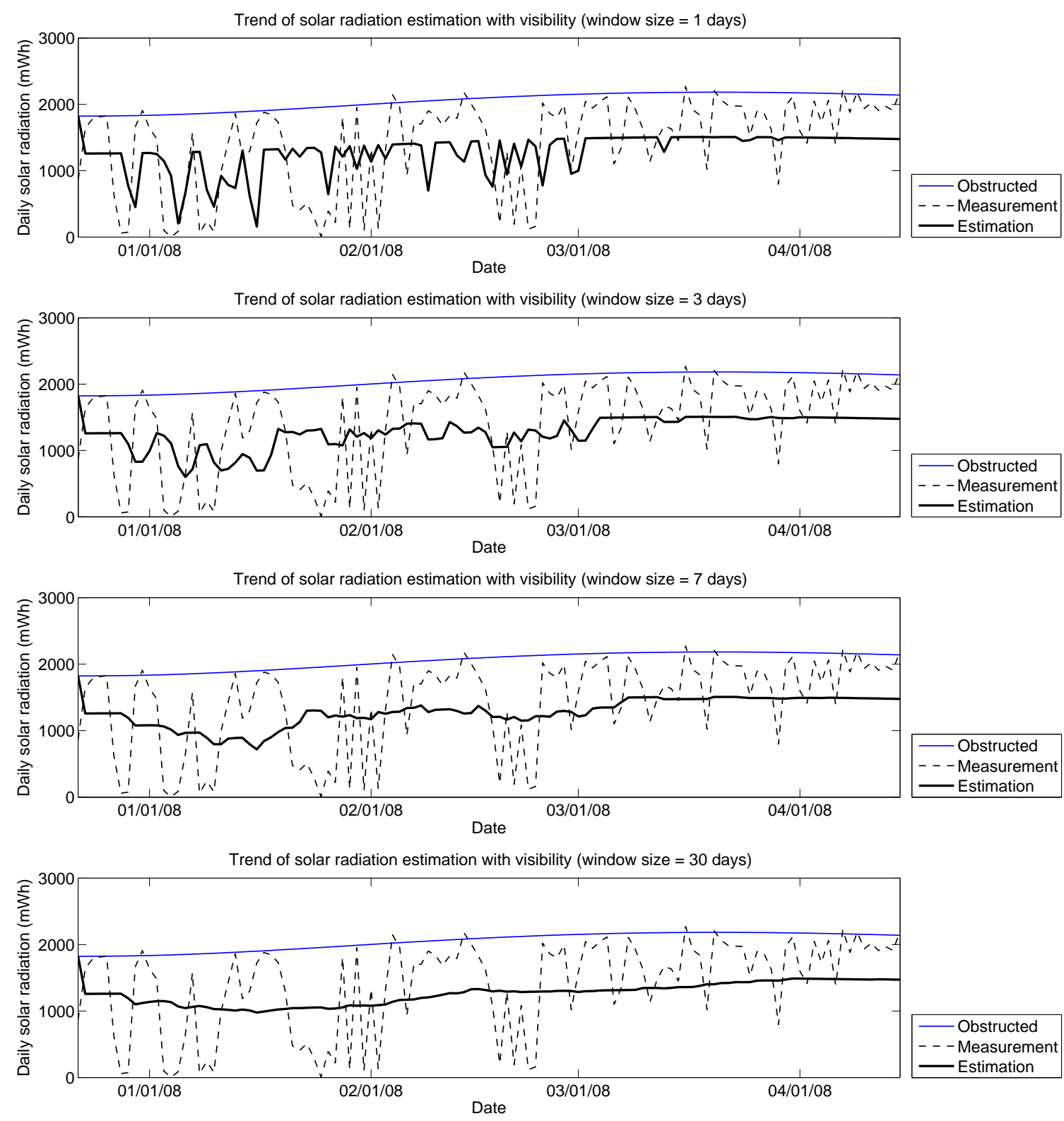

Figure 6.20: Solar radiation prediction with the visibility-based model with different window sizes (node A02 in the VLSB deployment from 12/22/2007 to 04/15/2008) 

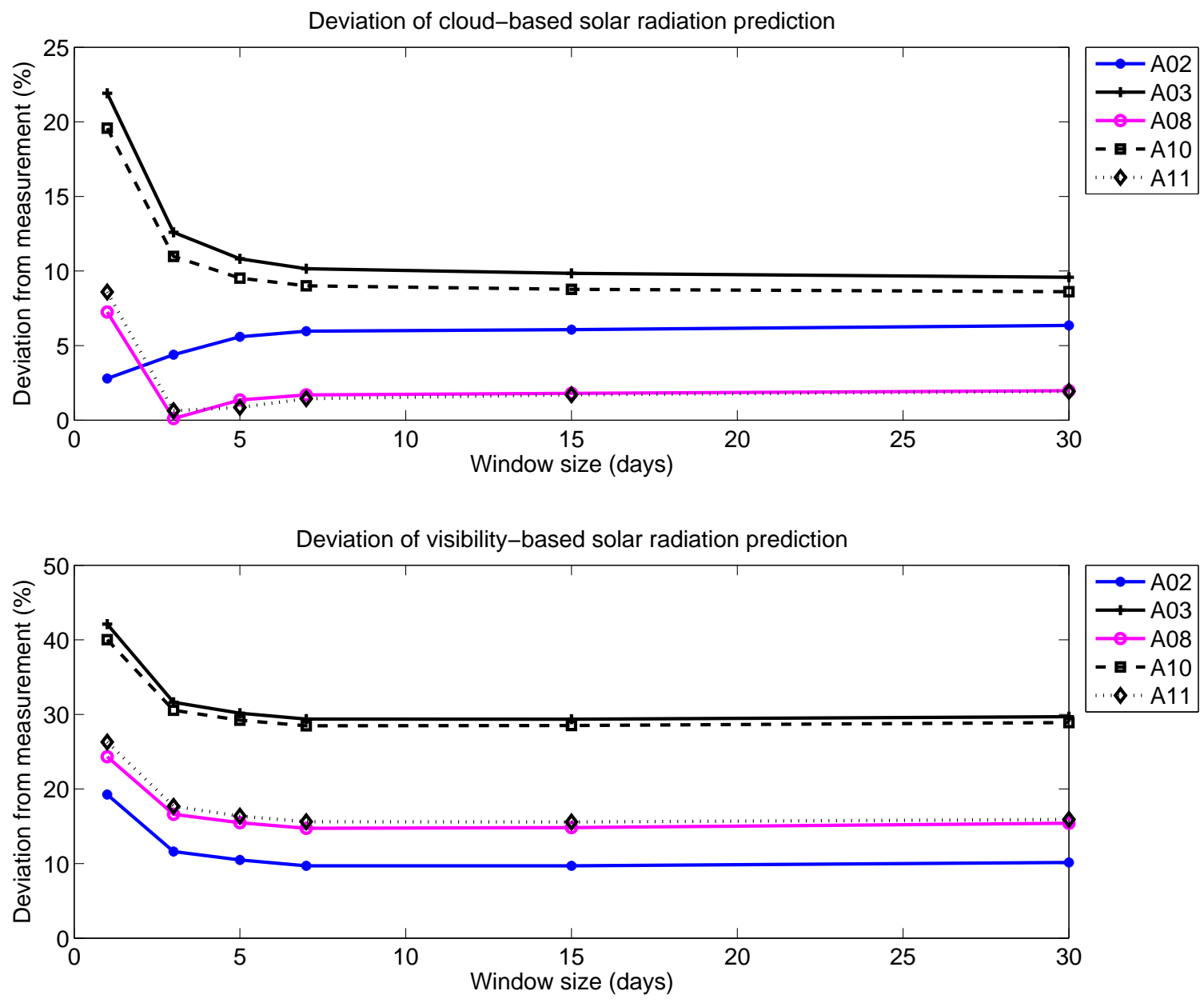

Figure 6.21: Deviation of solar radiation prediction with cloud-based and visibilitybased models 
an obstructed astronomical model using the probability distribution of the weather metric, which is built with samples of the solar radiation measurement and weather metric.

With the weather-metric model, we predicted the solar radiation by using a $k$-day history of the weather metric. With a cloud-based model, we were able to predict the solar radiation with a $6 \%$ margin of error while maintaining 3 or 7 days of history of the weather metric, while the prediction error is about $26 \%$ without a weather metric. With a visibility-based model, the prediction error was around $20 \%$.

One limitation of this weather-metric based model is that the prediction is for the average behavior over a certain window of time not for the behavior at a specific time. This is because our weather-metric model is a probabilistic model and the exact pattern of the weather metric for the future is not known. 


\section{Chapter 7}

\section{Extending the Simulator Beyond the Reference Design}

In previous chapters, a simulation model of micro-solar power systems was developed and validated. However, it is not just limited to the reference platform, as a simulation tool should be able to model hypothetical designs of micro-solar power systems and predict their behavior. First, this chapter extends the simulation model for a hypothetical design of micro-solar power systems and demonstrates its utility (Section 7.1). Then, it discusses possible ideas for extending the simulation model for two other renewable energy sources: wind and vibrations (Section 7.2).

\subsection{Extending the Simulator for a Micro-Solar Power System with Multi-Level Energy Storage}

In earlier chapters of this dissertation, we designed a simulator of the microsolar power system and validated it using our reference implementations, one with a supercapacitor and the other with two AA NiMH batteries. While these two examples demonstrated that our simulation model can be applied to a micro-solar power system with energy storage of different types and chemistry, they are demonstrated only with single-level energy storage. A few designs of micro-solar power system such as Prometheus [JPC05], Trio $\left[\mathrm{DHJ}^{+} 06\right]$ and AmbiMax [PC06] cannot be directly simulated with this simulation model. 


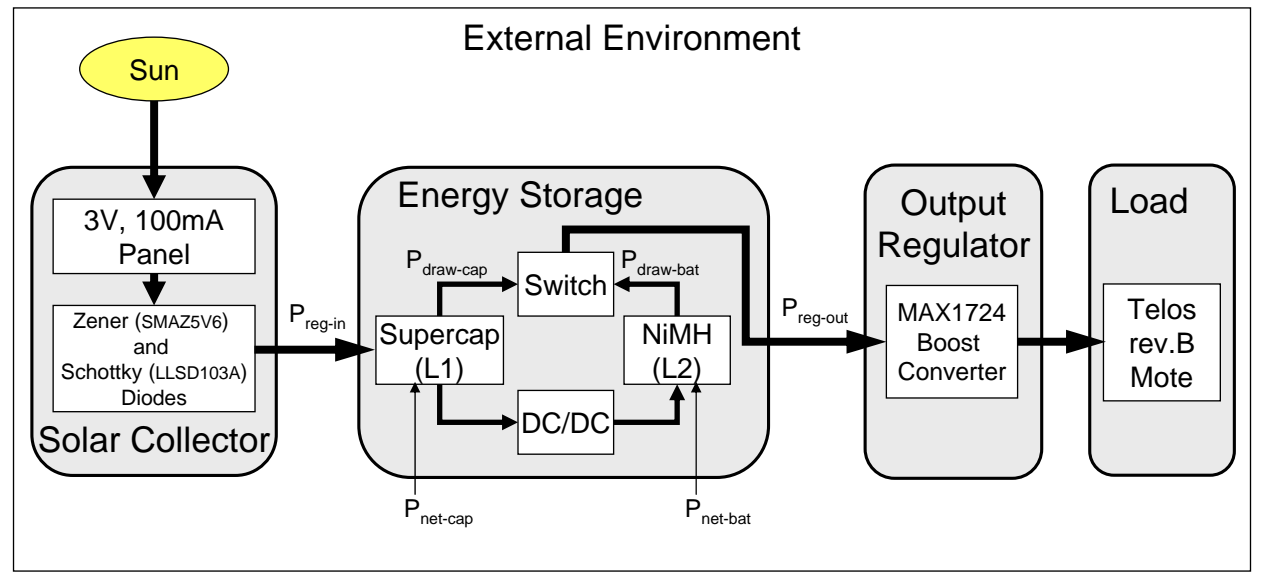

Figure 7.1: A model for micro-solar power system with multi-level energy storage

In this section, we develop a simulator for a micro-solar power system with twolevel energy storage consisting of a supercapacitor and NiMH batteries using the simulation modules we developed in Chapter 4 .

\subsubsection{A Simulation Model of Micro-Solar Power System with Two-Level Energy Storage}

Figure 7.1 is a model for the micro-solar power system with two-level energy storage. Compared to the design of a single-level energy storage system (Figure 5.4), the key difference is the energy storage. The energy storage has two storage elements: a supercapacitor as primary storage and two NiMH batteries in series as secondary storage. Other than the storage elements, the energy storage also has an output power switch and a DC/DC converter. The output power switch selects either the supercapacitor or the NiMH batteries to supply the power to the load. The DC/DC converter can be turned on or off. When turned on, the DC/DC converter makes the supercapacitor drive the charge to the NiMH batteries.

In order to describe the operation of the system, we annotated the model with energy flow as shown at Figure 7.1. From the solar collector to the energy storage, $P_{\text {reg-in }}$ of power is available, and the power consumption of the load that is seen by the energy storage is given as $P_{\text {reg-out }}$. Then, the charging logic determines how much 
power can be charged to the supercapacitor and the battery, $P_{n e t-c a p}$ and $P_{n e t-b a t}$ respectively. In the rest of this section, we describe the operation of the two-level energy storage simulator in terms of energy flow of the energy storage elements.

\section{Modeling Each Element of Two-Level Energy Storage}

The energy storage elements, supercapacitor and NiMH battery, are simulated using the variables that represent energy level, voltage, power draw and net charging power. The output power switch and the DC/DC converter are modeled using discrete states:

\section{- Supercap:}

- $E_{\text {cap }}$ : Energy level of the supercap

- $V_{\text {cap }}$ : Voltage level of the supercap

- $P_{\text {net-cap }}:$ Net power being charged to the supercap

- $P_{\text {draw-cap }}$ : Power draw from the supercap

\section{- Battery:}

- $E_{b a t}$ : Energy level of the battery

- $V_{\text {bat }}$ : Voltage level of the battery

- $P_{\text {net-bat }}$ : Net power being charged to the battery

- $P_{\text {draw-bat }}$ : Power draw from the battery

\section{- Output Power Switch:}

- $f_{\text {cap-run }}: 0$ when running on the battery, 1 when on the supercap

\section{- DC/DC Converter:}

$-f_{\text {bat-chg }}: 0$ when turned off, 1 when turned on

- Solar Collector:

$-P_{\text {reg-in }}:$ Available power from the solar collector 
- $V_{\text {sol }}$ : Solar panel voltage - positive if the panel is under radiation, 0 otherwise.

\section{- Load and Output Regulator:}

$-P_{\text {reg-out }}:$ Power draw from the load through the output regulator

The initial conditions for these state variables and other thresholds are listed below:

\section{- Supercap:}

- $E_{\text {cap-init }}$ : Initial energy level of the supercap

- $E_{\text {cap-max }}:$ Maximum energy level of the supercap

$-V_{c a p-l b}$ : Lower bound of the supercap voltage for battery charging

- $V_{c a p-u b}$ : Upper bound of the supercap voltage for battery charging

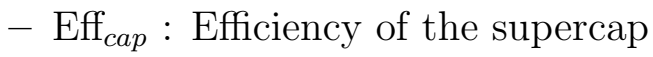

\section{- Battery:}

- $E_{\text {bat-init }}$ : Energy level of the battery

- $E_{\text {bat-max }}$ : Voltage level of the battery

$-V_{b a t-l b}:$ Lower bound of the battery voltage for battery charging

- $V_{b a t-u b}$ : Upper bound of the battery voltage for battery charging

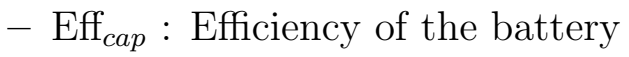

\section{- Output Power Switch:}

$-f_{\text {cap-run-init }}$ : Initial condition for the output power switch

\section{- DC/DC Converter:}

- $f_{\text {bat-chg-init }}$ : Initial condition for the DC/DC converter

- Imax-bat-chg : Maximum battery charging current

- $\mathrm{Eff}_{d c-d c}$ : Efficiency of the DC/DC converter 


\section{Algorithm for State Transition of Two-Level Energy Storage}

The algorithm that determines the state variables of the two-level energy storage is given at Figure 7.2. While most of the lines are self-explanatory, some of the lines need explanation:

- Line 10 : The function EtoVbat(), which gives the battery voltage for the given battery energy level, is described by calc_vout_NH15_2500() in Figure 4.19 .

- Line 11 : The function $\operatorname{EtoV} \operatorname{cap}()$, which gives the supercap voltage for the given supercap energy level, is described by formula 4.10 .

- Line 12 : Formula 4.9 describes the process that evaluates $P_{\text {reg-in }}[i]$, which is the available power from the solar collector.

- Line 14 : The function CalcNetPows() describes the procedure that determines the net power to the supercap and the battery (Figure 7.3).

- Line 15 : The function CalcFlags () describes the procedure that determines the discrete states for the output power switch and the DC/DC converter (Figure 7.5 .

CalcNetPows() can be divided into three cases depending on the discrete states of the output power switch and the DC/DC converter (Figure 7.4):

- Case 1 : Line 2 to 6

In this case, the system runs on the supercapacitor, and the battery is not being charged. From this condition, the draw from the battery and the net charge to the battery become zero. The input power from the solar collector to the supercapacitor is $P_{r e g-i n}$, and the draw from the supercapacitor for the load is $P_{\text {reg-out }}$. From this condition, the power available to the supercapacitor becomes $\left(P_{\text {reg-in }}-P_{\text {reg-out }}\right)$. We also need to consider that only Eff cap $_{\text {of }}$ the available power can be stored due to sub-unity efficiency of the supercap 1 .

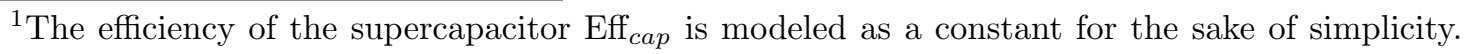
In a more accurate model, it can be represented as a function of current.
} 


$$
\begin{array}{ll}
\text { 1: } & f_{\text {next-bat-chg }} \leftarrow f_{\text {bat-chg-init }} \\
\text { 2: } & f_{\text {next-cap-run }} \leftarrow f_{\text {cap-run-init }} \\
\text { 3: } & E_{\text {next-bat }} \leftarrow E_{\text {bat-init }} \\
\text { 4: } & E_{\text {next-cap }} \leftarrow E_{\text {cap-init }} \\
\text { 5: } & \text { for } i=1 \text { to } \mathrm{N} \text { do } \\
\text { 6: } & f_{\text {bat-chg }}[i] \leftarrow f_{\text {next-bat-chg }} \\
\text { 7: } & f_{\text {cap-run }}[i] \leftarrow f_{\text {next-cap-run }} \\
\text { 8: } & E_{\text {bat }}[i] \leftarrow E_{\text {next-bat }} \\
\text { 9: } & E_{\text {cap }}[i] \leftarrow E_{\text {next-cap }} \\
\text { 10: } & V_{\text {bat }}[i] \leftarrow \mathbf{E t o V b a t}\left(E_{\text {bat }}[i]\right) \\
\text { 11: } & V_{\text {cap }}[i] \leftarrow \operatorname{EtoVcap}\left(E_{\text {cap }}[i]\right)
\end{array}
$$

12: $\quad$ Evaluate $P_{\text {reg-in }}[i]$.

13: $\quad P_{\text {reg-out }}[i] \leftarrow P_{\text {load }} / \mathrm{Eff}_{\text {reg-out }}$

14: $\quad\left[P_{\text {net-bat }}, P_{\text {net-cap }}\right] \leftarrow$ CalcNetPows $\left(f_{\text {bat-chg }}[i], f_{\text {cap-run }}[i]\right)$

15: $\quad\left[f_{\text {next-bat-chg }}, f_{\text {next-cap-run }}\right] \leftarrow \operatorname{CalcFlags}\left(V_{\text {bat }}[i], V_{\text {cap }}[i]\right)$

16: $\quad E_{\text {next-bat }} \leftarrow E_{\text {bat }}[i]+(t[i+1]-t[i]) \times P_{\text {net-bat }}[i]$

17: $\quad E_{\text {next-cap }} \leftarrow E_{\text {cap }}[i]+(t[i+1]-t[i]) \times P_{\text {net-cap }}[i]$

18: end for

Figure 7.2: Charging algorithm for two-level energy storage simulator 
Then, the net charge to the battery and the supercapacitor are as follows:

$$
\begin{aligned}
& P_{\text {net-bat }}=0 \\
& P_{\text {net-cap }}=\left(P_{\text {reg-in }}-P_{\text {reg-out }}\right) \times \mathrm{Eff}_{\text {cap }}
\end{aligned}
$$

- Case 2 : Line 7 to 11

In this case, the system runs on the supercapacitor, and the battery is being charged. The amount of current that flows into the battery is set by the maximum battery charge current $I_{\max -b a t-c h g}$, which is usually a characteristic of the DC/DC converter. Thus, the power that is drawn from the supercap is $V_{c a p} \times I_{\text {max-bat-chg}}$. If we consider that the battery has efficiency factor $\mathrm{Eff}_{b a t}$ and the DC/DC converter has efficiency factor Eff ${ }_{d c-d c}$, the power that is actually stored into the battery is the product of the incoming flow, the efficiency factor of the battery and the efficiency factor of the DC/DC converter. Thus, the net charge power to the battery and the supercapacitor can be given as follows:

$$
\begin{aligned}
& P_{\text {net-bat }}=V_{c a p} \times I_{\text {max-bat-chg }} \times \mathrm{Eff}_{\text {bat }} \times \mathrm{Eff}_{d c-d c} \\
& P_{\text {net-cap }}=\left(P_{\text {reg-in }}-P_{\text {reg-out }}\right) \times \mathrm{Eff}_{c a p}-V_{c a p} \times I_{\text {max-bat-chg }}
\end{aligned}
$$

- Case 3 : Line 12 to 16

In this case, the system runs on the battery, and the battery is not being charged. The battery has draw of $P_{\text {reg-out }}$ with no incoming power, and the supercapacitor has incoming power of $P_{r e g-i n}$ with no power draw. Thus, the net charge power to the battery and the supercapacitor can be given as follows: Preg-in.

$$
\begin{aligned}
& P_{\text {net-bat }}=-P_{\text {reg-out }} \\
& P_{\text {net-cap }}=P_{r e g-i n} \times \mathrm{Eff}_{c a p}
\end{aligned}
$$

The operation of CalcFlags() can be divided depending on the voltage level of the supercap.

- Stop Charging Condition : $V_{c a p}<V_{c a p-l b}$

It stops the battery charging. It runs on the battery if the battery voltage is above the minimum battery voltage. Otherwise, it runs on the supercap. 


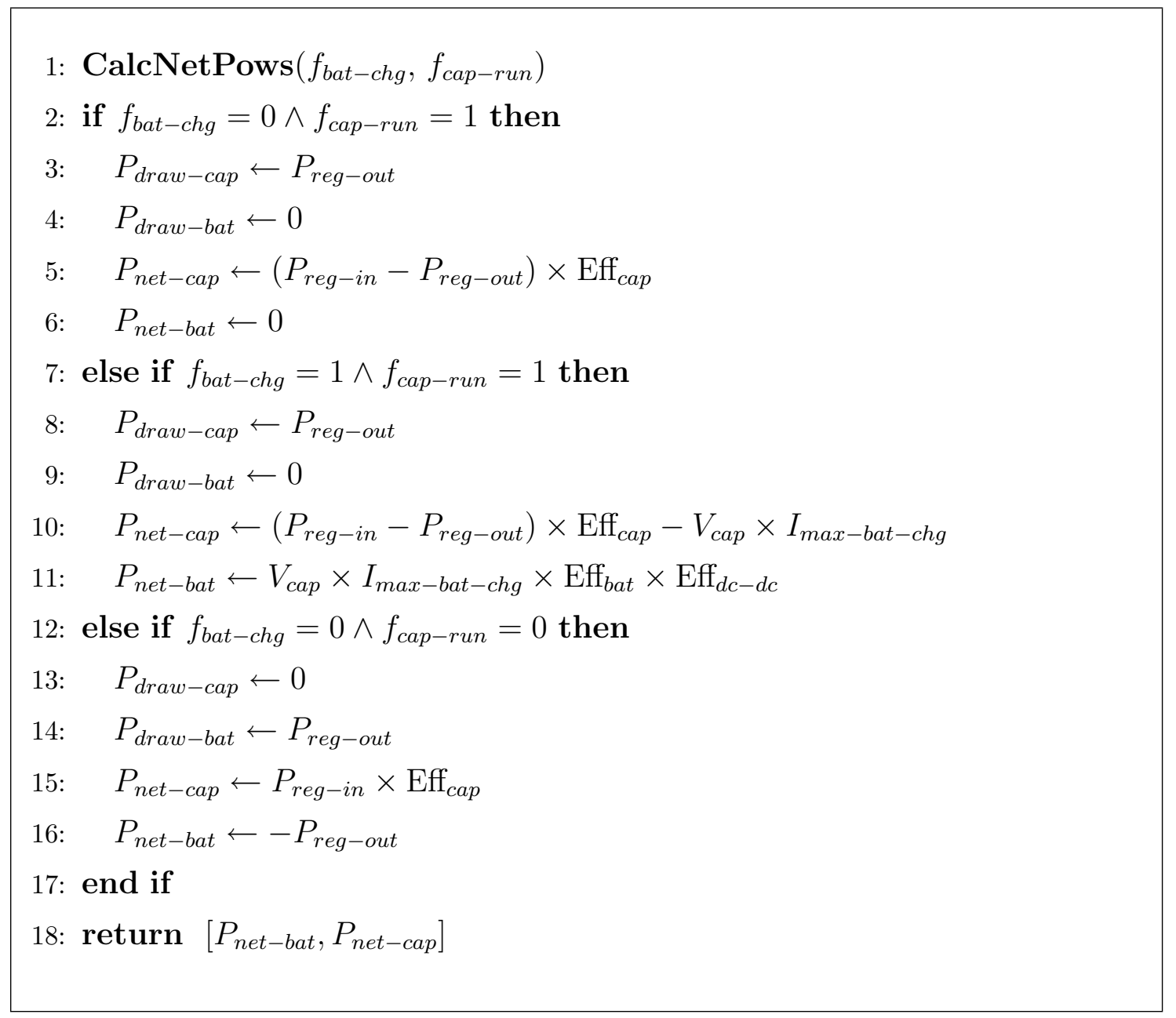

Figure 7.3: Algorithm that determines the net power to the energy storage 


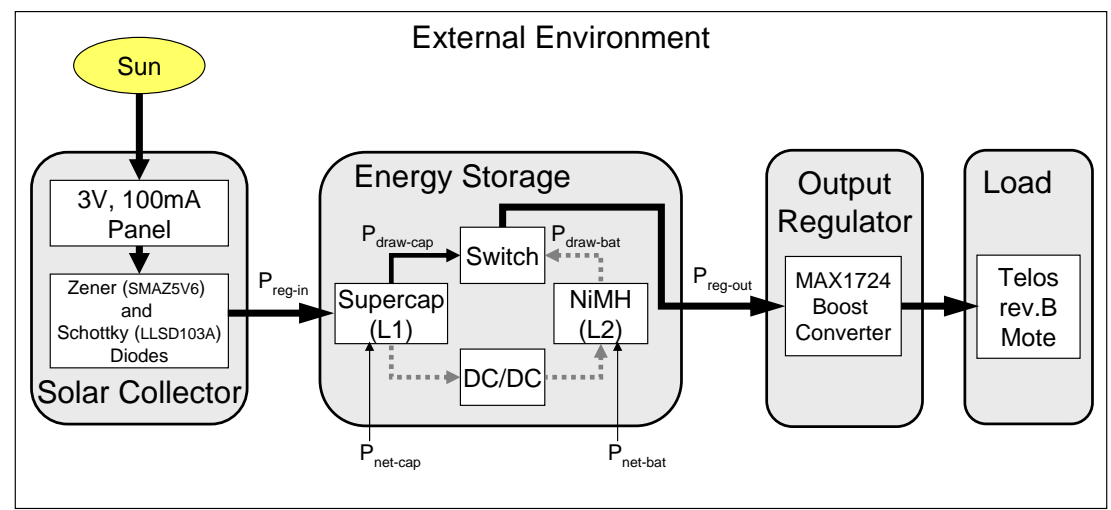

Case 1: Running on capacitor, not charging on battery.

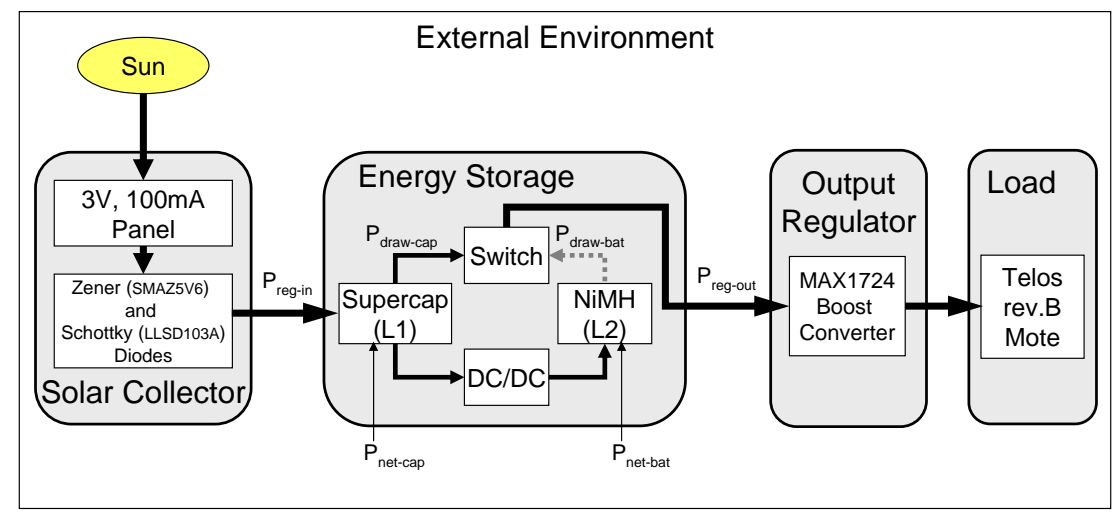

Case 2: Running on capacitor, charging on battery.

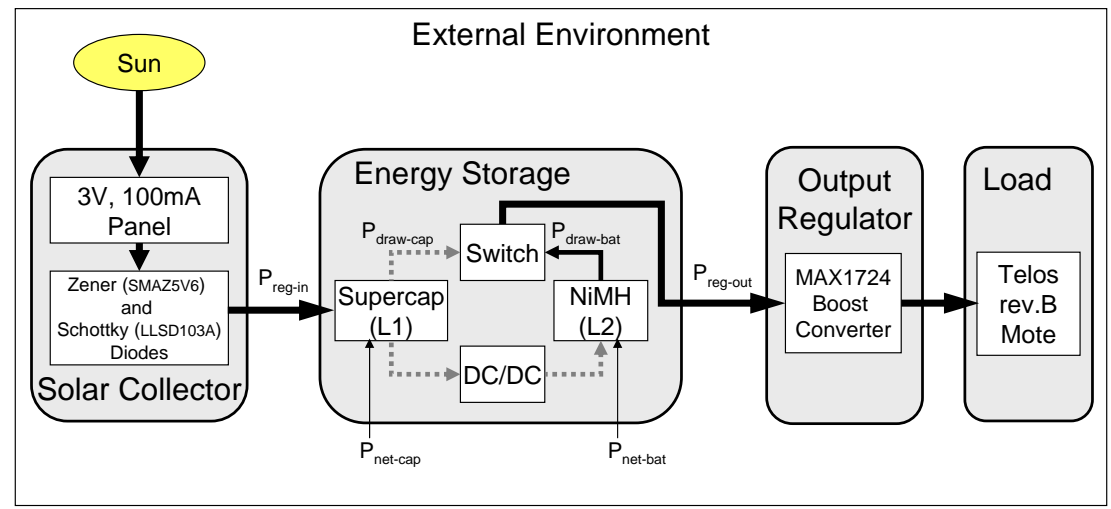

Case 3: Running on battery, not charging on battery.

Figure 7.4: Three cases for storage net charging power calculation 
- Start Charging Condition : $V_{c a p} \geq V_{c a p-l b}$

It runs on the supercap. It charges the battery if the battery voltage is below the maximum battery voltage and the solar panel is under radiation. Otherwise, it stops the battery charging.

- Keep Status Condition : $V_{c a p} \geq V_{c a p-l b} \wedge V_{c a p}<V_{c a p-u b}$

It runs on the supercap. It keeps the same charging status if the battery voltage is below the maximum battery voltage and the solar panel is under radiation. Otherwise, it stops the battery charging.

\subsubsection{Simulation of Micro-Solar Power System with Two- Level Energy Storage under Constant Radiation}

In order to validate the simulation model of the two-level energy storage, we set up the simulation as follows:

\section{Effect of Storage Size}

Two configurations of multi-level energy storage with a supercapacitor and NiMH batteries are considered by varying the capacity of the supercapacitor (Figure 7.6).

- Configuration 1a : 22F supercap + NiMH

- Configuration 1b : 140F supercap + NiMH 


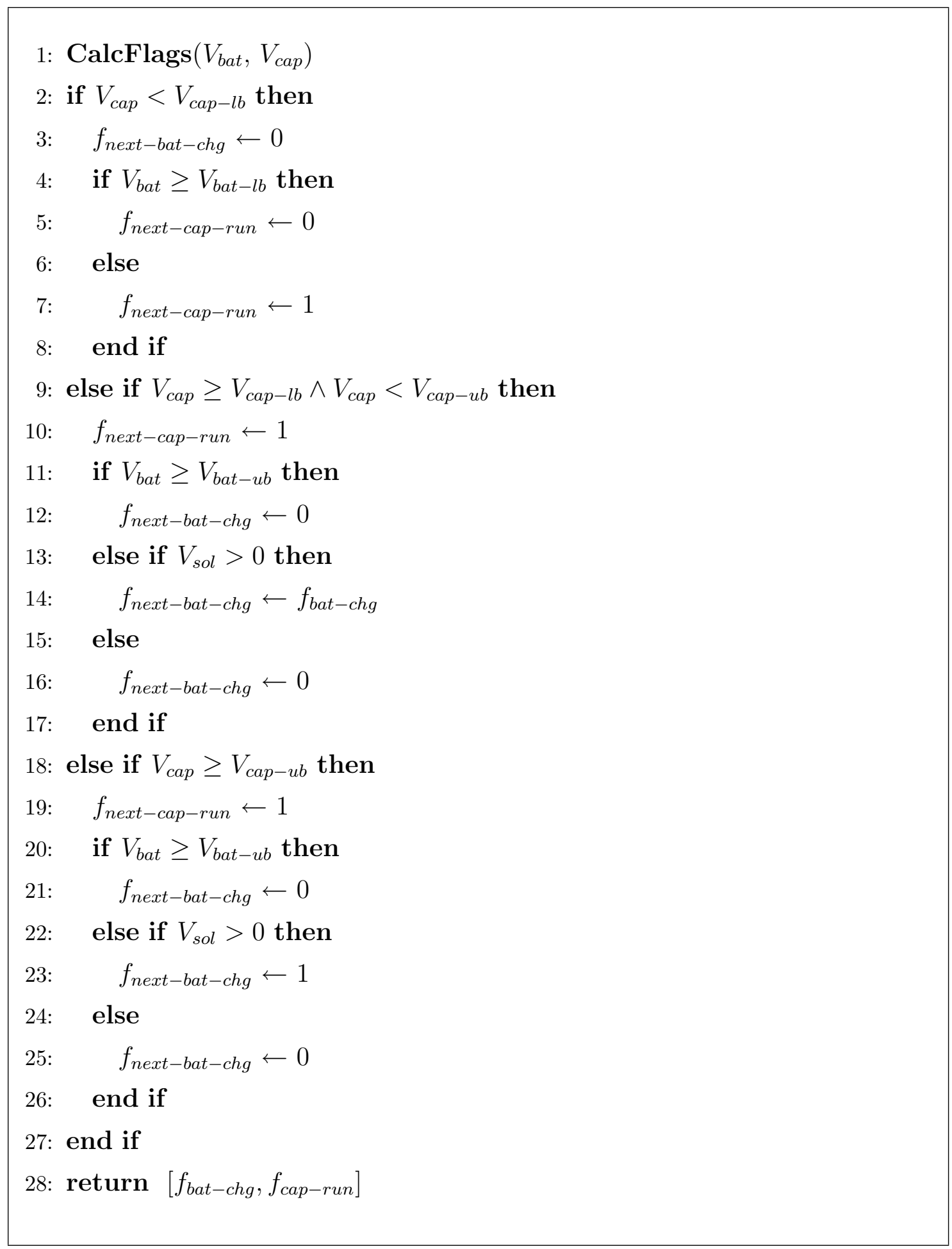

Figure 7.5: Algorithm that determines the discrete states for the output power switch and the $\mathrm{DC} / \mathrm{DC}$ converter 

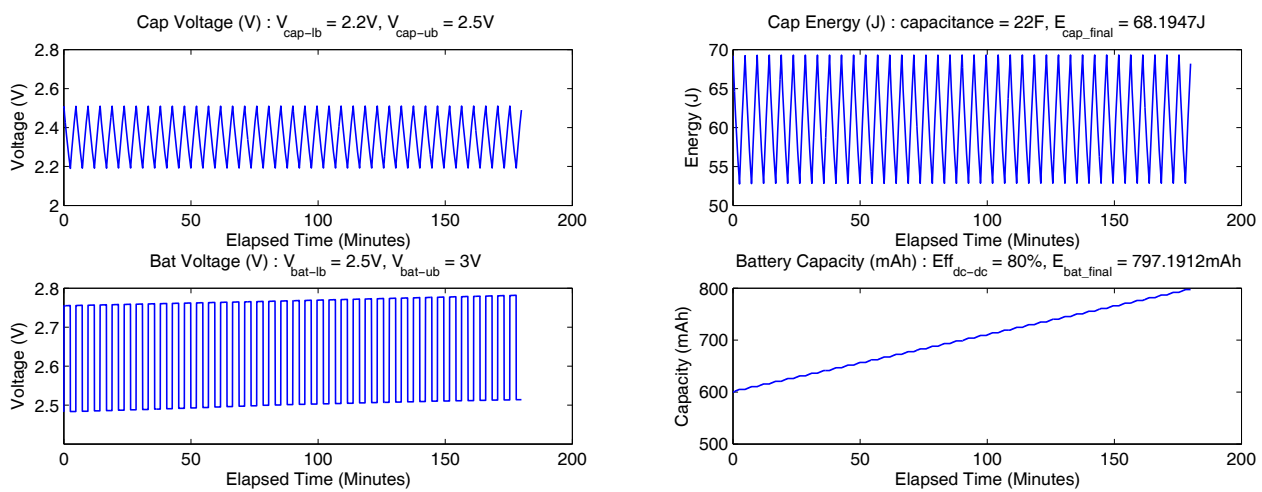

(a) Configuration 1a: 22F supercap $+\mathrm{NiMH}$
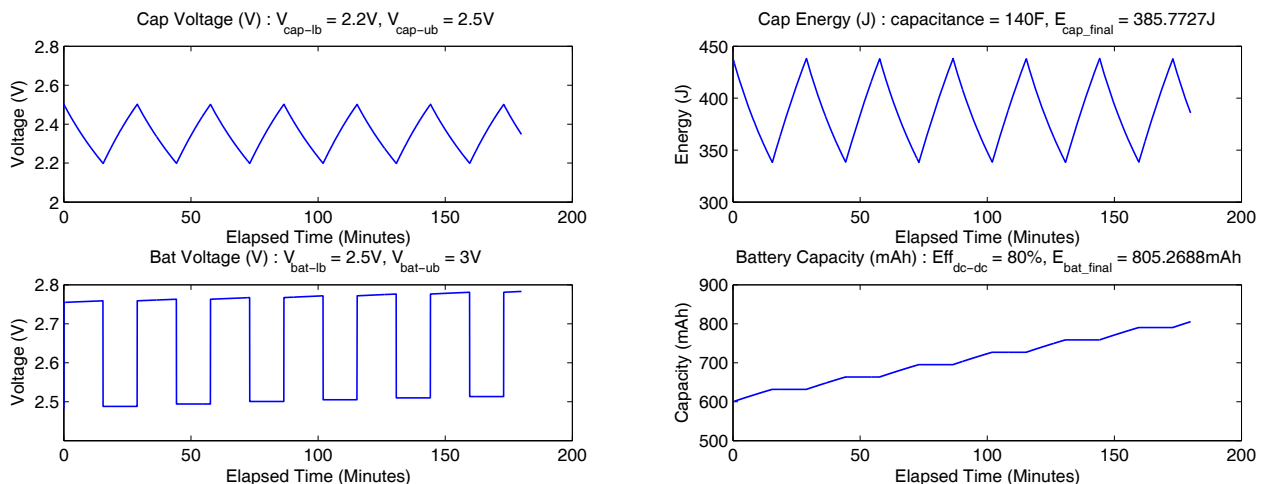

(b) Configuration 1b: 140F supercap $+\mathrm{NiMH}$

Figure 7.6: Effect of storage size 
The accumulated charge $Q=Q_{b a t}+Q_{c a p}$ for the two configurations can be calculated as follows:

For Configuration 1a,

$$
\begin{aligned}
Q & =Q_{\text {bat }}+Q_{\text {cap }} \\
& =\left(Q_{\text {bat-final }}-Q_{\text {bat-init }}\right)+\frac{E_{\text {cap-final }}-E_{\text {cap-init }}}{3.6 J / m W h \times V_{\text {cap-average }}} \\
& =(797.19 m A h-600 m A h)+\frac{-0.56 J}{3.6 J / m W h \times 2.35 V} \\
& =197.10 m A h
\end{aligned}
$$

For Configuration 1b,

$$
\begin{aligned}
Q & =Q_{\text {bat }}+Q_{\text {cap }} \\
& =\left(Q_{\text {bat-final }}-Q_{\text {bat-init }}\right)+\frac{E_{\text {cap-final }}-E_{\text {cap-init }}}{3.6 J / m W h \times V_{\text {cap-average }}} \\
& =(805.27 m A h-600 m A h)+\frac{-51.73 J}{3.6 J / m W h \times 2.35 V} \\
& =199.16 m A h
\end{aligned}
$$

The difference is $1.0 \%$ and they accumulate about the same level of charge in the battery. The difference is the frequency of the charging. For Configuration $1 \mathrm{~b}$, which has a bigger capacitor, it takes a longer time to charge the supercapacitor.

\section{Effect of Supercap Charging Range}

Three configurations of multi-level energy storage with a supercapacitor and NiMH batteries are considered by varying the supercapacitor charging range $\left[V_{c a p-l b}, V_{c a p-u b}\right]$ (Figure 7.7 and Figure 7.8).

- Configuration 2a : $22 \mathrm{~F}$ supercap $+\mathrm{NiMH}, V_{\text {cap-lb }}=2.2 \mathrm{~V}, V_{\text {cap-ub }}=2.5 \mathrm{~V}$

- Configuration 2b : $22 \mathrm{~F}$ supercap $+\mathrm{NiMH}, V_{c a p-l b}=2.3 \mathrm{~V}, V_{c a p-u b}=2.4 \mathrm{~V}$

- Configuration 2c : $22 \mathrm{~F}$ supercap $+\mathrm{NiMH}, V_{c a p-l b}=2.0 \mathrm{~V}, V_{c a p-u b}=2.3 \mathrm{~V}$ 

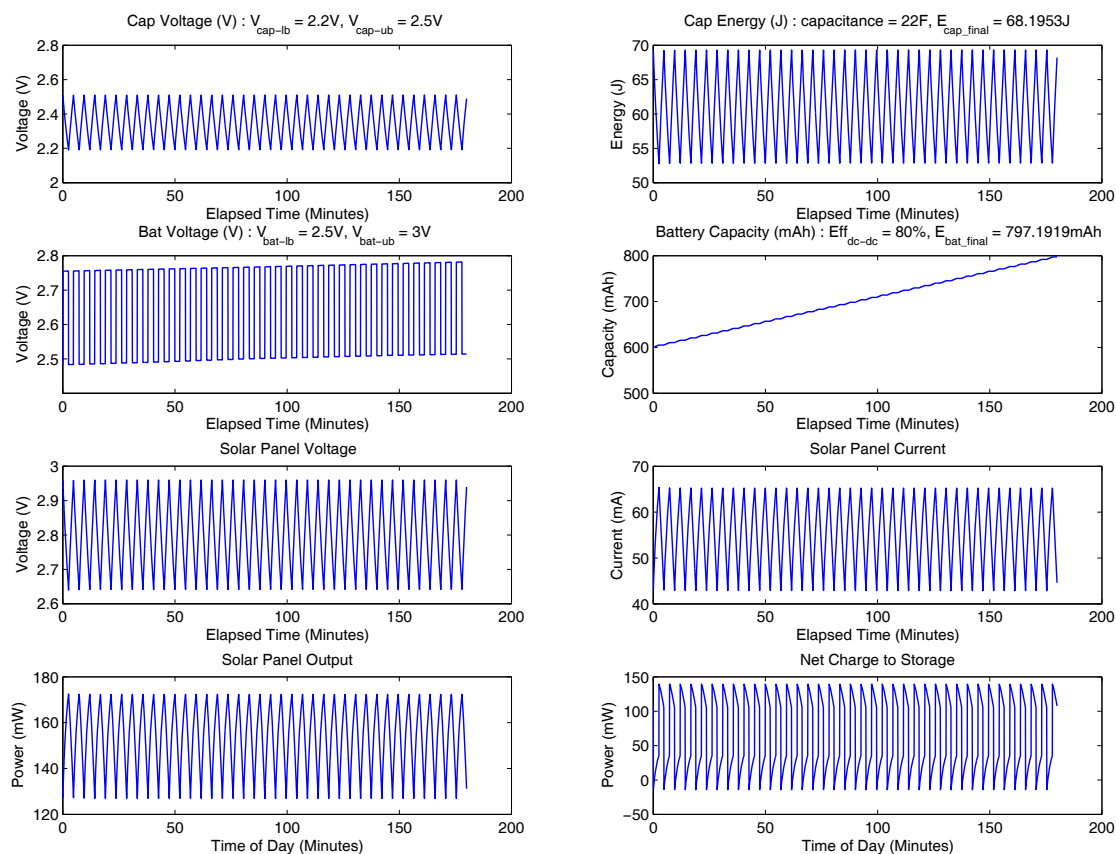

(a) Configuration 2a: supercap charging range $2.2 \mathrm{~V}-2.5 \mathrm{~V}$
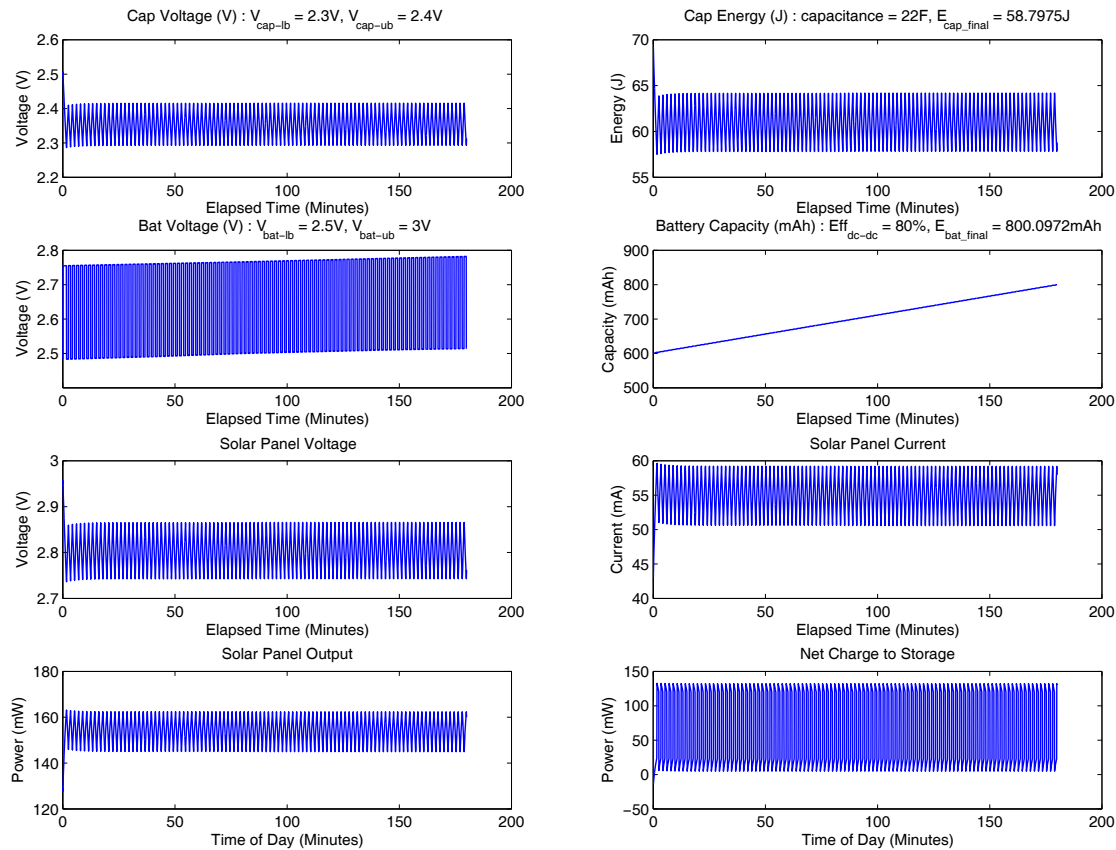

(b) Configuration 2b: supercap charging range $2.3 \mathrm{~V}-2.4 \mathrm{~V}$

Figure 7.7: Narrowing the supercap charging range 
Figure 7.7 compares the effect when the supercapacitor charging range is narrowed while the center point of the range is the same. The accumulated charge $Q$ for the two configurations $(2 \mathrm{a}$ and $2 \mathrm{~b})$ are as follows:

$$
\begin{aligned}
& \text { For Configuration 2a, } \quad Q=Q_{b a t}+Q_{c a p}=197.12 \mathrm{mAh} \\
& \text { For Configuration 2b, } \quad Q=Q_{b a t}+Q_{c a p}=198.92 \mathrm{mAh}
\end{aligned}
$$

In this case, they accumulate the same amount of charge in the battery while the frequency of charging is different. The one with the narrower range has a higher frequency of charging.

Figure 7.8 compares the effect when the supercapacitor charging range is shifted while the width of the range is the same.

$$
\begin{aligned}
& \text { For Configuration 2a, } \quad Q=Q_{b a t}+Q_{c a p}=197.12 m A h \\
& \text { For Configuration 2c, } \quad Q=Q_{b a t}+Q_{c a p}=222.33 m A h
\end{aligned}
$$

In this case, Configuration 2c accumulates $11.3 \%$ more charge in the battery than Configuration 2a. This is because Configuration 2c sets the operating voltage of the solar panel closer to its maximum power point, as shown in Figure 7.9

\section{Effect of Initial Supercap Voltage Level}

Four configurations of multi-level energy storage with a supercapacitor and NiMH batteries are considered by varying the initial supercapacitor voltage level and the capacity of the supercapacitor (Figure 7.10 and Figure 7.11).

- Configuration 3a : $22 \mathrm{~F}$ supercap $+\mathrm{NiMH}, V_{\text {cap-init }}=2.5 \mathrm{~V}$

- Configuration 3b : $22 \mathrm{~F}$ supercap $+\mathrm{NiMH}, V_{\text {cap-init }}=0.1 \mathrm{~V}$

- Configuration 3c : 140F supercap $+\mathrm{NiMH}, V_{\text {cap-init }}=2.5 \mathrm{~V}$

- Configuration 3d : 140F supercap $+\mathrm{NiMH}, V_{\text {cap-init }}=0.1 \mathrm{~V}$ 

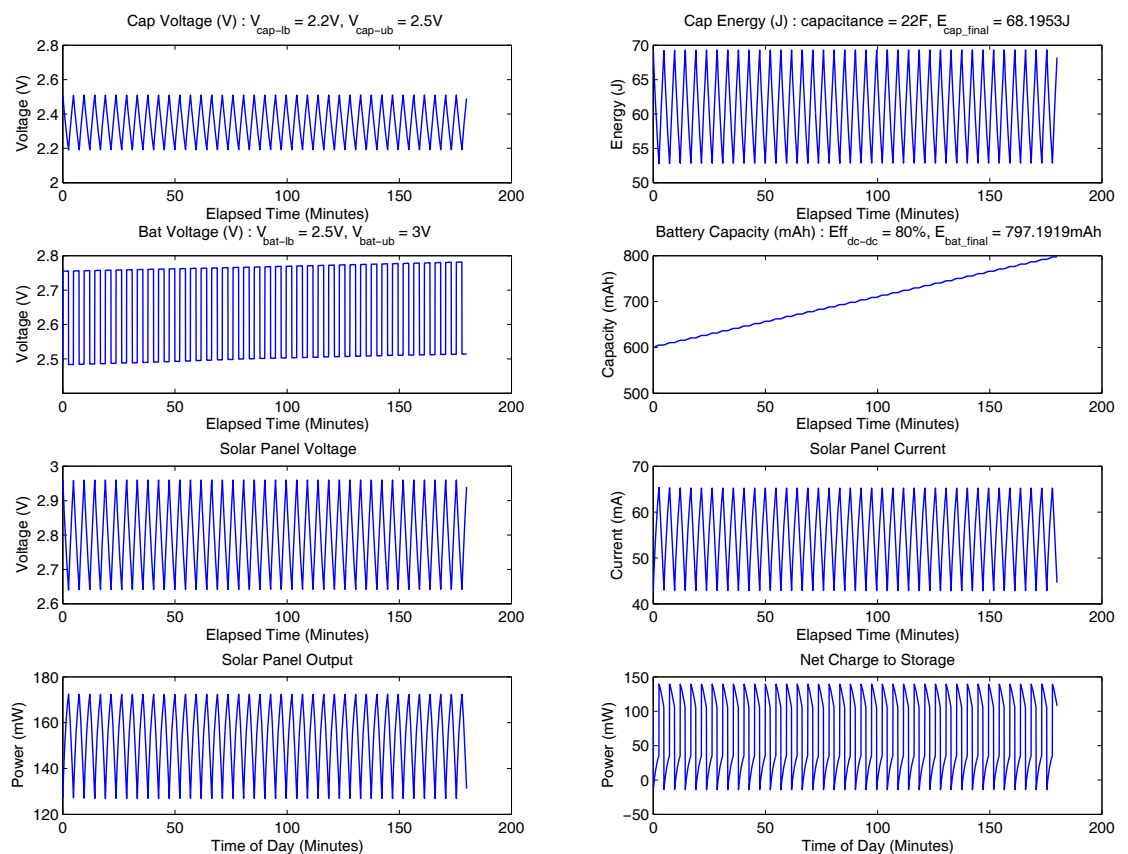

(a) Configuration 2a: supercap charging range $2.2 \mathrm{~V}-2.5 \mathrm{~V}$
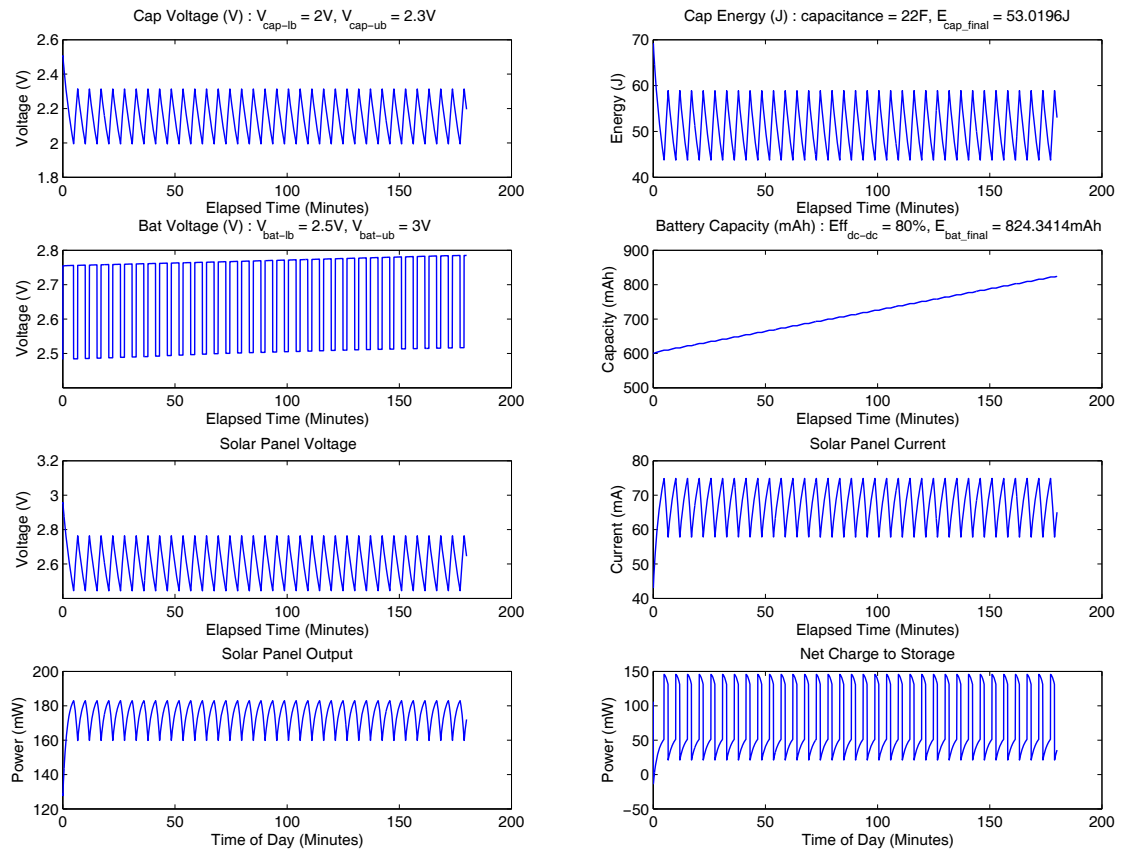

(b) Configuration 2c: supercap charging range $2.0 \mathrm{~V}-2.3 \mathrm{~V}$

Figure 7.8: Shifting the supercap charging range 


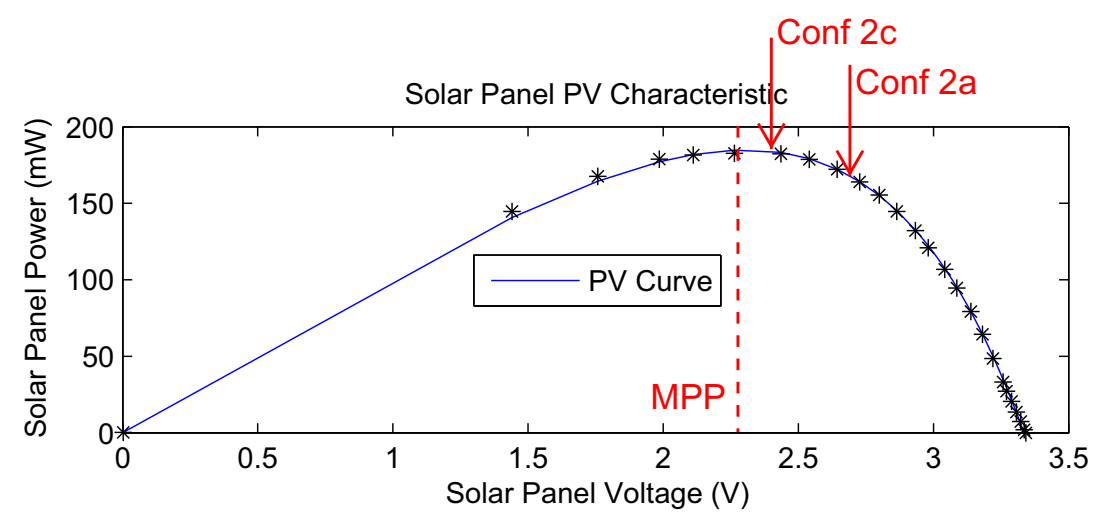

Figure 7.9: Comparison of solar panel operating voltage for Configuration $2 \mathrm{a}$ and $2 \mathrm{c}$
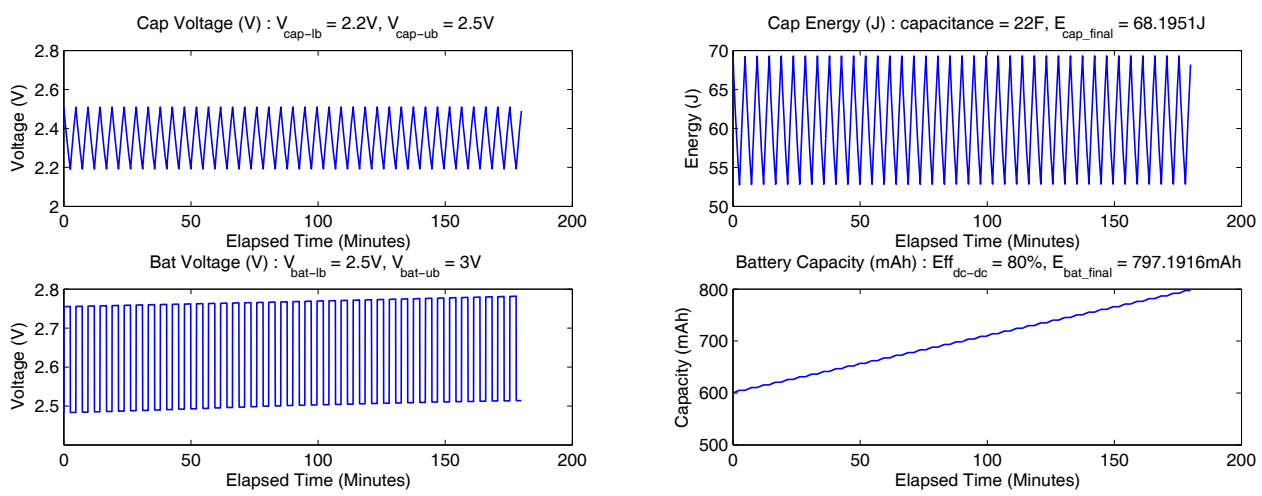

(a) Configuration 3a: $22 \mathrm{~F}$ supercap $+\mathrm{NiMH}, V_{\text {cap-init }}=2.5 \mathrm{~V}$
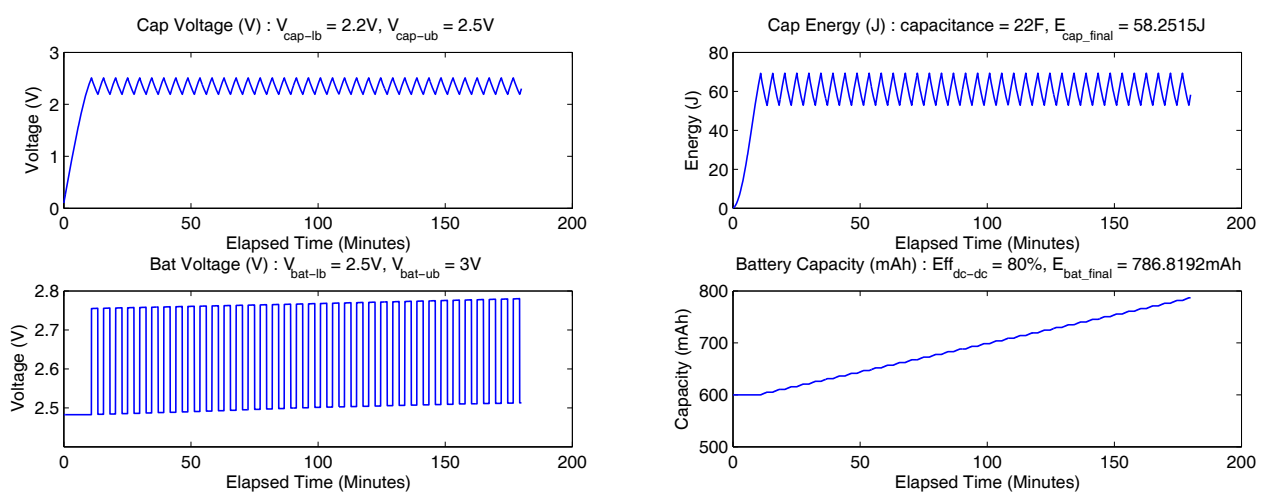

(b) Configuration 3b: 22F supercap $+\mathrm{NiMH}, V_{\text {cap-init }}=0.1 \mathrm{~V}$

Figure 7.10: Effect of initial battery voltage level (with $22 \mathrm{~F}$ supercap) 

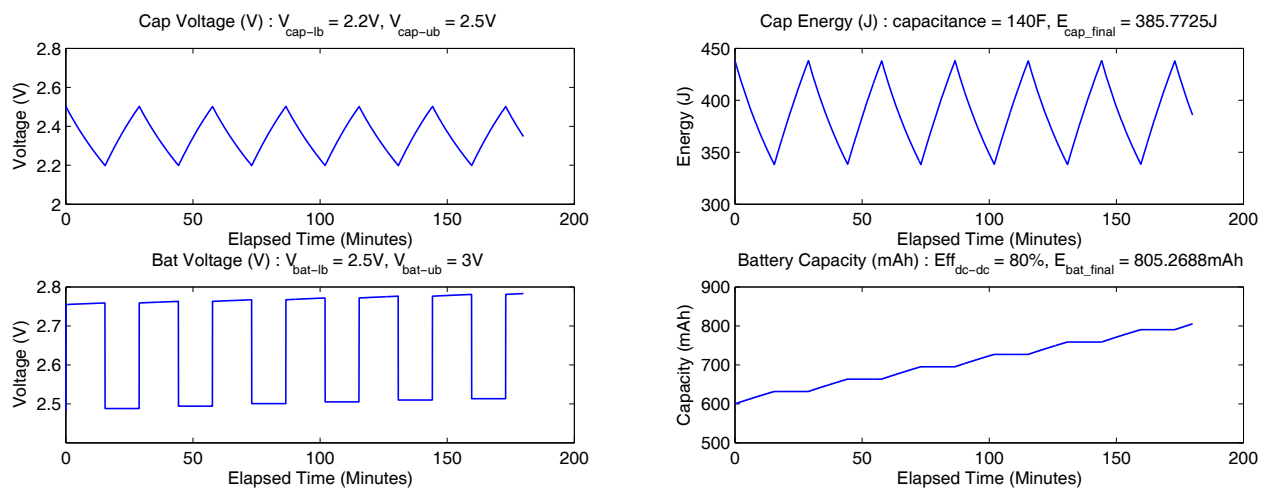

(a) Configuration 3c: $140 \mathrm{~F}$ supercap $+\mathrm{NiMH}, V_{\text {cap-init }}=2.5 \mathrm{~V}$
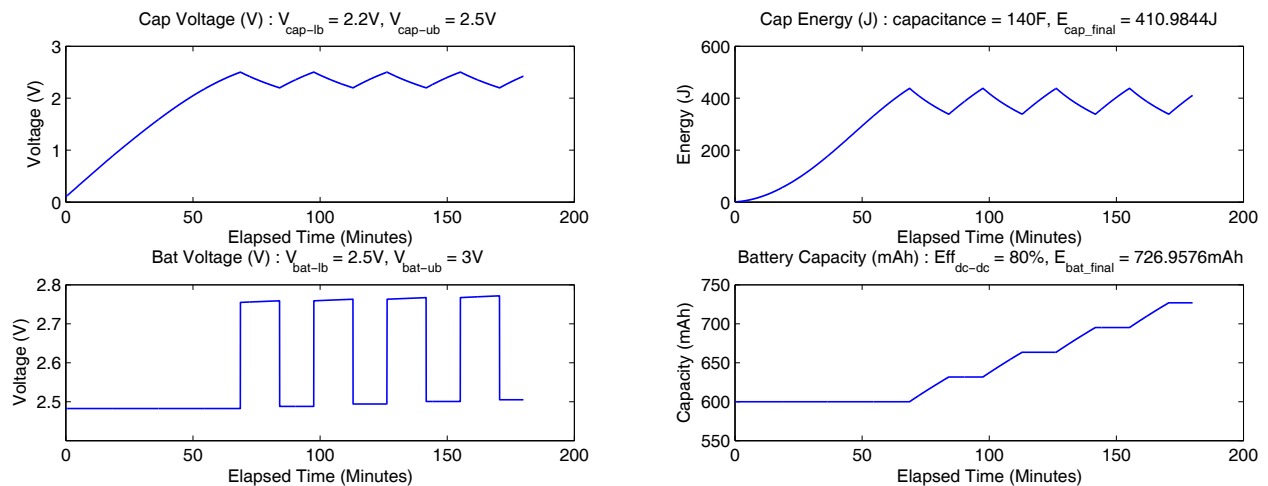

(b) Configuration 3d: $140 \mathrm{~F}$ supercap $+\mathrm{NiMH}, V_{\text {cap-init }}=0.1 \mathrm{~V}$

Figure 7.11: Effect of initial battery voltage level (with 140F supercap) 
When the supercapacitor is nearly empty (Configuration $3 \mathrm{~b}$ and $3 \mathrm{~d}$ ), it takes some amount time before the supercapacitor becomes full (11 minutes for Configuration 3b and 68 minutes for Configuration 3d). The time it takes to fully charge is proportional to the capacity of the supercapacitor.

$$
\begin{array}{r}
68 \min / 11 \min =6.18 \\
140 F / 22 F=6.36
\end{array}
$$

After the supercapacitor becomes full, it has the same behavior as the one that started from the full supercapacitor. (Configuration $3 \mathrm{a}$ and $3 \mathrm{~b}$ ).

For Configuration 3a, $\frac{Q_{\text {final }}-Q_{\text {cap-full }}}{T_{\text {final }}-T_{\text {cap-full }}}=\frac{197.19 \mathrm{mAh}}{180 \mathrm{~min}}=1.10 \mathrm{mAh} / \mathrm{min}$

For Configuration 3b, $\frac{Q_{\text {final }}-Q_{\text {cap-full }}}{T_{\text {final }}-T_{\text {cap-full }}}=\frac{186.81 \mathrm{mAh}}{169 \mathrm{~min}}=1.10 \mathrm{mAh} / \mathrm{min}$

\section{Effect of Initial Battery Voltage Level}

Two configurations of multi-level energy storage with a supercapacitor and NiMH batteries are considered by varying the initial battery voltage level (Figure 7.12).

- Configuration 4a : 22F supercap $+\mathrm{NiMH}, E_{\text {bat-init }}=600 \mathrm{mAh}$

- Configuration 4b : 22F supercap $+\mathrm{NiMH}, E_{b a t-i n i t}=2000 \mathrm{mAh}$

Configuration $4 \mathrm{a}$ and $4 \mathrm{~b}$ show that the solar panel and the supercapacitor operated at the same range while transferring the same amount of charge to the battery from the supercapacitor. The charge accumulation graph shows that the two configurations accumulated about the same amount of energy:

$$
\begin{aligned}
& \text { For Configuration 4a, } \quad Q=Q_{b a t}+Q_{c a p}=197.12 \mathrm{mAh} \\
& \text { For Configuration 4b, } \quad Q=Q_{b a t}+Q_{c a p}=197.09 \mathrm{mAh}
\end{aligned}
$$

\section{Effect of Maximum Battery Charging Current}

Four configurations of multi-level energy storage with a supercapacitor and NiMH batteries are considered by varying the maximum battery charging current and the capacity of the supercapacitor (Figure 7.13 and Figure 7.14). 

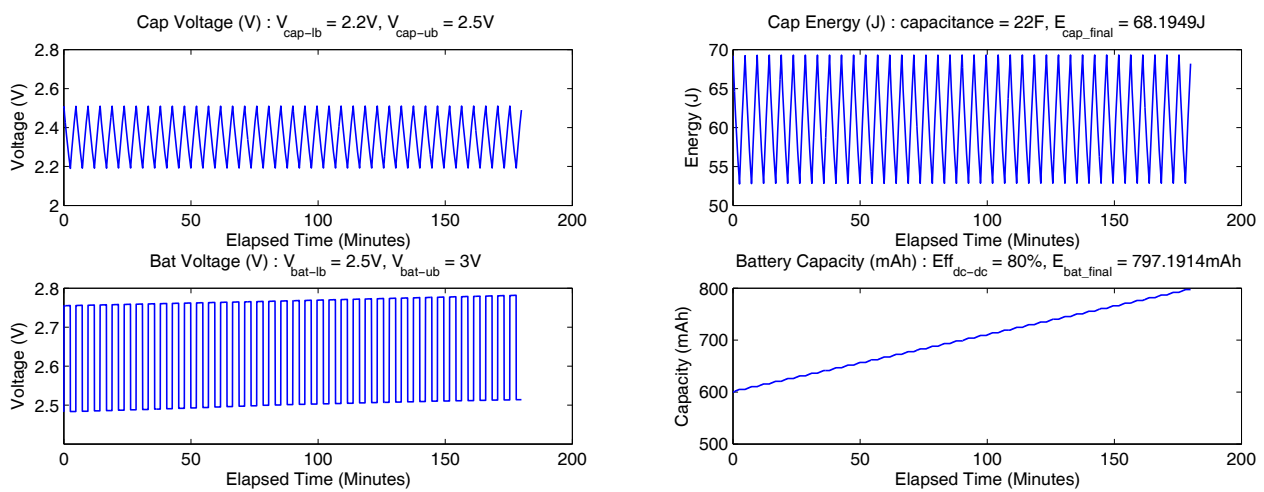

(a) Configuration 4a: $22 \mathrm{~F}$ supercap $+\mathrm{NiMH}, E_{\text {bat-init }}=600 \mathrm{mAh}$
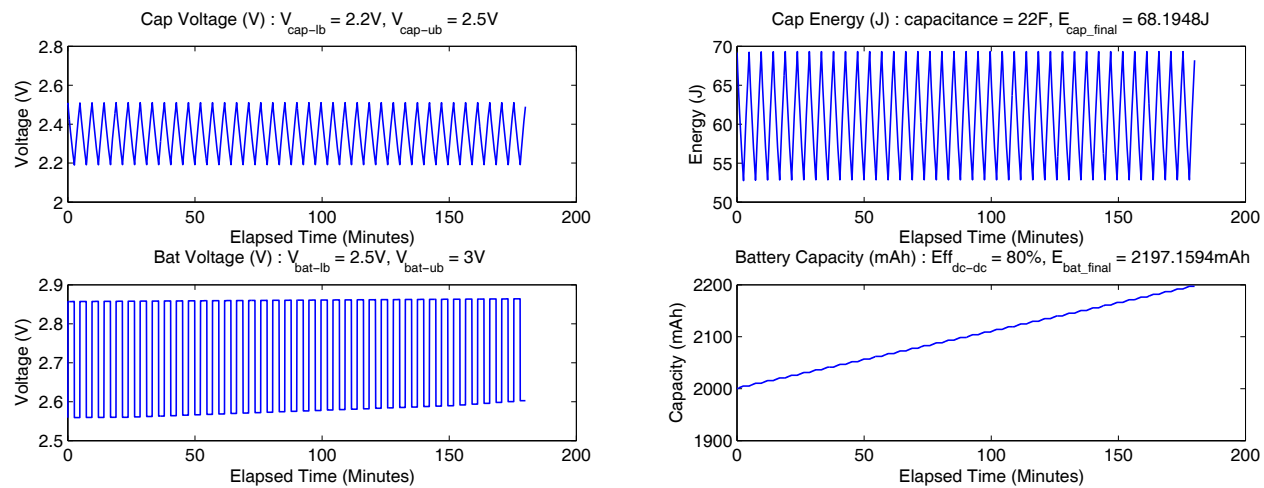

(b) Configuration 4b: 22F supercap $+\mathrm{NiMH,} E_{\text {bat-init }}=2000 \mathrm{mAh}$

Figure 7.12: Effect of initial battery voltage level 

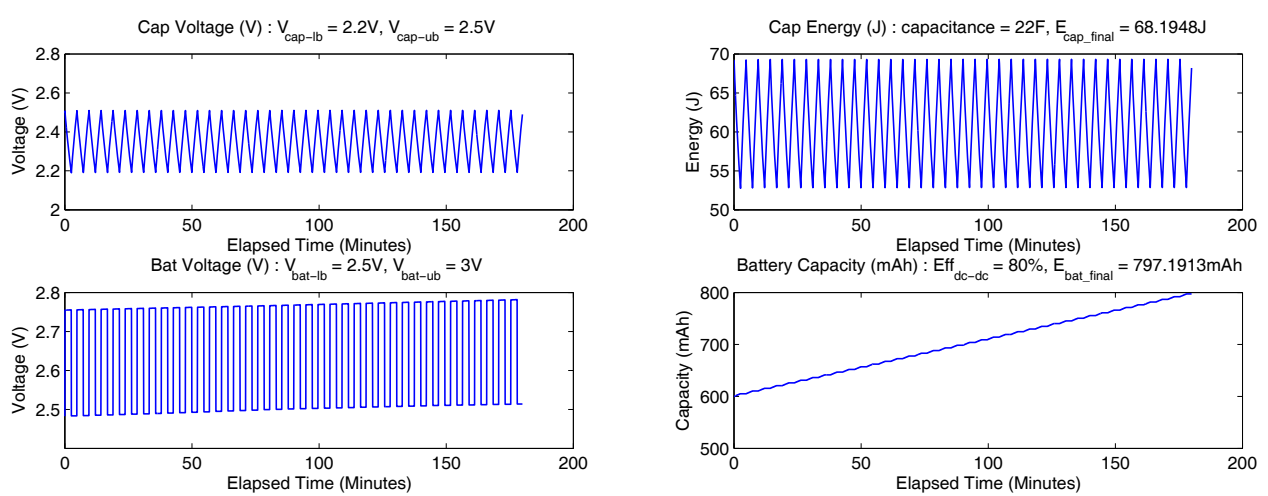

(a) Configuration 5a: 22F supercap $+\mathrm{NiMH}, I_{\max -b a t-c h g}=100 \mathrm{~mA}$
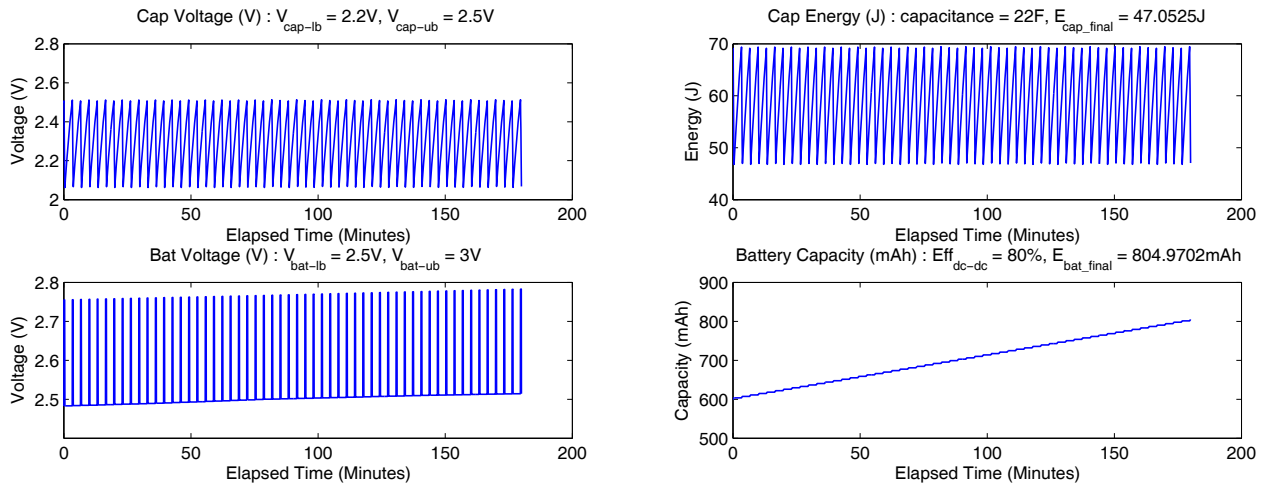

(b) Configuration 5b: 22F supercap $+\mathrm{NiMH}, I_{\max -b a t-c h g}=500 \mathrm{~mA}$

Figure 7.13: Effect of maximum battery charging current (with 22F supercap)

- Configuration 5a : 22F supercap $+\mathrm{NiMH}, I_{\text {max-bat-chg }}=100 \mathrm{~mA}$

- Configuration 5b : $22 \mathrm{~F}$ supercap $+\mathrm{NiMH}, I_{\text {max-bat-chg }}=500 \mathrm{~mA}$

- Configuration 5c : 140F supercap $+\mathrm{NiMH}, I_{\text {max-bat-chg }}=100 \mathrm{~mA}$

- Configuration 5d : 140F supercap $+\mathrm{NiMH}, I_{\max -b a t-c h g}=500 \mathrm{~mA}$ 

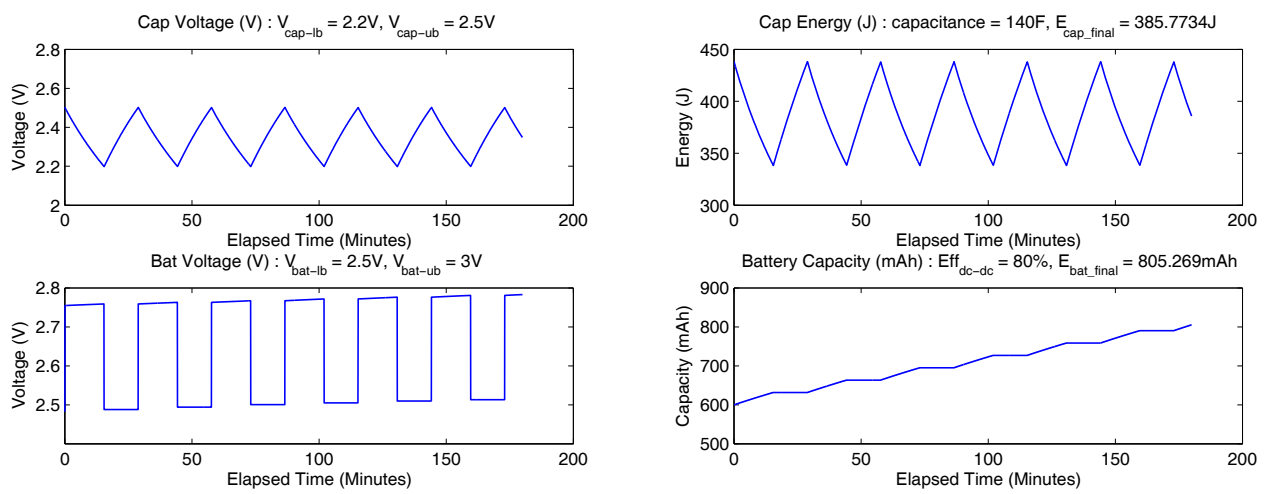

(a) Configuration 5c: $140 \mathrm{~F}$ supercap $+\mathrm{NiMH}, I_{\max -b a t-c h g}=100 \mathrm{~mA}$
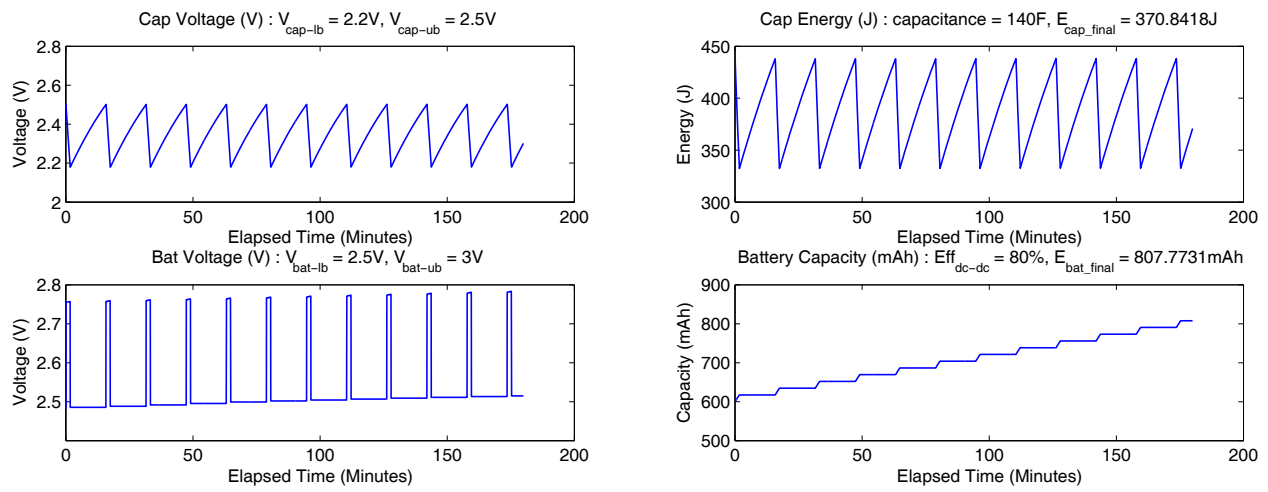

(b) Configuration 5d: 140F supercap $+\mathrm{NiMH}, I_{\max -b a t-c h g}=500 \mathrm{~mA}$

Figure 7.14: Effect of maximum battery charging current (with 140F supercap) 
The accumulated charge in the battery and the supercap are as follows:

$$
\begin{array}{ll}
\text { For Configuration 5a, } & Q=Q_{b a t}+Q_{c a p}=197.12 \mathrm{mAh} \\
\text { For Configuration 5b, } & Q=Q_{b a t}+Q_{c a p}=202.41 \mathrm{mAh} \\
\text { For Configuration 5c, } & Q=Q_{b a t}+Q_{c a p}=199.16 \mathrm{mAh} \\
\text { For Configuration 5d, } & Q=Q_{b a t}+Q_{c a p}=199.89 \mathrm{mAh}
\end{array}
$$

We can see that changing the maximum battery charging current does not change the accumulated charge in the battery and the supercap $(2.6 \%$ of difference for Configuration $5 \mathrm{a}$ and $5 \mathrm{~b}, 0.4 \%$ of difference for Configuration $5 \mathrm{c}$ and $5 \mathrm{~d}$ ). This is due to energy conservation. Any charge to the battery through the DC/DC converter is taken from the supercapacitor, so the overall accumulated charge in the supercapacitor is the same. The difference is that the supercapacitor discharge can happen in a shorter time when the maximum battery charging current increases.

\section{Effect of Efficiency of DC/DC Converter}

Two configurations of multi-level energy storage with a supercapacitor and NiMH batteries are considered by varying the efficiency of the DC/DC converter that charges to the battery (Figure 7.15).

- Configuration 6a : $22 \mathrm{~F}$ supercap $+\mathrm{NiMH}, \mathrm{Eff}_{d c-d c}=80 \%$

- Configuration 6b : $22 \mathrm{~F}$ supercap $+\mathrm{NiMH}, \mathrm{Eff}_{d c-d c}=50 \%$

The accumulated charge in the battery and the supercap are as follows:

$$
\begin{aligned}
& \text { For Configuration 6a, } \quad Q=Q_{b a t}+Q_{c a p}=197.12 m A h \\
& \text { For Configuration 6b, } \quad Q=Q_{b a t}+Q_{c a p}=123.16 m A h
\end{aligned}
$$

The efficiency of the DC/DC converter determines what percentage of extra charge from the supercapacitor can be charged to the battery. We can see that the ratio of 

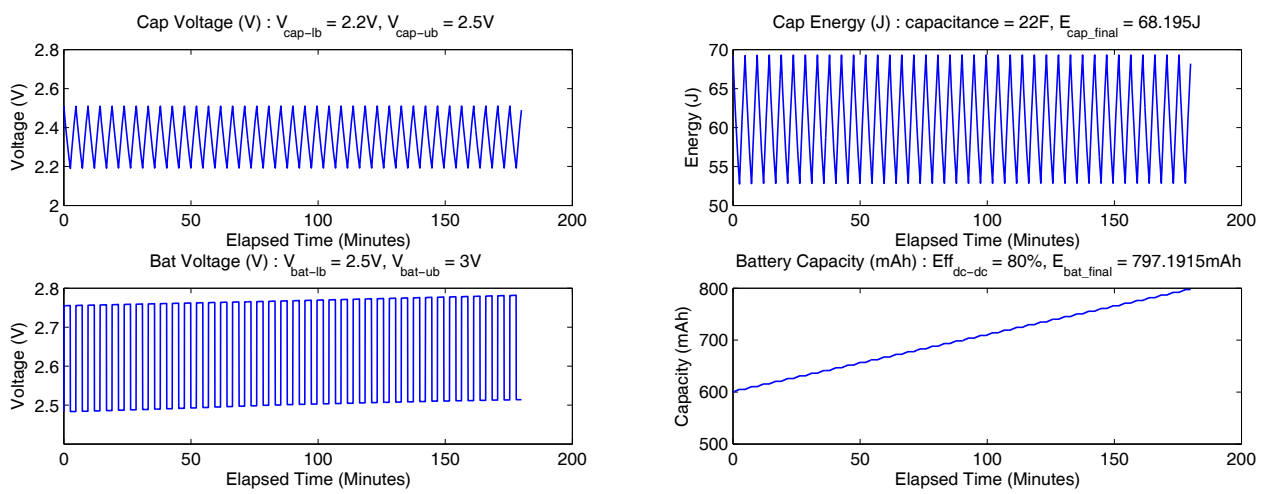

(a) Configuration 6a: 22F supercap + NiMH, $\mathrm{Eff}_{d c-d c}=80 \%$
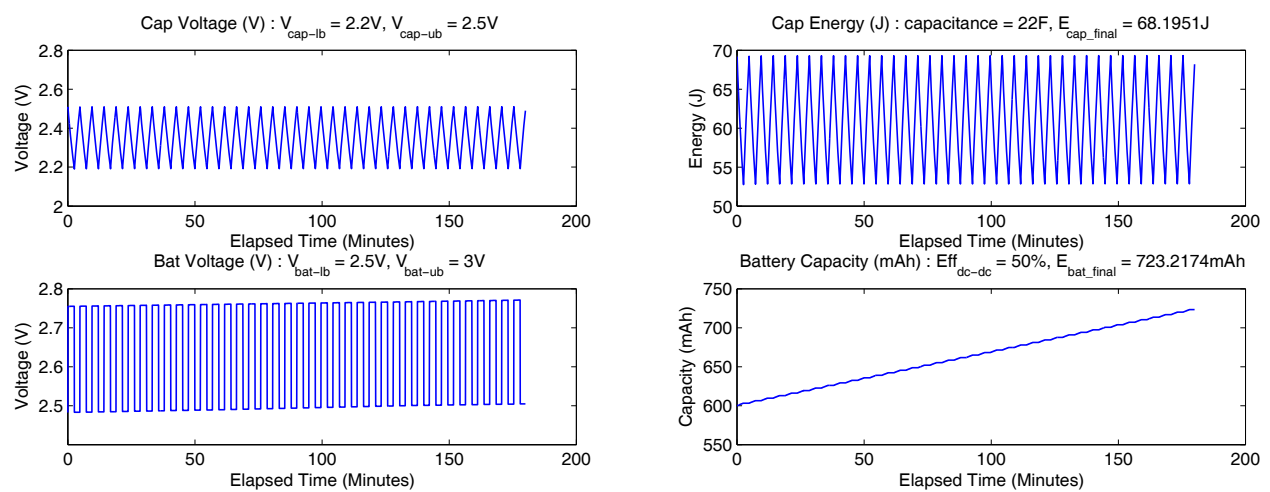

(b) Configuration 6b: 22F supercap $+\mathrm{NiMH}, \mathrm{Eff}_{d c-d c}=50 \%$

Figure 7.15: Effect of efficiency of DC/DC converter 
the accumulated charge for the two configurations are almost the same as the ratio of the efficiency factors of the DC/DC converter:

$$
\begin{aligned}
Q_{6 b} / Q_{6 a} & =123.16 m A h / 197.12 m A h=0.6248 \\
\mathrm{Eff}_{6 b} / \mathrm{Eff}_{6 a} & =50 \% / 80 \%=0.6250
\end{aligned}
$$

This shows that the accumulated charge is proportional to the efficiency factor of the DC-DC converter.

\section{Comparison with NiMH-only Storage}

A multi-level energy storage with a supercapacitor and NiMH batteries is compared with NiMH-only storage (Figure 7.16).

- Configuration 7a : 22F supercap + NiMH

- Configuration 7b : $\mathrm{NiMH}$

The accumulated charge in the energy storage are as follows:

$$
\begin{array}{ll}
\text { For Configuration 7a, } & Q=Q_{b a t}+Q_{c a p}=197.12 \mathrm{mAh} \\
\text { For Configuration 7b }, & Q=Q_{b a t}=46.35 \mathrm{mAh}
\end{array}
$$

We can see that the one with multi-level energy storage accumulates more charge than the one with the NiMH-only storage. This is because Configuration 7a (22F supercap $+\mathrm{NiMH})$ sets the operating point of the solar panel closer to the maximum power point than Configuration $7 \mathrm{~b}$ (NiMH only) under the indoor lamp light, as shown in Figure 7.17 .

\section{Operation During Darkness}

Two configurations of storage are compared with no radiation. The first one has a multi-level energy storage with a supercapacitor and the NiMH batteries. The other is NiMH-only storage (Figure 7.18):

- Configuration 8a : 22F supercap + NiMH 

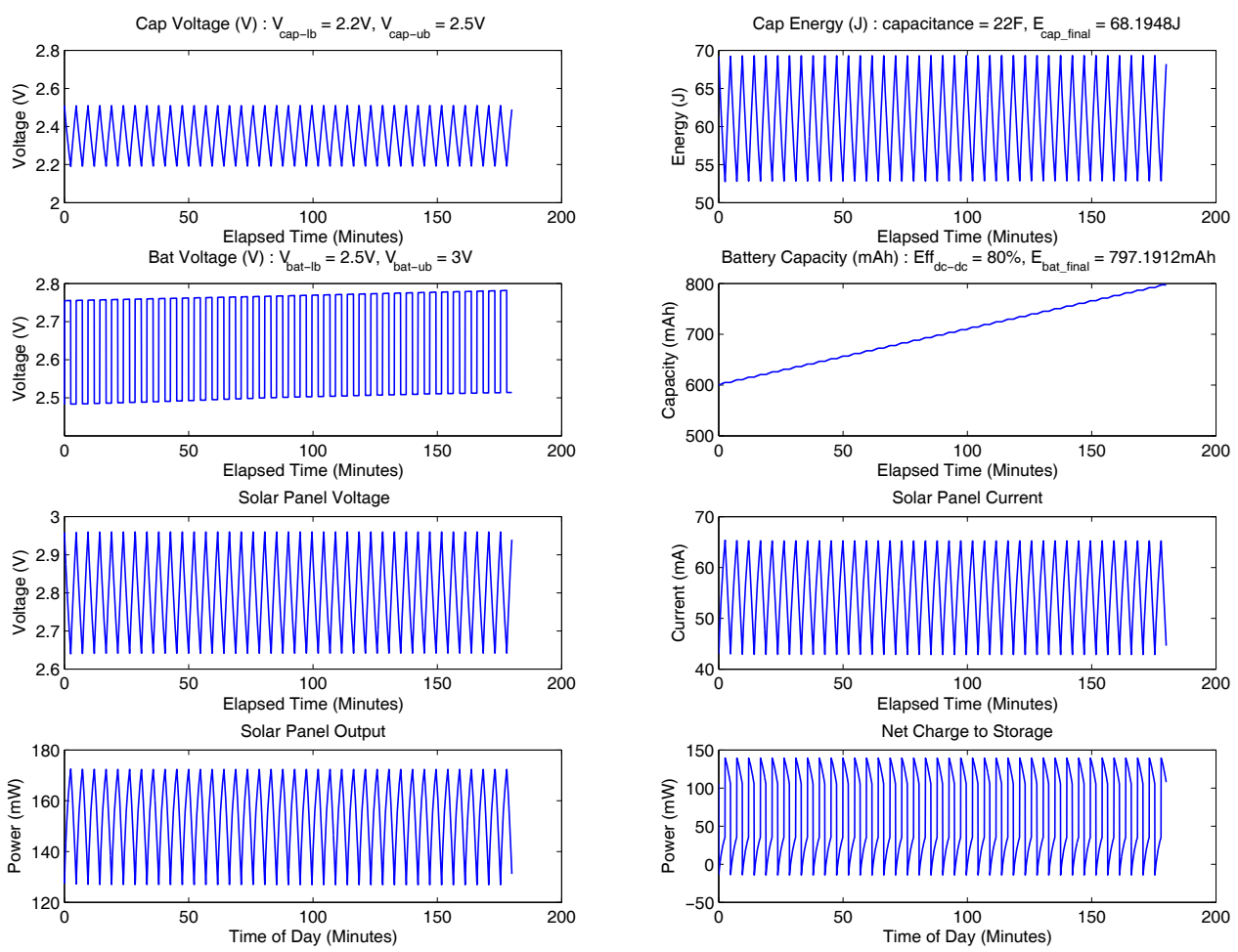

(a) Configuration 7a: $22 \mathrm{~F}$ supercap $+\mathrm{NiMH}$
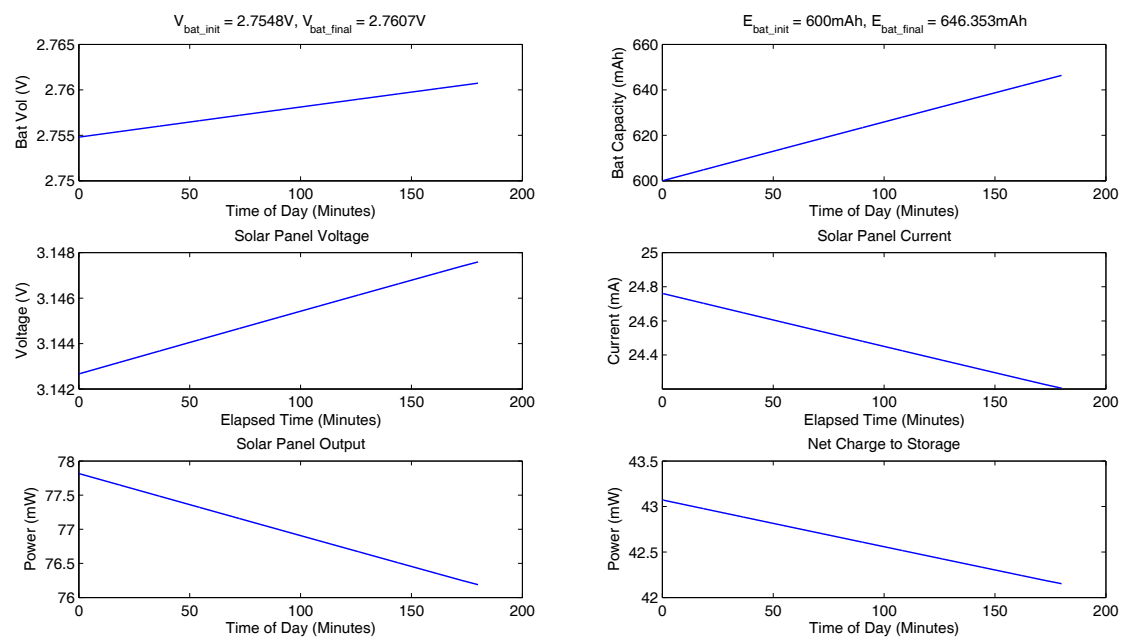

(b) Configuration 7b: NiMH

Figure 7.16: Comparison with NiMH-only storage 


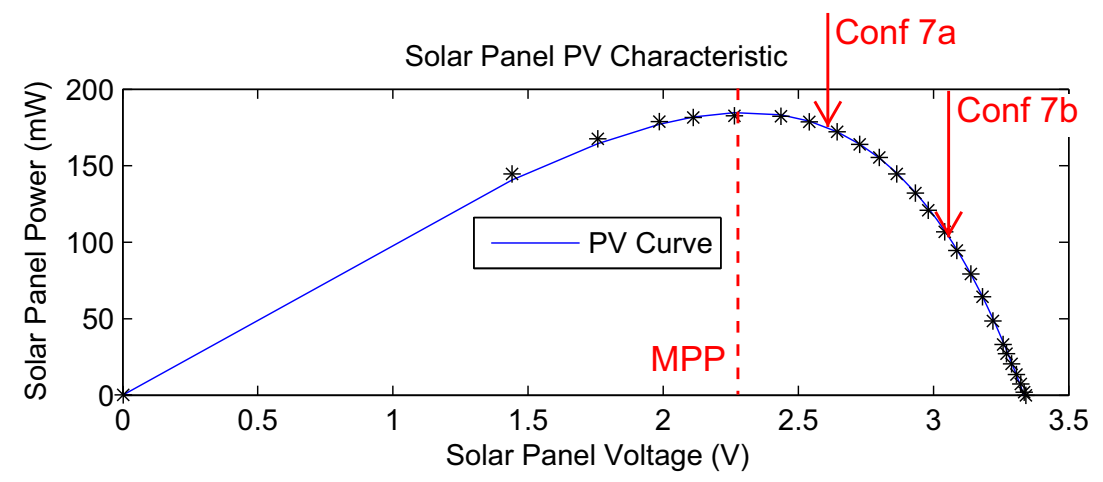

Figure 7.17: Comparison of solar panel operating voltage for Configuration 7a and $7 \mathrm{~b}$
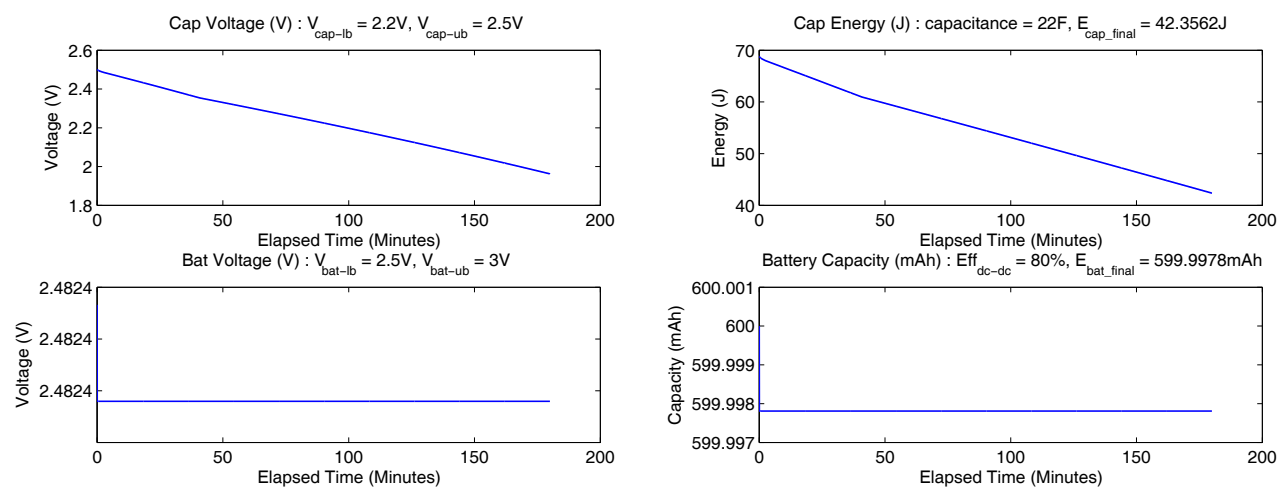

(a) Configuration 8a: 22F supercap $+\mathrm{NiMH}$
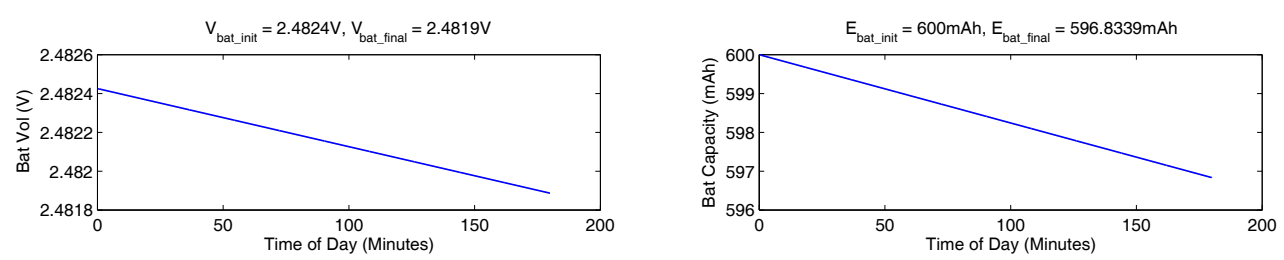

(b) Configuration 8b: NiMH

Figure 7.18: Operation during darkness 


\section{- Configuration 8b : NiMH}

The accumulated charge in the energy storage shows that $3.12 \mathrm{mAh}$ and $3.17 \mathrm{mAh}$ of charge was drawn from the storage for each case.

$$
\begin{array}{ll}
\text { For Configuration 8a, } & Q=Q_{b a t}+Q_{\text {cap }}=-3.12 m A h \\
\text { For Configuration 8b }, & Q=Q_{b a t}=-3.17 m A h
\end{array}
$$

This result matches the estimated charge consumption of the load through the output regulator.

$$
\begin{aligned}
Q_{\text {reg-out }} & =I_{\text {reg-out }} \times T \times \frac{1}{\mathrm{Eff}_{\text {reg-out }}} \\
& =0.53 \mathrm{~mA} \times 3 \mathrm{hr} \times \frac{1}{0.5} \\
& =3.18 \mathrm{mAh}
\end{aligned}
$$

\subsubsection{Summary of Simulation of Multi-Level Energy Storage}

In this section, we have demonstrated that we are able to simulate a hypothetical design of a micro-solar power system. As an example of a hypothetical design, we simulated a micro-solar power system with multi-level energy storage consisting of a supercapacitor and NiMH batteries. From the simulations, we observed the followings:

- The supercapacitor charging range affects the amount of charge transfer; more charge is transferred when the average supercapacitor voltage is matched closer to the maximum power point of the solar panel.

- The efficiency of the DC/DC converter determines how much charge from the supercapacitor is transferred to the battery.

- Other initial conditions do not affect the amount of charge transfer. They are the capacity of the supercapacitor, the width of the supercapacitor charging range, the initial voltage level of the supercapacitor, and the maximum battery charging current. 
We also found that our hypothetical design of a micro-solar power system with multi-level energy storage had a higher energy transfer rate than the system with the NiMH-only energy storage. This is because our hypothetical system had better matching for the maximum power point of the solar panel. While we demonstrated the case where our hypothetical design performs better than the system with the NiMHonly energy storage, the simulator can also produce the case where our hypothetical design performs worse. For example, setting the supercapacitor charging range farther from the optimum range makes the solar panel output power smaller.

\subsection{Extending the Simulator for Other Renewable Energy Sources}

This dissertation has focused on the use of outdoor solar energy for mote-class devices among various types of renewable energy sources. However, other renewable energy sources can also be considered in the absence of outdoor solar energy, although their power density is not as high as that of outdoor sunlight. In this section, we review two cases of other renewable energy sources - wind and vibrations. For each type of renewable energy source, we describe the characteristics of the energy source, the energy collector, and the rest of the energy harvesting system. Then, we will describe a brief idea on extending the micro-solar power simulator for each renewable energy source.

\subsubsection{Extension for a Wind Energy Harvesting System}

A wind energy harvesting system can be represented as a generic model in Figure 7.19, which consists of the following components: the external environment, an energy collector, input regulator, energy storage, output regulator, and a load. The wind energy from the environment is collected by a wind generator. The electricity generated from the wind generator is $\mathrm{AC} \mathrm{L}^{2}$ and it needs to be converted to DC before it is stored in the energy storage. The input regulator does this conversion: the

\footnotetext{
${ }^{2}$ As for wind generator, a three-phase permanent magnet synchronous generator is assumed, but other types of AC generator can be used.
} 


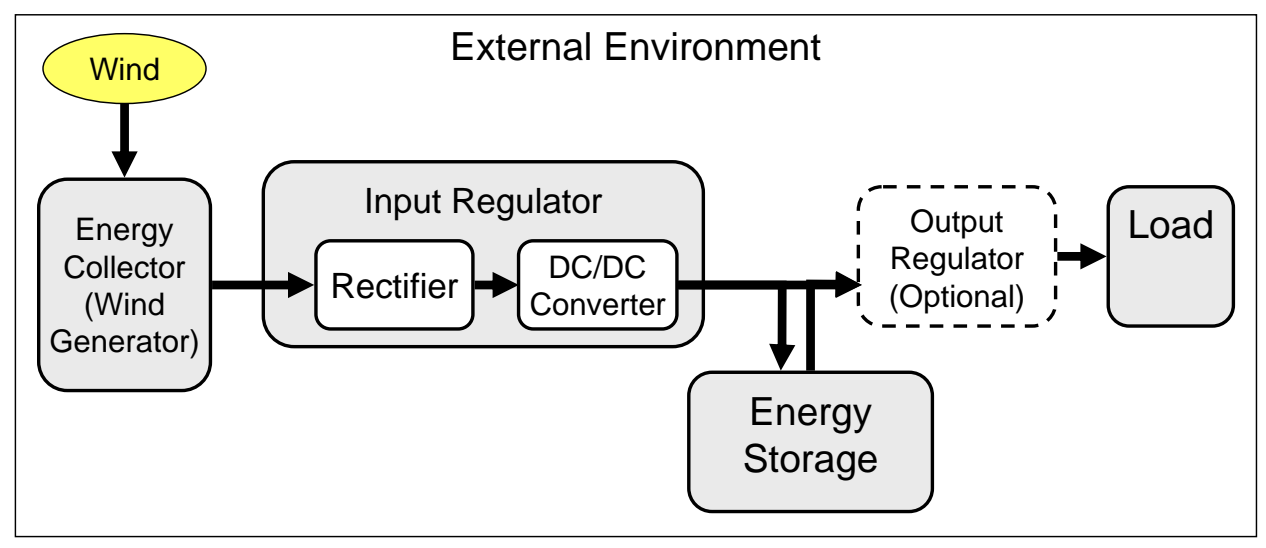

Figure 7.19: A generic model for wind energy harvesting system

rectifier converts $\mathrm{AC}$ to $\mathrm{DC}$, and the DC-DC converter adjusts the voltage level of the DC rectifier output to be within the charging range of the energy storage. The energy stored in the energy storage is used by the load and it can possibly go through an output regulator.

If we compare this with the architecture of a micro-solar power system, we can see that a wind energy harvesting system has different characteristics for the external environment, energy collector and input regulator. In the rest of this section, we will describe the characteristics of these components.

\section{External Environment: Wind}

Based on a fact that power from the wind is proportional to the cube of the wind speed $\left[\mathrm{GCD}^{+} 98\right]$, we can estimate the energy income by determining the wind speed at a particular location:

$$
P_{\text {wind }} \propto V_{\text {wind }}^{3}
$$

where the wind speed $V_{\text {wind }}$ can be measured or estimated from previous statistics.

\section{Energy Collector: Wind Generator}

The wind generator converts the mechanical energy from the wind to electrical energy. In order to model and characterize a wind generator, we use the experimental 
data by Gevorgian et al. $\left[\mathrm{GCD}^{+} 98\right]$ in Figure 7.20. From this experimental data, we know the followings:

- When the wind speed is between the lower bound (about $3 \mathrm{~m} / \mathrm{s}$ ) and the upper bound (about $8 \mathrm{~m} / \mathrm{s}$ ), the relationship between the wind speed and the wind generator output power follows the cubic curve.

- When the wind speed is above the upper bound, the output power is almost flat. This is because a wind generator is made not to exceed the maximum output voltage, which limits the wind generator output power above the upper bound.

- When the wind speed is below the lower bound, the output power is zero. This is when the output voltage of the wind generator is lower than the threshold voltage of the rectifier and is not usable.

From these findings, we can formulate the output of a wind generator as follows:

$$
P_{\text {wind-gen }}=\min \left\{\max \left\{k \cdot V_{\text {wind }}^{3}-P_{t h}, 0\right\}, P_{\max }\right\}
$$

\section{Input Regulator}

The input regulator for the wind energy harvesting system has two components: a rectifier and a DC-DC converter. We can characterize the input regulator with an operating range and a power efficiency for the rectifier and DC-DC converter.

Figure 7.21 summarizes the extension for the simulation with wind energy harvesting.

\subsubsection{Extension for Vibrational Energy Harvesting System}

A vibrational energy harvesting system can be represented as a generic model in Figure 7.22 , which is composed of the external environment, an energy collector, input regulator, energy storage, output regulator, and a load. The vibrational energy from the environment is collected by the vibration-to-electricity converter. Similar to the case of wind energy harvesting, the electricity generated from the vibration 


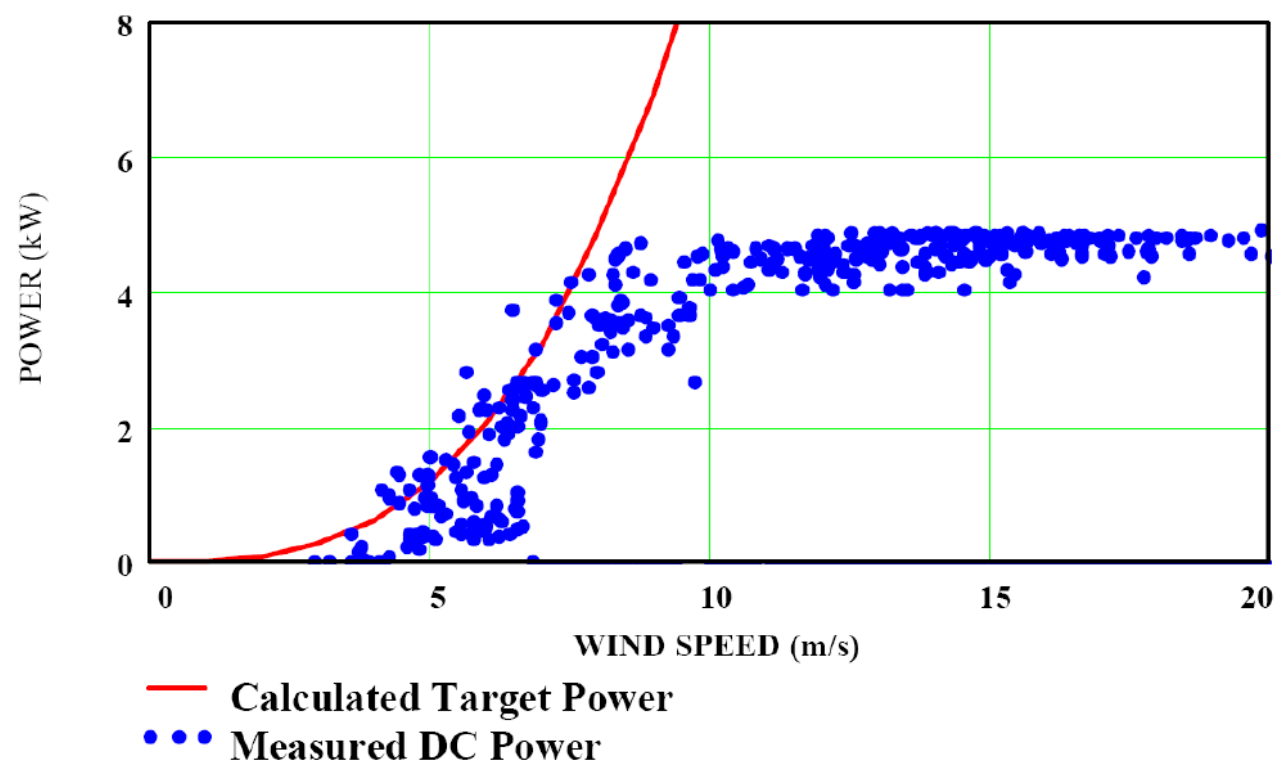

Figure 7.20: Output power from the wind generator for varying wind speed: courtesy of Gevorgian et al. [GCD $\left.{ }^{+} 98\right]$

is $\mathrm{AC}$ and it needs to be converted to DC before it is stored in the energy storage. The input regulator, which consists of a rectifier and a DC-DC converter, does the following: first, the rectifier converts $\mathrm{AC}$ to $\mathrm{DC}$, and second, the DC-DC converter adjusts the voltage level of the rectifier output to be within the charging range of the energy storage. The energy stored in the energy storage is used by the load and can possibly go through an output regulator.

We can see that the architecture of a vibrational energy harvesting system is similar to the wind energy harvesting system except for the external environment and the energy collector (vibration-to-electricity converter). In the rest of this section, we describe the characteristics of these two components.

\section{External Environment: Vibrations}

A vibration source is characterized by the amplitude and frequency of the dominant vibration mode. Table 7.1 by Roundy [Rou03] shows several examples of vibration sources that can be found in residential, office and industrial environments.

In most cases, a vibration source can be turned on or off by the user and a usage 


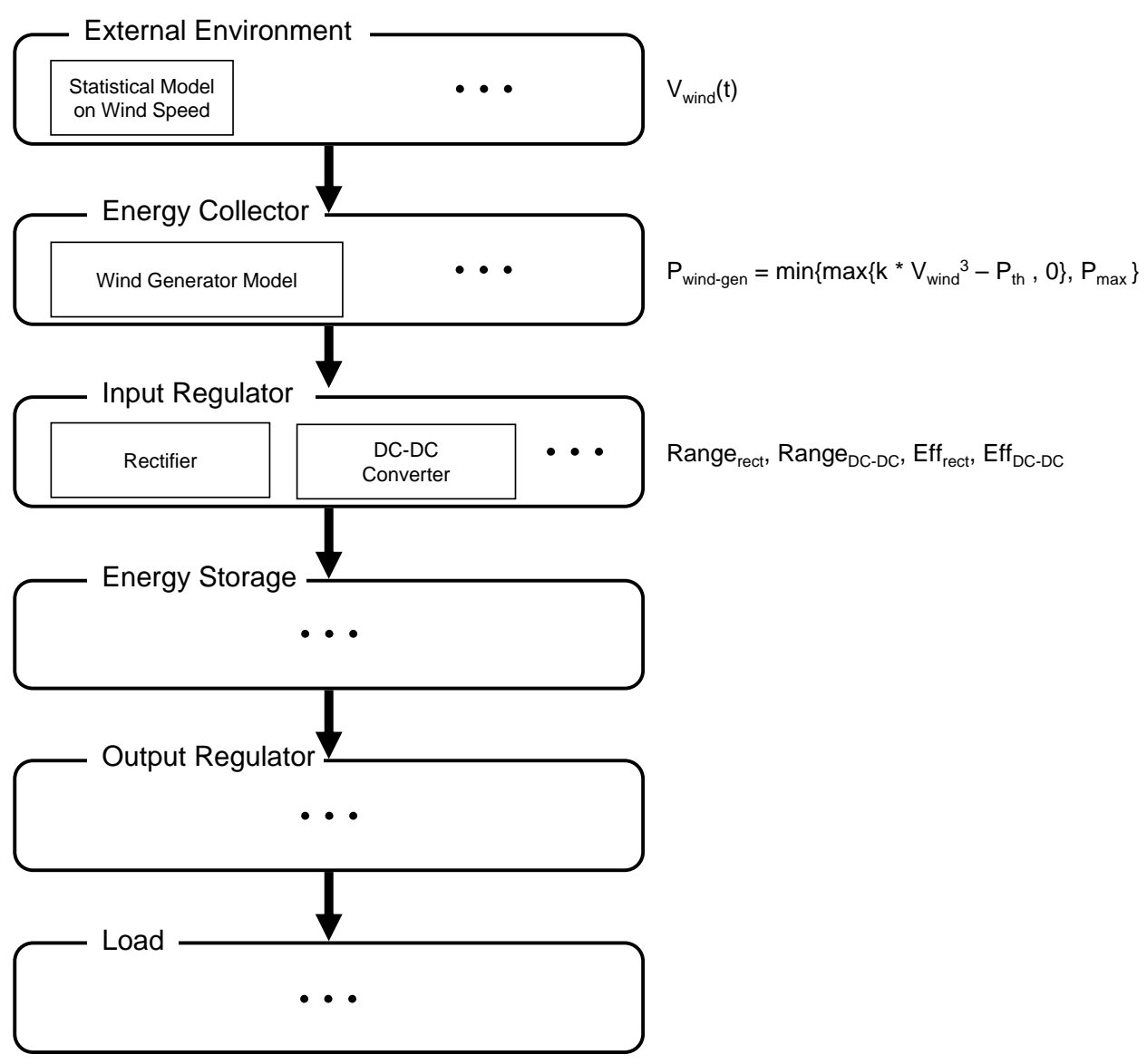

Figure 7.21: Extension for the simulation model with wind energy harvesting

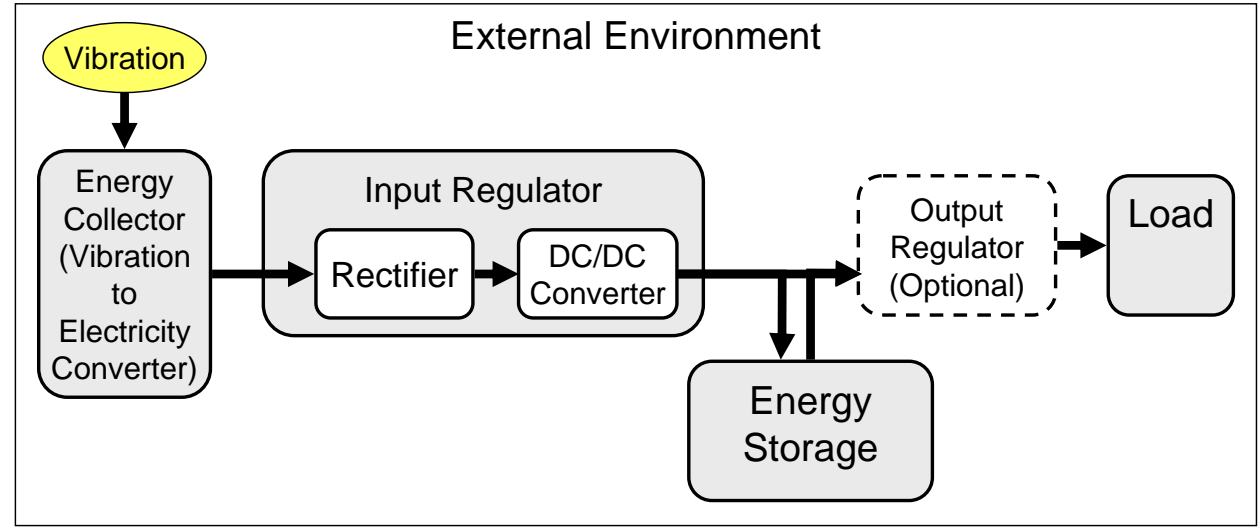

Figure 7.22: A generic model for vibrational energy harvesting system 
Table 7.1: List of vibration sources with the characteristics of the dominant vibration mode - courtesy of Roundy [Rou03]

\begin{tabular}{|l|l|l|}
\hline Vibration Source & $\begin{array}{l}\text { Acceleration } \\
\left(\mathbf{m} / \mathbf{s}^{2}\right)\end{array}$ & $\begin{array}{l}\text { Frequency } \\
(\mathbf{H z})\end{array}$ \\
\hline Base of 5 HP 3-axis machine tool with 36" bed & 10 & 70 \\
\hline Kitchen blender casing & 6.4 & 121 \\
\hline Clothes dryer & 3.5 & 121 \\
\hline Door frame just after door closes & 3 & 125 \\
\hline Small microwave oven & 2.25 & 121 \\
\hline HVAC vents in office building & $0.2-1.5$ & 60 \\
\hline Wooden deck with people walking & 1.3 & 385 \\
\hline Breadmaker & 1.03 & 121 \\
\hline External windows (size 2ft x 3ft) next to a busy street & 0.7 & 100 \\
\hline Notebook computer while CD is being read & 0.6 & 75 \\
\hline Washing Machine & 0.5 & 109 \\
\hline Second story floor of a wood frame office building & 0.2 & 100 \\
\hline Refrigerator & 0.1 & 240 \\
\hline
\end{tabular}

pattern needs to be considered. For example, HVAC vents can vibrate in the same pattern all day long, but they can have different patterns for day and night if air conditioning is turned off for energy saving during the night.

\section{Energy Collector: Vibration-to-Electricity Converter}

According to Roundy [Rou03], the output power of a vibration-to-electricity converter can be formulated as follows when the resonant frequency of the converter 
matches the frequency of the vibration $\omega$ :

$$
\begin{aligned}
|P| & =\frac{m \zeta_{e} A^{2}}{4 \omega \zeta_{T}^{2}} \\
m & : \text { mass } \\
\omega & : \text { driving frequency } \\
A & : \text { amplitude of acceleration } \\
\zeta_{e} & : \text { damping ratio (electric) } \\
\zeta_{T} & : \text { damping ratio (electric and mechanical) }
\end{aligned}
$$

From this equation, we can see the following: (a) the output power is proportional to the mass of the converter; (b) the output power is inversely proportional to the frequency of the driving vibration; (c) the output power is proportional to the square of the amplitude of the acceleration. The work of Roundy [Rou03] shows a concrete example of a vibration-to-electricity converter. He demonstrated that a piezo-electric vibration-to-electricity converter had an energy density of $335 \mathrm{uW} / \mathrm{cm}^{3}$ with a vibration source of $2.25 \mathrm{~m} / \mathrm{s}^{2}$ at $60 \mathrm{~Hz}$. Figure 7.23 summarizes the extension for the simulation with vibrational energy harvesting.

\section{$7.3 \quad$ Summary}

In this chapter, we explored two ideas for extending the micro-solar power system simulator. First, in order to demonstrate that the micro-solar power system simulator can be extended for a hypothetical design, we simulated a micro-solar power system with a multi-level energy storage consisting of a supercapacitor and $\mathrm{NiMH}$ batteries. From the simulations, we were able to determine which system parameters affected the system and how. Second, we extended the simulator to include wind and vibration energy to demonstrate that the simulation tool can be used for other renewable energy sources. The extended simulator is organized similarly to the micro-solar power system simulator: the external environment, an energy collector, input regulator, energy storage, output regulator, and a load. The differences are the characteristics of the external environment (e.g. wind and vibration) and the energy collector (e.g. wind generator and piezo-electric material). 


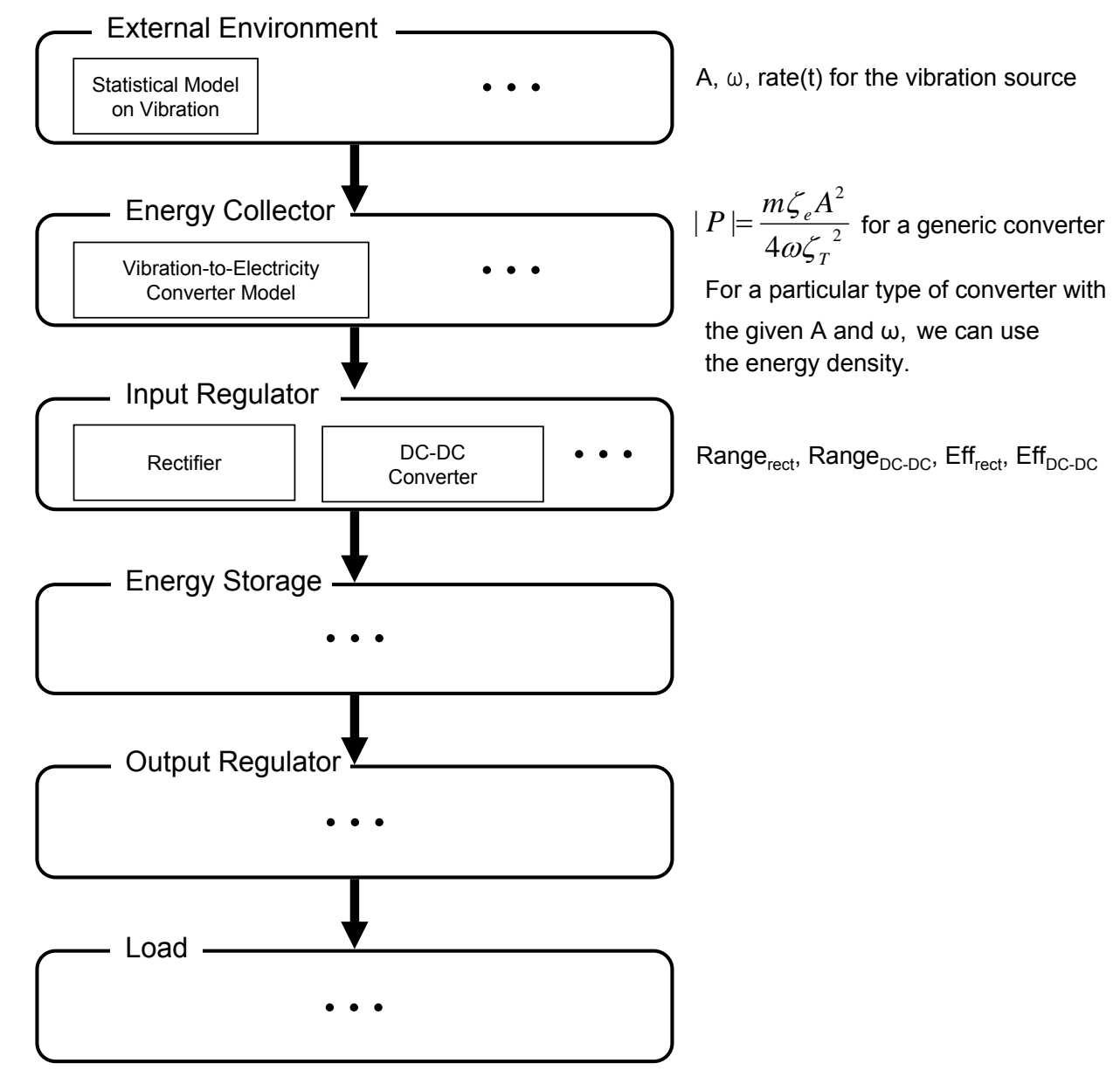

Figure 7.23: Extension for the simulation model with vibrational energy harvesting 


\section{Chapter 8}

\section{Extending the Simulator for a Meso-Solar Power System}

In this chapter, we extend our simulation tools for a meso-solar system to validate our model with a solar power system of a larger scale. For this purpose, we design a solar-powered web server by composing Watt-scale components (Section 8.1). We simulate this design by building a model for each component and integrating it into our simulator (Section 8.2). Then, we validate this design against the measurement of the implementation (Section 8.3) and use this result for long-term prediction (Section 8.4.

\subsection{System Architecture of Meso-Solar System}

The solar-powered web server is a PDA-class Linux box that has an 802.11 wireless interface and runs a web service (Figure 8.1). This meso-solar system has very similar components - solar panel, input regulator, energy storage, output regulator and load - and organization to a micro-solar power system (Figure 8.2). However, the scale and efficiency numbers are quite different. For example, the solar panel of the solarpowered web server is rated at $50 \mathrm{~W}$, whereas a typical solar panel for a micro-solar power system has $100 \mathrm{~mW}$ to $500 \mathrm{~mW}$ of output power. In the rest of the section, we look at the details of each component of the solar-powered web server and compare the numbers with those of a micro-solar power system. 


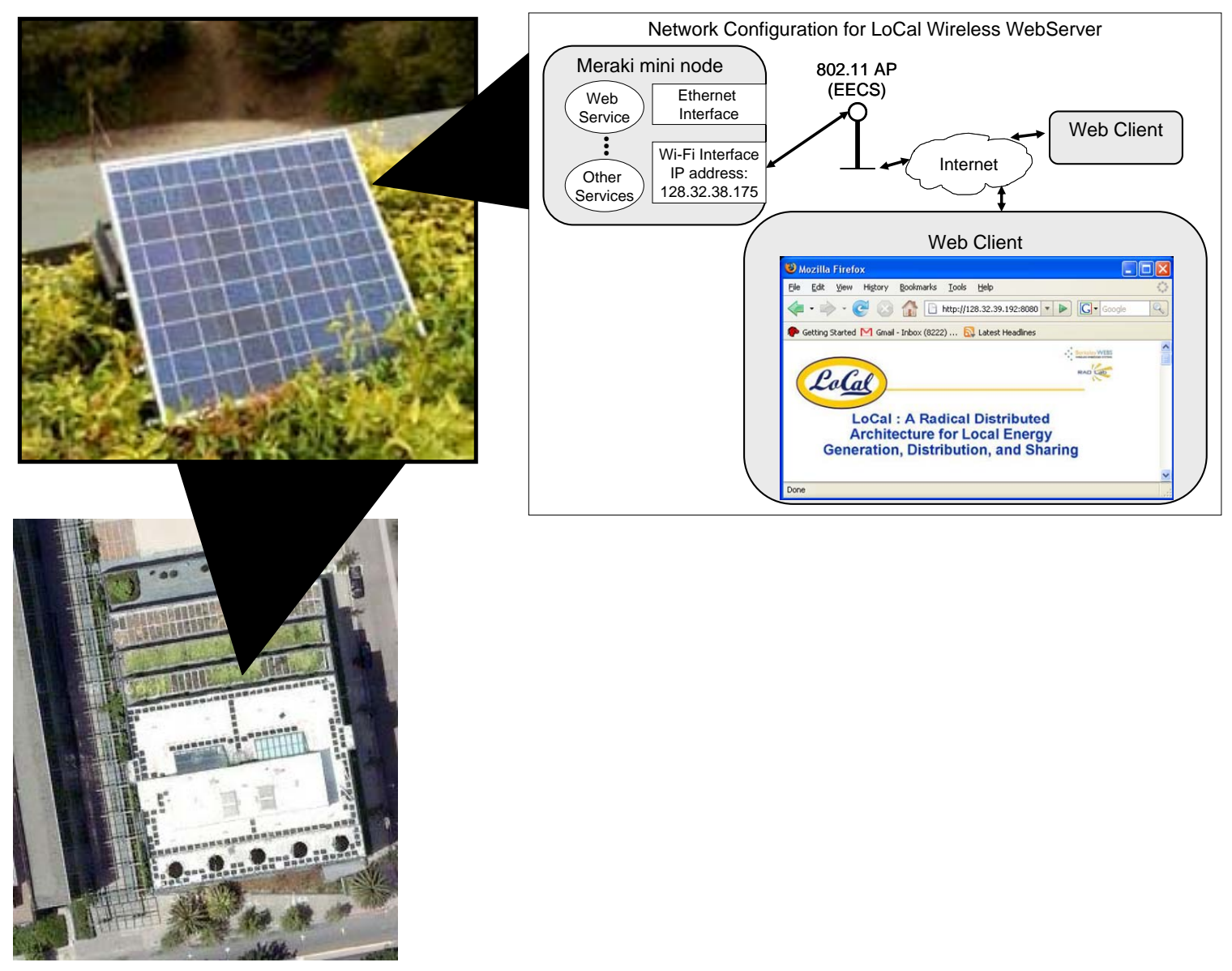

Figure 8.1: A solar-powered web server and its network architecture 

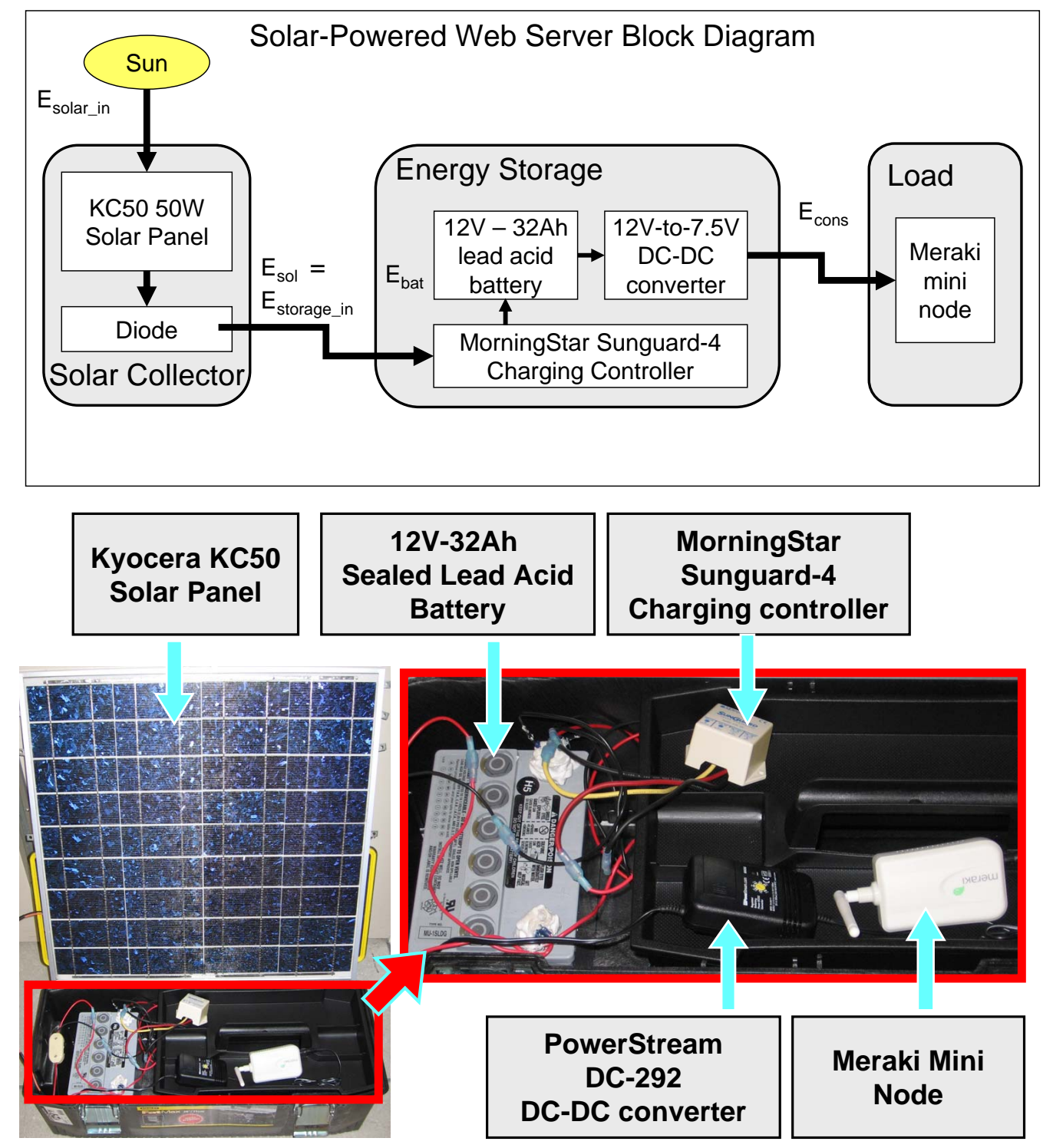

Figure 8.2: System architecture for a solar-powered web server: (b) Energy flow diagram, (b) Photograph of components 


\section{Load: Meraki Mini}

A Meraki Mini node [Mer], which is a PDA-class 802.11-wireless capable Linux box, is used as the load for the solar-powered web server. The power consumption of a Meraki Mini node provided by the manufacturer is typically $3.5 \mathrm{~W}$ and at maximum $7.5 \mathrm{~W}$. We measured the average power consumption of a Meraki Mini as $2.25 \mathrm{~W}$ (= $7.5 \mathrm{~V} * 0.3 \mathrm{~A})$. In contrast, the HydroWatch application, our reference implementation for a micro-solar power system, has an average power consumption of $1.5 \mathrm{~mW}$, more than 1000-times smaller than the power consumption of a meso-solar system.

\section{Solar Panel: Kyocera KC50 Panel}

The solar panel for the solar-powered web server is a Kyocera KC50 panel [Kyo]. The KC50 panel has a maximum power of $50 \mathrm{~W}$ at $16.7 \mathrm{~V}$ with $3.0 \mathrm{~A}$ and an open-circuit voltage of $21.5 \mathrm{~V}$ and a short-circuit current of 3.1A. The I-V and P-V characteristics of a KC50 panel are shown at Figure 8.3. Whereas, the HydroWatch application has a maximum of $300 \mathrm{~mW}$ of solar panel output, which is 100-times smaller than the solar panel output of the meso-solar system.

\section{Energy Storage: 12V-32Ah Sealed Lead Acid Battery}

As energy storage, a sealed-lead acid battery with a nominal operating voltage of $12 \mathrm{~V}$ and a capacity of $32 \mathrm{Ah}$ was used. The relationship between battery voltage and capacity is shown in Figure 8.4. This relationship is generated from a voltageto-capacity curve of a 12V lead acid battery [Per93] assuming total capacity of 32Ah, a charging rate of $\mathrm{C} / 20$ and a discharging rate of $\mathrm{C} / 100^{1}$. The charge-discharge efficiency is not shown for this particular model of battery, but it is known that a lead acid battery has an efficiency of $70 \%$ to $92 \%$ [Wik].

\section{Input Regulator: MorningStar Sunguard-4}

A MorningStar Sunguard-4 charging controller is used as an input regulator. This charging controller is designed for charging a $12 \mathrm{~V}$ lead-acid battery with a maximum

\footnotetext{
C.

${ }^{1} \mathrm{C}$ is the capacity rating of the battery. For example, $\mathrm{C} / 20$ is $1 / 20$ of $\mathrm{C}$ and $\mathrm{C} / 100$ is $1 / 100$ of
} 

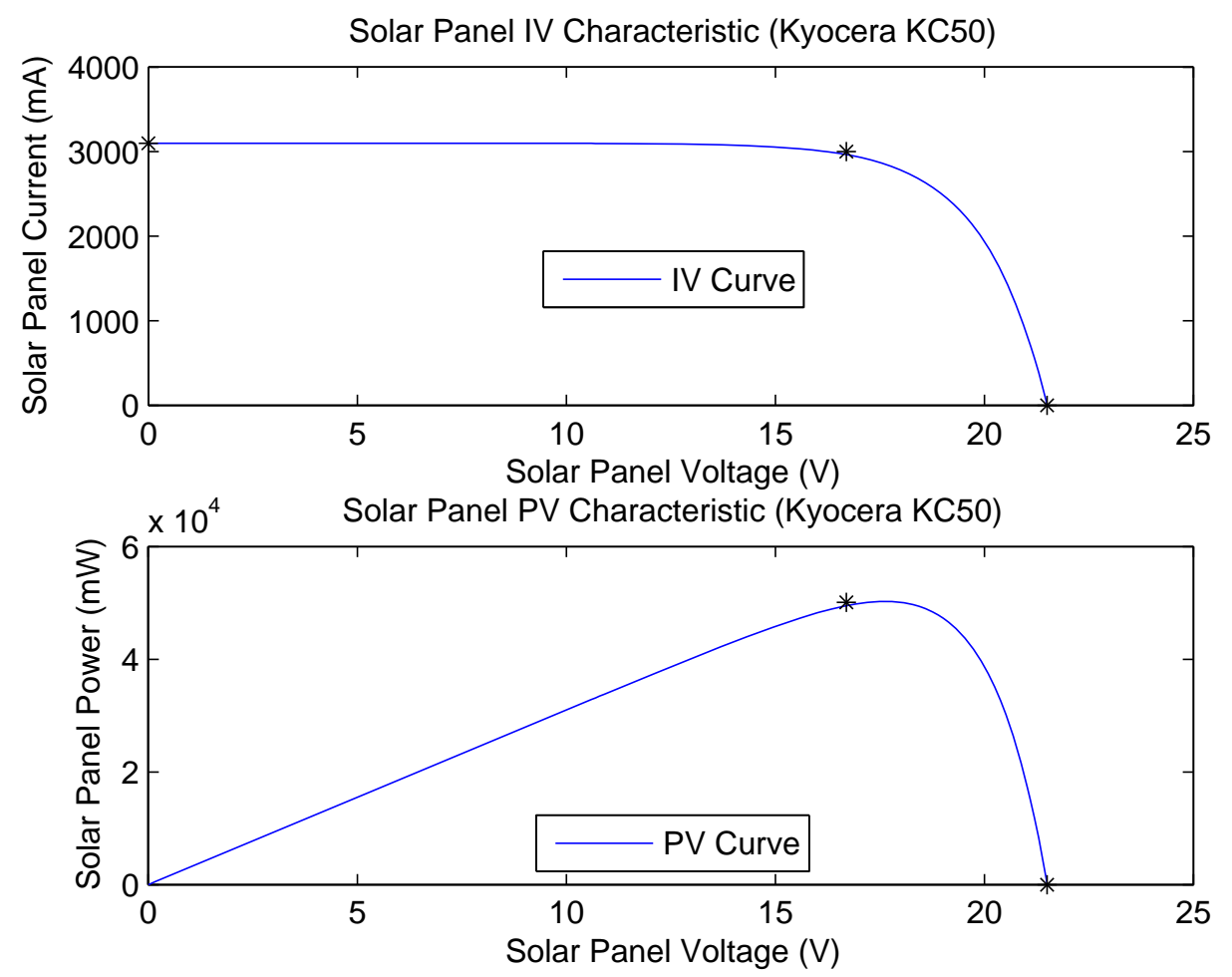

Figure 8.3: Characteristic for Kyocera KC50 solar panel. $I=I_{s c}-A \cdot(\exp (B \cdot V)-1)$ with $A=0.0063, B=0.6096, I_{s c}=3100$. Units of $V$ and $I$ are $V$ and $m A$.
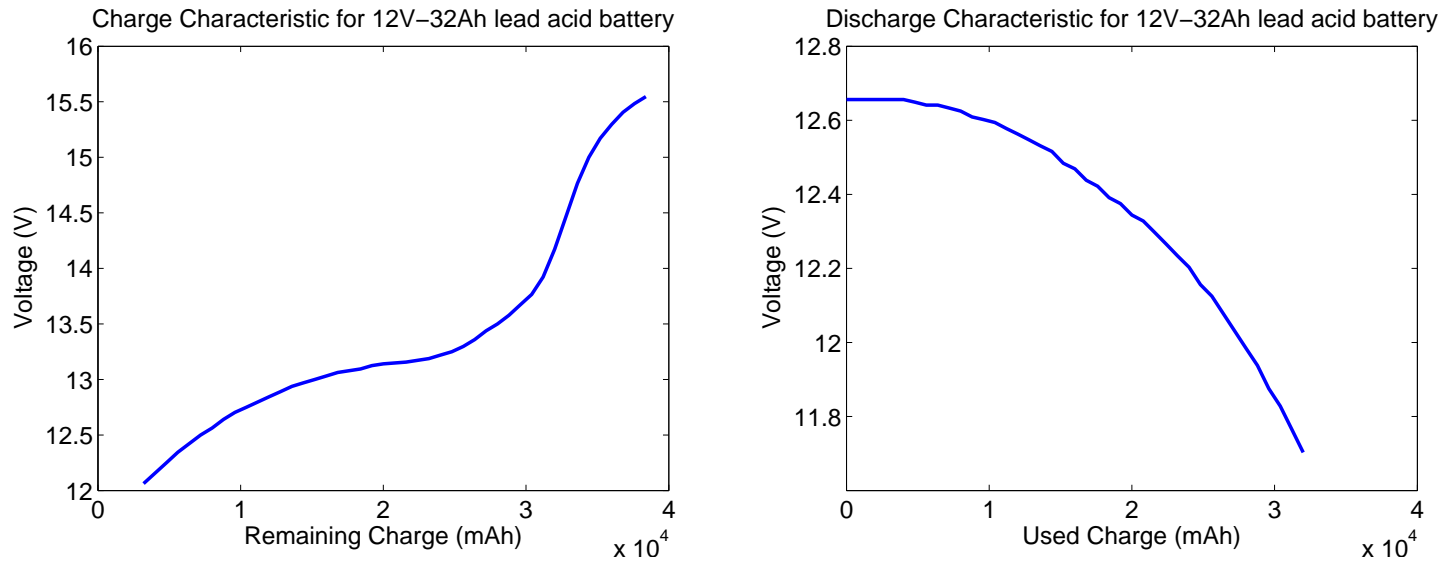

Figure 8.4: Voltage-to-capacity relationship for 12V-32Ah lead acid battery: (a) charging, (b) discharging 

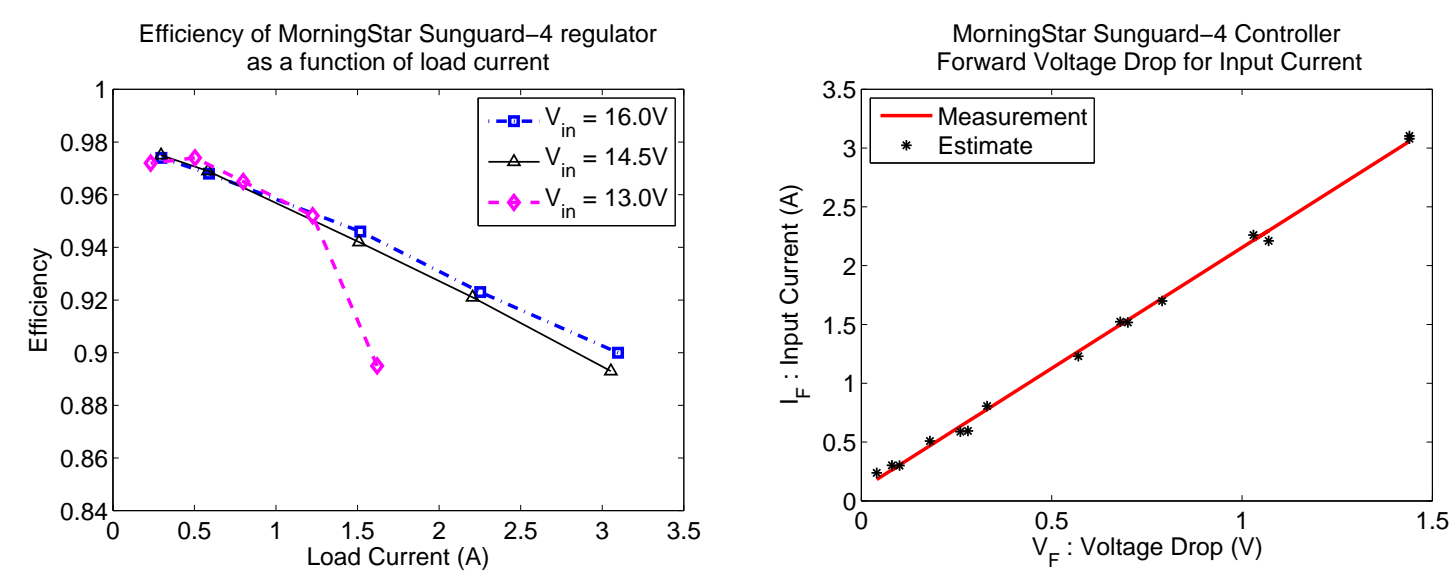

Figure 8.5: Characteristics of MorningStar Sunguard-4 regulator: (a) power efficiency, (b) operating points

input current of 4.5A. We measured the efficiency of this regulator at typical input voltage levels of the solar panel $(13 \mathrm{~V}, 14.5 \mathrm{~V}$ and $16 \mathrm{~V})$ with varying current between 0.29A to 3.1A. At this operating range, the MorningStar Sunguard-4 has an efficiency between $89 \%$ to $97 \%$ (Figure 8.5(a)). Notice that this is higher than the input regulator efficiency of a micro-solar power system. For example, the HydroWatch application has an input regulator efficiency of $60 \%$ when it is configured with an input regulator. The measurement of the operating points of the regulator (Figure 8.5(b)) shows that the forward voltage drop $V_{F}$ and the input current $I_{F}$ can be described by the following equation: $V_{F}=\max \left(\left(I_{F}-b\right) / a, 0\right)$ with $\mathrm{a}=2.0538$ and $\mathrm{b}=0.1$. This relation can be used to estimate the solar panel voltage (input of the charging controller) when the battery voltage (output of the charging controller) is given.

\section{Output Regulator: PowerStream PST-DC292}

As an output regulator, a PowerStream PST-DC292 [Pow] DC-DC converter was used. This regulator was designed to output several different voltage levels for $12 \mathrm{~V}$ or $24 \mathrm{~V}$ input, and we set it to output $7.5 \mathrm{~V}$. The output voltage was measured around $7.77 \mathrm{~V}$ within the operating range of the $12 \mathrm{~V}$ lead acid battery (Figure 8.6(a)). The power efficiency of this regulator was measured to be between $80 \%$ to $82 \%$ with a Meraki Mini node as load and a $12 \mathrm{~V}$ lead acid battery (Figure 8.6(b)). Notice that a micro-solar power system has a lower power efficiency for the output regulator, as 

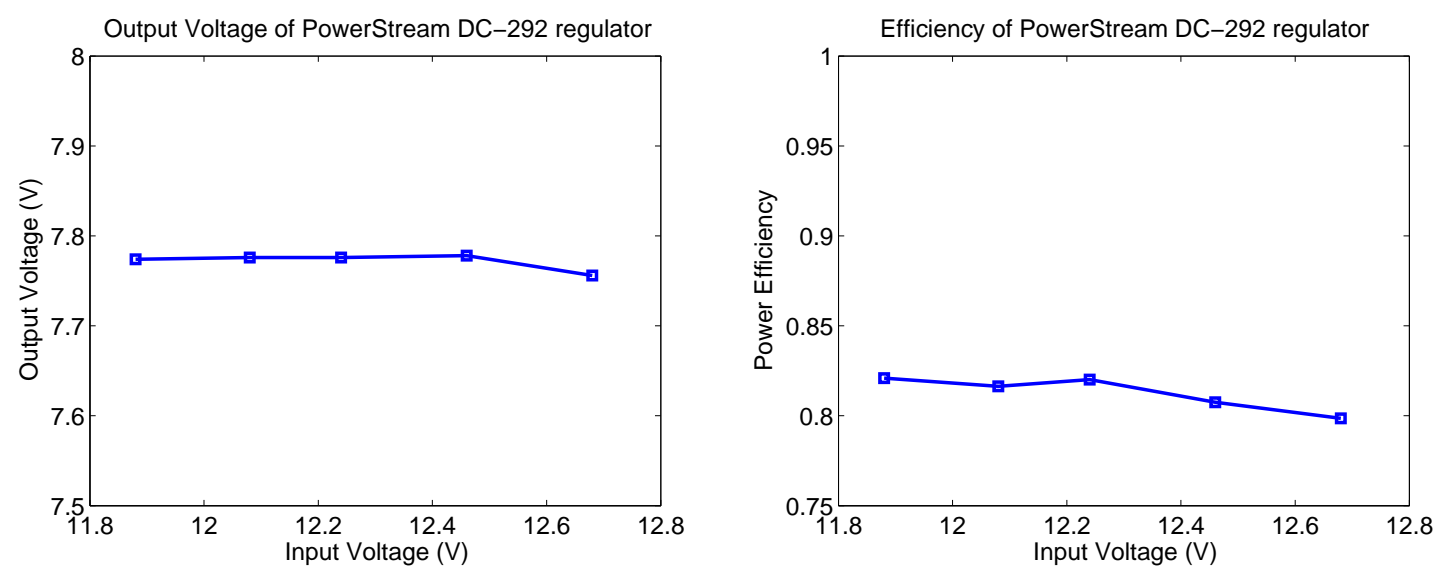

Figure 8.6: Characteristics of PowerStream PST-DC292 regulator: (a) output voltage, (b) power efficiency

the HydroWatch application has an output regulator efficiency of about $50 \%$. The characteristics of each component of the solar-powered web server is summarized in Table 8.1.

\section{Comparison of Meso-Solar System with Micro-solar System}

Table 8.2 compares a meso-solar system with a micro-solar power system using the Solar Web Server and the HydroWatch node as examples. From this data, we can observe the following: first, the two systems have the same categories of components and interconnections; second, the load of the meso-solar system is much higher than that of the micro-solar system, and this makes the meso-solar system require a higher capacity for the solar collector and the energy storage; third, components of the meso-solar system have a higher efficiency than the corresponding components of the micro-solar system. This observation reconfirms our statement in Section 2.2 .3 about the relationship between the micro-solar system and the meso-solar system: macrosolar and meso-solar power systems have the same basic component categories and the same interconnection as a micro-solar power system; the difference is the relative sizes, which translates into differences in the design and deployment of a micro-solar power system. 
Table 8.1: Components for solar-powered web server

\begin{tabular}{|c|c|}
\hline \multicolumn{2}{|c|}{ (a) Solar Panel (Kyocera KC50) } \\
\hline$V_{o c}, I_{s c}$ & $21.5 \mathrm{~V}, 3.1 \mathrm{~A}$ \\
\hline $\mathrm{MPP}$ & $50 \mathrm{~W}$ at $16.7 \mathrm{~V}$ with $3.0 \mathrm{~A}$ \\
\hline I-V curve & Figure 8.3 \\
\hline Dimension & $25.2 \mathrm{in} \times 25.7 \mathrm{in}$ \\
\hline Material, Efficiency & Polycrystalline silicon, $15 \%$ \\
\hline \multicolumn{2}{|c|}{ (b) Input Regulator (MorningStar Sunguard-4) } \\
\hline Measured efficiency & $89 \%$ to $97 \%$ \\
\hline \multicolumn{2}{|c|}{ (c) Energy Storage } \\
\hline Configuration & One Sealed Lead Acid Battery \\
\hline Voltage & $\begin{array}{l}12 \mathrm{~V} \text { nominal } \\
11.89 \mathrm{~V} \text { at } 0 \% \text { charge } \\
12.65 \mathrm{~V} \text { at } 100 \% \text { charge }\end{array}$ \\
\hline Capacity & $32 \mathrm{Ah}$ \\
\hline \multicolumn{2}{|c|}{ (d) Output Regulator (PowerStream PST-DC292) } \\
\hline Measured efficiency & $80 \%$ to $82 \%$ \\
\hline \multicolumn{2}{|c|}{ (e) Load (Meraki Mini) } \\
\hline Vec & $7.5 \mathrm{~V}$ \\
\hline $\begin{array}{l}\text { Manufacturer-provided } \\
\text { power consumption }\end{array}$ & 3.5W typical, $7.5 \mathrm{~W}$ maximum \\
\hline $\begin{array}{l}\text { Measured power } \\
\text { consumption }\end{array}$ & $2.25 \mathrm{~W}$ average $(=7.5 \mathrm{~V} * 0.3 \mathrm{~A})$ \\
\hline
\end{tabular}

Table 8.2: Comparison of a meso-solar system and a micro-solar system

\begin{tabular}{|l|l|l|l|}
\hline & $\begin{array}{l}\text { Meso-solar } \\
\text { (Solar Web Server) }\end{array}$ & $\begin{array}{l}\text { Micro-solar } \\
\text { (HydroWatch Node) }\end{array}$ & $\begin{array}{l}\text { Ratio of Scale } \\
\text { (meso / micro) }\end{array}$ \\
\hline $\begin{array}{l}\text { Maximum Solar } \\
\text { Panel Power }\end{array}$ & $50 \mathrm{~W}$ & $0.276 \mathrm{~W}$ & 181 times \\
\hline $\begin{array}{l}\text { Energy Storage } \\
\text { Capacity }\end{array}$ & $\begin{array}{l}384 \mathrm{Whr} \\
(=32 \mathrm{Ahr} \text { at } 12 \mathrm{~V})\end{array}$ & $\begin{array}{l}6 \mathrm{Whr} \\
(=2.5 \mathrm{Ahr} \text { at } 2.4 \mathrm{~V})\end{array}$ & 64 times \\
\hline $\begin{array}{l}\text { Average Load } \\
\text { Consumption }\end{array}$ & $2.25 \mathrm{~W}$ & $1.75 \times 10^{-3} \mathrm{~W}$ & 1286 times \\
\hline $\begin{array}{l}\text { Input Regulator } \\
\text { Efficiency }\end{array}$ & $89 \%$ to $97 \%$ & $55 \%$ to $65 \%$ & 1.55 times \\
\hline $\begin{array}{l}\text { Output Regula- } \\
\text { tor Efficiency }\end{array}$ & $80 \%$ to $82 \%$ & $50 \%$ to $52 \%$ & 1.59 times \\
\hline
\end{tabular}




\subsection{Simulating Meso-Solar System}

In this subsection, we simulate the behavior of the solar web server under a daily solar radiation. By comparing the daily energy accumulation and consumption, we can evaluate whether the meso-solar system can achieve sustainable operation.

\subsubsection{Simulation Set-up}

For the simulation, the following parameters are considered:

- External Environment: We simulate the meso-solar system that is deployed on the rooftop of the Computer Science building at UC Berkeley. For this situation, we can consider the astronomical model with $L=37.87, \phi_{p}=180$, $\tau=0.1$. We simulate the system varying two parameters: solar panel inclination $\theta_{p}$ and day of the year $n$.

- $\theta_{p}: 0$ (flat) and 45 (tilted $\left.45^{\circ}\right)$

- n: 80 (spring - average case of yearly variation), 356 (winter - worst case of yearly variation)

- Solar Panel: A Kyocera KC50 50W solar panel is used. The IV-curve in Figure 8.3 is used to simulate this solar panel.

- Input Regulator: A MorningStar Sunguard-4 12V lead acid battery charging controller is used. We model the input regulator as a device with $90 \%$ efficiency. The operating points of the input regulator is modeled after the $V_{F}$-to- $I_{F}$ relation in Figure 8.5(b).

- Energy Storage: A 12V-32Ah sealed lead acid battery. We estimate the voltage of the battery based on the charging status (charging or discharging) and the charge level of the battery. Figure 8.4 describes the charge-to-voltage relationship for the charging and discharging states.

- Output Regulator: We model the PowerStream PST-DC292 DC-DC converter as a device that has $80 \%$ efficiency and $7.77 \mathrm{~V}$ of output voltage. 
- Load: A Meraki Mini node is used with a steady state power consumption of $2.25 \mathrm{~W}$.

\subsubsection{Simulation Result}

In simulating the meso-solar system, we are to answer the following two questions:

- Q1 : How much energy can the meso-solar system accumulate, and how does this affect the sustainability?

- Q2 : How long should the charging period be in order for the system to accumulate the same amount of energy discharged during the night?

In order to answer Q1, we set the initial condition of the simulation so that the battery does not get full during the day. That way, we can find the daily energy accumulation without being affected by the battery saturation. In order to answer Q2, we set the initial condition of the simulation so that the final battery energy level is the same as the initial level.

\section{Spring}

Figure 8.7 shows the simulation result of the daily energy charge and discharge with the solar-powered web server during spring $(\mathrm{n}=80)$. Whether the solar panel is flat (Case 1) or tilted (Case 2), the system accumulates more energy than it consumes. We define the metric battery days as the ratio of battery capacity increase over battery discharge, and this metric implies how many days the system can operate without its energy level decreasing. The system has battery days of either 2.1 (Case 1) or 3.0 (Case 2) depending on panel inclination.

Figure 8.8 shows the simulation result of the daily charging and discharging time with the solar-powered web server during spring $(\mathrm{n}=80)$. Depending on the panel inclination, the system requires either $4 \mathrm{hr} 15 \mathrm{~min}$ (Case 1) or $3 \mathrm{hr} 45 \mathrm{~min}$ (Case 2) to fully charge the battery after darkness on the previous day. 

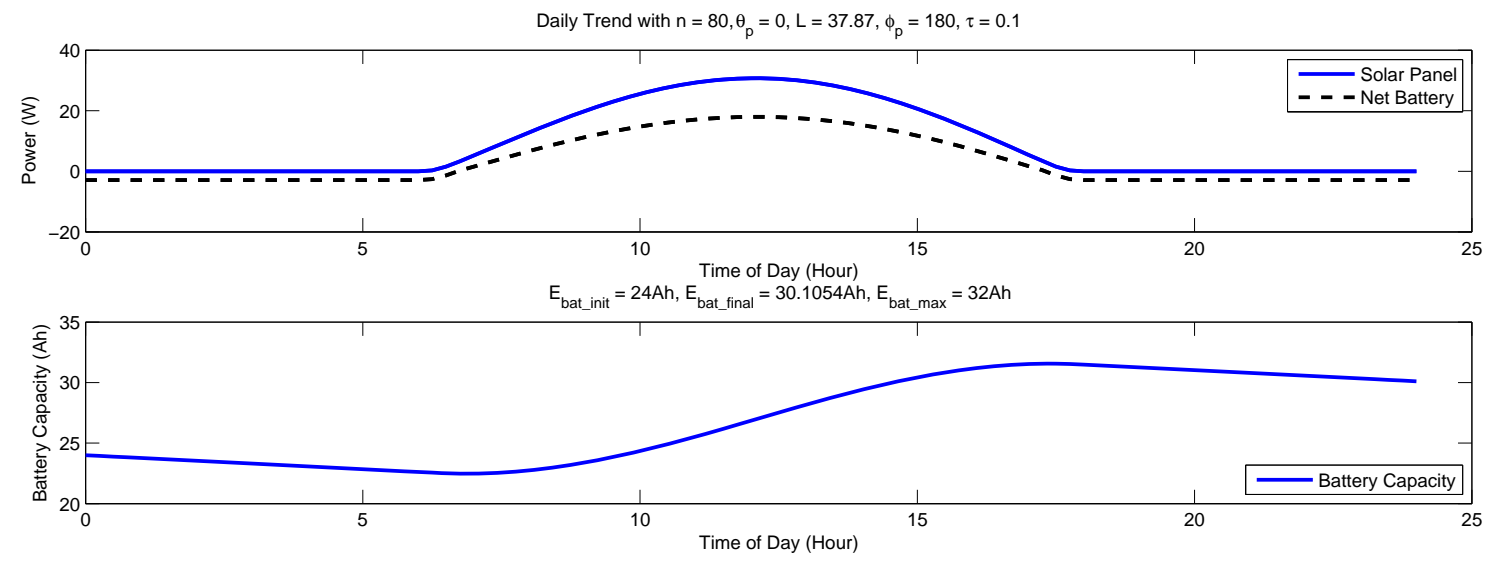

(a) Case 1: $n=80, \theta_{p}=0$
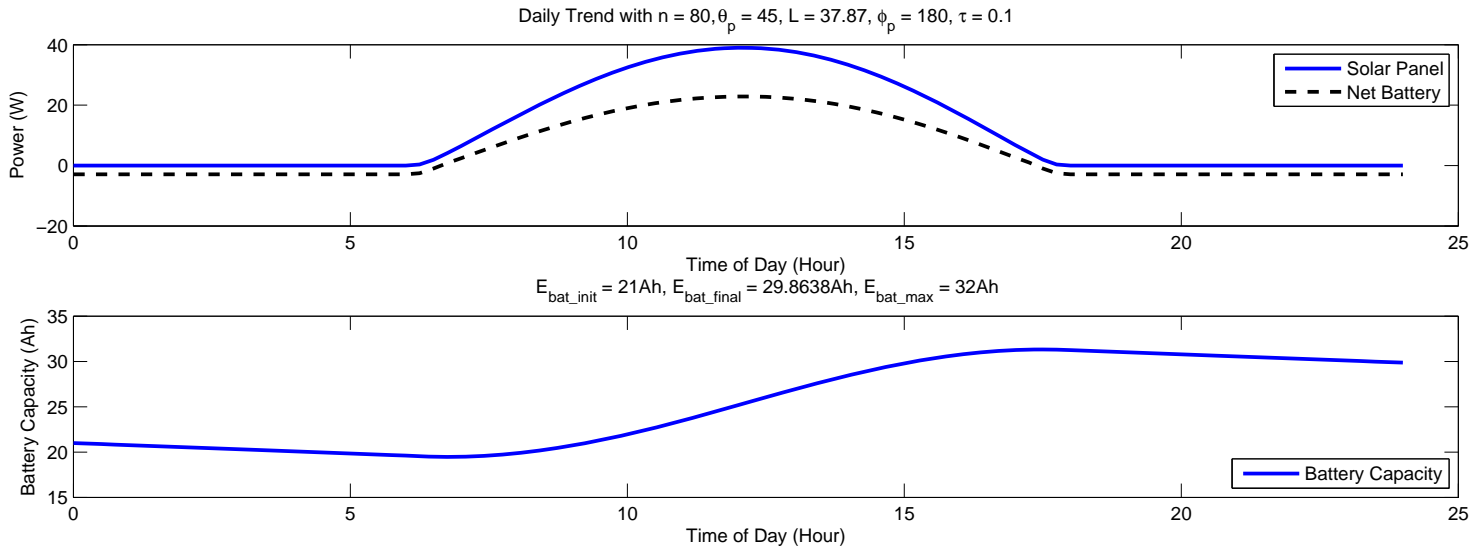

(b) Case 2: $n=80, \theta_{p}=45$

\begin{tabular}{|l|l|l|l|}
\hline & $\begin{array}{l}\text { Energy } \\
\text { Charged }\end{array}$ & $\begin{array}{l}\text { Energy } \\
\text { Discharged }\end{array}$ & $\begin{array}{l}\text { Battery } \\
\text { Days }\end{array}$ \\
\hline Case 1: panel flat & $9.1 \mathrm{Ah}$ & $3.0 \mathrm{Ah}$ & 2.1 days \\
Case 2: panel tilted $45^{\circ}$ & $11.8 \mathrm{Ah}$ & $3.0 \mathrm{Ah}$ & 3.0 days \\
\hline
\end{tabular}

Figure 8.7: Daily energy profile for the solar-powered web server with $n=80$. The battery is not saturated during the operation. 

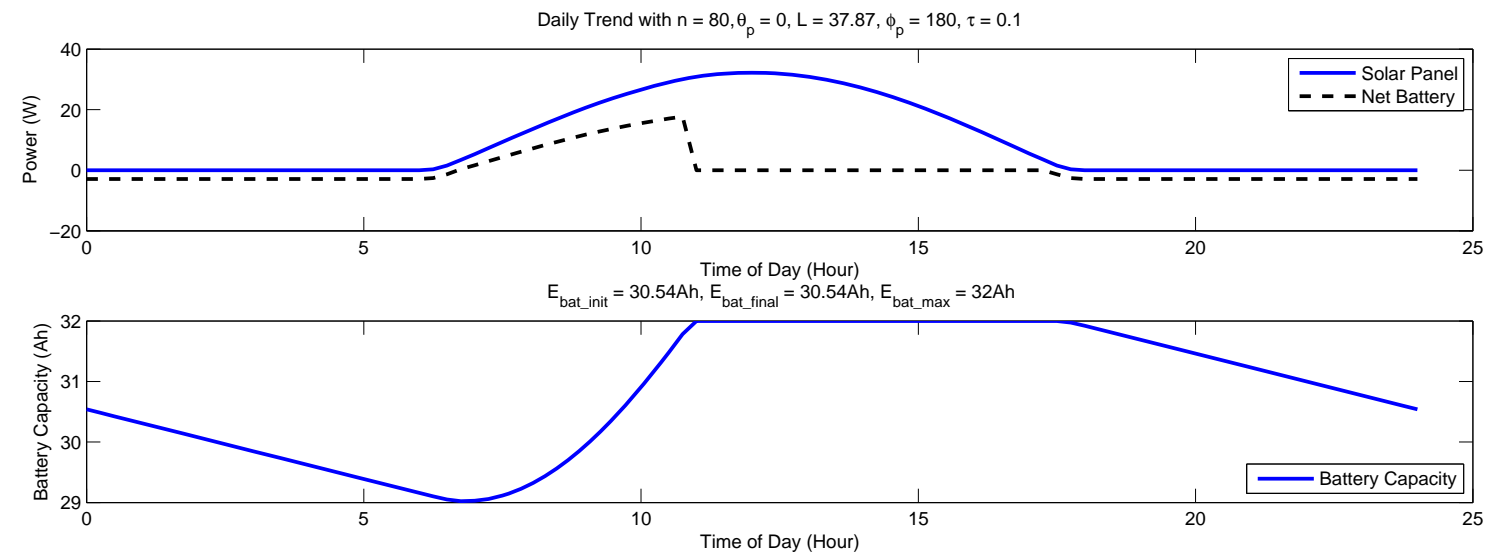

(a) Case 1: $n=80, \theta_{p}=0$
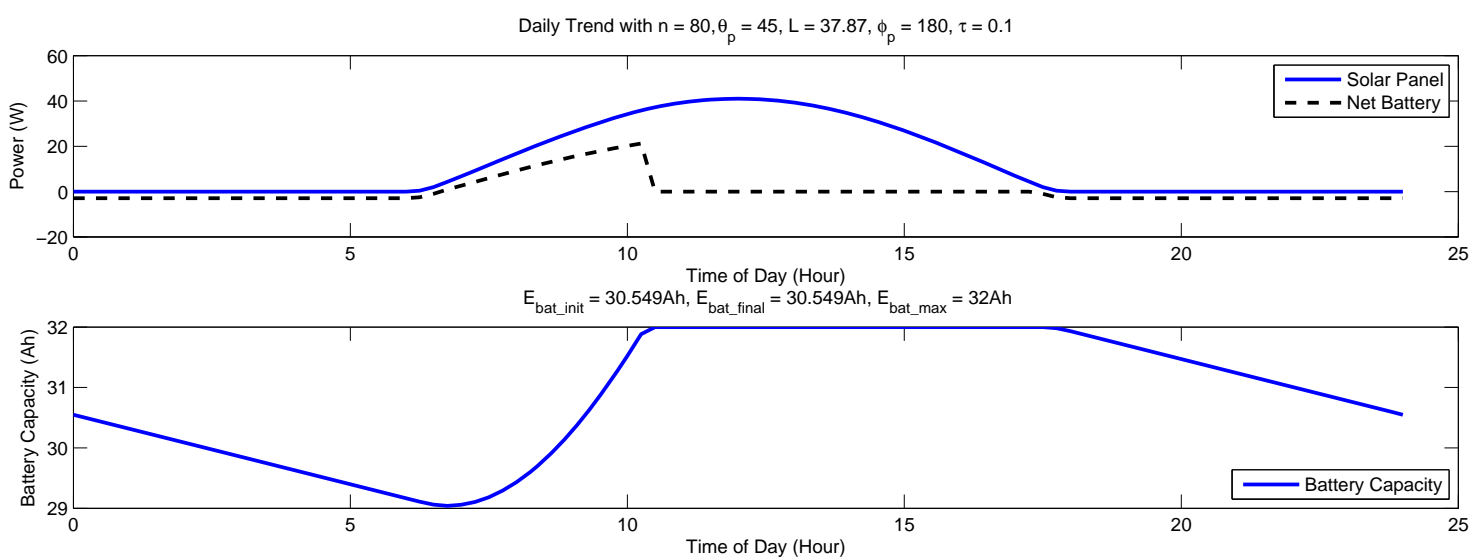

(b) Case 2: $n=80, \theta_{p}=45$

\begin{tabular}{|l|c|c|c|c|}
\hline & $\begin{array}{c}\text { Energy } \\
\text { Charged }\end{array}$ & $\begin{array}{c}\text { Energy } \\
\text { Discharged }\end{array}$ & $\begin{array}{c}\text { Charging } \\
\text { Time }\end{array}$ & $\begin{array}{c}\text { Discharging } \\
\text { Time }\end{array}$ \\
\hline Case 1: panel flat & $3.0 \mathrm{Ah}$ & $3.0 \mathrm{Ah}$ & $4 \mathrm{hr} 15 \mathrm{~min}$ & $13 \mathrm{hr} 15 \mathrm{~min}$ \\
Case 2: panel tilted $45^{\circ}$ & $3.0 \mathrm{Ah}$ & $3.0 \mathrm{Ah}$ & $3 \mathrm{hr} 45 \mathrm{~min}$ & $13 \mathrm{hr} 15 \mathrm{~min}$ \\
\hline
\end{tabular}

Figure 8.8: Daily energy profile for the solar-powered web server with $n=80$. The battery is saturated in middle of day and the final battery level is as the same as the initial level. 


\section{Winter}

Figure 8.9 shows the simulation result of the daily energy charge and discharge with the solar-powered web server during winter $(\mathrm{n}=356)$. The system accumulates more energy than it consumes when the solar panel is tilted (Case 2), and it consumes more energy when the solar panel is flat (Case 1). For Case 2, the system has 1.6 battery days.

Figure 8.10 shows the simulation result of the daily charging and discharging time with the solar-powered web server during winter $(\mathrm{n}=356)$. When the panel is tilted, the system requires $3 \mathrm{hr} 45 \mathrm{~min}$ to fully charge the battery after darkness on the previous day. We consider the case where the panel is tilted because the flat panel does not provide sufficient energy for sustainable operation.

\subsection{Refining the Simulation of a Meso-Solar Sys- tem Using Measurement Data}

Figure 8.11 shows the layout of the solar-powered web server deployment. It is located on the north-side of the balcony on the UC Berkeley Computer Science building with its solar panel tilted $45^{\circ}$ facing south. Thus, the solar panel is obstructed by the building during the day.

We estimated the daily trend of the solar panel output of the solar-powered web server using three models: (a) the astronomical model, (b) scaled astronomical model and (c) obstructed astronomical model. The estimations and the measurement are shown in Figure 8.12. The measurement result, which is closely captured by the obstructed astronomical model, is about $44 \%$ of the astronomical model estimation.

\subsection{Predicting the Behavior of Meso-Solar Sys- tems}

In order to see the long-term behavior of the solar-powered web server, we simulated the meso-solar system with the following parameters: 

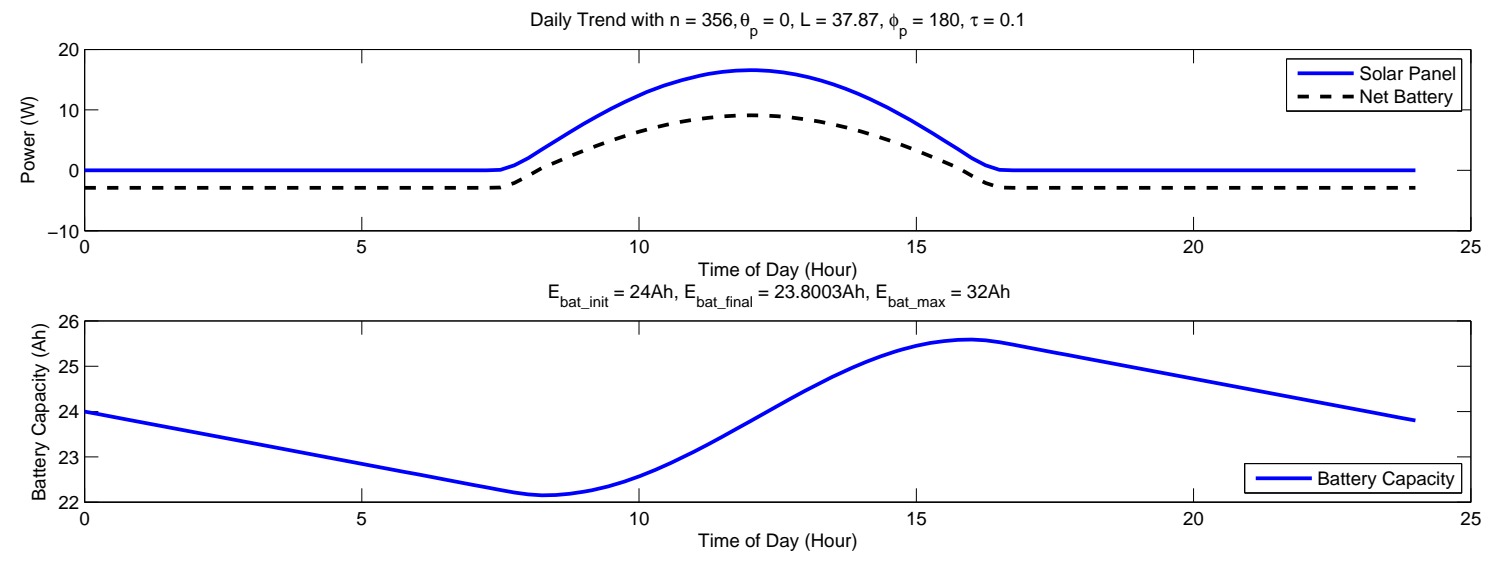

(a) Case 1: $n=356, \theta_{p}=0$
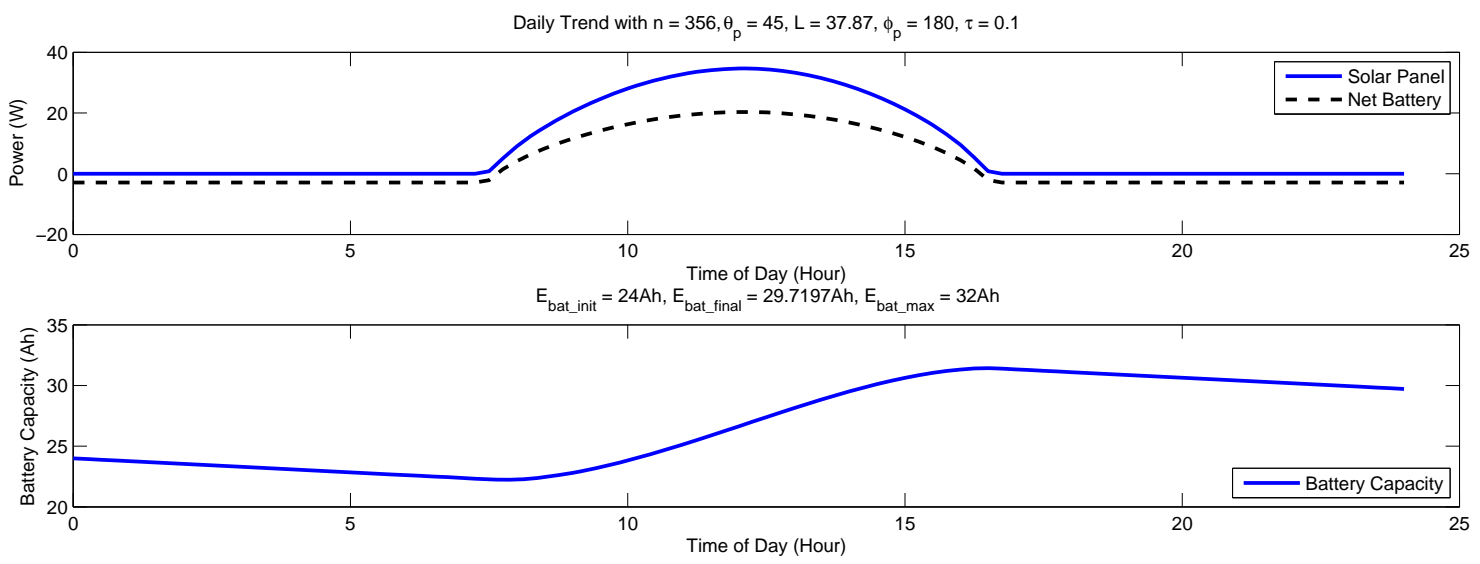

(b) Case 2: $n=356, \theta_{p}=45$

\begin{tabular}{|l|l|l|l|}
\hline & $\begin{array}{l}\text { Energy } \\
\text { Charged }\end{array}$ & $\begin{array}{l}\text { Energy } \\
\text { Discharged }\end{array}$ & $\begin{array}{l}\text { Battery } \\
\text { Days }\end{array}$ \\
\hline Case 1: panel flat & $3.4 \mathrm{Ah}$ & $3.6 \mathrm{Ah}$ & -0.1 days \\
Case 2: panel tilted $45^{\circ}$ & $9.2 \mathrm{Ah}$ & $3.5 \mathrm{Ah}$ & 1.6 days \\
\hline
\end{tabular}

Figure 8.9: Daily energy profile for the solar-powered web server with $n=356$. The battery is not saturated during the operation. 

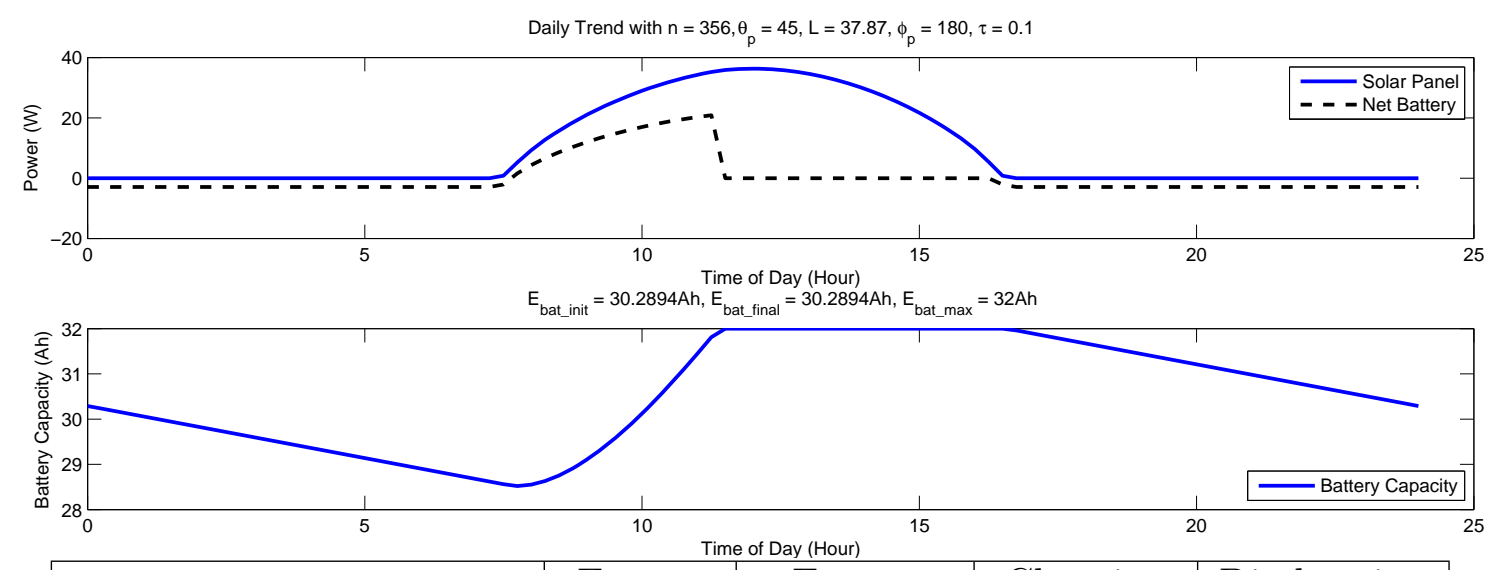

\begin{tabular}{|l|c|c|c|c|}
\hline & $\begin{array}{c}\text { Energy } \\
\text { Charged }\end{array}$ & $\begin{array}{c}\text { Energy } \\
\text { Discharged }\end{array}$ & $\begin{array}{c}\text { Charging } \\
\text { Time }\end{array}$ & $\begin{array}{c}\text { Discharging } \\
\text { Time }\end{array}$ \\
\hline Panel tilted $45^{\circ}$ & $3.5 \mathrm{Ah}$ & $3.5 \mathrm{Ah}$ & $3 \mathrm{hr} 45 \mathrm{~min}$ & $15 \mathrm{hr} 15 \mathrm{~min}$ \\
\hline
\end{tabular}

Figure 8.10: Daily energy profile for the solar-powered web server with $n=356$, $\theta_{p}=45$. The battery is saturated in middle of day and the final battery level is as the same as the initial level.
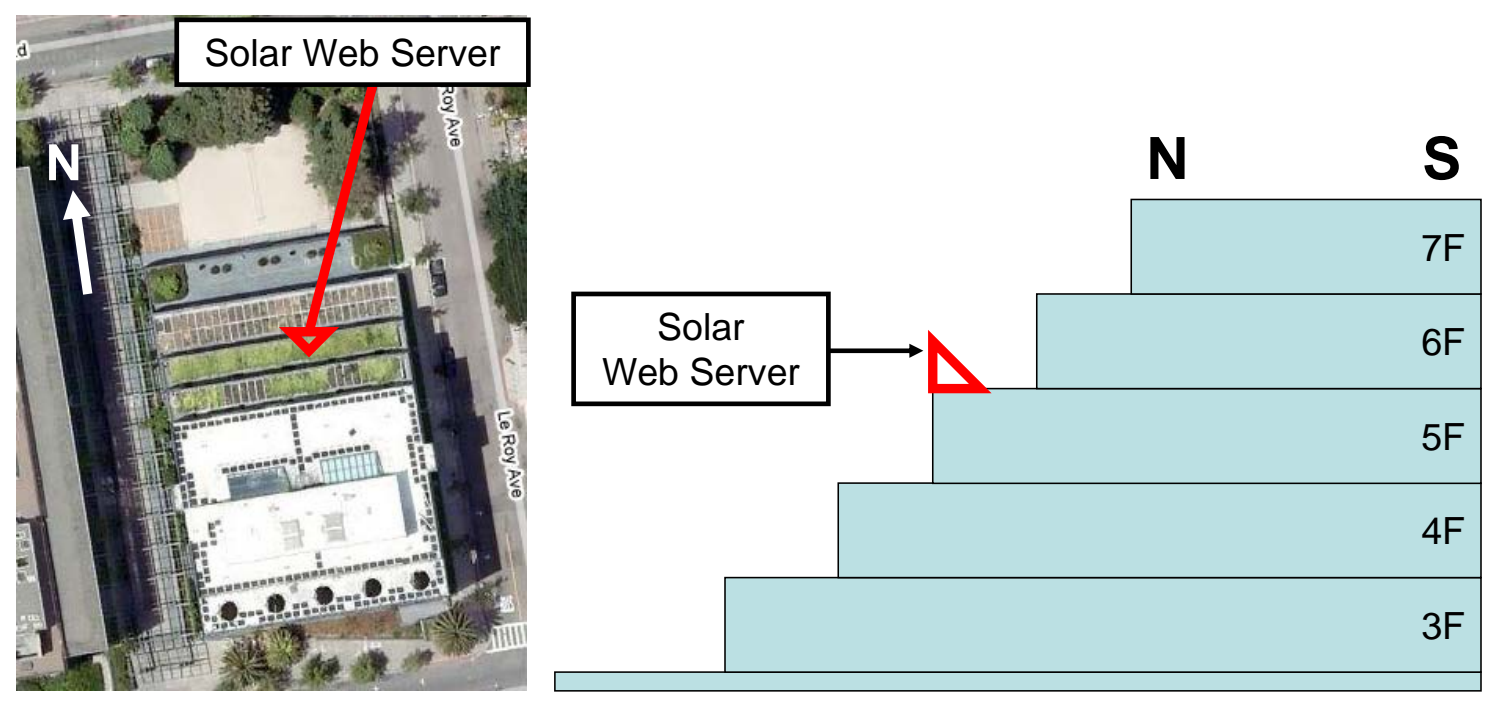

Figure 8.11: Layout of the solar-powered web server deployment 


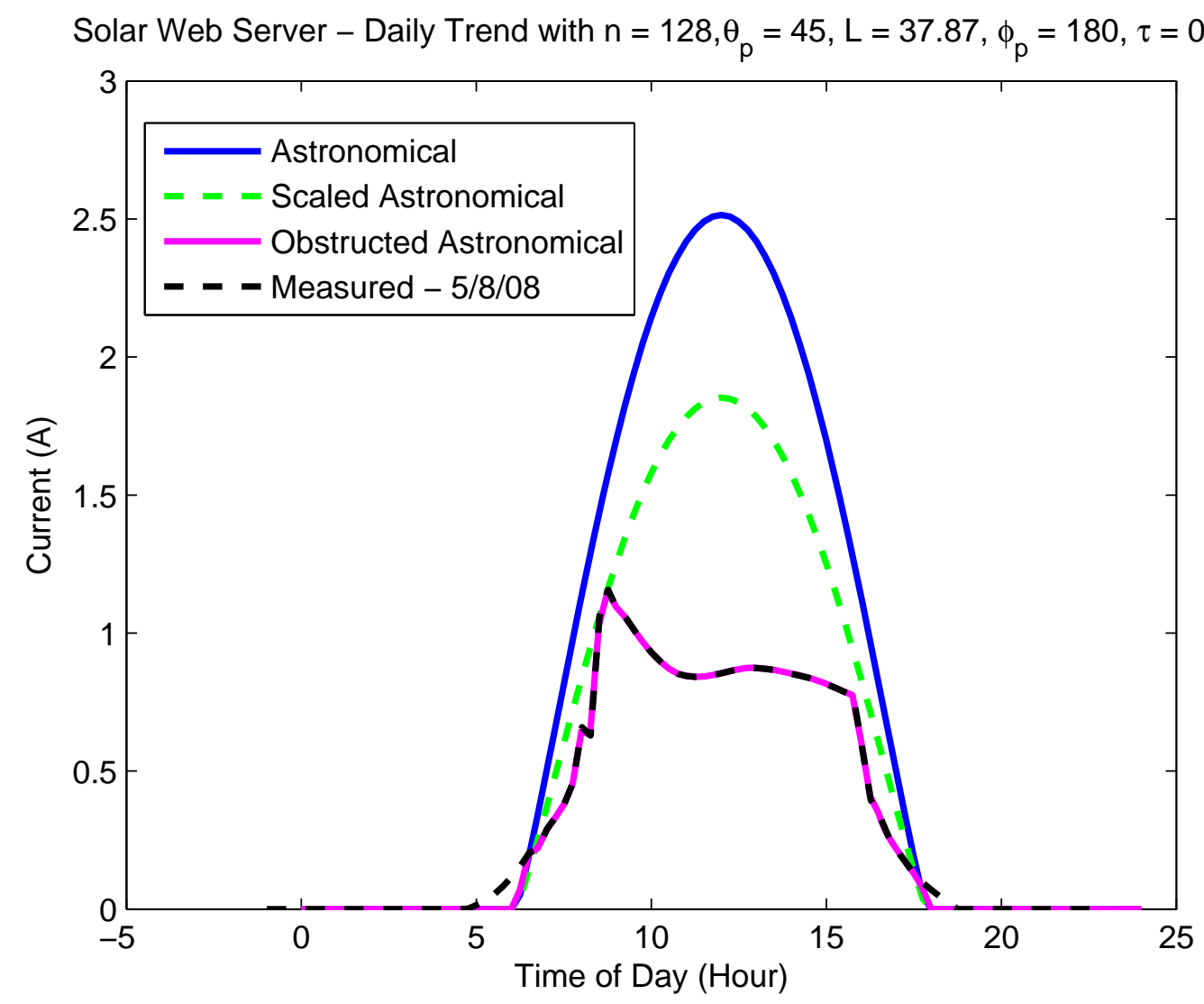

\begin{tabular}{|c|c|c|c|c|}
\hline & $\begin{array}{c}\text { Astronomical } \\
\text { Model }\end{array}$ & $\begin{array}{c}\text { Scaled } \\
\text { Astronomical }\end{array}$ & $\begin{array}{c}\text { Obstructed } \\
\text { Astronomical }\end{array}$ & Measurement \\
\hline Daily Radiation & $219.65 \mathrm{Wh}$ & $161.88 \mathrm{Wh}$ & $96.46 \mathrm{Wh}$ & $98.04 \mathrm{Wh}$ \\
\hline
\end{tabular}

Figure 8.12: Estimation of daily solar panel output for the solar-powered web server $\left(n=128, \theta_{p}=45, L=37.87, \phi_{p}=180\right)$ 
- Panel Parameters: panel inclination $\theta_{p}=45$, panel orientation $\phi_{p}=180$ (south), latitude $L=37.87$.

- Load Consumption: The effective daily load consumption seen by the solar panel is $P_{\text {load }} \cdot 24 \mathrm{~h} /\left(\mathrm{Eff}_{r e g-i n} \cdot \mathrm{Eff}_{\text {bat }} \cdot \mathrm{Eff}_{\text {reg-out }}\right)$. For the solar-powered web server, it is $107.1 \mathrm{Wh}(=87.3 \mathrm{Wh} /(0.9 * 0.8 * 0.7))$.

- Time: From 5/8/2008 $(\mathrm{n}=128)$ to $5 / 8 / 2009(\mathrm{n}=365+128)$.

- Estimation Models: Astronomical model, scaled astronomical model and obstructed astronomical model.

- Comparisons: Daily solar panel output energy and daily battery capacity.

Figure 8.13(a) shows the solar energy estimations and load consumption. While the daily solar energy for the astronomical model and the scaled astronomical model is always above the load consumption, the obstructed astronomical model has its daily solar energy below the load consumption during winter. Thus, the battery level will drop during winter.

Figure 8.13(b) shows the daily battery level at the end of each day. As we expected, the battery level stays almost full throughout the year for the astronomical and scaled astronomical model. For the obstructed astronomical model, the battery level stays near full capacity during spring, summer and fall. The battery level drops down to half of the total capacity in the middle of winter and it increases afterwards. This simulation result assumes clear weather, as depending on the weather conditions during winter, this system may consume more energy than its battery capacity and not survive during that time. 

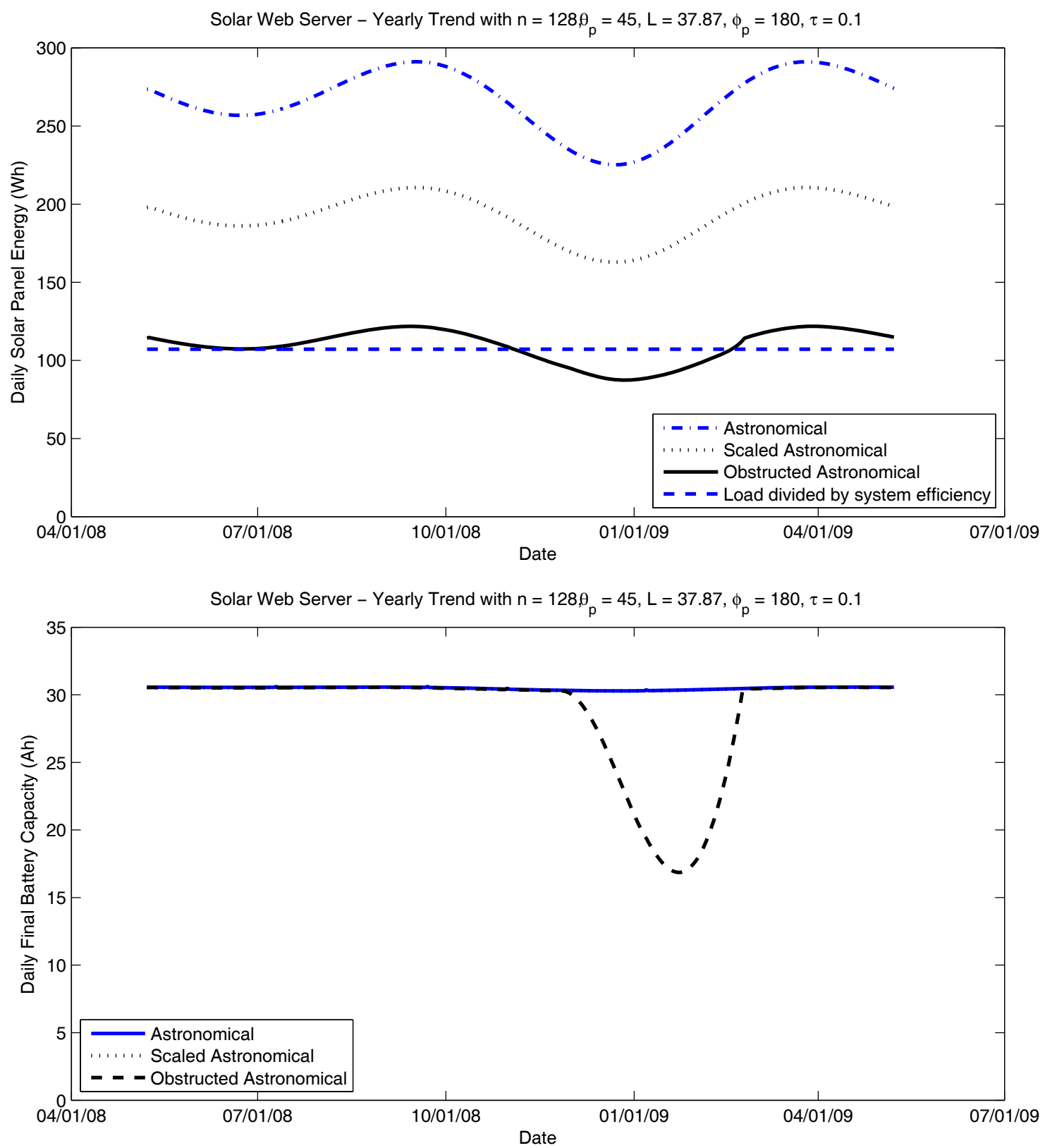

Figure 8.13: Yearly estimation for the solar-powered web server: (a) daily solar panel output, (b) daily battery capacity 


\section{Chapter 9}

\section{Concluding Remarks}

In this dissertation, we have presented a practical theory that enables a systematic design of a micro-solar power system. We summarize this dissertation as follows:

First, we developed a general model of micro-solar power systems. While the design space of micro-solar power systems is very large, it is essential we use the same simulation framework to represent such diversity in a scalable fashion. We presented a general model of micro-solar power systems that consisted of several lumped elements, such as the external environment, a solar collector, an input regulator, energy storage, output regulator and a load, and their relationship in terms of energy flow and efficiency factors. In this framework, designing a micro-solar power system implies setting the parameters of one or more of these lumped elements.

Second, we materialized this model into a simulation suite by formulating each component of a micro-solar power system as an analytical module and composing these modules into an instance of a micro-solar power system. To represent a microsolar power system in the simulation framework, its components need to be formulated. Depending on the availability and the characteristics of its behavior, each component is formulated either with an analytical formula, curve fitting, or piecewise interpolation. To represent the external environment, we proposed three methods: an astronomical model, an obstructed-astronomical model and a weather-metric model. The astronomical model estimates solar radiation using well-known formulas without any knowledge of the deployment site. This model works well on a clear day and can be used without knowledge of the deployment site, but its estimation error can 
be high on an overcast day. The obstructed-astronomical model refines the estimates of the astronomical model by using a few samples of local measurements incurring a modest estimation error. The weather-metric model further refines the obstructedastronomical model by adjusting the solar radiation by the amount of weather effects. Using all of these components, we have composed a few instances of micro-solar power systems. Using benchtop experiments, we have shown that this simulation tools work in a small time frame.

Third, we have implemented a reference platform of a micro-solar power system, the HydroWatch node, and validated the simulation suite by comparing solar energy estimates with empirical results in a realistic environment. The short-term deployment, where reference platform nodes were placed under varying solar profiles in urban neighborhoods and forest watershed environments, showed that our simulation tools could estimate the solar energy budget with only a small degree of error. The long-term deployment, where reference platform nodes were placed on an urban rooftop environment, showed that our simulation tools could estimate the solar energy budget of a system under varying weather conditions and that the difference between estimates and empirical results was bounded by roughly 30\%. We reduced this estimation error even further with a weather metric model, predicting the solar radiation with a $6 \%$ margin of error by maintaining a week of cloud condition history.

Finally, we extended the simulation suite for hypothetical designs of micro-solar power systems and meso-solar power systems, demonstrating that our model and simulation suite can be used beyond the reference design of micro-solar power systems. As an example of a hypothetical design, we simulated a micro-solar power system with multi-level energy storage consisting of a supercapacitor and NiMH batteries. This allowed us to find how parameters of a hypothetical system affect system performance. Next, we extended the simulator for wind and vibration energy. Our preliminary study shows that the simulator can be adapted for other renewable energy sources in a straightforward way by modeling the external environment and energy collector components. Lastly, as a meso-solar system, we designed a solar-powered web server by composing Watt-scale components and simulated this design by modeling each component and integrating it into the simulator. The measurements from the implementation validated our model with a solar power system of a larger scale. 


\section{Bibliography}

[Arc] Arch Rock Corporation. Arch Rock Corporation, Primer Pack / IP. http: //www.archrock.com/downloads/datasheet/primerpack_datasheet.pdf.

[BMS] Solar Radiation Research Laboratory. http://www.nrel.gov/midc/srrl_ $\mathrm{bms} /$.

[Cal] California Solar Initiative. Incentive Calculator. http://www.csi-epbb. $\mathrm{com}$.

[CH88] Theodore W. Cannon and Roland L. Hulstrom. The atmospheric optical calibration system. The Twentieth IEEE Photovoltaic Specialists Conference, September 1988.

[CJBM01] Benjie Chen, Kyle Jamieson, Hari Balakrishnan, and Robert Morris. Span: an energy-efficient coordination algorithm for topology maintenance in ad hoc wireless networks. The Seventh Annual International Conference on Mobile Computing and Networking (MobiCom '01), July 2001.

[COSKM05] Rachel Cardell-Oliver, Keith Smettem, Mark Kranz, and Kevin Mayer. A reactive soil moisture sensor network: Design and field evaluation. International Journal of Distributed Sensor Networks (IJDSN '05), March 2005.

[CVS ${ }^{+}$07] Peter Corke, Philip Valencia, Pavan Sikka, Tim Wark, and Les Overs. Long-duration solar-powered wireless sensor networks. The Fourth IEEE workshop on Embedded Networked Sensors (EmNets '07), June 2007. 
$\left[\mathrm{DHJ}^{+}\right.$06] Prabal Dutta, Jonathan Hui, Jaein Jeong, Sukun Kim, Cory Sharp, Jay Taneja, Gilman Tolle, Kamin Whitehouse, and David Culler. Trio: Enabling sustainable and scalable outdoor wireless sensor network deployments. The Fifth International Conference on Information Processing in Sensor Networks (IPSN/SPOTS '06), April 2006.

[DHM75] J. V. Dave, P. Halpern, and H. J. Myers. Computation of incident solar energy. IBM Journal of Research and Development, 19(6):539-549, 1975.

[Dus] Dust Networks. Technical Overview of Time Synchronized Mesh Protocol (TSMP). http://www.dustnetworks.com/docs/TSMP_Whitepaper. pdf.

[EV06] Sinem Coleri Ergen and Pravin Varaiya. Pedamacs: Power efficient and delay aware medium access protocol for sensor networks. IEEE Transactions on Mobile Computing, 5(7), July 2006.

[fin] FindSolar: Connecting You to Renewable Energy Professionals. http: //findsolar.com.

[GCD ${ }^{+98}$ Vahan Gevorgian, David A. Corbus, Stephen Drouilhet, Richard Holz, and Karen E. Thomas. Modeling, testing and economic analysis of a wind-electric battery charging station. Windpower '98, 1998.

[Har] Harvard University. MoteLab. http://motelab.eecs.harvard.edu/.

[HKWW06] Vlado Handziski, Andreas Köpke, Andreas Willig, and Adam Wolisz. Twist: a scalable and reconfigurable testbed for wireless indoor experiments with sensor networks. The 2nd international workshop on Multihop ad hoc networks: from theory to reality (REALMAN '06), 2006.

[HZKS06] Jason Hsu, Sadaf Zahedi, Aman Kansal, and Mani B Srivastava. Adaptive duty cycling for energy harvesting systems. International Symposium on Low Power Electronics and Design (ISLPED ‘06), 2006.

[Int] Intel Research, Berkeley. Mirage: Microeconomic resource allocation for sensornet testbeds. http://mirage.berkeley.intel-research.net. 
[Iow] Iowa Energy Center. Solar Data for Iowa Locations. http://www.energy. iastate.edu/renewable/solar/calculator.

[JPC05] Xiaofan Jiang, Joseph Polastre, and David Culler. Perpetual environmentally powered sensor networks. The Fourth International Symposium on Information Processing in Sensor Networks (IPSN/SPOTS '05), April 2005.

[KHZS07] Aman Kansal, Jason Hsu, Sadaf Zahedi, and Mani B. Srivastava. Power management in energy harvesting sensor networks. ACM Transactions in Embedded Computing Systems, 2007.

[Kim07] Sukun Kim. Wireless sensor networks for high frequency sampling. Ph.D Thesis, University of California at Berkeley, December 2007.

[KPS04] Aman Kansal, Dunny Potter, and Mani B. Srivastava. Performance aware tasking for environmentally powered sensor networks. The joint international conference on Measurement and modeling of computer systems (ACM SIGMETRICS '04), June 2004.

[Kyo] Kyocera. KC50 High Efficiency Multicrystal Photovoltaic Module. http://www.wholesalesolar.com/pdf.folder/module\%20pdf\%20folder/ KC50.pdf.

[LBV06] Koen Langendoen, Aline Baggio, and Otto Visser. Murphy loves potatoes: Experiences from a pilot sensor network deployment in precision agriculture. Workshop on Parallel and Distributed Real-Time Systems (WPDRTS ‘06), April 2006.

[LLWC03] Philip Levis, Nelson Lee, Matt Welsh, and David Culler. Tossim: Accurate and scalable simulation of entire tinyos applications. The First ACM Conference on Embedded Networked Sensor Systems (Sensys '03), 2003.

[LPCS04] P. Levis, N. Patel, D. Culler, and S. Shenker. Trickle: A self-regulating algorithm for code propagation and maintenance in wireless sensor net- 
works. The First Symposium on Networked Systems Design and Implementation (NSDI '04), March 2004.

[LWG05] Olaf Landsiedel, Klaus Wehrle, and Stefan Götz. Accurate prediction of power consumption in sensor networks. The Second IEEE workshop on Embedded Networked Sensors (EmNets '05), 2005.

[MBTB06a] C. Moser, B. Brunelli, L. Thiele, and L. Benini. Lazy scheduling for energy harvesting sensor nodes. IFIP Model-Driven Design to Resource Management for Distributed Embedded Systems, 2006.

[MBTB06b] C. Moser, B. Brunelli, L. Thiele, and L. Benini. Real-time scheduling with regenerative energy. The 18th Euromicro Conference on Real-Time Systems (ECRTS '06), 2006.

[Mer] Meraki. Meraki Mini. http://meraki.com/oursolution/hardware/mini/.

[Met] Meteonorm. Meteonorm. http://www.meteotest.ch/pdf/am/mn description.pdf.

[MFHH02] Samuel Madden, Michael J. Franklin, Joseph M. Hellerstein, and Wei Hong. Tag: a tiny aggregation service for ad-hoc sensor networks. The fifth symposium on Operating systems design and implementation (OSDI '02), December 2002.

[MKHC07] G. Montenegro, N. Kushalnagar, J. Hui, and D. Culler. Transmission of ipv6 packets over ieee 802.15.4 networks, September 2007. http://tools. ietf.org/html/rfc4944.

[Nat] National Renewable Energy Laboratory. PVWATTS: A Performance Calculator for Grid-Connected PV Systems. http://rredc.nrel.gov/solar/ codes_algs/PVWATTS.

[New78] Arthur Richard Newton. The simulation of large scale integrated circuits. Ph.D Thesis, University of California at Berkeley, Dec 1978. 
[NGSA04] Suman Nath, Phillip B. Gibbons, Srinivasan Seshan, and Zachary R. Anderson. Synopsis diffusion for robust aggregation in sensor networks. The Second ACM Conference on Embedded Networked Sensor Systems (Sensys '04), November 2004.

$\left[\mathrm{NLA}^{+} 07\right]$ Ani Nahapetian, Paolo Lombardo, Andrea Acquaviva, Luca Benini, and Majid Sarrafzadeh. Dynamic reconfiguration in sensor networks with regenerative energy sources. The conference on Design, automation and test in Europe (EDAA 'Or), 2007.

[NSR] National Solar Radiation Data Base. ftp://ftp3.ncdc.noaa.gov/pub/ data/nsrdb.

[Par06] Joseph A. Paradiso. Systems for human-powered mobile computing. IEEE Design Automation Conference (DAC '06), 2006.

[PC06] Chulsung Park and Pai H. Chou. Ambimax: Autonomous energy harvesting platform for multi-supply wireless sensor nodes. IEEE Sensor and Ad Hoc Communications and Networks (SECON '06), 2006.

[Per93] Richard Perez. Lead-acid battery state of charge vs. voltage. Home Power, 36, Aug/Sep 1993. http://www.arttec.net/Solar_Mower/4_ Electrical/Battery\%20Charging.pdf.

[PFR78] James T. Peterson, Edwin C. Flowers, and John H. Rudisill. Urban-rural solar radiation and atmospheric turbidity measurements in the los angeles basin. Journal of Applied Meteorology, 17(11):1595-1609, November 1978.

[PHC04] Joseph Polastre, Jason Hill, and David Culler. Versatile low power media access for wireless sensor networks. The Second ACM Conference on Embedded Networked Sensor Systems (Sensys '04), November 2004.

[PKR02] S. Sandeep Pradhan, Julius Kusuma, and Kannan Ramchandran. Distributed compression in a dense microsensor network. IEEE Signal Processing Magazine, March 2002. 
[Pow] PowerStream. PST-DC292. http://www.powerstream.com/dc6.htm.

[PSC05] Joseph Polastre, Robert Szewczyk, and David Culler. Telos: Enabling ultra-low power wireless research. The Fourth International Symposium on Information Processing in Sensor Networks (IPSN/SPOTS '05), April 2005.

[PSS00] Sung Park, Andreas Savvides, and Mani B. Srivastava. Sensorsim: A simulation framework for sensor networks. International Workshop on Modeling Analysis and Simulation of Wireless and Mobile Systems (MSWIM'00), 2000.

[PSS01] Sung Park, Andreas Savvides, and Mani B. Srivastava. Simulating networks of wireless sensors. Winter Simulation Conference, 2001.

$\left[\mathrm{RKH}^{+} 05\right]$ Vijay Raghunathan, Aman Kansal, Jason Hsu, Jonathan Friedman, and Mani Srivastava. Design considerations for solar energy harvesting wireless embedded systems. The Fourth International Symposium on Information Processing in Sensor Networks (IPSN/SPOTS '05), April 2005.

$\left[\mathrm{ROC}^{+}\right.$03] Shad Roundy, Brian P. Otis, Yuen-Hui Chee, Jan M. Rabaey, and Paul Wright. A 1.9ghz rf transmit beacon using environmentally scavenged energy. International Symposium on Low Power Electronics and Design (ISLPED '03), Aug. 2003.

[Rou03] Shad J. Roundy. Energy scavenging for wireless sensor nodes with a focus on vibration to electricity conversion. Ph.D Thesis, University of California at Berkeley, May 2003.

$\left[\mathrm{RSF}^{+} 04\right]$ Shad Roundy, Dan Steingart, Luc Frechette, Paul Wright, and Jan Rabaey. Power sources for wireless sensor networks. The First European Workshop on Wireless Sensor Networks (EWSN ‘04), January 2004.

[RV82] Elmer Robinson and Ralph J. Valente. Atmospheric turbidity over the united states from 1967 to 1976. U.S. Environmental Protection Agency Report 600382076, September 1982. 
[SC06] Farhan Simjee and Pai H. Chou. Everlast: Long-life, supercapacitoroperated wireless sensor node. International Symposium on Low Power Electronics and Design (ISLPED '06), October 2006.

$\left[\mathrm{SCV}^{+}\right.$06] Pavan Sikka, Peter Corke, Philip Valencia, Christopher Crossman, Dave Swain, and Greg Bishop-Hurley. Wireless adhoc sensor and actuator networks on the farm. The Fifth International Conference on Information Processing in Sensor Networks (IPSN/SPOTS '06), 2006.

[Sen] Sentilla. Sentilla Tmote Sky. http://www.sentilla.com/pdf/eol/ tmote-sky-datasheet.pdf.

$\left[\mathrm{SHC}^{+}\right.$04] Victor Shnayder, Mark Hempstead, Borrong Chen, Geoff Werner-Allen, and Matt Welsh. Simulating the power consumption of large-scale sensor network applications. The Second ACM Conference on Embedded Networked Sensor Systems (Sensys '04), November 2004.

[SKA04] Sameer Sundresh, Wooyoung Kim, and Gul Agha. Sens: a sensor, environment and network simulator. The 37th Annual Simulation Symposium (ANSS '04), 2004.

[SKG $\left.{ }^{+} 07\right]$ Jacob Sober, Alexander Kostadinov, Matthew Garber, Matthew Brennan, Mark D. Corner, and Emergy D. Berger. Eon: A language and runtime system for perpetual systems. The Fifth ACM Conference on Embedded Networked Sensor Systems (Sensys '07), 2007.

$\left[\mathrm{SMP}^{+} 04\right]$ Robert Szewczyk, Alan Mainwaring, Joseph Polastre, John Anderson, and David Culler. An analysis of a large scale habitat monitoring application. The Second ACM Conference on Embedded Networked Sensor Systems (Sensys '04), November 2004.

$\left[\mathrm{SOP}^{+} 04\right]$ Robert Szewczyk, Eric Osterweil, Joseph Polastre, Michael Hamilton, Alan Mainwaring, and Deborah Estrin. Habitat monitoring with sensor networks. Communications of the ACM, 47(6):34-40, 2004. 
[SS02] Anna Scaglione and Sergio Servetto. On the interdependence of routing and data compression in multi-hop sensor networks. The 8th Annual International Conference on Mobile Computing and Networking (MobiCom '02), 2002.

[Sun] Sunpower. Supower Solar Calculator. http://www.sunpowercorp.com/ For-Homes/How-To-Buy/Solar-Calculator.aspx.

[SVML03] Gyula Simon, Peter Volgyesi, Miklos Maroti, and Akos Ledeczi. Simulation-based optimization of communication protocols for largescale wireless sensor networks. IEEE Aerospace Conference, 2003.

[Tec] Linear Technology. LTC1751: Micropower, Regulated Charge Pump DC/DC Converter. http://www.linear.com/pc/downloadDocument.do? navId=H0,C1,C1003,C1039,C1133,P1904,D2062.

[TJC08] Jay Taneja, Jaein Jeong, and David Culler. Design, modeling, and capacity planning for micro-solar power sensor networks. The Seventh International Conference on Information Processing in Sensor Networks (IPSN/SPOTS '08), April 2008.

[TLP05] Ben L. Titzer, Daniel K. Lee, and Jens Palsberg. Avrora: scalable sensor network simulation with precise timing. The Fourth International Symposium on Information Processing in Sensor Networks (IPSN '05), 2005.

[TPS ${ }^{+}$05] Gilman Tolle, Joseph Polastre, Robert Szewczyk, David Culler, Neil Turner, Kevin Tu, Stephen Burgess, Todd Dawson, Phil Buonadonna, David Gay, and Wei Hong. A macroscope in the redwoods. The Third ACM Conference on Embedded Networked Sensor Systems (Sensys '05), November 2005.

[Unia] University of California, Berkeley. HydroWatch Project. http:// hydrowatch.cs.berkeley.edu.

[Unib] University of California, Berkeley. Smote Testbed. http://smote.cs. berkeley.edu. 
[vDL03] Tijs van Dam and Koen Langendoen. An adaptive energy-efficient mac protocol for wireless sensor networks. The First ACM Conference on Embedded Networked Sensor Systems (Sensys '03), November 2003.

[VGB07] Christopher M. Vigorito, Deepak Ganesan, and Andrew G. Barto. Adaptive control of duty cycling in energy-harvesting wireless sensor networks. IEEE Sensor and Ad Hoc Communications and Networks (SECON 'O'7), 2007.

[VXSB07] Maneesh Varshney, Defeng Xu, Mani Srivastava, and Rajive Bagrodia. squalnet: A scalable simulation and emulation environment for sensor networks. The Seventh International Conference on Information Processing in Sensor Networks (IPSN/SPOTS '07), 2007.

[WAJR ${ }^{+}$05] Geoffrey Werner-Allen, Jeff Johnson, Mario Ruiz, Jonathan Lees, and Matt Welsh. Monitoring volcanic eruptions with a wireless sensor network. The Second European Workshop on Wireless Sensor Networks (EWSN 05 ), January 2005.

[WASW05] Geoffrey Werner-Allen, Patrick Swieskowski, and Matt Welsh. Motelab: A wireless sensor network testbed. The Fourth International Symposium on Information Processing in Sensor Networks (IPSN/SPOTS ‘05), April 2005.

[Wea] Weather Underground. Solar Calculator. http://www.wunderground. com/calculators/solar.html.

[Wik] Wikipedia. Lead-acid battery. http://en.wikipedia.org/wiki/Lead_acid_ battery.

[Wun] Weather Underground. http://www.wunderground.com.

[YHE04] Wei Ye, John Heidemann, and Deborah Estrin. Medium access control with coordinated adaptive sleeping for wireless sensor networks. IEEE/ACM Transactions on Networking, 3, June 2004. 
[YSH06] Wei Ye, Fabio Silva, and John Heidemann. Ultra-low duty cycle mac with scheduled channel polling. The Fourth ACM Conference on Embedded Networked Sensor Systems (Sensys '06), November 2006.

[ZSLM04] Pei Zhang, Christopher M. Sadler, Stephen A. Lyon, and Margaret Martonosi. Hardware design experiences in zebranet. The Second ACM Conference on Embedded Networked Sensor Systems (Sensys '04), November 2004.

$\left[\mathrm{ZZH}^{+}\right.$07] Jingbin Zhang, Gang Zhou, Chengdu Huang, Sang H. Son, and John A. Stankovic. Tmmac: An energy efficient multi-channel mac protocol for ad hoc networks. IEEE International Conference on Communications (ICC '07), June 2007. 